\title{
1,5-Hydrogen Atom Transfer/Surzur-Tanner Rearrangement: A Radical Cascade Approach for the Synthesis of 1,6- Dioxaspiro[4.5]decane and 6,8-Dioxabicyclo[3.2.1]octane Scaffolds in Carbohydrate Systems
}

\author{
Elisa I. León, Ángeles Martín, Adrián S. Montes, Inés Pérez-Martín,* María del Sol Rodríguez, \\ and Ernesto Suárez*
}

Cite This: J. Org. Chem. 2021, 86, 14508-14552

Read Online

ABSTRACT: The 1,5-HAT-1,2-(ester)alkyl radical migration (Surzur-Tanner rearrangement) radical/polar sequence triggered by alkoxyl radicals has been studied on a series of $C$-glycosyl substrates with 3-C-( $\alpha, \beta$-D,L-glycopyranosyl $) 1$-propanol and $C$ - $(\alpha$ D,L-glycopyranosyl)methanol structures prepared from chiral pool D- and L-sugar. The use of acetoxy and diphenoxyphosphatoxy as leaving groups provides an efficient construction of 10-deoxy-1,6dioxaspiro[4.5] decane and 4-deoxy-6,8-dioxabicyclo[3.2.1] octane

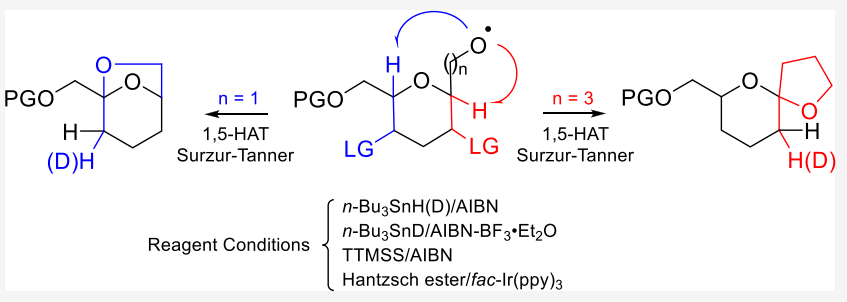
frameworks. The alkoxyl radicals were generated by the reaction of the corresponding $\mathrm{N}$-alkoxyphthalimides with group 14 hydrides $\left[n-\mathrm{Bu}_{3} \mathrm{SnH}(\mathrm{D})\right.$ and $\left.(\mathrm{TMS})_{3} \mathrm{SiH}\right]$, and in comparative terms, the reaction was also initiated by visible light photocatalysis using the Hantzsch ester $/ f a c-\operatorname{Ir}(\mathrm{ppy})_{3}$ procedure. Special attention was devoted to the influence of the relative stereochemistry of the centers involved in the radical sequence on the reaction outcome. The addition of $\mathrm{BF}_{3} \bullet \mathrm{Et}_{2} \mathrm{O}$ as a catalyst to the radical sequence resulted in a significant increase in the yields of the desired bicyclic ketals.

\section{INTRODUCTION}

The development of synthetic methodologies for bicyclic 1,6dioxaspiro[4.5] decane ${ }^{1}$ and 6,8-dioxabicyclo[3.2.1] octane $(6,8-\mathrm{DOBCO})^{2}$ scaffolds is largely stimulated by their occurrence as the structural core of highly active insect pheromones. ${ }^{3}$ They can also be widely found as subunits ${ }^{4}$ in the structure of other more complex and biologically important natural products such as steroids, ${ }^{5}$ polyether ionophores, ${ }^{6}$ and marine toxins. ${ }^{7}$ In some cases, both structural motifs are present in the same natural skeleton, as occurs in pinnatoxins and the related pteriatoxins, potent neurotoxins of a dinoflagellate origin. ${ }^{8}$ Moreover, both bicyclic ketals have attracted much interest from synthetic chemists as versatile building blocks in fine organic synthesis.

In the carbohydrate field, the preparation of spiro-heterocycles has been recently reviewed. ${ }^{10}$ Several naturally occurring 2,7 -anhydro- $\beta$-D-glyco-hept-2-ulopyranose sugars with 6,8 dioxabicyclo[3.2.1] octane structures have been described. The most representative example is sedoheptulosan (2,7anhydro- $\beta$-D-altro-hept-2-ulopyranose), although analogous compounds with D-gluco and D-manno stereochemistry are also known. ${ }^{11}$

In previous papers, we reported on a new procedure for the stereoselective construction of 1,6-dioxaspiro[4.5] decane ${ }^{12}$ and 6,8-dioxabicyclo[3.2.1] octane $^{13}$ frameworks on carbohydrate models as described in Scheme 1. Under mild oxidative conditions $\left(\mathrm{PhI}(\mathrm{OAc})_{2} / \mathrm{I}_{2}\right)$, the initially generated alkoxyl radicals (i.e., I and II, PGO) trigger a 1,5-hydrogen atom transfer $(1,5 \text {-HAT })^{14}$-radical oxidation-nucleophilic cyclization through a radical/polar crossover sequence that ultimately leads to the desired bicycles (i.e., III and IV, respectively) in a single step. In some cases, [4.5] spiroketal systems with a kinetic nonanomeric unstable configuration at the spiro center can be preferentially obtained using this methodology. Also using this simple procedure, natural $C$-glycosyl compounds of a $C$-(1,6-anhydro- $\beta$-D-glyco-1-ulopyranosyl)methanol structure (i.e., IV) with rare stereochemistries D-ido, D-gulo, and D-altro can be obtained from readily available D-gluco, D-galacto, and Dmanno chiral pool sugars, respectively. ${ }^{13}$

Otherwise, the generation of the above-mentioned alkoxyl radicals (i.e., I and II, PGO) under reductive conditions proceeds by a different mechanism that allows the preparation of interesting and highly versatile chiral synthons. Homolytic intermolecular allylmethalation of the intermediate $\mathrm{C} 1$ radical may lead to $C$-ketosides (i.e., $\mathrm{V}, \mathrm{R}=\mathrm{All}$ ). ${ }^{15}$ The regioselective

Received: June 10, 2021

Published: September 23, 2021 
Scheme 1. 1,5-HAT Reactions of 3-C-(Glycopyranosyl)propan-1-O-yl and C-(Glycopyranosyl)methan-1-O-yl Radicals ${ }^{a}$
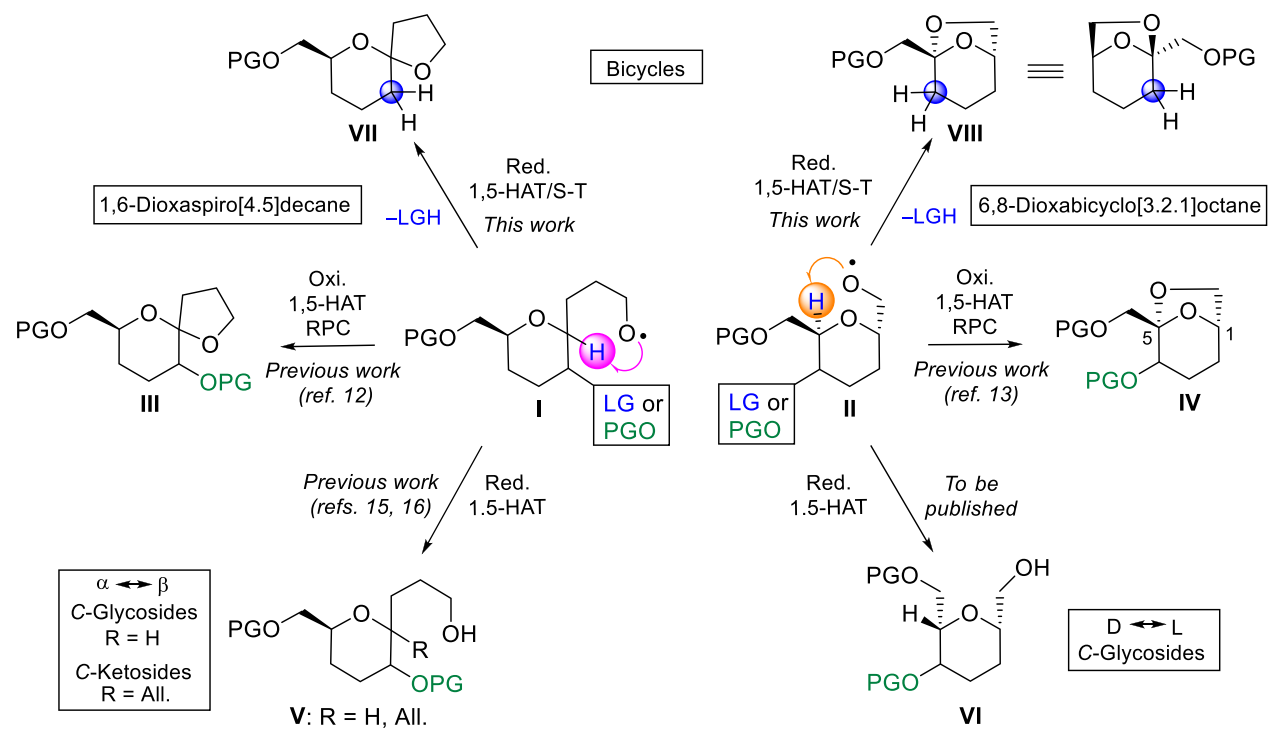

${ }^{a} \mathrm{~S}-\mathrm{T}=$ Surzur-Tanner; RPC $=$ radical polar crossover; HAT $=$ hydrogen atom transfer.

HAT by alkoxyl radicals of the H5 enables also the C5allylation and the possibility of preparing $C$-ketosides on both sides of the pyranosyl ring oxygen. ${ }^{16}$ Although, at first glance, the homolytic reduction of the $\mathrm{C} 1-$ and $\mathrm{C} 5$-radical intermediates might seem of little synthetic utility, it allows a diastereoselective interconversion between $\mathrm{D}$ - and L-C-glycosides (i.e., VI) ${ }^{17}$ and $\alpha$ - and $\beta$ - $C$-glycosides (i.e., $\mathrm{V}, \mathrm{R}=\mathrm{H}$ ), which is difficult to achieve using conventional methods. ${ }^{18}$

Since the discovery by Surzur and Teissier ${ }^{19}$ and by Tanner and $\mathrm{Law}^{20}$ in the 1960 s that $\beta$-(acyloxy)alkyl radicals undergo a 1,2-suprafacial migration of their ester group, this rearrangement has attracted considerable mechanistic and synthetic attention. ${ }^{21}$ The use of $\beta$-(phosphatoxy)alkyl radicals ${ }^{22}$ with a better leaving group (LG) and complexation with Lewis acids $^{23}$ notably increase the reaction rate and consequently its importance from a synthetic point of view.

In carbohydrate chemistry, this rearrangement has been exploited for a convenient synthesis of 2-deoxypyranoses from 1-pyranosyl radicals ${ }^{24}$ and in the stereoselective preparation of purine and pyrimidine $\alpha$-nucleosides. ${ }^{25}$ This rearrangement is also involved in the DNA and RNA strand scission from 2'and 4 '-radicals via the cleavage of the $\beta$-phosphate. ${ }^{26}$

It is evident that if we end the above-mentioned 1,5-HAT sequences with a $\beta$-(acyloxy)alkyl radical (i.e., I and II, LG), a simple and versatile preparation of 2-deoxy- $C$-glycosides on a 1-ulopyranose ring system (i.e., VII and VIII) could be achieved, where the HAT and the vicinal deoxygenation through an alkene radical-cation intermediate would occur in the same synthetic step. In fact, we would gain access to a series of ketoses with 5-deoxy-non-4-ulopyranose (i.e., VII) and 5-deoxy-hept-6-ulopyranose (also named 3-deoxy-hept-2ulopyranose) (i.e., VIII) structures by long-range selective oxidation at $\mathrm{C} 1$ and $\mathrm{C} 5$ ring carbon atoms, respectively. The synthetic interest is apparent; the 3-deoxy-hept-2-ulopyranose framework present in VIII is intimately related to the ring system of octulosonic (Kdo, Kdn) and sialic acids. ${ }^{27}$ Procedures for the preparation of analogous [4.5] spiroketals in 2-deoxy-pyranose systems using different methodologies have been described in previous publications. ${ }^{28}$ In general, deoxy-pyranoses are important targets and are frequently found in bioactive secondary metabolites of microbial origin. ${ }^{29}$

In this paper, the 1,5-HAT-Surzur-Tanner ( $\mathrm{S}-\mathrm{T}$ ) radical/ polar sequence has been studied principally on a series of $C$ glycosyl substrates with 3-C-(glyco)1-propanol (i.e., I, LG) and $C$-(glyco)methanol (i.e., II, LG) structures prepared from chiral pool $\mathrm{D}$ - and L-sugar and with $\alpha$ - and $\beta$-configurations at the anomeric center. The initial alkoxyl radicals were generated by homolytic cleavage of the corresponding $\mathrm{N}$-alkoxyphthalimide derivatives using the $n$ - $\mathrm{Bu}_{3} \mathrm{SnH} / \mathrm{AIBN}$ protocol under several different conditions. ${ }^{30}$ In most cases, the reaction finishes with an intramolecular nucleophilic 5-cyclization at the cine position of the radical-cation-LG anion pair intermediate to give the expected bicyclic acetal (i.e., VII or VIII) with a deoxygenated carbon atom at the vicinal position. ${ }^{21 \mathrm{e}}$

To unambiguously determine the fate of the radical throughout the cascade sequence, the experiments will also be performed with $n-\mathrm{Bu}_{3} \mathrm{SnD} / \mathrm{AIBN}$. This will allow us, among other things, to detect whether in the last step of the sequence the $\beta$-elimination of the ester takes place by the expected radical-polar $\beta$-(ester)alkyl shift mechanism or by a competitive pure radical $\beta$-(ester)alkyl fragmentation. ${ }^{21}$ Additionally, the influence of boron trifluoride as a catalyst on the sequence outcome will be addressed. In comparative terms, the reaction was also initiated by visible light photocatalysis using the Hantzsch ester $/ f a c-\operatorname{Ir}(\mathrm{ppy})_{3}$ procedure. ${ }^{31}$ In all cases, the reactions were allowed to proceed until the complete consumption of the radical precursors as indicated by TLC.

Due to the stereochemical requirements for the HAT reaction transition state, ${ }^{32}$ much attention has been paid to the not always apparent conformation of the sugar rings in these $C$ glycosyl compounds. For this purpose, the ${ }^{3} \mathrm{JCCH}_{\mathrm{HCH}}$ vicinal ring coupling constants were extracted from the experimental 1D ${ }^{1} \mathrm{H}$ NMR spectra by iterative simulation ${ }^{33}$ and compared with the values calculated on minimized structures in ${ }^{4} C_{1}$ and ${ }^{1} C_{4}$ conformations [see Tables $S 1$ and $S 2$ in the Supporting Information (SI) . $^{34}$

Previous examples of the HAT-S-T rearrangement sequence have been reported in the formation of tetrahydrofurans from $\beta$-(phosphatoxy)alkyl radicals ${ }^{35}$ and as a key 
Table 1. 1,5-HAT-S-T Sequence in 3-C-( $\alpha, \beta$-D-Glcp $)$ 1-propoxyphthalimides $1-4^{a}$

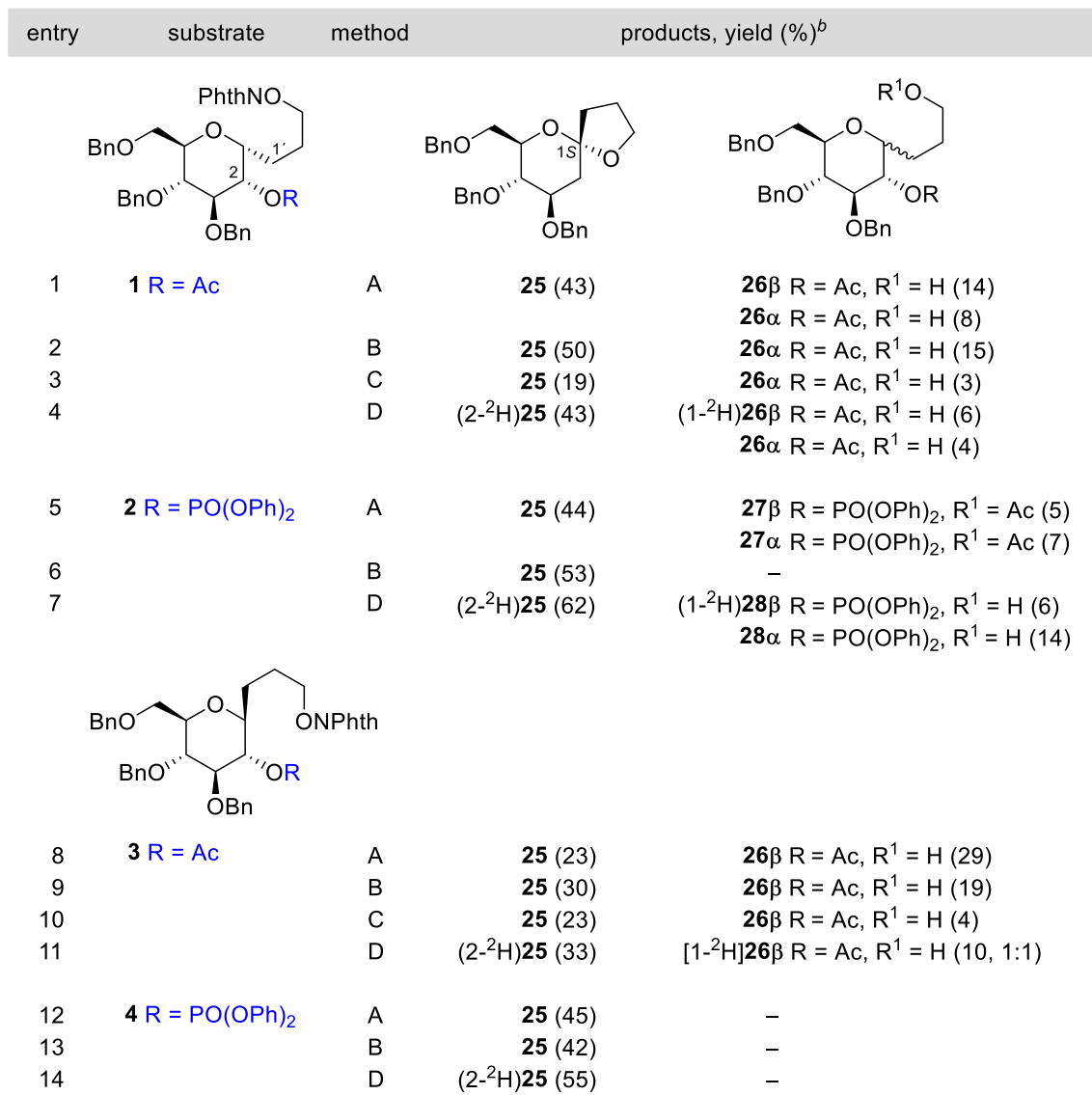

${ }^{a}$ Reagents and conditions: method $\mathrm{A}: n$ - $\mathrm{Bu}_{3} \mathrm{SnH}$ (1 equiv), $\mathrm{AIBN}\left(0.1\right.$ equiv), $\mathrm{PhCH}_{3}(0.013 \mathrm{M})$, reflux; method B: $n$ - $\mathrm{Bu} \mathrm{S}_{3} \mathrm{SnH}(1$ equiv/h), $\mathrm{AIBN}$ (0.1 equiv), $\mathrm{PhCH}_{3}(0.013 \mathrm{M})$, reflux; method C: TTMSS ( 1 equiv), AIBN (0.1 equiv), $\mathrm{PhCH}_{3}(0.013 \mathrm{M})$, reflux; and method $\mathrm{D}: n$-Bu $3 \mathrm{SnD}(1$ equiv), AIBN (0.1 equiv), $\mathrm{PhCH}_{3}(0.013 \mathrm{M})$, reflux. ${ }^{b}$ Values in parentheses are isolate yields; deuterium incorporation $\left({ }^{2} \mathrm{H} /{ }^{1} \mathrm{H}\right)$ is included in partially labeled compounds.

step in the synthesis of cephalosporolide E. ${ }^{36}$ We have also described another example of this sequence during the reaction of methyl 2,3,4-tri-O-acetyl-6-deoxy- $\alpha$-D-Talp-(1 $\rightarrow 4)$-2,3-di$O$-methyl- $\alpha$-D-Glcp-6-O-yl disaccharide radical. ${ }^{37}$ The initial 1,8-HAT $\left(6^{\mathrm{I}} \mathrm{O}^{\bullet} \rightarrow 5^{\mathrm{II}} \mathrm{C}^{\bullet}\right)$ between the two sugars generated a $4^{\mathrm{II}} \beta$-(acetoxy) $5^{\mathrm{II}}$-alkyl radical that led finally to the formation of a rare eight-membered $4^{\mathrm{II}}$-deoxy-1,3,5-trioxocane ring system. The use of $n-\mathrm{Bu}_{3} \mathrm{SnD}$ confirms that, at least in part, the last step of the sequence involves an $\mathrm{S}-\mathrm{T}$ rearrangement through a cine 8-exo-substitution mechanism. On the other hand, unsuccessful attempts to trap the intermediate alkene radical cation intramolecularly by carboxylate anions have been reported. $^{38}$

To obtain a complete picture of the stereochemical influence of the substituents in the course of the radical sequence, we have prepared 3-C-(glycopyranosyl)1-propoxyphthalimides with $\alpha, \beta$-D-gluco (1-4), $\alpha, \beta$-D-manno (5-8), $\alpha$-L-fuco (9 and 10), and $\alpha, \beta$-D-arabino (11 and 12) configurations (Scheme 4). ${ }^{39} \mathrm{~A}$ few examples of 3-C-( $\alpha$-D-ribofuranosyl)1-propoxyphthalimides $(13-15)$ have been included in this work to study the influence of the greater conformational flexibility of the five-membered ring (Scheme 5). Furthermore, $C$-(glycopyranosyl) $N$-methoxyphthalimides with $\alpha$-D-gluco (16-19), $\alpha$-Dgalacto (20 and 21), $\alpha$-L-rhamno (22), and $\alpha$-L-fuco (23 and 24) configurations (Scheme 6) have also been synthesized (Schemes 4-6 are presented later in this work). In most of these models, it has been possible to investigate the differences between the migratory capabilities of acetoxy and diphenoxyphosphatoxy groups and how they affect the final result of the sequence. $^{40}$

\section{RESULTS AND DISCUSSION}

Synthesis of 10-Deoxy-1,6-dioxaspiro[4.5]decane and 9-Deoxy-1,6-dioxaspiro[4.4]nonane Scaffolds. The results of the study with $3-C-(\alpha, \beta-\mathrm{D}-\mathrm{Gl} p p)$ propan-1-O-yl radicals using 2-acetyl and 2-diphenoxyphosphoryl as LGs are summarized in Table 1 . Initial experiments with 3-C-(2-Oacetyl- $\alpha$-D-Glcp)1-propoxyphthalimide precursor 1 employing conditions optimized for the generation of alkoxyl radicals from $N$-alkoxyphthalimides using $n-\mathrm{Bu}_{3} \mathrm{SnH}$ ( 1 equiv) in a dilute solution $(0.013 \mathrm{M})$ of toluene at reflux temperature and AIBN as the initiator gave a mixture of three compounds: 25 , $26 \beta$, and $26 \alpha$ (Table 1 , entry 1 ). The major product 25 was identified as the expected 1,5-HAT-S-T spiroketal. The minor components of the reaction are $26 \boldsymbol{\beta}$, which is formed by hydrogen abstraction at $\mathrm{C}-1$ and subsequent radical axial quenching with inversion of configuration, and isomeric alcohol $\mathbf{2 6} \alpha$, which could arise either by abstraction and retention of the configuration or simply by premature reduction of the alkoxyl radical. In the latter case, a combination of both mechanisms could be operative and cannot be ruled out at the present stage of the work. The yield 
Table 2. 1,5-HAT-S-T Sequence in 3-C-( $\alpha, \beta$-D-Man $p) 1$-propoxyphthalimides 5-8 ${ }^{a}$

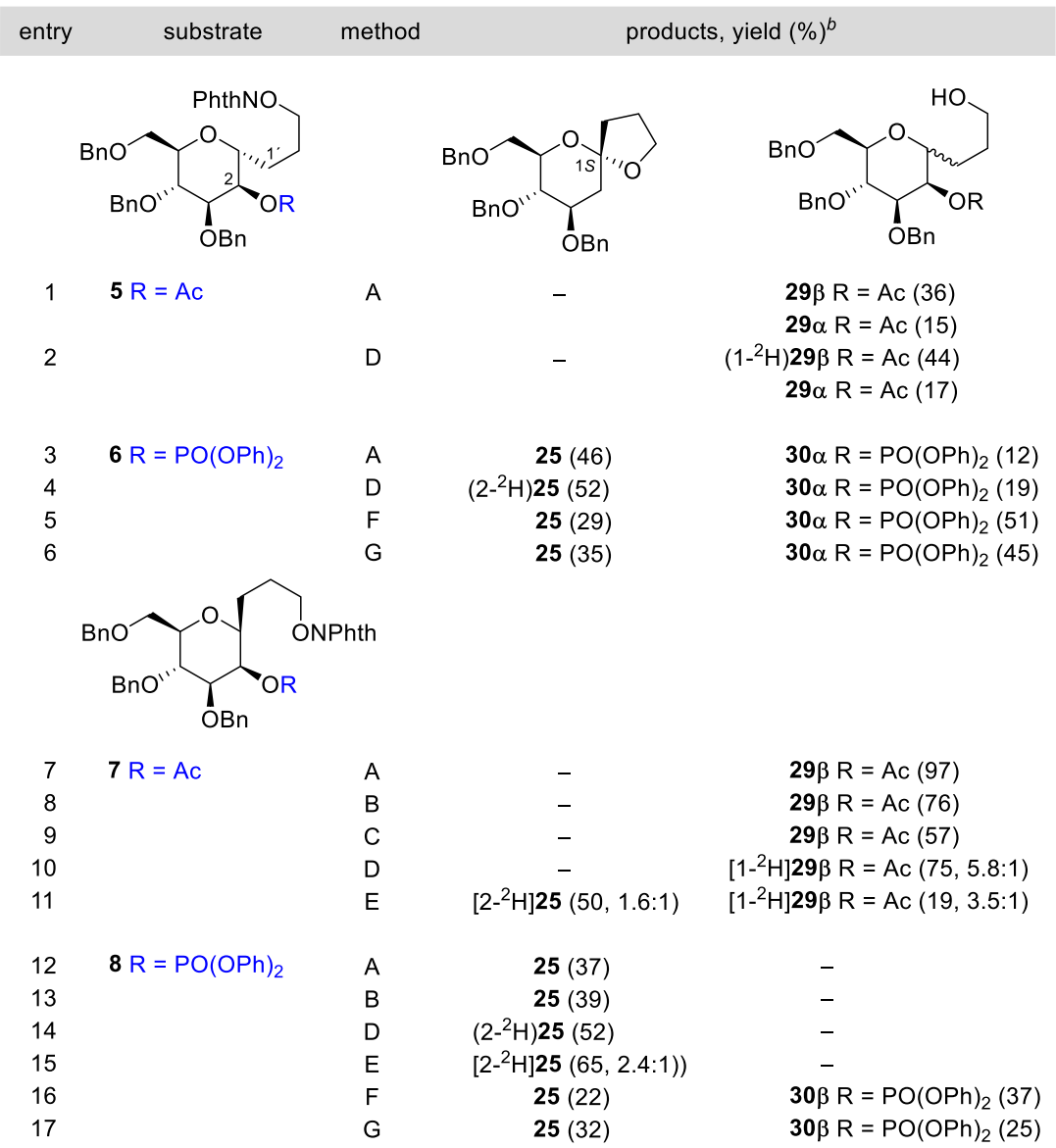

${ }^{a}$ Reagents and conditions: method A: $n$ - $\mathrm{Bu}_{3} \mathrm{SnH}$ (1 equiv), $\mathrm{AIBN}$ (0.1 equiv), $\mathrm{PhCH}_{3}(0.013 \mathrm{M})$, reflux; method B: $n$ - $\mathrm{Bu} 3 \mathrm{SnH}(1$ equiv/h), AIBN (0.1 equiv), $\mathrm{PhCH}_{3}(0.013 \mathrm{M})$, reflux; method C: TTMSS ( 1 equiv), $\mathrm{AIBN}$ (0.1 equiv), $\mathrm{PhCH}_{3}(0.013 \mathrm{M})$, reflux; method $\mathrm{D}: n$ - $\mathrm{Bu} \mathrm{u}_{3} \mathrm{SnD}(1$ equiv), AIBN (0.1 equiv), $\mathrm{PhCH}_{3}(0.013 \mathrm{M})$, reflux; method $\mathrm{E}: n-\mathrm{Bu}_{3} \mathrm{SnD}$ (1 equiv), $\mathrm{BF}_{3} \bullet \mathrm{Et}_{2} \mathrm{O}$ (0.2 equiv), $\mathrm{AIBN}\left(0.1\right.$ equiv), $\mathrm{PhCH}_{3}(0.013 \mathrm{M})$, reflux; method F: Hantzsch ester (1.1 equiv), fac-Ir(ppy) ${ }_{3}(0.01$ equiv), THF $(0.007 \mathrm{M})$, rt, blue LED; and method G: Hantzsch ester $(0.37$ equiv/h), fac$\operatorname{Ir}(\text { ppy })_{3}\left(0.01\right.$ equiv), THF $(0.007 \mathrm{M})$, rt, blue LED. ${ }^{b}$ Values in parentheses are isolate yields; deuterium incorporation $\left({ }^{2} \mathrm{H} /{ }^{1} \mathrm{H}\right)$ is included in partially labeled compounds.

of the cyclized product $\mathbf{2 5}$ was increased to $50 \%$ by lowering the tin hydride concentration with a syringe pump; under these conditions, the $C$-glucosyl compound $\mathbf{2 6} \boldsymbol{\beta}$ could not be detected (Table 1, entry 2). Attempts to improve the yield using (TMS) $)_{3} \mathrm{SiH}$ (TTMSS), a group 14 hydride with a smaller hydrogen donor capacity, ${ }^{41}$ to avoid the reduction of radical intermediates met with no success.

Considerable analytical and spectroscopic data were diagnostic of the spiroketal structure of 25, unambiguously expressing the presence of a quaternary ketal carbon and the additional methylene group as well as the disappearance of the acetyl group. In a minimized structure, the pyranose ring adopts preferentially a ${ }^{4} C_{1}$ chair conformation, from which the calculated coupling constants were in agreement with the experimental values (see Table S3 in the SI). The configuration of the spiro center was tentatively assigned as $1 S$, with the anomeric oxygen in an axial position, ${ }^{42}$ according to the downfield displacement observed for the $\mathrm{H} 3$ and $\mathrm{H} 5$ protons that in this conformation present 1,3-diaxial interactions with the $\mathrm{C} 1-\mathrm{O}$ bond. In addition, the absence of NOE interactions between $\mathrm{H} 1$ ' and $\mathrm{H} 5$ and/or $\mathrm{H} 3$ that were present in previously reported analogous [4.5] spiroketals in 2-deoxy-pyranose systems with $1 R$ stereochemistry may also support this assignment. ${ }^{28 a}$
The use of $n-\mathrm{Bu}_{3} \mathrm{SnD}$ showed the quantitative monodeuteration for $\left(2-{ }^{2} \mathrm{H}\right) 25$ and for the inverted product $\left(1-{ }^{2} \mathrm{H}\right) \mathbf{2 6} \boldsymbol{\beta}$ (Table 1, entry 4). Moreover, the early reduction of the alkoxyl radical was solely responsible for the unlabeled $26 \alpha$ and no retention at $\mathrm{C} 1$ could be detected in this experiment. The diastereoselective ratio $\left({ }^{2} \mathrm{H}_{\mathrm{ax}} /{ }^{2} \mathrm{H}_{\mathrm{eq}}, 7: 1\right)$ of deuterium at $\left(2-{ }^{2} \mathrm{H}\right) 25$ is mostly attributable to a $\beta$-facial preference for the radical quenching due to steric hindrance.

For comparative purposes, we have prepared $\mathrm{C}$-(2-Odiphenoxyphosphoryl- $\alpha$-D-Glc $p$ ) 1-propoxyphthalimide 2 . The rate constant of the $\beta$-(phosphatoxy)alkyl radical migration should be several orders of magnitude greater than that recorded for comparable acyloxy shifts. ${ }^{21 d}$ However, the reaction of 2 with $n-\mathrm{Bu}_{3} \mathrm{SnH} / \mathrm{AIBN}$ afforded the 1,5-HAT$\mathrm{S}-\mathrm{T}$ substitution product $\mathbf{2 5}$ in a similar yield (44\%) together with a mixture of alcohols that, after acetylation, were identified as $\mathbf{2 7 \boldsymbol { \beta }}$ and $\mathbf{2 7 \boldsymbol { \alpha }}$ (Table 1, entry 5). The slow addition of $n$ - $\mathrm{Bu}_{3} \mathrm{SnH}$ generates the bicycle 25 as a sole product in $53 \%$ yield (Table 1, entry 6 ). The reaction with $n$ $\mathrm{Bu}_{3} \mathrm{SnD}$ showed the complete monodeuteration for the spirocompound $\left(2 \beta-{ }^{2} \mathrm{H}\right) 25$ achieved in a significantly better yield $(62 \%)$ (Table 1 , entry 7$)$. The inseparable mixture of the complete labeling inverted product $\left(1-{ }^{2} \mathrm{H}\right) \mathbf{2 8} \boldsymbol{\beta}$ and the reduced unlabeled alcohol $\mathbf{2 8 \boldsymbol { \alpha }}$ was also obtained (20\%, 1:2.1). 
Table 3. 1,5-HAT-S-T Sequence in 3-C-( $\alpha$-L-Fucp)- and 3-C-(D-Arap)1-propoxyphthalimides 9-12 ${ }^{a}$

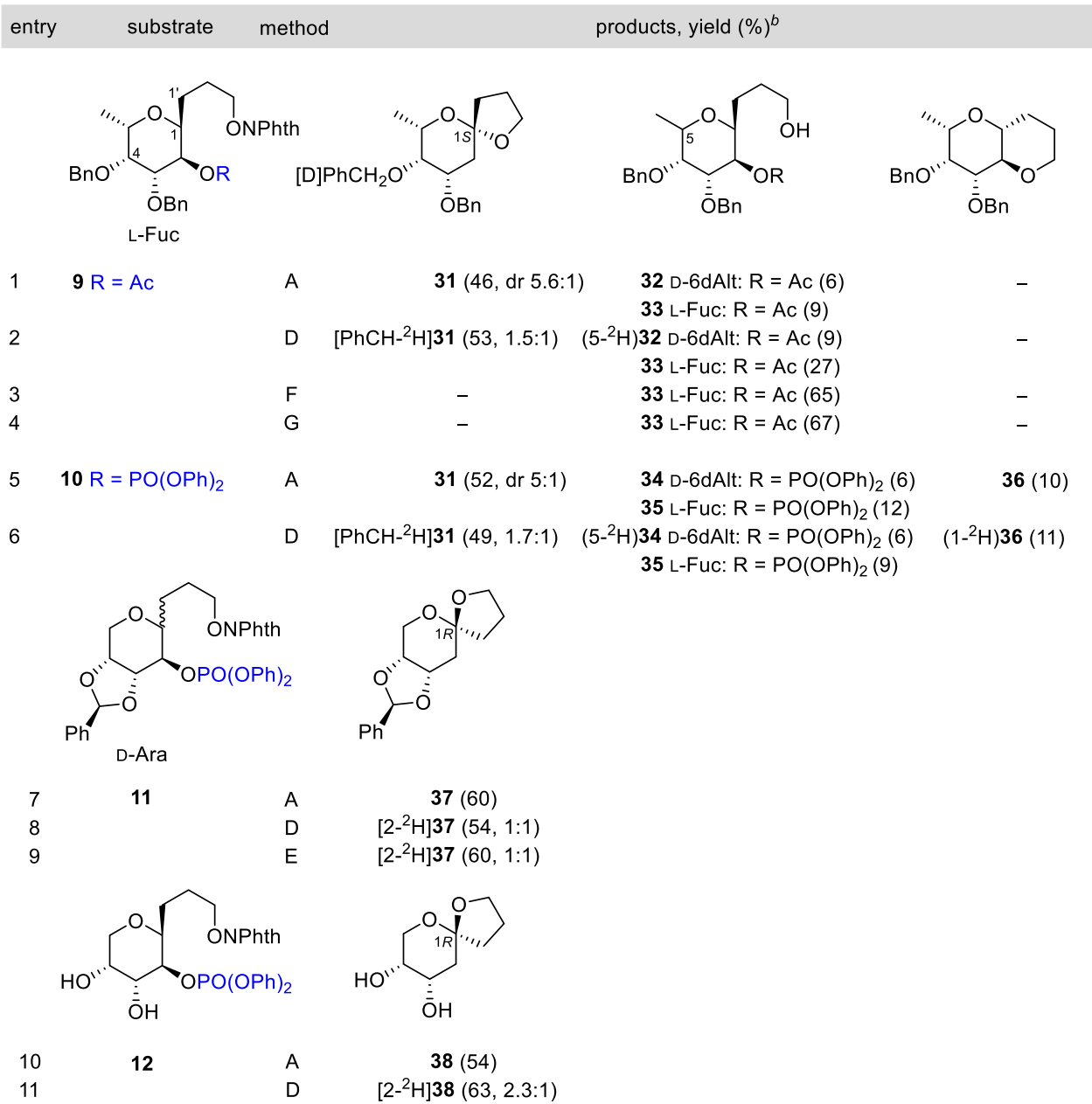

${ }^{a}$ Reagents and conditions: method A: $n-\mathrm{Bu}_{3} \mathrm{SnH}$ (1 equiv), $\mathrm{AIBN}$ (0.1 equiv), $\mathrm{PhCH}_{3}(0.013 \mathrm{M})$, reflux; method $\mathrm{D}: n$ - $\mathrm{Bu} 3 \mathrm{SnD}(1$ equiv), $\mathrm{AIBN}$ (0.1 equiv), $\mathrm{PhCH}_{3}(0.013 \mathrm{M})$, reflux; method $\mathrm{E}: n-\mathrm{Bu}_{3} \mathrm{SnD}$ (1 equiv), $\mathrm{BF}_{3} \bullet \mathrm{Et}_{2} \mathrm{O}$ (0.2 equiv), $\mathrm{AIBN}\left(0.1\right.$ equiv), $\mathrm{PhCH}_{3}(0.013 \mathrm{M})$, reflux; method F: Hantzsch ester (1.1 equiv), fac-Ir(ppy) 3 (0.01 equiv), THF $(0.007 \mathrm{M})$, rt, blue LED; and method G: Hantzsch ester (0.37 equiv/h), fac$\operatorname{Ir}(\text { ppy })_{3}\left(0.01\right.$ equiv), THF $(0.007 \mathrm{M})$, rt, blue LED. ${ }^{b}$ Values in parentheses are isolate yields; deuterium incorporation $\left({ }^{2} \mathrm{H} /{ }^{1} \mathrm{H}\right)$ is included in partially labeled compounds. $\mathrm{dr}=$ diastereomeric ratio; only the major isomer is shown.

This protocol was also applied to $C$-(2-O-acetyl- $\beta$-D-Glc $p) 1$ propoxyphthalimide 3 , where the pyranose ring adopts preferentially a ${ }^{4} C_{1}$ conformation with the three-carbon tether in an equatorial position and, consequently, the abstractable hydrogen atom at $\mathrm{C} 1$ is axially oriented (see Table $\mathrm{S} 1$ in the SI). Unfortunately, treatment of 3 under the same conditions mentioned above did not increase the yield of 25 (Table 1, compare entries 8-10 with 1-3). Now, the principal

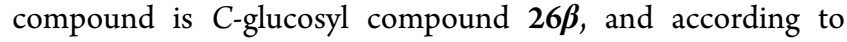
the reaction with $n-\mathrm{Bu}_{3} \mathrm{SnD}$, approximately $50 \%$ is formed by prereduction of the alkoxyl radical $\left\{\left[1-{ }^{2} \mathrm{H}\right] 26 \boldsymbol{\beta}\left({ }^{2} \mathrm{H} /{ }^{1} \mathrm{H}, 1: 1\right)\right\}$ (Table 1, entry 11). These results were rather unexpected since electrophilic radicals abstract axial hydrogen atoms much faster than the equatorial ones and the initial 1,5-HAT should be favored relative to our previous models 1 and $2 .{ }^{43}$ The different reactivity between $\mathbf{1}$ and 3 can be explained by a possible memory of chirality effect of the $\mathrm{C} 1$ radical after the 1,5-HAT reaction (Table 1 , compare entries 1 and 8). ${ }^{44}$

Moreover, the migration of a phosphatoxy group contributed to a marked improvement in the yield of $\mathbf{2 5}$ as shown in model 4 (Table 1, entries 12-14). Under these conditions, no appreciable amounts of $C$-glucosyl compounds resulting from the reduction of intermediate radicals were detected. As observed in previous models, the yield of the spiroketal increased significantly when changing from a hydride donor $\mathbf{2 5}$ to a less reactive deuteride donor $\left(2-{ }^{2} \mathrm{H}\right) \mathbf{2 5}$ (Table 1 , compare entries 12 and 14). These results probably reflect a kinetic isotope effect (KIE) in which a slower process permits the radical to reach the end of the sequence, avoiding prereduction and the formation of uncyclized products.

The reaction of an analogous series of 3-C-( $\alpha, \beta-\mathrm{D}-\mathrm{Man} p)$ propan-1-O-yl radicals using also 5-acetyl and 5-diphenoxyphosphatoxy as LGs is summarized in Table 2. The 3-C-(2-Oacetyl- $\alpha$-D-Man $p$ ) 1-propoxyphthalimide 5 under the classical tin hydride conditions afforded exclusively uncyclized compounds $29 \beta$ and $29 \alpha$, as confirmed by deuterium labeling experiments (Table 2, entries 1 and 2). The expected spiroketal 25 could not be detected.

The isomeric $\beta$-phthalimide 7 behaved similarly, with only $29 \boldsymbol{\beta}$ (97\%) being obtained (Table 2, entry 7 ). Under tin deuteride conditions, the reaction gave $\left[1{ }^{2} \mathrm{H}\right] 29 \boldsymbol{\beta}\left({ }^{2} \mathrm{H} /{ }^{1} \mathrm{H}\right.$, 5.8:1); the labeled compound originated by deuterium incorporation with retention after the 1,5-HAT and the unlabeled compound by direct reduction of the alkoxyl radical 
Scheme 2. Propagation Cycle of 3-C-( $\alpha$-L-Fucp $)$ propan-1-O-yl Radicals ${ }^{a}$

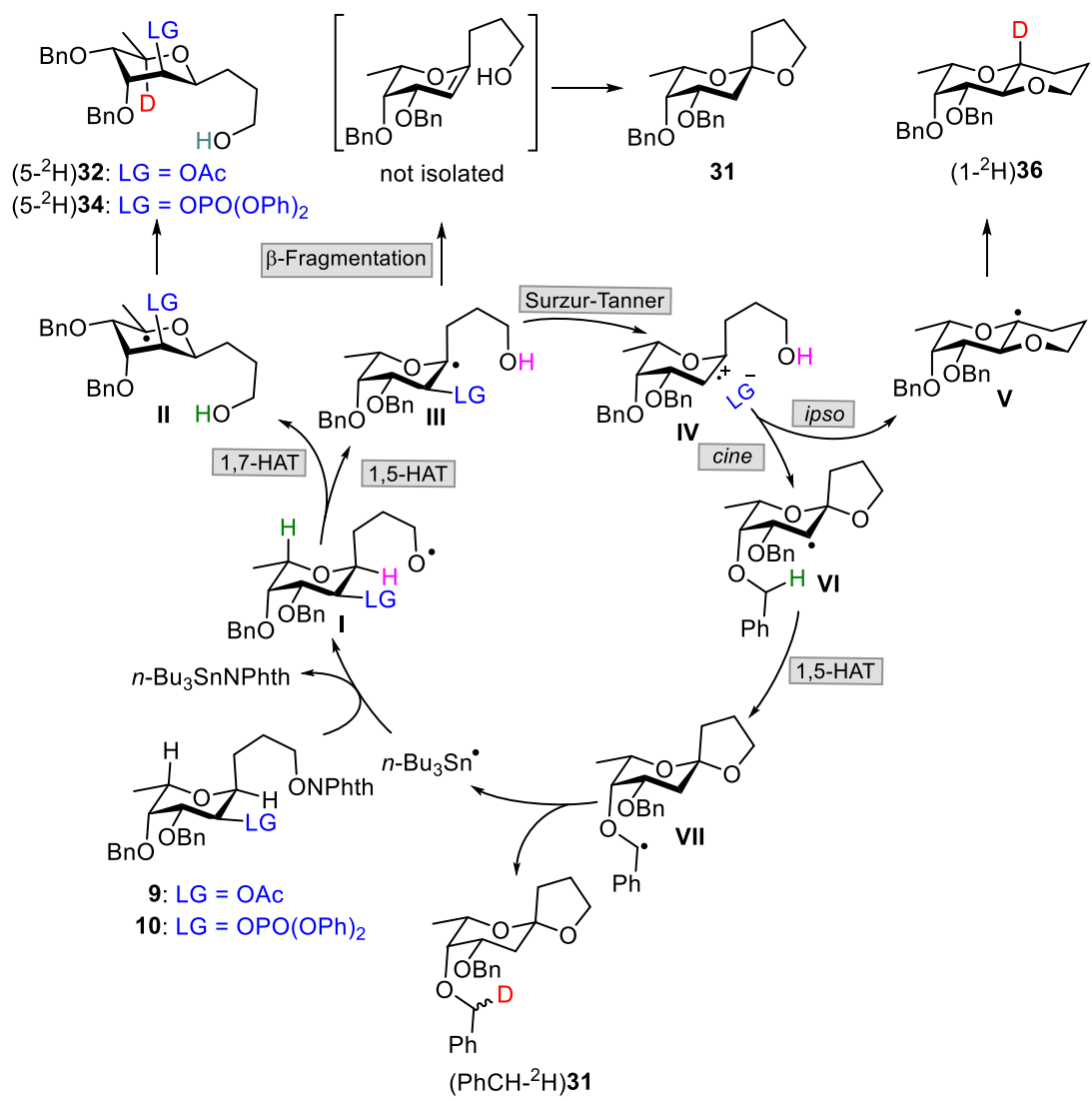

${ }^{a}$ The prereduction of the alkoxyl radical and the initiation and termination steps are omitted for clarity.

(Table 2, entry 10). Knowing that aluminum and scandium Lewis acid can efficiently enhance the rate of the $\mathrm{S}-\mathrm{T}$ rearrangement, ${ }^{23}$ we envisioned an experiment that under the same conditions $\left[n\right.$ - $\mathrm{Bu}_{3} \mathrm{SnD}$ ( 1 equiv), $\mathrm{AIBN}$ ( 0.1 equiv), and $\mathrm{PhCH}_{3}(0.013 \mathrm{M})$ at $\left.110{ }^{\circ} \mathrm{C}\right]$ contains a catalytic amount of $\mathrm{BF}_{3} \bullet \mathrm{Et}_{2} \mathrm{O}$ ( 0.2 equiv). To our delight, the radical sequence now proceeded nicely to the end, furnishing the desired product $\left[2-{ }^{2} \mathrm{H}\right] 25$ in $50 \%$ yield along with a small amount of $\left[1-{ }^{2} \mathrm{H}\right] 29 \beta(19 \%)$ (Table 2, entry 11). The ${ }^{1} \mathrm{H}$ NMR analysis of the deuterium incorporation at $\left[2-{ }^{2} \mathrm{H}\right] 25\left({ }^{2} \mathrm{H} /{ }^{1} \mathrm{H}, 1.6: 1\right)$ revealed that, in this case, the $\beta$-elimination of the ester could take place not only by the radical-polar $\beta$-(acyloxy)alkyl shift mechanism but also by a competitive pure radical $\beta$ (acyloxy)alkyl fragmentation. ${ }^{45}$ Alternatively, acid-catalyzed opening and recombination of the spiroketal ring through an unobserved glucal intermediate [3-C-(1,5-anhydro-2-deoxy-Darabino-hex-1-enopyranosyl)propan-1-ol] may also account for the loss of deuterium detected.

The 3-C-(2-O-diphenoxyphosphoryl- $\alpha, \beta$-D-Man $p) 1$-propoxyphthalimide models 6 and 8 with a faster migratory group gave, under standard tin hydride conditions, spiroketal 25 in moderate yields (Table 2, entries 3 and 12, respectively). The yields of $\left(2-{ }^{2} \mathrm{H}\right) 25$ improved using tin deuteride (52\% in both cases) and increased notably upon Lewis acid catalysis giving $\left[2-{ }^{2} \mathrm{H}\right] 25\left(65 \%,{ }^{2} \mathrm{H} /{ }^{1} \mathrm{H}, 2.4: 1\right)$ by partial labeling (Table 2, entries 4,14 , and 15). The difference in reactivity between 2 acetyl-D-gluco ( 1 and 3) and -D-manno derivatives (5 and 7) has been attributed to the observed lower migration efficiency of axial $\beta$-(acetoxy)alkyl radicals. ${ }^{24 \mathrm{~b}, 46}$
The formation of alkoxyl radicals from $N$-alkoxyphthalimide under photoredox catalysis conditions and their use in selective $\mathrm{C}\left(\mathrm{sp}^{3}\right)-\mathrm{H}$ functionalization through 1,5-HAT have been recently reported., ${ }^{31 \mathrm{~b} 47}$ As far as we know, this type of methodology has never been employed to initiate the 1,5HAT $-\mathrm{S}-\mathrm{T}$ sequence described in this paper. The blue LED irradiation of phthalimides 6 and 8 in the presence of a catalytic amount of $f a c-\left[\operatorname{Ir}(p p y)_{3}\right]$ and Hantzsch ester as the reductant afforded spirocycle $\mathbf{2 5}$ in a disappointingly low yield, with the prereduced alcohols $\mathbf{3 0 \alpha}$ and $\mathbf{3 0 \boldsymbol { \beta }}$, respectively, being the major products (Table 2, entries 5 and 16). Although the yield of $\mathbf{2 5}$ increased slightly by slowly adding the Hantzsch ester to the reaction mixture using a syringe pump, it is still clearly inferior to the results obtained with the tin hydrides (Table 2, entries 6 and 17).

Next, this study was extended to the acetyl and diphenoxyphosphoryl 3-C-( $\alpha$-L-Fucp $) 1$-propoxyphthalimides 9 and 10, respectively, as described in Table 3. When the 2acetyl precursor 9 was treated with $n-\mathrm{Bu}_{3} \mathrm{SnH} / \mathrm{AIBN}$, the main product was the expected spirocycle $31(46 \%)$ together with an inseparable mixture $(2: 3,15 \%)$ of two minor alcohols: 6deoxy-D-altro 32 and L-fuco 33 (Table 3, entry 1). Although both diastereomers can be tentatively identified by NMR analysis of the mixture, additional support for these structures came from the complete separation and characterization of diphenoxyphosphoryl analogues $\mathbf{3 4}$ and $\mathbf{3 5}$ achieved during the reaction of phthalimide $\mathbf{1 0}$ (Table 3 , entry 5 ).

The spiroketal 31 with a nonanomeric configuration at the spirocenter was isolated and contaminated with a small amount of the thermodynamic isomer $(1 S / 1 R, 85: 15)$. In 
both isomers, the pyranosyl ring preferentially adopts a ${ }^{1} C_{4}$ chair conformation (see Table S3 in the SI). The ${ }^{1} \mathrm{H}$ NMR spectrum of 31 shows a ${ }^{4} J_{\mathrm{w}}$ coupling $(1.3 \mathrm{~Hz} \text {, calcd } 1.3 \mathrm{~Hz})^{48}$ between $\mathrm{H} 2 \alpha$ and $\mathrm{H} 4$ equatorial hydrogens, which also supports the mentioned conformation (see Table S4 in the SI). The $1 S$ configuration of the major isomer (shown in Table 3) was established based on the NOE interactions observed between $\mathrm{H} 5$ and H1' and H2'. The downfield displacement observed for $\mathrm{H} 3(0.1 \mathrm{ppm})$ and $\mathrm{H} 5(0.3 \mathrm{ppm})$ in the ${ }^{1} \mathrm{H}$ NMR spectrum of the minor $1 R$-isomer lends further evidence to the proposed spiroketal stereochemistry.

Several interesting conclusions can be drawn from the results obtained during the deuteration experiment (Table 3, entry 2). Compound $\left[\mathrm{PhCH}-{ }^{2} \mathrm{H}\right] 31(\mathrm{D} / \mathrm{H}=1.5: 1 ; \mathrm{dr}=4: 1)$ showed no significant incorporation of deuterium at the $\mathrm{C} 2$ site, but instead a partial deuteration of a benzylic proton at $\mathrm{C} 4$ could be detected. The quantitative incorporation of deuterium confirmed that the 6-deoxy-D-altrose derivative $\left(5-{ }^{2} \mathrm{H}\right) 32$ was formed by the reductive inversion of configuration of a 5radical intermediate. Finally, the undeuterated alcohol 33 was formed exclusively by prereduction of the initial alkoxyl radical. All our attempts to obtain spirocyclic 31 by applying the photoredox conditions mentioned above were unsuccessful, with only prereduced alcohol 33 being isolated instead (Table 3 , entries 3 and 4).

The reaction of 3-C-(2-diphenoxyphosphoryl- $\alpha$-L-Fucp $) 1$ propoxyphthalimide 10 provided the desired bicycle 31 in better yield (52\%) together with small amounts of the 6-deoxyD-altro 34 and L-fuco 35 derivatives that could now be conveniently characterized. The 6 -deoxy- $\beta$-D-altropyranosyl ring in 34 exists preferentially in a ${ }^{4} C_{1}$ conformation with the two alkyl residues in an equatorial position, with the value of the ${ }^{3} J_{4,5}=9.8 \mathrm{~Hz}($ calcd $=8.4 \mathrm{~Hz})$ confirming the inversion of configuration at C5. Furthermore, a new compound 36 with a 2,7-dioxabicyclo[4.4.0] decane skeleton, hitherto undetected in the reaction of previous models, was also isolated in $10 \%$ yield (Table 3, entry 5). The structure and stereochemistry of $\mathbf{3 6}$, a constitutional isomer of $\mathbf{3 1}$, were readily established by analytic and spectroscopic means. Most significantly, the ${ }^{3} \mathrm{~J}$ fucopyranosyl ring coupling constants extracted by DAISY from the experimental spectrum and NOE interactions of $\mathrm{H} 1$ with $\mathrm{H} 3$ and $\mathrm{H} 5$, and $\mathrm{H} 2$ with $\mathrm{H}_{1}$ ' confirmed the trans-fused bis(pyran) proposed framework. ${ }^{49}$

A possible propagation cycle for the acetyl and diphenoxyphosphoryl 3-C-( $\alpha$-L-Fucp)propan-1-O-yl radical chain reactions, employing tin deuteride as reductant, is outlined in Scheme 2. The electrophilic alkoxyl radical (I) triggers two competitive hydrogen atom transfer reactions by abstraction of stereochemically accessible $1 \mathrm{H}$ (1,5-HAT) and 5H (1,7-HAT). Many examples of 1,5-hydrogen translocations are known; however, their 1,7-HAT counterparts are comparatively very scarce. $^{14,50}$ The 5-alkyl radical (II) leads finally to 3-C-(6deoxy- $\beta$-D-altropyranosyl $) 1$-propanol derivatives $\left(5-{ }^{2} \mathrm{H}\right) 32$ and $\left(5-{ }^{2} \mathrm{H}\right) 34$ with inversion of configuration. The 1 -alkyl radical (III) continues the cascade sequence by the two mechanisms mentioned before: pure radical $\beta$-fragmentation to give unlabeled 31, through a non-isolated olefin, and S-T rearrangement through the radical-polar intermediate (IV). ${ }^{45}$ When phosphate is used as LG, the reaction is directed toward two competitive pathways: cine and ipso intramolecular cyclization by the primary alcohol that now acts as a nucleophile. The minor ipso cyclization affords the bis(pyran) $\left(1-{ }^{2} \mathrm{H}\right) 36$ through radical $(\mathrm{V})$. Furthermore, the cine substitution provides 2-radical (VI) that regioselectively abstracts a benzylic hydrogen from the 4-OBn protecting group by means of another 1,5-HAT process. Consequently, no deuterium incorporation (within the limits of NMR detection) was observed at $\mathrm{C} 2$. Reductive quenching of radical VII leads to the quantitatively deuterated $\left(\mathrm{PhCH}_{-}{ }^{2} \mathrm{H}\right) 3 \mathbf{1}$, isolated together with the unlabeled 31 formed by the $\beta$ fragmentation mechanism.

The ${ }^{1} \mathrm{H}$ and ${ }^{13} \mathrm{C}\left\{{ }^{1} \mathrm{H}\right\}$ NMR spectra of isolated $\left[\mathrm{PhCH}_{-}{ }^{2} \mathrm{H}\right]$ $31(\mathrm{D} / \mathrm{H}, 1.5: 1 ; \mathrm{dr}=4: 1)$ deserve some comments. The deuteration at $4-\mathrm{OBn}$ is highly stereoselective, providing evidence for a steric hindered deuteride addition. Both diastereoisomeric deuterated benzyl ethers seem to adopt two different conformations that affect the chemical shift displacement of surrounding protons and carbons. Thus, for example, the 6-methyl group signal appears as three doublets of approximately the expected intensities (1.5:0.3:1.2): 1.130 ppm ( $J=6.3 \mathrm{~Hz}, \mathrm{D}$ major $), 1.133 \mathrm{ppm}(J=6.6 \mathrm{~Hz}, \mathrm{D}$ minor $)$, and $1.135 \mathrm{ppm}(J=6.3 \mathrm{~Hz}$, unlabeled) (see Table S5 and Figure S1 in the SI for details).

This anomalous behavior that may be attributable to the aromatic ring current effect can also be observed in its ${ }^{13} \mathrm{C}\left\{{ }^{1} \mathrm{H}\right\}$ NMR spectrum; the $\mathrm{C} 4$ atom appears as three signals: 74.97 ppm (D major), 75.02 ppm (D minor), and 75.09 ppm (unlabeled), also with intensities in accordance with the relative proportions (see Table S6 and Figure S3 in the SI for details).

This effect has not been detected in analogous monodeuterated 4-OBn compounds with a D-glucose configuration described in the literature. ${ }^{51}$ In an attempt to rationalize this unexpected NMR result, we prepared methyl 4-O-benzyl-6-Otert-butyldiphenylsilyl-2,3-di-O-methyl- $\alpha$-D- $\left[4-\mathrm{O}-\mathrm{PhCH}_{-}{ }^{2} \mathrm{H}\right]$ galactopyranoside $\left(\left[\mathrm{PhCH}_{-}{ }^{2} \mathrm{H}\right] 97\right)$ by the reaction of alcohol 96 with benzyl $\alpha$-[ $\left.{ }^{2} \mathrm{H}\right]-4$-methylbenzenesulfonate (Scheme 6). ${ }^{52}$ As expected for a D-sugar, $\left[\mathrm{PhCH}_{-}{ }^{2} \mathrm{H}\right] 97$ adopts a ${ }^{4} C_{1}$ conformation, while $\left[\mathrm{PhCH}^{-}{ }^{2} \mathrm{H}\right] 31$ exists preferentially in a ${ }^{1} C_{4}$ chair. Since the structures of $\mathrm{D}$-galactose and $\mathrm{L}$-fucose are in a pseudoenantiomeric relationship, 4-OBn would have a very similar stereochemical environment in $\left[\mathrm{PhCH}_{-}{ }^{2} \mathrm{H}\right] 97$ to that which it has in the structure of $\left[\mathrm{PhCH}^{-}{ }^{2} \mathrm{H}\right] 31$. Indeed, in the NMR spectra of labeled $\left[\mathrm{PhCH}_{-}{ }^{2} \mathrm{H}\right] 97(\mathrm{D} / \mathrm{H}, 7: 1 ; \mathrm{dr}=1: 1)$, it is also observed how both diastereoisomeric deuterated benzyl ethers affect the chemical displacement of the surrounding protons and carbons differently. For example, in the ${ }^{13} \mathrm{C}\left\{{ }^{1} \mathrm{H}\right\}$ NMR spectrum, the $\mathrm{C} 4$ atom analogously appears as three signals at $73.56 \mathrm{ppm}\left(\mathrm{D}_{1}\right), 73.59 \mathrm{ppm}\left(\mathrm{D}_{2}\right)$, and $73.64 \mathrm{ppm}$ (unlabeled) with the expected intensities (see Table S6 and Figure S4 in the SI for details).

The effectiveness of this methodology was also tested on a D-pentose structure. Thus, the reaction with $n$ - $\mathrm{Bu}_{3} \mathrm{SnH} / \mathrm{AIBN}$ of 3-C-(2-O-diphenoxyphosphoryl- $\alpha, \beta$-D-arabinopyranosyl) 1 propoxyphthalimide derivatives $\mathbf{1 1}$ as a mixture of anomers and its deprotected diastereoisomeric pure $\beta$-diol 12 afforded exclusively the desired spirocycles 37 and 38, respectively (Table 3, entries 7 and 10). The 2-deoxy-arabinopyranosyl ring adopted preferentially a ${ }^{1} C_{4}$ conformation (see Table S3 in the SI). Compound 38 was previously described by an alternative glycosylation method using thermodynamic conditions, and a $1 R$ anomeric stabilized configuration was assigned. ${ }^{28 \mathrm{c}}$ Consequently, we have not found NOE interaction between $\mathrm{H1}$ ' and $\mathrm{H} 3$ and/or $\mathrm{H} 5$ as in previous thermodynamic spiroketals prepared in this work. 
Table 4. 1,5-HAT-S-T Sequence in 3-C-( $\alpha$-D-Ribf $) 1$-propoxyphthalimides $13-15^{a}$

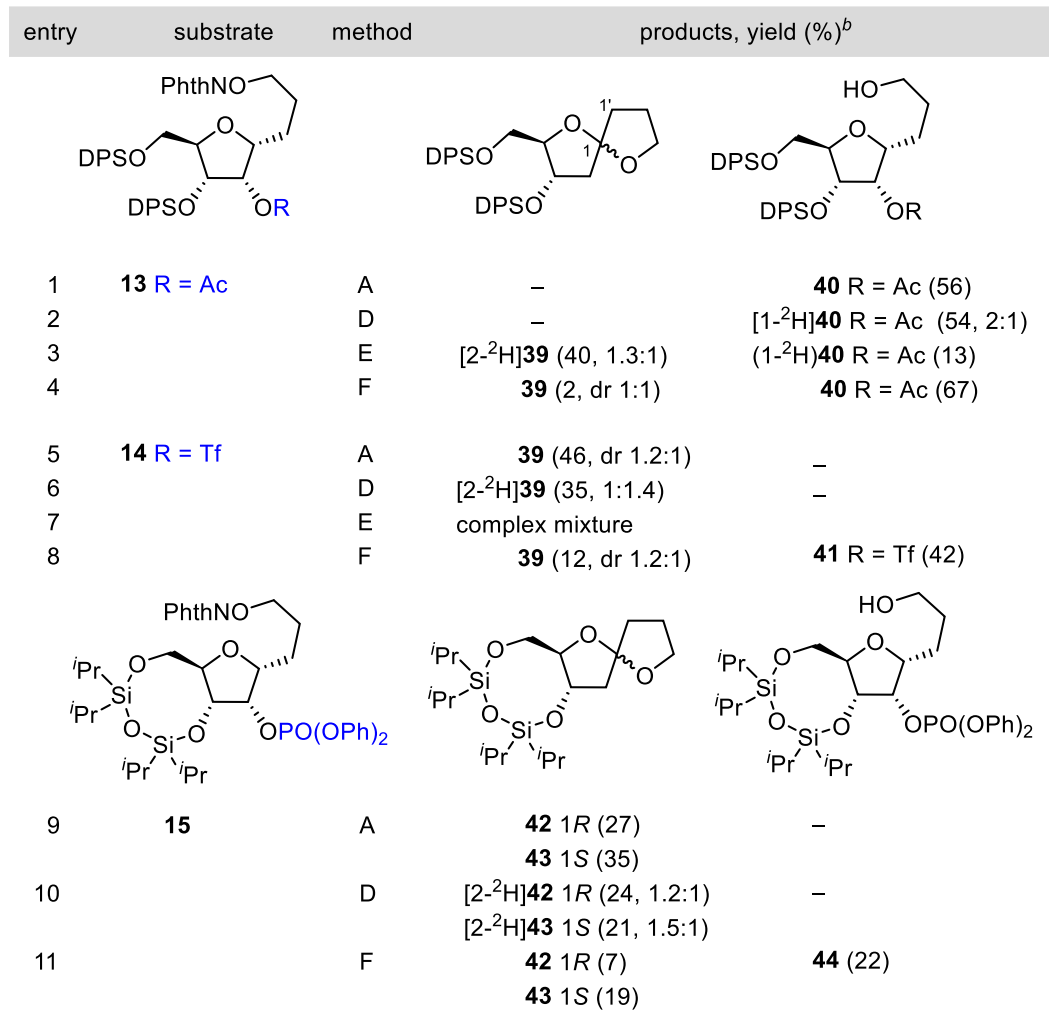

${ }^{a}$ Reagents and conditions: method A: $n-\mathrm{Bu}_{3} \mathrm{SnH}$ ( 1 equiv), $\mathrm{AIBN}$ (0.1 equiv), $\mathrm{PhCH}_{3}(0.013 \mathrm{M})$, reflux; method $\mathrm{D}: n$-Bu $3 \mathrm{SnD}(1$ equiv), $\mathrm{AIBN}$ (0.1 equiv), $\mathrm{PhCH}_{3}\left(0.013 \mathrm{M}\right.$ ), reflux; method $\mathrm{E}: n-\mathrm{Bu}_{3} \mathrm{SnD}$ (1 equiv), $\mathrm{BF}_{3} \bullet \mathrm{Et}_{2} \mathrm{O}$ (0.2 equiv), $\mathrm{AIBN}$ (0.1 equiv), $\mathrm{PhCH}_{3}(0.013 \mathrm{M}$ ), reflux; and method F: Hantzsch ester (1.1 equiv), fac-Ir(ppy) 3 (0.01 equiv), THF $(0.007 \mathrm{M})$, rt, blue LED. ${ }^{b}$ Values in parentheses are isolate yields; deuterium incorporation $\left({ }^{2} \mathrm{H} /{ }^{1} \mathrm{H}\right)$ is included in partially labeled compounds. $\mathrm{dr}=$ diastereomeric ratio.

The analysis of the isotopic distribution in $\left[2-{ }^{2} \mathrm{H}\right] 37$ $\left({ }^{2} \mathrm{H} /{ }^{1} \mathrm{H}, 1: 1\right)$ and $\left[2-{ }^{2} \mathrm{H}\right] 38\left({ }^{2} \mathrm{H} /{ }^{1} \mathrm{H}, 2.3: 1\right)$, obtained by reductive $n-\mathrm{Bu}_{3} \mathrm{SnD} / \mathrm{AIBN}$ with or without the $\mathrm{BF}_{3} \bullet \mathrm{Et}_{2} \mathrm{O}$ catalyst, showed a partial monodeuteration at $\mathrm{C} 2$, with the major isotopomer occupying the $\beta$-equatorial position (Table 3 , entries 8, 9, and 11).

For the sake of completeness, this methodology was also extended to a series of furanosyl models derived from 3-C- $(\alpha-$ D-ribofuranosyl)1-propanol as described in Table 4 . When the reaction of acetyl phthalimide $\mathbf{1 3}$ was carried out under the $n$ $\mathrm{Bu}_{3} \mathrm{SnH}(\mathrm{D}) / \mathrm{AIBN}$ conditions, no traces of any compound with a 1,6-dioxaspiro[4.4] nonane skeleton were detected. Only the alcohol 40 was obtained (Table 4, entries 1 and 2). The deuterium composition of $\left[1-{ }^{2} \mathrm{H}\right] 40\left({ }^{2} \mathrm{H} /{ }^{1} \mathrm{H}, 2: 1\right)$ indicates that a significant 1,5 -HAT reaction has taken place, but the $\mathrm{C} 1$ radical intermediate is reduced before the $\mathrm{S}-\mathrm{T}$ rearrangement occurs. An equimolecular mixture of spirocycles $\left[2-{ }^{2} \mathrm{H}\right] 39$ $\left({ }^{2} \mathrm{H} /{ }^{1} \mathrm{H}, 1.3: 1\right)$ was achieved in moderate yield by adding a catalytic amount of $\mathrm{BF}_{3} \bullet \mathrm{Et}_{2} \mathrm{O}$ to the reaction medium (Table 4 , entry 3). Also in this case, a substantial loss of deuterium at C2 indicated the possibility of competitive mechanisms with the 1,2- $\beta$-(acyloxy)alkyl radical migration. A change to a better LG such as triflate $\mathbf{1 4}$ increased the rate of S-T rearrangement, and the sequence could now be completed under standard tin hydride conditions (Table 4, entries 5 and 6). However, adding $\mathrm{BF}_{3} \bullet \mathrm{Et}_{2} \mathrm{O}$ to the reaction resulted in a very complex mixture containing alcohol $\mathbf{4 1}$ as the sole identifiable product. The initiation of the reaction under photoredox catalysis conditions on both phthalimides 13 and 14 afforded poorer results (Table 4 , entries 4 and 8 ).

The use of diphenylphosphate as in $\mathbf{1 5}$ gave access exclusively to spirocycles 42 and 43 , isolated as a separable mixture of anomers in $62 \%$ overall yield (Table 4, entries 9 and 10). Again, under photoredox conditions, lower yields of the spirocyclic compounds and significant amounts of prematurely reduced alcohol 44 were obtained (Table 4, entry 11).

Synthesis of 4-Deoxy-6,8-dioxabicyclo[3.2.1]octane Scaffolds. The objective of this section is the preparation of 4-deoxy carbohydrates with a 6,8-dioxabicyclo[3.2.1] octane skeleton by applying this $1,5-\mathrm{HAT}-\mathrm{S}-\mathrm{T}$ rearrangement sequence to $C$-glycosyl compounds of a $C-(\alpha-D, L-$ glycopyranosyl)methanol general structure, and the results are included in Tables 5 and 6 . The sequence was first attempted on the $C$-(4-O-acetyl-6-O-tert-butyldiphenylsilyl-2,3di-O-methyl- $\alpha$-D-glucopyranosyl) $N$-methoxyphthalimide (16) model. In this compound, the glucopyranosyl ring adopts preferentially a ${ }^{4} C_{1}$ chair conformation, and thus, the initial 1,5-HAT reaction should be favored (see Table S2 in the SI). However, the tin hydride conditions led to a mixture of four compounds, in which the desired bicycle $\mathbf{4 5}$ was isolated as a minor product. The other compounds were the unstable olefin 46 formed presumably by $\beta$-(acyloxy)alkyl fragmentation, and 47 and 48 generated by the premature reduction of intermediate radicals (Table 5 , entry 1 ).

The structural and stereochemical assignment of these compounds rests on spectroscopic and analytical data. Conformational evidence was obtained by extracting the ring 
Table 5. 1,5-HAT-S-T Sequence in $C$ - $\left(\alpha\right.$-D-Glcp)- and $C$ - $(\alpha$-D-Gal $p) N$-methoxyphthalimides $16-21^{a}$

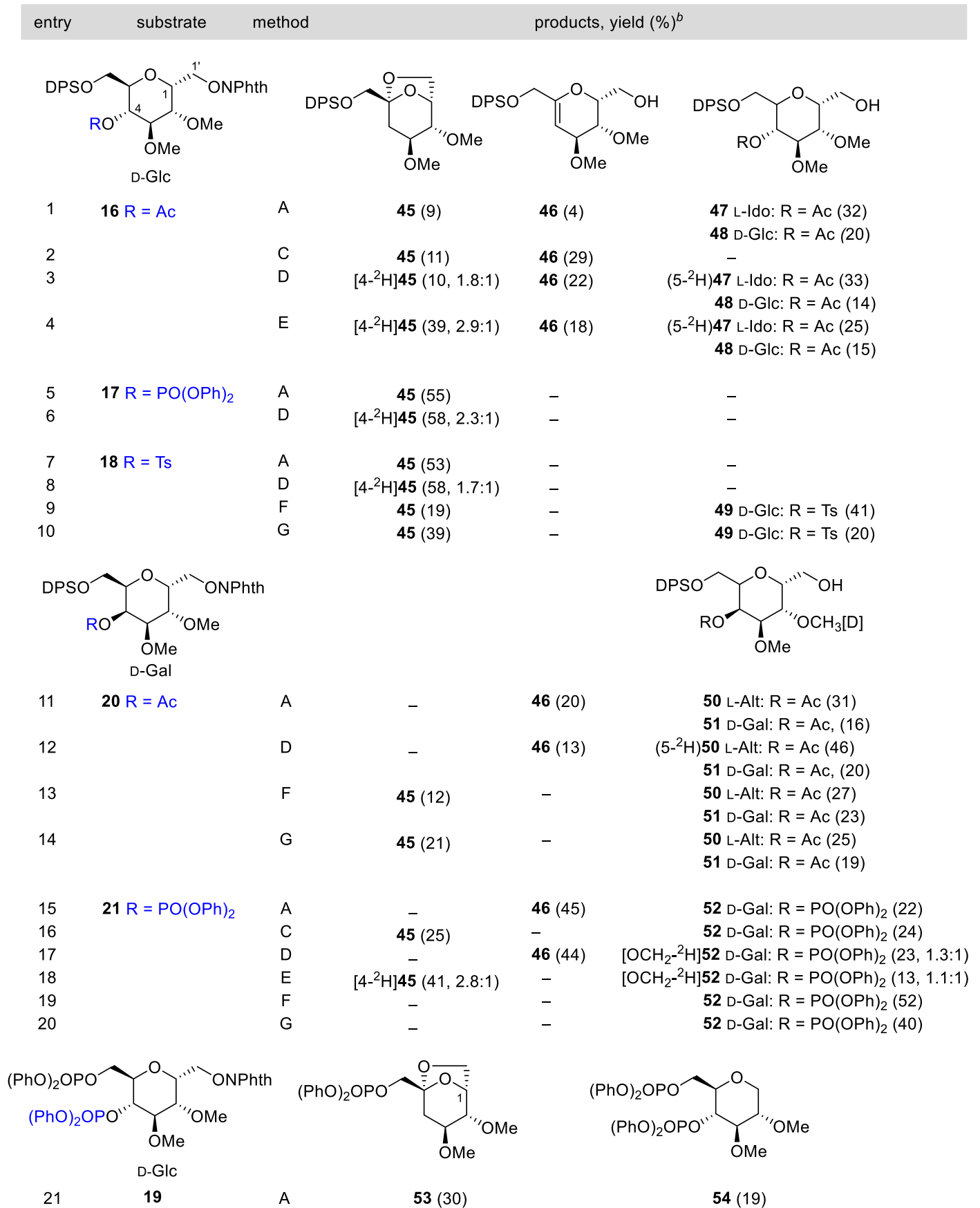

${ }^{a}$ Reagents and conditions: method A: $n$ - $\mathrm{Bu}_{3} \mathrm{SnH}$ (1 equiv), AIBN (0.1 equiv), $\mathrm{PhCH}_{3}(0.013 \mathrm{M})$, reflux; method C: TTMSS ( 1 equiv), AIBN (0.1 equiv), $\mathrm{PhCH}_{3}(0.013 \mathrm{M})$, reflux; method $\mathrm{D}: n-\mathrm{Bu}_{3} \mathrm{SnD}$ (1 equiv), $\mathrm{AIBN}$ (0.1 equiv), $\mathrm{PhCH}_{3}(0.013 \mathrm{M})$, reflux; method $\mathrm{E}: n$ - $\mathrm{Bu} \mathrm{H}_{3} \mathrm{SnD}(1$ equiv), $\mathrm{BF}_{3} \bullet \mathrm{Et}_{2} \mathrm{O}$ (0.2 equiv), $\mathrm{AIBN}$ (0.1 equiv), $\mathrm{PhCH}_{3}(0.013 \mathrm{M})$, reflux; method F: Hantzsch ester (1.1 equiv), fac- $\left.\operatorname{Ir}(\mathrm{ppy})\right)_{3}(0.01$ equiv), THF (0.007 $\mathrm{M})$, rt, blue LED; and method G: Hantzsch ester (0.37 equiv/h), fac-Ir(ppy) $)_{3}\left(0.01\right.$ equiv), THF (0.007 M), rt, blue LED. ${ }^{b}$ Values in parentheses are isolate yields; deuterium incorporation $\left({ }^{2} \mathrm{H} /{ }^{1} \mathrm{H}\right)$ is included in partially labeled compounds.

$J$-coupling from a simulated spectrum (see Table S7 in the SI). In addition, the long-range couplings ${ }^{4} J_{2,1},(1.1 \mathrm{~Hz}$, calcd 1.1 $\mathrm{Hz})^{48}$ observed in the spectrum of $\mathbf{4 5}$ and ${ }^{4} J_{2,4}(1.2 \mathrm{~Hz}$, calcd $1.2 \mathrm{~Hz})^{48}$ in 47 confirms the ${ }^{4} C_{1}$ and ${ }^{1} C_{4}$ conformations, respectively, for the sugar rings (see Table $\mathrm{S} 4$ in the $\mathrm{SI}$ ). Therefore, the main product 47 was assigned an L-Ido structure by the inversion of configuration at C5. Using TTMSS or $n-\mathrm{Bu}_{3} \mathrm{SnD}$ as reductants did not significantly improve the yield of the bicycle $\mathbf{4 5}$ but markedly increased the formation of the olefin 46 (Table 5, entries 2 and 3). As expected, the best yield was achieved by adding Lewis acid; $\left[4-{ }^{2} \mathrm{H}\right] 45$ (39\%; $\left.{ }^{2} \mathrm{H} /{ }^{1} \mathrm{H}, 2.9: 1 ; 4 R / 4 S, 1: 1.2\right)$ was formed with a high deuterium content but low stereoselectivity (Table 5, entry 4).

As shown in Table 5 (entries 5-8), we need better LGs for the sequence of reactions to reach the end. In these experiments, the starting 4-O-diphenoxyphosphoryl 17 and 4-O-tosyl-phthalimide 18 were exclusively transformed into 45 
Table 6. 1,5-HAT-S-T Sequence in $C$ - $(\alpha$-L-Fucp $)$ - and $C$ - $(\alpha$-L-Rhap $) N$-methoxyphthalimides $22-24^{a}$

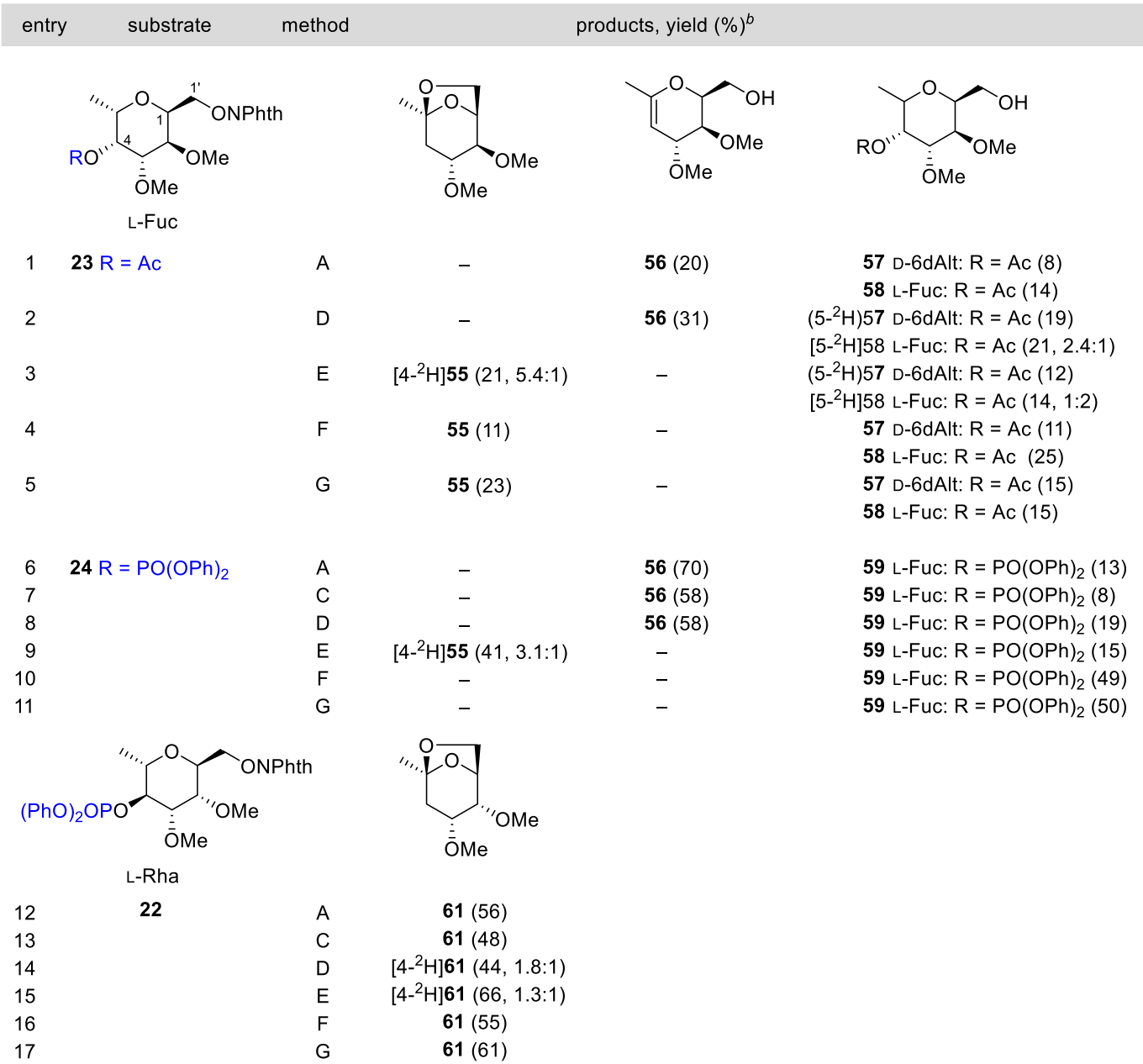

${ }^{a}$ Reagents and conditions: method A: $n$ - $\mathrm{Bu}_{3} \mathrm{SnH}$ (1 equiv), $\mathrm{AIBN}$ (0.1 equiv), $\mathrm{PhCH}_{3}(0.013 \mathrm{M})$, reflux; method C: TTMSS ( 1 equiv), AIBN (0.1 equiv), $\mathrm{PhCH}_{3}(0.013 \mathrm{M})$, reflux; method $\mathrm{D}: n-\mathrm{Bu}_{3} \mathrm{SnD}$ (1 equiv), $\mathrm{AIBN}$ (0.1 equiv), $\mathrm{PhCH}_{3}(0.013 \mathrm{M})$, reflux; method $\mathrm{E}: n$ - $\mathrm{Bu} \mathrm{S}_{3} \mathrm{SnD}(1$ equiv), $\mathrm{BF}_{3} \bullet \mathrm{Et}_{2} \mathrm{O}$ (0.2 equiv), AIBN (0.1 equiv), $\mathrm{PhCH}_{3}(0.013 \mathrm{M})$, reflux; method F: Hantzsch ester (1.1 equiv), fac-Ir(ppy) 3 (0.01 equiv), THF (0.007 $\mathrm{M})$, rt, blue LED; and method G: Hantzsch ester $\left(0.37\right.$ equiv/h), fac-Ir $(\mathrm{ppy})_{3}\left(0.01\right.$ equiv), THF $(0.007 \mathrm{M})$, rt, blue LED. ${ }^{b}$ Values in parentheses are isolate yields; deuterium incorporation $\left({ }^{2} \mathrm{H} /{ }^{1} \mathrm{H}\right)$ is included in partially labeled compounds.

or $\left[4{ }^{2} \mathrm{H}\right] 45$ and no prereduction or $\beta$-fragmentation byproducts were detected. Under photoredox catalysis conditions, tosyl derivative 18 gave the bicycle 45 in poor yield, which could be substantially enhanced by adding the Hantzsch ester slowly via a syringe pump (Table 5, entries 9 and 10).

The reaction of the models $\mathrm{C}$-(6-O-tert-butyldiphenylsilyl2,3-di- $O$-methyl- $\alpha$-D-galactopyranosyl $) N$-methoxyphthalimide (20 and 21) was then examined, and the results are presented in Table 5. Under the $n-\mathrm{Bu}_{3} \mathrm{SnH}(\mathrm{D})$ conditions and irrespective of whether the starting phthalimide was 20 or 21, no products with the 6,8-dioxabicyclo[3.2.1] octane skeleton were detected (Table 5, entries 11, 12, 15, and 17). In these experiments, the only relevant products isolated were olefin 46 and the L-altrose derivative 50 , both generated by the radical quenching at $\mathrm{C} 5$ prior to the $\mathrm{S}-\mathrm{T}$ rearrangement. Best results were ultimately attained using $n-\mathrm{Bu}_{3} \mathrm{SnD}$ under the Lewis acid catalyst, with compounds $\left[4-{ }^{2} \mathrm{H}\right] 45\left(41 \% ;{ }^{2} \mathrm{H} /{ }^{1} \mathrm{H}\right.$, $2.8: 1 ; 4 R / 4 S, 1: 1.2)$ and $\left[\mathrm{OCH}_{2}{ }^{2} \mathrm{H}\right] \mathbf{5 2}$ being obtained (Table 5 , entry 18). Analogously to the reaction of $16,\left[4-{ }^{2} \mathrm{H}\right] 45$ was formed with a high deuterium incorporation at $\mathrm{C} 4$ but with a low stereoselectivity (Table 5 , compare entries 4 and 18). The incorporation of deuterium in $\left[\mathrm{OCH}_{2}{ }^{2} \mathrm{H}\right] 52\left({ }^{2} \mathrm{H} /{ }^{1} \mathrm{H}, 1.1: 1\right)$ indicates a competitive abstraction of the H5 and the methoxyl group at C2 initiated by the alkoxyl radical through 1,5-HAT and 1,6-HAT processes, respectively. As would be expected, in this D-galactose model 21, which is less prone to undergo the 1,2-(ester)alkyl radical migration, the photoredox catalytic reaction gave only the prereduced alcohol 52, with compound 45 being undetectable by ${ }^{1} \mathrm{H}$ NMR (Table 5, entries 19 and 20).

A propagation cycle for the acetyl and diphenoxyphosphoryl $(\alpha$-D-Gal $p)$ methan-1-O-yl radical chain reactions, employing tin deuteride as the reductant, is shown in Scheme 3. The alkoxyl radical (I) initiated two competitive abstraction processes: 1,5-HAT of the $5 \mathrm{H}$ and 1,6-HAT of one hydrogen of the methoxyl group at $\mathrm{C} 2$. The radical II leads to $\left(\mathrm{OCH}_{2}{ }^{2} \mathrm{H}\right) 52$, whereas the radical at $\mathrm{C5}$ (III) may be stabilized by three different mechanisms: reduction with inversion of configuration giving rise to L-altrose derivative $\left(5-{ }^{2} \mathrm{H}\right) 50$ (path a), radical $\beta$-fragmentation of the LG that can explain the formation of olefin $\mathbf{4 6}$ and the unlabeled 45 (path b), ${ }^{45}$ or continuing the sequence by the radical-ionic mechanism that finally provided $\left(4-{ }^{2} \mathrm{H}\right) 45$ through the cine cyclization step (path $\mathrm{c}$ ). 
Scheme 3. Propagation Cycle of $C$ - $(\alpha-\mathrm{D}-\mathrm{Gal} p)$ methan-1-O-yl Radicals ${ }^{a}$

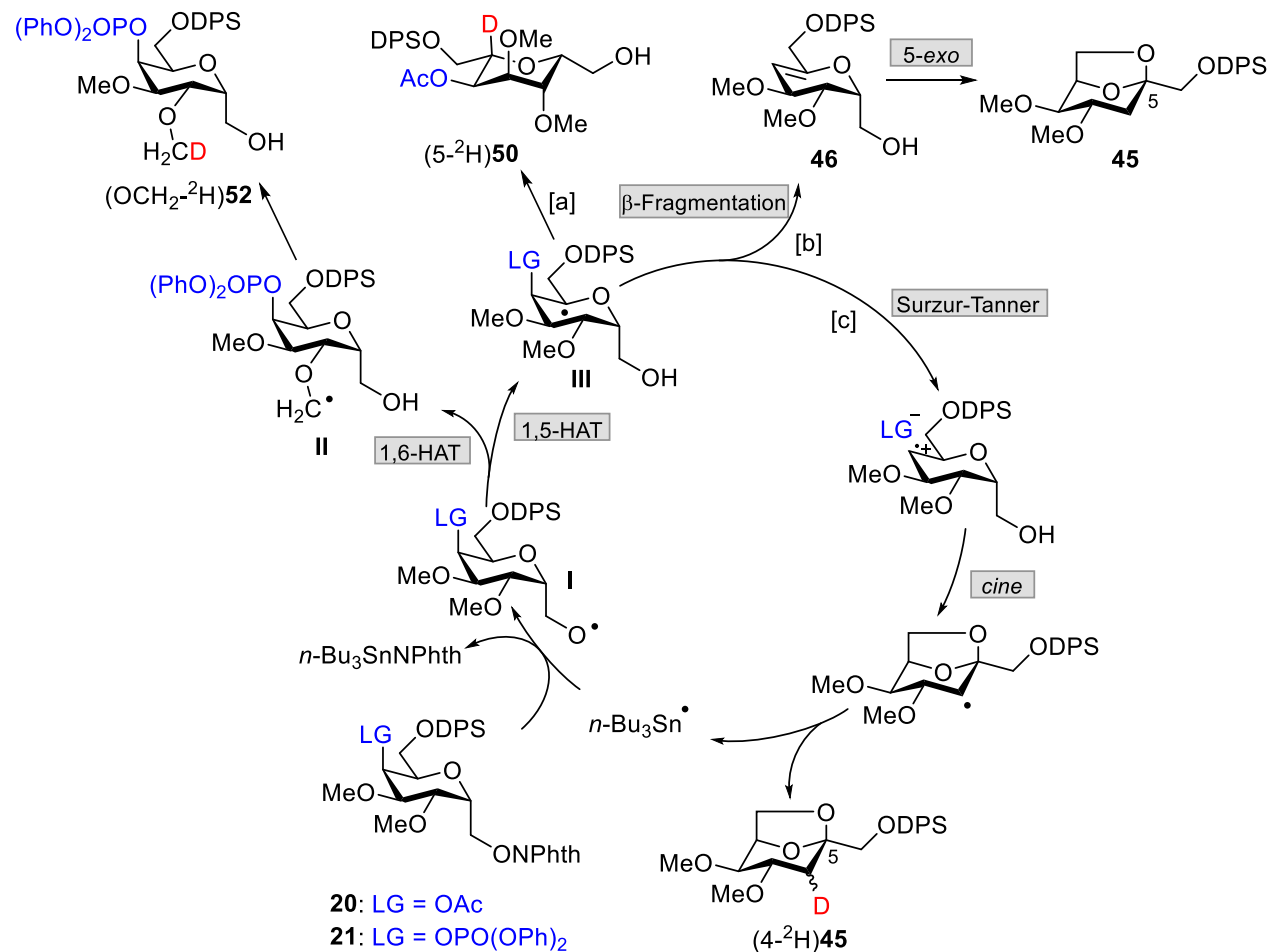

${ }^{a}$ The prereduction of the alkoxyl radical and the initiation and termination steps are omitted for clarity.

A phthalimide precursor 19 having two plausible LGs at C4 and C6 was also included in this study (Table 5, entry 21). Since the endocyclic alkene radical cation intermediate should be more stable than the exocyclic alternative, it is not surprising that we only obtained the 4-deoxy-bicycle 53 by $\mathrm{C} 4-\mathrm{OPO}(\mathrm{OPh})_{2}$ migration. This product was accompanied by D-glucitol derivative 54 as a result of the competitive $\beta$ fragmentation of the primary alkoxyl radical, which had not previously been observed in other members of this series., ${ }^{50 c 53}$

Examples with L-sugar frameworks such as $\alpha$-L-Fucp (23 and 24) and $\alpha$-L-Rhap (22) have also been accomplished, and the results are summarized in Table 6. Since $\alpha$-L-Fucp and $\alpha$-DGalp have a pseudoenantiomeric relationship, an analogous reaction pattern could be expected. Indeed, the results obtained for the L-Fucp derivatives (23 and 24) are quite similar to those observed for the previously studied D-Gal $p$ phthalimides 20 and 21 (compare Table 5, entries 11-20 with Table 6, entries 1-11). Thus, neither the acetyl 23 nor diphenoxyphosphoryl 24 precursor gave the desired 6,8dioxabicyclic compound when submitted to the $n-\mathrm{Bu}_{3} \mathrm{SnH}$ (D)/AIBN conditions in the absence of activating additives (Table 6, entries 1, 2, 6, and 8). In both cases, olefin 56 was the main product with a yield that reached a maximum of $70 \%$ using phosphatoxy as LG (Table 6, entry 6).

In the reaction of acetylphthalimide 23 , the olefin 56 was always accompanied by small amounts of $\mathbf{5 7}$ with an inverted 6-deoxy-D-altrose structure (Table 6, entries 1-5). The conversion of phthalimides 23 and $\mathbf{2 4}$ into 6,8-dioxabicyclic compound $\left[4-{ }^{2} \mathrm{H}\right] \mathbf{5 5}$ was only possible in the presence of a catalytic amount of $\mathrm{BF}_{3} \bullet \mathrm{Et}_{2} \mathrm{O}$ (Table 6 , entries 3 and 9). Under these conditions, a new anhydro-alditol 3-O-acetyl-2,6anhydro-1-deoxy-4,5-di-O-methyl-D- $\left(6-{ }^{2} \mathrm{H}\right)$ galactitol $\left[\left(1-{ }^{2} \mathrm{H}\right)\right.$ 60 ] was also isolated in a very low yield (3\%), probably generated by the $\beta$-fragmentation of the alkoxyl radical at the beginning of the sequence (not shown in Table 6, entry 3 ). Parallel to what occurred for the D-Galp derivatives 20 and 21, surprisingly, under photoredox conditions, the acetyl precursor 23 afforded the 6,8-dioxabicyclo 55 although in low yields, while the diphenoxyphosphoryl precursor 24 yielded exclusively the reduced alcohol 59 (compare Table 6, entries 4, 5, 10 , and 11 with Table 5, entries 13, 14, 19, and 20).

The phthalimides derived from $\alpha$-L-Rhap 22 and $\alpha$-D-Glcp 17 have a very similar stereochemical arrangement to the atoms involved in the radical sequence, and consequently, an analogous behavior should be expected (compare Table 5, entries 5 and 6 with Table 6 , entries 12 and 14). Indeed, the phthalimide 22 afforded exclusively the 6,8-dioxabicyclo 61 in good yield not only with tin hydride but also employing TTMSS or under the photoredox conditions.

\section{CONCLUSIONS}

In summary, the fate of the 3-C-( $\alpha, \beta$-D,L-glycopyranosyl $) 1$ propan- $O$-yl radical moves through the $C$-glycosyl skeleton by a 1,5-HAT-S-T rearrangement radical/polar sequence giving 1,4-anhydro-5-deoxy-non-4-ulopyranoses with a 10-deoxy-1,6dioxaspiro[4.5] decane structure.

The reaction under the tin hydride conditions appears to be reasonably independent of the axial or equatorial configuration of the abstractable $1 \mathrm{H}$ but is highly influenced by the nature and stereochemistry of the LGs (2-acetoxy or 2-phosphatoxy) used in the $\mathrm{S}-\mathrm{T}$ rearrangement at the end of the sequence. ${ }^{39}$ Thus, the 2-phosphatoxy LG in an equatorial position as in 3$C$-(2-O-diphenoxyphosphoryl- $\alpha, \beta$-D-Glc $p) 1$-propoxyphthalimides 2 and 4 was found to provide the best results (Table 1, entries 5-7 and 12-14). With a poorer 2-acetoxy LG axially disposed as in $C$-(2-O-acetyl- $\alpha, \beta$-D-Man $p) 1$-propoxyphthalimides 5 and 7 , the sequence did not reach the end and only the prereduced compound $\mathbf{2 9} \boldsymbol{\beta}$ was obtained (Table 2, entries 
Scheme 4. Synthesis of 3-C-(Glycopyranosyl)1-propoxyphthalimide (1-12) Precursors of 1,6-Dioxaspiro[4.5]decane Structures $^{a}$<smiles>[R]OCCC[C@@H]1O[C@H](COCc2ccccc2)[C@@H](O)[C@H](O)[C@H]1OCc1ccccc1</smiles>

62<smiles>[R]OCCC[C@H]1O[C@H](CO[B])[C@@H](O[B])[C@H](O)[C@@H]1OCc1ccccc1</smiles>

65<smiles>C=CC[C@H]1O[C@H](COCc2ccccc2)[C@@H](O)[C@H](O)[C@H]1O</smiles>

68<smiles>[R]CCC[C@H]1O[C@H](COCc2ccccc2)[C@@H](O)[C@H](O)[C@H]1OCc1ccccc1</smiles>

b 69: $\mathrm{R}=\mathrm{H}(53)$

$69: R=H(53)$
$70: R=$ NPhth $(84)$

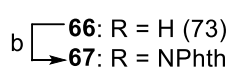

1: $R=A c(72)$

2: $\mathrm{R}=\mathrm{PO}(\mathrm{OPh})_{2}(76)$

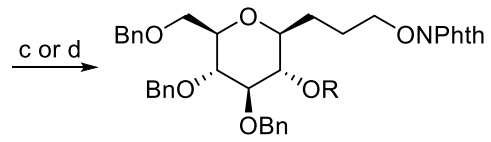

3: $R=A c(71)$

4: $\mathrm{R}=\mathrm{PO}(\mathrm{OPh})_{2}(51)$

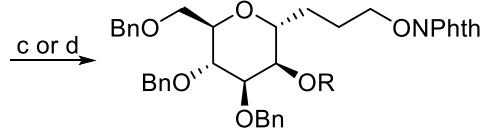

5: $R=A c(86)$ 6: $\mathrm{R}=\mathrm{PO}(\mathrm{OPh})_{2}(74)$<smiles>C=CC[C@H]1O[C@H](COBr)[C@@H](O)[C@H](O)[C@H]1O</smiles>

71<smiles>[R]CCC[C@H]1O[C@H](CO[B])[C@@H](O[B])[C@H](O)[C@H]1O</smiles>

b 72: $\mathrm{R}=\mathrm{H}(67)$

$\mathrm{b} \longrightarrow 73: \mathrm{R}=$ NPhth $(84)$ $\stackrel{\text { c or d }}{\longrightarrow}$

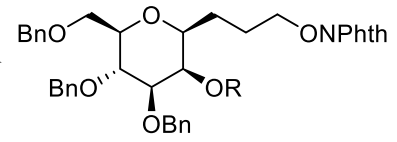

7: $\mathrm{R}=\mathrm{Ac}(80)$

8: $\mathrm{R}=\mathrm{PO}(\mathrm{OPh})_{2}(81)$<smiles>C=CC[C@H]1O[C@H](C)[C@@H](OCc2ccccc2)[C@H](O)[C@H]1Br</smiles>

74<smiles>C=CC[C@H]1O[C@H](C)[C@H](O)[C@H](O)[C@H]1O</smiles><smiles>[CH]C</smiles><smiles>[R]CCC[C@H]1O[C@H](C)[C@@H](O[B])[C@H](O)[C@H]1O[R]</smiles>

75 (63)

b 76: $\mathrm{R}=\mathrm{R}^{1}=\mathrm{H}(57)$

b $\longrightarrow 77: \mathrm{R}=$ NPhth, $\mathrm{R}^{1}=\mathrm{H}(94)$

c $\longrightarrow$ 9: $R=$ NPhth, $R^{1}=A c(58)$

10: $R=$ NPhth, $R^{1}=\mathrm{PO}(\mathrm{OPh})_{2}(56)$<smiles>CC(=O)O[C@H]1CO[C@H](OC(C)=O)[C@H](OC(C)=O)[C@H]1OC(C)=O</smiles>

78<smiles>C=CC[C@H]1OC[C@@H]2O[C@@H](c3ccccc3)O[C@H]2[C@H]1O</smiles><smiles>[R]OCCC[C@H]1OC[C@H]2O[C@@H](c3ccccc3)O[C@@H]2[C@H]1O[R]</smiles>

79: $\beta-P h(12)$

80: $\alpha-P h(41)$

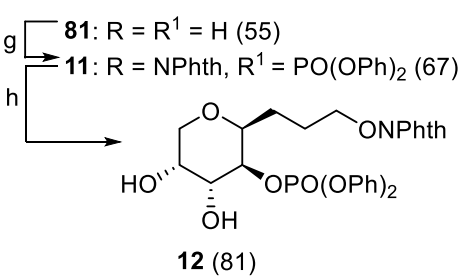

${ }^{a}$ Reagents and conditions: (a) (i) $\mathrm{BH}_{3} \bullet$ THF $1 \mathrm{M}$ complex, THF, $0{ }^{\circ} \mathrm{C}$ to rt, 1 h. (ii) $\mathrm{NaOH} 3 \mathrm{M}, ~_{2} \mathrm{O}_{2} 30 \%, 0{ }^{\circ} \mathrm{C}, 1 \mathrm{~h}$. (b) $\mathrm{HONPhth}, \mathrm{Ph}{ }_{3} \mathrm{P}$, DEAD, THF, $0^{\circ} \mathrm{C}$ to rt, $1-4$ h. (c) $\mathrm{Ac}_{2} \mathrm{O}$, Py, DMAP, $0{ }^{\circ} \mathrm{C}$ to rt, $1 \mathrm{~h}$. (d) $\mathrm{ClPO}(\mathrm{OPh})_{2}, \mathrm{DMAP}, \mathrm{CH}_{2} \mathrm{Cl}_{2}, 0^{\circ} \mathrm{C}$ to rt, $2 \mathrm{~h}$. (e) $(i) \mathrm{I}_{2}, \mathrm{CH}_{2} \mathrm{Cl}_{2}, \mathrm{rt}, 3 \mathrm{~h}$.

(ii) $\mathrm{Zn}$ dust, $\mathrm{AcOH}, \mathrm{Et}_{2} \mathrm{O}: \mathrm{MeOH}$, rt, overnight. (f) (i) allyltrimethylsilane, $\mathrm{BF}_{3} \bullet \mathrm{Et}_{2} \mathrm{O}, \mathrm{CH}_{3} \mathrm{CN}, 0{ }^{\circ} \mathrm{C}$ to rt, $1.5 \mathrm{~h}$. (ii) $\mathrm{Na}_{2} \mathrm{CO}_{3}, \mathrm{MeOH} \mathrm{rt}, 2.5 \mathrm{~h}$. (iii) $\mathrm{PhCH}(\mathrm{OMe})_{2}$, CSA, DMF, rt, overnight. (g) (i) HONPhth, $\mathrm{Ph}_{3} \mathrm{P}, \mathrm{DEAD}$, THF, $0{ }^{\circ} \mathrm{C}$ to rt, $0.5 \mathrm{~h}$. (ii) $\mathrm{ClPO}(\mathrm{OPh})_{2}, \mathrm{DMAP}^{\circ} \mathrm{CH}_{2} \mathrm{Cl}_{2}, 0{ }^{\circ} \mathrm{C}$ to rt, $1.5 \mathrm{~h}$. (h) $\mathrm{TFA} / \mathrm{H}_{2} \mathrm{O}, \mathrm{CH}_{2} \mathrm{Cl}_{2}, 0{ }^{\circ} \mathrm{C}$ to $\mathrm{rt}, 1 \mathrm{~h}$. ${ }^{b}$ Values in parentheses are isolate yields.

1, 2, and 7-10). In the two intermediate situationsequatorial 2-acetoxy phthalimides 1 and 3 (Table 1, entries 1-4 and 8-11) and axial 2-diphenoxyphosphoryl phthalimides 6 and 8 (Table 2, entries 3-6 and 12-17) - the spirocycle 25 is formed in significant amounts, indicating that the low migratory capacity of the LG can be compensated by favorable stereochemical effects and vice versa. A comparison of the best results obtained with the different LGs has been summarized in Table S9 at the SI.

Some other interesting facts can be culled from the data described in Tables 1 and 2. First, an expected KIE was observed during the formation of $\mathbf{2 5}$, with the yield increasing significantly in most cases when deuteride was used instead of hydride donors (e.g., Table 1, compare entries 8 and 11; see also Table $S 9$ in the $\mathrm{SI}$ ). The sequence yield was also 
Scheme 5. Synthesis of 3-C-( $\alpha$-D-Ribofuranosyl)1-propoxyphthalimide (13-15) Precursors of 1,6-Dioxaspiro[4.4]nonane Structures $^{a}$

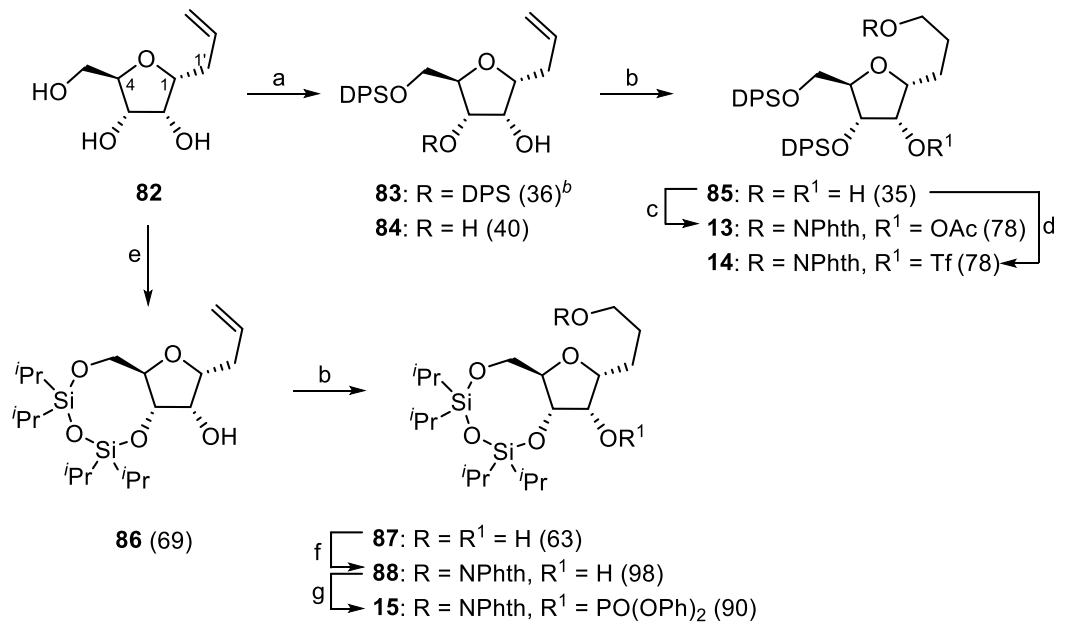

${ }^{a}$ Reagents and conditions: (a) DPSCl, imidazole, DMF, $0{ }^{\circ} \mathrm{C}, 0.5 \mathrm{~h}$. (b) $(\mathrm{i}) \mathrm{BH}_{3} \bullet$ THF $1 \mathrm{M}$ complex, THF, $0{ }^{\circ} \mathrm{C}$ to rt, $2.5 \mathrm{~h}$. (ii) $\mathrm{NaHCO}_{3}, \mathrm{H}_{2} \mathrm{O}_{2}$ $30 \%, 0{ }^{\circ} \mathrm{C}, 1$ h. (c) (i) HONPhth, $\mathrm{Ph}_{3} \mathrm{P}, \mathrm{DEAD}, 50^{\circ} \mathrm{C}, 2$ h. (ii) Ac $\mathrm{Ac}_{2} \mathrm{O}$, DMAP, Py, rt, 1 h. (d) (i) HONPhth, $\mathrm{Ph}_{3} \mathrm{P}, \mathrm{DEAD}, 50{ }^{\circ} \mathrm{C}, 2$ h. $(i i) \mathrm{Tf}_{2} \mathrm{O}$, Py, rt, 1 h. (e) 1,3-dichloro-1,1,3,3-tetraisopropyldisiloxane, Py, $0{ }^{\circ} \mathrm{C}, 20$ h. (f) $\mathrm{HONPhth} \mathrm{Ph}_{3} \mathrm{P}, \mathrm{DEAD}, 50{ }^{\circ} \mathrm{C}$, overnight. (g) $\mathrm{ClPO}(\mathrm{OPh})_{2}$, DMAP, $\mathrm{CH}_{2} \mathrm{Cl}_{2}$, rt, 3 h. ${ }^{b}$ Values in parentheses are isolate yields.

dramatically improved by complexation with $\mathrm{BF}_{3} \bullet \mathrm{Et}_{2} \mathrm{O}$ (Table 2, compare entries 10 and 11).

All these observations are in excellent agreement with experimental results obtained in the application of this methodology to $C$-glycopyranosyl models for the synthesis of either 10-deoxy-1,6-dioxaspiro[4.5] decane (Tables 1-3) or 4deoxy-6,8-dioxabicyclo[3.2.1] octane frameworks (Tables 5-6). For example, 3-C-( $\alpha$-L-Fuc $p) 1$-propoxyphthalimides 9 and 10 with the pyranosyl ring in a ${ }^{1} C_{4}$ conformation and the 2-acetoxy and 2-phosphatoxy LGs equatorially disposed afforded the spiroketal 31 in good yield (Table 3, entries 1, 2 and 5, 6). Nevertheless, $C$ - $(\alpha$-D-Gal $p) N$-methoxyphthalimides $\mathbf{2 0}$ and $\mathbf{2 1}$ with the pyranosyl ring in a ${ }^{4} C_{1}$ conformation and the 4-acetoxy and 4-phosphatoxy LGs axially oriented did not give the expected bicyclic ketal 45 , which was achieved only after the addition of $\mathrm{BF}_{3} \bullet \mathrm{Et}_{2} \mathrm{O}$ to the reaction media, as evidenced in the case of 21 (Table 5, entry 18). Also in this line, $C$ - $(\alpha$-L-Rhap $) N$-methoxyphthalimide $22\left({ }^{1} C_{4}\right.$, 4-phosphatoxy equatorially positioned) smoothly led to the desired compound 61 (Table 6, entries 12-15), whereas $C$ - $(\alpha$-L-Fucp) $\mathrm{N}$-methoxyphthalimides 23 and $24\left({ }^{1} C_{4}\right.$, axial LGs) reacted only under acid catalysis (Table 6 , entries 3 and 9).

In the reaction of $C$-(D,L-Glyp) $N$-methoxyphthalimides, a new olefin with a $C$-(hex-4-enopyranosyl)methanol structure was formed (Tables 5 and 6), appearing exclusively when the $\mathrm{S}-\mathrm{T}$ rearrangement is unfavored: with the 4-acetoxy group in the equatorial or axial disposition (compounds 16, 20, and 23; $4-31 \%)$ or with the 4-phosphatoxy group axially oriented (compounds 21 and 24; 44-70\%). It is not detected in favored S-T rearrangements (4-phosphatoxy or 4-p-toluenesulfonyloxy equatorial) (compounds 17, 18, and 22). Presumably due to the highly strained dioxabicyclo[3.2.1]octane system, a pure radical $\beta$-(ester)alkyl fragmentation competes, in some cases very favorably, with a radical-polar $\beta$ (ester)alkyl shift mechanism.

The results observed when the reaction is applied to Dpentoses deserve special comments (Tables 3 and 4). With 3$C$-(2-O-diphenoxyphosphoryl- $\alpha, \beta$-D-Arap $) 1$-propoxyphthalimides 11 and 12, the reaction behaved analogously and the expected spiroketals 37 and $\mathbf{3 8}$ were, respectively, formed in similar yields (Table 3, entries 7-11). Notwithstanding, some differences with these trends are observed during the reaction of D-pentofuranosyl substrates. The reaction of 3-C-(2-acetyl$\alpha$-D-Ribf)1-propoxyphthalimide 13 proceeds exclusively in the presence of $\mathrm{BF}_{3} \bullet \mathrm{Et}_{2} \mathrm{O}$ and the use of a better LG is necessary, as observed in compounds $\mathbf{1 4}$ and $\mathbf{1 5}$ (Table 4, entries 5-11).

In these more flexible five-membered rings, the configuration of the LGs does not appear to be as important. A pseudo-rotational analysis of compounds 13, 14, and 15 shows that the most populated conformers appear at phase angles of $P=354-9^{\circ}\left({ }^{3} T_{2}\right)$ in the northern region of the pseudorotational itinerary, leaving the $L G$ in a pseudo-axial configuration (see Table S8 in the SI for details).

When the sequences were initiated by visible light photocatalysis, low yields were observed in all $3-C-(\alpha, \beta$-D,LGlyp) 1-propoxyphthalimides, which were in general lower than those obtained with tin hydride (Tables 2 and 3, methods $F$ and $G$ ). The spirocycles were always accompanied by high percentages of prereduced products. A similar behavior was observed in most cases of C-(D,L-Glyp) N-methoxyphthalimides. Thus, in the reaction of $\mathbf{2 1}$, no traces of products resulting from the 1,5-HAT could be detected, with the prereduced alcohol $\mathbf{5 2}$ being formed exclusively (Table 5, entries 19 and 20). This means that, under these conditions, the six-membered TS of the 1,5-HAT cannot be reached probably due to conformational restrictions promoted by the bulky axially oriented diphenoxyphosphatoxy group. Paradoxically, with a poorer 2-acetoxy LG axially disposed as in $\mathrm{C}$-(4-Oacetyl- $\alpha$-D-Gal $p) N$-methoxyphthalimide 20, the [3.2.1] bicyclic 45 and the inverted L-altro derivative $\mathbf{5 0}$ were obtained in a $46 \%$ combined yield (Table 5, entries 13 and 14). This is presumably due to the smaller steric demands of the acetoxy group. The same occurred with $C$ - $(\alpha$-L-Fucp $) N$-methoxyphthalimides 23 and 24 with which 20 and 21 present a pseudoenantiomeric relationship (Table 6, entries 4, 5 and 10, 11). We have also noted that, under these photoredox conditions, no 4-enopyranosyl olefins (i.e., 46 or 56) were detected (Tables 5-6, methods F and G). 
Scheme 6. Synthesis of $C$-(Glycopyranosyl) $N$-methoxyphthalimide Precursors of 6,8-Dioxabicyclo[3.2.1] heptane Structures ${ }^{a}$<smiles>CO[C@H]1O[C@H](COS(=O)(=O)O)[C@@H](OC)[C@H](OC)[C@H]1O[Ga]</smiles><smiles>[R1]C[C@H]1O[C@H](CO[R5])[C@@H]([R6])[C@H](OC)[C@H]1OC</smiles>

$\mathrm{a} \longrightarrow$ 90: $\mathrm{R}=\mathrm{Ac}(94)^{b}$

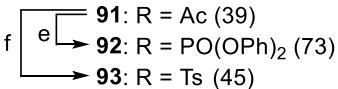

48: $\mathrm{R}=\mathrm{Ac}, \mathrm{R}^{1}=\mathrm{H}(64)$

$\mathrm{d} \longrightarrow$ 16: $R=A c, R^{1}=$ NPhth (66)

d 94: $\mathrm{R}=\mathrm{PO}(\mathrm{OPh})_{2}, \mathrm{R}^{1}=\mathrm{H}(52)$

$\mathrm{d} \longrightarrow$ 17: $\mathrm{R}=\mathrm{PO}(\mathrm{OPh})_{2}, \mathrm{R}^{1}=\mathrm{NPhth}(54)$

d 49: $R=T s, R^{1}=H(67)$

$\longrightarrow$ 18: $R=$ Ts, $R^{1}=$ NPhth $(80)$<smiles>CO[C@H]1[C@@H](CO)O[C@H](COP)[C@H](OC)[C@@H]1OC</smiles>

48

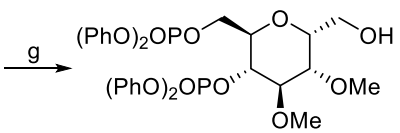

95 (37)

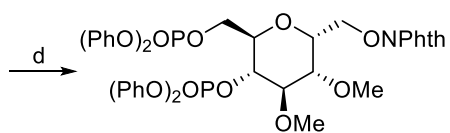

$19(62)$<smiles>CO[C@H]1O[C@H](COS(=O)(=O)O)[C@@H](OC)[C@H](OC)[C@@H]1O</smiles>

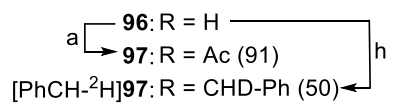<smiles>[R7]C[C@H]1O[C@H](CO[R5])[C@@H]([R6])[C@H](OC)[C@H]1OC</smiles>

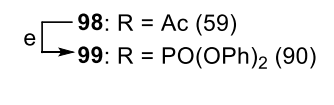

d 51: $R=A c, R^{1}=H(70)$

$\mathrm{d} \longrightarrow$ 20: $\mathrm{R}=\mathrm{Ac}, \mathrm{R}^{1}=$ NPhth (86)

52: $\mathrm{R}=\mathrm{PO}(\mathrm{OPh})_{2}, \mathrm{R}^{1}=\mathrm{H}(64)$

$\mathrm{d} \longrightarrow$ 21: R $=\mathrm{PO}(\mathrm{OPh})_{2}, \mathrm{R}^{1}=$ NPhth $(93)$

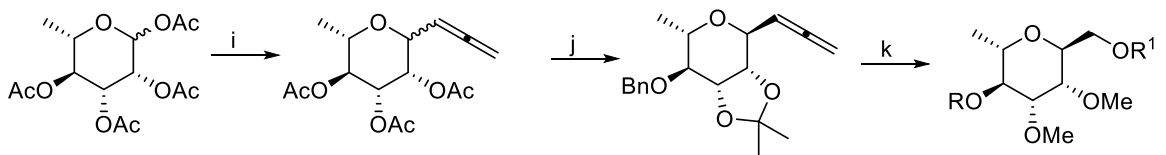

100

$101(1 S)(67)$ $102(1 R)(25)$

$103(54)$

104: $\mathrm{R}=\mathrm{Bn}, \mathrm{R}^{1}=\mathrm{H}(45)$

$\begin{aligned} \mathrm{I} \square \text { 105: } \mathrm{R} & =\mathrm{H}, \mathrm{R}^{1}=\text { NPhth }(57)\end{aligned}$

$\mathrm{m} \longrightarrow$ 22: $\mathrm{R}=\mathrm{PO}(\mathrm{OPh})_{2}, \mathrm{R}^{1}=$ NPhth (84)<smiles>CC(=O)O[C@H]1[C@H](OC(C)=O)[C@@H](C)O[C@H](OC(C)=O)[C@@H]1OC(C)=O</smiles>

106

$107(73)$<smiles>CCCCC</smiles><smiles>C=C1C[C@H]2O[C@H](C)[C@@H](O)[C@@H]2O[C@](C)(OC)[C@@]1(C)OC</smiles><smiles>C[18OH]</smiles><smiles>[R7]C[C@H]1O[C@H](C)[C@@H]([R9])[C@H](OC)[C@H]1OC</smiles>

108 (65)

\footnotetext{
$\begin{aligned} \text { 109: } R & =B n, R^{1}=H(43) \square \\ \text { 58: } R & =A c, R^{1}=H(66) \\ \text { 23: } R & =A c, R^{1}=\text { NPhth }(51 \Varangle \\ \text { a9: } & =P O(O P h)_{2}, R^{1}=H(60) \\ \text { d } & \text { 24: } R=P O(O P h)_{2}, R^{1}=\text { NPhth }(51)\end{aligned}$
}

${ }^{a}$ Reagents and conditions: (a) $\mathrm{Ac}_{2} \mathrm{O}$, Py, DMAP, rt, 0.5-1.5 h. (b) (i) propargyl trimethylsilane/Et $\mathrm{O}_{2} \mathrm{O} 39 \mathrm{v} / \mathrm{v}$, TMSOTf, CH $\mathrm{CN}_{3}$, sonication, rt, 1.5-3 h. (ii) DPSCl, imidazole, DMF, $0{ }^{\circ} \mathrm{C}, 2$ h. (c) (i) $\mathrm{O}_{3}, \mathrm{CH}_{2} \mathrm{Cl}_{2}-\mathrm{MeOH},-78{ }^{\circ} \mathrm{C}$. (ii) $\mathrm{NaBH}_{4}, 0{ }^{\circ} \mathrm{C}$ to rt, $1-3 \mathrm{~h}$. (d) $\mathrm{HONPhth}, \mathrm{Ph}_{3} \mathrm{P}, \mathrm{DEAD}$, $0{ }^{\circ} \mathrm{C}, 1.5 \mathrm{~h}$-overnight. (e) (i) $\mathrm{K}_{2} \mathrm{CO}_{3}, \mathrm{MeOH}$, rt, overnight. (ii) $\mathrm{ClPO}(\mathrm{OPh})_{2}, \mathrm{DMAP} \mathrm{CH}_{2} \mathrm{Cl}_{2}, \mathrm{rt}, 2.5-7$ h. (f) (i) $\mathrm{K}_{2} \mathrm{CO}_{3}, \mathrm{MeOH}$, rt, overnight.

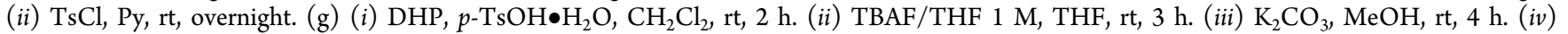
$\mathrm{ClPO}(\mathrm{OPh})_{2}, \mathrm{Py}$, rt, overnight. (h) $\mathrm{NaH} 60 \%$, p-TsO-CHD-Ph, DMF $/ \mathrm{CH}_{2} \mathrm{Cl}_{2}$, rt, 1 h. (i) BF $\bullet$ OEt 2 , TMSOTf, propargyl trimethylsilane,

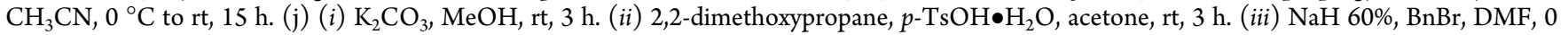
${ }^{\circ} \mathrm{C}, 3$ h. (k) (i) TFA $/ \mathrm{H}_{2} \mathrm{O}$, rt, 2 h. (ii) NaH 60\%, MeI, DMF, $0{ }^{\circ} \mathrm{C}, 1.5$ h. (iii) $\mathrm{O}_{3}, \mathrm{CH}_{2} \mathrm{Cl}_{2}-\mathrm{MeOH}-78{ }^{\circ} \mathrm{C}$. (iv) $\mathrm{NaBH}, 0{ }^{\circ} \mathrm{C}$ to rt, 0.75 h. (l) $($ i) $\mathrm{H}_{2}$, Pd/C 10\%, EtOAc, rt, overnight. (ii) HONPhth, $\mathrm{Ph}_{3} \mathrm{P}, \mathrm{DEAD}, 0{ }^{\circ} \mathrm{C}$ to rt, $3.5 \mathrm{~h} .(\mathrm{m}) \mathrm{ClPO}(\mathrm{OPh})_{2}, \mathrm{DMAP}, \mathrm{CH}_{2} \mathrm{Cl}_{2}, \mathrm{rt}, 2 \mathrm{~h} .(\mathrm{n})(i) \mathrm{K}_{2} \mathrm{CO}_{3}$, $\mathrm{MeOH}, \mathrm{rt}, 3 \mathrm{~h}$. (ii) 2,3-butanedione, $(\mathrm{MeO})_{3} \mathrm{CH}, \mathrm{BF}_{3} \bullet \mathrm{Et}_{2} \mathrm{O}, \mathrm{MeOH}, 60{ }^{\circ} \mathrm{C}, 4.5 \mathrm{~h}$. (o) (i) $\mathrm{NaH} 60 \%, \mathrm{BnBr}, \mathrm{DMF}, 0{ }^{\circ} \mathrm{C}, 2 \mathrm{~h}$. (ii) $\mathrm{TFA} / \mathrm{H}_{2} \mathrm{O}, 40{ }^{\circ} \mathrm{C}$, overnight. (iii) $\mathrm{NaH} 60 \%$, MeI, DMF, $0{ }^{\circ} \mathrm{C}$ to rt, 2 h. (iv) $\mathrm{O}_{3}, \mathrm{CH}_{2} \mathrm{Cl}_{2}-\mathrm{MeOH}-78{ }^{\circ} \mathrm{C}$. (v) $\mathrm{NaBH}, 0{ }^{\circ} \mathrm{C}$ to rt, 1 h. (p) (i) DPSCl, imidazole, DMF, rt, 3 h. (ii) $\mathrm{H}_{2}$, Pd/C 10\%, EtOAc, rt, overnight. (iii) Ac 2 O, Py, DMAP, rt, 0.5 h. (iv) TBAF/THF, 1 M, THF, rt, 4 h. (q) (i) DPSCl, imidazole, DMF, rt, 3 h. (ii) $\mathrm{H}_{2}$, Pd/C 10\%, EtOAc, rt, overnight. (iii) ClPO(OPh) ${ }_{2}$, DMAP, $\mathrm{CH}_{2} \mathrm{Cl}_{2}, \mathrm{rt}, 3.5$ h. (iv) TBAF/THF, $1 \mathrm{M}$, THF, rt, 3.5 h. ${ }^{b}$ Values in parentheses are isolate yields.

In the best of situations, $\mathrm{C}$-(4-O-diphenoxyphosphoryl-2,3di-O-methyl- $\alpha$-L-Rhap) $N$-methoxyphthalimide (22) $\left({ }^{1} C_{4}\right.$, phosphatoxy equatorial) with the Hantzsch ester introduced slowly by a syringe pump, the bicycle $\mathbf{6 1}$ is produced in a yield (61\%) comparable to that obtained with $n-\mathrm{Bu}_{3} \mathrm{SnD} / \mathrm{BF}_{3} \bullet \mathrm{Et}_{2} \mathrm{O}$ (Table 6, compare entries 15 and 17). These photocatalyzed reactions, carried out at room temperature, appear to be strongly influenced by the conformational equilibrium of the pyranosyl ring. However, under the tin hydride conditions (refluxing toluene, $110{ }^{\circ} \mathrm{C}$ ), the TS required for the HAT reaction can be more readily attained.

Preparation of 3-C-(Glycopyranosyl)1-propanol and 3-C-(Glycofuranosyl)1-propanol Models. C-Glycosyl compounds of the 3-C-( $\alpha, \beta$-D,L-glycopyranosyl) 1 -propene type 62 , $65,68,71$, and 75 were synthesized starting from perbenzylated D-glucose, D-mannose, or L-fucose, as required 
in each case, according to the procedure reported by Nicotra et al. (Scheme 4). ${ }^{54}$ Otherwise, for the D-arabinopyranose series, the allylation of $\mathbf{7 8}$ with allyltrimethylsilane and $\mathrm{BF}_{3} \bullet \mathrm{Et}_{2} \mathrm{O}$ gave an inseparable anomeric mixture of allyl derivatives in $69 \%$ yield. The saponification of the acetyl groups and the selective acetal protection by treatment overnight with $\mathrm{PhCH}(\mathrm{OMe})_{2}$ and CSA gave access to $\beta$ - and $\alpha$-phenyl benzylidene substituted products as a mixture of anomers 79 $(\beta / \alpha, 3: 1)$ and $\mathbf{8 0}(\beta / \alpha, 3.5: 1)$ with a free hydroxyl group at C2. Next, oxidative hydroboration of all the allyl compounds mentioned above gave efficiently the corresponding diols 63, $66,69,72,76$, and 81 , whose primary hydroxyl groups were converted selectively to $3-C$ - $(\alpha, \beta$-D,L-glycopyranosyl $) N$-propoxyphthalimides by the reaction with $N$-hydroxyphthalimide via Mitsunobu condensation yielding $64,67,70,73$, and $77 . .^{55}$ There only remains the subsequent protection of the free secondary hydroxyl group as a good LG. We thus prepared the acetyl derivatives $1,3,5,7$, and 9 and the phenyl phosphates 2 , 4, 6, 8, 10, and 11. Finally, acid hydrolysis of the benzylidene acetal in the diastereoisomeric mixture $\mathbf{1 1}$ provides, after chromatographic purification, the pure major $\beta$-diastereomer 12.

In the furanose series, we prepared the corresponding allyl ribose derivative $\mathbf{8 2}$ following a similar strategy to that described before for the D-arabinopyranose model (Scheme 5). ${ }^{56}$ Saponification of the acetyl groups and treatment of the corresponding triol with DPSCl and imidazole in dichloromethane at $0{ }^{\circ} \mathrm{C}$ produced the diprotected product 83 in $36 \%$ yield together with the diol $\mathbf{8 4}$ obtained in $40 \%$ yield. ${ }^{13,57}$ On the other hand, the reaction of 82 with 1,3-dichloro-1,1,3,3tetraisopropyldisiloxane in dry pyridine afforded the monoalcohol 86 in $69 \%$ yield. Once again, oxidative hydroboration of 83 and 86 gave the corresponding diols 85 and 87 . The conversion of the primary alcohol to an $N$-alkoxyphthalimide and the introduction of an LG at C2 afforded the required models: the acetate 13, triflate 14, and phenyl phosphate 15 .

Preparation of C-(Glycopyranosyl)methanol Models. The synthesis of 6,8-dioxabicyclo[3.2.1] heptane scaffolds commenced with the preparation of $C$ - $(4-O$-acetyl- $\alpha$-D,Lglycopyranosyl)allenes 91, 98, 101, 102, and 107 (Scheme 6). To achieve this with high $\alpha$-diastereoselectivity, we employed the ultrasound-assisted $C$-glycosylation described by Murphy et al. using propargyl trimethylsilane and a Lewis acid catalyst. ${ }^{58}$ Next, LG was interchanged from OAc to $\mathrm{PO}(\mathrm{OPh})_{2}$, yielding 92 and 99, and to the tosyl group, giving access to 93, by saponification of the acetyl group and treatment with the corresponding acid chloride in basic media. Subsequent reductive ozonolysis afforded the $C$ - $(\alpha$ glycopyranosyl)methanol derivatives 48, 94, 49, 51, and 52 in good yields. Product 48 was also used as a precursor to prepare a diphosphate substrate 95 to analyze whether competitive migrations of the LGs at C4 and C6 could occur. First, it was necessary to protect the primary $\mathrm{C1}$ '-alcohol as a tetrahydropyranyl (THP) ether; then removal of both the silyl and the acyl protectors gave a diol, which was transformed to a diphosphate by treatment with $\mathrm{ClPO}(\mathrm{OPh})_{2}$ in pyridine overnight. Acid hydrolysis of the THP protector afforded 95 in a $37 \%$ overall yield (four-step). Finally, Mitsunobu condensation of all the primary alcohols mentioned above with $N$ hydroxyphthalimide yielded $C$ - $(\alpha$-glycopyranosyl $) N$-methoxyphthalimide derivatives 16, 17, 18, 19, 20, and 21 in good to excellent yields.
In the case of the L-rhamnose 101 and L-fucose 107 derivatives, it was found necessary to hydrolyze the acetyl groups at C2, C3, and C4 to protect selectively the C2 and C3 hydroxyl groups as cyclic acetals to enable the ulterior introduction of the LG at C4. Once the isopropylidene group was introduced for the L-rhamnose derivative, benzylation of the free alcohol at C4 afforded 103 in 54\% yield. Acid hydrolysis of the transitory acetal assembly, methylation of both $\mathrm{C} 2$ and $\mathrm{C} 3$ hydroxyl groups, and reductive ozonolysis gave monoalcohol $\mathbf{1 0 4}$ in $45 \%$ overall yield. Afterward, palladium-catalyzed hydrogenolysis of the benzyl protective group gave the corresponding diol that was subsequently transformed into the $N$-alkoxyphthalimide 105 in $57 \%$ yield. The corresponding phenyl phosphate 22 was obtained efficiently after $2 \mathrm{~h}$ by treatment with $\mathrm{ClPO}(\mathrm{OPh})_{2}$ and DMAP at room temperature in dichloromethane.

For the L-fucose derivative, butane 2,3-bisacetal protection ${ }^{59}$ was selected to obtain 108 in $65 \%$ yield. Next, a similar strategy as described for the previous model was employed to afford monoalcohol 109 in $43 \%$ overall yield. Transient protection of the primary alcohol as a DPS ether allows the hydrogenolysis of the benzyl ether and the introduction of the LG at C4. Therefore, the formation of the acetate or phenyl phosphate followed by DPS removal (TBAF) allowed the generation of $\mathbf{5 8}$ and $\mathbf{5 9}$ in good overall yields. The conversion of the corresponding primary alcohols to $\mathrm{N}$-alkoxyphthalimides occurred in $51 \%$ yield for both substrates to generate 23 and 24, respectively.

\section{EXPERIMENTAL SECTION}

General Information. Commercially available reagents and solvents were analytical grade or were purified by standard procedures prior to use. Solvents for starting material preparation and radical reactions were dried before use. The spray reagents for TLC analysis were conducted with $0.5 \%$ vanillin in $\mathrm{H}_{2} \mathrm{SO}_{4}-\mathrm{EtOH}$ (4:1) or, in some specific cases, with the Pancaldi reagent $\left\{\left(\mathrm{NH}_{4}\right)_{6} \mathrm{MoO}_{4}, \mathrm{Ce}\left(\mathrm{SO}_{4}\right)_{2}\right.$, $\left.\mathrm{H}_{2} \mathrm{SO}_{4}, \mathrm{H}_{2} \mathrm{O}\right\}^{60}$ and further heating until the development of color. Melting points were determined with a hot-stage apparatus. Optical rotations were measured at the sodium line at the ambient temperature in $\mathrm{CHCl}_{3}$ solutions. IR spectra were measured as thin films on $\mathrm{CHCl}_{3}$ solutions. NMR spectra were determined at 500 or $400 \mathrm{MHz}$ for ${ }^{1} \mathrm{H}$ and at 125.7 or $100 \mathrm{MHz}$ for ${ }^{13} \mathrm{C}\left\{{ }^{1} \mathrm{H}\right\}$ in $\mathrm{CDCl}_{3}$ or $\mathrm{C}_{6} \mathrm{D}_{6}$ as stated. The chemical shifts are given in parts per million (ppm) relative to TMS at $\delta 0.00 \mathrm{ppm}$ or to residual $\mathrm{CDCl}_{3}$ at $\delta 7.26$ ppm for proton spectra and relative to $\mathrm{CDCl}_{3}$ at $\delta 77.00 \mathrm{ppm}$ for carbon spectra. NMR spectra were assigned with the aid of $1 \mathrm{D}$ and $2 \mathrm{D}$ techniques, including ${ }^{13} \mathrm{C}$ DEPT-135, COSY, HSQC, HMBC, and NOESY. The DAISY program as implemented in the TopSpin 4.0.6 software package was used for the simulation of ${ }^{1} \mathrm{H}$ NMR spectra. Low- and high-resolution mass spectra were recorded by using an electrospray $\left(\mathrm{ESI}^{+}\right)$and TOF analyzer. Flash column chromatography was performed on a Merck silica gel $60 \mathrm{PF}(0.063-0.2 \mathrm{~mm})$. For the chromatography of the radical reactions with $n-\mathrm{Bu}_{3} \mathrm{SnH}$ or $n-\mathrm{Bu}_{3} \mathrm{SnD}$, $10 \% \mathrm{KF}$ was added and mixed with the silica gel. Circular layers of 1 and $2 \mathrm{~mm}$ of the Merck silica gel $60 \mathrm{PF}_{254}$ were used on a Chromatotron for centrifugally assisted chromatography. HPLC separations were undertaken using a semipreparative $(10 \times 250$ $\mathrm{mm}$ ) Ascentis Si normal-phase column. An ultrasonic bath was used (2510E-DTH, Branson) for the synthesis of the allene precursors and for the deoxygenation of the THF for the photocatalytic reactions. Photochemical reactions were carried out with $15 \mathrm{~W}$ blue LEDs (468 $\mathrm{nm}$ peak wavelength, $25 \mathrm{~nm}$ spectral half-wave width, composed of 15 LED units each with $1 \mathrm{~W}, 3 \mathrm{~V}, 300 \mathrm{~mA}, 5 \mathrm{~cm}$ distance from the light source to the irradiation vessel). For convenience, the atomnumbering system used along this section and in the assignments of the Experimental Section corresponds to the one depicted in 
structures of the schemes and tables, although an IUPAC systematic nomenclature has been used throughout this paper. The IUPAC nomenclature for deuterated carbohydrates (2-Carb-16.6, with the parentheses indicating substitution and square brackets for partial labeling) has been used throughout the manuscript.

General Methods for Radical and Photoredox Reactions (Tables 1-6). Method A: Fast Addition of $n-\mathrm{Bu}_{3} \mathrm{SnH}$. A solution of the phthalimide $(1 \mathrm{mmol})$ in dry toluene $(75 \mathrm{~mL})$ was treated with $n$ $\mathrm{Bu}_{3} \mathrm{SnH}(269 \mu \mathrm{L}, 1 \mathrm{mmol})$ and $\mathrm{AIBN}(16.4 \mathrm{mg}, 0.1 \mathrm{mmol})$ and heated under reflux. Every hour, the same quantity of AIBN was added. In some cases, a supplementary addition of $n-\mathrm{Bu}_{3} \mathrm{SnH}(269$ $\mu \mathrm{L}, 1 \mathrm{mmol}$ ) was required as indicated. When all the starting material was consumed, the reaction mixture was directly poured into a column chromatography on a silica gel with $10 \% \mathrm{KF}$ (hexanes to hexanes-EtOAc) to give the corresponding products.

Method B: Slow Addition of $n-\mathrm{Bu}_{3} \mathrm{SnH}$ (1 equiv/h). A solution of the phthalimide $(1 \mathrm{mmol})$ in dry toluene $(75 \mathrm{~mL})$ was treated with AIBN (16.4 mg, $0.1 \mathrm{mmol})$, and $n$ - $\mathrm{Bu}_{3} \mathrm{SnH}(269 \mu \mathrm{L}, 1 \mathrm{mmol})$ was dropwise added during $1 \mathrm{~h}$ by means of a syringe pump under reflux. Every hour, the same quantity of AIBN was added. In some cases, a supplementary addition of $n-\mathrm{Bu}_{3} \mathrm{SnH}(269 \mu \mathrm{L}, 1 \mathrm{mmol})$ was required as indicated. When all the starting material was consumed, the reaction mixture was directly poured into a column chromatography on a silica gel with $10 \% \mathrm{KF}$ (hexanes to hexanes-EtOAc) to give the corresponding products.

Method C: Fast Addition of TTMSS. A solution of the phthalimide $(1 \mathrm{mmol})$ in dry toluene $(75 \mathrm{~mL})$ was treated with AIBN $(16.4 \mathrm{mg}$, $0.1 \mathrm{mmol})$ and TTMSS $(308.5 \mu \mathrm{L}, 1 \mathrm{mmol})$ and heated under reflux. Every hour, the same quantity of AIBN was added. In some cases, a supplementary addition of TTMSS $(308.5 \mu \mathrm{L}, 1 \mathrm{mmol})$ was required as indicated. When all the starting material was consumed, the reaction mixture was evaporated and purified by column chromatography (hexanes-EtOAc) to give the corresponding products.

Method D: Fast Addition of $n-B u_{3} S n D$. A solution of the phthalimide $(1 \mathrm{mmol})$ in dry toluene $(75 \mathrm{~mL})$ was treated with $n$ $\mathrm{Bu}_{3} \mathrm{SnD}(270.4 \mu \mathrm{L}, 1 \mathrm{mmol})$ and $\operatorname{AIBN}(16.4 \mathrm{mg}, 0.1 \mathrm{mmol})$ and heated under reflux. Every hour, the same quantity of AIBN was added. In some cases, a supplementary addition of $n-\mathrm{Bu}_{3} \mathrm{SnD}(270.4$ $\mu \mathrm{L}, 1 \mathrm{mmol}$ ) was required as indicated. When all the starting material was consumed, the reaction mixture was directly poured into a column chromatography on a silica gel with $10 \% \mathrm{KF}$ (hexanes to hexanes-EtOAc) to give the corresponding products.

Method E: Fast Addition of $n-B u_{3} S n D$ and $B F_{3} \bullet E t_{2} O$. A solution of the phthalimide $(1 \mathrm{mmol})$ in dry toluene $(75 \mathrm{~mL})$ was treated with $n-\mathrm{Bu}_{3} \mathrm{SnD}(270.4 \mu \mathrm{L}, 1 \mathrm{mmol}), \mathrm{BF}_{3} \bullet \mathrm{Et}_{2} \mathrm{O}(24.7 \mu \mathrm{L}, 0.2 \mathrm{mmol})$, and AIBN (16.4 mg, $0.11 \mathrm{mmol}$ ) and heated at $100{ }^{\circ} \mathrm{C}$. Every hour, the same quantity of AIBN was added. In some cases, a supplementary addition of $n-\mathrm{Bu}_{3} \mathrm{SnD}(270.4 \mu \mathrm{L}, 1 \mathrm{mmol})$ and $\mathrm{BF}_{3} \bullet \mathrm{Et}_{2} \mathrm{O}(24.7 \mu \mathrm{L}$, $0.2 \mathrm{mmol}$ ) was required as indicated. When all the starting material was consumed, the reaction mixture was directly poured into a column chromatography on a silica gel with $10 \% \mathrm{KF}$ (hexanes to hexanes-EtOAc) to give the corresponding products.

Method F: Photoredox Conditions. A deoxygenated solution of the phthalimide $(1 \mathrm{mmol})$, Hantzsch ester $(278.6 \mathrm{mg}, 1.1 \mathrm{mmol})$, and fac-Ir $(\mathrm{ppy})_{3}(6.5 \mathrm{mg}, 0.01 \mathrm{mmol})$ in dry THF $(148.7 \mathrm{~mL})$ was placed in a Schlenk tube under nitrogen and irradiated with blue LEDs at room temperature. The reaction mixture was concentrated and purified directly by chromatotron (hexanes-EtOAc) to give the corresponding products.

Method G: Photoredox Conditions with Slow Addition of Hantzsch Ester. A deoxygenated solution of the phthalimide (1 $\mathrm{mmol})$ and $f a c-\mathrm{Ir}(\mathrm{ppy})_{3}(6.5 \mathrm{mg}, 0.01 \mathrm{mmol})$ in dry THF $(116 \mathrm{~mL})$ was placed in a Schlenk tube under nitrogen and irradiated with blue LEDs at room temperature. A solution of Hantzsch ester $(279.1 \mathrm{mg}$, $1.1 \mathrm{mmol})$ in dry THF $(34.9 \mathrm{~mL})$ was then slowly added with a syringe pump over a period of $3 \mathrm{~h}$. The reaction mixture was concentrated and purified directly by chromatotron (hexanes-EtOAc, $8: 2$ to $1: 1)$ to give the corresponding products.

Synthesis of 10-Deoxy-1,6-dioxaspiro[4.5]decane Structures (Tables 1-3). Radical Reactions of 1. Method A. Following the general procedure, starting from substrate $1(62.8 \mathrm{mg}, 0.092$ mmol), after $2 \mathrm{~h}$ of reaction, a supplementary addition of $n-\mathrm{Bu}_{3} \mathrm{SnH}$ $(25 \mu \mathrm{L}, 0.092 \mathrm{mmol})$ was required. All the starting material was consumed after $3 \mathrm{~h}$. Column chromatography (hexanes to hexanesEtOAc, 1:1) gave (4S)-1,4-anhydro-6,7,9-tri-O-benzyl-2,3,5-trideoxyD-arabino-non-4-ulopyranose (25) (18.6 $\mathrm{mg}, 0.039 \mathrm{mmol}, 43 \%)$ as an amorphous solid, 3-C-(2-O-acetyl-3,4,6-tri-O-benzyl- $\beta$-Dglucopyranosyl)1-propanol (26ק) $(6.9 \mathrm{mg}, 0.013 \mathrm{mmol}, 14 \%)$ as a colorless oil, and 3-C-(2-O-acetyl-3,4,6-tri-O-benzyl- $\alpha$-D-

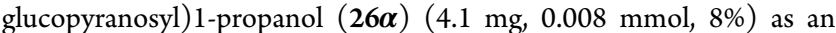
amorphous solid. Compound 25: $[\alpha]_{\mathrm{D}}=+40.0\left(c=0.18, \mathrm{CHCl}_{3}\right) .{ }^{1} \mathrm{H}$ NMR $\left(500 \mathrm{MHz}, \mathrm{CDCl}_{3}\right.$, simulated ring coupling constants using DAISY) $\delta_{\mathrm{H}} 7.34-7.18(\mathrm{~m}, 15 \mathrm{H}, \mathrm{Ar}), 4.89(\mathrm{~d}, J=11.1 \mathrm{~Hz}, 1 \mathrm{H}, \mathrm{OBn})$, $4.66(\mathrm{~d}, J=11.7 \mathrm{~Hz}, 1 \mathrm{H}, \mathrm{OBn}), 4.62(\mathrm{~d}, J=11.7 \mathrm{~Hz}, 1 \mathrm{H}, \mathrm{OBn}), 4.61$ $(\mathrm{d}, J=12.3 \mathrm{~Hz}, 1 \mathrm{H}, \mathrm{OBn}), 4.54(\mathrm{~d}, J=11.0 \mathrm{~Hz}, 1 \mathrm{H}, \mathrm{OBn}), 4.51(\mathrm{~d}, J$ $=12.3 \mathrm{~Hz}, 1 \mathrm{H}, \mathrm{OBn}$ ), 3.990 (ddd, $J=11.5,8.9,5.1 \mathrm{~Hz}, 1 \mathrm{H}, 3-\mathrm{H})$, 3.89 (ddd, $J=8.2,8.2,5.4 \mathrm{~Hz}, 1 \mathrm{H}, 3^{\prime}-\mathrm{H}_{\mathrm{b}}$ ), 3.83 (ddd, $J=8.2,8.2,6.3$ $\mathrm{Hz}, 1 \mathrm{H}, 3^{\prime}-\mathrm{H}_{\mathrm{a}}$ ), 3.79 (ddd, $\left.J=9.9,4.3,1.9 \mathrm{~Hz}, 1 \mathrm{H}, 5-\mathrm{H}\right), 3.74(\mathrm{dd}, J=$ $10.8,4.3 \mathrm{~Hz}, 1 \mathrm{H}, 6-\mathrm{H}_{\mathrm{b}}$ ), $3.64\left(\mathrm{dd}, J=10.8,1.9 \mathrm{~Hz}, 1 \mathrm{H}, 6-\mathrm{H}_{\mathrm{a}}\right), 3.57$ $(\mathrm{dd}, J=9.9,8.9 \mathrm{~Hz}, 1 \mathrm{H}, 4-\mathrm{H}), 2.222\left(\mathrm{dd}, J=12.7,5.1 \mathrm{~Hz}, 1 \mathrm{H}, 2-\mathrm{H}_{\mathrm{b}}\right)$, $2.12-2.00\left(\mathrm{~m}, 2 \mathrm{H}, 1^{\prime}-\mathrm{H}_{\mathrm{b}}, 2^{\prime}-\mathrm{H}_{\mathrm{b}}\right), 1.872(\mathrm{dd}, J=12.7,11.5 \mathrm{~Hz}, 1 \mathrm{H}, 2-$ $\left.\mathrm{H}_{\mathrm{a}}\right), 1.85\left(\mathrm{~m}, 1 \mathrm{H}, 2^{\prime}-\mathrm{H}_{\mathrm{a}}\right), 1.76 \mathrm{ppm}\left(\mathrm{m}, 1 \mathrm{H}, 11^{\prime}-\mathrm{H}_{\mathrm{a}}\right) .{ }^{13} \mathrm{C}\left\{{ }^{1} \mathrm{H}\right\} \mathrm{NMR}$ $\left(100.6 \mathrm{MHz}, \mathrm{CDCl}_{3}\right) \delta_{\mathrm{C}} 138.9(2 \times \mathrm{C}, \mathrm{Ar}), 138.5(\mathrm{C}, \mathrm{Ar}), 128.34(2$ $\times \mathrm{CH}, \mathrm{Ar}), 128.28(2 \times \mathrm{CH}, \mathrm{Ar}), 128.2(2 \times \mathrm{CH}, \mathrm{Ar}), 127.76(2 \times$ $\mathrm{CH}, \mathrm{Ar}), 127.74(2 \times \mathrm{CH}, \mathrm{Ar}), 127.6(2 \times \mathrm{CH}, \mathrm{Ar}), 127.5(2 \times \mathrm{CH}$, $\mathrm{Ar}), 127.4,(\mathrm{CH}, \mathrm{Ar}), 106.33$ (C, C-1), $79.2(\mathrm{CH}, \mathrm{C}-4), 78.51(\mathrm{CH}$, C-3), $74.7\left(\mathrm{CH}_{2}, \mathrm{OBn}\right), 73.3\left(\mathrm{CH}_{2}, \mathrm{OBn}\right), 71.8\left(\mathrm{CH}_{2}, \mathrm{OBn}\right), 71.8$ (CH, C-5), $69.4\left(\mathrm{CH}_{2}, \mathrm{C}-6\right), 67.3\left(\mathrm{CH}_{2}, \mathrm{C}-3\right), 38.62\left(\mathrm{CH}_{2}, \mathrm{C}-2\right)$, $37.2\left(\mathrm{CH}_{2}, \mathrm{C}^{\prime} 1^{\prime}\right), 23.5 \mathrm{ppm}\left(\mathrm{CH}_{2}, \mathrm{C}-2^{\prime}\right)$. IR $\left(\mathrm{CHCl}_{3}\right): \nu=3009$, 2939, 1456, $1089 \mathrm{~cm}^{-1}$. MS (ESI) $\mathrm{m} / z(\%)=497(100)[\mathrm{M}+\mathrm{Na}]^{+}$. HRMS (ESI) $m / z$ : $[\mathrm{M}+\mathrm{Na}]^{+}$calcd for $\mathrm{C}_{30} \mathrm{H}_{34} \mathrm{NaO}_{5}$ 497.2304; found 497.2303. Anal. calcd for $\mathrm{C}_{30} \mathrm{H}_{34} \mathrm{O}_{5}: \mathrm{C}, 75.92 ; \mathrm{H}, 7.12$. Found: C, 76.07; H, 7.21. Compound 26ק: $[\alpha]_{\mathrm{D}}=+12.3\left(c=0.43, \mathrm{CHCl}_{3}\right)$. ${ }^{1} \mathrm{H}$ NMR $\left(500 \mathrm{MHz}, \mathrm{CDCl}_{3}\right) \delta_{\mathrm{H}} 7.34-7.15(\mathrm{~m}, 15 \mathrm{H}, \mathrm{Ar}), 4.887(\mathrm{dd}$, $J=9.3,9.3 \mathrm{~Hz}, 1 \mathrm{H}, 2-\mathrm{H}), 4.81(\mathrm{~d}, J=11.4 \mathrm{~Hz}, 1 \mathrm{H}, \mathrm{OBn}), 4.77(\mathrm{~d}, J=$ $10.9 \mathrm{~Hz}, 1 \mathrm{H}, \mathrm{OBn}), 4.65(\mathrm{~d}, J=11.4 \mathrm{~Hz}, 1 \mathrm{H}, \mathrm{OBn}), 4.59(\mathrm{~d}, J=12.2$ $\mathrm{Hz}, 1 \mathrm{H}, \mathrm{OBn}), 4.53(\mathrm{~d}, J=12.2 \mathrm{~Hz}, 1 \mathrm{H}, \mathrm{OBn}), 4.52(\mathrm{~d}, J=10.6 \mathrm{~Hz}$, $1 \mathrm{H}, \mathrm{OBn}), 3.68\left(\mathrm{dd}, J=10.9,1.9 \mathrm{~Hz}, 1 \mathrm{H}, 6-\mathrm{H}_{\mathrm{b}}\right), 3.66-3.59(\mathrm{~m}, 5 \mathrm{H}$, 3 '- $\left.\mathrm{H}_{2}, 3-\mathrm{H}, 4-\mathrm{H}, 6-\mathrm{H}_{2}\right), 3.46(\mathrm{~m}, 1 \mathrm{H}, 5-\mathrm{H}), 3.34$ (ddd, $J=9.8,9.8,2.4$ $\mathrm{Hz}, 1 \mathrm{H}, 1-\mathrm{H}), 1.94(\mathrm{~s}, 3 \mathrm{H}, \mathrm{OAc}), 1.75-1.61\left(\mathrm{~m}, 3 \mathrm{H}, 1^{\prime}-\mathrm{H}_{\mathrm{b}}, 2^{\prime}-\mathrm{H}_{2}\right)$, $1.509 \mathrm{ppm}\left(\mathrm{m}, 1 \mathrm{H}, 1\right.$ '- $\left.\mathrm{H}_{\mathrm{a}}\right), 1 \mathrm{H}$ from $\mathrm{OH}$ is missing. ${ }^{13} \mathrm{C}\left\{{ }^{1} \mathrm{H}\right\} \mathrm{NMR}$ $\left(100.6 \mathrm{MHz}, \mathrm{CDCl}_{3}\right) \delta_{\mathrm{C}} 169.9$ (C, OAc), 138.4 (C, Ar), 138.1 (C, $\mathrm{Ar}), 138.0(\mathrm{C}, \mathrm{Ar}), 128.42(2 \times \mathrm{CH}, \mathrm{Ar}), 128.41(2 \times \mathrm{CH}, \mathrm{Ar})$, $128.37(2 \times \mathrm{CH}, \mathrm{Ar}), 128.0(2 \times \mathrm{CH}, \mathrm{Ar}), 127.8(3 \times \mathrm{CH}, \mathrm{Ar})$, $127.70(2 \times \mathrm{CH}, \mathrm{Ar}), 127.67(\mathrm{CH}, \mathrm{Ar}), 127.6(\mathrm{CH}, \mathrm{Ar}), 84.7(\mathrm{CH}$, C-3), $79.0(\mathrm{CH}, \mathrm{C}-4), 78.5(\mathrm{CH}, \mathrm{C}-5), 78.0(\mathrm{CH}, \mathrm{C}-1), 75.2\left(\mathrm{CH}_{2}\right.$ $\mathrm{OBn}), 75.0\left(\mathrm{CH}_{2}, \mathrm{OBn}\right), 73.81(\mathrm{CH}, \mathrm{C}-2), 73.5\left(\mathrm{CH}_{2}, \mathrm{OBn}\right), 69.1$ $\left(\mathrm{CH}_{2}, \mathrm{C}-6\right), 62.7\left(\mathrm{CH}_{2}, \mathrm{C}-3^{\prime}\right), 28.84\left(\mathrm{CH}_{2}, \mathrm{C}-2^{\prime}\right), 28.24\left(\mathrm{CH}_{2}, \mathrm{C}-1^{\prime}\right)$, $20.9 \mathrm{ppm}\left(\mathrm{CH}_{3}, \mathrm{OAc}\right)$. IR $\left(\mathrm{CHCl}_{3}\right): \nu=3430,3014,1738,1229$, $1039 \mathrm{~cm}^{-1}$. MS (ESI) $\mathrm{m} / z(\%)=557(100)[\mathrm{M}+\mathrm{Na}]^{+}$. HRMS (ESI) $m / z:[\mathrm{M}+\mathrm{Na}]^{+}$calcd for $\mathrm{C}_{32} \mathrm{H}_{38} \mathrm{NaO}_{7}$ 557.2515; found 557.2513. Anal. calcd for $\mathrm{C}_{32} \mathrm{H}_{38} \mathrm{O}_{7}$ : C, 71.89; $\mathrm{H}, 7.16$. Found: $\mathrm{C}$, 71.86; $\mathrm{H}$, 7.37. Compound 26 $\alpha$ : $[\alpha]_{\mathrm{D}}=+41.4\left(c=0.36, \mathrm{CHCl}_{3}\right) .{ }^{1} \mathrm{H}$ NMR $\left(500 \mathrm{MHz}, \mathrm{CDCl}_{3}\right) \delta_{\mathrm{H}} 7.34-7.14(\mathrm{~m}, 15 \mathrm{H}, \mathrm{Ar}), 5.03(\mathrm{dd}, J=8.5,5.4$ $\mathrm{Hz}, 1 \mathrm{H}, 2-\mathrm{H}), 4.75(\mathrm{~d}, J=11.7 \mathrm{~Hz}, 1 \mathrm{H}, \mathrm{OBn}), 4.70(\mathrm{~d}, J=11.0 \mathrm{~Hz}$, $1 \mathrm{H}, \mathrm{OBn}), 4.70(\mathrm{~d}, J=11.0 \mathrm{~Hz}, 1 \mathrm{H}, \mathrm{OBn}), 4.59(\mathrm{~d}, J=12.0 \mathrm{~Hz}, 1 \mathrm{H}$, OBn), $4.51(\mathrm{~d}, J=12.0 \mathrm{~Hz}, 1 \mathrm{H}, \mathrm{OBn}), 4.48(\mathrm{~d}, J=11.1 \mathrm{~Hz}, 1 \mathrm{H}$, OBn), $4.13(\mathrm{~m}, 1 \mathrm{H}, 1-\mathrm{H}), 3.81(\mathrm{dd}, J=8.2,8.2 \mathrm{~Hz}, 1 \mathrm{H}, 3-\mathrm{H}), 3.75$ (ddd, $J=8.2,3.8,3.8 \mathrm{~Hz}, 1 \mathrm{H}, 5-\mathrm{H}), 3.71-3.63\left(\mathrm{~m}, 4 \mathrm{H}, 3-\mathrm{H}_{2}, 6-\mathrm{H}_{2}\right)$, $3.60(\mathrm{dd}, J=8.2,8.2 \mathrm{~Hz}, 1 \mathrm{H}, 4-\mathrm{H}), 1.99(\mathrm{~s}, 3 \mathrm{H}, \mathrm{OAc}), 1.87(\mathrm{br} \mathrm{s}, 1 \mathrm{H}$, $\mathrm{OH}), 1.80\left(\mathrm{~m}, 1 \mathrm{H}, 1^{\prime}-\mathrm{H}_{\mathrm{b}}\right), 1.70-1.58\left(\mathrm{~m}, 2 \mathrm{H}, 2{ }^{\prime}-\mathrm{H}_{2}\right), 1.51 \mathrm{ppm}(\mathrm{m}$, $\left.1 \mathrm{H}, 1^{\prime}-\mathrm{H}_{\mathrm{a}}\right) .{ }^{13} \mathrm{C}\left\{{ }^{1} \mathrm{H}\right\} \mathrm{NMR}\left(100.6 \mathrm{MHz}, \mathrm{CDCl}_{3}\right) \delta_{\mathrm{C}} 170.0(\mathrm{C}, \mathrm{OAc})$, 138.3 (C, Ar), $138.0(2 \times \mathrm{C}, \mathrm{Ar}), 127.6-128.4(15 \times \mathrm{CH}, \mathrm{Ar}), 79.9$ (CH, C-3), $77.5(\mathrm{CH}, \mathrm{C}-4), 74.6\left(\mathrm{CH}_{2}, \mathrm{OBn}\right), 74.5\left(\mathrm{CH}_{2}, \mathrm{OBn}\right)$, $73.4\left(\mathrm{CH}_{2}, \mathrm{OBn}\right), 73.0(\mathrm{CH}, \mathrm{C}-2), 72.4(\mathrm{CH}, \mathrm{C}-1), 72.0(\mathrm{CH}, \mathrm{C}-5)$, 69.1 $\left(\mathrm{CH}_{2}, \mathrm{C}-6\right), 62.2\left(\mathrm{CH}_{2}, \mathrm{C}-3^{\prime}\right), 28.9\left(\mathrm{CH}_{2}, \mathrm{C}-2^{\prime}\right), 22.5\left(\mathrm{CH}_{2}, \mathrm{C}-\right.$ 1'), $20.9 \mathrm{ppm}\left(\mathrm{CH}_{3}, \mathrm{OAc}\right)$. IR $\left(\mathrm{CHCl}_{3}\right): \nu=3477,3014,2942,1740$, $1236,1100 \mathrm{~cm}^{-1}$. MS (ESI) $\mathrm{m} / z(\%)=557(100)[\mathrm{M}+\mathrm{Na}]^{+}$. HRMS (ESI) $m / z:[\mathrm{M}+\mathrm{Na}]^{+}$calcd for $\mathrm{C}_{32} \mathrm{H}_{38} \mathrm{NaO}_{7}$ 557.2515; found 
557.2515. Anal. calcd for $\mathrm{C}_{32} \mathrm{H}_{38} \mathrm{O}_{7}$ : C, 71.89; $\mathrm{H}, 7.16$. Found: $\mathrm{C}$, 71.57; H, 7.51.

Method B. Following the general procedure, starting from substrate $1(123.9 \mathrm{mg}, 0.18 \mathrm{mmol})$, after $2 \mathrm{~h}$ of reaction, two more equivalents of $n-\mathrm{Bu}_{3} \mathrm{SnH}(98 \mu \mathrm{L}, 0.36 \mathrm{mmol})$ added by a syringe pump were required. All the starting material was consumed after $14 \mathrm{~h}$. Column chromatography (hexanes to hexanes-EtOAc, 1:1) gave 25 (43.4 mg, $0.09 \mathrm{mmol}, 50 \%)$ and $\mathbf{2 6 \alpha}(14.1 \mathrm{mg}, 0.026 \mathrm{mmol}, 15 \%)$.

Method $C$. Following the general procedure, starting from substrate $1(62.8 \mathrm{mg}, 0.092 \mathrm{mmol})$, after $2 \mathrm{~h}$ of reaction, a supplementary addition of TTMSS $(28.5 \mu \mathrm{L}, 0.092 \mathrm{mmol})$ was required. All the starting material was consumed after $5 \mathrm{~h}$. Column chromatography (hexanes-EtOAc, 9:1 to 6:4) gave $25(8.4 \mathrm{mg}, 0.018 \mathrm{mmol}, 19 \%)$ and the reduced product $26 \alpha(1.5 \mathrm{mg}, 0.003 \mathrm{mmol}, 3 \%)$.

Method $D$. Following the general procedure, starting from substrate 1 ( $94 \mathrm{mg}, 0.14 \mathrm{mmol})$, after $2 \mathrm{~h}$ of reaction, a supplementary addition of $n-\mathrm{Bu}_{3} \mathrm{SnD}(37 \mu \mathrm{L}, 0.14 \mathrm{mmol})$ was required. All the starting material was consumed after $5 \mathrm{~h}$. Column chromatography (hexanes to hexanes-EtOAc, 1:1) gave (4S)-1,4-anhydro-6,7,9-tri-Obenzyl-2,3,5-trideoxy-D- $\left(5-{ }^{2} \mathrm{H}\right)$ arabino-non-4-ulopyranose [ $\left.\left(2-{ }^{2} \mathrm{H}\right) 25\right]$ $\left(28.5 \mathrm{mg}, 0.060 \mathrm{mmol}, 43 \%, 2 \beta-{ }^{2} \mathrm{H} / 2 \alpha-{ }^{2} \mathrm{H}, 7: 3\right)$ as an amorphous solid, 3-C-(2-O-acetyl-3,4,6-tri-O-benzyl- $\beta$-D- $\left(1-{ }^{2} \mathrm{H}\right)$ glucopyranosyl $) 1$ propanol $\left[\left(1-{ }^{2} \mathrm{H}\right) \mathbf{2 6 \boldsymbol { \beta }}\right](4.8 \mathrm{mg}, 0.009 \mathrm{mmol}, 6 \%)$ as a colorless oil, and the prematurely reduced product $26 \boldsymbol{\alpha}(3 \mathrm{mg}, 0.006 \mathrm{mmol}, 4 \%)$. Compound $\left(2-{ }^{2} \mathrm{H}\right) 25:{ }^{1} \mathrm{H}$ NMR $\left(400 \mathrm{MHz}, \mathrm{CDCl}_{3}\right.$, simulated ring coupling constants using DAISY) $\delta_{\mathrm{H}} 7.35-7.18(\mathrm{~m}, 15 \mathrm{H}, \mathrm{Ar}) .4 .89$ (d, $J=11.1 \mathrm{~Hz}, 1 \mathrm{H}, \mathrm{OBn}$ ), 4.66 (d, $J=11.7 \mathrm{~Hz}, 1 \mathrm{H}, \mathrm{OBn}), 4.61$ (d, $J$ $=12.3 \mathrm{~Hz}, 1 \mathrm{H}, \mathrm{OBn}), 4.61(\mathrm{~d}, J=12.3 \mathrm{~Hz}, 1 \mathrm{H}, \mathrm{OBn}), 4.54(\mathrm{~d}, J=$ $11.0 \mathrm{~Hz}, 1 \mathrm{H}, \mathrm{OBn}), 4.51(\mathrm{~d}, J=12.3 \mathrm{~Hz}, 1 \mathrm{H}, \mathrm{OBn}), 3.987$ [dd, $J=$ 8.9, $5.1 \mathrm{~Hz}, 0.7 \mathrm{H}, 3-\mathrm{H}\left(\right.$ from $\left.\left.2 \beta-{ }^{2} \mathrm{H}\right)\right], 3.987[\mathrm{dd}, J=11.5,8.9 \mathrm{~Hz}$, $0.3 \mathrm{H}, 3-\mathrm{H}\left(\right.$ from $\left.\left.2 \alpha{ }^{2} \mathrm{H}\right)\right], 3.89$ (ddd, $\left.J=8.2,8.2,5.3 \mathrm{~Hz}, 1 \mathrm{H}, 3^{\prime}-\mathrm{H}_{\mathrm{b}}\right)$, $3.83\left(\mathrm{ddd}, J=8.2,8.2,6.6 \mathrm{~Hz}, 1 \mathrm{H}, 3^{\prime}-\mathrm{H}_{\mathrm{a}}\right.$ ), 3.79 (ddd, $J=9.9,4.2,1.7$ $\mathrm{Hz}, 1 \mathrm{H}, 5-\mathrm{H}), 3.74\left(\mathrm{dd}, J=10.7,4.2 \mathrm{~Hz}, 1 \mathrm{H}, 6-\mathrm{H}_{\mathrm{b}}\right), 3.65(\mathrm{dd}, J=$ 10.7, $1.7 \mathrm{~Hz}, 1 \mathrm{H}, 6-\mathrm{H}_{\mathrm{a}}$ ), 3.57 (dd, $\left.J=9.9,8.9 \mathrm{~Hz}, 1 \mathrm{H}, 4-\mathrm{H}\right), 2.200$ (d, $J=5.1 \mathrm{~Hz}, 0.7 \mathrm{H}, 2 \alpha-\mathrm{H}), 2.13-1.99\left(\mathrm{~m}, 2 \mathrm{H}, 1^{\prime}-\mathrm{H}_{\mathrm{b}}, 2^{\prime}-\mathrm{H}_{\mathrm{b}}\right), 1.86(\mathrm{~m}$, $\left.1 \mathrm{H}, 2^{\prime}-\mathrm{H}_{\mathrm{a}}\right), 1.85(\mathrm{~d}, J=11.5 \mathrm{~Hz}, 0.3 \mathrm{H}, 2 \beta-\mathrm{H}), 1.74 \mathrm{ppm}\left(\mathrm{m}, 1 \mathrm{H}, \mathrm{l}^{\prime}-\right.$ $\left.\mathrm{H}_{\mathrm{a}}\right) .{ }^{13} \mathrm{C}\left\{{ }^{1} \mathrm{H}\right\}$ NMR $\left(100.6 \mathrm{MHz}, \mathrm{CDCl}_{3}\right) \delta_{\mathrm{C}} 138.9(2 \times \mathrm{C}, \mathrm{Ar})$, 138.5 (C, Ar), 128.33 ( $2 \times \mathrm{CH}, \mathrm{Ar}), 128.28(2 \times \mathrm{CH}, \mathrm{Ar}), 128.2(2 \times$ $\mathrm{CH}, \mathrm{Ar}), 127.76(2 \times \mathrm{CH}, \mathrm{Ar}), 127.74(2 \times \mathrm{CH}, \mathrm{Ar}), 127.6(2 \times \mathrm{CH}$, $\mathrm{Ar}), 127.5(2 \times \mathrm{CH}, \mathrm{Ar}), 127.4,(\mathrm{CH}, \mathrm{Ar}), 106.30$ (C, C-1), 79.1 (CH, C-4), $78.50(\mathrm{CH}, \mathrm{C}-3), 74.7\left(\mathrm{CH}_{2}, \mathrm{OBn}\right), 73.3\left(\mathrm{CH}_{2}, \mathrm{OBn}\right)$, $71.8\left(\mathrm{CH}_{2}, \mathrm{OBn}\right), 71.8(\mathrm{CH}, \mathrm{C}-5), 69.4\left(\mathrm{CH}_{2}, \mathrm{C}-6\right), 67.2\left(\mathrm{CH}_{2}, \mathrm{C}-\right.$ 3'), $38.26\left(\mathrm{CHD}, \mathrm{t}, J_{\mathrm{CD}}=19.7 \mathrm{~Hz}, \mathrm{C}-2\right), 37.1\left(\mathrm{CH}_{2}, \mathrm{C}-1\right.$ '), $23.5 \mathrm{ppm}$ $\left(\mathrm{CH}_{2}, \mathrm{C}-2^{\prime}\right)$. MS (ESI) $\mathrm{m} / z(\%)=498(100)[\mathrm{M}+\mathrm{Na}]^{+}$. HRMS (ESI) $m / z:[\mathrm{M}+\mathrm{Na}]^{+}$calcd for $\mathrm{C}_{30} \mathrm{H}_{33}{ }^{2} \mathrm{HNaO}_{5}$ 498.2367; found 498.2366. Compound $\left(1-{ }^{2} \mathrm{H}\right) 26 \boldsymbol{\beta}:{ }^{1} \mathrm{H}$ NMR $\left(400 \mathrm{MHz}, \mathrm{CDCl}_{3}\right) \delta_{\mathrm{H}}$ 7.34-7.14 (m, 15H, Ar), 4.881 (d, $J=8.8 \mathrm{~Hz}, 1 \mathrm{H}, 2-\mathrm{H}), 4.81(\mathrm{~d}, J=$ $11.4 \mathrm{~Hz}, 1 \mathrm{H}, \mathrm{OBn}), 4.77(\mathrm{~d}, J=10.8 \mathrm{~Hz}, 1 \mathrm{H}, \mathrm{OBn}), 4.65(\mathrm{~d}, J=11.4$ $\mathrm{Hz}, 1 \mathrm{H}, \mathrm{OBn}), 4.59$ (d, $J=12.2 \mathrm{~Hz}, 1 \mathrm{H}, \mathrm{OBn}), 4.55(\mathrm{~d}, J=10.2 \mathrm{~Hz}$, $1 \mathrm{H}, \mathrm{OBn}), 4.52(\mathrm{~d}, J=10.6 \mathrm{~Hz}, 1 \mathrm{H}, \mathrm{OBn}), 3.69(\mathrm{dd}, J=10.7,1.9 \mathrm{~Hz}$, $\left.1 \mathrm{H}, 6-\mathrm{H}_{\mathrm{b}}\right), 3.66-3.57\left(\mathrm{~m}, 5 \mathrm{H}, 3{ }^{\prime}-\mathrm{H}_{2}, 3-\mathrm{H}, 4-\mathrm{H}, 6-\mathrm{H}_{\mathrm{a}}\right), 3.46(\mathrm{~m}, 1 \mathrm{H}, 5-$ $\mathrm{H}), 1.94$ (s, 3H, OAc), 1.75-1.61 (m, 3H, 1'- $\left.\mathrm{H}_{\mathrm{b}}, 2^{\prime}-\mathrm{H}_{2}\right), 1.497 \mathrm{ppm}$ (ddd, $J=13.4,13.4,6.4 \mathrm{~Hz}, 1 \mathrm{H}, 1^{\prime}-\mathrm{H}_{\mathrm{a}}$ ), $1 \mathrm{H}$ from $\mathrm{OH}$ is missing. ${ }^{13} \mathrm{C}\left\{{ }^{1} \mathrm{H}\right\}$ NMR $\left(100.6 \mathrm{MHz}, \mathrm{CDCl}_{3}\right) \delta_{\mathrm{C}} 169.9$ (C, OAc), $138.4(\mathrm{C}$, Ar), 138.1 (C, Ar), 138.0 (C, Ar), $128.42(4 \times \mathrm{CH}, \mathrm{Ar}), 128.38(2 \times$ $\mathrm{CH}, \mathrm{Ar}), 128.0(2 \times \mathrm{CH}, \mathrm{Ar}), 127.8(3 \times \mathrm{CH}, \mathrm{Ar}), 127.70(2 \times \mathrm{CH}$, $\mathrm{Ar}), 127.67(\mathrm{CH}, \mathrm{Ar}), 127.65(\mathrm{CH}, \mathrm{Ar}), 84.6(\mathrm{CH}, \mathrm{C}-3), 78.9(\mathrm{CH}$, C-4), 78.5 ( $\mathrm{CH}, \mathrm{C}-5), 75.2\left(\mathrm{CH}_{2}, \mathrm{OBn}\right), 75.0\left(\mathrm{CH}_{2}, \mathrm{OBn}\right), 73.74$ (CH, C-2), $\left.73.5\left(\mathrm{CH}_{2}, \mathrm{OBn}\right), 69.1\left(\mathrm{CH}_{2}, \mathrm{C}-6\right), 62.7\left(\mathrm{CH}_{2}, \mathrm{C}-3\right)^{\prime}\right)$, $28.8\left(\mathrm{CH}_{2}, \mathrm{C}-2^{\prime}\right), 28.13\left(\mathrm{CH}_{2}, \mathrm{C}-1^{\prime}\right), 20.9 \mathrm{ppm}\left(\mathrm{CH}_{3}, \mathrm{OAc}\right) . \mathrm{MS}$ (ESI) $m / z(\%)=558(100)[\mathrm{M}+\mathrm{Na}]^{+}$. HRMS (ESI) $\mathrm{m} / z:[\mathrm{M}+$ $\mathrm{Na}]^{+}$calcd for $\mathrm{C}_{32} \mathrm{H}_{37}{ }^{2} \mathrm{HNaO}_{7}$ 558.2578; found 558.2574.

Radical Reactions of 2. Method A. Following the general procedure, starting from substrate $2(89.7 \mathrm{mg}, 0.10 \mathrm{mmol})$, after 2 $\mathrm{h}$ of reaction, a supplementary addition of $n-\mathrm{Bu}_{3} \mathrm{SnH}(29 \mu \mathrm{L}, 0.11$ $\mathrm{mmol}$ ) was required. All the starting material was consumed after $4 \mathrm{~h}$. Column chromatography (hexanes to hexanes-EtOAc, 1:1) gave 25 (20.8 $\mathrm{mg}, 0.044 \mathrm{mmol}, 44 \%$ ) and an inseparable mixture of alcohols (12.8 $\mathrm{mg}, 0.018 \mathrm{mmol}, 16 \%)$ that was elucidated by the usual acetylation to obtain 3-C-(3,4,6-tri-O-benzyl-2-O-diphenoxyphos- phoryl- $\beta$-D-glucopyranosyl) 1-propyl acetate $(\mathbf{2 7} \boldsymbol{\beta})(3.9 \mathrm{mg}, 0.005$ mmol, 5\% from 2) and 3-C-(3,4,6-tri-O-benzyl-2-O-diphenoxyphosphoryl- $\alpha$-D-glucopyranosyl)1-propyl acetate $(\mathbf{2 7 \alpha})(6.1 \mathrm{mg}, 0.008$ mmol, 7\% from 2), both as colorless oils. Compound 27ק: $[\alpha]_{\mathrm{D}}=$ $+16.2\left(c=0.33, \mathrm{CHCl}_{3}\right) .{ }^{1} \mathrm{H}$ NMR $\left(500 \mathrm{MHz}, \mathrm{CDCl}_{3}\right) \delta_{\mathrm{H}} 7.36-7.11$ (m, 25H, Ar), 4.59 (d, $J=12.3 \mathrm{~Hz}, 1 \mathrm{H}, \mathrm{OBn}), 4.52(\mathrm{~m}, 1 \mathrm{H}, 2-\mathrm{H})$, $4.52(\mathrm{~d}, J=12.0 \mathrm{~Hz}, 1 \mathrm{H}, \mathrm{OBn}), 4.51(\mathrm{~d}, J=12.0 \mathrm{~Hz}, 1 \mathrm{H}, \mathrm{OBn}), 4.46$ $(\mathrm{d}, J=12.3 \mathrm{~Hz}, 1 \mathrm{H}, \mathrm{OBn}), 4.43(\mathrm{~d}, J=12.0 \mathrm{~Hz}, 1 \mathrm{H}, \mathrm{OBn}), 4.13$ (d, $J$ $=12.3 \mathrm{~Hz}, 1 \mathrm{H}, \mathrm{OBn}), 4.09(\mathrm{dd}, J=2.5,2.5 \mathrm{~Hz}, 1 \mathrm{H}, 3-\mathrm{H}), 4.01-3.92$ (m, 3H, 5- $\left.\mathrm{H}, 3-\mathrm{H}_{2}\right), 3.78(\mathrm{~m}, 1 \mathrm{H}, 1-\mathrm{H}), 3.68(\mathrm{dd}, J=10.1,6.6 \mathrm{~Hz}$, $\left.1 \mathrm{H}, 6-\mathrm{H}_{\mathrm{b}}\right), 3.54\left(\mathrm{dd}, J=10.1,5.7 \mathrm{~Hz}, 1 \mathrm{H}, 6-\mathrm{H}_{\mathrm{a}}\right), 3.35(\mathrm{br} \mathrm{s}, 1 \mathrm{H}, 4-\mathrm{H})$, $1.98(\mathrm{~s}, 3 \mathrm{H}, \mathrm{OAc}), 1.83-1.75\left(\mathrm{~m}, 2 \mathrm{H}, 1^{\prime}-\mathrm{H}_{\mathrm{b}}, 2^{\prime}-\mathrm{H}_{\mathrm{b}}\right), 1.65-1.39 \mathrm{ppm}$ $\left(\mathrm{m}, 2 \mathrm{H}, 1^{\prime}-\mathrm{H}_{\mathrm{a}}, 2{ }^{\prime}-\mathrm{H}_{\mathrm{a}}\right) \cdot{ }^{13} \mathrm{C}\left\{{ }^{1} \mathrm{H}\right\} \operatorname{NMR}\left(125.7 \mathrm{MHz}, \mathrm{CDCl}_{3}\right) \delta_{\mathrm{C}} 171.1$ (C, OAc), 150.5 ( $2 \times \mathrm{C}, \mathrm{Ar}), 138.3$ (C, Ar), 137.7 (C, Ar), 137.4 (C, Ar), 120.0-129.7 (25 × CH, Ar), 77.2 (CH, C-2), $75.4(\mathrm{CH}, \mathrm{C}-5)$, $74.6\left(\mathrm{~d},{ }^{3} \mathrm{~J}_{\mathrm{PC}}=6.4 \mathrm{~Hz}, \mathrm{CH}, \mathrm{C}-1\right), 73.4\left(\mathrm{CH}_{2}, \mathrm{OBn}\right), 72.3\left(\mathrm{CH}_{2}\right.$, $\mathrm{OBn}), 71.7(\mathrm{CH}, \mathrm{C}-3$ or $\mathrm{C}-4), 71.6\left(\mathrm{CH}_{2}, \mathrm{OBn}\right), 71.5(\mathrm{CH}, \mathrm{C}-3$ or C-4), $69.8\left(\mathrm{CH}_{2}, \mathrm{C}-6\right), 64.3\left(\mathrm{CH}_{2}, \mathrm{C}-3^{\prime}\right), 27.5\left(\mathrm{CH}_{2}, \mathrm{C}-1^{\prime}\right.$ or C-2'), $24.9\left(\mathrm{CH}_{2}, \mathrm{C}-1^{\prime}\right.$ or $\left.\mathrm{C}-2^{\prime}\right), 20.9 \mathrm{ppm}\left(\mathrm{CH}_{3}, \mathrm{OAc}\right)$. IR $\left(\mathrm{CHCl}_{3}\right): \nu=$ $2927,1733,1491,1027 \mathrm{~cm}^{-1}$. MS (ESI) $\mathrm{m} / z(\%)=789(100)[\mathrm{M}+$ $\mathrm{Na}]^{+}$. HRMS (ESI) $m / z$ : $[\mathrm{M}+\mathrm{Na}]^{+}$calcd for $\mathrm{C}_{44} \mathrm{H}_{47} \mathrm{NaO}_{10} \mathrm{P}$ 789.2805; found 789.2831. Compound 27 $\alpha$ : $[\alpha]_{\mathrm{D}}=+22.0(c=0.30$, $\left.\mathrm{CHCl}_{3}\right) .{ }^{1} \mathrm{H}$ NMR $\left(500 \mathrm{MHz}, \mathrm{CDCl}_{3}\right) \delta_{\mathrm{H}} 7.32-7.08(\mathrm{~m}, 25 \mathrm{H}, \mathrm{Ar})$, $4.82(\mathrm{~d}, J=11.1 \mathrm{~Hz}, 1 \mathrm{H}, \mathrm{OBn}), 4.79$ (ddd, $J=8.3,5.7 \mathrm{~Hz},{ }^{3} J_{\mathrm{PH}}=8.3$ $\mathrm{Hz}, 1 \mathrm{H}, 2-\mathrm{H}), 4.74(\mathrm{~d}, J=10.7 \mathrm{~Hz}, 1 \mathrm{H}, \mathrm{OBn}), 4.73(\mathrm{~d}, J=11.1 \mathrm{~Hz}$, $1 \mathrm{H}, \mathrm{OBn}), 4.60(\mathrm{~d}, J=12.0 \mathrm{~Hz}, 1 \mathrm{H}, \mathrm{OBn}), 4.46(\mathrm{~d}, J=12.3 \mathrm{~Hz}, 1 \mathrm{H}$, OBn), $4.45(\mathrm{~d}, J=10.5 \mathrm{~Hz}, 1 \mathrm{H}, \mathrm{OBn}), 4.15(\mathrm{~m}, 1 \mathrm{H}, 1-\mathrm{H}), 4.02$ (ddd, $\left.J=10.7,10.7,6.3 \mathrm{~Hz}, 1 \mathrm{H}, 3^{\prime}-\mathrm{H}_{\mathrm{b}}\right), 3.98(\mathrm{ddd}, J=10.8,10.8,6.3 \mathrm{~Hz}$, $\left.1 \mathrm{H}, 3-\mathrm{H}_{\mathrm{a}}\right), 3.86(\mathrm{dd}, J=8.6,8.6 \mathrm{~Hz}, 1 \mathrm{H}, 3-\mathrm{H}), 3.70-3.60(\mathrm{~m}, 4 \mathrm{H}, 4-$ $\left.\mathrm{H}, 5-\mathrm{H}, 6-\mathrm{H}_{2}\right), 2.00(\mathrm{~s}, 3 \mathrm{H}, \mathrm{OAc}), 1.77-1.69\left(\mathrm{~m}, 2 \mathrm{H}, 1^{\prime}-\mathrm{H}_{\mathrm{b}}, 2^{\prime}-\mathrm{H}_{\mathrm{b}}\right)$, $1.62-1.49 \mathrm{ppm}\left(\mathrm{m}, 2 \mathrm{H}, 1\right.$ 1'- $\left.\mathrm{H}_{a}, 2^{\prime}-\mathrm{H}_{\mathrm{a}}\right) .{ }^{13} \mathrm{C}\left\{{ }^{1} \mathrm{H}\right\}$ NMR $(125.7 \mathrm{MHz}$, $\left.\mathrm{CDCl}_{3}\right) \delta_{\mathrm{C}} 171.1$ (C, OAc), 150.5 ( $\left.2 \times \mathrm{C}, \mathrm{Ar}\right), 138.0$ (C, Ar), 137.9 (C, Ar), 137.8 (C, Ar), $129.8(2 \times \mathrm{CH}, \mathrm{Ar}), 129.7(2 \times \mathrm{CH}, \mathrm{Ar})$, $128.41(2 \times \mathrm{CH}, \mathrm{Ar}), 128.37(2 \times \mathrm{CH}, \mathrm{Ar}), 128.3(2 \times \mathrm{CH}, \mathrm{Ar})$, $127.9(2 \times \mathrm{CH}, \mathrm{Ar}), 127.84(3 \times \mathrm{CH}, \mathrm{Ar}), 127.78(2 \times \mathrm{CH}, \mathrm{Ar})$, 127.7 (CH, Ar), 127.6 (CH, Ar), 125.5 (CH, Ar), 125.3 (CH, Ar), 120.14 (CH, Ar), 120.10 (CH, Ar), 120.0 (CH, Ar), 119.9 (CH, Ar), 119.9-129.8 (25 × CH, Ar), 80.5 (d, $\left.{ }^{3} \mathrm{~J}_{\mathrm{PC}}=6.4 \mathrm{~Hz}, \mathrm{CH}, \mathrm{C}-3\right), 78.4$ $\left(\mathrm{d},{ }^{3} \mathrm{~J}_{\mathrm{PC}}=7.4 \mathrm{~Hz}, \mathrm{CH}, \mathrm{C}-1\right), 77.7(\mathrm{CH}, \mathrm{C}-2), 75.1\left(\mathrm{CH}_{2}, \mathrm{OBn}\right), 74.9$ $\left(\mathrm{CH}_{2}, \mathrm{OBn}\right), 73.5\left(\mathrm{CH}_{2}, \mathrm{OBn}\right), 73.4(\mathrm{CH}, \mathrm{C}-5), 71.5(\mathrm{CH}, \mathrm{C}-4)$, $68.6\left(\mathrm{CH}_{2}, \mathrm{C}-6\right), 64.1\left(\mathrm{CH}_{2}, \mathrm{C}-3{ }^{\prime}\right), 24.5\left(\mathrm{CH}_{2}, \mathrm{C}-1^{\prime}\right.$ or $\left.\mathrm{C}-2^{\prime}\right), 24.5$ $\left(\mathrm{CH}_{2}, \mathrm{C}-1\right.$ ' or C-2'), $21.0 \mathrm{ppm}\left(\mathrm{CH}_{3}, \mathrm{OAc}\right) . \mathrm{IR}\left(\mathrm{CHCl}_{3}\right): \nu=3013$, $2928,1730,1026 \mathrm{~cm}^{-1}$. MS (ESI) $\mathrm{m} / z(\%)=789(100)[\mathrm{M}+\mathrm{Na}]^{+}$. HRMS (ESI) $m / z$ : $[\mathrm{M}+\mathrm{Na}]^{+}$calcd for $\mathrm{C}_{44} \mathrm{H}_{47} \mathrm{NaO}_{10} \mathrm{P} 789.2805$; found 789.2823 .

Method $B$. Following the general procedure, starting from substrate $2(56 \mathrm{mg}, 0.064 \mathrm{mmol})$, after $2 \mathrm{~h}$ of reaction, two more equivalents of $n-\mathrm{Bu}_{3} \mathrm{SnH}(35 \mu \mathrm{L}, 0.128 \mathrm{mmol})$ added by a syringe pump were required. All the starting material was consumed after $11 \mathrm{~h}$. Column chromatography (hexanes to hexanes-EtOAc, 1:1) gave 25 (16.2 mg, $0.034 \mathrm{mmol}, 53 \%)$.

Method D. Following the general procedure, starting from substrate 2 (66.3 mg, $0.076 \mathrm{mmol})$, after $2 \mathrm{~h}$ of reaction, a supplementary addition of $n-\mathrm{Bu}_{3} \mathrm{SnD}(21 \mu \mathrm{L}, 0.076 \mathrm{mmol})$ was required. All the starting material was consumed after $4 \mathrm{~h}$. Column chromatography (hexanes to hexanes-EtOAc, 1:1) gave $\left(2-{ }^{2} \mathrm{H}\right) 25$ (22.5 mg, $0.047 \mathrm{mmol}, 62 \%)$ and the inseparable mixture of $3-\mathrm{C}$ (3,4,6-tri- $O$-benzyl-2- $O$-diphenoxyphosphoryl- $\beta$-D- $\left(1-{ }^{2} \mathrm{H}\right)$ glucopyranosyl)1-propanol $\left[\left(1-{ }^{2} \mathrm{H}\right) 28 \boldsymbol{\beta}\right]$ and 3-C-(3,4,6-tri-O-benzyl-

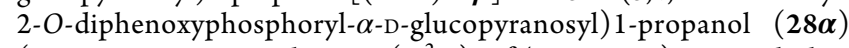
(11.1 $\left.\mathrm{mg}, 0.015 \mathrm{mmol}, 20 \%,\left(1{ }^{2} \mathrm{H}\right) \mathbf{2 8 \beta} / \mathbf{2 8 \alpha}, 1: 2.1\right)$ as a colorless oil. Compounds $\left(1{ }^{2} \mathrm{H}\right) \mathbf{2 8 \beta}$ and $28 \alpha$ : ${ }^{1} \mathrm{H}$ NMR $\left(500 \mathrm{MHz}, \mathrm{CDCl}_{3}\right.$, selected resolved signals of $\left(1-{ }^{2} \mathrm{H}\right) 28 \boldsymbol{\beta}$ from the mix spectrum) $\delta_{\mathrm{H}}$ $4.74(\mathrm{~d}, J=11.0 \mathrm{~Hz}, 1 \mathrm{H}, \mathrm{OBn}), 4.74(\mathrm{~d}, J=11.0 \mathrm{~Hz}, 1 \mathrm{H}, \mathrm{OBn}), 4.59$ (d, $J=12.0 \mathrm{~Hz}, 1 \mathrm{H}, \mathrm{OBn}), 4.532(\mathrm{~d}, J=2.6 \mathrm{~Hz}, 1 \mathrm{H}, 2-\mathrm{H}), 4.53(\mathrm{~d}, J$ $=12.0 \mathrm{~Hz}, 1 \mathrm{H}, \mathrm{OBn}), 4.46(\mathrm{~d}, J=11.0 \mathrm{~Hz}, 1 \mathrm{H}, \mathrm{OBn}), 4.42(\mathrm{~d}, J=$ $11.7 \mathrm{~Hz}, 1 \mathrm{H}, \mathrm{OBn}), 4.10(\mathrm{~d}, J=12.2 \mathrm{~Hz}, 1 \mathrm{H}, \mathrm{OBn}), 4.07$ (dd, $J=2.6$, $2.6 \mathrm{~Hz}, 1 \mathrm{H}, 3-\mathrm{H}), 3.44$ (dd, $J=10.1,0.0 \mathrm{~Hz}, 1 \mathrm{H}, 6-\mathrm{H}_{\mathrm{a}}$ ), $3.31 \mathrm{ppm}$ $(\mathrm{dd}, J=2.6,1.0 \mathrm{~Hz}, 1 \mathrm{H}, 4-\mathrm{H}) .{ }^{13} \mathrm{C}\left\{{ }^{1} \mathrm{H}\right\}$ NMR $\left(125.7 \mathrm{MHz}, \mathrm{CDCl}_{3}\right.$, selected resolved signals of $\left(1-{ }^{2} \mathrm{H}\right) \mathbf{2 8} \boldsymbol{\beta}$ from the mix spectrum) $\delta_{\mathrm{C}}$ 
$150.4\left(\mathrm{~d},{ }^{2} J_{\mathrm{PC}}=7.0 \mathrm{~Hz}, 2 \times \mathrm{C}, \mathrm{Ar}\right), 138.1$ (C, Ar), 137.6 (C, Ar), $137.3(\mathrm{C}, \mathrm{Ar}), 80.5\left(\mathrm{~d},{ }^{3} J_{\mathrm{PC}}=6.4 \mathrm{~Hz}, \mathrm{CH}, \mathrm{C}-3\right), 78.55\left(\mathrm{~d},{ }^{2} J_{\mathrm{PC}}=7.4\right.$ $\mathrm{Hz}, \mathrm{CH}, \mathrm{C}-2), 75.0$ (CH, C-5), $73.5\left(\mathrm{CH}_{2}, \mathrm{OBn}\right), 72.2\left(\mathrm{CH}_{2}, \mathrm{OBn}\right)$, $71.5\left(\mathrm{CH}_{2}, \mathrm{OBn}\right), 71.5(\mathrm{CH}, \mathrm{C}-4), 69.8\left(\mathrm{CH}_{2}, \mathrm{C}-6\right), 62.5\left(\mathrm{CH}_{2}, \mathrm{C}-\right.$ $\left.3^{\prime}\right), 29.68\left(\mathrm{CH}_{2}, \mathrm{C}-1^{\prime}\right.$ or $\left.\mathrm{C}-2^{\prime}\right), 28.08 \mathrm{ppm}\left(\mathrm{CH}_{2}, \mathrm{C}-1^{\prime}\right.$ or $\left.\mathrm{C}-2^{\prime}\right) .{ }^{1} \mathrm{H}$ NMR $\left(500 \mathrm{MHz}, \mathrm{CDCl}_{3}\right.$, selected resolved signals of $28 \alpha$ from the mix spectrum) $\delta_{\mathrm{H}} 4.80(\mathrm{~d}, J=11.0 \mathrm{~Hz}, 1 \mathrm{H}, \mathrm{OBn}), 4.770$ (ddd, $J=7.6$, $\left.4.5 \mathrm{~Hz},{ }^{3} J_{\mathrm{PH}}=6.3 \mathrm{~Hz}, 1 \mathrm{H}, 2-\mathrm{H}\right), 4.71(\mathrm{~d}, J=10.8 \mathrm{~Hz}, 1 \mathrm{H}, \mathrm{OBn}), 4.58$ (d, $J=12.0 \mathrm{~Hz}, 1 \mathrm{H}, \mathrm{OBn}$ ), 4.48 (d, $J=12.3 \mathrm{~Hz}, 1 \mathrm{H}, \mathrm{OBn}), 4.44$ (d, $J$ $=11.1 \mathrm{~Hz}, 1 \mathrm{H}, \mathrm{OBn}$ ), $4.23(\mathrm{ddd}, J=11.4,5.7,2.2 \mathrm{~Hz}, 1-\mathrm{H}), 3.85 \mathrm{ppm}$ (dd, $J=8.5,8.5 \mathrm{~Hz}, 1 \mathrm{H}, 3-\mathrm{H}) .{ }^{13} \mathrm{C}\left\{{ }^{1} \mathrm{H}\right\} \operatorname{NMR}\left(125.7 \mathrm{MHz}, \mathrm{CDCl}_{3}\right.$, selected resolved signals of $28 \alpha$ from the mix spectrum) $\delta_{\mathrm{C}} 150.5$ (d, $\left.{ }^{2} J_{\mathrm{PC}}=7.0 \mathrm{~Hz}, 2 \times \mathrm{C}, \mathrm{Ar}\right), 138.0(\mathrm{C}, \mathrm{Ar}), 137.8(2 \times \mathrm{C}, \mathrm{Ar}), 80.5(\mathrm{~d}$, $\left.{ }^{3} J_{\mathrm{PC}}=6.4 \mathrm{~Hz}, \mathrm{CH}, \mathrm{C}-3\right), 78.55\left(\mathrm{~d},{ }^{2} J_{\mathrm{PC}}=7.4 \mathrm{~Hz}, \mathrm{CH}, \mathrm{C}-2\right), 77.8$ ( $\mathrm{CH}, \mathrm{C}-5), 75.1\left(\mathrm{CH}_{2}, \mathrm{OBn}\right), 74.8\left(\mathrm{CH}_{2}, \mathrm{OBn}\right), 73.6(\mathrm{CH}, \mathrm{C}-1)$, $73.5\left(\mathrm{CH}_{2}, \mathrm{OBn}\right), 71.4(\mathrm{CH}, \mathrm{C}-4), 68.8\left(\mathrm{CH}_{2}, \mathrm{C}-6\right), 61.8\left(\mathrm{CH}_{2}, \mathrm{C}-\right.$ $\left.3^{\prime}\right), 28.56\left(\mathrm{CH}_{2}, \mathrm{C}-1^{\prime}\right.$ or $\left.\mathrm{C}-2^{\prime}\right), 20.69 \mathrm{ppm}\left(\mathrm{CH}_{2}, \mathrm{C}-1^{\prime}\right.$ or $\left.\mathrm{C}-2^{\prime}\right)$. IR $\left(\mathrm{CHCl}_{3}\right): \nu=3490,3422,2928,1550,1491,1192 \mathrm{~cm}^{-1}$. MS (ESI) $m / z(\%)=747(99.6)[\mathrm{M}+\mathrm{Na}]^{+}, 748(100)[\mathrm{M}+\mathrm{Na}]^{+}$. HRMS (ESI) $m / z:[\mathrm{M}+\mathrm{Na}]^{+}$calcd for $\mathrm{C}_{42} \mathrm{H}_{45} \mathrm{NaO}_{9} \mathrm{P}$ 747.2699; found 747.2697, $[\mathrm{M}+\mathrm{Na}]^{+}$calcd for $\mathrm{C}_{42} \mathrm{H}_{44}{ }^{2} \mathrm{HNaO}_{9} \mathrm{P}$ 748.2762; found 748.2764 .

Radical Reactions of 3. Method A. Following the general procedure, starting from substrate $3(89.2 \mathrm{mg}, 0.13 \mathrm{mmol})$, after 2 $\mathrm{h}$ of reaction, a supplementary addition of $n-\mathrm{Bu}_{3} \mathrm{SnH}(36 \mu \mathrm{L}, 0.13$ $\mathrm{mmol}$ ) was required. All the starting material was consumed after $4 \mathrm{~h}$. Column chromatography (hexanes to hexanes-EtOAc, 6:4) gave 25 (13.9 mg, $0.029 \mathrm{mmol}, 23 \%)$ and $\mathbf{2 6 \boldsymbol { \beta }}(20.4 \mathrm{mg}, 0.038 \mathrm{mmol}, 29 \%)$.

Method $B$. Following the general procedure, starting from substrate $3(110.2 \mathrm{mg}, 0.16 \mathrm{mmol})$, after $2 \mathrm{~h}$ of reaction, two more equivalents of $n-\mathrm{Bu}_{3} \mathrm{SnH}(88 \mu \mathrm{L}, 0.32 \mathrm{mmol})$ added by a syringe pump over $2 \mathrm{~h}$ were required. All the starting material was consumed after $5 \mathrm{~h}$. Column chromatography (hexanes to hexanes-EtOAc, 6:4) gave 25 $(22.4 \mathrm{mg}, 0.047 \mathrm{mmol}, 30 \%)$ and the reduced product $26 \boldsymbol{\beta}(16.4 \mathrm{mg}$, $0.031 \mathrm{mmol}, 19 \%)$.

Method $C$. Following the general procedure, starting from substrate 3 (96.2 mg, $0.14 \mathrm{mmol}$ ), after $2 \mathrm{~h}$ of reaction, a supplementary addition of TTMSS ( $66 \mu \mathrm{L}, 0.21 \mathrm{mmol})$ was required. All the starting material was consumed after $5 \mathrm{~h}$. Column chromatography (hexanesEtOAc, 9:1 to 6:4) gave $25(10.2 \mathrm{mg}, 0.022 \mathrm{mmol}, 23 \%)$ and the reduced product $26 \boldsymbol{\beta}$ (3 $\mathrm{mg}, 0.006 \mathrm{mmol}, 4 \%)$.

Method D. Following the general procedure, starting from substrate 3 (106.6 $\mathrm{mg}, 0.16 \mathrm{mmol}$ ), after $2 \mathrm{~h}$ of reaction, a supplementary addition of $n-\mathrm{Bu}_{3} \mathrm{SnD}(42 \mu \mathrm{L}, 0.16 \mathrm{mmol})$ was required. All the starting material was consumed after $5 \mathrm{~h}$. Column chromatography (hexanes to hexanes-EtOAc, 6:4) gave $\left(2-{ }^{2} \mathrm{H}\right) 25$ $(25.2 \mathrm{mg}, 0.053 \mathrm{mmol}, 33 \%)$ and $\left[1-{ }^{2} \mathrm{H}\right] \mathbf{2 6} \boldsymbol{\beta}(8.3 \mathrm{mg}, 0.016 \mathrm{mmol}$, $\left.10 \%,{ }^{2} \mathrm{H} /{ }^{1} \mathrm{H} 1: 1\right)$.

Radical Reactions of 4. Method A. Following the general procedure, starting from substrate $4(57.3 \mathrm{mg}, 0.066 \mathrm{mmol})$, after 2 $\mathrm{h}$ of reaction and again after $4 \mathrm{~h}$, a supplementary addition of $n$ $\mathrm{Bu}_{3} \mathrm{SnH}(18 \mu \mathrm{L}, 0.066 \mathrm{mmol})$ was required. All the starting material was consumed after $6 \mathrm{~h}$. Column chromatography (hexanes to hexanes-EtOAc, 8:2) gave 25 (14.2 $\mathrm{mg}, 0.030 \mathrm{mmol}, 45 \%)$.

Method $B$. Following the general procedure, starting from substrate $4(53 \mathrm{mg}, 0.061 \mathrm{mmol})$, after $2 \mathrm{~h}$ of reaction, a supplementary addition of $n-\mathrm{Bu}_{3} \mathrm{SnH}(16 \mu \mathrm{L}, 0.061 \mathrm{mmol})$ added by a syringe pump over $1 \mathrm{~h}$ was required. All the starting material was consumed after 6 h. Column chromatography (hexanes to hexanes-EtOAc, 8:2) gave 25 (12 mg, $0.025 \mathrm{mmol}, 42 \%)$.

Method $D$. Following the general procedure, starting from substrate $4(75 \mathrm{mg}, 0.086 \mathrm{mmol})$, after $2 \mathrm{~h}$ of reaction, a supplementary addition of $n-\mathrm{Bu}_{3} \mathrm{SnD}(23 \mu \mathrm{L}, 0.086 \mathrm{mmol})$ was required. All the starting material was consumed after $5 \mathrm{~h}$. Column chromatography (hexanes to hexanes-EtOAc, 8:2) gave $\left(2-{ }^{2} \mathrm{H}\right) 25$ (22.5 mg, $0.047 \mathrm{mmol}, 55 \%)$.

Radical Reactions of 5. Method A. Following the general procedure, starting from substrate $5(40.2 \mathrm{mg}, 0.059 \mathrm{mmol})$, after 2 $\mathrm{h}$ of reaction, a supplementary addition of $n-\mathrm{Bu}_{3} \mathrm{SnH}(16 \mu \mathrm{L}, 0.059$ $\mathrm{mmol}$ ) was required. All the starting material was consumed after $7 \mathrm{~h}$.
Column chromatography (hexanes to hexanes-EtOAc, 6:4) gave an inseparable mixture of isomers 3-C-(2-O-acetyl-3,4,6-tri-O-benzyl- $\beta$-Dmannopyranosyl)1-propanol $(29 \beta)$ and 3-C-(2-O-acetyl-3,4,6-tri- $O$ benzyl- $\alpha$-D-mannopyranosyl)1-propanol (29 $\alpha) \quad(16.1 \mathrm{mg}, 0.030$ mmol, $51 \%, 2.3: 1)$ as a colorless oil. Compounds $29 \beta$ and $29 \alpha:{ }^{1} \mathrm{H}$ NMR $(500 \mathrm{MHz}$, selected resolved signals of $29 \boldsymbol{\beta}$ from the mix spectrum) $\delta_{\mathrm{H}} 5.473(\mathrm{dd}, J=2.2,0.0 \mathrm{~Hz}, 1 \mathrm{H}, 2-\mathrm{H}), 4.85(\mathrm{~d}, J=10.8$ $\mathrm{Hz}, 1 \mathrm{H}, \mathrm{OBn}), 4.74(\mathrm{~d}, J=11.4 \mathrm{~Hz}, 1 \mathrm{H}, \mathrm{OBn}), 4.61(\mathrm{~d}, J=12.3 \mathrm{~Hz}$, $1 \mathrm{H}, \mathrm{OBn}), 4.54(\mathrm{~d}, J=12.0 \mathrm{~Hz}, 1 \mathrm{H}, \mathrm{OBn}), 4.50(\mathrm{~d}, J=11.1 \mathrm{~Hz}, 1 \mathrm{H}$, OBn), 4.49 (d, $J=11.4 \mathrm{~Hz}, 1 \mathrm{H}, \mathrm{OBn}), 4.48(\mathrm{~d}, J=10.4 \mathrm{~Hz}, 1 \mathrm{H}$, $\mathrm{OBn}), 2.17 \mathrm{ppm}(\mathrm{s}, 3 \mathrm{H}, \mathrm{OAc}) .{ }^{13} \mathrm{C}\left\{{ }^{1} \mathrm{H}\right\} \mathrm{NMR}\left(100.6 \mathrm{MHz}, \mathrm{CDCl}_{3}\right.$, selected resolved signals of $29 \beta$ from the mix spectrum) $\delta_{\mathrm{C}} 170.9(\mathrm{C}$, OAc), 138.3 (C, Ar), 138.2 (C, Ar), 137.8 (C, Ar), 81.9 (CH), 79.3 $(\mathrm{CH}), 77.2(\mathrm{CH}, \mathrm{C}-1), 75.1\left(\mathrm{CH}_{2}, \mathrm{OBn}\right), 74.7(\mathrm{CH}), 73.5\left(\mathrm{CH}_{2}\right.$, OBn $), 71.6\left(\mathrm{CH}_{2}, \mathrm{OBn}\right), 69.6\left(\mathrm{CH}_{2}, \mathrm{C}-6\right), 69.56(\mathrm{CH}, \mathrm{C}-2), 62.6$ $\left(\mathrm{CH}_{2}, \mathrm{C}-3^{\prime}\right), 29.5\left(\mathrm{CH}_{2}, \mathrm{C}-2^{\prime}\right), 29.3\left(\mathrm{CH}_{2}, \mathrm{C}-2^{\prime}\right), 28.17\left(\mathrm{CH}_{2}, \mathrm{C}-1^{\prime}\right)$, $21.0 \mathrm{ppm}\left(\mathrm{CH}_{3}, \mathrm{OAc}\right) .{ }^{1} \mathrm{H}$ NMR $\left(500 \mathrm{MHz}, \mathrm{CDCl}_{3}\right.$, selected resolved signals of $29 \alpha$ from the mix spectrum) $\delta_{\mathrm{H}} 5.245$ (dd, $J=2.8$, $2.8 \mathrm{~Hz}, 1 \mathrm{H}, 2-\mathrm{H}), 4.81(\mathrm{~d}, J=11.1 \mathrm{~Hz}, 1 \mathrm{H}, \mathrm{OBn}), 4.65(\mathrm{~d}, J=11.4$ $\mathrm{Hz}, 1 \mathrm{H}, \mathrm{OBn}), 4.61$ (d, $J=12.3 \mathrm{~Hz}, 1 \mathrm{H}, \mathrm{OBn}), 4.51(\mathrm{~d}, J=11.7 \mathrm{~Hz}$, $1 \mathrm{H}, \mathrm{OBn}), 4.47(\mathrm{~d}, J=11.1 \mathrm{~Hz}, 1 \mathrm{H}, \mathrm{OBn}), 3.99$ (ddd, $J=10.7,6.6$, $3.8 \mathrm{~Hz}, 1 \mathrm{H}, 1-\mathrm{H}), 3.85(\mathrm{dd}, J=8.2,3.2 \mathrm{~Hz}, 1 \mathrm{H}, 3-\mathrm{H}), 2.13 \mathrm{ppm}(\mathrm{s}$, $3 \mathrm{H}, \mathrm{OAc}) .{ }^{13} \mathrm{C}\left\{{ }^{1} \mathrm{H}\right\}$ NMR $\left(100.6 \mathrm{MHz}, \mathrm{CDCl}_{3}\right.$, selected resolved signals of $29 \alpha$ from the mix spectrum) $\delta_{\mathrm{C}} 170.6$ (C, OAc), 138.3 (C, Ar), $138.2(\mathrm{C}, \mathrm{Ar}) 137.8(\mathrm{C}, \mathrm{Ar}), 77.7(\mathrm{CH}), 75.3\left(\mathrm{CH}_{2}, \mathrm{OBn}\right), 74.8$ $(\mathrm{CH}), 74.7(\mathrm{CH}), 73.5\left(\mathrm{CH}_{2}, \mathrm{OBn}\right), 72.8(\mathrm{CH}), 71.9\left(\mathrm{CH}_{2}, \mathrm{OBn}\right)$, $70.9(\mathrm{CH}), 69.4\left(\mathrm{CH}_{2}, \mathrm{C}-6\right), 62.0\left(\mathrm{CH}_{2}, \mathrm{C}-3^{\prime}\right), 24.95\left(\mathrm{CH}_{2}, \mathrm{C}-1^{\prime}\right)$, $21.2 \mathrm{ppm}\left(\mathrm{CH}_{3}, \mathrm{OAc}\right)$. IR $\left(\mathrm{CHCl}_{3}\right): \nu=3496,3014,2928,1735$, $1238,1095 \mathrm{~cm}^{-1}$. MS (ESI) $\mathrm{m} / z(\%)=557(100)[\mathrm{M}+\mathrm{Na}]^{+}$. HRMS (ESI) $m / z:[\mathrm{M}+\mathrm{Na}]^{+}$calcd for $\mathrm{C}_{32} \mathrm{H}_{38} \mathrm{NaO}_{7}$ 557.2515; found 557.2515. Anal. calcd for $\mathrm{C}_{32} \mathrm{H}_{38} \mathrm{O}_{7}$ : C, 71.89; H, 7.16. Found: C, 71.55; H, 7.11.

Method $D$. Following the general procedure, starting from substrate 5 (30.2 $\mathrm{mg}, 0.044 \mathrm{mmol})$, after $2 \mathrm{~h}$ of reaction, a supplementary addition of $n-\mathrm{Bu}_{3} \mathrm{SnD}(12 \mu \mathrm{L}, 0.044 \mathrm{mmol})$ was required. All the starting material was consumed after $5 \mathrm{~h}$. Column chromatography (hexanes to hexanes-EtOAc, 7:3) gave the mixture of 3-C-(2-O-acetyl-3,4,6-tri-O-benzyl- $\beta$-D- $\left(1-{ }^{2} \mathrm{H}\right)$ mannopyranosyl) 1 propanol $\left[\left(1-{ }^{2} \mathrm{H}\right) 29 \boldsymbol{\beta}\right]$ and $29 \boldsymbol{\alpha}(14.4 \mathrm{mg}, 0.027 \mathrm{mmol}, 61 \%$, ${ }^{2} \mathrm{H} /{ }^{1} \mathrm{H}$ 1.8:1) as a colorless oil. Compounds $\left(1-{ }^{2} \mathrm{H}\right) 29 \boldsymbol{\beta}$ and $29 \alpha$ : ${ }^{1} \mathrm{H}$ NMR $\left(500 \mathrm{MHz}, \mathrm{CDCl}_{3}\right.$, selected resolved signals of $\left(1{ }^{2} \mathrm{H}\right) \mathbf{2 9} \boldsymbol{\beta}$ from the mix spectrum) $\delta_{\mathrm{H}} 4.74(\mathrm{~d}, J=11.0 \mathrm{~Hz}, 1 \mathrm{H}, \mathrm{OBn}), 4.74(\mathrm{~d}, J$ $=11.0 \mathrm{~Hz}, 1 \mathrm{H}, \mathrm{OBn}), 4.59(\mathrm{~d}, J=12.0 \mathrm{~Hz}, 1 \mathrm{H}, \mathrm{OBn}), 4.532(\mathrm{~d}, J=$ $2.6 \mathrm{~Hz}, 1 \mathrm{H}, 2-\mathrm{H}), 4.53(\mathrm{~d}, J=12.0 \mathrm{~Hz}, 1 \mathrm{H}, \mathrm{OBn}), 4.46(\mathrm{~d}, J=11.0$ $\mathrm{Hz}, 1 \mathrm{H}, \mathrm{OBn}), 4.42(\mathrm{~d}, J=11.7 \mathrm{~Hz}, 1 \mathrm{H}, \mathrm{OBn}), 4.10(\mathrm{~d}, J=12.2 \mathrm{~Hz}$, $1 \mathrm{H}, \mathrm{OBn}), 4.07(\mathrm{dd}, J=2.6,2.6 \mathrm{~Hz}, 1 \mathrm{H}, 3-\mathrm{H}), 3.44(\mathrm{dd}, J=10.1,0.0$ $\left.\mathrm{Hz}, 1 \mathrm{H}, 6-\mathrm{H}_{\mathrm{a}}\right), 3.31 \mathrm{ppm}(\mathrm{dd}, J=2.6,1.0 \mathrm{~Hz}, 1 \mathrm{H}, 4-\mathrm{H}) .{ }^{13} \mathrm{C}\left\{{ }^{1} \mathrm{H}\right\}$ NMR $\left(125.7 \mathrm{MHz}, \mathrm{CDCl}_{3}\right.$, selected resolved signals of $\left(1{ }^{2} \mathrm{H}\right) \mathbf{2 9} \boldsymbol{\beta}$ from the mix spectrum) $\delta_{\mathrm{C}} 150.4\left(\mathrm{~d},{ }^{2} J_{\mathrm{PC}}=7.0 \mathrm{~Hz}, 2 \times \mathrm{C}, \mathrm{Ar}\right), 138.1$ (C, Ar), 137.6 (C, Ar), 137.3 (C, Ar), 80.5 (d, ${ }^{3} J_{\mathrm{PC}}=6.4 \mathrm{~Hz}, \mathrm{CH}, \mathrm{C}-$ 3), $78.55\left(\mathrm{~d},{ }^{2} \mathrm{~J}_{\mathrm{PC}}=7.4 \mathrm{~Hz}, \mathrm{CH}, \mathrm{C}-2\right), 75.0(\mathrm{CH}, \mathrm{C}-5), 73.5\left(\mathrm{CH}_{2}\right.$, $\mathrm{OBn}), 72.2\left(\mathrm{CH}_{2}, \mathrm{OBn}\right), 71.5\left(\mathrm{CH}_{2}, \mathrm{OBn}\right), 71.5(\mathrm{CH}, \mathrm{C}-4), 69.8$ $\left(\mathrm{CH}_{2}, \mathrm{C}-6\right), 62.5\left(\mathrm{CH}_{2}, \mathrm{C}-3^{\prime}\right), 29.68\left(\mathrm{CH}_{2}, \mathrm{C}-1^{\prime}\right.$ or C-2'), $28.08 \mathrm{ppm}$ $\left(\mathrm{CH}_{2}, \mathrm{C}-1^{\prime}\right.$ or C-2'). IR $\left(\mathrm{CHCl}_{3}\right): \nu=3490,3422,2928,1550,1491$, $1192 \mathrm{~cm}^{-1}$. MS (ESI) $\mathrm{m} / z(\%)=747(99.6)[\mathrm{M}+\mathrm{Na}]^{+}, 748(100)$ $[\mathrm{M}+\mathrm{Na}]^{+}$. HRMS (ESI) $m / z:[\mathrm{M}+\mathrm{Na}]^{+}$calcd for $\mathrm{C}_{42} \mathrm{H}_{45} \mathrm{NaO}_{9} \mathrm{P}$ 747.2699; found 747.2697, $[\mathrm{M}+\mathrm{Na}]^{+}$calcd for $\mathrm{C}_{42} \mathrm{H}_{44}{ }^{2} \mathrm{HNaO}_{9} \mathrm{P}$ 748.2762; found 748.2764.

Radical Reactions of 6. Method A. Following the general procedure, starting from substrate $6(39 \mathrm{mg}, 0.045 \mathrm{mmol})$, after 2 $\mathrm{h}$ of reaction, a supplementary addition of $n-\mathrm{Bu}_{3} \mathrm{SnH}(12 \mu \mathrm{L}, 0.045$ $\mathrm{mmol}$ ) was required. All the starting material was consumed after $6 \mathrm{~h}$. Column chromatography (hexanes to hexanes-EtOAc, 1:1) gave 25 $(9.7 \mathrm{mg}, \quad 0.020 \mathrm{mmol}, 46 \%)$ and 3-C-(3,4,6-tri-O-benzyl-2-O-

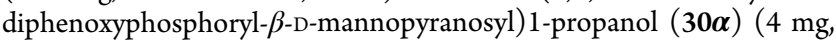
$0.006 \mathrm{mmol}, 12 \%)$ as a colorless oil. Compound $30 \alpha:[\alpha]_{\mathrm{D}}=+1.6(c$ $\left.=0.77, \mathrm{CHCl}_{3}\right) .{ }^{1} \mathrm{H}$ NMR $\left(500 \mathrm{MHz}, \mathrm{CDCl}_{3}\right) \delta_{\mathrm{H}} 7.36-7.11(\mathrm{~m}$, $25 \mathrm{H}, \mathrm{Ar}), 4.90\left(\mathrm{ddd}, J=3.2,3.2 \mathrm{~Hz},{ }^{3} J_{\mathrm{PH}}=6.3 \mathrm{~Hz}, 1 \mathrm{H}, 2-\mathrm{H}\right), 4.74(\mathrm{~d}$, $J=11.4 \mathrm{~Hz}, 1 \mathrm{H}, \mathrm{OBn}), 4.65(\mathrm{~d}, J=11.4 \mathrm{~Hz}, 1 \mathrm{H}, \mathrm{OBn}), 4.56(\mathrm{~d}, J=$ $12.0 \mathrm{~Hz}, 1 \mathrm{H}, \mathrm{OBn}), 4.52$ (d, $J=12.0 \mathrm{~Hz}, 1 \mathrm{H}, \mathrm{OBn}), 4.47$ (d, $J=11.4$ 
$\mathrm{Hz}, 1 \mathrm{H}, \mathrm{OBn}$ ), 4.38 (d, $J=11.1 \mathrm{~Hz}, 1 \mathrm{H}, \mathrm{OBn}), 4.04$ (ddd, $J=10.1$, $3.5,3.5 \mathrm{~Hz}, 1 \mathrm{H}, 1-\mathrm{H}$ ), 3.85 (ddd, $J=8.2,2.5 \mathrm{~Hz},{ }^{4} J_{\mathrm{PH}}=2.5 \mathrm{~Hz}, 1 \mathrm{H}, 3-$ $\mathrm{H}), 3.76$ (ddd, $J=8.5,5.4,3.5 \mathrm{~Hz}, 1 \mathrm{H}, 5-\mathrm{H}), 3.69-3.56(\mathrm{~m}, 5 \mathrm{H}, 3$ '$\mathrm{H}_{2}$, 4-H, 6- $\left.\mathrm{H}_{2}\right), 1.78-1.47$ ppm (m, 4H, 1'- $\left.\mathrm{H}_{2}, 2^{\prime}-\mathrm{H}_{2}\right), 1 \mathrm{H}$ from $\mathrm{OH}$ is missing. Stereochemistry was assigned as $1 \alpha$ since the starting phthalimide 6 was obtained by the reaction with $N$-hydroxyphthalimide under Mitsunobu conditions. ${ }^{13} \mathrm{C}\left\{{ }^{1} \mathrm{H}\right\}$ NMR $(125.7 \mathrm{MHz}$, $\left.\mathrm{CDCl}_{3}\right) \delta_{\mathrm{C}} 150.7$ (C, Ar), 150.5 (C, Ar), 138.1 (C, Ar), 138.0 (C, $\mathrm{Ar}), 137.6$ (C, Ar), $129.8(2 \times \mathrm{CH}, \mathrm{Ar}), 129.6(2 \times \mathrm{CH}, \mathrm{Ar}), 128.38$ $(2 \times \mathrm{CH}, \mathrm{Ar}), 128.36(2 \times \mathrm{CH}, \mathrm{Ar}), 128.3(2 \times \mathrm{CH}, \mathrm{Ar}), 128.2(2 \times$ $\mathrm{CH}, \mathrm{Ar}), 128.0(2 \times \mathrm{CH}, \mathrm{Ar}), 127.8(4 \times \mathrm{CH}, \mathrm{Ar}), 127.6(\mathrm{CH}, \mathrm{Ar})$, $125.4(\mathrm{CH}, \mathrm{Ar}), 125.1(\mathrm{CH}, \mathrm{Ar}), 120.4(\mathrm{CH}, \mathrm{Ar}), 120.3(\mathrm{CH}, \mathrm{Ar})$, 120.23 (CH, Ar), $120.19(\mathrm{CH}, \mathrm{Ar}), 77.7\left(\mathrm{~d},{ }^{2} J_{\mathrm{PC}}=6.4 \mathrm{~Hz}, \mathrm{CH}, \mathrm{C}-2\right)$, $77.6(2 \times \mathrm{CH}, \mathrm{C}-3, \mathrm{C}-4), 74.5\left(\mathrm{CH}_{2}, \mathrm{OBn}\right), 74.4(\mathrm{CH}, \mathrm{C}-1), 73.4$ $\left(\mathrm{CH}_{2}, \mathrm{OBn}\right), 73.1(\mathrm{CH}, \mathrm{C}-5), 72.1\left(\mathrm{CH}_{2}, \mathrm{OBn}\right), 69.1\left(\mathrm{CH}_{2}, \mathrm{C}-6\right)$, $61.8\left(\mathrm{CH}_{2}, \mathrm{C}-3^{\prime}\right), 29.4\left(\mathrm{CH}_{2}, \mathrm{C}-1\right.$ ' or C-2'), $29.1 \mathrm{ppm}\left(\mathrm{CH}_{2}, \mathrm{C}-1^{\prime}\right.$ or C-2'). IR $\left(\mathrm{CHCl}_{3}\right): \nu=3503,2928,1712,1491,1192 \mathrm{~cm}^{-1}$. MS (ESI) $\mathrm{m} / z(\%)=747(100)[\mathrm{M}+\mathrm{Na}]^{+}$. HRMS (ESI) $\mathrm{m} / z:[\mathrm{M}+$ $\mathrm{Na}]^{+}$calcd for $\mathrm{C}_{42} \mathrm{H}_{45} \mathrm{NaO}_{9} \mathrm{P}$ 747.2699; found 747.2692.

Method D. Following the general procedure, starting from substrate $6(30.2 \mathrm{mg}, 0.035 \mathrm{mmol})$, after $2 \mathrm{~h}$ of reaction, a supplementary addition of $n-\mathrm{Bu}_{3} \mathrm{SnD}(9 \mu \mathrm{L}, 0.035 \mathrm{mmol})$ was required. All the starting material was consumed after $4 \mathrm{~h}$. Column chromatography (hexanes to hexanes-EtOAc, 1:1) gave $\left(2-{ }^{2} \mathrm{H}\right) 25$ ( $8.6 \mathrm{mg}, 0.018 \mathrm{mmol}, 52 \%)$ and $30 \alpha(4.7 \mathrm{mg}, 0.005 \mathrm{mmol}, 19 \%)$.

Method $F$. Following the general procedure, starting from substrate $6(57.3 \mathrm{mg}, 0.066 \mathrm{mmol})$, all the starting material was consumed after $1 \mathrm{~h}$. Column chromatography (hexanes-EtOAc, 85:15 to 4:6) gave 25 (9 mg, $0.019 \mathrm{mmol}, 29 \%$ ) and $30 \alpha(24.2 \mathrm{mg}, 0.033 \mathrm{mmol}, 51 \%)$.

Method G. Following the general procedure, starting from substrate 6 (59 $\mathrm{mg}, 0.068 \mathrm{mmol}$ ), all the starting material was consumed after $3 \mathrm{~h}$. Column chromatography (hexanes-EtOAc, 8:2 to $4: 6)$ gave 25 ( $11.3 \mathrm{mg}, 0.024 \mathrm{mmol}, 35 \%)$ and $30 \alpha(22.1 \mathrm{mg}, 0.031$ $\mathrm{mmol}, 45 \%)$.

Radical Reactions of 7. Method A. Following the general procedure, starting from substrate $7(74.6 \mathrm{mg}, 0.11 \mathrm{mmol})$, after 2 $\mathrm{h}$ of reaction, a supplementary addition of $n-\mathrm{Bu}_{3} \mathrm{SnH}(30 \mu \mathrm{L}, 0.11$ mmol) was required. All the starting material was consumed after $4 \mathrm{~h}$. Column chromatography (hexanes to hexanes-EtOAc, 6:4) gave 3-C(2-O-acetyl-3,4,6-tri- $O$-benzyl- $\beta$-D-mannopyranosyl)1-propanol (29ק) $(56.8 \mathrm{mg}, 0.11 \mathrm{mmol}, 97 \%)$ as an amorphous solid: $[\alpha]_{\mathrm{D}}=-27.6(c=$ $\left.0.87, \mathrm{CHCl}_{3}\right) .{ }^{1} \mathrm{H}$ NMR $\left(500 \mathrm{MHz}, \mathrm{CDCl}_{3}\right) \delta_{\mathrm{H}} 7.35-7.15(\mathrm{~m}, 15 \mathrm{H}$, Ar), $5.474(\mathrm{dd}, J=2.2,0.0 \mathrm{~Hz}, 1 \mathrm{H}, 2-\mathrm{H}), 4.85(\mathrm{~d}, J=10.7 \mathrm{~Hz}, 1 \mathrm{H}$, OBn), $4.74(\mathrm{~d}, J=11.1 \mathrm{~Hz}, 1 \mathrm{H}, \mathrm{OBn}), 4.61(\mathrm{~d}, J=12.0 \mathrm{~Hz}, 1 \mathrm{H}$, OBn), 4.54 (d, $J=12.3 \mathrm{~Hz}, 1 \mathrm{H}, \mathrm{OBn}), 4.48$ (d, $J=11.0 \mathrm{~Hz}, 1 \mathrm{H}$, $\mathrm{OBn}), 4.48(\mathrm{~d}, J=11.0 \mathrm{~Hz}, 1 \mathrm{H}, \mathrm{OBn}), 3.74(\mathrm{dd}, J=10.7,1.9 \mathrm{~Hz}, 1 \mathrm{H}$, 6- $\mathrm{H}_{\mathrm{b}}$ ), 3.70-3.63 (m, 5H, 3-H, 4-H, 5- H, 6- $\mathrm{H}_{\mathrm{a}}, 3-\mathrm{H}_{\mathrm{b}}$ ), 3.51-3.46 (m, $\left.2 \mathrm{H}, 1-\mathrm{H}, 3-\mathrm{H}_{\mathrm{a}}\right), 2.17$ (s, 3H, OAc), 1.73-1.66 (m, 3H, 1'- $\left.\mathrm{H}_{\mathrm{b}}, 2^{\prime}-\mathrm{H}_{2}\right)$, $1.572 \mathrm{ppm}\left(\mathrm{m}, 1 \mathrm{H}, \mathrm{l}^{\prime}-\mathrm{H}_{\mathrm{a}}\right), 1 \mathrm{H}$ from $\mathrm{OH}$ is missing. ${ }^{13} \mathrm{C}\left\{{ }^{1} \mathrm{H}\right\}$ NMR $\left(125.7 \mathrm{MHz}, \mathrm{CDCl}_{3}\right) \delta_{\mathrm{C}} 170.9$ (C, OAc), 138.3 (C, Ar), $138.2(\mathrm{C}$, $\mathrm{Ar}), 137.8(\mathrm{C}, \mathrm{Ar}), 127.5-128.3(15 \times \mathrm{CH}, \mathrm{Ar}), 81.8(\mathrm{CH}), 79.2$ $(\mathrm{CH}), 77.1(\mathrm{CH}, \mathrm{C}-1), 75.1\left(\mathrm{CH}_{2}, \mathrm{OBn}\right), 74.7(\mathrm{CH}), 73.4\left(\mathrm{CH}_{2}\right.$, OBn $), 71.5\left(\mathrm{CH}_{2}, \mathrm{OBn}\right), 69.49(\mathrm{CH}, \mathrm{C}-2), 69.5\left(\mathrm{CH}_{2}, \mathrm{C}-6\right), 62.4$ $\left(\mathrm{CH}_{2}, \mathrm{C}-3^{\prime}\right), 29.3\left(\mathrm{CH}_{2}, \mathrm{C}-2^{\prime}\right), 28.08\left(\mathrm{CH}_{2}, \mathrm{C}-1^{\prime}\right), 20.9 \mathrm{ppm}\left(\mathrm{CH}_{3}\right.$, OAc). IR $\left(\mathrm{CHCl}_{3}\right): \nu=3430,3015,2936,1735,1091 \mathrm{~cm}^{-1}$. MS (ESI) $m / z(\%)=557(100)[\mathrm{M}+\mathrm{Na}]^{+}$. HRMS (ESI) $m / z:[\mathrm{M}+$ $\mathrm{Na}]^{+}$calcd for $\mathrm{C}_{32} \mathrm{H}_{38} \mathrm{NaO}_{7}$ 557.2515; found 557.2519. Anal. calcd for $\mathrm{C}_{32} \mathrm{H}_{38} \mathrm{O}_{7}$ : C, 71.89; $\mathrm{H}, 7.16$. Found: $\mathrm{C}, 71.93 ; \mathrm{H}, 7.08$.

Method $B$. Following the general procedure, starting from substrate $7(63.8 \mathrm{mg}, 0.094 \mathrm{mmol})$, after $2 \mathrm{~h}$ of reaction, a supplementary addition of $n-\mathrm{Bu}_{3} \mathrm{SnH}(13 \mu \mathrm{L}, 0.046 \mathrm{mmol})$ added by a syringe pump over $1 \mathrm{~h}$ was required. All the starting material was consumed after 5 h. Column chromatography (hexanes to hexanes-EtOAc, 6:4) gave $29 \beta$ (38.3 mg, $0.072 \mathrm{mmol}, 76 \%$ ).

Method C. Following the general procedure, starting from substrate $7(63.9 \mathrm{mg}, 0.094 \mathrm{mmol})$, after $3 \mathrm{~h}$ of reaction, a supplementary addition of TTMSS $(29 \mu \mathrm{L}, 0.094 \mathrm{mmol})$ was required. All the starting material was consumed after $5 \mathrm{~h}$. Column chromatography (hexanes-EtOAc, 7:3 to $1: 1$ ) gave $\mathbf{2 9 \beta}(28.8 \mathrm{mg}, 0.054 \mathrm{mmol}, 57 \%)$.
Method D. Following the general procedure, starting from substrate 7 (52.4 mg, $0.077 \mathrm{mmol})$, after $3 \mathrm{~h}$ of reaction, a supplementary addition of $n-\mathrm{Bu}_{3} \mathrm{SnD}(21 \mu \mathrm{L}, 0.077 \mathrm{mmol})$ was required. All the starting material was consumed after $6 \mathrm{~h}$. Column chromatography (hexanes to hexanes-EtOAc, 1:1) gave 3-C-(2-Oacetyl-3,4,6-tri- $O$-benzyl- $\beta$-D- $\left[1-{ }^{2} \mathrm{H}\right]$ mannopyranosyl) 1 -propanol $\left(\left[1-{ }^{2} \mathrm{H}\right] 29 \beta\right)\left(30.8 \mathrm{mg}, 0.057 \mathrm{mmol}, 75 \%,{ }^{2} \mathrm{H} /{ }^{1} \mathrm{H} \quad 5.8: 1\right)$ as an amorphous solid: ${ }^{1} \mathrm{H}$ NMR $\left(500 \mathrm{MHz}, \mathrm{CDCl}_{3}\right) \delta_{\mathrm{H}} 7.36-7.15(\mathrm{~m}$, $15 \mathrm{H}, \mathrm{Ar}), 5.466$ (d, $J=2.9 \mathrm{~Hz}, 1 \mathrm{H}, 2-\mathrm{H}), 4.85(\mathrm{~d}, J=10.7 \mathrm{~Hz}, 1 \mathrm{H}$, $\mathrm{OBn}$ ), $4.74(\mathrm{~d}, J=11.1 \mathrm{~Hz}, 1 \mathrm{H}, \mathrm{OBn}), 4.61(\mathrm{~d}, J=12.0 \mathrm{~Hz}, 1 \mathrm{H}$, $\mathrm{OBn}), 4.54(\mathrm{~d}, J=12.3 \mathrm{~Hz}, 1 \mathrm{H}, \mathrm{OBn}), 4.48(\mathrm{~d}, J=10.4 \mathrm{~Hz}, 1 \mathrm{H}$, OBn ), 4.48 (d, $J=10.4 \mathrm{~Hz}, 1 \mathrm{H}, \mathrm{OBn}), 3.73(\mathrm{dd}, J=10.8,1.9 \mathrm{~Hz}, 1 \mathrm{H}$, 6- $\mathrm{H}_{\mathrm{b}}$ ), 3.70-3.63 (m, 5H, 3-H, 4- $\mathrm{H}, 5-\mathrm{H}, 6-\mathrm{H}_{\mathrm{a}}, 3-\mathrm{H}_{\mathrm{b}}$ ), 3.47 (ddd, $J=$ 8.6, 6.0, $\left.1.9 \mathrm{~Hz}, 1 \mathrm{H}, 3{ }^{\prime}-\mathrm{H}_{\mathrm{a}}\right), 2.18(\mathrm{~s}, 3 \mathrm{H}, \mathrm{OAc}), 1.71-1.66\left(\mathrm{~m}, 3 \mathrm{H}, \mathrm{l}^{\prime}-\right.$ $\left.\mathrm{H}_{\mathrm{b}}, 2^{\prime}-\mathrm{H}_{2}\right), 1.566 \mathrm{ppm}\left(\mathrm{m}, 1 \mathrm{H}, 1^{\prime}-\mathrm{H}_{\mathrm{a}}\right), 1 \mathrm{H}$ from $\mathrm{OH}$ is missing. ${ }^{13} \mathrm{C}\left\{{ }^{1} \mathrm{H}\right\}$ NMR $\left(125.7 \mathrm{MHz}, \mathrm{CDCl}_{3}\right) \delta_{\mathrm{C}} 170.9$ (C, OAc), 138.3 (C, $\mathrm{Ar}), 138.2$ (C, Ar), 137.8 (C, Ar), 127.6-128.4 (15 × CH, Ar), 81.8 $(\mathrm{CH}), 79.2(\mathrm{CH}), 75.1\left(\mathrm{CH}_{2}, \mathrm{OBn}\right), 74.7(\mathrm{CH}), 73.4\left(\mathrm{CH}_{2}, \mathrm{OBn}\right)$, $71.5\left(\mathrm{CH}_{2}, \mathrm{OBn}\right), 69.50(\mathrm{CH}, \mathrm{C}-2), 69.4\left(\mathrm{CH}_{2}, \mathrm{C}-6\right), 62.5\left(\mathrm{CH}_{2}, \mathrm{C}-\right.$ 3'), $29.4\left(\mathrm{CH}_{2}, \mathrm{C}-2^{\prime}\right), 28.06\left(\mathrm{CH}_{2}, \mathrm{C}-1^{\prime}\right), 21.0 \mathrm{ppm}\left(\mathrm{CH}_{3}, \mathrm{OAc}\right), \mathrm{C}-1$ was undetectable. MS $($ ESI $) m / z(\%)=558(100)[\mathrm{M}+\mathrm{Na}]^{+}, 557$ (16) $[\mathrm{M}+\mathrm{Na}]^{+}$. HRMS (ESI) $m / z:[\mathrm{M}+\mathrm{Na}]^{+}$calcd for $\mathrm{C}_{32} \mathrm{H}_{37}{ }^{2} \mathrm{HNaO}_{7}$ 558.2578; found 558.2582, $[\mathrm{M}+\mathrm{Na}]^{+}$calcd for $\mathrm{C}_{32} \mathrm{H}_{38} \mathrm{NaO}_{7}$ 557.2515; found 557.2511.

Method E. Following the general procedure, starting from substrate $7(57.8 \mathrm{mg}, 0.085 \mathrm{mmol})$, after $2 \mathrm{~h}$ of reaction, a supplementary addition of $n-\mathrm{Bu}_{3} \mathrm{SnD}(23 \mu \mathrm{L}, 0.085 \mathrm{mmol})$ and $\mathrm{BF}_{3} \bullet \mathrm{Et}_{2} \mathrm{O}(2 \mu \mathrm{L}$, $0.017 \mathrm{mmol}$ ) was required. All the starting material was consumed after 5 h. Column chromatography (hexanes to hexanes-EtOAc, 1:1) gave $\left[2-{ }^{2} \mathrm{H}\right] 25\left(20.2 \mathrm{mg}, 0.043 \mathrm{mmol}, 50 \%,{ }^{2} \mathrm{H} /{ }^{1} \mathrm{H} 1.6: 1\right)$ and $\left[1-{ }^{2} \mathrm{H}\right]$ $29 \beta$ (8.6 mg, $0.016 \mathrm{mmol}, 19 \%,{ }^{2} \mathrm{H} /{ }^{1} \mathrm{H} 3.5: 1$ ).

Radical Reactions of 8 . Method A. Following the general procedure, starting from substrate $8(60.4 \mathrm{mg}, 0.07 \mathrm{mmol})$, after 2 $\mathrm{h}$ of reaction, a supplementary addition of $n-\mathrm{Bu}_{3} \mathrm{SnH}(19 \mu \mathrm{L}, 0.07$ $\mathrm{mmol}$ ) was required. All the starting material was consumed after $6 \mathrm{~h}$. Column chromatography (hexanes to hexanes-EtOAc, 8:2) gave 25 (12 mg, $0.025 \mathrm{mmol}, 37 \%$ ).

Method $B$. Following the general procedure, starting from substrate $8(64.6 \mathrm{mg}, 0.075 \mathrm{mmol})$, after $2 \mathrm{~h}$ of reaction, a supplementary addition of $n-\mathrm{Bu}_{3} \mathrm{SnH}(20 \mu \mathrm{L}, 0.075 \mathrm{mmol})$ added by a syringe pump over $1 \mathrm{~h}$ was required. All the starting material was consumed after 11 h. Column chromatography (hexanes to hexanes-EtOAc, 8:2) gave 25 (13.7 mg, $0.029 \mathrm{mmol}, 39 \%)$.

Method $D$. Following the general procedure, starting from substrate 8 (92.7 $\mathrm{mg}, 0.11 \mathrm{mmol})$, after $2 \mathrm{~h}$ of reaction, a supplementary addition of $n-\mathrm{Bu}_{3} \mathrm{SnD}(29 \mu \mathrm{L}, 0.11 \mathrm{mmol})$ was required. All the starting material was consumed after $3 \mathrm{~h}$. Column chromatography (hexanes to hexanes-EtOAc, 8:2) gave $\left(2-{ }^{2} \mathrm{H}\right) 25$ (26.3 mg, $0.055 \mathrm{mmol}, 52 \%)$.

Method E. Following the general procedure, starting from substrate $8(60 \mathrm{mg}, 0.069 \mathrm{mmol})$, after $2 \mathrm{~h}$ of reaction, a supplementary addition of $n-\mathrm{Bu}_{3} \mathrm{SnD}(17 \mu \mathrm{L}, 0.069 \mathrm{mmol})$ and $\mathrm{BF}_{3} \bullet \mathrm{Et}_{2} \mathrm{O}(2 \mu \mathrm{L}$, $0.016 \mathrm{mmol}$ ) was required. All the starting material was consumed after $3 \mathrm{~h}$. Column chromatography (hexanes to hexanes-EtOAc, 1:1) gave $\left[2-{ }^{2} \mathrm{H}\right] 25\left(20.2 \mathrm{mg}, 0.043 \mathrm{mmol}, 65 \%,{ }^{2} \mathrm{H} /{ }^{1} \mathrm{H} 2.4: 1\right)$.

Method F. Following the general procedure, starting from substrate $8(33.6 \mathrm{mg}, 0.039 \mathrm{mmol})$, all the starting material was consumed after $2 \mathrm{~h}$. Column chromatography (hexanes-EtOAc, 8:2 to 1:1) gave 25 $\left(4.1 \mathrm{mg}, 8.6 \cdot 10^{-3} \mathrm{mmol}, 22 \%\right)$ and $3-C$-(3,4,6-tri-O-benzyl-2-Odiphenoxyphosphoryl- $\beta$-D-mannopyranosyl)1-propanol (30 $\boldsymbol{\beta}) \quad(10.3$ $\mathrm{mg}, 0.014 \mathrm{mmol}, 37 \%)$ as a colorless oil. Compound 30 $\mathrm{\beta}:[\alpha]_{\mathrm{D}}=$ $-27.9\left(c=0.10, \mathrm{CHCl}_{3}\right) .{ }^{1} \mathrm{H}$ NMR $\left(400 \mathrm{MHz}, \mathrm{CDCl}_{3}\right) \delta_{\mathrm{H}} 7.39-7.05$ (m, 25H, Ar), $5.01(\mathrm{dd}, J=9.0,2.1,1 \mathrm{H}, 2-\mathrm{H}), 4.90(\mathrm{~d}, J=11.3 \mathrm{~Hz}$, $1 \mathrm{H}, \mathrm{OBn}), 4.63(\mathrm{~d}, J=11.1 \mathrm{~Hz}, 1 \mathrm{H}, \mathrm{OBn}), 4.60(\mathrm{~d}, J=12.5 \mathrm{~Hz}, 1 \mathrm{H}$, OBn ), $4.53(\mathrm{~d}, J=12.2 \mathrm{~Hz}, 1 \mathrm{H}, \mathrm{OBn}), 4.51(\mathrm{~d}, J=11.4 \mathrm{~Hz}, 1 \mathrm{H}$, OBn), 4.29 (d, $J=10.8 \mathrm{~Hz}, 1 \mathrm{H}, \mathrm{OBn}), 3.67$ (dd, $J=10.8,1.8 \mathrm{~Hz}$, $1 \mathrm{H}), 3.63-3.56(\mathrm{~m}, 3 \mathrm{H}), 3.54-3.49(\mathrm{~m}, 2 \mathrm{H}), 3.44-3.40(\mathrm{~m}, 2 \mathrm{H})$, $1.68-1.54 \mathrm{ppm}\left(\mathrm{m}, 4 \mathrm{H}, 1^{\prime}-\mathrm{H}_{2}, 2^{\prime}-\mathrm{H}_{2}\right), 1 \mathrm{H}$ from $\mathrm{OH}$ is missing. ${ }^{13} \mathrm{C}\left\{{ }^{1} \mathrm{H}\right\}$ NMR $\left(100.6 \mathrm{MHz}, \mathrm{CDCl}_{3}\right) \delta_{\mathrm{C}} 150.9\left(\mathrm{~d},{ }^{2} J_{\mathrm{PC}}=8.5 \mathrm{~Hz}, \mathrm{C}\right.$, Ar), $150.7\left(\mathrm{~d},{ }^{2} J_{\mathrm{PC}}=6.4 \mathrm{~Hz}, \mathrm{C}, \mathrm{Ar}\right), 138.2(\mathrm{C}, \mathrm{Ar}), 138.1$ (C, Ar), 
137.6 (C, Ar), $129.7(2 \times \mathrm{CH}, \mathrm{Ar}), 129.4(2 \times \mathrm{CH}, \mathrm{Ar}), 128.4(2 \times$ $\mathrm{CH}, \mathrm{Ar}), 128.33(4 \times \mathrm{CH}, \mathrm{Ar}), 128.26(2 \times \mathrm{CH}, \mathrm{Ar}), 128.0(2 \times \mathrm{CH}$, $\mathrm{Ar}), 127.8(2 \times \mathrm{CH}, \mathrm{Ar}), 127.70(\mathrm{CH}, \mathrm{Ar}), 127.65(\mathrm{CH}, \mathrm{Ar}), 127.6$ (CH, Ar), $125.3(\mathrm{CH}, \mathrm{Ar}), 124.8(\mathrm{CH}, \mathrm{Ar}), 120.4(\mathrm{CH}, \mathrm{Ar}), 120.3$ $(\mathrm{CH}, \mathrm{Ar}), 120.22(\mathrm{CH}, \mathrm{Ar}), 120.16(\mathrm{CH}, \mathrm{Ar}), 81.8(\mathrm{CH}, \mathrm{C}-3), 79.2$ $(\mathrm{CH}, \mathrm{C}-5), 77.3(\mathrm{CH}, \mathrm{C}-2), 77.2\left(\mathrm{~d},{ }^{3} J_{\mathrm{PC}}=7.8 \mathrm{~Hz}, \mathrm{CH}, \mathrm{C}-1\right), 75.2$ $\left(\mathrm{CH}_{2}, \mathrm{OBn}\right), 74.1(\mathrm{CH}, \mathrm{C}-4), 73.4\left(\mathrm{CH}_{2}, \mathrm{OBn}\right), 71.7\left(\mathrm{CH}_{2}, \mathrm{OBn}\right)$, 69.3 ( $\left.\mathrm{CH}_{2}, \mathrm{C}-6\right), 62.4\left(\mathrm{CH}_{2}, \mathrm{C}-3{ }^{\prime}\right), 29.3\left(\mathrm{CH}_{2}, \mathrm{C}-1^{\prime}\right.$ or C-2'), 28.1 ppm $\left(\mathrm{CH}_{2}, \mathrm{C}-1^{\prime}\right.$ or C-2'). IR $\left(\mathrm{CHCl}_{3}\right): \nu=3567,2928,2858,1490$, $1212 \mathrm{~cm}^{-1}$. MS (ESI) $\mathrm{m} / z(\%)=747(100)[\mathrm{M}+\mathrm{Na}]^{+}$. HRMS (ESI) $m / z:[\mathrm{M}+\mathrm{Na}]^{+}$calcd for $\mathrm{C}_{42} \mathrm{H}_{45} \mathrm{NaO}_{9} \mathrm{P}$ 747.2699; found 747.2709.

Method $G$. Following the general procedure, starting from substrate 8 (37.1 $\mathrm{mg}, 0.043 \mathrm{mmol}$ ), all the starting material was consumed after $3 \mathrm{~h}$. Column chromatography (hexanes-EtOAc, 8:2 to $1: 1)$ gave $25(6.5 \mathrm{mg}, 0.014 \mathrm{mmol}, 32 \%)$ and $\mathbf{3 0} \beta(7.8 \mathrm{mg}, 0.011$ mmol, 25\%).

Radical Reactions of 9. Method A. Following the general procedure, starting from substrate $9(64.4 \mathrm{mg}, 0.11 \mathrm{mmol})$, after 2 $\mathrm{h}$ of reaction, a supplementary addition of $n-\mathrm{Bu}_{3} \mathrm{SnH}(30 \mu \mathrm{L}, 0.11$ $\mathrm{mmol}$ ) was required. All the starting material was consumed after $5 \mathrm{~h}$. Column chromatography (hexanes to hexanes-EtOAc, 6:4) gave (4S)-1,4-anhydro-6,7-di-O-benzyl-2,3,5,9-tetradeoxy- $\beta$-L-lyxo-non-4ulopyranose $(31 S)$ contaminated with the thermodynamic isomer (4R)-1,4-anhydro-6,7-di-O-benzyl-2,3,5,9-tetradeoxy- $\alpha$-L-lyxo-non-4ulopyranose $(31 R)(19.2 \mathrm{mg}, 0.05 \mathrm{mmol}, 46 \%, S / R, 85: 15)$ as an amorphous solid and an inseparable mixture of 3-C-(2-O-acetyl-3,4di-O-benzyl-6-deoxy- $\beta$-D-altropyranosyl)1-propanol (32) and 3-C-(2$O$-acetyl-3,4-di-O-benzyl- $\alpha$-L-fucopyranosyl) 1-propanol (33) $(7.4 \mathrm{mg}$, $0.017 \mathrm{mmol}, 15 \%, 1: 1.7)$ as a colorless oil. Compound $31 \mathrm{~S}:{ }^{1} \mathrm{H}$ NMR $\left(500 \mathrm{MHz}, \mathrm{CDCl}_{3}\right.$, simulated ring coupling constants using DAISY) $\delta_{\mathrm{H}} 7.41-7.25(\mathrm{~m}, 10 \mathrm{H}, \mathrm{Ar}), 4.955(\mathrm{~d}, J=12.0 \mathrm{~Hz}, 1 \mathrm{H}, \mathrm{OBn}), 4.73(\mathrm{~d}$, $J=11.7 \mathrm{~Hz}, 1 \mathrm{H}, \mathrm{OBn}), 4.63(\mathrm{~d}, J=12.0 \mathrm{~Hz}, 1 \mathrm{H}, \mathrm{OBn}), 4.60(\mathrm{~d}, J=$ $12.0 \mathrm{~Hz}, 1 \mathrm{H}, \mathrm{OBn}$ ), 3.93 (ddd, $J=12.1,4.5,2.7 \mathrm{~Hz}, 1 \mathrm{H}, 3-\mathrm{H}$ ), 3.87 (ddd, $J=8.2,8.2,5.4 \mathrm{~Hz}, 1 \mathrm{H}, 3^{\prime}-\mathrm{H}_{\mathrm{b}}$ ), 3.82 (dddd, $J=6.5,6.5,6.5,1.7$ $\mathrm{Hz}, 1 \mathrm{H}, 5-\mathrm{H}$ ), 3.81 (ddd, $J=8.2,8.2,6.6 \mathrm{~Hz}, 1 \mathrm{H}, 3$ ' $-\mathrm{H}_{\mathrm{a}}$ ), 3.58 (ddd, $J$ $\left.=2.7,1.7 \mathrm{~Hz},{ }^{4} J_{2 \mathrm{a}, 4}=1.3 \mathrm{~Hz}, 1 \mathrm{H}, 4-\mathrm{H}\right), 2.32(\mathrm{dd}, J=12.3,12.1 \mathrm{~Hz}$, $\left.1 \mathrm{H}, 2-\mathrm{H}_{\mathrm{b}}\right), 2.13-2.00\left(\mathrm{~m}, 2 \mathrm{H}, 1^{\prime}-\mathrm{H}_{\mathrm{b}}, 2^{\prime}-\mathrm{H}_{\mathrm{b}}\right), 1.92(\mathrm{dd}, J=12.3,4.5$ $\left.\mathrm{Hz},{ }^{4} J_{2 \mathrm{a}, 4}=1.3 \mathrm{~Hz}, 1 \mathrm{H}, 2-\mathrm{H}_{\mathrm{a}}\right), 1.90\left(\mathrm{~m}, 1 \mathrm{H}, 2^{\prime}-\mathrm{H}_{\mathrm{a}}\right), 1.75\left(\mathrm{~m}, 1 \mathrm{H}, \mathrm{l}^{\prime}-\right.$ $\left.\mathrm{H}_{\mathrm{a}}\right), 1.14 \mathrm{ppm}\left(\mathrm{d}, J=6.5 \mathrm{~Hz}, 3 \mathrm{H}, 6-\mathrm{H}_{3}\right),{ }^{1} \mathrm{H}$ NMR $\left(500 \mathrm{MHz}, \mathrm{C}_{6} \mathrm{D}_{6}\right.$, simulated ring coupling constants using DAISY) $\delta_{\mathrm{H}} 7.44-7.42(\mathrm{~m}$, $2 \mathrm{H}, \mathrm{Ar}$ ), 7.38-7.35 (m, 2H, Ar), 7.22-7.13 (m, 6H, Ar), 5.05 (d, $J=$ $\left.11.5 \mathrm{~Hz}, 1 \mathrm{H}, \mathrm{C}_{4}-\mathrm{OBn}\right), 4.59$ (d, $\left.J=11.5 \mathrm{~Hz}, 1 \mathrm{H}, \mathrm{C}_{4}-\mathrm{OBn}\right), 4.44$ (d, $J$ $\left.=12.1 \mathrm{~Hz}, 1 \mathrm{H}, \mathrm{C}_{3}-\mathrm{OBn}\right), 4.40\left(\mathrm{~d}, J=12.1 \mathrm{~Hz}, 1 \mathrm{H}, \mathrm{C}_{3}-\mathrm{OBn}\right), 4.02$ (ddd, $J=12.0,4.5,2.7 \mathrm{~Hz}, 1 \mathrm{H}, 3-\mathrm{H}$ ), 3.87 (dddd, $J=6.5,6.5,6.5,1.4$ $\mathrm{Hz}, 1 \mathrm{H}, 5-\mathrm{H}), 3.79-3.73\left(\mathrm{~m}, 1 \mathrm{H}, 3^{\prime}-\mathrm{H}_{\mathrm{b}}\right), 3.71-3.64\left(\mathrm{~m}, 1 \mathrm{H}, 3^{\prime}-\mathrm{H}_{\mathrm{a}}\right)$, 3.36 (ddd, $\left.J=2.7,1.4 \mathrm{~Hz},{ }^{4} J_{2 \mathrm{a}, 4}=1.2 \mathrm{~Hz}, 1 \mathrm{H}, 4-\mathrm{H}\right), 2.49$ (dd, $J=12.1$, $\left.12.0 \mathrm{~Hz}, 1 \mathrm{H}, 2-\mathrm{H}_{\mathrm{b}}\right), 2.03-2.00(\mathrm{~m}, 1 \mathrm{H}), 1.94(\mathrm{ddd}, J=12.1,4.5 \mathrm{~Hz}$, $\left.{ }^{4} J_{2 \mathrm{a}, 4}=1.2 \mathrm{~Hz}, 1 \mathrm{H}, 2-\mathrm{H}_{\mathrm{a}}\right), 1.86-1.78(\mathrm{~m}, 1 \mathrm{H}), 1.46-1.40(\mathrm{~m}, 2 \mathrm{H})$, $1.28 \mathrm{ppm}\left(\mathrm{d}, J=6.5 \mathrm{~Hz}, 3 \mathrm{H}, 6-\mathrm{H}_{3}\right) .{ }^{13} \mathrm{C}\left\{{ }^{1} \mathrm{H}\right\} \mathrm{NMR}(125.7 \mathrm{MHz}$, $\left.\mathrm{CDCl}_{3}\right) \delta_{\mathrm{C}} 139.0(\mathrm{C}, \mathrm{Ar}), 138.8(\mathrm{C}, \mathrm{Ar}), 128.5(2 \times \mathrm{CH}, \mathrm{Ar}), 128.4$ $(2 \times \mathrm{CH}, \mathrm{Ar}), 128.1(2 \times \mathrm{CH}, \mathrm{Ar}), 127.2(2 \times \mathrm{CH}, \mathrm{Ar}), 127.2(2 \times$ $\mathrm{CH}, \mathrm{Ar}), 106.8$ (C, C-1), $77.3(\mathrm{CH}, \mathrm{C}-3), 75.0(\mathrm{CH}, \mathrm{C}-4), 74.16$ $\left(\mathrm{CH}_{2}, \mathrm{OBn}\right), 70.5\left(\mathrm{CH}_{2}, \mathrm{OBn}\right), 67.6(\mathrm{CH}, \mathrm{C}-5), 67.1\left(\mathrm{CH}_{2}, \mathrm{C}-3^{\prime}\right)$, $37.5\left(\mathrm{CH}_{2}, \mathrm{C}-1^{\prime}\right), 34.0\left(\mathrm{CH}_{2}, \mathrm{C}-2\right), 23.6\left(\mathrm{CH}_{2}, \mathrm{C}-2^{\prime}\right), 17.4 \mathrm{ppm}$ $\left(\mathrm{CH}_{3}, \mathrm{C}-6\right) .{ }^{13} \mathrm{C}\left\{{ }^{1} \mathrm{H}\right\}$ NMR $\left(125.7 \mathrm{MHz}, \mathrm{C}_{6} \mathrm{D}_{6}\right) \delta_{\mathrm{C}} 140.3(\mathrm{C}, \mathrm{Ar})$ some aromatic carbons were not observed, 140.0 (C, Ar), 107.4 (C, $\mathrm{C}-1), 78.0$ (CH, C-3), 76.9 (CH, C-4), $75.3\left(\mathrm{CH}_{2}, \mathrm{OBn}\right), 70.8\left(\mathrm{CH}_{2}\right.$, $\mathrm{OBn}), 68.5(\mathrm{CH}, \mathrm{C}-5), 67.6\left(\mathrm{CH}_{2}, \mathrm{C}-3{ }^{\prime}\right), 38.2\left(\mathrm{CH}_{2}, \mathrm{C}-1^{\prime}\right), 34.8$ $\left(\mathrm{CH}_{2}, \mathrm{C}-2\right), 24.4\left(\mathrm{CH}_{2}, \mathrm{C}-2{ }^{\prime}\right), 18.1 \mathrm{ppm}\left(\mathrm{CH}_{3}, \mathrm{C}-6\right)$. IR $\left(\mathrm{CHCl}_{3}\right): \nu$ $=2930,1226,1206 \mathrm{~cm}^{-1}$. MS (ESI) $\mathrm{m} / z(\%)=391(100)[\mathrm{M}+$ $\mathrm{Na}]^{+}$. HRMS (ESI) $m / z:[\mathrm{M}+\mathrm{Na}]^{+}$calcd for $\mathrm{C}_{23} \mathrm{H}_{28} \mathrm{NaO}_{4}$ 391.1885; found 391.1891. Compounds 32 and 33: ${ }^{1} \mathrm{H}$ NMR (500 MHz, $\mathrm{CDCl}_{3}$, selected signals of 32 from the mix spectrum) $\delta_{\mathrm{H}} 4.95(\mathrm{dd}, J=$ $3.8,1.6 \mathrm{~Hz}, 1 \mathrm{H}, 2-\mathrm{H}), 4.72(\mathrm{br} \mathrm{s}, 2 \mathrm{H}, \mathrm{OBn}), 4.45(\mathrm{~d}, J=11.7 \mathrm{~Hz}, 1 \mathrm{H}$, OBn), 4.34 (d, $J=11.7 \mathrm{~Hz}, 1 \mathrm{H}, \mathrm{OBn}$ ), 3.93 (dddd, $J=9.5,6.3,6.3$, $6.3 \mathrm{~Hz}, 1 \mathrm{H}, 5-\mathrm{H}$ ), 3.88 (ddd, $J=9.2,4.1,1.3 \mathrm{~Hz}, 1 \mathrm{H}, 1-\mathrm{H}$ ), 3.248 (dd, $J=9.8,3.2 \mathrm{~Hz}, 1 \mathrm{H}, 4-\mathrm{H}), 1.286 \mathrm{ppm}\left(\mathrm{d}, J=6.3 \mathrm{~Hz}, 3 \mathrm{H}, 6-\mathrm{H}_{3}\right)$. ${ }^{13} \mathrm{C}\left\{{ }^{1} \mathrm{H}\right\}$ NMR $\left(125.7 \mathrm{MHz}, \mathrm{CDCl}_{3}\right.$, selected signals of 32 from the mix spectrum) $\delta_{\mathrm{C}} 170.4(\mathrm{C}, \mathrm{OAc}), 138.0(\mathrm{C}, \mathrm{Ar}), 137.7$ (C, Ar), 77.67 (CH, C-4), 73.4 (CH, C-1), $72.6\left(\mathrm{CH}_{2}, \mathrm{OBn}\right), 71.7(\mathrm{CH}, \mathrm{C}-3)$,
71.4 (CH, C-2), 71.3 ( $\left.\mathrm{CH}_{2}, \mathrm{OBn}\right), 71.2(\mathrm{CH}, \mathrm{C}-5), 62.6\left(\mathrm{CH}_{2}, \mathrm{C}-3\right.$ '), $27.6\left(\mathrm{CH}_{2}, \mathrm{C}-1^{\prime}\right.$ or $\left.\mathrm{C}-2^{\prime}\right), 26.1\left(\mathrm{CH}_{2}, \mathrm{C}-1^{\prime}\right.$ or $\left.\mathrm{C}-2^{\prime}\right), 8.19 \mathrm{ppm}\left(\mathrm{CH}_{3}\right.$, C-6). ${ }^{1} \mathrm{H}$ NMR ( $500 \mathrm{MHz}, \mathrm{CDCl}_{3}$, selected signals of 33 from the mix spectrum) $\delta_{\mathrm{H}} 5.08(\mathrm{dd}, J=5.1,2.6 \mathrm{~Hz}, 1 \mathrm{H}, 2-\mathrm{H}), 4.76(\mathrm{~d}, J=$ $12.0 \mathrm{~Hz}, 1 \mathrm{H}, \mathrm{OBn}$ ), 4.67 (d, $J=12.0 \mathrm{~Hz}, 1 \mathrm{H}, \mathrm{OBn}), 4.63$ (d, $J=12.0$ $\mathrm{Hz}, 1 \mathrm{H}, \mathrm{OBn}$ ), 4.54 (d, $J=12.0 \mathrm{~Hz}, 1 \mathrm{H}, \mathrm{OBn}), 4.14-4.06$ (m, 2H, 1$\mathrm{H}, 5-\mathrm{H}), 3.75(\mathrm{dd}, J=4.8,3.2 \mathrm{~Hz}, 1 \mathrm{H}, 4-\mathrm{H}), 1.41 \mathrm{ppm}(\mathrm{d}, J=6.9 \mathrm{~Hz}$, $\left.3 \mathrm{H}, 6-\mathrm{H}_{3}\right) \cdot{ }^{13} \mathrm{C}\left\{{ }^{1} \mathrm{H}\right\}$ NMR $\left(125.7 \mathrm{MHz}, \mathrm{CDCl}_{3}\right.$, selected signals of 33 from the mix spectrum) $\delta_{\mathrm{C}} 170.3(\mathrm{C}, \mathrm{OAc}), 138.4(\mathrm{C}, \mathrm{Ar}), 138.2(\mathrm{C}$, $\mathrm{Ar}), 75.4(\mathrm{CH}, \mathrm{C}-3)$, $74.2(\mathrm{CH}, \mathrm{C}-4), 73.0\left(\mathrm{CH}_{2}, \mathrm{OBn}\right), 72.0\left(\mathrm{CH}_{2}\right.$, OBn), 71.8 (CH, C-2), $70.0(\mathrm{CH}, \mathrm{C}-5), 67.5(\mathrm{CH}, \mathrm{C}-1), 62.6\left(\mathrm{CH}_{2}\right.$, C-3'), $29.7\left(\mathrm{CH}_{2}, \mathrm{C}-1^{\prime}\right.$ or C-2'), $29.4\left(\mathrm{CH}_{2}, \mathrm{C}-1^{\prime}\right.$ or $\left.\mathrm{C}-2^{\prime}\right), 14.2 \mathrm{ppm}$ $\left(\mathrm{CH}_{3}, \mathrm{C}-6\right)$. IR $\left(\mathrm{CHCl}_{3}\right): \nu=3690,3018,1735,1222 \mathrm{~cm}^{-1} . \mathrm{MS}$ (ESI) $m / z(\%)=451(100)[\mathrm{M}+\mathrm{Na}]^{+}$. HRMS (ESI) $\mathrm{m} / z:[\mathrm{M}+$ $\mathrm{Na}]^{+}$calcd for $\mathrm{C}_{25} \mathrm{H}_{32} \mathrm{NaO}_{6} 451.2097$; found 451.2095.

Method $D$. Following the general procedure, starting from substrate $9(59.2 \mathrm{mg}, 0.10 \mathrm{mmol})$, after $2 \mathrm{~h}$ of reaction, a supplementary addition of $n-\mathrm{Bu}_{3} \mathrm{SnD}(28 \mu \mathrm{L}, 0.10 \mathrm{mmol})$ was required. All the starting material was consumed after $4 \mathrm{~h}$. Column chromatography (hexanes to hexanes-EtOAc, 6:4) gave $(4 S)$-1,4anhydro-6,7-di-O-benzyl-2,3,5,9-tetradeoxy- $\beta$-L- $\left[7-O-\mathrm{PhCH}_{-}{ }^{2} \mathrm{H}\right]$ lyxonon-4-ulopyranose $\left(\left[\mathrm{PhCH}_{-}{ }^{2} \mathrm{H}\right] 31\right)(19.5 \mathrm{mg}, 0.053 \mathrm{mmol}, 53 \%$, ${ }^{2} \mathrm{H} /{ }^{1} \mathrm{H}$ 1.5:1) and a mixture of 3-C-(2-O-acetyl-3,4-di-O-benzyl-6deoxy- $\beta$-D- $\left(5{ }^{2} \mathrm{H}\right)$ altropyranosyl $) 1$-propanol $\left(5-{ }^{2} \mathrm{H}\right) 32$ and 33 (15.8 $\left.\mathrm{mg}, 0.037 \mathrm{mmol}, 36 \%,{ }^{2} \mathrm{H} /{ }^{1} \mathrm{H} 1: 2.8\right)$ as colorless oils. Compound $\left[\mathrm{PhCH}_{-}{ }^{2} \mathrm{H}\right] 31:{ }^{1} \mathrm{H}$ NMR $\left(500 \mathrm{MHz}, \mathrm{CDCl}_{3}\right) \delta_{\mathrm{H}} 7.40-7.25(\mathrm{~m}, 10 \mathrm{H}$, $\mathrm{Ar}$ ), 4.95 (d, $J=12.0 \mathrm{~Hz}, 0.4 \mathrm{H}, \mathrm{OBn}$ ), 4.93 (br s, 0.5H, O-CHD-Ph), $4.72(\mathrm{~d}, J=12.0 \mathrm{~Hz}, 0.4 \mathrm{H}, \mathrm{OBn}), 4.71(\mathrm{br} \mathrm{s}, 0.1 \mathrm{H}, \mathrm{O}-\mathrm{CHD}-\mathrm{Ph}), 4.62$ $(\mathrm{d}, J=12.0 \mathrm{~Hz}, 1 \mathrm{H}, \mathrm{OBn}), 4.59(\mathrm{~d}, J=12.0 \mathrm{~Hz}, 1 \mathrm{H}, \mathrm{OBn}), 3.931$ (ddd, $J=12.3,4.4,2.5 \mathrm{~Hz}, 0.5 \mathrm{H}, 3-\mathrm{H}$ ), 3.929 (ddd, $J=12.3,5.0,2.8$ $\mathrm{Hz}, 0.5 \mathrm{H}, 3-\mathrm{H}$ ), 3.87 (ddd, $J=8.2,8.2,5.7 \mathrm{~Hz}, 1 \mathrm{H}, 3^{\prime}-\mathrm{H}_{\mathrm{b}}$ ), 3.82 (ddd, $\left.J=8.2,8.2,6.6 \mathrm{~Hz}, 1 \mathrm{H}, 3^{\prime}-\mathrm{H}_{\mathrm{a}}\right), 3.81(\mathrm{~m}, 1 \mathrm{H}, 5-\mathrm{H}), 3.57(\mathrm{br} \mathrm{s}, 1 \mathrm{H}, 4-$ $\mathrm{H}), 2.32\left(\mathrm{dd}, J=12.3,12.3 \mathrm{~Hz}, 1 \mathrm{H}, 2-\mathrm{H}_{\mathrm{b}}\right), 2.13-1.99\left(\mathrm{~m}, 2 \mathrm{H}, 1^{\prime}-\mathrm{H}_{\mathrm{b}}\right.$, $\left.2^{\prime}-\mathrm{H}_{\mathrm{b}}\right), 1.92\left(\mathrm{ddd}, J=12.3,4.7 \mathrm{~Hz},{ }^{4} J_{2 \mathrm{a}, 4}=1.0 \mathrm{~Hz}, 1 \mathrm{H}, 2-\mathrm{H}_{\mathrm{a}}\right), 1.85(\mathrm{~m}$, $\left.1 \mathrm{H}, 2^{\prime}-\mathrm{H}_{\mathrm{a}}\right), 1.74$ (ddd, $J=12.3,10.4,7.9 \mathrm{~Hz}, 1 \mathrm{H}, 1$ '- $\left.-\mathrm{H}_{\mathrm{a}}\right), 1.135(\mathrm{~d}, J=$ $\left.6.3 \mathrm{~Hz}, 1.5 \mathrm{H}, 6-\mathrm{H}_{3}\right), 1.133\left(\mathrm{~d}, J=6.6 \mathrm{~Hz}, 0.3 \mathrm{H}, 6-\mathrm{H}_{3}\right), 1.130 \mathrm{ppm}(\mathrm{d}$, $\left.J=6.3 \mathrm{~Hz}, 1.2 \mathrm{H}, 6-\mathrm{H}_{3}\right) .{ }^{13} \mathrm{C}\left\{{ }^{1} \mathrm{H}\right\} \mathrm{NMR}\left(125.7 \mathrm{MHz}, \mathrm{CDCl}_{3}\right) \delta_{\mathrm{C}}$ 139.0 (0.4C, Ar), 138.9 (0.6C, Ar), 138.8 (C, Ar), 128.49 (CH, Ar), 128.45 ( CH, Ar), $128.4(2 \times \mathrm{CH}, \mathrm{Ar}), 128.1(2 \times \mathrm{CH}, \mathrm{Ar}), 127.4(2$ $\times \mathrm{CH}, \mathrm{Ar}), 127.2(2 \times \mathrm{CH}, \mathrm{Ar}), 106.8(\mathrm{C}, \mathrm{C}-1), 77.7(\mathrm{CH}, \mathrm{C}-3)$, 75.09 (0.4CH, C-4), $75.02(0.1 \mathrm{CH}, \mathrm{C}-4), 74.97$ (0.5CH, C-4), 74.18 $\left(0.4 \mathrm{CH}_{2}, \mathrm{OBn}\right), 73.59\left(\mathrm{t}, J_{\mathrm{CD}}=22.1 \mathrm{~Hz}, 0.6 \mathrm{CHD}-\mathrm{Ph}\right), 70.5\left(\mathrm{CH}_{2}\right.$, OBn), 67.7 ( $\mathrm{CH}, \mathrm{C}-5), 67.1\left(\mathrm{CH}_{2}, \mathrm{C}-3^{\prime}\right), 37.5\left(\mathrm{CH}_{2}, \mathrm{C}-1^{\prime}\right), 34.0$ $\left(\mathrm{CH}_{2}, \mathrm{C}-2\right), 23.6\left(\mathrm{CH}_{2}, \mathrm{C}-2^{\prime}\right), 17.4 \mathrm{ppm}\left(\mathrm{CH}_{3}, \mathrm{C}-6\right) .{ }^{1} \mathrm{H}$ NMR $(500$ $\left.\mathrm{MHz}, \mathrm{C}_{6} \mathrm{D}_{6}\right) \delta_{\mathrm{H}} 7.44-7.42(\mathrm{~m}, 2 \mathrm{H}, \mathrm{Ar}), 7.36-7.35(\mathrm{~m}, 2 \mathrm{H}, \mathrm{Ar})$, $7.22-7.10(\mathrm{~m}, 6 \mathrm{H}, \mathrm{Ar}), 5.046(\mathrm{~d}, J=11.6 \mathrm{~Hz}, 0.4 \mathrm{H}, \mathrm{OBn}), 5.019(\mathrm{br}$ s, $0.5 \mathrm{H}, \mathrm{O}-\mathrm{CHD}-\mathrm{Ph}), 4.59(\mathrm{~d}, J=11.5 \mathrm{~Hz}, 0.4 \mathrm{H}, \mathrm{OBn}), 4.567$ (br s, $0.1 \mathrm{H}, \mathrm{O}-\mathrm{CHD}-\mathrm{Ph}$ ), 4.44 (d, $J=12.1 \mathrm{~Hz}, 1 \mathrm{H}, \mathrm{OBn}), 4.40$ (d, $J=11.9$ $\mathrm{Hz}, 1 \mathrm{H}, \mathrm{OBn}$ ), 4.02 (ddd, $J=12.1,4.8,2.8 \mathrm{~Hz}, 1 \mathrm{H}, 3-\mathrm{H}), 3.89-3.85$ $(\mathrm{m}, 1 \mathrm{H}, 5-\mathrm{H}), 3.78-3.74\left(\mathrm{~m}, 1 \mathrm{H}, 3^{\prime}-\mathrm{H}_{\mathrm{b}}\right), 3.79-3.66\left(\mathrm{~m}, 1 \mathrm{H}, 3^{\prime}-\mathrm{H}_{\mathrm{a}}\right)$, $3.36(\mathrm{~m}, 1 \mathrm{H}, 4-\mathrm{H}), 2.496\left(\mathrm{dd}, J=12.1,12.1 \mathrm{~Hz}, 0.5 \mathrm{H}, 2-\mathrm{H}_{\mathrm{b}}\right), 2.494$ $\left(\mathrm{dd}, J=12.1,12.1 \mathrm{~Hz}, 0.5 \mathrm{H}, 2-\mathrm{H}_{\mathrm{b}}\right), 2.02(\mathrm{~m}, 1 \mathrm{H}), 1.94(\mathrm{ddd}, J=12.6$, $\left.4.3 \mathrm{~Hz},{ }^{4} \mathrm{~J}_{2 \mathrm{a}, 4}=0.6 \mathrm{~Hz}, 1 \mathrm{H}, 2-\mathrm{H}_{\mathrm{a}}\right), 1.86-1.78(\mathrm{~m}, 1 \mathrm{H}), 1.46-1.40(\mathrm{~m}$, $2 \mathrm{H}), 1.280\left(\mathrm{~d}, J=6.6 \mathrm{~Hz}, 1.5 \mathrm{H}, 6-\mathrm{H}_{3}\right), 1.276 \mathrm{ppm}(\mathrm{d}, J=6.6 \mathrm{~Hz}$, $\left.1.5 \mathrm{H}, 6-\mathrm{H}_{3}\right) .{ }^{13} \mathrm{C}\left\{{ }^{1} \mathrm{H}\right\}$ NMR $\left(125.7 \mathrm{MHz}, \mathrm{C}_{6} \mathrm{D}_{6}\right) \delta_{\mathrm{C}}$ the aromatic carbons were not observed, $107.1 \mathrm{ppm}(\mathrm{C}, \mathrm{C}-1), 77.7$ (CH, C-3), $76.93(0.4 \mathrm{CH}, \mathrm{C}-4), 76.88$ (0.1CH, C-4), 76.85 (0.5CH, C-4), 75.26 $\left(0.4 \mathrm{CH}_{2}, \mathrm{OBn}\right), 74.85\left(\mathrm{t}, J_{\mathrm{CD}}=22.1 \mathrm{~Hz}, 0.6 \mathrm{CHD}-\mathrm{Ph}\right), 70.8\left(\mathrm{CH}_{2}\right.$, OBn), 68.5 (CH, C-5), $67.6\left(\mathrm{CH}_{2}, \mathrm{C}-3^{\prime}\right), 38.2\left(\mathrm{CH}_{2}, \mathrm{C}-1^{\prime}\right), 34.8$ $\left(\mathrm{CH}_{2}, \mathrm{C}-2\right), 24.4\left(\mathrm{CH}_{2}, \mathrm{C}-2\right), 18.2 \mathrm{ppm}\left(\mathrm{CH}_{3}, \mathrm{C}-6\right)$. MS (ESI) $\mathrm{m} / z$ $(\%)=392(100)[\mathrm{M}+\mathrm{Na}]^{+}, 391(48)[\mathrm{M}+\mathrm{Na}]^{+}$. HRMS (ESI) $\mathrm{m} / z$ : $[\mathrm{M}+\mathrm{Na}]^{+}$calcd for $\mathrm{C}_{23} \mathrm{H}_{27}{ }^{2} \mathrm{HNaO}_{4}$ 392.1948; found 392.1936, $[\mathrm{M}+$ $\mathrm{Na}]^{+}$calcd for $\mathrm{C}_{23} \mathrm{H}_{28} \mathrm{NaO}_{4}$ 391.1885; found 391.1891. Mixture of $\left(5-{ }^{2} \mathrm{H}\right) 32 / 33$ : ${ }^{1} \mathrm{H}$ NMR (400 $\mathrm{MHz}, \mathrm{CDCl}_{3}$, only the deuterated product $\left(5-^{2} \mathrm{H}\right) 32$ is described) $\delta_{\mathrm{H}} 7.40-7.22(\mathrm{~m}, 10 \mathrm{H}, \mathrm{Ar}), 4.95(\mathrm{dd}$, $J=3.5,1.2 \mathrm{~Hz}, 1 \mathrm{H}, 2-\mathrm{H}), 4.72($ br s, $2 \mathrm{H}, \mathrm{OBn}), 4.45(\mathrm{~d}, J=11.7 \mathrm{~Hz}$, $1 \mathrm{H}, \mathrm{OBn}), 4.34(\mathrm{~d}, J=11.7 \mathrm{~Hz}, 1 \mathrm{H}, \mathrm{OBn}), 3.88$ (ddd, $J=9.1,5.3,1.3$ $\mathrm{Hz}, 1 \mathrm{H}, 1-\mathrm{H}), 3.80$ (m, $1 \mathrm{H}, 3-\mathrm{H}), 3.69-3.58\left(\mathrm{~m}, 2 \mathrm{H}, 3{ }^{\prime}-\mathrm{H}_{2}\right), 3.248$ $(\mathrm{d}, J=3.1 \mathrm{~Hz}, 1 \mathrm{H}, 4-\mathrm{H}), 2.06$ (s, 3H, OAc), $1.67-1.45\left(\mathrm{~m}, 4 \mathrm{H}, 1^{\prime}-\mathrm{H}_{2}\right.$, $\left.2^{\prime}-\mathrm{H}_{2}\right), 1.280 \mathrm{ppm}\left(\mathrm{s}, 3 \mathrm{H}, 6-\mathrm{H}_{3}\right), 1 \mathrm{H}$ from $\mathrm{OH}$ is missing. ${ }^{13} \mathrm{C}\left\{{ }^{1} \mathrm{H}\right\}$ 
NMR $\left(100.6 \mathrm{MHz}, \mathrm{CDCl}_{3}\right.$, only the deuterated product $\left(5-{ }^{2} \mathrm{H}\right) 32$ is described) $\delta_{\mathrm{C}} 170.3$ (C, OAc), $138.0(\mathrm{C}, \mathrm{Ar}), 137.7$ (C, Ar), 127.5$128.3(10 \times \mathrm{CH}, \mathrm{Ar}), 77.48(\mathrm{CH}, \mathrm{C}-4), 73.3(\mathrm{CH}, \mathrm{C}-1), 72.7\left(\mathrm{CH}_{2}\right.$, $\mathrm{OBn}), 71.8(\mathrm{CH}, \mathrm{C}-3), 71.4(\mathrm{CH}, \mathrm{C}-2), 71.4\left(\mathrm{CH}_{2}, \mathrm{OBn}\right), 62.6$ $\left(\mathrm{CH}_{2}, \mathrm{C}-3^{\prime}\right), 27.6\left(\mathrm{CH}_{2}, \mathrm{C}-1^{\prime}\right.$ or C-2'), $26.1\left(\mathrm{CH}_{2}, \mathrm{C}-1\right.$ ' or C-2'), 21.0 $\left(\mathrm{CH}_{3}, \mathrm{OAc}\right), 18.07 \mathrm{ppm}\left(\mathrm{CH}_{3}, \mathrm{C}-6\right)$. MS (ESI) $\mathrm{m} / z(\%)=452(100)$ $[\mathrm{M}+\mathrm{Na}]^{+}, 451(75)[\mathrm{M}+\mathrm{Na}]^{+}$. HRMS (ESI) $m / z:[\mathrm{M}+\mathrm{Na}]^{+}$calcd for $\mathrm{C}_{25} \mathrm{H}_{31}{ }^{2} \mathrm{HNaO}_{6} 452.2159$; found $452.2159,[\mathrm{M}+\mathrm{Na}]^{+}$calcd for $\mathrm{C}_{25} \mathrm{H}_{32} \mathrm{NaO}_{6}$ 451.2097; found 451.2098 .

Method F. Following the general procedure, starting from substrate $9(17.9 \mathrm{mg}, 0.031 \mathrm{mmol})$, all the starting material was consumed after $3 \mathrm{~h}$. Column chromatography (hexanes-EtOAc, 6:4 to 4:6) gave 33 (8.7 mg, $0.020 \mathrm{mmol}, 65 \%$ ).

Method G. Following the general procedure, starting from substrate $9(18.2 \mathrm{mg}, 0.032 \mathrm{mmol})$, all the starting material was consumed after $3 \mathrm{~h}$. Column chromatography (hexanes-EtOAc, 6:4 to $4: 6)$ gave 33 ( $13.1 \mathrm{mg}, 0.031 \mathrm{mmol}, 67 \%)$.

Radical Reactions of 10. Method A. Following the general procedure, starting from substrate $10(95.8 \mathrm{mg}, 0.13 \mathrm{mmol})$, after $2 \mathrm{~h}$ of reaction, a supplementary addition of $n-\mathrm{Bu}_{3} \mathrm{SnH}(15 \mu \mathrm{L}, 0.07$ $\mathrm{mmol}$ ) was required. All the starting material was consumed after $4 \mathrm{~h}$. Column chromatography (hexanes to hexanes-EtOAc, 4:6) gave 31 (24.7 mg, $0.07 \mathrm{mmol}, 52 \%, S / R, 83: 17$ ), 2,6:5,9-di-anhydro-3,4-di-Obenzyl-1,7,8-trideoxy-D-glycero-D-galacto-nonitol (36) (4.6 mg, 0.013 mmol, 10\%), 3-C-(3,4-di-O-benzyl-6-deoxy-2-O-diphenoxyphosphoryl- $\beta$-D-altropyranosyl) 1-propanol (34) (5.1 mg, $0.008 \mathrm{mmol}, 6 \%)$, and 3 - $C$-(3,4-di-O-benzyl-2-O-diphenoxyphosphoryl- $\alpha$-L-fucopyranosyl) 1 propanol (35) $(9.7 \mathrm{mg}, 0.016 \mathrm{mmol}, 12 \%)$, all as colorless oils. Compound 36: $[\alpha]_{\mathrm{D}}=+2.2\left(c=0.27, \mathrm{CHCl}_{3}\right) .{ }^{1} \mathrm{H}$ NMR $(500 \mathrm{MHz}$, $\mathrm{CDCl}_{3}$, simulated ring coupling constants using DAISY) $\delta_{\mathrm{H}} 7.42-$ $7.27(\mathrm{~m}, 10 \mathrm{H}, \mathrm{Ar}), 4.98$ (d, $J=11.7 \mathrm{~Hz}, 1 \mathrm{H}, \mathrm{OBn}), 4.88(\mathrm{~d}, J=12.3$ $\mathrm{Hz}, 1 \mathrm{H}, \mathrm{OBn}), 4.71(\mathrm{~d}, J=11.7 \mathrm{~Hz}, 1 \mathrm{H}, \mathrm{OBn}), 4.70(\mathrm{~d}, J=12.3 \mathrm{~Hz}$, $1 \mathrm{H}, \mathrm{OBn}), 3.98\left(\mathrm{~m}, 1 \mathrm{H}, 3^{\prime}-\mathrm{H}_{\mathrm{b}}\right), 3.629(\mathrm{dd}, J=9.8,9.1 \mathrm{~Hz}, 1 \mathrm{H}, 2-\mathrm{H})$, $3.62(\mathrm{dd}, J=3.0,1.3 \mathrm{~Hz}, 1 \mathrm{H}, 4-\mathrm{H}), 3.52(\mathrm{dd}, J=9.8,3.0 \mathrm{~Hz}, 1 \mathrm{H}, 3-$ $\mathrm{H}), 3.51$ (dddd, $J=6.4,6.4,6.4,1.3 \mathrm{~Hz}, 1 \mathrm{H}, 5-\mathrm{H}), 3.46\left(\mathrm{~m}, 1 \mathrm{H}, 3^{\prime}-\right.$ $\mathrm{H}_{\mathrm{a}}$ ), 3.06 (ddd, $\left.J=11.1,9.1,4.3 \mathrm{~Hz}, 1 \mathrm{H}, 1-\mathrm{H}\right), 2.038\left(\mathrm{~m}, 1 \mathrm{H}, \mathrm{l}^{\prime}-\mathrm{H}_{\mathrm{b}}\right)$, $1.75-1.70\left(\mathrm{~m}, 2 \mathrm{H}, 2^{\prime}-\mathrm{H}_{2}\right), 1.611\left(\mathrm{~m}, 1 \mathrm{H}, 1^{\prime}-\mathrm{H}_{\mathrm{a}}\right), 1.16 \mathrm{ppm}(\mathrm{d}, J=6.4$ $\left.\mathrm{Hz}, 3 \mathrm{H}, 6-\mathrm{H}_{3}\right) .{ }^{13} \mathrm{C}\left\{{ }^{1} \mathrm{H}\right\} \mathrm{NMR}\left(125.7 \mathrm{MHz}, \mathrm{CDCl}_{3}\right) \delta_{\mathrm{C}} 139.0(\mathrm{C}$, $\mathrm{Ar}), 138.7$ (C, Ar), $128.6(2 \times \mathrm{CH}, \mathrm{Ar}), 128.4(2 \times \mathrm{CH}, \mathrm{Ar}), 128.2(2$ $\times \mathrm{CH}, \mathrm{Ar}), 127.6(\mathrm{CH}, \mathrm{Ar}), 127.52(2 \times \mathrm{CH}, \mathrm{Ar}), 127.47(\mathrm{CH}, \mathrm{Ar})$, $81.9(\mathrm{CH}, \mathrm{C}-3), 79.60(\mathrm{CH}, \mathrm{C}-2$ or C-4), $77.88(\mathrm{CH}, \mathrm{C}-2$ or $\mathrm{C}-4)$, 76.4 (CH, C-1), 74.9 (CH, C-5), $74.9\left(\mathrm{CH}_{2}, \mathrm{OBn}\right), 73.0\left(\mathrm{CH}_{2}\right.$, OBn), $67.9\left(\mathrm{CH}_{2}, \mathrm{C}-3^{\prime}\right), 29.12\left(\mathrm{CH}_{2}, \mathrm{C}-1^{\prime}\right), 25.6\left(\mathrm{CH}_{2}, \mathrm{C}-2^{\prime}\right), 17.3$ $\operatorname{ppm}\left(\mathrm{CH}_{3}, \mathrm{C}-6\right)$. IR $\left(\mathrm{CHCl}_{3}\right): \nu=3017,1454,1220 \mathrm{~cm}^{-1}$. MS (ESI) $m / z(\%)=391(100)[\mathrm{M}+\mathrm{Na}]^{+}$. HRMS (ESI) $m / z:[\mathrm{M}+\mathrm{Na}]^{+}$ calcd for $\mathrm{C}_{23} \mathrm{H}_{28} \mathrm{NaO}_{4}$ 391.1885; found 391.1878. Compound 34: $[\alpha]_{\mathrm{D}}=+10.0\left(c=0.50, \mathrm{CHCl}_{3}\right) .{ }^{1} \mathrm{H} \mathrm{NMR}\left(500 \mathrm{MHz}, \mathrm{CDCl}_{3}\right) \delta_{\mathrm{H}}$ $7.37-7.20(\mathrm{~m}, 20 \mathrm{H}, \mathrm{Ar}), 4.68(\mathrm{~d}, J=12.0 \mathrm{~Hz}, 1 \mathrm{H}, \mathrm{OBn}), 4.62(\mathrm{~d}, J=$ $12.0 \mathrm{~Hz}, 1 \mathrm{H}, \mathrm{OBn}), 4.59(\mathrm{~m}, 1 \mathrm{H}, 2-\mathrm{H}), 4.21(\mathrm{~d}, J=11.7 \mathrm{~Hz}, 1 \mathrm{H}$, OBn), $4.11(\mathrm{~d}, J=11.7 \mathrm{~Hz}, 1 \mathrm{H}, \mathrm{OBn}), 4.03(\mathrm{dd}, J=3.2,3.2 \mathrm{~Hz}, 1 \mathrm{H}$, 3-H), 3.90-3.81 (m, 2H, 1-H, 5-H), 3.57-3.54 (m, 2H, 3'- $\left.\mathrm{H}_{2}\right), 3.240$ (dd, $J=9.8,2.9 \mathrm{~Hz}, 1 \mathrm{H}, 4-\mathrm{H}), 1.63-1.50\left(\mathrm{~m}, 4 \mathrm{H}, 1^{\prime}-\mathrm{H}_{2}, 2^{\prime}-\mathrm{H}_{2}\right), 1.262$ ppm $\left(\mathrm{d}, J=6.3 \mathrm{~Hz}, 3 \mathrm{H}, 6-\mathrm{H}_{3}\right), 1 \mathrm{H}$ from $\mathrm{OH}$ is missing. ${ }^{13} \mathrm{C}\left\{{ }^{1} \mathrm{H}\right\}$ NMR $\left(125.7 \mathrm{MHz}, \mathrm{CDCl}_{3}\right) \delta_{\mathrm{C}} 150.5(2 \times \mathrm{C}, \mathrm{Ar}), 137.8(2 \times \mathrm{C}, \mathrm{Ar})$, $129.9(2 \times \mathrm{CH}, \mathrm{Ar}), 129.8(2 \times \mathrm{CH}, \mathrm{Ar}), 128.4(2 \times \mathrm{CH}, \mathrm{Ar}), 128.3$ $(2 \times \mathrm{CH}, \mathrm{Ar}), 128.1(2 \times \mathrm{CH}, \mathrm{Ar}), 127.9(\mathrm{CH}, \mathrm{Ar}), 127.7(3 \times \mathrm{CH}$, $\mathrm{Ar}), 125.6(2 \times \mathrm{CH}, \mathrm{Ar}), 120.2(\mathrm{CH}, \mathrm{Ar}), 120.1(\mathrm{CH}, \mathrm{Ar}), 120.09$ (CH, Ar), 120.06 (CH, Ar), 77.99 (CH, C-4), 77.3 (CH, C-2), 73.5 $\left(\mathrm{d},{ }^{3} \mathrm{P}_{\mathrm{PC}}=6.4 \mathrm{~Hz}, \mathrm{CH}, \mathrm{C}-1\right), 73.0\left(\mathrm{CH}_{2}, \mathrm{OBn}\right), 72.2(\mathrm{CH}, \mathrm{C}-3), 71.5$ $\left(\mathrm{CH}_{2}, \mathrm{OBn}\right), 71.1(\mathrm{CH}, \mathrm{C}-5), 62.6\left(\mathrm{CH}_{2}, \mathrm{C}-3^{\prime}\right), 29.3\left(\mathrm{CH}_{2}, \mathrm{C}-1^{\prime}\right)$, $27.5\left(\mathrm{CH}_{2}, \mathrm{C}-2\right), 18.23 \mathrm{ppm}\left(\mathrm{CH}_{3}, \mathrm{C}-6\right)$. IR $\left(\mathrm{CHCl}_{3}\right): \nu=3426$, 3022, 1490, $1220 \mathrm{~cm}^{-1}$. MS (ESI) $\mathrm{m} / z(\%)=641(100)[\mathrm{M}+\mathrm{Na}]^{+}$. HRMS (ESI) $m / z:[\mathrm{M}+\mathrm{Na}]^{+}$calcd for $\mathrm{C}_{35} \mathrm{H}_{39} \mathrm{NaO}_{8} \mathrm{P}$ 641.2280; found 641.2288. Compound 35: $[\alpha]_{\mathrm{D}}=-24.8\left(c=0.72, \mathrm{CHCl}_{3}\right) .{ }^{1} \mathrm{H}$ $\operatorname{NMR}\left(500 \mathrm{MHz}, \mathrm{CDCl}_{3}\right) \delta_{\mathrm{H}} 7.33-7.16(\mathrm{~m}, 20 \mathrm{H}, \mathrm{Ar}), 4.83$ (ddd, $J=$ $\left.6.0,2.8 \mathrm{~Hz},{ }^{3} J_{\mathrm{PH}}=8.5 \mathrm{~Hz}, 1 \mathrm{H}, 2-\mathrm{H}\right), 4.70(\mathrm{~d}, J=11.7 \mathrm{~Hz}, 1 \mathrm{H}, \mathrm{OBn})$, $4.63(\mathrm{~d}, J=11.7 \mathrm{~Hz}, 1 \mathrm{H}, \mathrm{OBn}), 4.54(\mathrm{~d}, J=12.0 \mathrm{~Hz}, 1 \mathrm{H}, \mathrm{OBn}), 4.41$ (d, $J=11.7 \mathrm{~Hz}, 1 \mathrm{H}, \mathrm{OBn}), 4.09-4.04(\mathrm{~m}, 2 \mathrm{H}, 1-\mathrm{H}, 5-\mathrm{H}), 3.92(\mathrm{dd}, J$ $=5.7,3.2 \mathrm{~Hz}, 1 \mathrm{H}, 3-\mathrm{H}), 3.721(\mathrm{dd}, J=3.8,3.8 \mathrm{~Hz}, 1 \mathrm{H}, 4-\mathrm{H}), 3.55(\mathrm{br}$ s, $\left.2 \mathrm{H}, 3^{\prime}-\mathrm{H}_{2}\right), 1.84($ br s, $1 \mathrm{H}, \mathrm{OH}), 1.63-1.52\left(\mathrm{~m}, 4 \mathrm{H}, 1^{\prime}-\mathrm{H}_{2}, 2^{\prime}-\mathrm{H}_{2}\right)$,
$1.361 \mathrm{ppm}\left(\mathrm{d}, J=6.9 \mathrm{~Hz}, 3 \mathrm{H}, 6-\mathrm{H}_{3}\right) .{ }^{13} \mathrm{C}\left\{{ }^{1} \mathrm{H}\right\}$ NMR $(125.7 \mathrm{MHz}$, $\left.\mathrm{CDCl}_{3}\right) \delta_{\mathrm{C}} 150.5\left(\mathrm{~d},{ }^{2} J_{\mathrm{PC}}=7.4 \mathrm{~Hz}, \mathrm{C}, \mathrm{Ar}\right), 150.5\left(\mathrm{~d},{ }^{2} J_{\mathrm{PC}}=7.4 \mathrm{~Hz}, \mathrm{C}\right.$, $\mathrm{Ar}), 138.2$ (C, Ar), 138.0 (C, Ar), 129.81 ( $2 \times \mathrm{CH}, \mathrm{Ar}), 129.76(2 \times$ $\mathrm{CH}, \mathrm{Ar}), 128.4(2 \times \mathrm{CH}, \mathrm{Ar}), 128.3(2 \times \mathrm{CH}, \mathrm{Ar}), 127.7(3 \times \mathrm{CH}$, $\mathrm{Ar}), 127.62(\mathrm{CH}, \mathrm{Ar}), 127.56(2 \times \mathrm{CH}, \mathrm{Ar}), 125.4(2 \times \mathrm{CH}, \mathrm{Ar})$, $120.2(\mathrm{CH}, \mathrm{Ar}), 120.12(\mathrm{CH}, \mathrm{Ar}), 120.06(\mathrm{CH}, \mathrm{Ar}), 120.0(\mathrm{CH}, \mathrm{Ar})$, $77.5(\mathrm{CH}, \mathrm{C}-2), 75.9(\mathrm{CH}, \mathrm{C}-3), 74.65(\mathrm{CH}, \mathrm{C}-4), 73.2\left(\mathrm{CH}_{2}, \mathrm{OBn}\right)$, $72.4\left(\mathrm{CH}_{2}, \mathrm{OBn}\right), 69.6(\mathrm{CH}, \mathrm{C}-5), 68.5\left(\mathrm{~d},{ }^{3} \mathrm{~J}_{\mathrm{PC}}=4.2 \mathrm{~Hz}, \mathrm{CH}, \mathrm{C}-1\right)$, $62.4\left(\mathrm{CH}_{2}, \mathrm{C}-3^{\prime}\right), 29.7\left(\mathrm{CH}_{2}, \mathrm{C}-1^{\prime}\right), 29.2\left(\mathrm{CH}_{2}, \mathrm{C}-2^{\prime}\right), 14.44 \mathrm{ppm}$ $\left(\mathrm{CH}_{3}, \mathrm{C}-6\right)$. IR $\left(\mathrm{CHCl}_{3}\right): \nu=3454,3015,1490,1205 \mathrm{~cm}^{-1}$. MS (ESI) $\mathrm{m} / z(\%)=641(100)[\mathrm{M}+\mathrm{Na}]^{+}$. HRMS (ESI) $\mathrm{m} / z:[\mathrm{M}+$ $\mathrm{Na}]^{+}$calcd for $\mathrm{C}_{35} \mathrm{H}_{39} \mathrm{NaO}_{8} \mathrm{P}$ 641.2280; found 641.2278.

Method $D$. Following the general procedure, starting from substrate $10(85.2 \mathrm{mg}, 0.11 \mathrm{mmol})$, after $2 \mathrm{~h}$ of reaction, a supplementary addition of $n-\mathrm{Bu}_{3} \mathrm{SnD}(15 \mu \mathrm{L}, 0.07 \mathrm{mmol})$ was required. All the starting material was consumed after $4 \mathrm{~h}$. Column chromatography (hexanes to hexanes-EtOAc, 4:6) gave $\left[\mathrm{PhCH}_{-}{ }^{2} \mathrm{H}\right]$ 31 (20.1 mg, $0.05 \mathrm{mmol}, 49 \%,{ }^{2} \mathrm{H} /{ }^{1} \mathrm{H}$ 1.7:1), 2,6:5,9-di-anhydro-3,4di-O-benzyl-1,7,8-trideoxy-D-[6- $\left.{ }^{2} \mathrm{H}\right]$ glycero-D-galacto-nonitol $\left[\left(1-{ }^{2} \mathrm{H}\right)\right.$ 36] $\left(4.5 \mathrm{mg}, 0.012 \mathrm{mmol}, 11 \%,{ }^{2} \mathrm{H} /{ }^{1} \mathrm{H}\right.$ 8:1), and 3-C-(3,4-di-Obenzyl-6-deoxy-2-O-diphenoxyphosphoryl- $\beta$-D- $\left(5-{ }^{2} \mathrm{H}\right)$ altropyranosyl)1-propanol $\left[\left(5-{ }^{2} \mathrm{H}\right) 34\right](4.3 \mathrm{mg}, 0.007 \mathrm{mmol}, 6 \%)$ as colorless oils and $35(6 \mathrm{mg}, 0.01 \mathrm{mmol}, 9 \%)$. Compound $\left(1-{ }^{2} \mathrm{H}\right) 36:{ }^{1} \mathrm{H}$ NMR $(500$ $\mathrm{MHz}$, only the deuterated product is described) $\delta_{\mathrm{H}} 7.42-7.26(\mathrm{~m}$, $10 \mathrm{H}, \mathrm{Ar}), 4.98(\mathrm{~d}, J=11.7 \mathrm{~Hz}, 1 \mathrm{H}, \mathrm{OBn}), 4.88(\mathrm{~d}, J=12.3 \mathrm{~Hz}, 1 \mathrm{H}$, OBn), $4.71(\mathrm{~d}, J=11.7 \mathrm{~Hz}, 1 \mathrm{H}, \mathrm{OBn}), 4.70(\mathrm{~d}, J=12.3 \mathrm{~Hz}, 1 \mathrm{H}$, $\mathrm{OBn}), 3.98\left(\mathrm{~m}, 1 \mathrm{H}, 3^{\prime}-\mathrm{H}_{\mathrm{b}}\right), 3.637(\mathrm{~d}, J=9.8 \mathrm{~Hz}, 1 \mathrm{H}, 2-\mathrm{H}), 3.62$ (dd, $J$ $=2.8,1.0 \mathrm{~Hz}, 1 \mathrm{H}, 4-\mathrm{H}), 3.52(\mathrm{dd}, J=9.8,2.8 \mathrm{~Hz}, 1 \mathrm{H}, 3-\mathrm{H}), 3.51$ (dddd, $J=6.3,6.3,6.3,1.3 \mathrm{~Hz}, 1 \mathrm{H}, 5-\mathrm{H}), 3.46\left(\mathrm{~m}, 1 \mathrm{H}, 3^{\prime}-\mathrm{H}_{\mathrm{a}}\right), 2.029$ $\left(\mathrm{m}, 1 \mathrm{H}, \mathrm{l}^{\prime}-\mathrm{H}_{\mathrm{b}}\right), 1.74-1.70\left(\mathrm{~m}, 2 \mathrm{H}, 2^{\prime}-\mathrm{H}_{2}\right), 1.606\left(\mathrm{~m}, 1 \mathrm{H}, \mathrm{l}^{\prime}-\mathrm{H}_{\mathrm{a}}\right), 1.16$ ppm $\left(\mathrm{d}, J=6.7 \mathrm{~Hz}, 3 \mathrm{H}, 6-\mathrm{H}_{3}\right) .{ }^{13} \mathrm{C}\left\{{ }^{1} \mathrm{H}\right\}$ NMR $\left(125.7 \mathrm{MHz}, \mathrm{CDCl}_{3}\right.$, only the deuterated product is described) $\delta_{\mathrm{C}} 138.9(\mathrm{C}, \mathrm{Ar}), 138.6(\mathrm{C}$, $\mathrm{Ar}), 128.5(2 \times \mathrm{CH}, \mathrm{Ar}), 128.3(2 \times \mathrm{CH}, \mathrm{Ar}), 128.1(2 \times \mathrm{CH}, \mathrm{Ar})$, 127.54 (CH, Ar), $127.49(2 \times \mathrm{CH}, \mathrm{Ar}), 127.4(\mathrm{CH}, \mathrm{Ar}), 81.9(\mathrm{CH}$, C-3), 79.49 (CH, C-2), 77.84 (CH, C-4), $74.8(\mathrm{CH}, \mathrm{C}-5), 74.8\left(\mathrm{CH}_{2}\right.$, $\mathrm{OBn}), 73.0\left(\mathrm{CH}_{2}, \mathrm{OBn}\right), 67.9\left(\mathrm{CH}_{2}, \mathrm{C}-3{ }^{\prime}\right), 28.99\left(\mathrm{CH}_{2}, \mathrm{C}-1^{\prime}\right), 25.6$ $\left(\mathrm{CH}_{2}, \mathrm{C}-2^{\prime}\right), 17.3 \mathrm{ppm}\left(\mathrm{CH}_{3}, \mathrm{C}-6\right)$. MS (ESI) $\mathrm{m} / z(\%)=392(100)$ $[\mathrm{M}+\mathrm{Na}]^{+}, 391(12)[\mathrm{M}+\mathrm{Na}]^{+}$. HRMS (ESI) $m / z:[\mathrm{M}+\mathrm{Na}]^{+}$calcd for $\mathrm{C}_{23} \mathrm{H}_{27}{ }^{2} \mathrm{HNaO}_{4}$ 392.1948; found 392.1954. Compound $\left(5-{ }^{2} \mathrm{H}\right) 34$ : ${ }^{1} \mathrm{H}$ NMR $\left(500 \mathrm{MHz}, \mathrm{CDCl}_{3}\right) \delta_{\mathrm{H}} 7.37-7.20(\mathrm{~m}, 20 \mathrm{H}, \mathrm{Ar}), 4.68(\mathrm{~d}, J=$ $12.0 \mathrm{~Hz}, 1 \mathrm{H}, \mathrm{OBn}), 4.62(\mathrm{~d}, J=12.0 \mathrm{~Hz}, 1 \mathrm{H}, \mathrm{OBn}), 4.57(\mathrm{~m}, 1 \mathrm{H}, 2$ $\mathrm{H}), 4.20(\mathrm{~d}, J=11.7 \mathrm{~Hz}, 1 \mathrm{H}, \mathrm{OBn}), 4.11(\mathrm{~d}, J=11.7 \mathrm{~Hz}, 1 \mathrm{H}, \mathrm{OBn})$, $4.02(\mathrm{dd}, J=3.5,3.5 \mathrm{~Hz}, 1 \mathrm{H}, 3-\mathrm{H}), 3.83(\mathrm{~m}, 1 \mathrm{H}, 1-\mathrm{H}), 3.57-3.54(\mathrm{~m}$, $\left.2 \mathrm{H}, 3{ }^{\prime}-\mathrm{H}_{2}\right), 3.235(\mathrm{~d}, J=2.9 \mathrm{~Hz}, 1 \mathrm{H}, 4-\mathrm{H}), 2.00(\mathrm{br} \mathrm{s}, 1 \mathrm{H}, \mathrm{OH})$, 1.64-1.50 (m, 4H, 1'- $\left.\mathrm{H}_{2}, 2^{\prime}-\mathrm{H}_{2}\right), 1.248 \mathrm{ppm}\left(\mathrm{s}, 3 \mathrm{H}, 6-\mathrm{H}_{3}\right) .{ }^{13} \mathrm{C}\left\{{ }^{1} \mathrm{H}\right\}$ NMR $\left(125.7 \mathrm{MHz}, \mathrm{CDCl}_{3}\right) \delta_{\mathrm{C}} 150.5(2 \times \mathrm{C}, \mathrm{Ar}), 137.9(2 \times \mathrm{C}, \mathrm{Ar})$, $129.9(2 \times \mathrm{CH}, \mathrm{Ar}), 129.8(2 \times \mathrm{CH}, \mathrm{Ar}), 128.4(2 \times \mathrm{CH}, \mathrm{Ar}), 128.3$ $(2 \times \mathrm{CH}, \mathrm{Ar}), 128.1(2 \times \mathrm{CH}, \mathrm{Ar}), 127.9(\mathrm{CH}, \mathrm{Ar}), 127.7(2 \times \mathrm{CH}$, $\mathrm{Ar}), 127.4(\mathrm{CH}, \mathrm{Ar}), 125.5(2 \times \mathrm{CH}, \mathrm{Ar}), 120.15(\mathrm{CH}, \mathrm{Ar}), 120.10$ (CH, Ar), 120.08 (CH, Ar), $120.04(\mathrm{CH}, \mathrm{Ar}), 77.91$ (CH, C-4), 77.3 $(\mathrm{CH}, \mathrm{C}-2), 73.5\left(\mathrm{~d},{ }^{3} J_{\mathrm{PC}}=6.4 \mathrm{~Hz}, \mathrm{CH}, \mathrm{C}-1\right), 73.0\left(\mathrm{CH}_{2}, \mathrm{OBn}\right), 72.2$ $(\mathrm{CH}, \mathrm{C}-3), 71.5\left(\mathrm{CH}_{2}, \mathrm{OBn}\right), 62.6\left(\mathrm{CH}_{2}, \mathrm{C}-3{ }^{\prime}\right), 29.3\left(\mathrm{CH}_{2}, \mathrm{C}-1^{\prime}\right)$, $27.5\left(\mathrm{CH}_{2}, \mathrm{C}-2^{\prime}\right), 18.10 \mathrm{ppm}\left(\mathrm{CH}_{3}, \mathrm{C}-6\right)$. MS (ESI) $\mathrm{m} / z(\%)=642$ (100) $[\mathrm{M}+\mathrm{Na}]^{+}$. HRMS (ESI) $m / z:[\mathrm{M}+\mathrm{Na}]^{+}$calcd for $\mathrm{C}_{35} \mathrm{H}_{38}{ }^{2} \mathrm{HNaO}_{8} \mathrm{P}$ 642.2280; found 642.2291.

Radical Reactions of 11. Method A. Following the general procedure, starting from substrate $\mathbf{1 1}(68.2 \mathrm{mg}, 0.10 \mathrm{mmol})$, after $2 \mathrm{~h}$ of reaction, a supplementary addition of $n-\mathrm{Bu}_{3} \mathrm{SnH}(29 \mu \mathrm{L}, 0.10$ $\mathrm{mmol}$ ) was required. All the starting material was consumed after $3 \mathrm{~h}$. Column chromatography (hexanes to hexanes-EtOAc, 9:1) gave (4S)-1,4-anhydro-6,7-O-benzylidene-2,3,5-trideoxy-D-erythro-oct-4ulopyranose $(37)(15.8 \mathrm{mg}, 0.06 \mathrm{mmol}, 60 \%)$ as a colorless oil: $[\alpha]_{\mathrm{D}}$ $=-109.3\left(c=0.28, \mathrm{CHCl}_{3}\right) \cdot{ }^{1} \mathrm{H} \mathrm{NMR}\left(500 \mathrm{MHz}, \mathrm{CDCl}_{3}\right.$, simulated ring coupling constants using DAISY) $\delta_{\mathrm{H}} 7.48-7.46(\mathrm{~m}, 2 \mathrm{H}, \mathrm{Ar})$, $7.40-7.35$ (m, 3H, Ar), 6.20 (s, 1H, PhCH), 4.700 (ddd, J = 8.7, 6.3, $5.5 \mathrm{~Hz}, 1 \mathrm{H}, 3-\mathrm{H}$ ), 4.10 (ddd, $J=5.5,2.4,1.0 \mathrm{~Hz}, 1 \mathrm{H}, 4-\mathrm{H}$ ), 4.03 (dd, $\left.J=13.3,1.0 \mathrm{~Hz}, 1 \mathrm{H}, 5-\mathrm{H}_{\mathrm{b}}\right), 4.02\left(\mathrm{dd}, J=13.3,2.4 \mathrm{~Hz}, 1 \mathrm{H}, 5-\mathrm{H}_{\mathrm{a}}\right)$, 3.95 (ddd, $J=8.2,8.2,5.7 \mathrm{~Hz}, 1 \mathrm{H}, 3{ }^{\prime}-\mathrm{H}_{\mathrm{b}}$ ), 3.88 (ddd, $J=8.2,8.2,6.3$ $\left.\mathrm{Hz}, 1 \mathrm{H}, 3-\mathrm{H}_{\mathrm{a}}\right), 2.11\left(\mathrm{dd}, J=13.5,8.7 \mathrm{~Hz}, 1 \mathrm{H}, 2-\mathrm{H}_{\mathrm{b}}\right), 2.05(\mathrm{dd}, J=$ 13.5, $\left.6.3 \mathrm{~Hz}, 1 \mathrm{H}, 2-\mathrm{H}_{\mathrm{a}}\right), 2.18-2.04\left(\mathrm{~m}, 2 \mathrm{H}, 1^{\prime}-\mathrm{H}_{\mathrm{b}}, 2^{\prime}-\mathrm{H}_{\mathrm{b}}\right), 1.93(\mathrm{~m}$, 
$1 \mathrm{H}, 2$ '- $\mathrm{H}_{\mathrm{a}}$ ), $1.80 \mathrm{ppm}$ (ddd, $J=12.3,10.1,7.9 \mathrm{~Hz}, 1 \mathrm{H}, 1^{\prime}-\mathrm{H}_{\mathrm{a}}$ ). ${ }^{13} \mathrm{C}\left\{{ }^{1} \mathrm{H}\right\}$ NMR $\left(125.7 \mathrm{MHz}, \mathrm{CDCl}_{3}\right) \delta_{\mathrm{C}} 139.3(\mathrm{C}, \mathrm{Ar}), 128.9(\mathrm{CH}$, $\mathrm{Ar}), 128.4(2 \times \mathrm{CH}, \mathrm{Ar}), 126.1(2 \times \mathrm{CH}, \mathrm{Ar}), 105.75$ (C, C-1), 102.6 $(\mathrm{CH}, \mathrm{PhCH}), 71.83(\mathrm{CH}, \mathrm{C}-4), 71.72(\mathrm{CH}, \mathrm{C}-3), 67.4\left(\mathrm{CH}_{2}, \mathrm{C}-3\right.$ '), $60.6\left(\mathrm{CH}_{2}, \mathrm{C}-5\right), 37.9\left(\mathrm{CH}_{2}, \mathrm{C}-1^{\prime}\right), 34.0\left(\mathrm{CH}_{2}, \mathrm{C}-2\right), 23.6 \mathrm{ppm}\left(\mathrm{CH}_{2}\right.$, C-2'). IR $\left(\mathrm{CHCl}_{3}\right): \nu=3024,1458,1210 \mathrm{~cm}^{-1}$. MS (ESI) $\mathrm{m} / z(\%)=$ $285(100)[\mathrm{M}+\mathrm{Na}]^{+}$. HRMS (ESI) $\mathrm{m} / z:[\mathrm{M}+\mathrm{Na}]^{+}$calcd for $\mathrm{C}_{15} \mathrm{H}_{18} \mathrm{NaO}_{4}$ 285.1103; found 285.1103. Anal. calcd for $\mathrm{C}_{15} \mathrm{H}_{18} \mathrm{O}_{4}$ : C, 68.68; H, 6.92. Found: C, 68.68; H, 7.00.

Method $D$. Following the general procedure, starting from substrate 11 (72.6 $\mathrm{mg}, 0.11 \mathrm{mmol})$, after $2 \mathrm{~h}$, all the starting material was consumed. Column chromatography (hexanes to hexanesEtOAc, 9:1) gave (4S)-1,4-anhydro-6,7-O-benzylidene-2,3,5-trideoxyD- $\left[5-{ }^{2} \mathrm{H}\right]$ erythro-oct-4-ulopyranose $\left(\left[2-{ }^{2} \mathrm{H}\right] 37\right)(15.7 \mathrm{mg}, 0.06 \mathrm{mmol}$, $\left.54 \%,{ }^{2} \mathrm{H} /{ }^{1} \mathrm{H} 1: 1\right)$ as a colorless oil: ${ }^{1} \mathrm{H}$ NMR $\left(500 \mathrm{MHz}, \mathrm{CDCl}_{3}\right.$, only the deuterated compound is described) $\delta_{\mathrm{H}} 7.48-7.46(\mathrm{~m}, 2 \mathrm{H}, \mathrm{Ar})$, $7.40-7.34$ (m, 3H, Ar), 6.20 (s, 1H, PhCH), 4.701 (dd, $J=8.6,5.4$ $\mathrm{Hz}, 1 \mathrm{H}, 3-\mathrm{H}), 4.11$ (ddd, $J=5.4,2.3,1.3 \mathrm{~Hz}, 1 \mathrm{H}, 4-\mathrm{H}), 4.03$ (dd, $J=$ 13.6, $\left.1.0 \mathrm{~Hz}, 1 \mathrm{H}, 5-\mathrm{H}_{\mathrm{b}}\right), 4.00\left(\mathrm{dd}, J=13.3,2.2 \mathrm{~Hz}, 1 \mathrm{H}, 5-\mathrm{H}_{\mathrm{a}}\right), 3.94$ (ddd, $\left.J=8.2,8.2,5.7 \mathrm{~Hz}, 1 \mathrm{H}, 3^{\prime}-\mathrm{H}_{\mathrm{b}}\right), 3.88(\mathrm{ddd}, J=8.2,8.2,6.3 \mathrm{~Hz}$, $\left.1 \mathrm{H}, 3^{\prime}-\mathrm{H}_{\mathrm{a}}\right), 2.18-2.04\left(\mathrm{~m}, 3 \mathrm{H}, 2-\mathrm{H}, 1^{\prime}-\mathrm{H}_{\mathrm{b}}, 2^{\prime}-\mathrm{H}_{\mathrm{b}}\right), 1.92\left(\mathrm{~m}, 1 \mathrm{H}, 2^{\prime}-\right.$ $\left.\mathrm{H}_{\mathrm{a}}\right), 1.79 \mathrm{ppm}\left(\mathrm{ddd}, J=12.3,10.1,7.9 \mathrm{~Hz}, 1 \mathrm{H}, 1^{\prime}-\mathrm{H}_{\mathrm{a}}\right) .{ }^{13} \mathrm{C}\left\{{ }^{1} \mathrm{H}\right\} \mathrm{NMR}$ $\left(125.7 \mathrm{MHz}, \mathrm{CDCl}_{3}\right.$, only the deuterated compound is described) $\delta_{\mathrm{C}}$ 139.3 (C, Ar), 128.9 ( CH, Ar), $128.4(2 \times \mathrm{CH}, \mathrm{Ar}), 126.1(2 \times \mathrm{CH}$, $\mathrm{Ar}), 105.72(\mathrm{C}, \mathrm{C}-1), 102.5(\mathrm{CH}, \mathrm{PhCH}), 71.80(\mathrm{CH}, \mathrm{C}-3$ or $\mathrm{C}-4)$, $71.67(\mathrm{CH}, \mathrm{C}-3$ or $\left.\mathrm{C}-4), 67.4\left(\mathrm{CH}_{2}, \mathrm{C}-3\right)^{\prime}\right), 60.6\left(\mathrm{CH}_{2}, \mathrm{C}-5\right), 37.9$ $\left(\mathrm{CH}_{2}, \mathrm{C}-1^{\prime}\right), 33.69\left(\mathrm{t}, J_{\mathrm{CD}}=20.1 \mathrm{~Hz}, \mathrm{CHD}, \mathrm{C}-2\right), 23.6 \mathrm{ppm}\left(\mathrm{CH}_{2}, \mathrm{C}-\right.$ $\left.2^{\prime}\right)$. MS (ESI) $m / z(\%)=286(100)[\mathrm{M}+\mathrm{Na}]^{+}, 285(83)[\mathrm{M}+\mathrm{Na}]^{+}$. HRMS (ESI) $m / z:[\mathrm{M}+\mathrm{Na}]^{+}$calcd for $\mathrm{C}_{15} \mathrm{H}_{17}{ }^{2} \mathrm{HNaO}_{4}$ 286.1166; found 286.1154, $[\mathrm{M}+\mathrm{Na}]^{+}$calcd for $\mathrm{C}_{15} \mathrm{H}_{18} \mathrm{NaO}_{4}$ 285.1103; found 285.1107.

Method E. Following the general procedure, starting from substrate $11(60 \mathrm{mg}, 0.091 \mathrm{mmol})$, after $2 \mathrm{~h}$, all the starting material was consumed. Column chromatography (hexanes to hexanes-EtOAc, 9:1) gave $\left[2-{ }^{2} \mathrm{H}\right] 37\left(14.4 \mathrm{mg}, 0.055 \mathrm{mmol}, 60 \%,{ }^{2} \mathrm{H} /{ }^{1} \mathrm{H} 1: 1\right)$.

Radical Reactions of 12. Method A. Following the general procedure, starting from substrate $12(65.2 \mathrm{mg}, 0.11 \mathrm{mmol})$, all the starting material was consumed after $2 \mathrm{~h}$. Column chromatography (DCM to DCM-MeOH, 97:3) gave (4S)-1,4-anhydro-2,3,5trideoxy-D-erythro-oct-4-ulopyranose (38) $(10.4 \mathrm{mg}, 0.06 \mathrm{mmol}$, $54 \%)$ as a colorless oil: $[\alpha]_{\mathrm{D}}=-57.3\left(c=0.62, \mathrm{CHCl}_{3}\right) .{ }^{1} \mathrm{H}$ NMR $\left(400 \mathrm{MHz}, \mathrm{CDCl}_{3}\right.$, simulated ring coupling constants using DAISY) $\delta_{\mathrm{H}} 4.055(\mathrm{ddd}, J=11.7,5.3,3.5 \mathrm{~Hz}, 1 \mathrm{H}, 3-\mathrm{H}), 3.90(\mathrm{dd}, J=12.5,1.6$ $\left.\mathrm{Hz}, 1 \mathrm{H}, 5-\mathrm{H}_{\mathrm{b}}\right), 3.96-3.83\left(\mathrm{~m}, 2 \mathrm{H}, 3\right.$ ' $\left.-\mathrm{H}_{2}\right), 3.80(\mathrm{br} \mathrm{s}, 1 \mathrm{H}, 4-\mathrm{H}), 3.72$ $\left(\mathrm{dd}, J=12.5,2.3 \mathrm{~Hz}, 1 \mathrm{H}, 5-\mathrm{H}_{\mathrm{a}}\right), 2.31(\mathrm{br} \mathrm{s}, 1 \mathrm{H}, \mathrm{OH}), 2.17(\mathrm{br} \mathrm{s}, 1 \mathrm{H}$, $\mathrm{OH}), 2.09-2.01\left(\mathrm{~m}, 2 \mathrm{H}, 1^{\prime}-\mathrm{H}_{\mathrm{b}}, 2^{\prime}-\mathrm{H}_{\mathrm{b}}\right), 1.99(\mathrm{dd}, J=12.8,11.7 \mathrm{~Hz}$, $\left.1 \mathrm{H}, 2-\mathrm{H}_{\mathrm{b}}\right), 1.88\left(\mathrm{~m}, 1 \mathrm{H}, 2-\mathrm{H}_{\mathrm{a}}\right), 1.86\left(\mathrm{dd}, J=12.8,5.3 \mathrm{~Hz}, 1 \mathrm{H}, 2-\mathrm{H}_{\mathrm{a}}\right)$, $1.72 \mathrm{ppm}\left(\mathrm{m}, 1 \mathrm{H}, 11^{\prime}-\mathrm{H}_{\mathrm{a}}\right) .{ }^{13} \mathrm{C}\left\{{ }^{1} \mathrm{H}\right\}$ NMR $\left(125.7 \mathrm{MHz}, \mathrm{CDCl}_{3}\right) \delta_{\mathrm{C}}$ $\left.106.63(\mathrm{C}, \mathrm{C}-1), 67.9(\mathrm{CH}, \mathrm{C}-4), 67.5\left(\mathrm{CH}_{2}, \mathrm{C}-3\right)^{\prime}\right), 66.59(\mathrm{CH}, \mathrm{C}-3)$, $63.7\left(\mathrm{CH}_{2}, \mathrm{C}-5\right), 37.5\left(\mathrm{CH}_{2}, \mathrm{C}-1^{\prime}\right), 36.83\left(\mathrm{CH}_{2}, \mathrm{C}-2\right), 23.3 \mathrm{ppm}$ $\left(\mathrm{CH}_{2}, \mathrm{C}-2^{\prime}\right)$. IR $\left(\mathrm{CHCl}_{3}\right): \nu=3685,3571,3020,1056 \mathrm{~cm}^{-1}$. MS (ESI) $m / z(\%)=197(100)[\mathrm{M}+\mathrm{Na}]^{+}$. HRMS (ESI) $\mathrm{m} / z:[\mathrm{M}+$ $\mathrm{Na}]^{+}$calcd for $\mathrm{C}_{8} \mathrm{H}_{14} \mathrm{NaO}_{4}$ 197.0790; found 197.0789. Anal. calcd for $\mathrm{C}_{8} \mathrm{H}_{14} \mathrm{O}_{4}$ : C, 55.16; H, 8.10. Found: C, 54.97; H, 7.92.

Method $D$. Following the general procedure, starting from substrate 12 (64.2 $\mathrm{mg}, 0.11 \mathrm{mmol})$, after $2 \mathrm{~h}$, all the starting material was consumed. Column chromatography (DCM to DCM-MeOH, 97:3) gave (4S)-1,4-anhydro-2,3,5-trideoxy-D-[5- $\left.{ }^{2} \mathrm{H}\right]$ erythro-oct-4-ulopyranose $\left(\left[2{ }^{2} \mathrm{H}\right] 38\right)\left(12.2 \mathrm{mg}, 0.07 \mathrm{mmol}, 63 \%,{ }^{2} \mathrm{H} /{ }^{1} \mathrm{H} 2.3: 1\right)$ as a colorless oil: ${ }^{1} \mathrm{H}$ NMR $\left(500 \mathrm{MHz}, \mathrm{CDCl}_{3}\right.$, only the deuterated compound is described) $\delta_{\mathrm{H}} 4.049(\mathrm{~m}, 1 \mathrm{H}, 3-\mathrm{H}), 3.95-3.85(\mathrm{~m}, 2 \mathrm{H}$, 3'- $\left.\mathrm{H}_{2}\right), 3.90\left(\mathrm{dd}, J=12.6,1.3 \mathrm{~Hz}, 1 \mathrm{H}, 5-\mathrm{H}_{\mathrm{b}}\right), 3.80(\mathrm{br} \mathrm{s}, 1 \mathrm{H}, 4-\mathrm{H})$, $3.72\left(\mathrm{dd}, J=12.6,2.2 \mathrm{~Hz}, 1 \mathrm{H}, 5-\mathrm{H}_{\mathrm{a}}\right), 2.22(\mathrm{br} \mathrm{s}, 1 \mathrm{H}, \mathrm{OH}), 2.09-2.00$ $\left(\mathrm{m}, 2 \mathrm{H}, 1^{\prime}-\mathrm{H}_{\mathrm{b}}, 2^{\prime}-\mathrm{H}_{\mathrm{b}}\right), 1.92-1.85\left(\mathrm{~m}, 2 \mathrm{H}, 2-\mathrm{H}, 2^{\prime}-\mathrm{H}_{\mathrm{a}}\right), 1.72 \mathrm{ppm}(\mathrm{m}$, $\left.1 \mathrm{H}, 1^{\prime}-\mathrm{H}_{\mathrm{a}}\right), 1 \mathrm{H}$ from $\mathrm{OH}$ is missing. ${ }^{13} \mathrm{C}\left\{{ }^{1} \mathrm{H}\right\}$ NMR $(125.7 \mathrm{MHz}$, $\mathrm{CDCl}_{3}$, only the deuterated compound is described) $\delta_{\mathrm{C}} 106.57$ (C, C1), $\left.67.9\left(\mathrm{CH}_{2}, \mathrm{C}-4\right), 67.5\left(\mathrm{CH}_{2}, \mathrm{C}-3\right)^{\prime}\right), 66.52(\mathrm{CH}, \mathrm{C}-3), 63.7(\mathrm{CH}$, $\mathrm{C}-5), 37.4\left(\mathrm{CH}_{2}, \mathrm{C}-1^{\prime}\right), 23.3 \mathrm{ppm}\left(\mathrm{CH}_{2}, \mathrm{C}-2^{\prime}\right), \mathrm{C}-2$ was undetectable. MS (ESI) $m / z(\%)=198(100)[\mathrm{M}+\mathrm{Na}]^{+}, 197(44)[\mathrm{M}+\mathrm{Na}]^{+}$. HRMS (ESI) $m / z$ : $[\mathrm{M}+\mathrm{Na}]^{+}$calcd for $\mathrm{C}_{8} \mathrm{H}_{13}{ }^{2} \mathrm{HNaO}_{4}$ 198.0853; found 198.0856, $[\mathrm{M}+\mathrm{Na}]^{+}$calcd for $\mathrm{C}_{8} \mathrm{H}_{14} \mathrm{NaO}_{4}$ 197.0790; found 197.0796.

Synthesis of 9-Deoxy-1,6-dioxaspiro[4.4]nonane structures (Table 4). Radical Reactions of 13. Method A. Following the general procedure, starting from substrate $13(102 \mathrm{mg}, 0.12 \mathrm{mmol})$, after $2 \mathrm{~h}$ of reaction, a supplementary addition of $n-\mathrm{Bu}_{3} \mathrm{SnH}(32 \mu \mathrm{L}, 0.12$ $\mathrm{mmol}$ ) was required. All the starting material was consumed after $3 \mathrm{~h}$. Column chromatography on a silica gel without KF (hexanes to hexanes-EtOAc, 8:2) gave 3-C-(2-O-acetyl-3,5-di-O-tert-butyldiphenylsilyl- $\alpha$-D-ribofuranosyl)1-propanol (40) $(51.2 \mathrm{mg}, 0.072 \mathrm{mmol}$, $56 \%)$ as a colorless oil: $[\alpha]_{\mathrm{D}}=+46.0\left(c=0.61, \mathrm{CHCl}_{3}\right) .{ }^{1} \mathrm{H}$ NMR $\left(500 \mathrm{MHz}, \mathrm{CDCl}_{3}\right) \delta_{\mathrm{H}} 7.65-7.26(\mathrm{~m}, 20 \mathrm{H}, \mathrm{Ar}), 5.12(\mathrm{dd}, J=4.4,4.4$ $\mathrm{Hz}, 1 \mathrm{H}, 2-\mathrm{H}), 4.61$ (dd, $J=6.7,4.5 \mathrm{~Hz}, 1 \mathrm{H}, 3-\mathrm{H}), 4.03(\mathrm{~m}, 1 \mathrm{H}, 4-\mathrm{H})$, $3.97(\mathrm{~m}, 1 \mathrm{H}, 1-\mathrm{H}), 3.66-3.60\left(\mathrm{~m}, 3 \mathrm{H}, 5-\mathrm{H}_{\mathrm{b}}, 3-\mathrm{H}_{2}\right), 3.32(\mathrm{dd}, J=$ $\left.11.4,3.5 \mathrm{~Hz}, 1 \mathrm{H}, 5-\mathrm{H}_{\mathrm{a}}\right), 2.13$ (s, 3H, OAc), 1.69-1.53 (m, 4H, 1'- $\mathrm{H}_{2}$, $\left.2^{2}-\mathrm{H}_{2}\right), 1.02\left(\mathrm{~s}, 9 \mathrm{H},{ }^{t} \mathrm{Bu}\right), 0.91 \mathrm{ppm}\left(\mathrm{s}, 9 \mathrm{H},{ }^{t} \mathrm{Bu}\right) .{ }^{13} \mathrm{C}\left\{{ }^{1} \mathrm{H}\right\}$ NMR $\left(125.7 \mathrm{MHz}, \mathrm{CDCl}_{3}\right) \delta_{\mathrm{C}} 170.4(\mathrm{C}, \mathrm{OAc}) .135 .78(2 \times \mathrm{CH}, \mathrm{Ar})$, $135.72(2 \times \mathrm{CH}, \mathrm{Ar}), 135.60(2 \times \mathrm{CH}, \mathrm{Ar}), 135.55(2 \times \mathrm{CH}, \mathrm{Ar})$, 133.3 (C, Ar), 133.24 (C, Ar), 133.22 (C, Ar), 132.7 (C, Ar), 129.98 (CH, Ar), $129.96(\mathrm{CH}, \mathrm{Ar}), 129.5(2 \times \mathrm{CH}, \mathrm{Ar}), 127.76(2 \times \mathrm{CH}$, $\mathrm{Ar}), 127.72(2 \times \mathrm{CH}, \mathrm{Ar}), 127.56(2 \times \mathrm{CH}, \mathrm{Ar}), 127.54(2 \times \mathrm{CH}$, $\mathrm{Ar}), 82.8(\mathrm{CH}, \mathrm{C}-4), 79.5(\mathrm{CH}, \mathrm{C}-1), 75.0(\mathrm{CH}, \mathrm{C}-2), 72.8(\mathrm{CH}, \mathrm{C}-$ 3), $63.6\left(\mathrm{CH}_{2}, \mathrm{C}-5\right), 62.6\left(\mathrm{CH}_{2}, \mathrm{C}-3^{\prime}\right), 29.6\left(\mathrm{CH}_{2}, \mathrm{C}-1^{\prime}\right.$ or $\left.\mathrm{C}-2{ }^{\prime}\right), 26.8$ $\left(3 \times \mathrm{CH}_{3},{ }^{t} \mathrm{Bu}\right), 26.7\left(3 \times \mathrm{CH}_{3},{ }^{t} \mathrm{Bu}\right), 26.5\left(\mathrm{CH}_{2}, \mathrm{C}-1\right.$ ' or $\left.\mathrm{C}-2{ }^{\prime}\right), 21.0$ $\left(\mathrm{CH}_{3}, \mathrm{OAc}\right), 19.2\left(\mathrm{C},{ }^{t} \mathrm{Bu}\right), 19.1 \mathrm{ppm}\left(\mathrm{C},{ }^{t} \mathrm{Bu}\right) . \mathrm{IR}\left(\mathrm{CHCl}_{3}\right): \nu=$ $3451,2932,1735,1216,1113 \mathrm{~cm}^{-1}$. MS (ESI) $\mathrm{m} / z(\%)=733(100)$ $[\mathrm{M}+\mathrm{Na}]^{+}$. HRMS (ESI) $m / z:[\mathrm{M}+\mathrm{Na}]^{+}$calcd for $\mathrm{C}_{42} \mathrm{H}_{54} \mathrm{NaO}_{6} \mathrm{Si}_{2}$ 733.3357; found 733.3360. Anal. calcd for $\mathrm{C}_{42} \mathrm{H}_{54} \mathrm{O}_{6} \mathrm{Si}_{2}: \mathrm{C}$, 70.95; $\mathrm{H}$, 7.66. Found: C, 71.05; H, 7.82.

Method $D$. Following the general procedure, starting from substrate $13(98.4 \mathrm{mg}, 0.12 \mathrm{mmol})$, after $2 \mathrm{~h}$ of reaction and again after $4 \mathrm{~h}$, a supplementary addition of $n-\mathrm{Bu}_{3} \mathrm{SnD}(31 \mu \mathrm{L}, 0.12 \mathrm{mmol})$ was required. All the starting material was consumed after $5 \mathrm{~h}$. Column chromatography on a silica gel without KF (hexanes to hexanes-EtOAc, 8:2) gave 3-C-(2-O-acetyl-3,5-di-O-tert-butyldiphenylsilyl- $\alpha$-D- $\left[1-{ }^{2} \mathrm{H}\right]$ ribofuranosyl) 1-propanol $\left(\left[1-{ }^{2} \mathrm{H}\right] 40\right)(46.1 \mathrm{mg}$, $0.065 \mathrm{mmol}, 54 \%,{ }^{2} \mathrm{H} /{ }^{1} \mathrm{H}$ 2:1) as a colorless oil: ${ }^{1} \mathrm{H}$ NMR (500 $\mathrm{MHz}, \mathrm{CDCl}_{3}$, only the deuterated compound is described) $\delta_{\mathrm{H}} 7.65-$ $7.26(\mathrm{~m}, 20 \mathrm{H}, \mathrm{Ar}), 5.117(\mathrm{~d}, J=4.7 \mathrm{~Hz}, 1 \mathrm{H}, 2-\mathrm{H}), 4.61(\mathrm{dd}, J=6.9$, $4.7 \mathrm{~Hz}, 1 \mathrm{H}, 3-\mathrm{H}), 4.03(\mathrm{~m}, 1 \mathrm{H}, 4-\mathrm{H}), 3.66-3.60\left(\mathrm{~m}, 3 \mathrm{H}, 5-\mathrm{H}_{\mathrm{b}}, 3\right.$ '$\left.\mathrm{H}_{2}\right), 3.31\left(\mathrm{dd}, J=11.4,3.5 \mathrm{~Hz}, 1 \mathrm{H}, 5-\mathrm{H}_{\mathrm{a}}\right), 2.13(\mathrm{~s}, 3 \mathrm{H}, \mathrm{OAc}), 1.69-$ $1.53\left(\mathrm{~m}, 4 \mathrm{H}, 1^{\prime}-\mathrm{H}_{2}, 2^{\prime}-\mathrm{H}_{2}\right), 1.02\left(\mathrm{~s}, 9 \mathrm{H},{ }^{t} \mathrm{Bu}\right), 0.91 \mathrm{ppm}\left(\mathrm{s}, 9 \mathrm{H},{ }^{t} \mathrm{Bu}\right)$. ${ }^{13} \mathrm{C}\left\{{ }^{1} \mathrm{H}\right\}$ NMR $\left(100.6 \mathrm{MHz}, \mathrm{CDCl}_{3}\right) \delta_{\mathrm{C}} 170.4$ (C, OAc), $135.79(2 \times$ $\mathrm{CH}, \mathrm{Ar}), 135.73(2 \times \mathrm{CH}, \mathrm{Ar}), 135.61(2 \times \mathrm{CH}, \mathrm{Ar}), 135.56(2 \times$ CH, Ar), 133.4 (C, Ar), 133.28 (C, Ar), 133.25 (C, Ar), 132.7 (C, $\mathrm{Ar}), 129.98(\mathrm{CH}, \mathrm{Ar}), 129.95(\mathrm{CH}, \mathrm{Ar}), 129.5(2 \times \mathrm{CH}, \mathrm{Ar}), 127.76$ $(2 \times \mathrm{CH}, \mathrm{Ar}), 127.72(2 \times \mathrm{CH}, \mathrm{Ar}), 127.56(2 \times \mathrm{CH}, \mathrm{Ar}), 127.54(2$ $\times \mathrm{CH}, \mathrm{Ar}), 82.8(\mathrm{CH}, \mathrm{C}-4), 74.96(\mathrm{CH}, \mathrm{C}-2), 72.8(\mathrm{CH}, \mathrm{C}-3), 63.7$ $\left(\mathrm{CH}_{2}, \mathrm{C}-5\right), 62.6\left(\mathrm{CH}_{2}, \mathrm{C}-3{ }^{\prime}\right), 29.6\left(\mathrm{CH}_{2}, \mathrm{C}-1^{\prime}\right.$ or $\left.\mathrm{C}-2^{\prime}\right), 26.8(3 \times$ $\left.\mathrm{CH}_{3},{ }^{t} \mathrm{Bu}\right), 26.7\left(3 \times \mathrm{CH}_{3},{ }^{t} \mathrm{Bu}\right), 26.5\left(\mathrm{CH}_{2}, \mathrm{C}-1\right.$ ' or $\left.\mathrm{C}-2\right), 21.0\left(\mathrm{CH}_{3}\right.$, OAc), $19.2\left(\mathrm{C},{ }^{t} \mathrm{Bu}\right), 19.1 \mathrm{ppm}\left(\mathrm{C},{ }^{t} \mathrm{Bu}\right)$. MS (ESI) $\mathrm{m} / z(\%)=734$ (100) $[\mathrm{M}+\mathrm{Na}]^{+}, 733(34)[\mathrm{M}+\mathrm{Na}]^{+}$. HRMS (ESI) $\mathrm{m} / z:[\mathrm{M}+$ $\mathrm{Na}]^{+}$calcd for $\mathrm{C}_{42} \mathrm{H}_{53}{ }^{2} \mathrm{HNaO}_{6} \mathrm{Si}_{2}$ 734.3419; found 734.3417, [M + $\mathrm{Na}]^{+}$calcd for $\mathrm{C}_{42} \mathrm{H}_{54} \mathrm{NaO}_{6} \mathrm{Si}_{2}$ 733.3357; found 733.3351.

Method $E$. Following the general procedure, starting from substrate $13(128.6 \mathrm{mg}, 0.15 \mathrm{mmol})$, after $2 \mathrm{~h}$, a supplementary addition of $n$ $\mathrm{Bu}_{3} \mathrm{SnD}(41 \mu \mathrm{L}, 0.15 \mathrm{mmol})$ and $\mathrm{BF}_{3} \bullet \mathrm{Et}_{2} \mathrm{O}(4 \mu \mathrm{L}, 0.03 \mathrm{mmol})$ was required. All the starting material was consumed after $5 \mathrm{~h}$. Column chromatography on a silica gel without KF (hexanes to hexanesEtOAc, 75:25) gave (4RS)-1,4-anhydro-5-O-acetyl-6,8-bis-O-tertbutyldiphenylsilyl-2,3-dideoxy-D- $\left[5-{ }^{2} \mathrm{H}\right]$ ribo-oct-4-ulofuranose $\left(\left[2-{ }^{2} \mathrm{H}\right] 39\right)\left(39.1 \mathrm{mg}, 0.06 \mathrm{mmol}, 40 \%,{ }^{2} \mathrm{H} /{ }^{1} \mathrm{H} 1.3: 1,1 R / 1 \mathrm{~S} 1: 1\right)$ as a colorless oil and $\left(1-{ }^{2} \mathrm{H}\right) 40(13.9 \mathrm{mg}, 0.02 \mathrm{mmol}, 13 \%)$. Compound $\left[2-{ }^{2} \mathrm{H}\right] 39:{ }^{1} \mathrm{H}$ NMR $\left(500 \mathrm{MHz}, \mathrm{CDCl}_{3}\right.$, only nondeuterated products of both isomers are described) $\delta_{\mathrm{H}} 7.72-7.25(\mathrm{~m}, 40 \mathrm{H}, \mathrm{Ar}), 4.44(\mathrm{~m}$, $2 \mathrm{H}, 3-\mathrm{H}), 4.12-4.08(\mathrm{~m}, 2 \mathrm{H}, 4-\mathrm{H}), 4.00$ (ddd, $J=8.5,8.5,5.4 \mathrm{~Hz}$, $\left.1 \mathrm{H}, 3^{\prime}-\mathrm{H}_{\mathrm{b}}\right), 3.90\left(\mathrm{ddd}, J=7.6,7.6,7.6 \mathrm{~Hz}, 1 \mathrm{H}, 3^{\prime}-\mathrm{H}_{\mathrm{a}}\right), 3.83(\mathrm{ddd}, J=$ $8.2,8.2,4.5 \mathrm{~Hz}, 1 \mathrm{H}, 3^{\prime}-\mathrm{H}_{\mathrm{b}}$ ), 3.70 (ddd, $J=7.6,7.6,7.6 \mathrm{~Hz}, 1 \mathrm{H}, 3^{\prime}-\mathrm{H}_{\mathrm{a}}$ ), $3.62\left(\mathrm{dd}, J=11.4,2.6 \mathrm{~Hz}, 1 \mathrm{H}, 5-\mathrm{H}_{\mathrm{b}}\right), 3.50(\mathrm{dd}, J=10.8,5.4 \mathrm{~Hz}, 1 \mathrm{H}$, $\left.5-\mathrm{H}_{\mathrm{b}}\right), 3.45\left(\mathrm{dd}, J=11.0,5.7 \mathrm{~Hz}, 1 \mathrm{H}, 5-\mathrm{H}_{\mathrm{a}}\right), 3.40(\mathrm{dd}, J=11.0,3.5$ 
$\mathrm{Hz}, 1 \mathrm{H}, 5-\mathrm{H}_{\mathrm{a}}$ ), 2.22 (dd, $J=9.5,9.5 \mathrm{~Hz}, 1 \mathrm{H}, 1^{\prime}-\mathrm{H}_{\mathrm{b}}$ ), 2.12 (m, 1H, 2'$\left.\mathrm{H}_{\mathrm{b}}\right), 2.10-1.96\left(\mathrm{~m}, 6 \mathrm{H}, 1^{\prime}-\mathrm{H}_{\mathrm{b}}, 2 \times 2^{\prime}-\mathrm{H}_{2}, 2^{\prime}-\mathrm{H}_{\mathrm{a}}, 2 \times 2-\mathrm{H}_{\mathrm{b}}\right), 1.88-1.81$ $\left(\mathrm{m}, 3 \mathrm{H}, 1^{\prime}-\mathrm{H}_{a}, 2 \times 2-\mathrm{H}_{\mathrm{a}}\right), 1.73\left(\mathrm{ddd}, J=12.0,8.9,8.9 \mathrm{~Hz}, 1 \mathrm{H}, 1^{\prime}-\mathrm{H}_{\mathrm{a}}\right)$, $1.06\left(\mathrm{~s}, 18 \mathrm{H},{ }^{t} \mathrm{Bu}\right), 0.96\left(\mathrm{~s}, 9 \mathrm{H},{ }^{t} \mathrm{Bu}\right), 0.93 \mathrm{ppm}\left(\mathrm{s}, 9 \mathrm{H},{ }^{t} \mathrm{Bu}\right) .{ }^{13} \mathrm{C}\left\{{ }^{1} \mathrm{H}\right\}$ NMR (125.7 MHz, $\mathrm{CDCl}_{3}$, only nondeuterated products of both isomers are described) $\delta_{\mathrm{C}} 134.07(\mathrm{C}, \mathrm{Ar}), 133.87(\mathrm{C}, \mathrm{Ar}), 133.81(\mathrm{C}$, Ar), 133.63 ( $2 \times \mathrm{C}, \mathrm{Ar}), 133.61$ (C, Ar), 133.59 (C, Ar), 133.5 (C, $\mathrm{Ar}), 127.5-135.9(40 \times \mathrm{CH}, \mathrm{Ar}), 114.8(\mathrm{C}, \mathrm{C}-1), 114.2(\mathrm{C}, \mathrm{C}-1)$, 86.9 (CH, C-4), 85.9 (CH, C-4), 74.1 (CH, C-3), 72.9 (CH, C-3), $67.4\left(\mathrm{CH}_{2}, \mathrm{C}-3^{\prime}\right), 67.1\left(\mathrm{CH}_{2}, \mathrm{C}-3^{\prime}\right), 65.0\left(\mathrm{CH}_{2}, \mathrm{C}-5\right), 63.4\left(\mathrm{CH}_{2}, \mathrm{C}-\right.$ 5), $44.3\left(\mathrm{CH}_{2}, \mathrm{C}-2^{\prime}\right), 43.5\left(\mathrm{CH}_{2}, \mathrm{C}-2^{\prime}\right), 36.8\left(\mathrm{CH}_{2}, \mathrm{C}-1^{\prime}\right), 36.1\left(\mathrm{CH}_{2}\right.$, $\left.\mathrm{C}-1^{\prime}\right), 27.0\left(3 \times \mathrm{CH}_{3},{ }^{t} \mathrm{Bu}\right), 26.9\left(3 \times \mathrm{CH}_{3},{ }^{t} \mathrm{Bu}\right), 26.8\left(3 \times \mathrm{CH}_{3}\right.$, $\left.{ }^{t} \mathrm{Bu}\right), 26.7\left(3 \times \mathrm{CH}_{3},{ }^{t} \mathrm{Bu}\right), 24.22\left(\mathrm{CH}_{2}, \mathrm{C}-2\right), 24.20\left(\mathrm{CH}_{2}, \mathrm{C}-2\right)$, $19.19\left(\mathrm{C},{ }^{t} \mathrm{Bu}\right), 19.16\left(2 \times \mathrm{C},{ }^{t} \mathrm{Bu}\right), 19.1 \mathrm{ppm}\left(\mathrm{C},{ }^{t} \mathrm{Bu}\right) . \mathrm{IR}\left(\mathrm{CHCl}_{3}\right): \nu$ $=2932,1428,1222,1113 \mathrm{~cm}^{-1}$. MS (ESI) $\mathrm{m} / z(\%)=674(100)[\mathrm{M}$ $+\mathrm{Na}]^{+}, 673(33)[\mathrm{M}+\mathrm{Na}]^{+}$. HRMS (ESI) $m / z:[\mathrm{M}+\mathrm{Na}]^{+}$calcd for $\mathrm{C}_{40} \mathrm{H}_{49}{ }^{2} \mathrm{HNaO}_{4} \mathrm{Si}_{2}$ 674.3208; found 674.3206, $[\mathrm{M}+\mathrm{Na}]^{+}$calcd for $\mathrm{C}_{40} \mathrm{H}_{50} \mathrm{NaO}_{4} \mathrm{Si}_{2}$ 673.3145; found 673.3163.

Method $F$. Following the general procedure, starting from substrate 13 (40.8 $\mathrm{mg}, 0.048 \mathrm{mmol}$ ), all the starting material was consumed after 75 h. Chromatotron chromatography (hexanes-EtOAc, 7:3) gave (4RS)-1,4-anhydro-5-O-acetyl-6,8-bis-O-tert-butyldiphenylsilyl2,3-dideoxy-D-ribo-oct-4-ulofuranose (39) $\left(0.6 \mathrm{mg}, 9.6 \cdot 10^{-4} \mathrm{mmol}\right.$, $2 \%, 1 R / 1 S 1: 1)$ and $40(22.8 \mathrm{mg}, 0.032 \mathrm{mmol}, 67 \%)$ as colorless oils. Compound 39: ${ }^{1} \mathrm{H}$ NMR (500 MHz, $\mathrm{CDCl}_{3}$, descrived above for the $\left.\left[2{ }^{2} \mathrm{H}\right] 39\right) .{ }^{13} \mathrm{C}\left\{{ }^{1} \mathrm{H}\right\}$ NMR $\left(125.7 \mathrm{MHz}, \mathrm{CDCl}_{3}\right.$, descrived above for the $\left.\left[2-{ }^{2} \mathrm{H}\right] 39\right)$. MS (ESI) $m / z(\%)=673(100)[\mathrm{M}+\mathrm{Na}]^{+}$. HRMS (ESI) $m / z:[\mathrm{M}+\mathrm{Na}]^{+}$calcd for $\mathrm{C}_{40} \mathrm{H}_{50} \mathrm{NaO}_{4} \mathrm{Si}_{2}: 673.3145[\mathrm{M}+$ $\mathrm{Na}]^{+}$; found 673.3146. $\mathrm{C}_{40} \mathrm{H}_{50} \mathrm{NaO}_{4} \mathrm{Si}_{2}$ (650.99): calcd. C 73.80, H 7.74; found: C 73.70, H 7.74.

Radical Reactions of 14. Method A. Following the general procedure, starting from substrate $14(56.9 \mathrm{mg}, 0.06 \mathrm{mmol})$, after $2 \mathrm{~h}$ of reaction, a supplementary addition of $n-\mathrm{Bu}_{3} \mathrm{SnH}(16 \mu \mathrm{L}, 0.06$ $\mathrm{mmol}$ ) was required. All the starting material was consumed after $4 \mathrm{~h}$. Column chromatography on a silica gel without KF (hexanes to hexanes-EtOAc, 7:3) gave $39(18 \mathrm{mg}, 0.028 \mathrm{mmol}, 46 \%, 1 \mathrm{R} / 1 \mathrm{~S}$ 1.2:1).

Method D. Following the general procedure, starting from substrate 14 (54.5 mg, $0.06 \mathrm{mmol})$, after $2 \mathrm{~h}$ of reaction, a supplementary addition of $n-\mathrm{Bu}_{3} \mathrm{SnD}(16 \mu \mathrm{L}, 0.06 \mathrm{mmol})$ was required. All the starting material was consumed after $5 \mathrm{~h}$. Column chromatography on a silica gel without KF (hexanes to hexanesEtOAc, $7: 3)$ gave $\left[2-{ }^{2} \mathrm{H}\right] 39\left(13 \mathrm{mg}, 0.02 \mathrm{mmol}, 35 \%,{ }^{2} \mathrm{H} /{ }^{1} \mathrm{H} 1: 1.4\right)$.

Method E. Following the general procedure, starting from substrate $14(49.8 \mathrm{mg}, 0.05 \mathrm{mmol})$, after $4 \mathrm{~h}$, the reaction was discarded since although the remaining starting material was present, several more polar products were detected in the TLC.

Method F. Following the general procedure, starting from substrate $14(57.4 \mathrm{mg}, 0.06 \mathrm{mmol})$, all the starting material was consumed after 0.75 h. Chromatotron chromatography (hexanes-EtOAc, 97:3 to $7: 3)$ gave 39 (4.8 mg, $0.007 \mathrm{mmol}, 12 \%, 1 R / 1 S 1.2: 1)$ and 3-C-(3,5di-O-tert-butyldiphenylsilyl-2-O-trifluoromethanesulfonyl- $\alpha$-Dribofuranosyl)1-propanol (41) (20.3 $\mathrm{mg}, 0.025 \mathrm{mmol}, 42 \%)$ as a colorless oil. Compound 41: $[\alpha]_{\mathrm{D}}=+18.8\left(c=1.3, \mathrm{CHCl}_{3}\right) .{ }^{1} \mathrm{H}$ NMR $\left(500 \mathrm{MHz}, \mathrm{CDCl}_{3}\right) \delta_{\mathrm{H}} 7.45-7.22(\mathrm{~m}, 20 \mathrm{H}, \mathrm{Ar}), 5.26(\mathrm{dd}, J=4.1,4.1$ $\mathrm{Hz}, 1 \mathrm{H}, 2-\mathrm{H}$ ), 4.67 (dd, $J=4.8,4.8 \mathrm{~Hz}, 1 \mathrm{H}, 3-\mathrm{H}), 4.17$ (ddd, $J=10.1$, $3.2,3.2 \mathrm{~Hz}, 1 \mathrm{H}, 1-\mathrm{H}$ ), 3.95 (ddd, $J=5.4,2.9,2.9 \mathrm{~Hz}, 1 \mathrm{H}, 4-\mathrm{H}), 3.65$ $\left(\mathrm{m}, 2 \mathrm{H}, 3^{\prime}-\mathrm{H}_{2}\right), 3.34\left(\mathrm{dd}, J=11.7,2.2 \mathrm{~Hz}, 1 \mathrm{H}, 5-\mathrm{H}_{\mathrm{b}}\right), 2.75(\mathrm{dd}, J=$ $\left.11.7,3.2 \mathrm{~Hz}, 1 \mathrm{H}, 5-\mathrm{H}_{\mathrm{a}}\right), 1.86\left(\mathrm{~m}, 1 \mathrm{H}, \mathrm{l}^{\prime}-\mathrm{H}_{\mathrm{b}}\right), 1.77-1.65\left(\mathrm{~m}, 3 \mathrm{H}, \mathrm{l}^{\prime}-\right.$ $\left.\mathrm{H}_{\mathrm{a}}, 2^{2}-\mathrm{H}_{2}\right), 1.06\left(\mathrm{~s}, 9 \mathrm{H},{ }^{t} \mathrm{Bu}\right), 0.87 \mathrm{ppm}\left(\mathrm{s}, 9 \mathrm{H},{ }^{t} \mathrm{Bu}\right) .{ }^{13} \mathrm{C}\left\{{ }^{1} \mathrm{H}\right\} \mathrm{NMR}$ $\left(125.7 \mathrm{MHz}, \mathrm{CDCl}_{3}\right) \delta_{\mathrm{C}} 135.9(2 \times \mathrm{CH}, \mathrm{Ar}), 135.8(2 \times \mathrm{CH}, \mathrm{Ar})$, 135.5 (4 × CH, Ar), 133.1 (C, Ar), 132.96 (C, Ar), 132.95 (C, Ar), 131.7 (C, Ar), $130.15(\mathrm{CH}, \mathrm{Ar}), 130.10(\mathrm{CH}, \mathrm{Ar}), 129.6(2 \times \mathrm{CH}$, $\mathrm{Ar}), 127.9(2 \times \mathrm{CH}, \mathrm{Ar}), 127.8(2 \times \mathrm{CH}, \mathrm{Ar}), 127.6(4 \times \mathrm{CH}, \mathrm{Ar})$, $89.1(\mathrm{CH}, \mathrm{C}-2), 82.8(\mathrm{CH}, \mathrm{C}-4), 78.4(\mathrm{CH}, \mathrm{C}-1), 73.3(\mathrm{CH}, \mathrm{C}-3)$, $63.7\left(\mathrm{CH}_{2}, \mathrm{C}-5\right), 62.4\left(\mathrm{CH}_{2}, \mathrm{C}-3{ }^{\prime}\right), 29.4\left(\mathrm{CH}_{2}, \mathrm{C}-1^{\prime}\right), 26.7\left(3 \times \mathrm{CH}_{3}\right.$, $\left.{ }^{t} \mathrm{Bu}\right), 26.6\left(3 \times \mathrm{CH}_{3},{ }^{t} \mathrm{Bu}\right), 26.5\left(\mathrm{CH}_{2}, \mathrm{C}-2^{\prime}\right), 19.2\left(\mathrm{C},{ }^{t} \mathrm{Bu}\right), 19.0 \mathrm{ppm}$ $\left(\mathrm{C},{ }^{t} \mathrm{Bu}\right), 1 \mathrm{C}$ from $\mathrm{CF}_{3}$ group is missing. IR $\left(\mathrm{CHCl}_{3}\right): \nu=3694,3429$, $3020,2933,2254,1778,1740,1224,1113 \mathrm{~cm}^{-1}$. MS (ESI) $\mathrm{m} / z(\%)=$ $823(100)[\mathrm{M}+\mathrm{Na}]^{+}$. HRMS (ESI) $\mathrm{m} / z:[\mathrm{M}+\mathrm{Na}]^{+}$calcd for
$\mathrm{C}_{41} \mathrm{H}_{51} \mathrm{~F}_{3} \mathrm{NaO}_{7} \mathrm{SSi}_{2}$ 823.2744; found 823.2750. Anal. calcd for $\mathrm{C}_{41} \mathrm{H}_{51} \mathrm{~F}_{3} \mathrm{O}_{7} \mathrm{SSi}_{2}$ : C, 61.47; H, 6.42; S, 4.00. Found: C, 61.20; $\mathrm{H}$, 6.44; S, 3.62 .

Radical Reactions of 15. Method A. Following the general procedure, starting from substrate $15(93.8 \mathrm{mg}, 0.12 \mathrm{mmol})$, after $2 \mathrm{~h}$ of reaction, a supplementary addition of $n-\mathrm{Bu}_{3} \mathrm{SnH}(31 \mu \mathrm{L}, 0.12$ $\mathrm{mmol}$ ) was required. All the starting material was consumed after $3 \mathrm{~h}$. Column chromatography on a silica gel without KF (hexanes to hexanes-EtOAc, 97:3) gave (4R)-1,4-anhydro-2,3,5-trideoxy-6,8-bis$O$-(1,1,3,3-tetraisopropyldisiloxanyl)-D-erythro-oct-4-ulofuranose (42) (13 mg, $0.031 \mathrm{mmol}, 27 \%)$ and (4S)-1,4-anhydro-2,3,5-trideoxy-6,8bis-O-(1,1,3,3-tetraisopropyldisiloxanyl)-D-erythro-oct-4-ulofuranose (43) (16.8 mg, $0.040 \mathrm{mmol}, 35 \%)$, both as colorless oils. Compound 42: $[\alpha]_{\mathrm{D}}=-56.6\left(c=0.53, \mathrm{CHCl}_{3}\right) .{ }^{1} \mathrm{H} \mathrm{NMR}\left(500 \mathrm{MHz}, \mathrm{CDCl}_{3}\right) \delta_{\mathrm{H}}$ 4.64 (ddd, $J=8.9,7.3,5.4 \mathrm{~Hz}, 1 \mathrm{H}, 3-\mathrm{H}), 3.95$ (dd, $J=10.1,2.5 \mathrm{~Hz}$, $\left.1 \mathrm{H}, 5-\mathrm{H}_{\mathrm{b}}\right), 3.89\left(\mathrm{ddd}, J=8.2,8.2,5.4 \mathrm{~Hz}, 1 \mathrm{H}, 3^{\prime}-\mathrm{H}_{\mathrm{b}}\right), 3.84-3.77(\mathrm{~m}$, $\left.3 \mathrm{H}, 4-\mathrm{H}, 5-\mathrm{H}_{\mathrm{a}}, 3^{\prime}-\mathrm{H}_{\mathrm{a}}\right), 2.35\left(\mathrm{dd}, J=12.3,7.3 \mathrm{~Hz}, 1 \mathrm{H}, 2-\mathrm{H}_{\mathrm{b}}\right), 2.18(\mathrm{dd}$, $\left.J=12.7,8.9 \mathrm{~Hz}, 1 \mathrm{H}, 2-\mathrm{H}_{\mathrm{a}}\right), 2.06\left(\mathrm{ddd}, J=11.7,11.7,3.2 \mathrm{~Hz}, 1 \mathrm{H}, 1^{\prime}-\right.$ $\left.\mathrm{H}_{\mathrm{b}}\right), 2.02\left(\mathrm{~m}, 1 \mathrm{H}, 2^{\prime}-\mathrm{H}_{\mathrm{b}}\right), 1.93-1.82\left(\mathrm{~m}, 2 \mathrm{H}, 1^{\prime}-\mathrm{H}_{\mathrm{a}}, 2^{\prime}-\mathrm{H}_{\mathrm{a}}\right), 1.10-0.99$ ppm $\left(\mathrm{m}, 28 \mathrm{H},{ }^{i} \mathrm{Pr}\right) .{ }^{13} \mathrm{C}\left\{{ }^{1} \mathrm{H}\right\} \mathrm{NMR}\left(125.7 \mathrm{MHz}, \mathrm{CDCl}_{3}\right) \delta_{\mathrm{C}} 113.1$ (C, C-1), $84.4(\mathrm{CH}, \mathrm{C}-4), 74.7$ (CH, C-3), $67.3\left(\mathrm{CH}_{2}, \mathrm{C}-3^{\prime}\right), 66.1$ $\left(\mathrm{CH}_{2}, \mathrm{C}-5\right), 44.0\left(\mathrm{CH}_{2}, \mathrm{C}-2\right), 34.9\left(\mathrm{CH}_{2}, \mathrm{C}-1^{\prime}\right), 23.9\left(\mathrm{CH}_{2}, \mathrm{C}-2^{\prime}\right)$, $17.6\left(\mathrm{CH}_{3},{ }^{i} \mathrm{Pr}\right), 17.4\left(3 \times \mathrm{CH}_{3},{ }^{i} \mathrm{Pr}\right), 17.3\left(\mathrm{CH}_{3},{ }^{i} \mathrm{Pr}\right), 17.1\left(\mathrm{CH}_{3}\right.$, $\left.{ }^{i} \mathrm{Pr}\right), 17.02\left(\mathrm{CH}_{3},{ }^{i} \mathrm{Pr}\right), 16.99\left(\mathrm{CH}_{3},{ }^{i} \mathrm{Pr}\right), 13.4\left(2 \times \mathrm{CH},{ }^{i} \mathrm{Pr}\right), 12.8$ $\left(\mathrm{CH},{ }^{i} \mathrm{Pr}\right), 12.6 \mathrm{ppm}\left(\mathrm{CH},{ }^{i} \mathrm{Pr}\right)$. IR $\left(\mathrm{CHCl}_{3}\right): \nu=2947,2868,1464$, $1136,1035 \mathrm{~cm}^{-1}$. MS (ESI) $m / z(\%)=439(100)[\mathrm{M}+\mathrm{Na}]^{+}$. HRMS (ESI) $m / z:[\mathrm{M}+\mathrm{Na}]^{+}$calcd for $\mathrm{C}_{20} \mathrm{H}_{40} \mathrm{NaO}_{5} \mathrm{Si}_{2}$ 439.2312; found 439.2308. Anal. calcd for $\mathrm{C}_{20} \mathrm{H}_{40} \mathrm{O}_{5} \mathrm{Si}_{2}$ : C, 57.65; H, 9.68. Found: $\mathrm{C}$, 57.39; H, 9.46. Compound 43: $[\alpha]_{\mathrm{D}}=+20.8\left(c=0.89, \mathrm{CHCl}_{3}\right) .{ }^{1} \mathrm{H}$ NMR $\left(500 \mathrm{MHz}, \mathrm{CDCl}_{3}\right) \delta_{\mathrm{H}} 4.33(\mathrm{ddd}, J=8.2,8.2,6.9 \mathrm{~Hz}, 1 \mathrm{H}, 3-$ $\mathrm{H}), 3.99\left(\mathrm{dd}, J=11.4,2.2 \mathrm{~Hz}, 1 \mathrm{H}, 5-\mathrm{H}_{\mathrm{b}}\right), 3.94-3.89\left(\mathrm{~m}, 2 \mathrm{H}, 3^{\prime}-\mathrm{H}_{2}\right)$, $3.86-3.80\left(\mathrm{~m}, 2 \mathrm{H}, 4-\mathrm{H}, 5-\mathrm{H}_{\mathrm{a}}\right), 2.38\left(\mathrm{dd}, J=13.3,8.2 \mathrm{~Hz}, 1 \mathrm{H}, 2-\mathrm{H}_{\mathrm{b}}\right)$, $2.23\left(\mathrm{dd}, J=13.2,7.3 \mathrm{~Hz}, 1 \mathrm{H}, 2-\mathrm{H}_{\mathrm{a}}\right), 2.08-2.01\left(\mathrm{~m}, 2 \mathrm{H}, 1^{\prime}-\mathrm{H}_{\mathrm{b}}, 2^{\prime}-\right.$ $\left.\mathrm{H}_{\mathrm{b}}\right), 1.91-1.82\left(\mathrm{~m}, 2 \mathrm{H}, 1^{\prime}-\mathrm{H}_{\mathrm{a}}, 2^{\prime}-\mathrm{H}_{\mathrm{a}}\right), 1.10-0.99 \mathrm{ppm}\left(\mathrm{m}, 28 \mathrm{H},{ }^{i} \mathrm{Pr}\right)$. ${ }^{13} \mathrm{C}\left\{{ }^{1} \mathrm{H}\right\}$ NMR $\left(125.7 \mathrm{MHz}, \mathrm{CDCl}_{3}\right) \delta_{\mathrm{C}} 112.7(\mathrm{C}, \mathrm{C}-1), 82.9(\mathrm{CH}$, C-4), 71.5 (CH, C-3), $67.3\left(\mathrm{CH}_{2}, \mathrm{C}-3\right.$ '), $62.3\left(\mathrm{CH}_{2}, \mathrm{C}-5\right), 43.3\left(\mathrm{CH}_{2}\right.$, $\mathrm{C}-2), 36.5\left(\mathrm{CH}_{2}, \mathrm{C}-1^{\prime}\right), 24.2\left(\mathrm{CH}_{2}, \mathrm{C}-2^{\prime}\right), 17.5\left(\mathrm{CH}_{3},{ }^{i} \mathrm{Pr}\right), 17.36(2 \times$ $\left.\mathrm{CH}_{3},{ }^{i} \mathrm{Pr}\right), 17.35\left(\mathrm{CH}_{3},{ }^{i} \mathrm{Pr}\right), 17.27\left(\mathrm{CH}_{3},{ }^{i} \mathrm{Pr}\right), 17.2\left(\mathrm{CH}_{3},{ }^{i} \mathrm{Pr}\right), 17.0$ $\left(\mathrm{CH}_{3},{ }^{i} \mathrm{Pr}\right), 16.9\left(\mathrm{CH}_{3},{ }^{i} \mathrm{Pr}\right), 13.5\left(\mathrm{CH},{ }^{i} \mathrm{Pr}\right), 13.2\left(\mathrm{CH},{ }^{i} \mathrm{Pr}\right), 12.8$ $\left(\mathrm{CH},{ }^{i} \mathrm{Pr}\right), 12.6 \mathrm{ppm}\left(\mathrm{CH},{ }^{i} \mathrm{Pr}\right)$. IR $\left(\mathrm{CHCl}_{3}\right): \nu=2947,2868,1465$, $1210,1133,1043 \mathrm{~cm}^{-1}$. MS (ESI) $\mathrm{m} / z(\%)=439(100)[\mathrm{M}+\mathrm{Na}]^{+}$. HRMS (ESI) $m / z:[\mathrm{M}+\mathrm{Na}]^{+}$calcd for $\mathrm{C}_{20} \mathrm{H}_{40} \mathrm{NaO}_{5} \mathrm{Si}_{2}$ 439.2312; found 439.2312. Anal. calcd for $\mathrm{C}_{20} \mathrm{H}_{40} \mathrm{O}_{5} \mathrm{Si}_{2}$ : C, 57.65; H, 9.68. Found: C, 57.39; H, 9.46.

Method $D$. Following the general procedure, starting from substrate 15 (93.7 mg, $0.12 \mathrm{mmol})$, after $2 \mathrm{~h}$ of reaction, a supplementary addition of $n-\mathrm{Bu}_{3} \mathrm{SnD}(31 \mu \mathrm{L}, 0.12 \mathrm{mmol})$ was required. All the starting material was consumed after $6 \mathrm{~h}$. Column chromatography on a silica gel without KF (hexanes to hexanesEtOAc, 97:3) gave (4R)-1,4-anhydro-2,3,5-trideoxy-6,8-bis- $O$ (1,1,3,3-tetraisopropyldisiloxanyl)- $\beta$-D- $\left[5-{ }^{2} \mathrm{H}\right]$ erythro-oct-4-ulofuranose $\left(\left[2-{ }^{2} \mathrm{H}\right] 42\right)\left(11.7 \mathrm{mg}, 0.02 \mathrm{mmol}, 24 \%,{ }^{2} \mathrm{H} /{ }^{1} \mathrm{H}\right.$ 1.2:1) and $(4 S)$ 1,4-anhydro-2,3,5-trideoxy-6,8-bis-O-(1,1,3,3-tetraisopropyldisiloxanyl) $\beta$-D- $\left[5-{ }^{2} \mathrm{H}\right]$ erythro-oct-4-ulofuranose $\left(\left[2-{ }^{2} \mathrm{H}\right] 43\right)(10 \mathrm{mg}, 0.024$ mmol, $\left.21 \%,{ }^{2} \mathrm{H} /{ }^{1} \mathrm{H} 1.5: 1\right)$, which was obtained as a $1: 1.2$ mixture with $\left[2-{ }^{2} \mathrm{H}\right] 42$. Compound $\left[2-{ }^{2} \mathrm{H}\right] 42:{ }^{1} \mathrm{H}$ NMR $\left(500 \mathrm{MHz}, \mathrm{CDCl}_{3}\right.$, only deuterated $2 R S$ isomers are described) $\delta_{\mathrm{H}} 4.64(\mathrm{~m}, 1 \mathrm{H}, 3-\mathrm{H}), 3.96-$ $3.87\left(\mathrm{~m}, 2 \mathrm{H}, 5-\mathrm{H}_{\mathrm{b}}, 3^{\prime}-\mathrm{H}_{\mathrm{b}}\right), 3.84-3.77\left(\mathrm{~m}, 3 \mathrm{H}, 4-\mathrm{H}, 5-\mathrm{H}_{\mathrm{a}}, 3^{\prime}-\mathrm{H}_{\mathrm{a}}\right)$, $2.336(\mathrm{~d}, J=7.3 \mathrm{~Hz}, 1 \mathrm{H}, 2-\mathrm{H}, 2 \mathrm{R}$ isomer $), 2.166(\mathrm{~d}, J=9.2 \mathrm{~Hz}, 1 \mathrm{H}$, $2-\mathrm{H}, 2 \mathrm{~S}$ isomer), 2.06 (ddd, $J=11.4,11.4,2.9 \mathrm{~Hz}, 1 \mathrm{H}, 1^{\prime}-\mathrm{H}_{\mathrm{b}}$ ), 2.01 $\left(\mathrm{m}, 1 \mathrm{H}, 2^{\prime}-\mathrm{H}_{\mathrm{b}}\right), 1.93-1.83\left(\mathrm{~m}, 2 \mathrm{H}, 1^{\prime}-\mathrm{H}_{\mathrm{a}}, 2^{\prime}-\mathrm{H}_{\mathrm{a}}\right), 1.10-0.99 \mathrm{ppm}(\mathrm{m}$, $\left.28 \mathrm{H},{ }^{i} \mathrm{Pr}\right) .{ }^{13} \mathrm{C}\left\{{ }^{1} \mathrm{H}\right\}$ NMR $\left(125.7 \mathrm{MHz}, \mathrm{CDCl}_{3}\right.$, only deuterated $2 R S$ isomers are described) $\delta_{\mathrm{C}} 113.1(\mathrm{C}, \mathrm{C}-1), 84.4(\mathrm{CH}, \mathrm{C}-4), 74.60$ $(\mathrm{CH}, \mathrm{C}-3), 67.3\left(\mathrm{CH}_{2}, \mathrm{C}-3^{\prime}\right), 66.1\left(\mathrm{CH}_{2}, \mathrm{C}-5\right), 43.68\left(\mathrm{t}, J_{\mathrm{CD}}=22.2\right.$ $\mathrm{Hz}, \mathrm{CHD}, \mathrm{C}-2), 34.9\left(\mathrm{CH}_{2}, \mathrm{C}-1^{\prime}\right), 23.9\left(\mathrm{CH}_{2}, \mathrm{C}-2^{\prime}\right), 17.6\left(\mathrm{CH}_{3},{ }^{i} \mathrm{Pr}\right)$, $17.4\left(3 \times \mathrm{CH}_{3},{ }^{i} \mathrm{Pr}\right), 17.3\left(\mathrm{CH}_{3},{ }^{i} \mathrm{Pr}\right), 17.1\left(\mathrm{CH}_{3},{ }^{i} \mathrm{Pr}\right), 17.02\left(\mathrm{CH}_{3}\right.$, $\left.{ }^{i} \mathrm{Pr}\right), 16.99\left(\mathrm{CH}_{3},{ }^{i} \mathrm{Pr}\right), 13.4\left(2 \times \mathrm{CH},{ }^{i} \mathrm{Pr}\right), 12.8\left(\mathrm{CH},{ }^{i} \mathrm{Pr}\right), 12.6 \mathrm{ppm}$ $\left(\mathrm{CH},{ }^{i} \mathrm{Pr}\right)$. MS (ESI) $\mathrm{m} / z(\%)=440(100)[\mathrm{M}+\mathrm{Na}]^{+}, 439(68)[\mathrm{M}$ $+\mathrm{Na}]^{+}$. HRMS (ESI) $m / z:[\mathrm{M}+\mathrm{Na}]^{+}$calcd for $\mathrm{C}_{20} \mathrm{H}_{39}{ }^{2} \mathrm{HNaO}_{5} \mathrm{Si}_{2}$ 440.2375; found 440.2372, $[\mathrm{M}+\mathrm{Na}]^{+}$calcd for $\mathrm{C}_{20} \mathrm{H}_{40} \mathrm{NaO}_{5} \mathrm{Si}_{2}$ 
439.2312; found 439.2300. Compound $\left[2-{ }^{2} \mathrm{H}\right] 43$ : ${ }^{1} \mathrm{H}$ NMR (500 $\mathrm{MHz}, \mathrm{CDCl}_{3}$, only deuterated $2 \mathrm{RS}$ isomers are described) $\delta_{\mathrm{H}} 4.33$ (ddd, $J=8.2,8.2,6.9 \mathrm{~Hz}, 1 \mathrm{H}, 3-\mathrm{H}), 3.99(\mathrm{dd}, J=11.4,2.2 \mathrm{~Hz}, 1 \mathrm{H}, 5-$ $\mathrm{H}_{\mathrm{b}}$ ), 3.94-3.89 (m, 2H, 3'- $\left.\mathrm{H}_{2}\right), 3.86-3.80\left(\mathrm{~m}, 2 \mathrm{H}, 4-\mathrm{H}, 5-\mathrm{H}_{\mathrm{a}}\right), 2.365$ $(\mathrm{d}, J=8.5 \mathrm{~Hz}, 1 \mathrm{H}, 2-\mathrm{H}), 2.228(\mathrm{~d}, J=6.9 \mathrm{~Hz}, 1 \mathrm{H}, 2-\mathrm{H}), 2.08-2.01$ $\left(\mathrm{m}, 2 \mathrm{H}, 1^{\prime}-\mathrm{H}_{\mathrm{b}}, 2^{\prime}-\mathrm{H}_{\mathrm{b}}\right), 1.91-1.82\left(\mathrm{~m}, 2 \mathrm{H}, 1^{\prime}-\mathrm{H}_{\mathrm{a}}, 2^{\prime}-\mathrm{H}_{\mathrm{a}}\right), 1.10-0.99$ ppm (m, 28H, $\left.{ }^{i} \mathrm{Pr}\right) .{ }^{13} \mathrm{C}\left\{{ }^{1} \mathrm{H}\right\}$ NMR (125.7 $\left.\mathrm{MHz}, \mathrm{CDCl}_{3}\right) \delta_{\mathrm{C}} 112.7$ (C, C-1), 82.9 (CH, C-4), 71.5 ( $\mathrm{CH}, \mathrm{C}-3), 67.3\left(\mathrm{CH}_{2}, \mathrm{C}-{ }^{\prime}\right), 62.3$ $\left(\mathrm{CH}_{2}, \mathrm{C}-5\right), 42.95\left(\mathrm{t}, J_{\mathrm{CD}}=21.2 \mathrm{~Hz}, \mathrm{CHD}, \mathrm{C}-2\right), 36.5\left(\mathrm{CH}_{2}, \mathrm{C}-1^{\prime}\right)$, $\left.24.2\left(\mathrm{CH}_{2}, \mathrm{C}-2\right)\right), 17.5\left(\mathrm{CH}_{3},{ }^{i} \mathrm{Pr}\right), 17.4\left(2 \times \mathrm{CH}_{3},{ }^{i} \mathrm{Pr}\right), 17.3(2 \times$ $\left.\mathrm{CH}_{3},{ }^{i} \mathrm{Pr}\right), 17.2\left(\mathrm{CH}_{3},{ }^{i} \mathrm{Pr}\right), 17.0\left(\mathrm{CH}_{3},{ }^{i} \mathrm{Pr}\right), 16.9\left(\mathrm{CH}_{3},{ }^{i} \mathrm{Pr}\right), 13.5$ $\left(\mathrm{CH},{ }^{i} \mathrm{Pr}\right), 13.2\left(\mathrm{CH},{ }^{i} \mathrm{Pr}\right), 12.8\left(\mathrm{CH},{ }^{i} \mathrm{Pr}\right), 12.6 \mathrm{ppm}\left(\mathrm{CH},{ }^{i} \mathrm{Pr}\right) . \mathrm{IR}$ $\left(\mathrm{CHCl}_{3}\right): \nu=2947,2868,1465,1210,1133,1043 \mathrm{~cm}^{-1}$. MS (ESI) $\mathrm{m} / z(\%)=440(100)[\mathrm{M}+\mathrm{Na}]^{+}, 439(55)[\mathrm{M}+\mathrm{Na}]^{+}$. HRMS (ESI) $m / z:[\mathrm{M}+\mathrm{Na}]^{+}$calcd for $\mathrm{C}_{20} \mathrm{H}_{39}{ }^{2} \mathrm{HNaO}_{5} \mathrm{Si}_{2}$ 440.2375; found 440.2374, $[\mathrm{M}+\mathrm{Na}]^{+}$calcd for $\mathrm{C}_{20} \mathrm{H}_{40} \mathrm{NaO}_{5} \mathrm{Si}_{2}$ 439.2312; found 439.2306 .

Method F. Following the general procedure, starting from substrate $15(53.8 \mathrm{mg}, 0.066 \mathrm{mmol})$, all the starting material was consumed after $0.5 \mathrm{~h}$. Chromatotron chromatography (hexanes-EtOAc, 95:5 to 1:1) gave 42 and 43 (7.3 mg, $0.018 \mathrm{mmol}, 26 \%, 1 R / 1 \mathrm{~S} 1: 2.5)$, and 3$\mathrm{C}$-(2-O-diphenoxyphosphoryl-3,5-bis-O-(1,1,3,3-tetraisopropyldisiloxanyl)- $\alpha$-D-ribofuranosyl) 1 -propanol (44) (9.5 mg, $0.014 \mathrm{mmol}, 22 \%)$ as a colorless oil. Compound 44: $[\alpha]_{\mathrm{D}}=+12.9\left(c=0.71, \mathrm{CHCl}_{3}\right) .{ }^{1} \mathrm{H}$ NMR $\left(500 \mathrm{MHz}, \mathrm{CDCl}_{3}\right) \delta_{\mathrm{H}} 7.33-7.15(\mathrm{~m}, 10 \mathrm{H}, \mathrm{Ar}), 5.13$ (ddd, $J=$ $\left.3.8,3.8 \mathrm{~Hz},{ }^{3} J_{\mathrm{PH}}=7.9 \mathrm{~Hz}, 1 \mathrm{H}, 2-\mathrm{H}\right), 4.47(\mathrm{~m}, 1 \mathrm{H}, 3-\mathrm{H}), 4.12(\mathrm{~m}, 1 \mathrm{H}$, $1-\mathrm{H}), 4.00\left(\mathrm{dd}, J=12.6,2.8 \mathrm{~Hz}, 1 \mathrm{H}, 5-\mathrm{H}_{\mathrm{b}}\right), 3.95-3.91(\mathrm{~m}, 2 \mathrm{H}, 4-\mathrm{H}$, 5- $\left.\mathrm{H}_{\mathrm{a}}\right), 3.51-3.49\left(\mathrm{~m}, 2 \mathrm{H}, 3^{\prime}-\mathrm{H}_{2}\right), 1.66-1.51\left(\mathrm{~m}, 4 \mathrm{H}, 1^{\prime}-\mathrm{H}_{2}, 2^{\prime}-\mathrm{H}_{2}\right)$, $1.09-0.81 \mathrm{ppm}\left(\mathrm{m}, 28 \mathrm{H},{ }^{i} \mathrm{Pr}\right), 1 \mathrm{H}$ from $\mathrm{OH}$ is missing. ${ }^{13} \mathrm{C}\left\{{ }^{1} \mathrm{H}\right\}$ NMR $\left(125.7 \mathrm{MHz}, \mathrm{CDCl}_{3}\right) \delta_{\mathrm{C}} 150.9\left(\mathrm{~d},{ }^{2} J_{\mathrm{PC}}=7.4 \mathrm{~Hz}, \mathrm{C}, \mathrm{Ar}\right), 150.6$ $\left(\mathrm{d},{ }^{2} J_{\mathrm{PC}}=7.4 \mathrm{~Hz}, \mathrm{C}, \mathrm{Ar}\right), 129.7(2 \times \mathrm{CH}, \mathrm{Ar}), 129.6(2 \times \mathrm{CH}, \mathrm{Ar})$, $125.3(\mathrm{CH}, \mathrm{Ar}), 125.1(\mathrm{CH}, \mathrm{Ar}), 120.19(\mathrm{CH}, \mathrm{Ar}), 120.14(\mathrm{CH}, \mathrm{Ar})$, $120.0(\mathrm{CH}, \mathrm{Ar}), 119.9(\mathrm{CH}, \mathrm{Ar}), 81.6\left(\mathrm{~d},{ }^{2} J_{\mathrm{PC}}=6.4 \mathrm{~Hz}, \mathrm{CH}, \mathrm{C}-2\right)$, $79.8(\mathrm{CH}, \mathrm{C}-4), 79.5\left(\mathrm{~d},{ }^{3} \mathrm{~J}_{\mathrm{PC}}=6.3 \mathrm{~Hz}, \mathrm{CH}, \mathrm{C}-1\right), 71.6(\mathrm{CH}, \mathrm{C}-3)$, $62.5\left(\mathrm{CH}_{2}, \mathrm{C}-3^{\prime}\right), 60.9\left(\mathrm{CH}_{2}, \mathrm{C}-5\right), 29.2\left(\mathrm{CH}_{2}, \mathrm{C}-1^{\prime}\right.$ or $\left.\mathrm{C}-2^{\prime}\right), 26.7$ $\left(\mathrm{CH}_{2}, \mathrm{C}-1^{\prime}\right.$ or C-2'), $17.4\left(\mathrm{CH}_{3},{ }^{i} \mathrm{Pr}\right), 17.28\left(\mathrm{CH}_{3},{ }^{i} \mathrm{Pr}\right), 17.27\left(\mathrm{CH}_{3}\right.$, $\left.{ }^{i} \mathrm{Pr}\right), 17.25\left(\mathrm{CH}_{3},{ }^{i} \mathrm{Pr}\right), 17.0\left(2 \times \mathrm{CH}_{3},{ }^{i} \mathrm{Pr}\right), 16.8\left(\mathrm{CH}_{3},{ }^{i} \mathrm{Pr}\right), 16.7$ $\left(\mathrm{CH}_{3},{ }^{i} \mathrm{Pr}\right), 13.5\left(\mathrm{CH},{ }^{i} \mathrm{Pr}\right), 13.1\left(\mathrm{CH},{ }^{i} \mathrm{Pr}\right), 12.6\left(\mathrm{CH},{ }^{i} \mathrm{Pr}\right), 12.4 \mathrm{ppm}$ $\left(\mathrm{CH},{ }^{i} \mathrm{Pr}\right)$. IR $\left(\mathrm{CHCl}_{3}\right): \nu=3692,3610,3022,2948,1490.1210,1039$ $\mathrm{cm}^{-1}$. MS (ESI) $\mathrm{m} / z(\%)=689(100)[\mathrm{M}+\mathrm{Na}]^{+}$. HRMS (ESI) $\mathrm{m} / z$ : $[\mathrm{M}+\mathrm{Na}]^{+}$calcd for $\mathrm{C}_{32} \mathrm{H}_{51} \mathrm{NNaO}_{9} \mathrm{PSi}_{2}$ 689.2707; found 689.2706. Anal. calcd for $\mathrm{C}_{32} \mathrm{H}_{51} \mathrm{NO}_{9} \mathrm{PSi}_{2}$ : C, 57.63; H, 7.71. Found: C, 57.61; $\mathrm{H}, 8.07$.

Synthesis of 4-Deoxy-6,8-dioxabicyclo[3.2.1]heptane Structures (Tables 5 and 6). Radical Reactions of 16. Method A. Following the general procedure, starting from substrate $16(49 \mathrm{mg}$, $0.076 \mathrm{mmol}$ ), after $2 \mathrm{~h}$ of reaction, a supplementary addition of $n$ $\mathrm{Bu}_{3} \mathrm{SnH}(20 \mu \mathrm{L}, 0.076 \mathrm{mmol})$ was required. All the starting material was consumed after $5 \mathrm{~h}$. Column chromatography (hexanes to hexanes-EtOAc, 6:4) gave (2S)-2,7-anhydro-1-O-tert-butyldiphenylsilyl-3-deoxy-4,5-di-O-methyl- $\beta$-D-xylo-hept-2-ulopyranose (45) (3.1 $\mathrm{mg}, 0.007 \mathrm{mmol}, 9 \%)$, an inseparable mixture of 48 (7.6 $\mathrm{mg}, 0.015$ $\mathrm{mmol}, 20 \%)$ and unstable $C$-(6-O-tert-butyldiphenylsilyl-4-deoxy-2,3di- $O$-methyl- $\beta$-L-threo-hex-4-enopyranosyl)methanol (46) $(1.5 \mathrm{mg}$, $0.03 \mathrm{mmol}, 4 \%$ ), and $\mathrm{C}$-(4-O-acetyl-6-O-tert-butyldiphenylsilyl-2,3-di$O$-methyl- $\beta$-L-idopyranosyl)methanol (47) $(12.1 \mathrm{mg}, 0.024 \mathrm{mmol}$, $32 \%)$, all as colorless oils. Compound 45: $[\alpha]_{\mathrm{D}}=+14.5(c=0.38$, $\left.\mathrm{CHCl}_{3}\right) .{ }^{1} \mathrm{H}$ NMR $\left(500 \mathrm{MHz}, \mathrm{CDCl}_{3}\right.$, simulated ring coupling constants using DAISY) $\delta_{\mathrm{H}} 7.70-7.68(\mathrm{~m}, 4 \mathrm{H}, \mathrm{Ar}), 7.45-7.37(\mathrm{~m}$, $6 \mathrm{H}, \mathrm{Ar}$ ), 4.56 (ddd, $J=5.0,4.3,0.0 \mathrm{~Hz}, 1 \mathrm{H}, 1-\mathrm{H}), 4.03$ (dd, $J=7.6$, $\left.0.0 \mathrm{~Hz}, 1 \mathrm{H}, 1^{\prime}-\mathrm{H}_{\mathrm{b}}\right), 3.75\left(\mathrm{~d}, J=11.0 \mathrm{~Hz}, 1 \mathrm{H}, 6-\mathrm{H}_{\mathrm{b}}\right), 3.73(\mathrm{~d}, J=10.7$ $\left.\mathrm{Hz}, 1 \mathrm{H}, 6-\mathrm{H}_{\mathrm{a}}\right), 3.68\left(\mathrm{dd}, J=7.5,5.0 \mathrm{~Hz},{ }^{4} J_{2,1 \mathrm{a}}=1.1 \mathrm{~Hz}, 1 \mathrm{H}, 1^{\prime}-\mathrm{H}_{\mathrm{a}}\right)$, 3.571 (ddd, $J=10.1,8.2,6.6 \mathrm{~Hz}, 1 \mathrm{H}, 3-\mathrm{H}$ ), 3.50 (s, $3 \mathrm{H}, \mathrm{OMe}$ ), 3.42 $(\mathrm{s}, 3 \mathrm{H}, \mathrm{OMe}), 3.40$ (ddd, $\left.J=8.2,4.4 \mathrm{~Hz},{ }^{4} J_{2,1 \mathrm{a}}=1.1 \mathrm{~Hz}, 1 \mathrm{H}, 2-\mathrm{H}\right)$, $2.36\left(\mathrm{dd}, J=13.0,6.6 \mathrm{~Hz}, 1 \mathrm{H}, 4-\mathrm{H}_{\mathrm{b}}\right), 1.70(\mathrm{dd}, J=13.0,10.1 \mathrm{~Hz}, 1 \mathrm{H}$, 4- $\left.\mathrm{H}_{\mathrm{a}}\right), 1.08 \mathrm{ppm}\left(\mathrm{s}, 9 \mathrm{H},{ }^{t} \mathrm{Bu}\right) .{ }^{13} \mathrm{C}\left\{{ }^{1} \mathrm{H}\right\} \mathrm{NMR}\left(100.6 \mathrm{MHz}, \mathrm{CDCl}_{3}\right)$ $\delta_{\mathrm{C}} 135.68(2 \times \mathrm{CH}, \mathrm{Ar}), 135.67(2 \times \mathrm{CH}, \mathrm{Ar}), 133.2(2 \times \mathrm{C}, \mathrm{Ar})$, $129.7(2 \times \mathrm{CH}, \mathrm{Ar}), 127.7(4 \times \mathrm{CH}, \mathrm{Ar}), 107.94(\mathrm{C}, \mathrm{C}-5), 81.0(\mathrm{CH}$, C-2), 77.78 (CH, C-3), 73.7 ( $\mathrm{CH}, \mathrm{C}-1), 66.8\left(\mathrm{CH}_{2}, \mathrm{C}-6\right), 65.8\left(\mathrm{CH}_{2}\right.$, C-1'), $58.4\left(\mathrm{CH}_{3}, \mathrm{OMe}\right), 57.2\left(\mathrm{CH}_{3}, \mathrm{OMe}\right), 37.08\left(\mathrm{CH}_{2}, \mathrm{C}-4\right), 26.8$ $\left(3 \times \mathrm{CH}_{3}\right.$, DPS $), 19.3 \mathrm{ppm}$ (C, DPS). IR $\left(\mathrm{CHCl}_{3}\right): \nu=2931,1464$, $1113 \mathrm{~cm}^{-1}$. MS (ESI) $\mathrm{m} / z(\%)=465(100)[\mathrm{M}+\mathrm{Na}]^{+}$. HRMS (ESI) $m / z:[\mathrm{M}+\mathrm{Na}]^{+}$calcd for $\mathrm{C}_{25} \mathrm{H}_{34} \mathrm{NaO}_{5} \mathrm{Si}$ 465.2073; found 465.2071. Anal. calcd for $\mathrm{C}_{25} \mathrm{H}_{34} \mathrm{O}_{5} \mathrm{Si}$ : C, 67.84; $\mathrm{H}, 7.74$. Found: C, 67.63; H, 7.68. Compound 46: could not be purified perfectly due to its instability. ${ }^{1} \mathrm{H}$ NMR ( $500 \mathrm{MHz}, \mathrm{CDCl}_{3}$, simulated coupling constants of the allylic system using DAISY) $\delta_{\mathrm{H}} 7.74-7.34(\mathrm{~m}, 10 \mathrm{H}, \mathrm{Ar}), 5.23$ (dddd, $J=4.9 \mathrm{~Hz},{ }^{4} J=1.5,1.5,1.0 \mathrm{~Hz}, 1 \mathrm{H}, 4-\mathrm{H}$ ), 4.16 (ddd, $J=13.9$ $\left.\mathrm{Hz},{ }^{4} J=1.0 \mathrm{~Hz},{ }^{5} J=1.6 \mathrm{~Hz}, 1 \mathrm{H}, 6-\mathrm{H}_{\mathrm{b}}\right), 4.12\left(\mathrm{ddd}, J=13.9 \mathrm{~Hz},{ }^{4} J=\right.$ $\left.1.5 \mathrm{~Hz},{ }^{5} \mathrm{~J}=0.7 \mathrm{~Hz}, 1 \mathrm{H}, 6-\mathrm{H}_{\mathrm{a}}\right), 3.98-3.92\left(\mathrm{~m}, 2 \mathrm{H}, 1-\mathrm{H}, \mathrm{1}^{\prime}-\mathrm{H}_{\mathrm{b}}\right), 3.81$ $\left(\mathrm{m}, 1 \mathrm{H}, 1^{\prime}-\mathrm{H}_{\mathrm{a}}\right.$ ), 3.72 (dddd, $J=4.9,2.3 \mathrm{~Hz},{ }^{5} J=1.6,0.7 \mathrm{~Hz}, 1 \mathrm{H}, 3-$ $\mathrm{H}), 3.454(\mathrm{~s}, 3 \mathrm{H}, \mathrm{OMe}), 3.450(\mathrm{~m}, 1 \mathrm{H}, 2-\mathrm{H}), 3.42(\mathrm{~s}, 3 \mathrm{H}, \mathrm{OMe})$, $1.08 \mathrm{ppm}\left(\mathrm{s}, 9 \mathrm{H},{ }^{t} \mathrm{Bu}\right), 1 \mathrm{H}$ from $\mathrm{OH}$ is missing. ${ }^{13} \mathrm{C}\left\{{ }^{1} \mathrm{H}\right\} \operatorname{NMR}(100.6$ $\left.\mathrm{MHz}, \mathrm{CDCl}_{3}\right) \delta_{\mathrm{C}} 156.0(\mathrm{C}, \mathrm{C}-5), 135.58(2 \times \mathrm{CH}, \mathrm{Ar}), 135.56(2 \times$ $\mathrm{CH}, \mathrm{Ar}), 133.3(2 \times \mathrm{C}, \mathrm{Ar}), 129.7(2 \times \mathrm{CH}, \mathrm{Ar}), 127.7(4 \times \mathrm{CH}, \mathrm{Ar})$, 92.8 (CH, C-4), 76.5 (CH, C-3), $74.1(\mathrm{CH}, \mathrm{C}-1), 69.4(\mathrm{CH}, \mathrm{C}-2)$, $62.7\left(2 \times \mathrm{CH}_{2}, \mathrm{C}-1, \mathrm{C}-6\right), 58.0\left(\mathrm{CH}_{3}, \mathrm{OMe}\right), 55.4\left(\mathrm{CH}_{3}, \mathrm{OMe}\right), 26.8$ $\left(3 \times \mathrm{CH}_{3}, \mathrm{DPS}\right), 19.3 \mathrm{ppm}$ (C, DPS). IR $\left(\mathrm{CHCl}_{3}\right): \nu=3674,3504$, 2931, $1113 \mathrm{~cm}^{-1}$. MS (ESI) $\mathrm{m} / z(\%)=465(100)[\mathrm{M}+\mathrm{Na}]^{+}$. HRMS (ESI) $m / z:[\mathrm{M}+\mathrm{Na}]^{+}$calcd for $\mathrm{C}_{25} \mathrm{H}_{34} \mathrm{NaO}_{5} \mathrm{Si}$ 465.2073; found 465.2061. Compound 47: $[\alpha]_{\mathrm{D}}=+0.4\left(c=1.20, \mathrm{CHCl}_{3}\right) .{ }^{1} \mathrm{H}$ NMR $\left(500 \mathrm{MHz}, \mathrm{CDCl}_{3}\right.$, simulated ring coupling constants using DAISY) $\delta_{\mathrm{H}} 7.64-7.61(\mathrm{~m}, 4 \mathrm{H}, \mathrm{Ar}), 7.45-7.35(\mathrm{~m}, 6 \mathrm{H}, \mathrm{Ar}), 5.074$ (ddd, $J=$ 2.6, $\left.1.9,{ }^{4} J_{2,4}=1.2 \mathrm{~Hz}, 1 \mathrm{H}, 4-\mathrm{H}\right), 4.01$ (ddd, $J=9.1,5.2,1.6 \mathrm{~Hz}, 1 \mathrm{H}$, 5-H), $3.94\left(\mathrm{dd}, J=11.7,8.0 \mathrm{~Hz}, 1 \mathrm{H}, 1^{\prime}-\mathrm{H}_{\mathrm{b}}\right), 3.81(\mathrm{dd}, J=9.8,5.2 \mathrm{~Hz}$, $\left.1 \mathrm{H}, 6-\mathrm{H}_{\mathrm{b}}\right), 3.79$ (ddd, $\left.J=8.0,4.0,1.6 \mathrm{~Hz}, 1 \mathrm{H}, 1-\mathrm{H}\right), 3.78(\mathrm{dd}, J=$ $\left.11.7,9.1 \mathrm{~Hz}, 1 \mathrm{H}, 6-\mathrm{H}_{\mathrm{a}}\right), 3.74(\mathrm{dd}, J=2.7,2.6 \mathrm{~Hz}, 1 \mathrm{H}, 3-\mathrm{H}), 3.63(\mathrm{dd}$, $\left.J=11.7,4.0 \mathrm{~Hz}, 1 \mathrm{H}, 1^{\prime}-\mathrm{H}_{\mathrm{a}}\right), 3.55$ (s, 3H, OMe), 3.36 (s, $\left.3 \mathrm{H}, \mathrm{OMe}\right)$, 3.20 (ddd, $\left.J=2.7,1.6,{ }^{4} J_{2,4}=1.2 \mathrm{~Hz}, 1 \mathrm{H}, 2-\mathrm{H}\right), 2.03(\mathrm{~s}, 3 \mathrm{H}, \mathrm{OAc}$ ), 1.85 (br s, $1 \mathrm{H}, \mathrm{OH}), 1.04 \mathrm{ppm}\left(\mathrm{s}, 9 \mathrm{H},{ }^{t} \mathrm{Bu}\right) .{ }^{13} \mathrm{C}\left\{{ }^{1} \mathrm{H}\right\}$ NMR $(100.6$ $\left.\mathrm{MHz}, \mathrm{CDCl}_{3}\right) \delta_{\mathrm{C}} 170.8(\mathrm{C}, \mathrm{OAc}), 135.6(2 \times \mathrm{CH}, \mathrm{Ar}), 135.5(2 \times$ $\mathrm{CH}, \mathrm{Ar}), 133.3$ (C, Ar), 133.2 (C, Ar), 129.74 (CH, Ar), $129.72(\mathrm{CH}$, $\mathrm{Ar}), 127.7(4 \times \mathrm{CH}, \mathrm{Ar}), 76.3(\mathrm{CH}, \mathrm{C}-1$ or $\mathrm{C}-2), 76.1(\mathrm{CH}, \mathrm{C}-1$ or $\mathrm{C}-2), 74.6(\mathrm{CH}, \mathrm{C}-5), 71.7(\mathrm{CH}, \mathrm{C}-3), 66.20(\mathrm{CH}, \mathrm{C}-4), 62.6\left(\mathrm{CH}_{2}\right.$, C-1'), $61.62\left(\mathrm{CH}_{2}, \mathrm{C}-6\right), 58.1\left(\mathrm{CH}_{3}, \mathrm{OMe}\right), 58.0\left(\mathrm{CH}_{3}, \mathrm{OMe}\right), 26.8$ $\left(3 \times \mathrm{CH}_{3}\right.$, DPS $), 21.0\left(\mathrm{CH}_{3}, \mathrm{OAc}\right), 19.1 \mathrm{ppm}(\mathrm{C}, \mathrm{DPS})$. IR $\left(\mathrm{CHCl}_{3}\right)$ : $\nu=3675,3594,2933,1731,1103 \mathrm{~cm}^{-1}$. MS (ESI) $\mathrm{m} / z(\%)=525$ (100) $[\mathrm{M}+\mathrm{Na}]^{+}$. HRMS (ESI) $m / z:[\mathrm{M}+\mathrm{Na}]^{+}$calcd for $\mathrm{C}_{27} \mathrm{H}_{38} \mathrm{NaO}_{7} \mathrm{Si}$ 525.2285; found 525.2276. Anal. calcd for $\mathrm{C}_{27} \mathrm{H}_{38} \mathrm{O}_{7} \mathrm{Si}$ : C, 64.51; H, 7.62. Found: C, 64.81; H, 7.86.

Method C. Following the general procedure, starting from substrate $16(54.5 \mathrm{mg}, 0.084 \mathrm{mmol})$, after $2 \mathrm{~h}$ of reaction, a supplementary addition of TTMSS $(26 \mu \mathrm{L}, 0.084 \mathrm{mmol})$ was required. All the starting material was consumed after $7 \mathrm{~h}$. Column chromatography (hexanes-EtOAc, 9:1 to 7:3) gave $45(4.1 \mathrm{mg}, 0.009 \mathrm{mmol}, 11 \%)$ and 46 (10.8 $\mathrm{mg}, 0.024 \mathrm{mmol}, 29 \%)$.

Method $D$. Following the general procedure, starting from substrate $16(69.9 \mathrm{mg}, 0.11 \mathrm{mmol})$, after $2 \mathrm{~h}$ of reaction, a supplementary addition of $n-\mathrm{Bu}_{3} \mathrm{SnD}(29 \mu \mathrm{L}, 0.11 \mathrm{mmol})$ was required. All the starting material was consumed after $4 \mathrm{~h}$. Column chromatography (hexanes to hexanes-EtOAc, 1:1) gave 2,7-anhydro1-O-tert-butyldiphenylsilyl-3-deoxy-4,5-di- $O$-methyl- $\beta$-D- $\left[3-{ }^{2} \mathrm{H}\right] x y l o-$ hept-2-ulopyranose $\left(\left[4-{ }^{2} \mathrm{H}\right] 45\right)\left(5.3 \mathrm{mg}, 0.011 \mathrm{mmol}, 10 \%,{ }^{2} \mathrm{H} /{ }^{1} \mathrm{H}\right.$ 1.8:1, 4R/4S 1:1.2), an inseparable mixture of reduced alcohol 48 (7.6 $\mathrm{mg}, 0.015 \mathrm{mmol}, 14 \%)$ and olefin $46(10.7 \mathrm{mg}, 0.024 \mathrm{mmol}, 22 \%)$, and $\mathrm{C}$-(4-O-acetyl-6-O-tert-butyldiphenylsilyl-2,3-di- $O$-methyl- $\beta$-L$\left(5{ }^{2} \mathrm{H}\right)$ idopyranosyl $)$ methanol $\left[\left(5{ }^{2} \mathrm{H}\right) 47\right](18.3 \mathrm{mg}, 0.036 \mathrm{mmol}$, $33 \%)$, all as colorless oils. Compound $\left[4-{ }^{2} \mathrm{H}\right] 45:{ }^{1} \mathrm{H}$ NMR $(500 \mathrm{MHz}$, $\mathrm{CDCl}_{3}$, only deuterated $4 R S$ isomers are described) $\delta_{\mathrm{H}} 7.70-7.65(\mathrm{~m}$, $4 \mathrm{H}, \mathrm{DPS}$ ), 7.45-7.37 (m, 6H, DPS), 4.56 (ddd, $J=4.4,4.4,0.0 \mathrm{~Hz}$, $1 \mathrm{H}, 1-\mathrm{H}), 4.03\left(\mathrm{dd}, J=7.3,0.0 \mathrm{~Hz}, 1 \mathrm{H}, 1^{\prime}-\mathrm{H}_{\mathrm{b}}\right), 3.75(\mathrm{~d}, J=11.0 \mathrm{~Hz}$, $\left.1 \mathrm{H}, 6-\mathrm{H}_{\mathrm{b}}\right), 3.73\left(\mathrm{~d}, J=11.0 \mathrm{~Hz}, 1 \mathrm{H}, 6-\mathrm{H}_{\mathrm{a}}\right), 3.70-3.66\left(\mathrm{~m}, 1 \mathrm{H}, 1^{\prime}-\mathrm{H}_{\mathrm{a}}\right)$, $3.60-3.54(\mathrm{~m}, 1 \mathrm{H}, 3-\mathrm{H}), 3.50$ (s, 3H, OMe), 3.43 (s, 3H, OMe), 3.42 $(\mathrm{dd}, J=8.8,3.5 \mathrm{~Hz}, 1 \mathrm{H}, 2-\mathrm{H}), 2.35(\mathrm{~d}, J=6.6 \mathrm{~Hz}, 1 \mathrm{H}, 4-\mathrm{H}, 4 R$ isomer), $1.69(\mathrm{~d}, J=10.1 \mathrm{~Hz}, 1 \mathrm{H}, 4-\mathrm{H}, 4 \mathrm{~S}$ isomer), $1.08 \mathrm{ppm}(\mathrm{s}, 18 \mathrm{H}$, $\left.{ }^{t} \mathrm{Bu}\right) .{ }^{13} \mathrm{C}\left\{{ }^{1} \mathrm{H}\right\}$ NMR $\left(100.6 \mathrm{MHz}, \mathrm{CDCl}_{3}\right.$, only deuterated $4 R S$ isomers are described) $\delta_{\mathrm{C}} 135.68(2 \times \mathrm{CH}, \mathrm{DPS}), 135.66(2 \times \mathrm{CH}$, DPS), 133.27 (C, DPS), 133.20 (C, DPS), $129.7(2 \times \mathrm{CH}, \mathrm{DPS})$, $127.7(4 \times \mathrm{CH}, \mathrm{DPS}), 107.90$ (C, C-5), $81.0(\mathrm{CH}, \mathrm{C}-2), 77.73(\mathrm{CH}$, $\mathrm{C}-3,4 R$ or $4 S$ isomer), $77.70(\mathrm{CH}, \mathrm{C}-3,4 R$ or $4 S$ isomer $), 73.7(\mathrm{CH}$, 
C-1), $66.8\left(\mathrm{CH}_{2}, \mathrm{C}-6\right), 65.8\left(\mathrm{CH}_{2}, \mathrm{C}-1^{\prime}\right), 58.4\left(\mathrm{CH}_{3}, \mathrm{OMe}\right), 57.2$ $\left(\mathrm{CH}_{3}, \mathrm{OMe}\right), 36.75\left(\mathrm{t}, J_{\mathrm{CD}}=19.1 \mathrm{~Hz}, \mathrm{CHD}, \mathrm{C}-4\right), 26.8\left(3 \times \mathrm{CH}_{3}\right.$, DPS), $19.3 \mathrm{ppm}$ (C, DPS). MS (ESI) $\mathrm{m} / z(\%)=466(100)[\mathrm{M}+$ $\mathrm{Na}]^{+}, 465(46)[\mathrm{M}+\mathrm{Na}]^{+}$. HRMS (ESI) $\mathrm{m} / z:[\mathrm{M}+\mathrm{Na}]^{+}$calcd for $\mathrm{C}_{25} \mathrm{H}_{33}{ }^{2} \mathrm{HNaO}_{5} \mathrm{Si}$ 466.2136; found 466.2141, $[\mathrm{M}+\mathrm{Na}]^{+}$calcd for $\mathrm{C}_{25} \mathrm{H}_{34} \mathrm{NaO}_{5} \mathrm{Si}$ 465.2073; found 465.2060. Compound $\left(5-{ }^{2} \mathrm{H}\right) 47:{ }^{1} \mathrm{H}$ NMR $\left(400 \mathrm{MHz}, \mathrm{CDCl}_{3}\right.$, simulated ring coupling constants using DAISY) $\delta_{\mathrm{H}} 7.64-7.61(\mathrm{~m}, 4 \mathrm{H}, \mathrm{Ar}), 7.45-7.35(\mathrm{~m}, 6 \mathrm{H}, \mathrm{Ar}), 5.070$ $\left(\mathrm{dd}, J=2.7 \mathrm{~Hz},{ }^{4} J_{2,4}=1.2 \mathrm{~Hz}, 1 \mathrm{H}, 4-\mathrm{H}\right), 3.93(\mathrm{dd}, J=11.7,8.1 \mathrm{~Hz}$, $\left.1 \mathrm{H}, 1^{\prime}-\mathrm{H}_{\mathrm{b}}\right), 3.81\left(\mathrm{~d}, J=9.6 \mathrm{~Hz}, 1 \mathrm{H}, 6-\mathrm{H}_{\mathrm{a}}\right), 3.79(\mathrm{~d}, J=9.6 \mathrm{~Hz}, 1 \mathrm{H}, 6-$ $\mathrm{H}_{\mathrm{b}}$ ), 3.79 (ddd, $\left.J=8.1,4.0,1.6 \mathrm{~Hz}, 1 \mathrm{H}, 1-\mathrm{H}\right), 3.73(\mathrm{dd}, J=2.7,2.7$ $\mathrm{Hz}, 1 \mathrm{H}, 3-\mathrm{H}), 3.62\left(\mathrm{dd}, J=11.7,4.0 \mathrm{~Hz}, 1 \mathrm{H}, 1\right.$ '- $\left.\mathrm{H}_{\mathrm{a}}\right), 3.55(\mathrm{~s}, 3 \mathrm{H}$, OMe), 3.36 (s, $3 \mathrm{H}, \mathrm{OMe}$ ), 3.19 (ddd, $J=2.7,1.6 \mathrm{~Hz},{ }^{4} J_{2,4}=1.2 \mathrm{~Hz}$, $1 \mathrm{H}, 2-\mathrm{H}), 2.03(\mathrm{~s}, 3 \mathrm{H}, \mathrm{OAc}), 1.04 \mathrm{ppm}\left(\mathrm{s}, 9 \mathrm{H},{ }^{t} \mathrm{Bu}\right), 1 \mathrm{H}$ from $\mathrm{OH}$ is missing. ${ }^{13} \mathrm{C}\left\{{ }^{1} \mathrm{H}\right\}$ NMR (100.6 MHz, $\left.\mathrm{CDCl}_{3}\right) \delta_{\mathrm{C}} 170.8$ (C, OAc), $135.6(2 \times \mathrm{CH}, \mathrm{Ar}), 135.5(2 \times \mathrm{CH}, \mathrm{Ar}), 133.3$ (C, Ar), $133.2(\mathrm{C}$, $\mathrm{Ar}), 129.74(\mathrm{CH}, \mathrm{Ar}), 129.71(\mathrm{CH}, \mathrm{Ar}), 127.7(4 \times \mathrm{CH}, \mathrm{Ar}), 76.2$ (CH, C-1 or C-2), $76.1(\mathrm{CH}, \mathrm{C}-1$ or $\mathrm{C}-2), 71.7(\mathrm{CH}, \mathrm{C}-3), 66.14$ $(\mathrm{CH}, \mathrm{C}-4), 62.6\left(\mathrm{CH}_{2}, \mathrm{C}-1{ }^{\prime}\right), 61.54\left(\mathrm{CH}_{2}, \mathrm{C}-6\right), 58.1\left(\mathrm{CH}_{3}, \mathrm{OMe}\right)$, $58.0\left(\mathrm{CH}_{3}, \mathrm{OMe}\right), 26.8\left(3 \times \mathrm{CH}_{3}\right.$, DPS $), 21.0\left(\mathrm{CH}_{3}, \mathrm{OAc}\right), 19.1 \mathrm{ppm}$ (C, DPS). MS (ESI) $m / z(\%)=526(100)[\mathrm{M}+\mathrm{Na}]^{+}$. HRMS (ESI) $m / z:[\mathrm{M}+\mathrm{Na}]^{+}$calcd for $\mathrm{C}_{27} \mathrm{H}_{37}{ }^{2} \mathrm{HNaO}_{7} \mathrm{Si}$ 526.2347; found 526.2346 .

Method E. Following the general procedure, starting from substrate $16(38.3 \mathrm{mg}, 0.059 \mathrm{mmol})$, after $2 \mathrm{~h}$, a supplementary addition of $n$ $\mathrm{Bu}_{3} \mathrm{SnD}(16 \mu \mathrm{L}, 0.059 \mathrm{mmol})$ and $\mathrm{BF}_{3} \bullet \mathrm{Et}_{2} \mathrm{O}(2 \mu \mathrm{L}, 0.012 \mathrm{mmol})$ was required. All the starting material was consumed after $4 \mathrm{~h}$. Column chromatography (hexanes to hexanes-EtOAc, $1: 1$ ) gave $\left[4{ }^{2} \mathrm{H}\right] 45$ (10.3 mg, $\left.0.023 \mathrm{mmol}, 39 \%,{ }^{2} \mathrm{H} /{ }^{1} \mathrm{H} \quad 2.9: 1,4 R / 4 S \quad 1: 1.2\right)$, an inseparable mixture of $48(4.6 \mathrm{mg}, 0.009 \mathrm{mmol}, 15 \%)$ and unstable $46(4.6 \mathrm{mg}, 0.010 \mathrm{mmol}, 18 \%)$, and $\left(5-{ }^{2} \mathrm{H}\right) 47(7.3 \mathrm{mg}, 0.015 \mathrm{mmol}$, $25 \%)$.

Radical Reactions of 17. Method A. Following the general procedure, starting from substrate $17(49 \mathrm{mg}, 0.058 \mathrm{mmol})$, after $2 \mathrm{~h}$ of reaction, a supplementary addition of $n-\mathrm{Bu}_{3} \mathrm{SnH}(16 \mu \mathrm{L}, 0.058$ $\mathrm{mmol}$ ) was required. All the starting material was consumed after $5 \mathrm{~h}$. Column chromatography on a silica gel without KF (hexanes to hexanes-EtOAc, 9:1) gave 45 (14 mg, $0.032 \mathrm{mmol}, 55 \%)$.

Method $D$. Following the general procedure, starting from substrate $17(59.6 \mathrm{mg}, 0.071 \mathrm{mmol})$, after $2 \mathrm{~h}$ of reaction, a supplementary addition of $n-\mathrm{Bu}_{3} \mathrm{SnD}(19 \mu \mathrm{L}, 0.071 \mathrm{mmol})$ was required. All the starting material was consumed after $4 \mathrm{~h}$. Column chromatography (hexanes to hexanes-EtOAc, 9:1) gave $\left[4-{ }^{2} \mathrm{H}\right] 45$ (18.1 mg, $0.041 \mathrm{mmol}, 58 \%,{ }^{2} \mathrm{H} /{ }^{1} \mathrm{H}$ 2.3:1, 4R/4S 1:1.2).

Radical Reactions of 18. Method A. Following the general procedure, starting from substrate $18(38 \mathrm{mg}, 0.05 \mathrm{mmol})$, after $2 \mathrm{~h}$ of reaction, a supplementary addition of $n-\mathrm{Bu}_{3} \mathrm{SnH}(13 \mu \mathrm{L}, 0.05 \mathrm{mmol})$ was required. All the starting material was consumed after $6 \mathrm{~h}$. Column chromatography on a silica gel without KF (hexanes to hexanes-EtOAc, 9:1) gave $45(11.7 \mathrm{mg}, 0.027 \mathrm{mmol}, 53 \%)$.

Method $D$. Following the general procedure, starting from substrate 18 (38.8 $\mathrm{mg}, 0.05 \mathrm{mmol})$, after $2 \mathrm{~h}$ of reaction, a supplementary addition of $n-\mathrm{Bu}_{3} \mathrm{SnD}(14 \mu \mathrm{L}, 0.05 \mathrm{mmol})$ was required. All the starting material was consumed after $4 \mathrm{~h}$. Column chromatography (hexanes to hexanes-EtOAc, 9:1) gave $\left[4{ }^{2} \mathrm{H}\right] 45$ (13 mg, $0.029 \mathrm{mmol}, 58 \%,{ }^{2} \mathrm{H} /{ }^{1} \mathrm{H}$ 1.7:1, 4R/4S 1:1.2).

Method F. Following the general procedure, starting from substrate $18(12.4 \mathrm{mg}, 0.016 \mathrm{mmol})$, all the starting material was consumed after $1.5 \mathrm{~h}$. Chromatotron chromatography (hexanes-EtOAc, 8:2 to 4:6) gave 45 ( $1.4 \mathrm{mg}, 0.003 \mathrm{mmol}, 19 \%)$ and 49 (4.1 mg, 0.007 $\mathrm{mmol}, 41 \%)$.

Method G. Following the general procedure, starting from substrate $18(14.1 \mathrm{mg}, 0.019 \mathrm{mmol})$, all the starting material was consumed after $3 \mathrm{~h}$. Chromatotron chromatography (hexanesEtOAc, $8: 2$ to $4: 6)$ gave $45(3.2 \mathrm{mg}, 0.007 \mathrm{mmol}, 39 \%)$ and 49 (2.3 mg, $0.004 \mathrm{mmol}, 20 \%)$.

Radical Reactions of 19. Method A. Following the general procedure, starting from substrate $19(72.6 \mathrm{mg}, 0.087 \mathrm{mmol})$, after 2 $\mathrm{h}$, a supplementary addition of $n-\mathrm{Bu}_{3} \mathrm{SnH}(24 \mu \mathrm{L}, 0.087 \mathrm{mmol})$ was required. All the starting material was consumed after $3 \mathrm{~h}$. Column chromatography (hexanes to hexanes-EtOAc, 2:8) gave 2,7-anhydro3-deoxy-1-O-diphenoxyfosforyl-4,5-di- $O$-methyl- $\beta$-D-xylo-hept-2-ulopyranose (53) (11.5 mg, $0.026 \mathrm{mmol}, 30 \%)$ and 1,5-anhydro-4,6-bis$O$-diphenoxyphosphoryl-2,3-di-O-methyl-D-glucitol (54) (11 mg, $0.017 \mathrm{mmol}, 19 \%)$ as colorless oils. Compound 53: $[\alpha]_{\mathrm{D}}=+2.6(c$ $\left.=0.46, \mathrm{CHCl}_{3}\right) .{ }^{1} \mathrm{H} \mathrm{NMR}\left(400 \mathrm{MHz}, \mathrm{CDCl}_{3}\right.$, simulated ring coupling constants using DAISY) $\delta_{\mathrm{H}} 7.36-7.17(\mathrm{~m}, 10 \mathrm{H}, \mathrm{Ar}), 4.54$ (ddd, $J=$ 5.1, 3.9, $0.0 \mathrm{~Hz}, 1 \mathrm{H}, 1-\mathrm{H}), 4.29\left(\mathrm{~d},{ }^{3} J_{\mathrm{PH}}=8.2 \mathrm{~Hz}, 2 \mathrm{H}, 6-\mathrm{H}_{2}\right), 4.02$ (dd, $J=7.7,0.0 \mathrm{~Hz}, 1 \mathrm{H}, 1^{\prime}-\mathrm{H}_{\mathrm{b}}$ ), 3.65 (ddd, $J=7.5,5.1 \mathrm{~Hz},{ }^{4} J_{2,1 \mathrm{a}}=1.1$ $\left.\mathrm{Hz}, 1 \mathrm{H}, 1^{\prime}-\mathrm{H}_{\mathrm{a}}\right), 3.528$ (ddd, $\left.J=10.0,7.8,6.5 \mathrm{~Hz}, 1 \mathrm{H}, 3-\mathrm{H}\right), 3.47(\mathrm{~s}$, $3 \mathrm{H}, \mathrm{OMe}$ ), 3.36 (ddd, $\left.J=7.8,3.9 \mathrm{~Hz},{ }^{4} J_{2,1} \mathrm{a}=1.1 \mathrm{~Hz}, 1 \mathrm{H}, 2-\mathrm{H}\right), 3.36$ (s, 3H, OMe), 2.29 (dd, $\left.J=12.9,6.5 \mathrm{~Hz}, 1 \mathrm{H}, 4-\mathrm{H}_{\mathrm{b}}\right), 1.52 \mathrm{ppm}(\mathrm{dd}, J$ $\left.=12.9,10.0 \mathrm{~Hz}, 1 \mathrm{H}, 4-\mathrm{H}_{\mathrm{a}}\right) .{ }^{13} \mathrm{C}\left\{{ }^{1} \mathrm{H}\right\} \operatorname{NMR}\left(125.7 \mathrm{MHz}, \mathrm{CDCl}_{3}\right) \delta_{\mathrm{C}}$ $150.5\left(\mathrm{~d},{ }^{2} J_{\mathrm{PC}}=6.3 \mathrm{~Hz}, 2 \times \mathrm{C}, \mathrm{Ar}\right), 120.1-129.8(10 \times \mathrm{CH}, \mathrm{Ar})$, $105.65\left(\mathrm{~d},{ }^{3} \mathrm{~J}_{\mathrm{PC}}=7.4 \mathrm{~Hz}, \mathrm{C}, \mathrm{C}-5\right), 80.4(\mathrm{CH}, \mathrm{C}-2), 77.31(\mathrm{CH}, \mathrm{C}-3)$, $73.9(\mathrm{CH}, \mathrm{C}-1), 69.3\left(\mathrm{~d},{ }^{2} J_{\mathrm{PC}}=5.3 \mathrm{~Hz}, \mathrm{CH}_{2}, \mathrm{C}-6\right), 66.2\left(\mathrm{CH}_{2}, \mathrm{C}-1^{\prime}\right)$, $58.5\left(\mathrm{CH}_{3}, \mathrm{OMe}\right), 57.1\left(\mathrm{CH}_{3}, \mathrm{OMe}\right), 36.76 \mathrm{ppm}\left(\mathrm{CH}_{2}, \mathrm{C}-4\right)$. IR $\left(\mathrm{CHCl}_{3}\right): \nu=2929,1490,1232 \mathrm{~cm}^{-1}$. MS (ESI) $\mathrm{m} / z(\%)=459$ (100) $[\mathrm{M}+\mathrm{Na}]^{+}$. HRMS (ESI) $\mathrm{m} / z:[\mathrm{M}+\mathrm{Na}]^{+}$calcd for $\mathrm{C}_{21} \mathrm{H}_{25} \mathrm{NaO}_{8} \mathrm{P}$ 459.1185; found 459.1175. Compound 54: $[\alpha]_{\mathrm{D}}=$ $+27.0\left(c=0.70, \mathrm{CHCl}_{3}\right) .{ }^{1} \mathrm{H} \mathrm{NMR}\left(500 \mathrm{MHz}, \mathrm{CDCl}_{3}\right.$, simulated ring coupling constants using DAISY) $\delta_{\mathrm{H}} 7.33-7.10(\mathrm{~m}, 20 \mathrm{H}, \mathrm{Ar}), 4.42$ (ddd, $J=11.6,2.0 \mathrm{~Hz},{ }^{3} J_{\mathrm{PH}}=8.2 \mathrm{~Hz}, 1 \mathrm{H}, 6-\mathrm{H}_{\mathrm{b}}$ ), 4.36 (ddd, $J=9.7$, $\left.9.1 \mathrm{~Hz},{ }^{3} J_{\mathrm{PH}}=9.4 \mathrm{~Hz}, 1 \mathrm{H}, 4-\mathrm{H}\right), 4.13\left(\mathrm{ddd}, J=11.6,5.9 \mathrm{~Hz},{ }^{3} J_{\mathrm{PH}}=\right.$ $\left.9.8 \mathrm{~Hz}, 1 \mathrm{H}, 6-\mathrm{H}_{\mathrm{a}}\right), 3.950\left(\mathrm{dd}, J=11.3,5.2 \mathrm{~Hz}, 1 \mathrm{H}, 1-\mathrm{H}_{\mathrm{b}}\right.$ ), 3.51 (ddd, $J$ $=9.7,5.9,2.0 \mathrm{~Hz}, 1 \mathrm{H}, 5-\mathrm{H}), 3.45(\mathrm{~s}, 3 \mathrm{H}, \mathrm{OMe}), 3.43(\mathrm{~s}, 3 \mathrm{H}, \mathrm{OMe})$, 3.29 (dd, $J=9.1,8.8 \mathrm{~Hz}, 1 \mathrm{H}, 3-\mathrm{H}), 3.207$ (ddd, $J=10.6,8.8,5.2 \mathrm{~Hz}$, $1 \mathrm{H}, 2-\mathrm{H}), 3.040 \mathrm{ppm}\left(\mathrm{dd}, J=11.3,10.6 \mathrm{~Hz}, 1 \mathrm{H}, 1-\mathrm{H}_{\mathrm{a}}\right) .{ }^{13} \mathrm{C}\left\{{ }^{1} \mathrm{H}\right\}$ NMR $\left(125.7 \mathrm{MHz}, \mathrm{CDCl}_{3}\right) \delta_{\mathrm{C}} 150.7\left(\mathrm{~d},{ }^{2} J_{\mathrm{PC}}=7.4 \mathrm{~Hz}, \mathrm{C}, \mathrm{Ar}\right), 150.6$ $\left(\mathrm{d},{ }^{2} J_{\mathrm{PC}}=7.4 \mathrm{~Hz}, \mathrm{C}, \mathrm{Ar}\right), 150.5\left(\mathrm{~d},{ }^{2} J_{\mathrm{PC}}=7.4 \mathrm{~Hz}, \mathrm{C}, \mathrm{Ar}\right), 150.4(\mathrm{~d}$, $\left.{ }^{2} J_{\mathrm{PC}}=7.4 \mathrm{~Hz}, \mathrm{C}, \mathrm{Ar}\right), 120.0-129.8(20 \times \mathrm{CH}, \mathrm{Ar}), 84.9(\mathrm{CH}, \mathrm{C}-3)$, $79.82(\mathrm{CH}, \mathrm{C}-2), 76.7(\mathrm{CH}, \mathrm{C}-5), 75.8\left(\mathrm{~d},{ }^{2} \mathrm{~J}_{\mathrm{PC}}=6.3 \mathrm{~Hz}, \mathrm{CH}, \mathrm{C}-4\right)$, $67.5\left(\mathrm{~d},{ }^{2} J_{\mathrm{PC}}=6.4 \mathrm{~Hz}, \mathrm{CH}_{2}, \mathrm{C}-6\right), 67.19\left(\mathrm{CH}_{2}, \mathrm{C}-1\right), 60.5\left(\mathrm{CH}_{3}\right.$, $\mathrm{OMe}), 58.7 \mathrm{ppm}\left(\mathrm{CH}_{3}, \mathrm{OMe}\right)$. IR $\left(\mathrm{CHCl}_{3}\right): \nu=3020,2929,1490$, $1218 \mathrm{~cm}^{-1}$. MS (ESI) $\mathrm{m} / z(\%)=679(100)[\mathrm{M}+\mathrm{Na}]^{+}$. HRMS (ESI) $m / z:[\mathrm{M}+\mathrm{Na}]^{+}$calcd for $\mathrm{C}_{32} \mathrm{H}_{34} \mathrm{NaO}_{11} \mathrm{P}_{2}$ 679.1474; found 679.1474.

Radical Reactions of 20. Method A. Following the general procedure, starting from substrate $20(89 \mathrm{mg}, 0.14 \mathrm{mmol})$, after $2 \mathrm{~h}$ of reaction, a supplementary addition of $n-\mathrm{Bu}_{3} \mathrm{SnH}(37 \mu \mathrm{L}, 0.14 \mathrm{mmol})$ was required. All the starting material was consumed after $5 \mathrm{~h}$. Column chromatography on a silica gel without KF (hexanes to hexanes-EtOAc, 75:25) gave an inseparable mixture of $C$-(4-Oacetyl-6-O-tert-butyldiphenylsilyl-2,3-di-O-methyl- $\beta$-L-altropyranosyl)methanol (50) and $\mathbf{5 1}(33 \mathrm{mg}, 0.066 \mathrm{mmol}, 47 \%, 2: 1)$ as a colorless oil, and 46 (12.1 mg, $0.027 \mathrm{mmol}, 20 \%)$. Compounds 50 and $51:{ }^{1} \mathrm{H}$ NMR $\left(500 \mathrm{MHz}, \mathrm{CDCl}_{3}\right.$, only $\mathbf{5 0}$ is described) $\delta_{\mathrm{H}} 7.73-7.63(\mathrm{~m}, 4 \mathrm{H}$, Ar), 7.46-7.35 (m, 6H, Ar), $5.144(\mathrm{dd}, J=10.1,2.9 \mathrm{~Hz}, 1 \mathrm{H}, 4-\mathrm{H})$, 3.93-3.77 (m, 7H, 1-H, 3-H, 5-H, 6- $\left.\mathrm{H}_{2}, 1^{\prime}-\mathrm{H}_{2}\right), 3.46$ (s, 3H, OMe), $3.45(\mathrm{~s}, 3 \mathrm{H}, \mathrm{OMe}), 3.37(\mathrm{dd}, J=3.8,1.0 \mathrm{~Hz}, 1 \mathrm{H}, 2-\mathrm{H}), 2.03(\mathrm{~s}, 3 \mathrm{H}$, $\mathrm{OAc}), 1.05 \mathrm{ppm}\left(\mathrm{s}, 9 \mathrm{H},{ }^{t} \mathrm{Bu}\right), 1 \mathrm{H}$ from $\mathrm{OH}$ is missing. ${ }^{13} \mathrm{C}\left\{{ }^{1} \mathrm{H}\right\}$ NMR $\left(100.6 \mathrm{MHz}, \mathrm{CDCl}_{3}\right.$, only 50 is described) $\delta_{\mathrm{C}} 169.9$ (C, OAc), 135.8 $(2 \times \mathrm{CH}, \mathrm{Ar}), 135.6(2 \times \mathrm{CH}, \mathrm{Ar}), 133.9$ (C, Ar), $133.6(\mathrm{C}, \mathrm{Ar})$, $129.5(2 \times \mathrm{CH}, \mathrm{Ar}), 127.6(2 \times \mathrm{CH}, \mathrm{Ar}), 127.5(2 \times \mathrm{CH}, \mathrm{Ar}), 77.5$ $(\mathrm{CH}, \mathrm{C}-2), 74.7(\mathrm{CH}, \mathrm{C}-1$ or $\mathrm{C}-5), 74.4(\mathrm{CH}, \mathrm{C}-1$ or $\mathrm{C}-5), 74.4$ (CH, C-3), $68.55(\mathrm{CH}, \mathrm{C}-4), 63.88\left(\mathrm{CH}_{2}, \mathrm{C}-6\right), 62.8\left(\mathrm{CH}_{2}, \mathrm{C}-1\right.$ '), $59.2\left(\mathrm{CH}_{3}, \mathrm{OMe}\right), 58.2\left(\mathrm{CH}_{3}, \mathrm{OMe}\right), 26.7\left(3 \times \mathrm{CH}_{3}, \mathrm{DPS}\right), 20.9$ $\left(\mathrm{CH}_{3}, \mathrm{OAc}\right), 19.3 \mathrm{ppm}$ (C, DPS). IR $\left(\mathrm{CHCl}_{3}\right): \nu=3690,3567,2933$, $1737,1217 \mathrm{~cm}^{-1}$. MS (ESI) $\mathrm{m} / z(\%)=525(100)[\mathrm{M}+\mathrm{Na}]^{+}$. HRMS (ESI) $m / z:[\mathrm{M}+\mathrm{Na}]^{+}$calcd for $\mathrm{C}_{27} \mathrm{H}_{38} \mathrm{NaO}_{7} \mathrm{Si}$ 525.2285; found 525.2267. Anal. calcd for $\mathrm{C}_{27} \mathrm{H}_{38} \mathrm{O}_{7} \mathrm{Si}$ : C, 64.51; H, 7.62. Found: C, 64.58 ; H. 7.84 .

Method D. Following the general procedure, starting from substrate $20(38 \mathrm{mg}, 0.06 \mathrm{mmol})$, after $2 \mathrm{~h}$ of reaction and again after $4 \mathrm{~h}$, a supplementary addition of $n-\mathrm{Bu}_{3} \mathrm{SnD}(16 \mu \mathrm{L}, 0.06 \mathrm{mmol})$ was required. All the starting material was consumed after $9 \mathrm{~h}$. Column chromatography on a silica gel without KF (hexanes to hexanes-EtOAc, 8:2) gave an inseparable mixture of three compounds, $\left(5-{ }^{2} \mathrm{H}\right) \mathbf{5 0}$ and 51 (19.3 $\left.\mathrm{mg}, 0.04 \mathrm{mmol}, 66 \%, 2.3: 1\right)$ and olefin $46(3.3 \mathrm{mg}, 0.007 \mathrm{mmol}, 13 \%)$, as a colorless oil. Mixture of $\left(5-{ }^{2} \mathrm{H}\right) 50 / 51 / 46:{ }^{1} \mathrm{H} \mathrm{NMR}\left(400 \mathrm{MHz}, \mathrm{CDCl}_{3}\right.$, only $\left(5-{ }^{2} \mathrm{H}\right) 50$ is 
described) $\delta_{\mathrm{H}} 7.73-7.60(\mathrm{~m}, 4 \mathrm{H}, \mathrm{Ar}), 7.44-7.32(\mathrm{~m}, 6 \mathrm{H}, \mathrm{Ar}), 5.137$ (d, $J=3.1 \mathrm{~Hz}, 1 \mathrm{H}, 4-\mathrm{H}), 3.94-3.76\left(\mathrm{~m}, 6 \mathrm{H}, 1-\mathrm{H}, 3-\mathrm{H}, 6-\mathrm{H}_{2}, 1^{\prime}-\mathrm{H}_{2}\right)$, $3.45(\mathrm{~s}, 6 \mathrm{H}, 2 \times \mathrm{OMe}), 3.37(\mathrm{dd}, J=3.8,1.0 \mathrm{~Hz}, 1 \mathrm{H}, 2-\mathrm{H}), 2.03(\mathrm{~s}$, $3 \mathrm{H}, \mathrm{OAc}), 1.05 \mathrm{ppm}\left(\mathrm{s}, 9 \mathrm{H},{ }^{t} \mathrm{Bu}\right), 1 \mathrm{H}$ from $\mathrm{OH}$ is missing. ${ }^{13} \mathrm{C}\left\{{ }^{1} \mathrm{H}\right\}$ NMR $\left(100.6 \mathrm{MHz}, \mathrm{CDCl}_{3}\right.$, only $\left(5{ }^{2} \mathrm{H}\right) 50$ is described) $\delta_{\mathrm{C}} 169.9$ (C, OAc), 135.8 ( $4 \times \mathrm{CH}, \mathrm{Ar}), 133.4$ (C, Ar), 133.3 (C, Ar), 129.5 ( $2 \times$ $\mathrm{CH}, \mathrm{Ar}), 127.5(4 \times \mathrm{CH}, \mathrm{Ar}), 77.6(\mathrm{CH}, \mathrm{C}-2), 74.7(\mathrm{CH}, \mathrm{C}-1), 74.5$ (CH, C-3), $68.53(\mathrm{CH}, \mathrm{C}-4), 63.84\left(\mathrm{CH}_{2}, \mathrm{C}-6\right), 62.7\left(\mathrm{CH}_{2}, \mathrm{C}-1^{\prime}\right)$, $59.3\left(\mathrm{CH}_{3}, \mathrm{OMe}\right), 58.2\left(\mathrm{CH}_{3}, \mathrm{OMe}\right), 26.7\left(3 \times \mathrm{CH}_{3}, \mathrm{DPS}\right), 20.9$ $\left(\mathrm{CH}_{3}, \mathrm{OAc}\right), 19.3 \mathrm{ppm}$ (C, DPS). MS (ESI) $\mathrm{m} / z(\%)=526(100)$ $[\mathrm{M}+\mathrm{Na}]^{+}, 525(48)[\mathrm{M}+\mathrm{Na}]^{+}, 465(54)[\mathrm{M}+\mathrm{Na}]^{+}$. HRMS (ESI) $m / z:[\mathrm{M}+\mathrm{Na}]^{+}$calcd for $\mathrm{C}_{27} \mathrm{H}_{37}{ }^{2} \mathrm{HNaO}_{7} \mathrm{Si}$ 526.2358; found 526.2358, $[\mathrm{M}+\mathrm{Na}]^{+}$calcd for $\mathrm{C}_{27} \mathrm{H}_{38} \mathrm{NaO}_{7} \mathrm{Si}$ 525.2285; found 525.2282, $[\mathrm{M}+\mathrm{Na}]^{+}$calcd for $\mathrm{C}_{25} \mathrm{H}_{34} \mathrm{NaO}_{5} \mathrm{Si}$ 465.2073; found 465.2089.

Method $F$. Following the general procedure, starting from substrate 20 (57 mg, $0.088 \mathrm{mmol}$ ), all the starting material was consumed after $2 \mathrm{~h}$. Chromatotron chromatography (hexanes-EtOAc, 8:2 to 7:3) gave $45(4.67 \mathrm{mg}, 0.011 \mathrm{mmol}, 12 \%)$ and an inseparable mixture of 50 and 51 (22.1 mg, $0.044 \mathrm{mmol}, 50 \%, 1.2: 1)$.

Method G. Following the general procedure, starting from substrate 20 (43 mg, $0.066 \mathrm{mmol}$ ), all the starting material was consumed after $3 \mathrm{~h}$. Chromatotron chromatography (hexanesEtOAc, 8:2 to $7: 3)$ gave $45(6.1 \mathrm{mg}, 0.014 \mathrm{mmol}, 21 \%)$ and an inseparable mixture of 50 and 51 (14.6 mg, $0.029 \mathrm{mmol}, 44 \%, 1.3: 1)$.

Radical Reactions of 21. Method A. Following the general procedure, starting from substrate $21(75.7 \mathrm{mg}, 0.09 \mathrm{mmol})$, all the starting material was consumed after $2 \mathrm{~h}$. Column chromatography on a silica gel without KF (hexanes to hexanes-EtOAc, 7:3) gave olefin $46(18.1 \mathrm{mg}, 0.041 \mathrm{mmol}, 45 \%)$ and alcohol 52 (13.8 mg, 0.020 mmol, 22\%).

Method C. Following the general procedure, starting from substrate $21(73 \mathrm{mg}, 0.087 \mathrm{mmol})$, after $2 \mathrm{~h}$ of reaction, a supplementary addition of TTMSS $(27 \mu \mathrm{L}, 0.087 \mathrm{mmol})$ was required. All the starting material was consumed after $4 \mathrm{~h}$. Column chromatography (hexanes-EtOAc, 8:2) gave $\mathbf{4 5}(9.6 \mathrm{mg}, 0.022 \mathrm{mmol}, 25 \%)$ and $\mathbf{5 2}$ (14.5 mg, $0.021 \mathrm{mmol}, 24 \%$ ).

Method D. Following the general procedure, starting from substrate 21 ( $74.3 \mathrm{mg}, 0.089 \mathrm{mmol})$, all the starting material was consumed after $2 \mathrm{~h}$. Column chromatography on a silica gel without $\mathrm{KF}$ (hexanes to hexanes-EtOAc, 1:1) gave C-(6-O-tert-butyldiphenylsilyl-4-O-diphenoxyphosphoryl-2,3-di-O-methyl- $\alpha$-D-[2-OMe- ${ }^{2} \mathrm{H}$ galactopyranosyl)methanol $\left(\left[\mathrm{OCH}_{2}{ }^{2} \mathrm{H}\right] 52\right)(14.2 \mathrm{mg}, 0.020 \mathrm{mmol}$, $\left.23 \%,{ }^{2} \mathrm{H} /{ }^{1} \mathrm{H} 1.3: 1\right)$ and olefin 46 (17.3 mg, $\left.0.039 \mathrm{mmol}, 44 \%\right)$, both as colorless oils. Compound $\left[\mathrm{OCH}_{2}{ }^{2} \mathrm{H}\right]$ 52: ${ }^{1} \mathrm{H}$ NMR $(500 \mathrm{MHz}$, $\mathrm{CDCl}_{3}$, only the deuterated product is described) $\delta_{\mathrm{H}} 7.65-7.05(\mathrm{~m}$, $20 \mathrm{H}, \mathrm{Ar}$ ), 5.05 (ddd, $\left.J=2.8,2.2 \mathrm{~Hz},{ }^{3} J_{\mathrm{PH}}=8.8 \mathrm{~Hz}, 1 \mathrm{H}, 4-\mathrm{H}\right), 4.09$ (ddd, $J=7.3,5.7,5.7 \mathrm{~Hz}, 1 \mathrm{H}, 1-\mathrm{H}), 3.80-3.70\left(\mathrm{~m}, 5 \mathrm{H}, 5-\mathrm{H}, 6-\mathrm{H}_{2}, 1\right.$ '$\left.\mathrm{H}_{2}\right), 3.57(\mathrm{dd}, J=8.5,5.7 \mathrm{~Hz}, 1 \mathrm{H}, 2-\mathrm{H}), 3.38(\mathrm{~m}, 1 \mathrm{H}, 3-\mathrm{H}), 3.372(\mathrm{t}$, $\left.J=1.6 \mathrm{~Hz}, 2 \mathrm{H}, \mathrm{OCH}_{2} \mathrm{D}\right), 3.35(\mathrm{~s}, 3 \mathrm{H}, \mathrm{OMe}), 1.03 \mathrm{ppm}\left(\mathrm{s}, 9 \mathrm{H},{ }^{t} \mathrm{Bu}\right)$, $1 \mathrm{H}$ from $\mathrm{OH}$ is missing. ${ }^{13} \mathrm{C}\left\{{ }^{1} \mathrm{H}\right\} \mathrm{NMR}\left(125.7 \mathrm{MHz}, \mathrm{CDCl}_{3}\right.$, only the deuterated product is described) $\delta_{\mathrm{C}} 150.8\left(\mathrm{~d},{ }^{2} J_{\mathrm{PC}}=7.4 \mathrm{~Hz}, \mathrm{C}, \mathrm{Ar}\right)$, $150.4\left(\mathrm{~d},{ }^{2} J_{\mathrm{PC}}=7.4 \mathrm{~Hz}, \mathrm{C}, \mathrm{Ar}\right), 135.6(2 \times \mathrm{CH}, \mathrm{Ar}), 135.5(2 \times \mathrm{CH}$, $\mathrm{Ar}), 133.3$ (C, Ar), 133.2 (C, Ar), 129.8 (CH, Ar), $129.72(\mathrm{CH}, \mathrm{Ar})$, $129.65(2 \times \mathrm{CH}, \mathrm{Ar}), 129.4(2 \times \mathrm{CH}, \mathrm{Ar}), 127.7(4 \times \mathrm{CH}, \mathrm{Ar}), 125.2$ $(\mathrm{CH}, \mathrm{Ar}), 125.1(\mathrm{CH}, \mathrm{Ar}), 120.34(\mathrm{CH}, \mathrm{Ar}), 120.30(\mathrm{CH}, \mathrm{Ar})$, $120.02(\mathrm{CH}, \mathrm{Ar}), 119.98(\mathrm{CH}, \mathrm{Ar}), 78.7(\mathrm{CH}, \mathrm{C}-3), 77.0(\mathrm{CH}, \mathrm{C}-2)$, $74.2\left(\mathrm{~d},{ }^{2} J_{\mathrm{PC}}=6.3 \mathrm{~Hz}, \mathrm{CH}, \mathrm{C}-4\right), 72.9\left(\mathrm{~d},{ }^{3} J_{\mathrm{PC}}=5.3 \mathrm{~Hz}, \mathrm{CH}, \mathrm{C}-5\right)$, $72.6(\mathrm{CH}, \mathrm{C}-1), 62.2\left(\mathrm{CH}_{2}, \mathrm{C}-6\right), 59.3\left(\mathrm{CH}_{2}, \mathrm{C}-1^{\prime}\right), 59.26\left(\mathrm{CH}_{2} \mathrm{D}\right)$, $57.7\left(\mathrm{CH}_{3}, \mathrm{OMe}\right), 26.7\left(3 \times \mathrm{CH}_{3}, \mathrm{DPS}\right), 19.1 \mathrm{ppm}$ (C, DPS). MS (ESI) $m / z(\%)=716(100)[\mathrm{M}+\mathrm{Na}]^{+}, 715(67)[\mathrm{M}+\mathrm{Na}]^{+}$. HRMS (ESI) $m / z:[\mathrm{M}+\mathrm{Na}]^{+}$calcd for $\mathrm{C}_{37} \mathrm{H}_{44}{ }^{2} \mathrm{HNaO}_{9} \mathrm{PSi} 716.2531$; found 716.2554, $M+\mathrm{Na}]^{+}$calcd for $\mathrm{C}_{37} \mathrm{H}_{45} \mathrm{NaO}_{9} \mathrm{PSi}$ 715.2468; found 715.2471 .

Method $E$. Following the general procedure, starting from substrate $21(81.8 \mathrm{mg}, 0.098 \mathrm{mmol})$, after $2 \mathrm{~h}$, a supplementary addition of $n$ $\mathrm{Bu}_{3} \mathrm{SnD}(26 \mu \mathrm{L}, 0.098 \mathrm{mmol})$ and $\mathrm{BF}_{3} \bullet \mathrm{Et}_{2} \mathrm{O}(3 \mu \mathrm{L}, 0.024 \mathrm{mmol})$ was required. All the starting material was consumed after $3 \mathrm{~h}$. Column chromatography on a silica gel without KF (hexanes to hexanesEtOAc, 1:1) gave $\left[4-{ }^{2} \mathrm{H}\right] 45\left(17.8 \mathrm{mg}, 0.040 \mathrm{mmol}, 41 \%,{ }^{2} \mathrm{H} /{ }^{1} \mathrm{H} 2.8: 1\right.$,
$4 R / 4 S$ 1:1.2) and $\left[\mathrm{OCH}_{2}{ }^{2} \mathrm{H}\right] \mathbf{5 2}\left(8.6 \mathrm{mg}, 0.012 \mathrm{mmol}, 13 \%,{ }^{2} \mathrm{H} /{ }^{1} \mathrm{H}\right.$ $1.1: 1)$.

Method F. Following the general procedure, starting from substrate $21(36.9 \mathrm{mg}, 0.044 \mathrm{mmol})$, all the starting material was consumed after $3 \mathrm{~h}$. Chromatotron chromatography (hexanes-EtOAc, 8:2 to 4:6) gave $\mathbf{5 2}(15.8 \mathrm{mg}, 0.023 \mathrm{mmol}, 52 \%)$.

Method $G$. Following the general procedure, starting from substrate 21 ( $40.5 \mathrm{mg}, 0.048 \mathrm{mmol}$ ), all the starting material was consumed after $3 \mathrm{~h}$. Chromatotron chromatography (hexanesEtOAc, 8:2 to $4: 6)$ gave $\mathbf{5 2}(13.5 \mathrm{mg}, 0.019 \mathrm{mmol}, 40 \%)$.

Radical Reactions of 22. Method A. Following the general procedure, starting from substrate $22(106.8 \mathrm{mg}, 0.18 \mathrm{mmol})$, after 2 h, a supplementary addition of $n-\mathrm{Bu}_{3} \mathrm{SnH}(49 \mu \mathrm{L}, 0.18 \mathrm{mmol})$ was required. All the starting material was consumed after $5 \mathrm{~h}$. Column chromatography (hexanes to hexanes-EtOAc, 1:1) gave 2,7-anhydro1,3-dideoxy-4,5-di- $O$-methyl- $\beta$-L-ribo-hept-2-ulopyranose (61) (18.9 $\mathrm{mg}, 0.10 \mathrm{mmol}, 56 \%)$ as a colorless oil. $[\alpha]_{\mathrm{D}}=+0.02(c=0.34$, $\left.\mathrm{CHCl}_{3}\right) \cdot{ }^{1} \mathrm{H}$ NMR $\left(500 \mathrm{MHz}, \mathrm{CDCl}_{3}\right.$, simulated ring coupling constants using DAISY) $\delta_{\mathrm{H}} 4.75$ (ddd, $\left.J=5.8,2.8,0.9 \mathrm{~Hz}, 1 \mathrm{H}, 1-\mathrm{H}\right)$, $3.82\left(\mathrm{dd}, J=7.7,5.8 \mathrm{~Hz}, 1 \mathrm{H}, 1^{\prime}-\mathrm{H}_{\mathrm{b}}\right), 3.67\left(\mathrm{dd}, J=7.7,0.9 \mathrm{~Hz}, 1 \mathrm{H}, 1^{\prime}-\right.$ $\mathrm{H}_{\mathrm{a}}$ ), 3.626 (ddd, $\left.J=11.1,6.0,4.1 \mathrm{~Hz}, 1 \mathrm{H}, 3-\mathrm{H}\right), 3.46(\mathrm{dd}, J=4.1,2.8$ $\mathrm{Hz}, 1 \mathrm{H}, 2-\mathrm{H}), 3.56$ (s, 3H, OMe), 3.38 (s, 3H, OMe), 2.121 (dd, $J=$ $\left.12.5,6.0 \mathrm{~Hz}, 1 \mathrm{H}, 4-\mathrm{H}_{\mathrm{b}}\right), 1.816\left(\mathrm{dd}, J=12.5,11.1 \mathrm{~Hz}, 1 \mathrm{H}, 4-\mathrm{H}_{\mathrm{a}}\right), 1.51$ ppm (s, 3H, 6- $\left.\mathrm{H}_{3}\right) .{ }^{13} \mathrm{C}\left\{{ }^{1} \mathrm{H}\right\}$ NMR (125.7 MHz, $\left.\mathrm{CDCl}_{3}\right) \delta_{\mathrm{C}} 106.96$ (C, C-5), 75.4 ( $\mathrm{CH}, \mathrm{C}-2), 74.1(\mathrm{CH}, \mathrm{C}-1), 73.78$ (CH, C-3), 65.8 $\left(\mathrm{CH}_{2}, \mathrm{C}-1^{\prime}\right), 57.9\left(\mathrm{CH}_{3}, \mathrm{OMe}\right), 56.2\left(\mathrm{CH}_{3}, \mathrm{OMe}\right), 38.69\left(\mathrm{CH}_{2}, \mathrm{C}-4\right)$, $23.8 \mathrm{ppm}\left(\mathrm{CH}_{3}, \mathrm{C}-6\right)$. IR $\left(\mathrm{CHCl}_{3}\right): \nu=3015,2934,1389,1198 \mathrm{~cm}^{-1}$. MS (ESI) $m / z(\%)=211(100)[\mathrm{M}+\mathrm{Na}]^{+}$. HRMS (ESI) $\mathrm{m} / z:[\mathrm{M}+$ $\mathrm{Na}]^{+}$calcd for $\mathrm{C}_{9} \mathrm{H}_{16} \mathrm{NaO}_{4}$ 211.0946; found 211.0948. Anal. calcd for $\mathrm{C}_{9} \mathrm{H}_{16} \mathrm{O}_{4}$ : C, 57.43; $\mathrm{H}, 8.57$. Found: C, 57.63; H, 8.63.

Method C. Following the general procedure, starting from substrate $22(58.8 \mathrm{mg}, 0.10 \mathrm{mmol})$, after $2 \mathrm{~h}$ of reaction, a supplementary addition of TTMSS $(31 \mu \mathrm{L}, 0.10 \mathrm{mmol})$ was required. All the starting material was consumed after $4 \mathrm{~h}$. Column chromatography (hexanesEtOAc, 9:1 to $6: 4)$ gave 61 (9 mg, $0.048 \mathrm{mmol}, 48 \%)$.

Method $D$. Following the general procedure, starting from substrate $22(61.5 \mathrm{mg}, 0.105 \mathrm{mmol})$, after $2 \mathrm{~h}$, a supplementary addition of $n-\mathrm{Bu}_{3} \mathrm{SnD}(29 \mu \mathrm{L}, 0.105 \mathrm{mmol})$ was required. All the starting material was consumed after $5 \mathrm{~h}$. Column chromatography (hexanes to hexanes-EtOAc, 1:1) gave 2,7-anhydro-1,3-dideoxy-4,5di-O-methyl- $\beta$-L- $\left[4-{ }^{2} \mathrm{H}\right]$ ribo-hept-2-ulopyranose $\left(\left[4-{ }^{2} \mathrm{H}\right] 61\right)(8.7 \mathrm{mg}$, $0.046 \mathrm{mmol}, 44 \%,{ }^{2} \mathrm{H} /{ }^{1} \mathrm{H}$ 1.8:1, $4 R / 4 S$ 1:1.3) as a colorless oil: ${ }^{1} \mathrm{H}$ NMR $\left(500 \mathrm{MHz}, \mathrm{CDCl}_{3}\right.$, only deuterated isomers are described) $\delta_{\mathrm{H}}$ 4.75 (ddd, $J=5.7,2.8,0.0 \mathrm{~Hz}, 1 \mathrm{H}, 1-\mathrm{H}), 3.82(\mathrm{dd}, J=7.6,5.7 \mathrm{~Hz}$, $\left.1 \mathrm{H}, 1^{\prime}-\mathrm{H}_{\mathrm{b}}\right), 3.67$ (dd, $\left.J=7.9,0.0 \mathrm{~Hz}, 1 \mathrm{H}, 1^{\prime}-\mathrm{H}_{\mathrm{a}}\right), 3.63-3.60(\mathrm{~m}, 1 \mathrm{H}$, 3-H), 3.55 (s, 3H, OMe), 3.47-3.44 (m, $1 \mathrm{H}, 2-\mathrm{H}), 3.38(\mathrm{~s}, 3 \mathrm{H}$, $\mathrm{OMe}$ ), 2.101 (d, $J=5.7 \mathrm{~Hz}, 1 \mathrm{H}, 4-\mathrm{H}, 4 S$ isomer), 1.793 (d, $J=11.1$ $\mathrm{Hz}, 1 \mathrm{H}, 4-\mathrm{H}, 4 \mathrm{R}$ isomer), $1.51 \mathrm{ppm}\left(\mathrm{s}, 3 \mathrm{H}, 6-\mathrm{H}_{3}\right) .{ }^{13} \mathrm{C}\left\{{ }^{1} \mathrm{H}\right\}$ NMR $\left(125.7 \mathrm{MHz}, \mathrm{CDCl}_{3}\right.$, only deuterated isomers are described) $\delta_{\mathrm{C}}$ 106.92 (C, C-5), 75.4 (CH, C-2), 74.1 (CH, C-1), 73.69 (CH, C-3), $65.8\left(\mathrm{CH}_{2}, \mathrm{C}-1^{\prime}\right), 57.8\left(\mathrm{CH}_{3}, \mathrm{OMe}\right), 56.2\left(\mathrm{CH}_{3}, \mathrm{OMe}\right), 38.52\left(\mathrm{t}, J_{\mathrm{CD}}\right.$ $=20.1 \mathrm{~Hz}, \mathrm{CHD}, \mathrm{C}-4), 23.8 \mathrm{ppm}\left(\mathrm{CH}_{3}, \mathrm{C}-6\right)$. MS (ESI) $\mathrm{m} / z(\%)=$ $212(100)[\mathrm{M}+\mathrm{Na}]^{+}, 211(100)[\mathrm{M}+\mathrm{Na}]^{+}$. HRMS (ESI) $\mathrm{m} / z:[\mathrm{M}$ $+\mathrm{Na}]^{+}$calcd for $\mathrm{C}_{9} \mathrm{H}_{15}{ }^{2} \mathrm{HNaO}_{4}$ 212.1009; found 212.1005, $[\mathrm{M}+$ $\mathrm{Na}]^{+}$calcd for $\mathrm{C}_{9} \mathrm{H}_{16} \mathrm{NaO}_{4}$ 211.0946; found 211.0948.

Method E. Following the general procedure, starting from substrate $22(77 \mathrm{mg}, 0.13 \mathrm{mmol})$, after $2 \mathrm{~h}$, a supplementary addition of $n$ $\mathrm{Bu}_{3} \mathrm{SnD}(35 \mu \mathrm{L}, 0.13 \mathrm{mmol})$ and $\mathrm{BF}_{3} \cdot \mathrm{Et}_{2} \mathrm{O}(3.3 \mu \mathrm{L}, 0.026 \mathrm{mmol})$ was required. All the starting material was consumed after $7 \mathrm{~h}$. Column chromatography (hexanes to hexanes-EtOAc, $6: 4$ ) gave $\left[4{ }^{2} \mathrm{H}\right] 61$ (16.2 mg, $0.086 \mathrm{mmol}, 66 \%,{ }^{2} \mathrm{H} /{ }^{1} \mathrm{H}$ 1.3:1, $4 R / 4 S$ 1:1.3).

Method $F$. Following the general procedure, starting from substrate $22(28.9 \mathrm{mg}, 0.05 \mathrm{mmol})$, all the starting material was consumed after $1.5 \mathrm{~h}$. Chromatotron chromatography (hexanes-EtOAc, 4:6 to 3:7) gave 61 (5.2 mg, $0.028 \mathrm{mmol}, 55 \%)$.

Method G. Following the general procedure, starting from substrate $22(29.1 \mathrm{mg}, 0.050 \mathrm{mmol})$, all the starting material was consumed after $3 \mathrm{~h}$. Chromatotron chromatography (hexanesEtOAc, 4:6 to $3: 7)$ gave $61(5.7 \mathrm{mg}, 0.030 \mathrm{mmol}, 61 \%)$. 
Radical Reactions of 23. Method A. Following the general procedure, starting from substrate $23(88 \mathrm{mg}, 0.22 \mathrm{mmol})$, after $2 \mathrm{~h}$, a supplementary addition of $n-\mathrm{Bu}_{3} \mathrm{SnH}(60 \mu \mathrm{L}, 0.22 \mathrm{mmol})$ was required. All the starting material was consumed after $6 \mathrm{~h}$. Column chromatography (hexanes to hexanes-EtOAc, 4:6) gave $C$ - $(4,6-$ dideoxy-2,3-di- $O$-methyl- $\beta$-D-threo-hex-4-enopyranosyl)methanol (56) $(8.3 \mathrm{mg}, 0.044 \mathrm{mmol}, 20 \%)$ and $\mathrm{C}$-(4-O-acetyl-2,3-6-deoxy-di-Omethyl- $\beta$-D-altropyranosyl)methanol (57) $(4.4 \mathrm{mg}, 0.018 \mathrm{mmol}, 8 \%)$ as colorless oils, and $58(7.8 \mathrm{mg}, 0.031 \mathrm{mmol}, 14 \%)$. Compound 56: $[\alpha]_{\mathrm{D}}=-119.1\left(c=0.45, \mathrm{CHCl}_{3}\right) .{ }^{1} \mathrm{H} \mathrm{NMR}\left(500 \mathrm{MHz}, \mathrm{CDCl}_{3}\right.$, simulated ring coupling constants using DAISY) $\delta_{\mathrm{H}} 4.78$ (br ddd, $J=$ $\left.5.3 \mathrm{~Hz},{ }^{4} J=2.0,0.9 \mathrm{~Hz}, 1 \mathrm{H}, 4-\mathrm{H}\right), 4.01(\mathrm{dd}, J=11.4,6.4 \mathrm{~Hz}, 1 \mathrm{H}, 1$ ' $\mathrm{H}_{\mathrm{b}}$ ), 3.94 (ddd, $\left.J=6.4,4.1,1.5 \mathrm{~Hz}, 1 \mathrm{H}, 1-\mathrm{H}\right), 3.86$ (br dd, $J=11.4$, $4.1 \mathrm{~Hz}, 1 \mathrm{H}, \mathrm{l}^{\prime}-\mathrm{H}_{\mathrm{a}}$ ), 3.60 (ddd, $\left.J=5.3,2.0 \mathrm{~Hz},{ }^{5} J=1.0 \mathrm{~Hz}, 1 \mathrm{H}, 3-\mathrm{H}\right)$, $3.45(\mathrm{~s}, 3 \mathrm{H}, \mathrm{OMe}), 3.40\left(\mathrm{ddd}, J=2.0,1.5 \mathrm{~Hz},{ }^{4} J=2.0 \mathrm{~Hz}, 1 \mathrm{H}, 2-\mathrm{H}\right)$, 3.39 (s, 3H, OMe), 2.25 (br s, $1 \mathrm{H}, \mathrm{OH}), 1.86 \mathrm{ppm}\left(\mathrm{dd},{ }^{5} J=1.0 \mathrm{~Hz},{ }^{4} J\right.$ $\left.=0.9 \mathrm{~Hz}, 3 \mathrm{H}, 6-\mathrm{H}_{3}\right) .{ }^{13} \mathrm{C}\left\{{ }^{1} \mathrm{H}\right\} \mathrm{NMR}\left(125.7 \mathrm{MHz}, \mathrm{CDCl}_{3}\right) \delta_{\mathrm{C}} 154.7$ (C, C-5), $93.7(\mathrm{CH}, \mathrm{C}-4), 75.7(\mathrm{CH}, \mathrm{C}-2), 73.8(\mathrm{CH}, \mathrm{C}-1), 69.7$ $(\mathrm{CH}, \mathrm{C}-3), 63.0\left(\mathrm{CH}_{2}, \mathrm{C}-1^{\prime}\right), 58.1\left(\mathrm{CH}_{3}, \mathrm{OMe}\right), 55.4\left(\mathrm{CH}_{3}, \mathrm{OMe}\right)$, $20.0 \mathrm{ppm}\left(\mathrm{CH}_{3}, \mathrm{C}-6\right)$. IR $\left(\mathrm{CHCl}_{3}\right): \nu=3691,3602,3013,2933$, $1672,1226 \mathrm{~cm}^{-1}$. MS (ESI) $m / z(\%)=211(100)[\mathrm{M}+\mathrm{Na}]^{+}$. HRMS (ESI) $m / z:[\mathrm{M}+\mathrm{Na}]^{+}$calcd for $\mathrm{C}_{9} \mathrm{H}_{16} \mathrm{NaO}_{4}$ 211.0946; found 211.0942. Anal. calcd for $\mathrm{C}_{9} \mathrm{H}_{16} \mathrm{O}_{4}$ : C, 57.43; $\mathrm{H}, 8.57$. Found: $\mathrm{C}$ 57.10; $\mathrm{H}, 8.27$. Compound 57: $[\alpha]_{\mathrm{D}}=+50.3\left(c=0.35, \mathrm{CHCl}_{3}\right) .{ }^{1} \mathrm{H}$ NMR $\left(500 \mathrm{MHz}, \mathrm{CDCl}_{3}\right) \delta_{\mathrm{H}} 4.799(\mathrm{dd}, J=10.1,3.2 \mathrm{~Hz}, 1 \mathrm{H}, 4-\mathrm{H})$, 3.92-3.84 (m, 4H, 1-H, 2-H, 5-H, 1'- $\left.\mathrm{H}_{\mathrm{b}}\right), 3.67\left(\mathrm{~m}, 1 \mathrm{H}, \mathrm{l}^{\prime}-\mathrm{H}_{\mathrm{a}}\right), 3.464$ (s, 3H, OMe), $3.462(\mathrm{~s}, 3 \mathrm{H}, \mathrm{OMe}), 3.40(\mathrm{dd}, J=3.8,1.0 \mathrm{~Hz}, 1 \mathrm{H}, 3-$ $\mathrm{H}), 2.12(\mathrm{~s}, 3 \mathrm{H}, \mathrm{OAc}), 1.201 \mathrm{ppm}\left(\mathrm{d}, J=6.4 \mathrm{~Hz}, 3 \mathrm{H}, 6-\mathrm{H}_{3}\right), 1 \mathrm{H}$ from $\mathrm{OH}$ is missing. ${ }^{13} \mathrm{C}\left\{{ }^{1} \mathrm{H}\right\}$ NMR $\left(125.7 \mathrm{MHz}, \mathrm{CDCl}_{3}\right) \delta_{\mathrm{C}} 170.2(\mathrm{C}$, OAc), 77.7 (CH, C-3), $74.7(\mathrm{CH}, \mathrm{C}-2), 74.2(\mathrm{CH}, \mathrm{C}-1), 73.40(\mathrm{CH}$, C-4), 69.68 (CH, C-5), $62.7\left(\mathrm{CH}_{2}, \mathrm{C}-1\right.$ '), $59.3\left(\mathrm{CH}_{3}, \mathrm{OMe}\right), 58.4$ $\left(\mathrm{CH}_{3}, \mathrm{OMe}\right), 21.1\left(\mathrm{CH}_{3}, \mathrm{OAc}\right), 17.79 \mathrm{ppm}\left(\mathrm{CH}_{3}, \mathrm{C}-6\right)$. IR $\left(\mathrm{CHCl}_{3}\right)$ : $\nu=3690,3603,3018,2935,1734,1220 \mathrm{~cm}^{-1}$. MS (ESI) $\mathrm{m} / z(\%)=$ $271(100)[\mathrm{M}+\mathrm{Na}]^{+}$. HRMS (ESI) $\mathrm{m} / z:[\mathrm{M}+\mathrm{Na}]^{+}$calcd for $\mathrm{C}_{11} \mathrm{H}_{20} \mathrm{NaO}_{6}$ 271.1158; found 271.1167. Anal. calcd for $\mathrm{C}_{11} \mathrm{H}_{20} \mathrm{O}_{6}$ : $\mathrm{C}$, 53.21; H, 8.12. Found: C, 52.92; H, 7.97.

Method $D$. Following the general procedure, starting from substrate 23 ( $62 \mathrm{mg}, 0.16 \mathrm{mmol})$, after $2 \mathrm{~h}$, a supplementary addition of $n-\mathrm{Bu}_{3} \mathrm{SnD}(43 \mu \mathrm{L}, 0.16 \mathrm{mmol})$ was required. All the starting material was consumed after $4 \mathrm{~h}$. Column chromatography (hexanes to hexanes-EtOAc, 3:7) gave 56 (9.4 $\mathrm{mg}, 0.05 \mathrm{mmol}, 31 \%), \mathrm{C}-(4-\mathrm{O}$ acetyl-2,3-6-deoxy-di- $O$-methyl- $\beta$-D- $\left(5-{ }^{2} \mathrm{H}\right)$ altropyranosyl $)$ methanol $\left[\left(5{ }^{2} \mathrm{H}\right) 57\right](7.6 \mathrm{mg}, 0.031 \mathrm{mmol}, 19 \%)$, and $\mathrm{C}$-(4-O-acetyl-2,3-di-Omethyl- $\alpha$-D- $\left[5-{ }^{2} \mathrm{H}\right]$ fucopyranosyl)methanol $\left.\left(5-{ }^{2} \mathrm{H}\right] 58\right)(8.4 \mathrm{mg}, 0.034$ mmol, $\left.21 \%,{ }^{2} \mathrm{H} /{ }^{1} \mathrm{H} 2.4: 1\right)$ as colorless oils. Compound $\left(5-{ }^{2} \mathrm{H}\right) 57:{ }^{1} \mathrm{H}$ NMR $\left(500 \mathrm{MHz}, \mathrm{CDCl}_{3}\right) \delta_{\mathrm{H}} 4.796(\mathrm{~d}, J=2.9 \mathrm{~Hz}, 1 \mathrm{H}, 4-\mathrm{H}), 3.92-$ $3.84\left(\mathrm{~m}, 3 \mathrm{H}, 1-\mathrm{H}, 2-\mathrm{H}, 1^{\prime}-\mathrm{H}_{\mathrm{b}}\right), 3.67\left(\mathrm{~m}, 1 \mathrm{H}, 1^{\prime}-\mathrm{H}_{\mathrm{a}}\right), 3.463(\mathrm{~s}, 3 \mathrm{H}$, $\mathrm{OMe}), 3.461(\mathrm{~s}, 3 \mathrm{H}, \mathrm{OMe}), 3.40(\mathrm{dd}, J=3.5,1.0 \mathrm{~Hz}, 1 \mathrm{H}, 3-\mathrm{H}), 2.12$ $(\mathrm{s}, 3 \mathrm{H}, \mathrm{OAc}), 1.192 \mathrm{ppm}\left(\mathrm{s}, 3 \mathrm{H}, 6-\mathrm{H}_{3}\right), 1 \mathrm{H}$ from $\mathrm{OH}$ is missing. ${ }^{13} \mathrm{C}\left\{{ }^{1} \mathrm{H}\right\}$ NMR $\left(125.7 \mathrm{MHz}, \mathrm{CDCl}_{3}\right) \delta_{\mathrm{C}} 170.2(\mathrm{C}, \mathrm{OAc}), 77.7(\mathrm{CH}$, C-3), $74.7(\mathrm{CH}, \mathrm{C}-2), 74.2(\mathrm{CH}, \mathrm{C}-1), 73.33(\mathrm{CH}, \mathrm{C}-4), 69.26(\mathrm{t}$, $\left.J_{\mathrm{CD}}=21.2 \mathrm{~Hz}, \mathrm{C}, \mathrm{C}-5\right), 62.7\left(\mathrm{CH}_{2}, \mathrm{C}-1^{\prime}\right), 59.3\left(\mathrm{CH}_{3}, \mathrm{OMe}\right), 58.4$ $\left(\mathrm{CH}_{3}, \mathrm{OMe}\right), 21.1\left(\mathrm{CH}_{3}, \mathrm{OAc}\right), 17.66 \mathrm{ppm}\left(\mathrm{CH}_{3}, \mathrm{C}-6\right) . \mathrm{MS}(\mathrm{ESI})$ $m / z(\%)=272(100)[\mathrm{M}+\mathrm{Na}]^{+}$. HRMS (ESI) $m / z:[\mathrm{M}+\mathrm{Na}]^{+}$ calcd for $\mathrm{C}_{11} \mathrm{H}_{19}{ }^{2} \mathrm{HNaO}_{6}$ 272.1220; found 272.1219. Compound [5- $\left.{ }^{2} \mathrm{H}\right] 58:{ }^{1} \mathrm{H}$ NMR $\left(500 \mathrm{MHz}, \mathrm{CDCl}_{3}\right.$, only the deuterated product is described) $\delta_{\mathrm{H}} 5.323(\mathrm{~d}, J=3.2 \mathrm{~Hz}, 1 \mathrm{H}, 4-\mathrm{H}), 4.23(\mathrm{~m}, 1 \mathrm{H}, 1-\mathrm{H})$, $3.91-3.83\left(\mathrm{~m}, 2 \mathrm{H}, 1^{\prime}-\mathrm{H}_{2}\right), 3.70(\mathrm{dd}, J=9.1,6.0 \mathrm{~Hz}, 1 \mathrm{H}, 2-\mathrm{H}), 3.50$ (dd, $J=9.1,3.5 \mathrm{~Hz}, 1 \mathrm{H}, 3-\mathrm{H}), 3.50(\mathrm{~s}, 3 \mathrm{H}, \mathrm{OMe}), 3.42(\mathrm{~s}, 3 \mathrm{H}, \mathrm{OMe})$, $2.17(\mathrm{~s}, 3 \mathrm{H}, \mathrm{OAc}), 1.169 \mathrm{ppm}\left(\mathrm{s}, 3 \mathrm{H}, 6-\mathrm{H}_{3}\right), 1 \mathrm{H}$ from $\mathrm{OH}$ is missing. ${ }^{13} \mathrm{C}\left\{{ }^{1} \mathrm{H}\right\}$ NMR $\left(125.7 \mathrm{MHz}, \mathrm{CDCl}_{3}\right.$, only the deuterated product is described) $\delta_{\mathrm{C}} 170.7(\mathrm{C}, \mathrm{OAc}) .78 .8(\mathrm{CH}, \mathrm{C}-3), 77.1(\mathrm{CH}, \mathrm{C}-2), 73.4$ (CH, C-1), $69.46(\mathrm{CH}, \mathrm{C}-4), 59.7\left(\mathrm{CH}_{2}, \mathrm{C}-1^{\prime}\right), 59.4\left(\mathrm{CH}_{3}, \mathrm{OMe}\right)$, $57.5\left(\mathrm{CH}_{3}, \mathrm{OMe}\right), 20.8\left(\mathrm{CH}_{3}, \mathrm{OAc}\right), 16.37 \mathrm{ppm}\left(\mathrm{CH}_{3}, \mathrm{C}-6\right)$. MS (ESI) $m / z(\%)=272(71)[\mathrm{M}+\mathrm{Na}]^{+}, 271(28)[\mathrm{M}+\mathrm{Na}]^{+}$. HRMS (ESI) $m / z:[\mathrm{M}+\mathrm{Na}]^{+}$calcd for $\mathrm{C}_{11} \mathrm{H}_{19}{ }^{2} \mathrm{HNaO}_{6}$ 272.1220; found 272.1222, $[\mathrm{M}+\mathrm{Na}]^{+}$calcd for $\mathrm{C}_{11} \mathrm{H}_{20} \mathrm{NaO}_{6}$ 271.1158; found 271.1164 .

Method E. Following the general procedure, starting from substrate $23(126 \mathrm{mg}, 0.32 \mathrm{mmol})$, after $2 \mathrm{~h}$, a supplementary addition of $n$ -
$\mathrm{Bu}_{3} \mathrm{SnD}(87 \mu \mathrm{L}, 0.32 \mathrm{mmol})$ and $\mathrm{BF}_{3} \bullet \mathrm{Et}_{2} \mathrm{O}(8 \mu \mathrm{L}, 0.064 \mathrm{mmol})$ was required. All the starting material was consumed after $4 \mathrm{~h}$. Column chromatography (hexanes to hexanes-EtOAc, 3:7) gave $\left(5-{ }^{2} \mathrm{H}\right) 57$ (9.5 mg, $0.038 \mathrm{mmol}, 12 \%),\left[5{ }^{2} \mathrm{H}\right] 58$ (11.4 mg, $0.046 \mathrm{mmol}, 14 \%$, ${ }^{2} \mathrm{H} /{ }^{1} \mathrm{H}$ 1:2), the unstable and volatile 2,7 -anhydro-1,3-dideoxy-4,5-di$O$-methyl- $\beta$-L- $\left[3-{ }^{2} \mathrm{H}\right] x y l o$-hept-2-ulopyranose $\left(\left[4-{ }^{2} \mathrm{H}\right] 55\right)(13 \mathrm{mg}$, $0.069 \mathrm{mmol}, \quad 21 \%,{ }^{2} \mathrm{H} /{ }^{1} \mathrm{H}$ 5.4:1), and 3-O-acetyl-2,6-anhydro-1deoxy-4,5-di-O-methyl-D- $\left(6{ }^{2} \mathrm{H}\right)$ galactitol $\left[\left(1{ }^{2} \mathrm{H}\right) 60\right](2 \mathrm{mg}, 0.009$ mmol, $3 \%)$ as colorless oils. Compound $\left[4-{ }^{2} \mathrm{H}\right] 55$ : ${ }^{1} \mathrm{H}$ NMR (500 $\left.\mathrm{MHz}, \mathrm{CDCl}_{3}\right) \delta_{\mathrm{H}} 4.51$ (ddd, $\left.J=4.7,4.7,0.0 \mathrm{~Hz}, 1 \mathrm{H}, 1-\mathrm{H}\right), 3.99$ (dd, $J$ $\left.=7.6,0.0 \mathrm{~Hz}, 1 \mathrm{H}, 1^{\prime}-\mathrm{H}_{\mathrm{b}}\right), 3.74\left(\mathrm{dd}, J=7.6,5.4 \mathrm{~Hz}, 1 \mathrm{H}, 1^{\prime}-\mathrm{H}_{\mathrm{a}}\right), 3.53-$ $3.48(\mathrm{~m}, 1 \mathrm{H}, 3-\mathrm{H}), 3.48$ (s, 3H, OMe), 3.40 (s, 3H, OMe), 3.35 (dd, $J$ $=8.2,4.1 \mathrm{~Hz}, 1 \mathrm{H}, 2-\mathrm{H}), 2.33\left(\mathrm{dd}, J=13.2,6.7 \mathrm{~Hz}, 1 \mathrm{H}, 4-\mathrm{H}_{\mathrm{b}}\right), 2.32$ $(\mathrm{d}, J=6.6 \mathrm{~Hz}, 1 \mathrm{H}, 4-\mathrm{HD}), 1.44\left(\mathrm{~m}, 1 \mathrm{H}, 4-\mathrm{H}_{\mathrm{a}}\right), 1.49 \mathrm{ppm}(\mathrm{s}, 3 \mathrm{H}, 6-$ $\left.\mathrm{H}_{3}\right) .{ }^{13} \mathrm{C}\left\{{ }^{1} \mathrm{H}\right\}$ NMR $\left(125.7 \mathrm{MHz}, \mathrm{CDCl}_{3}\right) \delta_{\mathrm{C}} 107.0(\mathrm{C}, \mathrm{C}-5), 80.7$ (CH, C-2), 77.3 (CH, C-3), $73.5(\mathrm{CH}, \mathrm{C}-1), 65.8\left(\mathrm{CH}_{2}, \mathrm{C}-1\right.$ '), 58.3 $\left(\mathrm{CH}_{3}, \mathrm{OMe}\right), 57.1\left(\mathrm{CH}_{3}, \mathrm{OMe}\right), 36.5\left(\mathrm{CH}_{2}, \mathrm{C}-4\right.$ reduced product $)$, $23.5 \mathrm{ppm}\left(\mathrm{CH}_{3}, \mathrm{C}-6\right)$, expected triplet for C-4 was imperceptible for the deuterated product. IR $\left(\mathrm{CHCl}_{3}\right): \nu=3022,2929,1226 \mathrm{~cm}^{-1}$. MS (ESI) $m / z(\%)=212(100)[\mathrm{M}+\mathrm{Na}]^{+}, 211(18)[\mathrm{M}+\mathrm{Na}]^{+}$. HRMS (ESI) $m / z:[\mathrm{M}+\mathrm{Na}]^{+}$calcd for $\mathrm{C}_{9} \mathrm{H}_{15}{ }^{2} \mathrm{HNaO}_{4}$ 212.1009; found 212.1015, $[\mathrm{M}+\mathrm{Na}]^{+}$calcd for $\mathrm{C}_{9} \mathrm{H}_{16} \mathrm{NaO}_{4}$ 211.0946; found 211.0950. Compound $\left(1-^{2} \mathrm{H}\right) 60:[\alpha]_{\mathrm{D}}=-11.1\left(c=0.45, \mathrm{CHCl}_{3}\right)$. ${ }^{1} \mathrm{H}$ NMR $\left(500 \mathrm{MHz}, \mathrm{CDCl}_{3}\right.$, simulated ring coupling constants using DAISY) $\delta_{\mathrm{H}} 5.34(\mathrm{dd}, J=3.4,1.31 \mathrm{~Hz}, 1 \mathrm{H}, 4-\mathrm{H}), 4.10(\mathrm{br} \mathrm{d}, J=4.4$ $\mathrm{Hz}, 1 \mathrm{H}, 1-\mathrm{H}$ ), 3.58 (dddd, $J=6.6,6.6,6.6,1.1 \mathrm{~Hz}, 1 \mathrm{H}, 5-\mathrm{H}), 3.52(\mathrm{~m}$, $1 \mathrm{H}, 2-\mathrm{H}), 3.50$ (s, 3H, OMe), $3.43(\mathrm{~s}, 3 \mathrm{H}, \mathrm{OMe}), 3.24(\mathrm{dd}, J=9.3$, $3.4 \mathrm{~Hz}, 1 \mathrm{H}, 3-\mathrm{H}), 2.18(\mathrm{~s}, 3 \mathrm{H}, \mathrm{OAc}), 1.17 \mathrm{ppm}(\mathrm{d}, J=6.6 \mathrm{~Hz}, 3 \mathrm{H}, 6-$ $\left.\mathrm{H}_{3}\right) .{ }^{13} \mathrm{C}\left\{{ }^{1} \mathrm{H}\right\}$ NMR (125.7 MHz, $\left.\mathrm{CDCl}_{3}\right) \delta_{\mathrm{C}} 170.8$ (C, OAc), 82.8 (CH, C-3), $75.4(\mathrm{CH}, \mathrm{C}-2), 73.5$ (CH, C-5), 69.4 (CH, C-4), 59.0 $\left(\mathrm{CH}_{3}, \mathrm{OMe}\right), 57.4\left(\mathrm{CH}_{3}, \mathrm{OMe}\right), 20.8\left(\mathrm{CH}_{3}, \mathrm{OAc}\right), 16.8 \mathrm{ppm}\left(\mathrm{CH}_{3}\right.$, C-6), C-1 was imperceptible. IR $\left(\mathrm{CHCl}_{3}\right): \nu=3016,2932,1226$ $\mathrm{cm}^{-1}$. MS (ESI) $m / z(\%)=242(100)[\mathrm{M}+\mathrm{Na}]^{+}$. HRMS (ESI) $m / z$ : $[\mathrm{M}+\mathrm{Na}]^{+}$calcd for $\mathrm{C}_{10} \mathrm{H}_{17}{ }^{2} \mathrm{HNaO}_{5}$ 242.1115; found 242.1111.

Method $F$. Following the general procedure, starting from substrate $23(13.9 \mathrm{mg}, 0.035 \mathrm{mmol}$ ), all the starting material was consumed after $3 \mathrm{~h}$. Chromatotron chromatography (hexanes-EtOAc, 4:6 to $0: 1)$ gave $57(1 \mathrm{mg}, 0.004 \mathrm{mmol}, 11 \%), 58(2.2 \mathrm{mg}, 0.09 \mathrm{mmol}$, $25 \%)$, and product $55(0.7 \mathrm{mg}, 0.004 \mathrm{mmol}, 11 \%)$.

Method G. Following the general procedure, starting from substrate $23(13.7 \mathrm{mg}, 0.035 \mathrm{mmol})$, all the starting material was consumed after $3 \mathrm{~h}$. Chromatotron chromatography (hexanesEtOAc, 4:6 to $0: 1)$ gave $57(1.3 \mathrm{mg}, 0.030 \mathrm{mmol}, 15 \%), 58(1.3$ $\mathrm{mg}, 0.005 \mathrm{mmol}, 15 \%)$, and product $55(1.5 \mathrm{mg}, 0.008 \mathrm{mmol}, 23 \%)$.

Radical Reactions of 24. Method A. Following the general procedure, starting from substrate $24(80.5 \mathrm{mg}, 0.18 \mathrm{mmol})$, after $2 \mathrm{~h}$, a supplementary addition of $n-\mathrm{Bu}_{3} \mathrm{SnH}(50 \mu \mathrm{L}, 0.18 \mathrm{mmol})$ was required. All the starting material was consumed after $3 \mathrm{~h}$. Column chromatography (hexanes to hexanes-EtOAc, 4:6) gave 56 (23.6 mg, $0.126 \mathrm{mmol}, 70 \%)$ and $59(10.2 \mathrm{mg}, 0.023 \mathrm{mmol}, 13 \%)$.

Method C. Following the general procedure, starting from substrate $24(62 \mathrm{mg}, 0.11 \mathrm{mmol})$, after $2 \mathrm{~h}$ of reaction, a supplementary addition of TTMSS ( $33 \mu \mathrm{L}, 0.11 \mathrm{mmol}$ ) was required. All the starting material was consumed after $4 \mathrm{~h}$. Column chromatography (hexanesEtOAc, 6:4 to $3: 7)$ gave $\mathbf{5 6}(12 \mathrm{mg}, 0.064 \mathrm{mmol}, \mathbf{5 8 \%})$ and $\mathbf{5 9}(3.7$ $\mathrm{mg}, 0.008 \mathrm{mmol}, 8 \%)$.

Method D. Following the general procedure, starting from substrate $24(50 \mathrm{mg}, 0.086 \mathrm{mmol})$, after $2 \mathrm{~h}$, a supplementary addition of $n-\mathrm{Bu}_{3} \mathrm{SnD}(23 \mu \mathrm{L}, 0.086 \mathrm{mmol})$ was required. All the starting material was consumed after $4 \mathrm{~h}$. Column chromatography (hexanes to hexanes-EtOAc, 3:7) gave $56(12 \mathrm{mg}, 0.064 \mathrm{mmol}, 58 \%)$ and 59 (7.2 $\mathrm{mg}, 0.016 \mathrm{mmol}, 19 \%)$.

Method E. Following the general procedure, starting from substrate $24(37.7 \mathrm{mg}, 0.066 \mathrm{mmol})$, after $2 \mathrm{~h}$, a supplementary addition of $n$ $\mathrm{Bu}_{3} \mathrm{SnD}(17 \mu \mathrm{L}, 0.066 \mathrm{mmol})$ and $\mathrm{BF}_{3} \bullet \mathrm{Et}_{2} \mathrm{O}(2 \mu \mathrm{L}, 0.016 \mathrm{mmol})$ was required. All the starting material was consumed after $4 \mathrm{~h}$. Column chromatography (hexanes to hexanes-EtOAc, 3:7) gave $\mathbf{5 9}(4.2 \mathrm{mg}$, $0.009 \mathrm{mmol}, 15 \%)$ and $\left[4{ }^{2} \mathrm{H}\right] 55\left(5.1 \mathrm{mg}, 0.027 \mathrm{mmol}, 41 \%,{ }^{2} \mathrm{H} /{ }^{1} \mathrm{H}\right.$ 3.1:1). 
Method F. Following the general procedure, starting from substrate 24 (53 mg, $0.091 \mathrm{mmol})$, all the starting material was consumed after $2 \mathrm{~h}$. Chromatotron chromatography (hexanes-EtOAc, 3:7) gave 59 (19.7 mg, $0.045 \mathrm{mmol}, 49 \%$ ).

Method G. Following the general procedure, starting from substrate $24(61 \mathrm{mg}, 0.105 \mathrm{mmol})$, all the starting material was consumed after $3 \mathrm{~h}$. Chromatotron chromatography (hexanesEtOAc, 3:7) gave $\mathbf{5 9}(22.9 \mathrm{mg}, 0.052 \mathrm{mmol}, 50 \%)$.

3-C-(3,4-Di-O-benzyl- $\alpha$-L-fucopyranosyl)1-propene (75). 3$C$-(2,3,4-Tri-O-benzyl- $\alpha$-L-fucopyranosyl)1-propene $(74)^{61}(1.56 \mathrm{~g}$, $3.41 \mathrm{mmol})$ was dissolved in dry $\mathrm{CH}_{2} \mathrm{Cl}_{2}(68 \mathrm{~mL})$ under a $\mathrm{N}_{2}$ atmosphere, and $\mathrm{I}_{2}(8.6 \mathrm{~g}, 34.1 \mathrm{mmol})$ was added. The mixture was stirred at room temperature for $3 \mathrm{~h}$, and then it was poured over an aqueous solution of $\mathrm{Na}_{2} \mathrm{~S}_{2} \mathrm{O}_{3}$ and extracted with $\mathrm{CH}_{2} \mathrm{Cl}_{2}$. The combined extracts were dried over $\mathrm{Na}_{2} \mathrm{SO}_{4}$ and concentrated under reduced pressure. The resulting crude was dissolved in $\mathrm{Et}_{2} \mathrm{O} / \mathrm{MeOH}$ $(1: 1)(35 \mathrm{~mL})$, and $\mathrm{Zn}$ dust $(2.04 \mathrm{~g}, 31.2 \mathrm{mmol})$ and $\mathrm{AcOH}(357 \mu \mathrm{L})$ were subsequently added, with the mixture stirred overnight at room temperature. Then, it was filtered over Celite, evaporated, poured over a saturated aqueous solution of $\mathrm{NaHCO}_{3}$, and extracted with $\mathrm{CH}_{2} \mathrm{Cl}_{2}$. The organic layers were dried over $\mathrm{Na}_{2} \mathrm{SO}_{4}$ and concentrated under reduced pressure. Column chromatography of the residue (hexanesEtOAc, 8:2) gave $75(791.8 \mathrm{mg}, 2.15 \mathrm{mmol}, 63 \%)$ as an amorphous solid: $[\alpha]_{\mathrm{D}}=-57.1\left(c=0.42, \mathrm{CHCl}_{3}\right) .{ }^{1} \mathrm{H}$ NMR $\left(500 \mathrm{MHz}, \mathrm{CDCl}_{3}\right)$ $\delta_{\mathrm{H}} 7.35-7.24(\mathrm{~m}, 10 \mathrm{H}, \mathrm{Ar}), 5.81$ (dddd, $J=17.1,10.1,6.7,6.7 \mathrm{~Hz}$, $\left.1 \mathrm{H}, 2^{\prime}-\mathrm{H}\right), 5.10\left(\mathrm{dd}, J=17.1,1.0 \mathrm{~Hz}, 1 \mathrm{H}, 3^{\prime}-\mathrm{H}_{\mathrm{b}}\right), 5.05(\mathrm{dd}, J=10.1$, $\left.0.0 \mathrm{~Hz}, 1 \mathrm{H}, 3^{\prime}-\mathrm{H}_{\mathrm{a}}\right), 4.78(\mathrm{~d}, J=12.0 \mathrm{~Hz}, 1 \mathrm{H}, \mathrm{OBn}), 4.73(\mathrm{~d}, J=12.0$ $\mathrm{Hz}, 1 \mathrm{H}, \mathrm{OBn}), 4.59(\mathrm{~d}, J=12.0 \mathrm{~Hz}, 1 \mathrm{H}, \mathrm{OBn}), 4.58(\mathrm{~d}, J=11.9 \mathrm{~Hz}$, $1 \mathrm{H}, \mathrm{OBn}), 4.11(\mathrm{~m}, 1 \mathrm{H}, 1-\mathrm{H}), 4.04(\mathrm{br} \mathrm{s}, 1 \mathrm{H}, 2-\mathrm{H}), 3.94(\mathrm{~m}, 1 \mathrm{H}, 5-$ $\mathrm{H}), 3.77(\mathrm{dd}, J=3.2,3.2 \mathrm{~Hz}, 1 \mathrm{H}, 4-\mathrm{H}), 3.73(\mathrm{dd}, J=7.0,2.9 \mathrm{~Hz}, 1 \mathrm{H}$, $3-\mathrm{H}$ ), 2.33 (dd, $J=7.6,7.6 \mathrm{~Hz}, 2 \mathrm{H}, 1^{\prime}-\mathrm{H}_{2}$ ), 2.18 (br s, $\left.1 \mathrm{H}, \mathrm{OH}\right), 1.31$ $\operatorname{ppm}\left(\mathrm{d}, J=6.6 \mathrm{~Hz}, 3 \mathrm{H}, 6-\mathrm{H}_{3}\right) .{ }^{13} \mathrm{C}\left\{{ }^{1} \mathrm{H}\right\}$ NMR $\left(100.6 \mathrm{MHz}, \mathrm{CDCl}_{3}\right)$ $\delta_{\mathrm{C}} 138.4(\mathrm{C}, \mathrm{Ar}), 138.3(\mathrm{C}, \mathrm{Ar}), 135.0\left(\mathrm{CH}, \mathrm{C}-2^{\prime}\right), 128.4(2 \times \mathrm{CH}$, $\mathrm{Ar}), 128.3(2 \times \mathrm{CH}, \mathrm{Ar}), 127.7(3 \times \mathrm{CH}, \mathrm{Ar}), 127.6(3 \times \mathrm{CH}, \mathrm{Ar})$, $116.7\left(\mathrm{CH}_{2}, \mathrm{C}-3{ }^{\prime}\right), 78.8(\mathrm{CH}, \mathrm{C}-3), 75.0(\mathrm{CH}, \mathrm{C}-4), 73.0\left(\mathrm{CH}_{2}\right.$, $\mathrm{OBn}), 72.6\left(\mathrm{CH}_{2}, \mathrm{OBn}\right), 70.8(\mathrm{CH}, \mathrm{C}-1), 69.1(\mathrm{CH}, \mathrm{C}-5), 68.9(\mathrm{CH}$, C-2), $31.9\left(\mathrm{CH}_{2}, \mathrm{C}-1^{\prime}\right), 15.4 \mathrm{ppm}\left(\mathrm{CH}_{3}, \mathrm{C}-6\right)$. IR $\left(\mathrm{CHCl}_{3}\right): \nu=3580$, $3021,1210 \mathrm{~cm}^{-1}$. MS (ESI) $\mathrm{m} / z(\%)=391(100)[\mathrm{M}+\mathrm{Na}]^{+}$. HRMS (ESI) $m / z:[\mathrm{M}+\mathrm{Na}]^{+}$calcd for $\mathrm{C}_{23} \mathrm{H}_{28} \mathrm{NaO}_{4}$ 391.1885; found 391.1889.

3-C-(3,4-Di-O-benzylidene- $\alpha, \beta$-D-arabinopyranosyl) 1-propene (79 and 80$)$. Tetra-O-acetyl-D-arabinopyranose $(78)^{62}(3.35 \mathrm{~g}$, $10.54 \mathrm{mmol}$ ) was dissolved in dry $\mathrm{CH}_{3} \mathrm{CN}(129 \mathrm{~mL})$ under a $\mathrm{N}_{2}$ atmosphere, and allyltrimethylsilane $(9.8 \mathrm{~mL}, 61.51 \mathrm{mmol})$ and $\mathrm{BF}_{3} \bullet \mathrm{Et}_{2} \mathrm{O}(6.2 \mathrm{~mL}, 49.2 \mathrm{mmol})$ were dropwise added at $0{ }^{\circ} \mathrm{C}$. Then, the mixture was stirred at room temperature for $1.5 \mathrm{~h}$. Subsequently, the solution was poured over a saturated aqueous solution of $\mathrm{NaCl}$, extracted with EtOAc, dried over $\mathrm{Na}_{2} \mathrm{SO}_{4}$, and evaporated. Column chromatography of the residue (hexanes-EtOAc, 7:3) gave the allyl derivative (2.18 g, $7.27 \mathrm{mmol}, 69 \%, 1 \mathrm{~S} / 1 \mathrm{R}$ isomers 6.8:1) as a colorless oil, which was subsequently dissolved in dry $\mathrm{MeOH}$ (34 $\mathrm{mL})$, and $\mathrm{Na}_{2} \mathrm{CO}_{3}(1.23 \mathrm{~g}, 11.60 \mathrm{mmol})$ was added. The mixture was stirred at room temperature for $2.5 \mathrm{~h}$, and then it was filtered, neutralized with the Amberlyst $15 \mathrm{H}^{+}$ion exchange resin, and evaporated. The crude was submitted to the benzylidene protection by treatment overnight with $\mathrm{PhCH}(\mathrm{OMe})_{2}(1.5 \mathrm{~mL}, 10.91 \mathrm{mmol})$ and CSA $(17 \mathrm{mg}, 0.07 \mathrm{mmol})$ in dry DMF $(7.3 \mathrm{~mL})$ at room temperature under a $\mathrm{N}_{2}$ atmosphere. The reaction was evaporated in a high vacuum rotovap and purified by column chromatography (hexanes-EtOAc, $8: 2)$ to give $79(228 \mathrm{mg}, 0.87 \mathrm{mmol}, 12 \%$, d.r., 3:1) and 80 (780.8 g, $2.98 \mathrm{mmol}, 41 \%$, d.r., 3.5:1) as colorless oils. Compound 79: ${ }^{1} \mathrm{H}$ NMR ( $500 \mathrm{MHz}, \mathrm{CDCl}_{3}$, only the major isomer is described) $\delta_{\mathrm{H}} 7.48-7.34(\mathrm{~m}, 5 \mathrm{H}, \mathrm{Ar}), 6.23(\mathrm{~s}, 1 \mathrm{H}, \mathrm{PhCH}), 5.86$ (dddd, $\left.J=17.0,10.1,7.0,7.0 \mathrm{~Hz}, 1 \mathrm{H}, 2^{\prime}-\mathrm{H}\right), 5.18$ (dd, $J=17.0,1.6$ $\mathrm{Hz}, 1 \mathrm{H}, 3$ ' $-\mathrm{H}_{\mathrm{b}}$ ), 5.13 (dd, $J=10.1,1.6 \mathrm{~Hz}, 1 \mathrm{H}, 3^{\prime}-\mathrm{H}_{\mathrm{a}}$ ), 4.57 (ddd, $J=$ 9.2, 6.7, $5.1 \mathrm{~Hz}, 1 \mathrm{H}, 4-\mathrm{H}), 4.30(\mathrm{dd}, J=5.1,2.9 \mathrm{~Hz}, 1 \mathrm{H}, 3-\mathrm{H}), 4.10$ (dd, $\left.J=12.0,6.7 \mathrm{~Hz}, 1 \mathrm{H}, 5-\mathrm{H}_{\mathrm{b}}\right), 3.97($ br d, $J=5.4 \mathrm{~Hz}, 1 \mathrm{H}, 2-\mathrm{H})$, $3.73(\mathrm{ddd}, J=7.9,6.7,1.6 \mathrm{~Hz}, 1 \mathrm{H}, 1-\mathrm{H}), 3.55(\mathrm{dd}, J=12.0,9.1 \mathrm{~Hz}$, $\left.1 \mathrm{H}, 5-\mathrm{H}_{\mathrm{a}}\right), 2.48\left(\mathrm{~m}, 1 \mathrm{H}, 1^{\prime}-\mathrm{H}_{\mathrm{b}}\right), 2.36 \mathrm{ppm}\left(\mathrm{m}, 1 \mathrm{H}, 1^{\prime}-\mathrm{H}_{\mathrm{a}}\right), 1 \mathrm{H}$ from $\mathrm{OH}$ is missing. ${ }^{13} \mathrm{C}\left\{{ }^{1} \mathrm{H}\right\}$ NMR (125.7 MHz, $\mathrm{CDCl}_{3}$, only the major isomer is described) $\delta_{\mathrm{C}} 139.0(\mathrm{C}, \mathrm{Ar}), 134.0\left(\mathrm{CH}, \mathrm{C}-2^{\prime}\right), 129.1(\mathrm{CH}$, $\mathrm{Ar}), 128.4(2 \times \mathrm{CH}, \mathrm{Ar}), 125.9(2 \times \mathrm{CH}, \mathrm{Ar}), 117.6\left(\mathrm{CH}_{2}, \mathrm{C}-3^{\prime}\right)$, $103.3(\mathrm{CH}, \mathrm{PhCH}), 75.7(\mathrm{CH}, \mathrm{C}-3), 75.0(\mathrm{CH}, \mathrm{C}-1), 70.1(\mathrm{CH}, \mathrm{C}-$ 4), $67.5(\mathrm{CH}, \mathrm{C}-2), 60.0\left(\mathrm{CH}_{2}, \mathrm{C}-5\right), 34.6 \mathrm{ppm}\left(\mathrm{CH}_{2}, \mathrm{C}-1\right.$ '). IR $\left(\mathrm{CHCl}_{3}\right): \nu=3567,3452,1643,1457,1100 \mathrm{~cm}^{-1}$. MS (ESI) $\mathrm{m} / z(\%)$ $=285(100)[\mathrm{M}+\mathrm{Na}]^{+}$. HRMS (ESI) $\mathrm{m} / z:[\mathrm{M}+\mathrm{Na}]^{+}$calcd for $\mathrm{C}_{15} \mathrm{H}_{18} \mathrm{NaO}_{4}$ 285.1103; found 285.1099. Compound 80: ${ }^{1} \mathrm{H}$ NMR $\left(500 \mathrm{MHz}, \mathrm{CDCl}_{3}\right.$, only the major isomer is described) $\delta_{\mathrm{H}} 7.52-7.39$ (m, 5H, Ar), 5.95 (s, 1H, PhCH), 5.84 (dddd, $J=17.4,10.1,7.3,7.3$ $\left.\mathrm{Hz}, 1 \mathrm{H}, 2^{\prime}-\mathrm{H}\right), 5.18\left(\mathrm{brd}, J=17.2 \mathrm{~Hz}, 1 \mathrm{H}, 3^{\prime}-\mathrm{H}_{\mathrm{b}}\right), 5.11(\mathrm{br} \mathrm{d}, J=10.1$ $\left.\mathrm{Hz}, 1 \mathrm{H}, 3-\mathrm{H}_{\mathrm{a}}\right), 4.43(\mathrm{~m}, 1 \mathrm{H}, 4-\mathrm{H}), 4.36(\mathrm{dd}, J=5.7,2.9 \mathrm{~Hz}, 1 \mathrm{H}, 3-$ $\mathrm{H}), 4.08\left(\mathrm{dd}, J=12.0,6.3 \mathrm{~Hz}, 1 \mathrm{H}, 5-\mathrm{H}_{\mathrm{b}}\right), 4.00(\mathrm{dd}, J=2.5,1.9 \mathrm{~Hz}$, $1 \mathrm{H}, 2-\mathrm{H}), 3.73(\mathrm{ddd}, J=8.2,6.6,1.9 \mathrm{~Hz}, 1 \mathrm{H}, 1-\mathrm{H}), 3.50(\mathrm{dd}, J=12.0$, $\left.8.5 \mathrm{~Hz}, 1 \mathrm{H}, 5-\mathrm{H}_{\mathrm{a}}\right), 2.45\left(\mathrm{~m}, 1 \mathrm{H}, 1^{\prime}-\mathrm{H}_{\mathrm{b}}\right), 2.34 \mathrm{ppm}\left(\mathrm{m}, 1 \mathrm{H}, \mathrm{l}^{\prime}-\mathrm{H}_{\mathrm{a}}\right), 1 \mathrm{H}$ from $\mathrm{OH}$ is missing. ${ }^{13} \mathrm{C}\left\{{ }^{1} \mathrm{H}\right\} \mathrm{NMR}\left(125.7 \mathrm{MHz}, \mathrm{CDCl}_{3}\right.$, only the major isomer is described) $\delta_{\mathrm{C}} 137.1(\mathrm{C}, \mathrm{Ar}), 134.0(\mathrm{CH}, \mathrm{C}-2$ '), 129.1 $(\mathrm{CH}, \mathrm{Ar}), 128.4(2 \times \mathrm{CH}, \mathrm{Ar}), 126.4(2 \times \mathrm{CH}, \mathrm{Ar}), 117.6\left(\mathrm{CH}_{2}, \mathrm{C}-\right.$ 3'), $104.3(\mathrm{CH}, \mathrm{PhCH}), 77.7(\mathrm{CH}, \mathrm{C}-3), 75.0(\mathrm{CH}, \mathrm{C}-1), 69.2(\mathrm{CH}$, C-4), $68.0\left(\mathrm{CH}_{2}, \mathrm{C}-5\right), 67.5(\mathrm{CH}, \mathrm{C}-2), 34.8 \mathrm{ppm}\left(\mathrm{CH}_{2}, \mathrm{C}-1^{\prime}\right)$. IR $\left(\mathrm{CHCl}_{3}\right): \nu=3567,3422,3023,1643,1459,1068 \mathrm{~cm}^{-1}$. MS (ESI) $m / z(\%)=285(100)[\mathrm{M}+\mathrm{Na}]^{+}$. HRMS $(\mathrm{ESI}) m / z:[\mathrm{M}+\mathrm{Na}]^{+}$ calcd for $\mathrm{C}_{15} \mathrm{H}_{18} \mathrm{NaO}_{4}$ 285.1103; found 285.1097.

3-C-(3,5-Di-O-tert-butyldiphenylsilyl- $\alpha$-D-ribofuranosyl) 1propene (83). To a solution of 3-C-( $\alpha$-D-ribofuranosyl $) 1$-propene $(82)^{56,57}(5.24 \mathrm{~g}, 30.11 \mathrm{mmol}, 87 \%)$ in dry $\mathrm{CH}_{2} \mathrm{Cl}_{2}(145 \mathrm{~mL})$ at $0{ }^{\circ} \mathrm{C}$ were sequentially added imidazole $(3.07 \mathrm{~g}, 45.17 \mathrm{mmol})$ and DPSCl $(7.72 \mathrm{~mL}, 30.11 \mathrm{mmol})$. The resulting mixture was stirred at $0{ }^{\circ} \mathrm{C}$ for $3 \mathrm{~h}$, treated with saturated aqueous $\mathrm{NH}_{4} \mathrm{Cl}$, and extracted with $\mathrm{CH}_{2} \mathrm{Cl}_{2}$. Purification by column chromatography (hexanes-EtOAc, $97: 3$ to $6: 4)$ afforded monoalcohol $83(7.11 \mathrm{~g}, 10.94 \mathrm{mmol}, 36 \%)$ and known diol 3-C-(5-O-tert-butyldiphenylsilyl- $\alpha$-D-ribofuranosyl)1-propene $(84)^{13,57}(4.92 \mathrm{~g}, 11.94 \mathrm{mmol}, 40 \%)$ as colorless oils. Compound 83: $[\alpha]_{\mathrm{D}}=+33.1\left(c=0.58, \mathrm{CHCl}_{3}\right) .{ }^{1} \mathrm{H}$ NMR $(500$ $\left.\mathrm{MHz}, \mathrm{CDCl}_{3}\right) \delta_{\mathrm{H}} 7.57-7.15(\mathrm{~m}, 20 \mathrm{H}, \mathrm{Ar}$ ), 5.77 (dddd, $J=17.0,10.1$, $\left.7.0,7.0 \mathrm{~Hz}, 1 \mathrm{H}, 2^{\prime}-\mathrm{H}\right), 5.04$ (br d, $J=17.4 \mathrm{~Hz}, 1 \mathrm{H}, 3^{\prime}-\mathrm{H}_{\mathrm{b}}$ ), 4.97 (br d, $\left.J=10.1 \mathrm{~Hz}, 1 \mathrm{H}, 3^{\prime}-\mathrm{H}_{\mathrm{a}}\right), 4.49(\mathrm{dd}, J=5.4,5.4 \mathrm{~Hz}, 1 \mathrm{H}, 3-\mathrm{H}), 3.87(\mathrm{~m}$, $1 \mathrm{H}, 4-\mathrm{H}), 3.84(\mathrm{~m}, 1 \mathrm{H}, 1-\mathrm{H}), 3.77(\mathrm{dd}, J=4.7,4.7 \mathrm{~Hz}, 1 \mathrm{H}, 2-\mathrm{H})$, $3.50\left(\mathrm{dd}, J=11.5,2.2 \mathrm{~Hz}, 1 \mathrm{H}, 5-\mathrm{H}_{\mathrm{b}}\right), 3.14(\mathrm{dd}, J=11.4,3.2 \mathrm{~Hz}, 1 \mathrm{H}$, 5- $\left.\mathrm{H}_{\mathrm{a}}\right), 2.76(\mathrm{br} \mathrm{s}, 1 \mathrm{H}, \mathrm{OH}), 2.38\left(\mathrm{dd}, J=6.9,6.9 \mathrm{~Hz}, 2 \mathrm{H}, 1^{\prime}-\mathrm{H}_{2}\right), 1.00$ (s, 9H, $\left.{ }^{t} \mathrm{Bu}\right), 0.80 \mathrm{ppm}\left(\mathrm{s}, 9 \mathrm{H},{ }^{t} \mathrm{Bu}\right) .{ }^{13} \mathrm{C}\left\{{ }^{1} \mathrm{H}\right\}$ NMR $(125.7 \mathrm{MHz}$, $\left.\mathrm{CDCl}_{3}\right) \delta_{\mathrm{C}} 135.7(2 \times \mathrm{CH}, \mathrm{Ar}), 135.63(2 \times \mathrm{CH}, \mathrm{Ar}), 135.61(\mathrm{CH}$, C-2'), 135.5 ( $2 \times \mathrm{CH}, \mathrm{Ar}), 135.1(2 \times \mathrm{CH}, \mathrm{Ar}), 133.4$ (C, Ar), 133.2 (C, Ar), 132.6 (C, Ar), 132.4 (C, Ar), 130.20 (CH, Ar), $130.16(\mathrm{CH}$, $\mathrm{Ar}), 129.5(2 \times \mathrm{CH}, \mathrm{Ar}), 128.0(2 \times \mathrm{CH}, \mathrm{Ar}), 127.8(2 \times \mathrm{CH}, \mathrm{Ar})$, $\left.127.5(4 \times \mathrm{CH}, \mathrm{Ar}), 116.6\left(\mathrm{CH}_{2}, \mathrm{C}-3\right)^{\prime}\right), 83.1(\mathrm{CH}, \mathrm{C}-4), 81.0(\mathrm{CH}$, $\mathrm{C}-1)$, 74.5 (CH, C-3), $72.7(\mathrm{CH}, \mathrm{C}-2), 64.0\left(\mathrm{CH}_{2}, \mathrm{C}-5\right), 34.1\left(\mathrm{CH}_{2}\right.$, C-1'), $26.9\left(3 \times \mathrm{CH}_{3},{ }^{t} \mathrm{Bu}\right), 26.7\left(3 \times \mathrm{CH}_{3},{ }^{t} \mathrm{Bu}\right), 19.2\left(\mathrm{C},{ }^{t} \mathrm{Bu}\right), 19.0$ $\mathrm{ppm}\left(\mathrm{C},{ }^{t} \mathrm{Bu}\right)$. IR $\left(\mathrm{CHCl}_{3}\right): \nu=3673,3541,2932,2860,1428,1113$ $\mathrm{cm}^{-1}$. MS (ESI) $m / z(\%)=673(100)[\mathrm{M}+\mathrm{Na}]^{+}$. HRMS (ESI) $m / z$ : $[\mathrm{M}+\mathrm{Na}]^{+}$calcd for $\mathrm{C}_{40} \mathrm{H}_{50} \mathrm{NaO}_{4} \mathrm{Si}_{2}$ 673.3145; found 673.3141.

3-C-(3,5-Di-O-1, 1,3,3-tetraisopropyldisiloxanyl- $\alpha$-Dribofuranosyl)1-propene (86). 1,3-Dichloro-1,1,3,3-tetraisopropyldisiloxane $(1.1 \mathrm{~mL}, 3.44 \mathrm{mmol})$ was added to a stirred solution of triol $82^{56,57}(300 \mathrm{mg}, 1.72 \mathrm{mmol})$ in dry pyridine $(53 \mathrm{~mL})$ at $0{ }^{\circ} \mathrm{C}$. The reaction mixture was stirred at room temperature for $20 \mathrm{~h}$, and then the pyridine was evaporated under reduced pressure. The residue was poured over $10 \% \mathrm{HCl}$ and extracted with EtOAc. The combined organic extracts were washed with a saturated solution of $\mathrm{NaHCO}_{3}$, dried over $\mathrm{Na}_{2} \mathrm{SO}_{4}$, filtered, and evaporated. The crude was subjected to chromatography (hexanes-EtOAc, 95:5) to afford monoalcohol 86 $(496.4 \mathrm{mg}, 1.19 \mathrm{mmol}, 69 \%)$ as a colorless oil: $[\alpha]_{\mathrm{D}}=-17.6(c=$ $\left.0.51, \mathrm{CHCl}_{3}\right) .{ }^{1} \mathrm{H}$ NMR $\left(500 \mathrm{MHz}, \mathrm{CDCl}_{3}\right) \delta_{\mathrm{H}} 5.86(1 \mathrm{H}, \mathrm{dddd}, J=$ $\left.17.1,10.1,7.0,7.0 \mathrm{~Hz}, 1 \mathrm{H}, 2^{\prime}-\mathrm{H}\right), 5.15$ (br d, $J=17.1 \mathrm{~Hz}, 1 \mathrm{H}, 3^{\prime}-\mathrm{H}_{\mathrm{b}}$ ), $5.06\left(\right.$ br d, $\left.J=10.1 \mathrm{~Hz}, 1 \mathrm{H}, 3^{\prime}-\mathrm{H}_{\mathrm{a}}\right), 4.37(\mathrm{dd}, J=7.3,4.8 \mathrm{~Hz}, 1 \mathrm{H}, 3-$ $\mathrm{H}), 4.10(\mathrm{dd}, J=4.4,4.4 \mathrm{~Hz}, 1 \mathrm{H}, 2-\mathrm{H}), 4.01-3.97(\mathrm{~m}, 2 \mathrm{H}, 1-\mathrm{H}, 5-$ $\mathrm{H}_{\mathrm{b}}$ ), 3.93 (ddd, $\left.J=7.0,7.0,3.5 \mathrm{~Hz}, 1 \mathrm{H}, 4-\mathrm{H}\right), 3.83(\mathrm{dd}, J=11.7,6.3$ $\left.\mathrm{Hz}, 1 \mathrm{H}, 5-\mathrm{H}_{\mathrm{a}}\right), 2.52\left(\mathrm{ddd}, J=14.2,6.9,6.9 \mathrm{~Hz}, 1 \mathrm{H}, 1^{\prime}-\mathrm{H}_{\mathrm{b}}\right), 2.42(\mathrm{ddd}$, $\left.J=14.5,7.3,7.3 \mathrm{~Hz}, 1 \mathrm{H}, 1^{\prime}-\mathrm{H}_{2}\right), 1.10-0.89 \mathrm{ppm}\left(\mathrm{m}, 28 \mathrm{H},{ }^{i} \mathrm{Pr}\right), 1 \mathrm{H}$ from $\mathrm{OH}$ is missing. ${ }^{13} \mathrm{C}\left\{{ }^{1} \mathrm{H}\right\}$ NMR $\left(125.7 \mathrm{MHz}, \mathrm{CDCl}_{3}\right) \delta_{\mathrm{C}} 134.6$ $\left(\mathrm{CH}, \mathrm{C}-2\right.$ ') $117.1\left(\mathrm{CH}_{2}, \mathrm{C}-3^{\prime}\right), 80.5(\mathrm{CH}, \mathrm{C}-1$ or $\mathrm{C}-4), 80.3(\mathrm{CH}, \mathrm{C}-$ 
1 or C-4), 74.9 (CH, C-3), $72.4(\mathrm{CH}, \mathrm{C}-2), 63.4\left(\mathrm{CH}_{2}, \mathrm{C}-5\right), 33.8$ $\left(\mathrm{CH}_{2}, \mathrm{C}-1^{\prime}\right), 17.5\left(\mathrm{CH}_{3},{ }^{i} \mathrm{Pr}\right), 17.38\left(\mathrm{CH}_{3},{ }^{i} \mathrm{Pr}\right), 17.35\left(2 \times \mathrm{CH}_{3},{ }^{i} \mathrm{Pr}\right)$, 17.2 $\left(\mathrm{CH}_{3},{ }^{i} \mathrm{Pr}\right), 17.1\left(2 \times \mathrm{CH}_{3},{ }^{i} \mathrm{Pr}\right), 17.0\left(\mathrm{CH}_{3},{ }^{i} \mathrm{Pr}\right), 13.4\left(\mathrm{CH},{ }^{i} \mathrm{Pr}\right)$, $13.2\left(\mathrm{CH},{ }^{i} \mathrm{Pr}\right), 12.9\left(\mathrm{CH},{ }^{i} \mathrm{Pr}\right), 12.6 \mathrm{ppm}\left(\mathrm{CH},{ }^{i} \mathrm{Pr}\right)$. IR $\left(\mathrm{CHCl}_{3}\right): \nu=$ $3671,3540,2949,1732,1643,1465,1120 \mathrm{~cm}^{-1}$. MS (ESI) $\mathrm{m} / z(\%)=$ $439(100)[\mathrm{M}+\mathrm{Na}]^{+}$. HRMS (ESI) $\mathrm{m} / z:[\mathrm{M}+\mathrm{Na}]^{+}$calcd for $\mathrm{C}_{20} \mathrm{H}_{40} \mathrm{NaO}_{5} \mathrm{Si}_{2}$ 439.2312; found 439.2310.

General Procedure of Hydroboration to Give 63, 66, 69, 72, $76,81,85$, and 87 . The corresponding allyl derivative $(1 \mathrm{mmol})$ was dissolved in dry THF $(10.5 \mathrm{~mL}) . \mathrm{BH}_{3} \cdot$ THF $1 \mathrm{M}$ complex $(4 \mathrm{~mL}, 4$ mmol) was added under a $\mathrm{N}_{2}$ atmosphere at $0{ }^{\circ} \mathrm{C}$, and then the reaction was stirred at room temperature for $1 \mathrm{~h}$. At $0{ }^{\circ} \mathrm{C}$, an aqueous solution of $\mathrm{NaOH} 3 \mathrm{M}(20 \mathrm{~mL})$ was dropwise added followed by $\mathrm{H}_{2} \mathrm{O}_{2} 30 \%(20 \mathrm{~mL})$ and stirring was continued during $1 \mathrm{~h}$ at that temperature. The reaction was poured into brine and extracted with $\mathrm{CH}_{2} \mathrm{Cl}_{2}$. The organic layers were dried over $\mathrm{Na}_{2} \mathrm{SO}_{4}$ and concentrated under reduced pressure. The residue was purified by column chromatography (hexanes-EtOAc) to give the corresponding alcohol.

3-C-(3,4,6-Tri-O-benzyl- $\alpha$-D-glucopyranosyl)1-propanol (63). Following the general procedure for the hydroboration, starting from 3-C-(3,4,6-tri-O-benzyl- $\alpha$-D-glucopyranosyl)1-propene $(62)^{54}$ (700 $\mathrm{mg}, 1.48 \mathrm{mmol}$ ) and purification by column chromatography (hexanes-EtOAc, 8:2), the alcohol $63(478 \mathrm{mg}, 0.97 \mathrm{mmol}, 66 \%)$ was obtained as a crystalline solid: $\mathrm{mp} 101.5-102.3^{\circ} \mathrm{C}$ ( $n$-hexaneEtOAc); $[\alpha]_{\mathrm{D}}=+31.6\left(c=0.31, \mathrm{CHCl}_{3}\right) .{ }^{1} \mathrm{H} \mathrm{NMR}(400 \mathrm{MHz}$, $\left.\mathrm{CDCl}_{3}\right) \delta_{\mathrm{H}} 7.35-7.20(\mathrm{~m}, 15 \mathrm{H}, \mathrm{Ar}), 4.67(\mathrm{~d}, J=11.7 \mathrm{~Hz}, 1 \mathrm{H}, \mathrm{OBn})$, $4.63(\mathrm{~d}, J=11.4 \mathrm{~Hz}, 1 \mathrm{H}, \mathrm{OBn}), 4.59$ (d, $J=11.8 \mathrm{~Hz}, 1 \mathrm{H}, \mathrm{OBn}), 4.54$ $(\mathrm{d}, J=12.2 \mathrm{~Hz}, 1 \mathrm{H}, \mathrm{OBn}), 4.53(\mathrm{~d}, J=11.2 \mathrm{~Hz}, 1 \mathrm{H}, \mathrm{OBn}), 4.50$ (d, $J$ $=12.1 \mathrm{~Hz}, 1 \mathrm{H}, \mathrm{OBn}$ ), 4.00 (ddd, $J=5.1,5.1,5.1 \mathrm{~Hz}, 1 \mathrm{H}, 5-\mathrm{H}), 3.90$ (ddd, $J=9.5,3.2,3.2 \mathrm{~Hz}, 1 \mathrm{H}, 1-\mathrm{H}), 3.79$ (dd, $J=10.1,6.0 \mathrm{~Hz}, 1 \mathrm{H}, 6-$ $\left.\mathrm{H}_{\mathrm{b}}\right), 3.73(\mathrm{dd}, J=5.8,5.8 \mathrm{~Hz}, 1 \mathrm{H}, 3-\mathrm{H}), 3.68-3.61\left(\mathrm{~m}, 4 \mathrm{H}, 3{ }^{\prime}-\mathrm{H}_{2}, 2-\right.$ $\left.\mathrm{H}, 6-\mathrm{H}_{\mathrm{a}}\right), 3.58(\mathrm{dd}, J=5.4,5.4 \mathrm{~Hz}, 1 \mathrm{H}, 4-\mathrm{H}), 2.96(\mathrm{br} \mathrm{s}, 1 \mathrm{H}, \mathrm{OH})$, 2.15 (br s, $1 \mathrm{H}, \mathrm{OH}), 1.80-1.61 \mathrm{ppm}\left(\mathrm{m}, 4 \mathrm{H}, 1^{\prime}-\mathrm{H}_{2}, 2^{\prime}-\mathrm{H}_{2}\right) .{ }^{13} \mathrm{C}\left\{{ }^{1} \mathrm{H}\right\}$ NMR $\left(100.6 \mathrm{MHz}, \mathrm{CDCl}_{3}\right) \delta_{\mathrm{C}} 138.0(2 \times \mathrm{C}, \mathrm{Ar}), 137.4(\mathrm{C}, \mathrm{Ar})$, 127.6-128.5 (15 × CH, Ar), 78.1 (CH, C-3), 75.2 (CH, C-4), 73.5 $\left(\mathrm{CH}_{2}, \mathrm{OBn}\right), 73.3\left(\mathrm{CH}_{2}, \mathrm{OBn}\right), 73.3(\mathrm{CH}, \mathrm{C}-5), 73.0\left(\mathrm{CH}_{2}, \mathrm{OBn}\right)$, 71.9 (CH, C-1), $\left.70.0(\mathrm{CH}, \mathrm{C}-2), 68.2\left(\mathrm{CH}_{2}, \mathrm{C}-6\right), 62.6\left(\mathrm{CH}_{2}, \mathrm{C}-3\right)^{\prime}\right)$, $29.2\left(\mathrm{CH}_{2}, \mathrm{C}-2^{\prime}\right), 24.8 \mathrm{ppm}\left(\mathrm{CH}_{2}, \mathrm{C}-1^{\prime}\right)$, IR $\left(\mathrm{CHCl}_{3}\right): \nu=3496$, 2938, 1455, $1086 \mathrm{~cm}^{-1}$. MS (ESI) $\mathrm{m} / z(\%)=515(100)[\mathrm{M}+\mathrm{Na}]^{+}$. HRMS (ESI) $m / z:[\mathrm{M}+\mathrm{Na}]^{+}$calcd for $\mathrm{C}_{30} \mathrm{H}_{36} \mathrm{NaO}_{6}$ 515.2410; found 515.2407. Anal. calcd for $\mathrm{C}_{30} \mathrm{H}_{36} \mathrm{O}_{6}: \mathrm{C}, 73.15 ; \mathrm{H}, 7.37$. Found: C, $72.90 ; \mathrm{H}, 7.26$.

3-C-(3,4,6-Tri-O-benzyl- $\beta$-D-glucopyranosyl) 1-propanol (66). Following the general procedure for the hydroboration, starting from 3$C$-(3,4,6-tri-O-benzyl- $\beta$-D-glucopyranosyl) 1 -propene $(65)^{50 c}(103 \mathrm{mg}$, $0.22 \mathrm{mmol}$ ) and purification by column chromatography (hexanesEtOAc, 7:3), the alcohol $66(77.3 \mathrm{mg}, 0.16 \mathrm{mmol}, 73 \%)$ was obtained as a crystalline solid: $\mathrm{mp} 112.0-112.7^{\circ} \mathrm{C}\left(n\right.$-hexane-EtOAc); $[\alpha]_{\mathrm{D}}=$ $+35.7\left(c=0.63, \mathrm{CHCl}_{3}\right) .{ }^{1} \mathrm{H} \mathrm{NMR}\left(400 \mathrm{MHz}, \mathrm{CDCl}_{3}\right) \delta_{\mathrm{H}} 7.28-7.18$ (m, 13H, Ar), 7.12-7.10 (m, 2H, Ar), $4.88(\mathrm{~d}, J=11.6 \mathrm{~Hz}, 1 \mathrm{H}$, OBn), 4.71 (d, $J=10.8 \mathrm{~Hz}, 1 \mathrm{H}, \mathrm{OBn}), 4.67(\mathrm{~d}, J=11.7 \mathrm{~Hz}, 1 \mathrm{H}$, OBn), $4.53(\mathrm{~d}, J=12.2 \mathrm{~Hz}, 1 \mathrm{H}, \mathrm{OBn}), 4.49(\mathrm{~d}, J=10.8 \mathrm{~Hz}, 1 \mathrm{H}$, OBn), 4.45 (d, $J=12.1 \mathrm{~Hz}, 1 \mathrm{H}, \mathrm{OBn}$ ), 3.61 (ddd, $J=10.8,10.8,2.2$ $\mathrm{Hz}, 1 \mathrm{H}, 5-\mathrm{H}), 3.58-3.55\left(\mathrm{~m}, 3 \mathrm{H}, 3^{\prime}-\mathrm{H}_{2}, 6-\mathrm{H}_{\mathrm{b}}\right), 3.51$ (dd, $J=9.3,9.3$ $\mathrm{Hz}, 1 \mathrm{H}, 4-\mathrm{H}), 3.39$ (dd, $J=8.9,8.9 \mathrm{~Hz}, 1 \mathrm{H}, 3-\mathrm{H}), 3.37$ (m, 1H, 6$\mathrm{H}_{\mathrm{a}}$ ), $3.26(\mathrm{dd}, J=9.2,9.2 \mathrm{~Hz}, 1 \mathrm{H}, 2-\mathrm{H}), 3.15$ (ddd, $J=8.5,8.5,2.4$ $\mathrm{Hz}, 1 \mathrm{H}, 1-\mathrm{H}), 2.22(\mathrm{br} \mathrm{s}, 2 \mathrm{H}, 2 \times \mathrm{OH}), 1.92\left(\mathrm{~m}, 1 \mathrm{H}, \mathrm{l}^{\prime}-\mathrm{H}_{\mathrm{b}}\right), 1.68-$ $1.62\left(\mathrm{~m}, 2 \mathrm{H}, 2^{\prime}-\mathrm{H}_{2}\right), 1.49 \mathrm{ppm}$ (dddd, $J=7.7,7.7,7.7,7.7 \mathrm{~Hz}, 1 \mathrm{H}, 1^{\prime}$ $\left.\mathrm{H}_{\mathrm{a}}\right),{ }^{13} \mathrm{C}\left\{{ }^{1} \mathrm{H}\right\}$ NMR $\left(100.6 \mathrm{MHz}, \mathrm{CDCl}_{3}\right) \delta_{\mathrm{C}} 138.6(\mathrm{C}, \mathrm{Ar}), 138.0(2$ $\times \mathrm{C}, \mathrm{Ar}), 128.6(2 \times \mathrm{CH}, \mathrm{Ar}), 128.4(2 \times \mathrm{CH}, \mathrm{Ar}), 128.3(2 \times \mathrm{CH}$, $\mathrm{Ar}), 127.89(3 \times \mathrm{CH}, \mathrm{Ar}), 127.84(2 \times \mathrm{CH}, \mathrm{Ar}), 127.79(2 \times \mathrm{CH}$, $\mathrm{Ar}), 127.75$ (CH, Ar), 127.6 (CH, Ar), 86.8 (CH, C-3), 79.4 (CH, C4), $78.8(\mathrm{CH}, \mathrm{C}-5), 78.4(\mathrm{CH}, \mathrm{C}-1), 75.2\left(\mathrm{CH}_{2}, \mathrm{OBn}\right), 74.7\left(\mathrm{CH}_{2}\right.$, OBn), 73.7 ( $\mathrm{CH}, \mathrm{C}-2), 73.5\left(\mathrm{CH}_{2}, \mathrm{OBn}\right), 69.0\left(\mathrm{CH}_{2}, \mathrm{C}-6\right), 62.7$ $\left(\mathrm{CH}_{2}, \mathrm{C}-3^{\prime}\right), 28.8\left(\mathrm{CH}_{2}, \mathrm{C}-2^{\prime}\right), 28.5 \mathrm{ppm}\left(\mathrm{CH}_{2}, \mathrm{C}-1^{\prime}\right)$. IR $\left(\mathrm{CHCl}_{3}\right): \nu$ $=3588,3500,2928,1455,1052 \mathrm{~cm}^{-1}$. MS (ESI) $\mathrm{m} / z(\%)=515$ (100) $[\mathrm{M}+\mathrm{Na}]^{+}$. HRMS (ESI) $m / z:[\mathrm{M}+\mathrm{Na}]^{+}$calcd for $\mathrm{C}_{30} \mathrm{H}_{36} \mathrm{NaO}_{6}$ 515.2410; found 515.2412.
3-C-(3,4,6-Tri-O-benzyl- $\alpha$-D-mannopyranosyl)1-propanol (69). Following the general procedure for the hydroboration, starting from 3-C-(3,4,6-tri- $O$-benzyl- $\alpha$-D-mannopyranosyl) 1 -propene $(68)^{54,63}(675.6 \mathrm{mg}, 1.42 \mathrm{mmol})$ and purification by column chromatography (hexanes-EtOAc, 1:1 to 3:7), the alcohol 69 (368.4 mg, $0.75 \mathrm{mmol}, 53 \%$ ) was obtained as an amorphous solid: $[\alpha]_{\mathrm{D}}=+34.0\left(c=0.45, \mathrm{CHCl}_{3}\right) .{ }^{1} \mathrm{H} \mathrm{NMR}\left(500 \mathrm{MHz}, \mathrm{CDCl}_{3}\right) \delta_{\mathrm{H}}$ $7.35-7.25(\mathrm{~m}, 13 \mathrm{H}, \mathrm{Ar}), 7.22-7.20(\mathrm{~m}, 2 \mathrm{H}, \mathrm{Ar}), 4.71(\mathrm{~d}, J=11.1 \mathrm{~Hz}$, $1 \mathrm{H}, \mathrm{OBn}), 4.61(\mathrm{~d}, J=11.7 \mathrm{~Hz}, 1 \mathrm{H}, \mathrm{OBn}), 4.57(\mathrm{~d}, J=10.1 \mathrm{~Hz}, 1 \mathrm{H}$, OBn), $4.55(\mathrm{~d}, J=11.1 \mathrm{~Hz}, 1 \mathrm{H}, \mathrm{OBn}), 4.52(\mathrm{~d}, J=11.4 \mathrm{~Hz}, 1 \mathrm{H}$, $\mathrm{OBn}), 4.51(\mathrm{~d}, J=12.0 \mathrm{~Hz}, 1 \mathrm{H}, \mathrm{OBn}), 3.89(\mathrm{~m}, 1 \mathrm{H}, 1-\mathrm{H}), 3.82-3.80$ $(\mathrm{m}, 2 \mathrm{H}, 2-\mathrm{H}, 3-\mathrm{H}), 3.77-3.75(\mathrm{~m}, 2 \mathrm{H}, 4-\mathrm{H}, 5-\mathrm{H}), 3.70(\mathrm{dd}, J=10.1$, $\left.5.4 \mathrm{~Hz}, 1 \mathrm{H}, 6-\mathrm{H}_{\mathrm{b}}\right), 3.68-3.60\left(\mathrm{~m}, 3 \mathrm{H}, 3-\mathrm{H}_{2}, 6-\mathrm{H}_{\mathrm{a}}\right), 2.25(\mathrm{br} \mathrm{s}, 2 \mathrm{H}, 2$ $\times \mathrm{OH}), 1.76-1.59 \mathrm{ppm}\left(\mathrm{m}, 4 \mathrm{H}, 1^{\prime}-\mathrm{H}_{2}, 2^{\prime}-\mathrm{H}_{2}\right) .{ }^{13} \mathrm{C}\left\{{ }^{1} \mathrm{H}\right\} \mathrm{NMR}(100.6$ $\left.\mathrm{MHz}, \mathrm{CDCl}_{3}\right) \delta_{\mathrm{C}} 138.1(2 \times \mathrm{C}, \mathrm{Ar}), 137.6(\mathrm{C}, \mathrm{Ar}), 127.6-128.6(15$ $\times \mathrm{CH}, \mathrm{Ar}), 79.2(\mathrm{CH}, \mathrm{C}-3), 75.1(\mathrm{CH}, \mathrm{C}-1), 74.2(\mathrm{CH}, \mathrm{C}-5), 74.0$ $\left(\mathrm{CH}_{2}, \mathrm{OBn}\right), 73.4\left(\mathrm{CH}_{2}, \mathrm{OBn}\right), 72.8(\mathrm{CH}, \mathrm{C}-4), 72.3\left(\mathrm{CH}_{2}, \mathrm{OBn}\right)$, $69.4(\mathrm{CH}, \mathrm{C}-2), 69.0\left(\mathrm{CH}_{2}, \mathrm{C}-6\right), 62.2\left(\mathrm{CH}_{2}, \mathrm{C}-3^{\prime}\right), 29.2\left(\mathrm{CH}_{2}, \mathrm{C}-\right.$ $\left.2^{\prime}\right), 25.8 \mathrm{ppm}\left(\mathrm{CH}_{2}, \mathrm{C}-1^{\prime}\right)$. IR $\left(\mathrm{CHCl}_{3}\right): \nu=3562,3500,2933,1094$ $\mathrm{cm}^{-1}$. MS (ESI) $\mathrm{m} / z(\%)=515(100)[\mathrm{M}+\mathrm{Na}]^{+}$. HRMS (ESI) $\mathrm{m} / z$ : $[\mathrm{M}+\mathrm{Na}]^{+}$calcd for $\mathrm{C}_{30} \mathrm{H}_{36} \mathrm{NaO}_{6}$ 515.2410; found 515.2403. Anal. calcd for $\mathrm{C}_{30} \mathrm{H}_{36} \mathrm{O}_{6}$ : C, 78.15; H, 7.87. Found $\mathrm{C}, 78.07 ; \mathrm{H}, 7.60$.

3-C-(3,4,6-Tri-O-benzyl- $\beta$-D-mannopyranosyl)1-propanol (72). Following the general procedure for the hydroboration, starting from 3-C-(3,4,6-tri- $O$-benzyl- $\beta$-D-mannopyranosyl) 1 -propene $(71)^{54,63}(106 \mathrm{mg}, 0.22 \mathrm{mmol})$ and purification by column chromatography (hexanes-EtOAc, 7:3), the alcohol $72(72.6 \mathrm{mg}$, $0.15 \mathrm{mmol}, 67 \%)$ was obtained as a colorless oil: $[\alpha]_{\mathrm{D}}=+2.1(c=$ $\left.0.48, \mathrm{CHCl}_{3}\right) .{ }^{1} \mathrm{H}$ NMR $\left(400 \mathrm{MHz}, \mathrm{CDCl}_{3}\right) \delta_{\mathrm{H}} 7.37-7.17(\mathrm{~m}, 15 \mathrm{H}$, $\mathrm{Ar}), 4.83(\mathrm{~d}, J=10.8 \mathrm{~Hz}, 1 \mathrm{H}, \mathrm{OBn}), 4.71(\mathrm{~d}, J=11.6 \mathrm{~Hz}, 1 \mathrm{H}, \mathrm{OBn})$, $4.64(\mathrm{~d}, J=11.7 \mathrm{~Hz}, 1 \mathrm{H}, \mathrm{OBn}), 4.57(\mathrm{~d}, J=12.1 \mathrm{~Hz}, 1 \mathrm{H}, \mathrm{OBn}), 4.52$ (d, $J=12.1 \mathrm{~Hz}, 1 \mathrm{H}, \mathrm{OBn}$ ), 4.49 (d, $J=10.8 \mathrm{~Hz}, 1 \mathrm{H}, \mathrm{OBn}$ ), 3.90 (dd, $J=2.9,0.0 \mathrm{~Hz}, 1 \mathrm{H}, 2-\mathrm{H}), 3.74(\mathrm{dd}, J=9.6,9.6 \mathrm{~Hz}, 1 \mathrm{H}, 4-\mathrm{H}), 3.70$ $\left(\mathrm{dd}, J=8.0,1.6 \mathrm{~Hz}, 1 \mathrm{H}, 6-\mathrm{H}_{\mathrm{b}}\right), 3.63\left(\mathrm{~m}, 3 \mathrm{H}, 6-\mathrm{H}_{\mathrm{a}}, 3-\mathrm{H}_{2}\right), 3.57$ (dd, $J$ $=9.0,3.2 \mathrm{~Hz}, 1 \mathrm{H}, 3-\mathrm{H}), 3.40(\mathrm{ddd}, J=9.8,5.4,1.9 \mathrm{~Hz}, 1 \mathrm{H}, 5-\mathrm{H})$, 3.35 (ddd, $J=9.2,3.8,0.0 \mathrm{~Hz}, 1 \mathrm{H}, 1-\mathrm{H}), 2.94(\mathrm{br} \mathrm{s}, 2 \mathrm{H}, 2 \times \mathrm{OH})$, $1.88\left(\mathrm{~m}, 1 \mathrm{H}, \mathrm{l}^{\prime}-\mathrm{H}_{\mathrm{b}}\right), 1.75-1.64 \mathrm{ppm}\left(\mathrm{m}, 3 \mathrm{H}, 1^{\prime}-\mathrm{H}_{\mathrm{a}}, 2^{\prime}-\mathrm{H}_{2}\right) .{ }^{13} \mathrm{C}\left\{{ }^{1} \mathrm{H}\right\}$ NMR $\left(100.6 \mathrm{MHz}, \mathrm{CDCl}_{3}\right) \delta_{\mathrm{C}} 138.2(\mathrm{C}, \mathrm{Ar}), 138.1$ (C, Ar), 137.8 (C, Ar), 127.6-128.5 (15 × CH, Ar), $83.4(\mathrm{CH}, \mathrm{C}-3), 79.0(\mathrm{CH}, \mathrm{C}-$ 1), 78.1 ( $\mathrm{CH}, \mathrm{C}-5), 75.1\left(\mathrm{CH}_{2}, \mathrm{OBn}\right), 74.7(\mathrm{CH}, \mathrm{C}-4), 73.4\left(\mathrm{CH}_{2}\right.$, $\mathrm{OBn}), 71.6\left(\mathrm{CH}_{2}, \mathrm{OBn}\right), 69.4\left(\mathrm{CH}_{2}, \mathrm{C}-6\right), 68.7(\mathrm{CH}, \mathrm{C}-2), 62.6$ $\left(\mathrm{CH}_{2}, \mathrm{C}-3^{\prime}\right), 29.4\left(\mathrm{CH}_{2}, \mathrm{C}-2^{\prime}\right), 28.0 \mathrm{ppm}\left(\mathrm{CH}_{2}, \mathrm{C}-1^{\prime}\right)$. IR $\left(\mathrm{CHCl}_{3}\right): \nu$ $=3461,2869,1455,1093 \mathrm{~cm}^{-1}$. MS (ESI) $\mathrm{m} / z(\%)=515(100)[\mathrm{M}$ $+\mathrm{Na}]^{+}$. HRMS (ESI) $m / z:[\mathrm{M}+\mathrm{Na}]^{+}$calcd for $\mathrm{C}_{30} \mathrm{H}_{36} \mathrm{NaO}_{6}$ 515.2410; found 515.2403. Anal. calcd for $\mathrm{C}_{30} \mathrm{H}_{36} \mathrm{O}_{6}$ : C, 73.15; $\mathrm{H}$, 7.37. Found: C, 73.36; H, 7.67 .

3-C-(3,4-Di-O-benzyl- $\alpha$-L-fucopyranosyl) 1-propanol (76). Following the general procedure for the hydroboration, starting from 75 (744.3 $\mathrm{mg}, 2.02 \mathrm{mmol}$ ) and purification by column chromatography (hexanes-EtOAc, 2:8), the alcohol $76(442.9 \mathrm{mg}, 1.15 \mathrm{mmol}, 57 \%)$ was obtained as a colorless oil: $[\alpha]_{\mathrm{D}}=-51.4\left(c=0.52, \mathrm{CHCl}_{3}\right) .{ }^{1} \mathrm{H}$ NMR $\left(500 \mathrm{MHz}, \mathrm{CDCl}_{3}\right) \delta_{\mathrm{H}} 7.35-7.25(\mathrm{~m}, 10 \mathrm{H}, \mathrm{Ar}), 4.78$ (d, $J=$ $11.7 \mathrm{~Hz}, 1 \mathrm{H}, \mathrm{OBn}$ ), 4.75 (d, $J=11.7 \mathrm{~Hz}, 1 \mathrm{H}, \mathrm{OBn}), 4.60$ (d, $J=11.7$ $\mathrm{Hz}, 1 \mathrm{H}, \mathrm{OBn}), 4.58(\mathrm{~d}, J=11.7 \mathrm{~Hz}, 1 \mathrm{H}, \mathrm{OBn}), 4.07-4.06(\mathrm{~m}, 2 \mathrm{H}, 1-$ $\mathrm{H}, 2-\mathrm{H}), 3.91(\mathrm{~m}, 1 \mathrm{H}, 5-\mathrm{H}), 3.76(\mathrm{dd}, J=3.2,3.2 \mathrm{~Hz}, 1 \mathrm{H}, 4-\mathrm{H}), 3.71$ (dd, $J=6.6,2.5 \mathrm{~Hz}, 1 \mathrm{H}, 3-\mathrm{H}), 3.67-3.61\left(\mathrm{~m}, 2 \mathrm{H}, 3{ }^{\prime}-\mathrm{H}_{2}\right), 2.40$ (br s, $1 \mathrm{H}, \mathrm{OH}), 2.21(\mathrm{br} \mathrm{s}, 1 \mathrm{H}, \mathrm{OH}), 1.69-1.62\left(\mathrm{~m}, 4 \mathrm{H}, 1^{\prime}-\mathrm{H}_{2}, 2^{\prime}-\mathrm{H}_{2}\right), 1.31$ ppm $\left(\mathrm{d}, J=6.6 \mathrm{~Hz}, 3 \mathrm{H}, 6-\mathrm{H}_{3}\right) .{ }^{13} \mathrm{C}\left\{{ }^{1} \mathrm{H}\right\} \mathrm{NMR}\left(100.6 \mathrm{MHz}, \mathrm{CDCl}_{3}\right)$ $\delta_{\mathrm{C}} 138.4(\mathrm{C}, \mathrm{Ar}), 138.2(\mathrm{C}, \mathrm{Ar}), 128.5(2 \times \mathrm{CH}, \mathrm{Ar}), 128.3(2 \times \mathrm{CH}$, $\mathrm{Ar}), 127.7(3 \times \mathrm{CH}, \mathrm{Ar}), 127.6(3 \times \mathrm{CH}, \mathrm{Ar}), 79.0(\mathrm{CH}, \mathrm{C}-3), 75.1$ (CH, C-4), $73.2\left(\mathrm{CH}_{2}, \mathrm{OBn}\right), 72.6\left(\mathrm{CH}_{2}, \mathrm{OBn}\right), 71.8(\mathrm{CH}, \mathrm{C}-1$ or $\mathrm{C}-$ 2), $69.1(\mathrm{CH}, \mathrm{C}-5), 68.9\left(\mathrm{CH}, \mathrm{C}-1\right.$ or C-2), $62.6\left(\mathrm{CH}_{2}, \mathrm{C}-3\right.$ '), $23.6(2$ $\left.\times \mathrm{CH}_{2}, \mathrm{C}-1^{\prime}, \mathrm{C}-2^{\prime}\right), 15.6 \mathrm{ppm}\left(\mathrm{CH}_{3}, \mathrm{C}-6\right) . \mathrm{IR}\left(\mathrm{CHCl}_{3}\right): \nu=3585$, $3422,3016,1228 \mathrm{~cm}^{-1}$. MS (ESI) $\mathrm{m} / z(\%)=409(100)\left[\mathrm{M}+\mathrm{Na}^{+}\right.$. HRMS (ESI) $m / z:[\mathrm{M}+\mathrm{Na}]^{+}$calcd for $\mathrm{C}_{23} \mathrm{H}_{30} \mathrm{NaO}_{5}$ 409.1991; found 409.1990 .

3-C-(3,4-Di-O-benzylidene- $\alpha, \beta$-D-arabinopyranosyl) 1-propanol (81). Following the general procedure for the hydroboration, starting from $79(180 \mathrm{mg}, 0.69 \mathrm{mmol})$ and purification by column chromatography (hexanes-EtOAc, 2:8), the alcohol 81 (106.1 mg, 
$0.38 \mathrm{mmol}, 55 \%)$ was obtained as a colorless oil: ${ }^{1} \mathrm{H}$ NMR $(500 \mathrm{MHz}$, $\mathrm{CDCl}_{3}$, only the major $1 S$ isomer is described) $\delta_{\mathrm{H}} 7.47-7.37(\mathrm{~m}, 5 \mathrm{H}$, Ar), $6.22($ br s, $1 \mathrm{H}, \mathrm{PhCH}), 4.57$ (ddd, $J=8.9,6.4,5.1 \mathrm{~Hz}, 1 \mathrm{H}, 4-\mathrm{H})$, $4.29(\mathrm{dd}, J=5.1,2.3 \mathrm{~Hz}, 1 \mathrm{H}, 3-\mathrm{H}), 4.08(\mathrm{dd}, J=11.7,6.6 \mathrm{~Hz}, 1 \mathrm{H}, 5-$ $\mathrm{H}_{\mathrm{b}}$ ), 3.95 (br s, $\left.1 \mathrm{H}, 2-\mathrm{H}\right), 3.70-3.66\left(\mathrm{~m}, 3 \mathrm{H}, 3^{\prime}-\mathrm{H}_{2}, 1-\mathrm{H}\right), 3.54$ (dd, $J$ $\left.=11.7,8.8 \mathrm{~Hz}, 1 \mathrm{H}, 5-\mathrm{H}_{\mathrm{a}}\right), 2.5(\mathrm{br} \mathrm{s}, 1 \mathrm{H}, \mathrm{OH}), 2.66(\mathrm{br} \mathrm{s}, 1 \mathrm{H}, \mathrm{OH})$, 1.82-1.66 ppm (m, 4H, 1'- $\left.\mathrm{H}_{2}, 2^{\prime}-\mathrm{H}_{2}\right) .{ }^{13} \mathrm{C}\left\{{ }^{1} \mathrm{H}\right\}$ NMR (125.7 MHz, $\mathrm{CDCl}_{3}$, only the major $1 \mathrm{~S}$ isomer is described) $\delta_{\mathrm{C}} 139.0(\mathrm{C}, \mathrm{Ar})$, $129.1(\mathrm{CH}, \mathrm{Ar}), 128.4(2 \times \mathrm{CH}, \mathrm{Ar}), 125.9(2 \times \mathrm{CH}, \mathrm{Ar}), 103.3$ $(\mathrm{CH}, \mathrm{PhCH}), 75.7(\mathrm{CH}, \mathrm{C}-1$ or $\mathrm{C}-3), 75.6(\mathrm{CH}, \mathrm{C}-1$ or $\mathrm{C}-3), 70.1$ (CH, C-4), $68.0(\mathrm{CH}, \mathrm{C}-2), 65.9\left(\mathrm{CH}_{2}, \mathrm{C}-5\right), 62.6\left(\mathrm{CH}_{2}, \mathrm{C}-3^{\prime}\right), 28.9$ $\left(\mathrm{CH}_{2}, \mathrm{C}-1^{\prime}\right.$ or C-2'), $26.7 \mathrm{ppm}\left(\mathrm{CH}_{2}, \mathrm{C}-1^{\prime}\right.$ or C-2'). IR $\left(\mathrm{CHCl}_{3}\right): \nu=$ $3613,3417,2927,1208,1070 \mathrm{~cm}^{-1}$. MS (ESI) $\mathrm{m} / z(\%)=303(100)$ $[\mathrm{M}+\mathrm{Na}]^{+}$. HRMS (ESI) $m / z:[\mathrm{M}+\mathrm{Na}]^{+}$calcd for $\mathrm{C}_{15} \mathrm{H}_{20} \mathrm{NaO}_{5}$ 303.1208; found 303.1203.

3-C-(3,5-Di-O-tert-butyldiphenylsilyl- $\alpha$-D-ribofuranosyl) 1-propanol (85). The general procedure for the hydroboration starting from 83 (3.55 g, $5.46 \mathrm{mmol}$ ) but adding dropwise at $0{ }^{\circ} \mathrm{C}$ an aqueous saturated solution of $\mathrm{NaHCO}_{3}(35.5 \mathrm{~mL})$ instead of the $\mathrm{NaOH}$ solution followed by $\mathrm{H}_{2} \mathrm{O}_{2} 30 \%(18 \mathrm{~mL})$ gave, after purification by column chromatography (hexanes-EtOAc, 97:3 to 7:3), the alcohol $85(1.28 \mathrm{mg}, 1.92 \mathrm{mmol}, 35 \%)$ as a colorless oil: $[\alpha]_{\mathrm{D}}=+23.0(c=$ 0.64, $\left.\mathrm{CHCl}_{3}\right) .{ }^{1} \mathrm{H}$ NMR $\left(500 \mathrm{MHz}, \mathrm{CDCl}_{3}\right) \delta_{\mathrm{H}} 7.65-7.26(\mathrm{~m}, 20 \mathrm{H}$, Ar), $4.53(\mathrm{dd}, J=5.4,5.4 \mathrm{~Hz}, 1 \mathrm{H}, 3-\mathrm{H}), 3.97(\mathrm{~m}, 1 \mathrm{H}, 4-\mathrm{H}), 3.88(\mathrm{~m}$, $1 \mathrm{H}, 1-\mathrm{H}), 3.82(\mathrm{dd}, J=5.1,5.1 \mathrm{~Hz}, 1 \mathrm{H}, 2-\mathrm{H}), 3.71-3.62(\mathrm{~m}, 2 \mathrm{H}, 3$ ' $\left.\mathrm{H}_{2}\right), 3.57\left(\mathrm{dd}, J=11.4,2.2 \mathrm{~Hz}, 1 \mathrm{H}, 5-\mathrm{H}_{\mathrm{b}}\right), 3.25(\mathrm{dd}, J=11.4,3.8 \mathrm{~Hz}$, $\left.1 \mathrm{H}, 5-\mathrm{H}_{\mathrm{a}}\right), 1.82-1.77\left(\mathrm{~m}, 2 \mathrm{H}, 1^{\prime}-\mathrm{H}_{2}\right), 1.71-1.66\left(\mathrm{~m}, 2 \mathrm{H}, 2^{\prime}-\mathrm{H}_{2}\right), 1.61$ (br s, 2H, OH), $1.08\left(\mathrm{~s}, 9 \mathrm{H},{ }^{t} \mathrm{Bu}\right), 0.91 \mathrm{ppm}\left(\mathrm{s}, 9 \mathrm{H},{ }^{t} \mathrm{Bu}\right) .{ }^{13} \mathrm{C}\left\{{ }^{1} \mathrm{H}\right\}$ NMR $\left(125.7 \mathrm{MHz}, \mathrm{CDCl}_{3}\right) \delta_{\mathrm{C}} 135.67(2 \times \mathrm{CH}, \mathrm{Ar}), 135.62(2 \times$ $\mathrm{CH}, \mathrm{Ar}), 135.58(2 \times \mathrm{CH}, \mathrm{Ar}), 135.53(2 \times \mathrm{CH}, \mathrm{Ar}), 133.3(\mathrm{C}, \mathrm{Ar})$, 133.2 (C, Ar), 132.5 (C, Ar), 132.3 (C, Ar), 130.25 (CH, Ar), 130.20 (CH, Ar), $129.5(2 \times \mathrm{CH}, \mathrm{Ar}), 128.0(2 \times \mathrm{CH}, \mathrm{Ar}), 127.9(2 \times \mathrm{CH}$, $\mathrm{Ar}), 127.6(4 \times \mathrm{CH}, \mathrm{Ar}), 83.0(\mathrm{CH}, \mathrm{C}-4), 81.5(\mathrm{CH}, \mathrm{C}-1), 74.6(\mathrm{CH}$, C-3), $73.0(\mathrm{CH}, \mathrm{C}-2), 64.0\left(\mathrm{CH}_{2}, \mathrm{C}-5\right), 62.8\left(\mathrm{CH}_{2}, \mathrm{C}-3\right), 29.6\left(\mathrm{CH}_{2}\right.$, $\left.\mathrm{C}-2^{\prime}\right), 26.9\left(3 \times \mathrm{CH}_{3},{ }^{t} \mathrm{Bu}\right), 26.7\left(3 \times \mathrm{CH}_{3},{ }^{t} \mathrm{Bu}\right), 26.1\left(\mathrm{CH}_{2}, \mathrm{C}-1^{\prime}\right)$, $19.2\left(\mathrm{C},{ }^{t} \mathrm{Bu}\right), 19.1 \mathrm{ppm}\left(\mathrm{C},{ }^{t} \mathrm{Bu}\right)$. IR $\left(\mathrm{CHCl}_{3}\right): \nu=3532,2932,1428$, $1206,1113 \mathrm{~cm}^{-1}$. MS (ESI) $\mathrm{m} / z(\%)=691(100)[\mathrm{M}+\mathrm{Na}]^{+}$. HRMS (ESI) $m / z:[\mathrm{M}+\mathrm{Na}]^{+}$calcd for $\mathrm{C}_{40} \mathrm{H}_{52} \mathrm{NaO}_{5} \mathrm{Si}_{2}$ 691.3251; found 691.3250 .

3-C-(3,5-Di-O-1,1,3,3-tetraisopropyldisiloxanyl- $\alpha-D-$ ribofuranosyl)1-propanol (87). The general procedure for the hydroboration starting from $86(228 \mathrm{mg}, 0.55 \mathrm{mmol})$ but adding dropwise at $0{ }^{\circ} \mathrm{C}$ an aqueous saturated solution of $\mathrm{NaHCO}_{3}(3.1 \mathrm{~mL})$ instead of the $\mathrm{NaOH}$ solution followed by $\mathrm{H}_{2} \mathrm{O}_{2} 30 \%(1.6 \mathrm{~mL})$ gave, after purification by column chromatography (hexanes-EtOAc, 1:1), the alcohol $87(150.47 \mathrm{mg}, 0.35 \mathrm{mmol}, 63 \%)$ as a colorless oil: $[\alpha]_{\mathrm{D}}=$ $-10.8\left(c=0.62, \mathrm{CHCl}_{3}\right) .{ }^{1} \mathrm{H} \mathrm{NMR}\left(500 \mathrm{MHz}, \mathrm{CDCl}_{3}\right) \delta_{\mathrm{H}} 4.38(\mathrm{dd}, J$ $=7.3,4.8 \mathrm{~Hz}, 1 \mathrm{H}, 3-\mathrm{H}), 5.00(\mathrm{dd}, J=4.7,4.7 \mathrm{~Hz}, 1 \mathrm{H}, 2-\mathrm{H}), 4.00-$ $3.96\left(\mathrm{~m}, 2 \mathrm{H}, 1-\mathrm{H}, 5-\mathrm{H}_{\mathrm{b}}\right), 3.91$ (ddd, $J=7.3,7.3,3.5 \mathrm{~Hz}, 1 \mathrm{H}, 4-\mathrm{H}$ ), 3.64 (dd, $\left.J=12.0,6.3 \mathrm{~Hz}, 1 \mathrm{H}, 5-\mathrm{H}_{\mathrm{a}}\right), 3.69-3.63\left(\mathrm{~m}, 2 \mathrm{H}, 3{ }^{\prime}-\mathrm{H}_{2}\right), 2.23$ (br s, $2 \mathrm{H}, \mathrm{OH}$ ), 1.84 (ddd, $J=14.2,7.6,7.6 \mathrm{~Hz}, 1 \mathrm{H}, 1^{\prime}-\mathrm{H}_{\mathrm{b}}$ ), $1.79-$ $1.64\left(\mathrm{~m}, 3 \mathrm{H}, 11^{\prime}-\mathrm{H}_{\mathrm{a}}, 2^{\prime}-\mathrm{H}_{2}\right), 1.11-0.95 \mathrm{ppm}\left(\mathrm{m}, 28 \mathrm{H},{ }^{i} \mathrm{Pr}\right) .{ }^{13} \mathrm{C}\left\{{ }^{1} \mathrm{H}\right\}$ NMR $\left(125.7 \mathrm{MHz}, \mathrm{CDCl}_{3}\right) \delta_{\mathrm{C}} 80.8(\mathrm{CH}, \mathrm{C}-1), 80.3(\mathrm{CH}, \mathrm{C}-4), 74.8$ (CH, C-3), 72.8 (CH, C-2), $63.2\left(\mathrm{CH}_{2}, \mathrm{C}-5\right), 62.7\left(\mathrm{CH}_{2}, \mathrm{C}-3\right), 29.3$ $\left(\mathrm{CH}_{2}, \mathrm{C}-2^{\prime}\right), 25.8\left(\mathrm{CH}_{2}, \mathrm{C}-1{ }^{\prime}\right), 17.4\left(\mathrm{CH}_{3},{ }^{i} \mathrm{Pr}\right), 17.33\left(\mathrm{CH}_{3},{ }^{i} \mathrm{Pr}\right)$, $17.30\left(2 \times \mathrm{CH}_{3},{ }^{i} \mathrm{Pr}\right), 17.19\left(\mathrm{CH}_{3},{ }^{i} \mathrm{Pr}\right), 17.02\left(2 \times \mathrm{CH}_{3},{ }^{i} \mathrm{Pr}\right), 16.93$ $\left(\mathrm{CH}_{3},{ }^{i} \mathrm{Pr}\right), 13.4\left(\mathrm{CH},{ }^{i} \mathrm{Pr}\right), 13.2\left(\mathrm{CH},{ }^{i} \mathrm{Pr}\right), 12.8\left(\mathrm{CH},{ }^{i} \mathrm{Pr}\right), 12.6 \mathrm{ppm}$ $\left(\mathrm{CH},{ }^{i} \mathrm{Pr}\right)$. IR $\left(\mathrm{CHCl}_{3}\right): \nu=3622,3528,2948,2870,1465,1041$ $\mathrm{cm}^{-1}$. MS (ESI) $m / z(\%)=457(100)[\mathrm{M}+\mathrm{Na}]^{+}$. HRMS (ESI) $\mathrm{m} / z$ : $[\mathrm{M}+\mathrm{Na}]^{+}$calcd for $\mathrm{C}_{20} \mathrm{H}_{42} \mathrm{NaO}_{6} \mathrm{Si}_{2}$ 457.2418; found 457.2413.

General Procedure for the Mitsunobu Reaction to Give Phthalimide Derivatives 11, 13, 14, 64, 67, 70, 73, 77, and 88 . DEAD ( $449 \mu \mathrm{L}, 2.58 \mathrm{mmol})$ was added dropwise to a stirred solution of the alcohol (1 mmol), $N$-hydroxyphthalimide $(420 \mathrm{mg}, 2.58$ $\mathrm{mmol})$, and $\mathrm{PPh}_{3}(670 \mathrm{mg}, 2.58 \mathrm{mmol})$ in dry THF $(10.3 \mathrm{~mL})$, and the resulting solution was stirred at $0{ }^{\circ} \mathrm{C}$ for $1-4 \mathrm{~h}$. Then, the solvent was removed and the crude was quenched with water and extracted with $\mathrm{CHCl}_{3}$. The combined extracts were dried over $\mathrm{Na}_{2} \mathrm{SO}_{4}$ and concentrated under reduced pressure. Column chromatography of the residue (hexanes-EtOAc) gave the corresponding phthalimide.
3-C-(3,4-Di-O-benzylidene-2-O-diphenoxyphosphoryl- $\alpha, \beta-D$ arabinopyranosyl)1-propoxyphthalimide (11). Following the general procedure starting from alcohol $\mathbf{8 1}(95.2 \mathrm{mg}, 0.34 \mathrm{mmol})$ stirring at $0{ }^{\circ} \mathrm{C}$ for $0.5 \mathrm{~h}$, after purification by column chromatography (hexanes-EtOAc, 6:4), a phthalimide intermediate $(202.4 \mathrm{mg}$ ) was obtained as a yellow oil. The crude $(202.4 \mathrm{mg})$ was dissolved in dry $\mathrm{CH}_{2} \mathrm{Cl}_{2}(10.2 \mathrm{~mL})$ under a $\mathrm{N}_{2}$ atmosphere. $\mathrm{ClPO}(\mathrm{OPh})_{2}(324 \mu \mathrm{L}$, $1.6 \mathrm{mmol})$ and DMAP $(195.5 \mathrm{mg}, 1.6 \mathrm{mmol})$ were added at $0{ }^{\circ} \mathrm{C}$, and after $5 \mathrm{~min}$, the mixture was stirred at room temperature for $1.5 \mathrm{~h}$. The reaction was quenched with a saturated aqueous $\mathrm{NH}_{4} \mathrm{Cl}$ solution and extracted with $\mathrm{CH}_{2} \mathrm{Cl}_{2}$. The organic extracts were dried over $\mathrm{Na}_{2} \mathrm{SO}_{4}$ and concentrated under reduced pressure. Column chromatography of the residue $\left(\mathrm{PhCH}_{3}-\mathrm{EtOAc}, 9: 1\right)$ gave 11 (149.4 mg, $0.23 \mathrm{mmol}, 67 \%, 1 \mathrm{~S} / 1 \mathrm{R} \mathrm{4.7:1)}$ as a colorless oil. ${ }^{1} \mathrm{H}$ NMR $\left(500 \mathrm{MHz}, \mathrm{CDCl}_{3}\right.$, only the major $1 S$ isomer is described, simulated ring coupling constants using DAISY) $\delta_{\mathrm{H}} 7.84-7.81(\mathrm{~m}$, $2 \mathrm{H}, \mathrm{Ar}), 7.75-7.72(\mathrm{~m}, 2 \mathrm{H}, \mathrm{Ar}), 7.40-7.14(\mathrm{~m}, 15 \mathrm{H}, \mathrm{Ar}), 6.20(\mathrm{~s}$, $1 \mathrm{H}, \mathrm{PhCH}), 4.88$ (ddd, $\left.J=3.3,2.0 \mathrm{~Hz},{ }^{3} J_{\mathrm{PH}}=9.2 \mathrm{~Hz}, 1 \mathrm{H}, 2-\mathrm{H}\right), 4.38$ (ddd, $J=7.9,6.0,5.2 \mathrm{~Hz}, 1 \mathrm{H}, 4-\mathrm{H}), 4.32$ (dd, $J=5.2,3.3 \mathrm{~Hz}, 1 \mathrm{H}, 3-$ $\mathrm{H}), 4.13$ (ddd, $\left.J=6.7,6.7,1.3 \mathrm{~Hz}, 2 \mathrm{H}, 3{ }^{\prime}-\mathrm{H}_{2}\right), 4.04(\mathrm{dd}, J=12.2,6.0$ $\left.\mathrm{Hz}, 1 \mathrm{H}, 5-\mathrm{H}_{\mathrm{b}}\right), 3.83(\mathrm{~m}, 1 \mathrm{H}, 1-\mathrm{H}), 3.59$ (dd, $J=12.2,7.9 \mathrm{~Hz}, 1 \mathrm{H}, 5-$ $\left.\mathrm{H}_{\mathrm{a}}\right), 1.92\left(\mathrm{~m}, 1 \mathrm{H}, 2^{\prime}-\mathrm{H}_{\mathrm{b}}\right), 1.85-1.76\left(\mathrm{~m}, 2 \mathrm{H}, 1^{\prime}-\mathrm{H}_{\mathrm{b}}, 2^{\prime}-\mathrm{H}_{\mathrm{a}}\right), 1.67 \mathrm{ppm}$ $\left(\mathrm{m}, 1 \mathrm{H}, 1\right.$ ' $\left.-\mathrm{H}_{\mathrm{a}}\right) \cdot{ }^{13} \mathrm{C}\left\{{ }^{1} \mathrm{H}\right\}$ NMR $\left(125.7 \mathrm{MHz}, \mathrm{CDCl}_{3}\right.$, only the major $1 S$ isomer is described $) \delta_{\mathrm{C}} 163.6(2 \times \mathrm{C}, \mathrm{CO}), 150.4\left(\mathrm{~d},{ }^{2} J_{\mathrm{PC}}=8.4\right.$ $\mathrm{Hz}, \mathrm{C}, \mathrm{Ar}), 150.3$ (d, $\left.{ }^{2} J_{\mathrm{PC}}=7.4 \mathrm{~Hz}, \mathrm{C}, \mathrm{Ar}\right), 138.6$ (C, Ar), $134.4(2 \times$ $\mathrm{CH}, \mathrm{Ar}), 129.9$ ( $2 \times \mathrm{CH}, \mathrm{Ar}), 129.8(2 \times \mathrm{CH}, \mathrm{Ar}), 129.1(2 \times \mathrm{C}, \mathrm{Ar})$, $129.0(\mathrm{CH}, \mathrm{Ar}), 128.4(2 \times \mathrm{CH}, \mathrm{Ar}), 126.0(2 \times \mathrm{CH}, \mathrm{Ar}), 125.58$ (CH, Ar), 125.55 (CH, Ar), $123.5(2 \times \mathrm{CH}, \mathrm{Ar}), 120.2(2 \times \mathrm{CH}, \mathrm{Ar})$, $120.1(2 \times \mathrm{CH}, \mathrm{Ar}), 103.4(\mathrm{CH}, \mathrm{PhCH}), 77.9\left(\mathrm{CH}_{2}, \mathrm{C}-3^{\prime}\right), 75.3(\mathrm{~d}$, $\left.{ }^{2} J_{\mathrm{PC}}=6.3 \mathrm{~Hz}, \mathrm{CH}, \mathrm{C}-2\right), 73.6\left(\mathrm{~d},{ }^{3} \mathrm{~J}_{\mathrm{PC}}=5.3 \mathrm{~Hz}, \mathrm{CH}, \mathrm{C}-1\right.$ or C-3), $73.5\left(\mathrm{~d},{ }^{3} \mathrm{~J}_{\mathrm{PC}}=2.1 \mathrm{~Hz}, \mathrm{CH}, \mathrm{C}-1\right.$ or C-3), $70.5(\mathrm{CH}, \mathrm{C}-4), 64.6\left(\mathrm{CH}_{2}\right.$, C-5), $25.8\left(\mathrm{CH}_{2}, \mathrm{C}-1^{\prime}\right), 24.5 \mathrm{ppm}\left(\mathrm{CH}_{2}, \mathrm{C}-2^{\prime}\right)$. IR $\left(\mathrm{CHCl}_{3}\right): \nu=$ $3018,1791,1734,1226 \mathrm{~cm}^{-1}$. MS (ESI) $\mathrm{m} / z(\%)=680(100)[\mathrm{M}+$ $\mathrm{Na}]^{+}$. HRMS (ESI) $m / z$ : $[\mathrm{M}+\mathrm{Na}]^{+}$calcd for $\mathrm{C}_{35} \mathrm{H}_{32} \mathrm{NNaO}_{10} \mathrm{P}$ 680.1662; found 680.1661. Anal. calcd for $\mathrm{C}_{35} \mathrm{H}_{32} \mathrm{NO}_{10} \mathrm{P}: \mathrm{C}, 63.92$; $\mathrm{H}, 4.90: \mathrm{N}, 2.13$. Found: C, 64.10; H, 5.16; N, 2.33 .

3-C-(2-O-Acetyl-3,5-di-O-tert-butyldiphenylsilyl- $\alpha-D-$ ribofuranosyl)1-propoxyphthalimide (13). Following the general procedure starting from alcohol $85(604 \mathrm{mg}, 0.90 \mathrm{mmol})$ and stirring at $50{ }^{\circ} \mathrm{C}$ for $2 \mathrm{~h}$, after purification by column chromatography (hexanes-EtOAc, 8:2), a phthalimide intermediate was obtained (715.4 mg, $0.88 \mathrm{mmol}, 98 \%)$ as a colorless oil. Phthalimide (715.4 $\mathrm{mg}, 0.88 \mathrm{mmol}$ ) was dissolved in dry pyridine $(3.4 \mathrm{~mL})$, and $\mathrm{Ac}_{2} \mathrm{O}$ $(1.15 \mathrm{~mL})$ and DMAP $\left(1.1 \mathrm{mg}, 9.0 \cdot 10^{-3} \mathrm{mmol}\right)$ were added. The reaction was stirred at room temperature for $1 \mathrm{~h}$, and then it was evaporated in a high vacuum rotovap, quenched with an aqueous solution of $\mathrm{HCl} 10 \%$, and extracted with $\mathrm{CH}_{2} \mathrm{Cl}_{2}$. The combined organic extracts were dried over $\mathrm{Na}_{2} \mathrm{SO}_{4}$ and concentrated under reduced pressure. Column chromatography of the residue (hexanesEtOAc, 8:2) gave $13(589.9 \mathrm{mg}, 0.69 \mathrm{mmol}, 78 \%)$ as a white solid. $\mathrm{mp} 42.3-43.7{ }^{\circ} \mathrm{C}\left(n\right.$-hexane-EtOAc); $[\alpha]_{\mathrm{D}}=+33.2(c=0.76$, $\left.\mathrm{CHCl}_{3}\right) .{ }^{1} \mathrm{H} \mathrm{NMR}\left(500 \mathrm{MHz}, \mathrm{CDCl}_{3}\right.$, simulated ring coupling constants using DAISY) $\delta_{\mathrm{H}} 7.82-7.71(\mathrm{~m}, 4 \mathrm{H}, \mathrm{Ar}), 7.67-7.27(\mathrm{~m}$, $20 \mathrm{H}, \mathrm{Ar}), 5.17(\mathrm{dd}, J=4.7,3.4 \mathrm{~Hz}, 1 \mathrm{H}, 2-\mathrm{H}), 4.64(\mathrm{dd}, J=6.7,4.7$ $\mathrm{Hz}, 1 \mathrm{H}, 3-\mathrm{H}), 4.22-4.17\left(\mathrm{~m}, 2 \mathrm{H}, 3\right.$ ' $\left.-\mathrm{H}_{2}\right), 4.03-3.98(\mathrm{~m}, 2 \mathrm{H}, 1-\mathrm{H}, 4-$ $\mathrm{H}), 3.61\left(\mathrm{dd}, J=11.4,2.2 \mathrm{~Hz}, 1 \mathrm{H}, 5-\mathrm{H}_{\mathrm{b}}\right), 3.31(\mathrm{dd}, J=11.4,3.2 \mathrm{~Hz}$, $\left.1 \mathrm{H}, 5-\mathrm{H}_{\mathrm{a}}\right), 2.15(\mathrm{~s}, 3 \mathrm{H}, \mathrm{OAc}), 1.95\left(\mathrm{~m}, 1 \mathrm{H}, 2^{\prime}-\mathrm{H}_{\mathrm{b}}\right), 1.81-1.68(\mathrm{~m}$, $\left.3 \mathrm{H}, 1^{\prime}-\mathrm{H}_{2}, 2{ }^{\prime}-\mathrm{H}_{\mathrm{a}}\right), 1.04\left(\mathrm{~s}, 9 \mathrm{H},{ }^{t} \mathrm{Bu}\right), 0.90 \mathrm{ppm}\left(\mathrm{s}, 9 \mathrm{H},{ }^{t} \mathrm{Bu}\right) .{ }^{13} \mathrm{C}\left\{{ }^{1} \mathrm{H}\right\}$ NMR $\left(125.7 \mathrm{MHz}, \mathrm{CDCl}_{3}\right) \delta_{\mathrm{C}} 170.4(\mathrm{C}, \mathrm{OAc}), 163.6(2 \times \mathrm{C}, \mathrm{CO})$, $135.84(2 \times \mathrm{CH}, \mathrm{Ar}), 135.79(2 \times \mathrm{CH}, \mathrm{Ar}), 135.64(2 \times \mathrm{CH}, \mathrm{Ar})$, $135.59(2 \times \mathrm{CH}, \mathrm{Ar}), 134.4(2 \times \mathrm{CH}, \mathrm{Ar}), 133.5$ (C, Ar), $133.4(\mathrm{C}$, $\mathrm{Ar}), 133.3$ (C, Ar), 132.8 (C, Ar), 129.98 (CH, Ar), 129.95 (CH, Ar), $129.5(2 \times \mathrm{CH}, \mathrm{Ar}), 129.0(2 \times \mathrm{C}, \mathrm{Ar}), 127.78(2 \times \mathrm{CH}, \mathrm{Ar}), 127.74$ $(2 \times \mathrm{CH}, \mathrm{Ar}), 127.57(2 \times \mathrm{CH}, \mathrm{Ar}), 127.55(2 \times \mathrm{CH}, \mathrm{Ar}), 123.5(2 \times$ $\mathrm{CH}, \mathrm{Ar}), 82.8$ (CH, C-4), $\left.79.0(\mathrm{CH}, \mathrm{C}-1), 78.3\left(\mathrm{CH}_{2}, \mathrm{C}-3\right)^{\prime}\right), 74.9$ $(\mathrm{CH}, \mathrm{C}-2), 72.8(\mathrm{CH}, \mathrm{C}-3), 63.6\left(\mathrm{CH}_{2}, \mathrm{C}-5\right), 26.8\left(3 \times \mathrm{CH}_{3},{ }^{t} \mathrm{Bu}\right)$, $26.7\left(3 \times \mathrm{CH}_{3},{ }^{t} \mathrm{Bu}\right), 26.1\left(\mathrm{CH}_{2}, \mathrm{C}-1^{\prime}\right), 25.0\left(\mathrm{CH}_{2}, \mathrm{C}-2{ }^{\prime}\right), 21.0\left(\mathrm{CH}_{3}\right.$, OAc), $19.2\left(\mathrm{C},{ }^{t} \mathrm{Bu}\right), 19.1 \mathrm{ppm}\left(\mathrm{C},{ }^{t} \mathrm{Bu}\right)$. IR $\left(\mathrm{CHCl}_{3}\right): \nu=2932,2860$, $1791,1731,1428,1242,1113 \mathrm{~cm}^{-1}$. MS (ESI) $\mathrm{m} / z(\%)=878(100)$ 
$[\mathrm{M}+\mathrm{Na}]^{+}$. HRMS (ESI) $m / z:[\mathrm{M}+\mathrm{Na}]^{+}$calcd for $\mathrm{C}_{50} \mathrm{H}_{57} \mathrm{NNaO}_{8} \mathrm{Si}_{2}$ 878.3520; found 878.3530 .

3-C-(3,5-Di-O-tert-butyldiphenylsilyl-2-O-trifluoromethylsulfon$y l-\alpha$-D-ribofuranosyl)1-propoxyphthalimide (14). Following the general procedure starting from alcohol $85(604 \mathrm{mg}, 0.90 \mathrm{mmol})$ and stirring at $50{ }^{\circ} \mathrm{C}$ for $2 \mathrm{~h}$, after purification by column chromatography (hexanes-EtOAc, 8:2), a phthalimide intermediate was obtained $(715.4 \mathrm{mg}, 0.88 \mathrm{mmol}, 98 \%)$ as a colorless oil. Phthalimide (715.4 mg, $0.88 \mathrm{mmol})$ was dissolved in dry pyridine $(0.26 \mathrm{~mL})$, and $\mathrm{Tf}_{2} \mathrm{O}(22 \mu \mathrm{L}, 0.13 \mathrm{mmol})$ was added. The reaction was stirred at room temperature for $1 \mathrm{~h}$, and then it was evaporated in a high vacuum rotovap, quenched with an aqueous solution of $\mathrm{HCl}$ $10 \%$, and extracted with $\mathrm{CH}_{2} \mathrm{Cl}_{2}$. The combined organic extracts were dried over $\mathrm{Na}_{2} \mathrm{SO}_{4}$ and concentrated under reduced pressure. Column chromatography of the residue (hexanes-EtOAc, 8:2) gave 14 (48.4 $\mathrm{mg}, 0.051 \mathrm{mmol}, 78 \%)$ as a white solid. $\mathrm{mp} 42.6-43.9^{\circ} \mathrm{C}$ ( $n$-hexaneEtOAc); $[\alpha]_{\mathrm{D}}=+20.8\left(c=0.63, \mathrm{CHCl}_{3}\right) .{ }^{1} \mathrm{H}$ NMR $(500 \mathrm{MHz}$, $\mathrm{CDCl}_{3}$, simulated ring coupling constants using DAISY) $\delta_{\mathrm{H}} 7.44-$ $7.42(\mathrm{~m}, 4 \mathrm{H}, \mathrm{Ar}), 7.41-7.24(\mathrm{~m}, 20 \mathrm{H}, \mathrm{Ar}), 5.30(\mathrm{dd}, J=4.6,4.1 \mathrm{~Hz}$, $1 \mathrm{H}, 2-\mathrm{H}), 4.71(\mathrm{dd}, J=5.7,4.6 \mathrm{~Hz}, 1 \mathrm{H}, 3-\mathrm{H}), 4.25-4.16(\mathrm{~m}, 3 \mathrm{H}, 1-$ $\mathrm{H}, 3$ ' $\left.-\mathrm{H}_{2}\right), 3.95(\mathrm{~m}, 1 \mathrm{H}, 4-\mathrm{H}), 3.35\left(\mathrm{dd}, J=11.6,2.0 \mathrm{~Hz}, 1 \mathrm{H}, 5-\mathrm{H}_{\mathrm{b}}\right)$, $2.76\left(\mathrm{dd}, J=11.6,3.1 \mathrm{~Hz}, 1 \mathrm{H}, 5-\mathrm{H}_{\mathrm{a}}\right), 2.05-1.92\left(\mathrm{~m}, 3 \mathrm{H}, 1^{\prime}-\mathrm{H}_{2}\right.$ or $2^{\prime}-$ $\mathrm{H}_{2}, 1^{\prime}-\mathrm{H}_{\mathrm{b}}$ or $\left.2^{\prime}-\mathrm{H}_{\mathrm{b}}\right), 1.82\left(\mathrm{dddd}, J=12.9,12.9,6.3,6.3 \mathrm{~Hz}, 1 \mathrm{H}, 1^{\prime}-\mathrm{H}_{\mathrm{a}}\right.$ or $\left.2{ }^{\prime}-\mathrm{H}_{\mathrm{a}}\right), 1.06\left(\mathrm{~s}, 9 \mathrm{H},{ }^{t} \mathrm{Bu}\right), 0.85 \mathrm{ppm}\left(\mathrm{s}, 9 \mathrm{H},{ }^{t} \mathrm{Bu}\right) .{ }^{13} \mathrm{C}\left\{{ }^{1} \mathrm{H}\right\} \mathrm{NMR}$ $\left(125.7 \mathrm{MHz}, \mathrm{CDCl}_{3}\right) \delta_{\mathrm{C}} 163.5(2 \times \mathrm{C}, \mathrm{CO}), 135.9(2 \times \mathrm{CH}, \mathrm{Ar})$, $135.8(2 \times \mathrm{CH}, \mathrm{Ar}), 135.5(4 \times \mathrm{CH}, \mathrm{Ar}), 134.4(2 \times \mathrm{CH}, \mathrm{Ar}), 133.2$ (C, Ar), 133.03 (C, Ar), 132.97 (C, Ar), 131.6 (C, Ar), $130.13(\mathrm{CH}$, $\mathrm{Ar}), 130.06$ (CH, Ar), 129.58 (CH, Ar), 129.56 (CH, Ar), $129.0(2 \times$ C, Ar), $127.9(2 \times \mathrm{CH}, \mathrm{Ar}), 127.7(2 \times \mathrm{CH}, \mathrm{Ar}), 127.55(4 \times \mathrm{CH}$, $\mathrm{Ar}), 123.5(2 \times \mathrm{CH}, \mathrm{Ar}), 89.3(\mathrm{CH}, \mathrm{C}-2), 82.6(\mathrm{CH}, \mathrm{C}-4), 77.84$ $(\mathrm{CH}, \mathrm{C}-1), 77.81\left(\mathrm{CH}_{2}, \mathrm{C}-3^{\prime}\right), 73.1(\mathrm{CH}, \mathrm{C}-3), 63.5\left(\mathrm{CH}_{2}, \mathrm{C}-5\right)$, $26.65\left(3 \times \mathrm{CH}_{3},{ }^{t} \mathrm{Bu}\right), 26.63\left(3 \times \mathrm{CH}_{3},{ }^{t} \mathrm{Bu}\right), 25.9\left(\mathrm{CH}_{2}, \mathrm{C}-1\right.$ ' or $\mathrm{C}-$ $\left.2^{\prime}\right), 24.8\left(\mathrm{CH}_{2}, \mathrm{C}-1\right.$ ' or $\mathrm{C}-2$ '), $19.2\left(\mathrm{C},{ }^{t} \mathrm{Bu}\right), 19.0 \mathrm{ppm}\left(\mathrm{C},{ }^{t} \mathrm{Bu}\right), 1 \mathrm{C}$ from $\mathrm{CF}_{3}$ group is missing. IR $\left(\mathrm{CHCl}_{3}\right): \nu=2932,1791,1734,1113$ $\mathrm{cm}^{-1}$. MS (ESI) $\mathrm{m} / z(\%)=968(100)[\mathrm{M}+\mathrm{Na}]^{+}$. HRMS (ESI) $\mathrm{m} / z$ : $[\mathrm{M}+\mathrm{Na}]^{+}$calcd for $\mathrm{C}_{49} \mathrm{H}_{54} \mathrm{~F}_{3} \mathrm{NNaO}_{9} \mathrm{SSi}_{2}$ 968.2908; found 968.2907. Anal. calcd for $\mathrm{C}_{49} \mathrm{H}_{54} \mathrm{~F}_{3} \mathrm{NO}_{9} \mathrm{SSi}_{2}$ : C, 62.20; H, 5.75; N, 1.48; S, 3.39. Found: C, 62.11; H, 5.97; N, 1.52; S, 3.19.

3-C-(3,4,6-Tri-O-benzyl- $\alpha$-D-glucopyranosyl) 1-propoxyphthalimide (64). Following the general procedure starting from alcohol 63 $(85.6 \mathrm{mg}, 0.18 \mathrm{mmol}$ ) and purification by column chromatography (hexanes- $\left.\mathrm{Et}_{2} \mathrm{O}, 1: 1\right)$, product $64(107 \mathrm{mg}, 0.17 \mathrm{mmol}, 93 \%)$ was obtained as a colorless oil: $[\alpha]_{\mathrm{D}}=+12.3\left(c=0.26, \mathrm{CHCl}_{3}\right) .{ }^{1} \mathrm{H}$ NMR $\left(400 \mathrm{MHz}, \mathrm{CDCl}_{3}\right) \delta_{\mathrm{H}} 7.82-7.71(\mathrm{~m}, 4 \mathrm{H}, \mathrm{Ar}), 7.34-7.21(\mathrm{~m}, 15 \mathrm{H}$, $\mathrm{Ar}), 4.69(\mathrm{~d}, J=11.5 \mathrm{~Hz}, 1 \mathrm{H}, \mathrm{OBn}), 4.63(\mathrm{~d}, J=11.4 \mathrm{~Hz}, 1 \mathrm{H}, \mathrm{OBn})$, $4.62(\mathrm{~d}, J=11.7 \mathrm{~Hz}, 1 \mathrm{H}, \mathrm{OBn}), 4.56(\mathrm{~d}, J=12.4 \mathrm{~Hz}, 1 \mathrm{H}, \mathrm{OBn}), 4.56$ (d, $J=12.4 \mathrm{~Hz}, 1 \mathrm{H}, \mathrm{OBn}), 4.48(\mathrm{~d}, J=12.0 \mathrm{~Hz}, 1 \mathrm{H}, \mathrm{OBn}), 4.26-4.21$ $\left(\mathrm{m}, 2 \mathrm{H}, 3^{\prime}-\mathrm{H}_{2}\right), 4.02-3.96(\mathrm{~m}, 2 \mathrm{H}, 1-\mathrm{H}, 2-\mathrm{H}), 3.82(\mathrm{dd}, J=10.2,5.7$ $\left.\mathrm{Hz}, 1 \mathrm{H}, 6-\mathrm{H}_{\mathrm{b}}\right), 3.77(\mathrm{dd}, J=5.8,5.8 \mathrm{~Hz}, 1 \mathrm{H}, 4-\mathrm{H}), 3.73-3.68(\mathrm{~m}$, $\left.2 \mathrm{H}, 5-\mathrm{H}, 6-\mathrm{H}_{\mathrm{a}}\right), 3.63(\mathrm{dd}, J=5.3,5.3 \mathrm{~Hz}, 1 \mathrm{H}, 3-\mathrm{H}), 1.99-1.76 \mathrm{ppm}$ $\left(\mathrm{m}, 4 \mathrm{H}, 1^{\prime}-\mathrm{H}_{2}, 2^{\prime}-\mathrm{H}_{2}\right), 1 \mathrm{H}$ from $\mathrm{OH}$ is missing. ${ }^{13} \mathrm{C}\left\{{ }^{1} \mathrm{H}\right\}$ NMR $(100.6$ $\left.\mathrm{MHz}, \mathrm{CDCl}_{3}\right) \delta_{\mathrm{C}} 163.6(2 \times \mathrm{C}, \mathrm{CO}), 138.1(2 \times \mathrm{C}, \mathrm{Ar}), 137.5(\mathrm{C}$, $\mathrm{Ar}), 134.3(2 \times \mathrm{CH}, \mathrm{Ar}), 129.0(2 \times \mathrm{C}, \mathrm{Ar}), 128.5(2 \times \mathrm{CH}, \mathrm{Ar})$, $128.4(2 \times \mathrm{CH}, \mathrm{Ar}), 128.3(2 \times \mathrm{CH}, \mathrm{Ar}), 127.84(3 \times \mathrm{CH}, \mathrm{Ar})$, $127.79(\mathrm{CH}, \mathrm{Ar}), 127.7(2 \times \mathrm{CH}, \mathrm{Ar}), 127.58(2 \times \mathrm{CH}, \mathrm{Ar}), 127.56$ $(\mathrm{CH}, \mathrm{Ar}), 123.4(2 \times \mathrm{CH}, \mathrm{Ar}), 78.3\left(\mathrm{CH}_{2}, \mathrm{C}-3^{\prime}\right), 78.1(\mathrm{CH}, \mathrm{C}-3)$, $75.3(\mathrm{CH}, \mathrm{C}-4), 73.5\left(\mathrm{CH}_{2}, \mathrm{OBn}\right), 73.3\left(\mathrm{CH}_{2}, \mathrm{OBn}\right), 73.3(\mathrm{CH}, \mathrm{C}-$ 5), $72.9\left(\mathrm{CH}_{2}, \mathrm{OBn}\right), 71.4(\mathrm{CH}, \mathrm{C}-1), 69.8(\mathrm{CH}, \mathrm{C}-2), 68.3\left(\mathrm{CH}_{2}, \mathrm{C}-\right.$ 6), $24.5\left(\mathrm{CH}_{2}, \mathrm{C}-1^{\prime}\right), 24.3 \mathrm{ppm}\left(\mathrm{CH}_{2}, \mathrm{C}-2^{\prime}\right)$. IR $\left(\mathrm{CHCl}_{3}\right): \nu=3514$, $2935,2871,1792,1737,1372,1083 \mathrm{~cm}^{-1}$. MS (E/I $\left.70 \mathrm{eV}\right): \mathrm{m} / z(\%)$ $=546$ (6) $\left[\mathrm{M}-\mathrm{C}_{7} \mathrm{H}_{7}\right]^{+}, 529(51)\left[\mathrm{M}-\mathrm{C}_{7} \mathrm{H}_{8} \mathrm{O}\right]^{+}, 91$ (100) $\left[\mathrm{C}_{7} \mathrm{H}_{7}\right]^{+}$. HRMS (E/I): $\mathrm{m} / z$ : $\left[\mathrm{M}-\mathrm{C}_{7} \mathrm{H}_{8} \mathrm{O}\right]^{+}$calcd for $\mathrm{C}_{31} \mathrm{H}_{31} \mathrm{NO}_{7}$ 529.2101; found 529.2122.

3-C-(3,4,6-Tri-O-benzyl- $\beta$-D-glucopyranosyl) 1-propoxyphthalimide (67). Following the general procedure starting from alcohol 66 $(810 \mathrm{mg}, 1.64 \mathrm{mmol})$ and purification by column chromatography (hexanes-EtOAc, 1:1), product $67(790 \mathrm{mg}, 1.24 \mathrm{mmol}, 76 \%)$ was obtained as a colorless oil: $[\alpha]_{\mathrm{D}}=+14.5\left(c=0.53, \mathrm{CHCl}_{3}\right) .{ }^{1} \mathrm{H}$ NMR $\left(400 \mathrm{MHz}, \mathrm{CDCl}_{3}\right) \delta_{\mathrm{H}} 7.82-7.79(\mathrm{~m}, 2 \mathrm{H}, \mathrm{Ar}), 7.75-7.72(\mathrm{~m}, 2 \mathrm{H}$, Ar), 7.37-7.18 (m, $15 \mathrm{H}, \mathrm{Ar}), 4.95(\mathrm{~d}, J=11.6 \mathrm{~Hz}, 1 \mathrm{H}, \mathrm{OBn}), 4.80$ $(\mathrm{d}, J=11.0 \mathrm{~Hz}, 1 \mathrm{H}, \mathrm{OBn}), 4.80(\mathrm{~d}, J=11.0 \mathrm{~Hz}, 1 \mathrm{H}, \mathrm{OBn}), 4.61(\mathrm{~d}, J$ $=12.2 \mathrm{~Hz}, 1 \mathrm{H}, \mathrm{OBn}), 4.58(\mathrm{~d}, J=10.7 \mathrm{~Hz}, 1 \mathrm{H}, \mathrm{OBn}), 4.53(\mathrm{~d}, J=$ $12.3 \mathrm{~Hz}, 1 \mathrm{H}, \mathrm{OBn}$ ), 4.24 (ddd, $J=9.5,6.6,1.2 \mathrm{~Hz}, 2 \mathrm{H}, 3^{\prime}-\mathrm{H}_{2}$ ), 3.71 (br s, $\left.2 \mathrm{H}, 6-\mathrm{H}_{2}\right), 3.61(\mathrm{dd}, J=9.2,9.2 \mathrm{~Hz}, 1 \mathrm{H}, 4-\mathrm{H}), 3.52(\mathrm{dd}, J=8.6$, $8.6 \mathrm{~Hz}, 1 \mathrm{H}, 3-\mathrm{H}$ ), 3.42 (ddd, $J=9.6,2.9,2.9 \mathrm{~Hz}, 1 \mathrm{H}, 5-\mathrm{H}), 3.39$ (dd, $J=9.1,9.1 \mathrm{~Hz}, 1 \mathrm{H}, 2-\mathrm{H}), 3.28(\mathrm{ddd}, J=8.2,8.2,2.2 \mathrm{~Hz}, 1 \mathrm{H}, 1-\mathrm{H})$, 2.14-1.98 (m, 2H, 1'- $\left.\mathrm{H}_{\mathrm{b}}, 2^{\prime}-\mathrm{H}_{\mathrm{b}}\right), 1.87\left(\mathrm{~m}, 1 \mathrm{H}, 2^{\prime}-\mathrm{H}_{\mathrm{a}}\right), 1.71 \mathrm{ppm}(\mathrm{m}$, $\left.1 \mathrm{H}, \mathrm{l}^{\prime}-\mathrm{H}_{\mathrm{a}}\right), 1 \mathrm{H}$ from $\mathrm{OH}$ is missing. ${ }^{13} \mathrm{C}\left\{{ }^{1} \mathrm{H}\right\}$ NMR $(100.6 \mathrm{MHz}$, $\left.\mathrm{CDCl}_{3}\right) \delta_{\mathrm{C}} 163.7(2 \times \mathrm{C}, \mathrm{CO}), 138.7(\mathrm{C}, \mathrm{Ar}), 138.2(2 \times \mathrm{C}, \mathrm{Ar})$, $134.8(2 \times \mathrm{CH}, \mathrm{Ar}), 129.4(2 \times \mathrm{C}, \mathrm{Ar}), 129.0(2 \times \mathrm{CH}, \mathrm{Ar}), 128.8(2$ $\times \mathrm{CH}, \mathrm{Ar}), 128.7(2 \times \mathrm{CH}, \mathrm{Ar}), 128.31(2 \times \mathrm{CH}, \mathrm{Ar}), 128.25(2 \times$ $\mathrm{CH}, \mathrm{Ar}), 128.2(\mathrm{CH}, \mathrm{Ar}), 128.14(2 \times \mathrm{CH}, \mathrm{Ar}), 128.11(\mathrm{CH}, \mathrm{Ar})$, $127.9(\mathrm{CH}, \mathrm{Ar}), 123.9(2 \times \mathrm{CH}, \mathrm{Ar}), 87.0(\mathrm{CH}, \mathrm{C}-3), 79.1(\mathrm{CH}, \mathrm{C}-1$ or C-5), $78.9\left(\mathrm{CH}, \mathrm{C}-1\right.$ or C-5), $\left.78.6\left(\mathrm{CH}_{2}, \mathrm{C}-3\right)^{\prime}\right), 78.4(\mathrm{CH}, \mathrm{C}-4)$, $75.2\left(\mathrm{CH}_{2}, \mathrm{OBn}\right), 74.8\left(\mathrm{CH}_{2}, \mathrm{OBn}\right), 73.9(\mathrm{CH}, \mathrm{C}-2), 73.5\left(\mathrm{CH}_{2}\right.$, $\mathrm{OBn}), 69.1\left(\mathrm{CH}_{2}, \mathrm{C}-6\right), 27.7\left(\mathrm{CH}_{2}, \mathrm{C}-1\right.$ '), $24.0 \mathrm{ppm}\left(\mathrm{CH}_{2}, \mathrm{C}-2^{\prime}\right)$. IR $\left(\mathrm{CHCl}_{3}\right): \nu=3565,2926,2860,1791,1737,1370,1097 \mathrm{~cm}^{-1}$. MS (ESI) $\mathrm{m} / z(\%)=660(100)[\mathrm{M}+\mathrm{Na}]^{+}$. HRMS (ESI) $\mathrm{m} / z:[\mathrm{M}+$ $\mathrm{Na}]^{+}$calcd for $\mathrm{C}_{38} \mathrm{H}_{39} \mathrm{NNaO}_{8} 660.2573$; found 660.2558 .

3-C-(3,4,6-Tri-O-benzyl- $\alpha$-D-mannopyranosyl) 1-propoxyphthalimide (70). Following the general procedure starting from alcohol 69 $(178.8 \mathrm{mg}, 0.36 \mathrm{mmol})$ and purification by column chromatography (hexanes-EtOAc, 1:1), product $70(206 \mathrm{mg}, 0.32 \mathrm{mmol}, 90 \%)$ was obtained as a colorless oil: $[\alpha]_{\mathrm{D}}=+13.5\left(c=1.50, \mathrm{CHCl}_{3}\right) .{ }^{1} \mathrm{H}$ NMR $\left(500 \mathrm{MHz}, \mathrm{CDCl}_{3}\right) \delta_{\mathrm{H}} 7.83-7.81(\mathrm{~m}, 2 \mathrm{H}, \mathrm{Ar}), 7.74-7.73(\mathrm{~m}, 2 \mathrm{H}$, $\mathrm{Ar}), 7.34-7.20(\mathrm{~m}, 15 \mathrm{H}, \mathrm{Ar}), 4.73(\mathrm{~d}, J=11.4 \mathrm{~Hz}, 1 \mathrm{H}, \mathrm{OBn}), 4.65$ (d, $J=12.3 \mathrm{~Hz}, 1 \mathrm{H}, \mathrm{OBn}$ ), 4.63 (d, $J=11.7 \mathrm{~Hz}, 1 \mathrm{H}, \mathrm{OBn}), 4.56$ (d, $J$ $=12.0 \mathrm{~Hz}, 1 \mathrm{H}, \mathrm{OBn}), 4.53(\mathrm{~d}, J=11.4 \mathrm{~Hz}, 1 \mathrm{H}, \mathrm{OBn}), 4.50(\mathrm{~d}, J=$ $12.0 \mathrm{~Hz}, 1 \mathrm{H}, \mathrm{OBn}), 4.27-4.19\left(\mathrm{~m}, 2 \mathrm{H}, 3^{\prime}-\mathrm{H}_{2}\right), 3.97(\mathrm{~m}, 1 \mathrm{H}, 1-\mathrm{H})$, $3.89(\mathrm{dd}, J=3.6,2.9 \mathrm{~Hz}, 1 \mathrm{H}, 2-\mathrm{H}), 3.83(\mathrm{dd}, J=7.2,3.1 \mathrm{~Hz}, 1 \mathrm{H}, 3-$ $\mathrm{H}), 3.81(\mathrm{dd}, J=6.6,6.6 \mathrm{~Hz}, 1 \mathrm{H}, 4-\mathrm{H}), 3.77(\mathrm{~m}, 1 \mathrm{H}, 5-\mathrm{H}), 3.73(\mathrm{dd}$, $\left.J=10.4,5.1 \mathrm{~Hz}, 1 \mathrm{H}, 6-\mathrm{H}_{\mathrm{b}}\right), 3.68\left(\mathrm{dd}, J=10.4,3.5 \mathrm{~Hz}, 1 \mathrm{H}, 6-\mathrm{H}_{\mathrm{a}}\right)$, $1.94\left(\mathrm{~m}, 1 \mathrm{H}, 2^{\prime}-\mathrm{H}_{\mathrm{b}}\right), 1.88-1.78 \mathrm{ppm}\left(\mathrm{m}, 3 \mathrm{H}, 1^{\prime}-\mathrm{H}_{2}, 2^{\prime}-\mathrm{H}_{\mathrm{a}}\right), 1 \mathrm{H}$ from $\mathrm{OH}$ is missing. ${ }^{13} \mathrm{C}\left\{{ }^{1} \mathrm{H}\right\} \mathrm{NMR}\left(100.6 \mathrm{MHz}, \mathrm{CDCl}_{3}\right) \delta_{\mathrm{C}} 163.6(2 \times \mathrm{C}$, CO), 138.3 (C, Ar), 138.2 (C, Ar), 137.7 (C, Ar), $134.4(2 \times \mathrm{CH}$, Ar), $129.0(2 \times \mathrm{C}, \mathrm{Ar}), 128.6(2 \times \mathrm{CH}, \mathrm{Ar}), 128.4(2 \times \mathrm{CH}, \mathrm{Ar})$, $128.3(2 \times \mathrm{CH}, \mathrm{Ar}), 128.0(\mathrm{CH}, \mathrm{Ar}), 127.9(4 \times \mathrm{CH}, \mathrm{Ar}), 127.73(2$ $\times \mathrm{CH}, \mathrm{Ar}), 127.68(\mathrm{CH}, \mathrm{Ar}), 127.5(\mathrm{CH}, \mathrm{Ar}), 123.5(2 \times \mathrm{CH}, \mathrm{Ar})$, $79.4\left(\mathrm{CH}, \mathrm{C}-3\right.$ or C-4), $78.0\left(\mathrm{CH}_{2}, \mathrm{C}-3^{\prime}\right), 74.8(\mathrm{CH}, \mathrm{C}-1), 74.3(\mathrm{CH}$, $\mathrm{C}-5), 74.1\left(\mathrm{CH}_{2}, \mathrm{OBn}\right), 73.4\left(\mathrm{CH}_{2}, \mathrm{OBn}\right), 72.8(\mathrm{CH}, \mathrm{C}-3$ or $\mathrm{C}-4)$, $72.3\left(\mathrm{CH}_{2}, \mathrm{OBn}\right), 69.3(\mathrm{CH}, \mathrm{C}-2), 69.1\left(\mathrm{CH}_{2}, \mathrm{C}-6\right), 25.5\left(\mathrm{CH}_{2}, \mathrm{C}-1^{\prime}\right.$ or C-2'), $24.6 \mathrm{ppm}\left(\mathrm{CH}_{2}, \mathrm{C}-1^{\prime}\right.$ or C-2'). IR $\left(\mathrm{CHCl}_{3}\right): \nu=3559,2930$, $1789,1731,1082 \mathrm{~cm}^{-1}$. MS (ESI) $\mathrm{m} / z(\%)=660(100)[\mathrm{M}+\mathrm{Na}]^{+}$. HRMS (ESI) $m / z$ : $[\mathrm{M}+\mathrm{Na}]^{+}$calcd for $\mathrm{C}_{38} \mathrm{H}_{39} \mathrm{NNaO}_{8}$ 660.2573; found 660.2578 .

3-C-(3,4,6-Tri-O-benzyl- $\beta$-D-mannopyranosyl) 1-propoxyphthalimide (73). Following the general procedure starting from alcohol 72 $(72.6 \mathrm{mg}, 0.15 \mathrm{mmol}$ ) and purification by column chromatography (hexanes- $\left.\mathrm{Et}_{2} \mathrm{O}, 1: 1\right)$, product $73(80 \mathrm{mg}, 0.13 \mathrm{mmol}, 84 \%)$ was obtained as a colorless oil: $[\alpha]_{\mathrm{D}}=+5.3\left(c=0.34, \mathrm{CHCl}_{3}\right) .{ }^{1} \mathrm{H}$ NMR $\left(500 \mathrm{MHz}, \mathrm{CDCl}_{3}\right.$, simulated ring coupling constants using DAISY) $\delta_{\mathrm{H}} 7.83-7.80(\mathrm{~m}, 2 \mathrm{H}, \mathrm{Ar}), 7.75-7.72(\mathrm{~m}, 2 \mathrm{H}, \mathrm{Ar}), 7.38-7.18(\mathrm{~m}$ $15 \mathrm{H}, \mathrm{Ar}), 4.85(\mathrm{~d}, J=10.7 \mathrm{~Hz}, 1 \mathrm{H}, \mathrm{OBn}), 4.75(\mathrm{~d}, J=11.7 \mathrm{~Hz}, 1 \mathrm{H}$, OBn ), $4.67(\mathrm{~d}, J=11.7 \mathrm{~Hz}, 1 \mathrm{H}, \mathrm{OBn}), 4.60(\mathrm{~d}, J=12.3 \mathrm{~Hz}, 1 \mathrm{H}$, OBn), $4.53(\mathrm{~d}, J=10.4 \mathrm{~Hz}, 1 \mathrm{H}, \mathrm{OBn}), 4.53(\mathrm{~d}, J=12.3 \mathrm{~Hz}, 1 \mathrm{H}$, $\mathrm{OBn}$ ), 4.29-4.20 (m, 2H, 3'- $\left.\mathrm{H}_{2}\right), 3.98(\mathrm{dd}, J=2.9,0.6 \mathrm{~Hz}, 1 \mathrm{H}, 2-\mathrm{H})$, $3.78(\mathrm{dd}, J=9.4,9.2 \mathrm{~Hz}, 1 \mathrm{H}, 4-\mathrm{H}), 3.72(\mathrm{dd}, J=11.3,1.9 \mathrm{~Hz}, 1 \mathrm{H}, 6-$ $\mathrm{H}_{\mathrm{b}}$ ), $3.68\left(\mathrm{dd}, J=11.3,5.0 \mathrm{~Hz}, 1 \mathrm{H}, 6-\mathrm{H}_{\mathrm{a}}\right), 3.63(\mathrm{dd}, J=9.2,2.9 \mathrm{~Hz}$, $1 \mathrm{H}, 3-\mathrm{H}), 3.47(\mathrm{ddd}, J=7.9,4.1,0.6 \mathrm{~Hz}, 1 \mathrm{H}, 1-\mathrm{H}), 3.41(\mathrm{ddd}, J=9.4$, 5.0, $1.9 \mathrm{~Hz}, 1 \mathrm{H}, 5-\mathrm{H}), 2.31(\mathrm{br} \mathrm{s}, 1 \mathrm{H}, \mathrm{OH}), 2.04-1.94\left(\mathrm{~m}, 2 \mathrm{H}, \mathrm{l}^{\prime}-\mathrm{H}_{\mathrm{b}}\right.$, $\left.2^{\prime}-\mathrm{H}_{\mathrm{b}}\right), 1.92-1.84 \mathrm{ppm}\left(\mathrm{m}, 2 \mathrm{H}, 1^{\prime}-\mathrm{H}_{a}, 2^{\prime}-\mathrm{H}_{\mathrm{a}}\right) .{ }^{13} \mathrm{C}\left\{{ }^{1} \mathrm{H}\right\} \operatorname{NMR}(100.6$ $\left.\mathrm{MHz}, \mathrm{CDCl}_{3}\right) \delta_{\mathrm{C}} 163.6(2 \times \mathrm{C}, \mathrm{CO}), 138.4(2 \times \mathrm{C}, \mathrm{Ar}), 137.9(\mathrm{C}$, $\mathrm{Ar}), 134.4(2 \times \mathrm{CH}, \mathrm{Ar}), 129.0(2 \times \mathrm{C}, \mathrm{Ar}), 128.5(2 \times \mathrm{CH}, \mathrm{Ar})$, $128.30(2 \times \mathrm{CH}, \mathrm{Ar}), 128.26(2 \times \mathrm{CH}, \mathrm{Ar}), 127.93(2 \times \mathrm{CH}, \mathrm{Ar})$, $127.88(3 \times \mathrm{CH}, \mathrm{Ar}), 127.8(2 \times \mathrm{CH}, \mathrm{Ar}), 127.6(\mathrm{CH}, \mathrm{Ar}), 127.5$ $(\mathrm{CH}, \mathrm{Ar}), 123.4(2 \times \mathrm{CH}, \mathrm{Ar}), 83.6(\mathrm{CH}, \mathrm{C}-3), 79.2(\mathrm{CH}, \mathrm{C}-1), 78.3$ $\left.\left(\mathrm{CH}_{2}, \mathrm{C}-3\right)^{\prime}\right), 77.3(\mathrm{CH}, \mathrm{C}-5), 75.1\left(\mathrm{CH}_{2}, \mathrm{OBn}\right), 74.8(\mathrm{CH}, \mathrm{C}-4), 73.4$ $\left(\mathrm{CH}_{2}, \mathrm{OBn}\right), 71.6\left(\mathrm{CH}_{2}, \mathrm{OBn}\right), 69.5\left(\mathrm{CH}_{2}, \mathrm{C}-6\right), 68.4(\mathrm{CH}, \mathrm{C}-2)$, $27.1\left(\mathrm{CH}_{2}, \mathrm{C}-2^{\prime}\right), 24.7 \mathrm{ppm}\left(\mathrm{CH}_{2}, \mathrm{C}-1^{\prime}\right)$. IR $\left(\mathrm{CHCl}_{3}\right): \nu=3569$, $2927,2862,1792,1737,1118 \mathrm{~cm}^{-1}$. MS (ESI) $m / z(\%)=660(100)$ 
$[\mathrm{M}+\mathrm{Na}]^{+}$. HRMS (ESI) $m / z:[\mathrm{M}+\mathrm{Na}]^{+}$calcd for $\mathrm{C}_{38} \mathrm{H}_{39} \mathrm{NNaO}_{8}$ 660.2573; found 660.2576. Anal. calcd for $\mathrm{C}_{38} \mathrm{H}_{39} \mathrm{NO}_{8}: \mathrm{C}, 71.57 ; \mathrm{H}$, 6.16; N, 2.20. Found: C, 71.62; H, 6.30; N, 1.95.

3-C-(3,4-Di-O-benzyl- $\alpha$-L-fucopyranosyl) 1-propoxyphthalimide (77). Following the general procedure starting from alcohol 76 (413 $\mathrm{mg}, 1.07 \mathrm{mmol}$ ) and purification by column chromatography (hexanes-EtOAc, 7:3), product 77 (536.9 $\mathrm{mg}, 1.01 \mathrm{mmol}$, 94\%) was obtained as a colorless oil: $[\alpha]_{\mathrm{D}}=-14.4\left(c=0.80, \mathrm{CHCl}_{3}\right) .{ }^{1} \mathrm{H}$ NMR $\left(500 \mathrm{MHz}, \mathrm{CDCl}_{3}\right) \delta_{\mathrm{H}} 7.83-7.81(\mathrm{~m}, 2 \mathrm{H}, \mathrm{Ar}), 7.74-7.73(\mathrm{~m}$, $2 \mathrm{H}, \mathrm{Ar}), 7.37-7.24(\mathrm{~m}, 10 \mathrm{H}, \mathrm{Ar}), 4.79(\mathrm{~d}, J=11.7 \mathrm{~Hz}, 1 \mathrm{H}, \mathrm{OBn})$, $4.76(\mathrm{~d}, J=11.9 \mathrm{~Hz}, 1 \mathrm{H}, \mathrm{OBn}), 4.62(\mathrm{~d}, J=12.0 \mathrm{~Hz}, 1 \mathrm{H}, \mathrm{OBn}), 4.61$ $(\mathrm{d}, J=12.0 \mathrm{~Hz}, 1 \mathrm{H}, \mathrm{OBn}), 4.28-4.21\left(\mathrm{~m}, 2 \mathrm{H}, 3^{\prime}-\mathrm{H}_{2}\right), 4.14-4.07(\mathrm{~m}$, $2 \mathrm{H}, 1-\mathrm{H}, 2-\mathrm{H}), 3.94(\mathrm{~m}, 1 \mathrm{H}, 5-\mathrm{H}), 3.79(\mathrm{dd}, J=2.8,2.8 \mathrm{~Hz}, 1 \mathrm{H}, 4-$ $\mathrm{H}), 3.76(\mathrm{dd}, J=7.3,2.9 \mathrm{~Hz}, 1 \mathrm{H}, 3-\mathrm{H}), 2.52($ br s, $1 \mathrm{H}, \mathrm{OH}), 2.04-$ $1.77\left(\mathrm{~m}, 4 \mathrm{H}, \mathrm{l}^{\prime}-\mathrm{H}_{2}, 2^{\prime}-\mathrm{H}_{2}\right), 1.30 \mathrm{ppm}\left(\mathrm{d}, J=6.7 \mathrm{~Hz}, 3 \mathrm{H}, 6-\mathrm{H}_{3}\right)$. ${ }^{13} \mathrm{C}\left\{{ }^{1} \mathrm{H}\right\}$ NMR $\left(125.7 \mathrm{MHz}, \mathrm{CDCl}_{3}\right) \delta_{\mathrm{C}} 163.6(2 \times \mathrm{C}, \mathrm{CO}), 138.5$ (C, Ar), 138.3 (C, Ar), $128.9(2 \times \mathrm{C}, \mathrm{Ar}), 128.5(2 \times \mathrm{CH}, \mathrm{Ar}), 128.4$ $(3 \times \mathrm{CH}, \mathrm{Ar}), 128.2(2 \times \mathrm{CH}, \mathrm{Ar}), 127.7(2 \times \mathrm{CH}, \mathrm{Ar}), 127.6(\mathrm{CH}$, $\mathrm{Ar}), 127.5(2 \times \mathrm{CH}, \mathrm{Ar}), 123.4(2 \times \mathrm{CH}, \mathrm{Ar}), 79.2(\mathrm{CH}, \mathrm{C}-3), 78.3$ $\left(\mathrm{CH}_{2}, \mathrm{C}-3^{\prime}\right), 75.3(\mathrm{CH}, \mathrm{C}-4), 73.2\left(\mathrm{CH}_{2}, \mathrm{OBn}\right), 72.5\left(\mathrm{CH}_{2}, \mathrm{OBn}\right)$, $71.9(\mathrm{CH}, \mathrm{C}-2), 68.7(\mathrm{CH}, \mathrm{C}-1), 68.6(\mathrm{CH}, \mathrm{C}-5), 24.9\left(\mathrm{CH}_{2}, \mathrm{C}-1\right.$ ' or C-2'), $22.8\left(\mathrm{CH}_{2}, \mathrm{C}-1^{\prime}\right.$ or C-2'), $15.7 \mathrm{ppm}\left(\mathrm{CH}_{3}, \mathrm{C}-6\right)$. IR $\left(\mathrm{CHCl}_{3}\right): \nu$ $=3675,3574,3015,1790,1733,1120 \mathrm{~cm}^{-1}$. MS (ESI) $\mathrm{m} / z(\%)=$ $554(100)[\mathrm{M}+\mathrm{Na}]^{+}$. HRMS (ESI) $\mathrm{m} / z:[\mathrm{M}+\mathrm{Na}]^{+}$calcd for $\mathrm{C}_{31} \mathrm{H}_{33} \mathrm{NNaO}_{7}$ 554.2155; found 554.2147.

3-C-(3,5-Di-O-1, 1, 3, 3-tetraisopropyldisiloxanyl- $\alpha-D-$ ribofuranosyl)1-propoxyphthalimide (88). Following the general procedure starting from alcohol $87(350 \mathrm{mg}, 0.81 \mathrm{mmol})$ and purification by column chromatography (hexanes-EtOAc, 8:2), product 88 (457.9 $\mathrm{mg}, 0.79 \mathrm{mmol}, 98 \%)$ was obtained as a colorless oil: $[\alpha]_{\mathrm{D}}=-13.0\left(c=0.73, \mathrm{CHCl}_{3}\right) .{ }^{1} \mathrm{H} \mathrm{NMR}\left(400 \mathrm{MHz}, \mathrm{CDCl}_{3}\right) \delta_{\mathrm{H}}$ $7.83-7.72(\mathrm{~m}, 4 \mathrm{H}, \mathrm{Ar}), 4.36(\mathrm{dd}, J=7.2,4.8 \mathrm{~Hz}, 1 \mathrm{H}, 3-\mathrm{H}), 4.25-$ $4.20\left(\mathrm{~m}, 2 \mathrm{H}, 3^{\prime}-\mathrm{H}_{2}\right), 4.11(\mathrm{dd}, J=4.8,3.4 \mathrm{~Hz}, 1 \mathrm{H}, 2-\mathrm{H}), 4.04(\mathrm{~m}, 1 \mathrm{H}$, $1-\mathrm{H}), 3.95$ (dd, $J=11.4,3.2 \mathrm{~Hz}, 1 \mathrm{H}, 5-\mathrm{H}_{\mathrm{b}}$ ), 3.89 (ddd, $J=6.1,6.1,3.4$ $\mathrm{Hz}, 1 \mathrm{H}, 4-\mathrm{H}), 3.83\left(\mathrm{dd}, J=11.6,6.1 \mathrm{~Hz}, 1 \mathrm{H}, 5-\mathrm{H}_{\mathrm{a}}\right), 1.96-1.80(\mathrm{~m}$, $4 \mathrm{H}, 1$ ' $\left.-\mathrm{H}_{2}, 2{ }^{\prime}-\mathrm{H}_{2}\right), 1.09-0.97 \mathrm{ppm}\left(\mathrm{m}, 28 \mathrm{H},{ }^{i} \mathrm{Pr}\right), 1 \mathrm{H}$ from $\mathrm{OH}$ is missing. ${ }^{13} \mathrm{C}\left\{{ }^{1} \mathrm{H}\right\}$ NMR $\left(125.7 \mathrm{MHz}, \mathrm{CDCl}_{3}\right) \delta_{\mathrm{C}} 163.6(2 \times \mathrm{C}, \mathrm{CO})$, $134.4(2 \times \mathrm{CH}, \mathrm{Ar}), 129.0(2 \times \mathrm{C}, \mathrm{Ar}), 123.4(2 \times \mathrm{CH}, \mathrm{Ar}), 80.2$ $\left(\mathrm{CH}, \mathrm{C}-1\right.$ or C-4), $80.2(\mathrm{CH}, \mathrm{C}-1$ or $\mathrm{C}-4), 78.3\left(\mathrm{CH}_{2}, \mathrm{C}-3{ }^{\prime}\right), 74.6$ $(\mathrm{CH}, \mathrm{C}-3), 72.5(\mathrm{CH}, \mathrm{C}-2), 63.1\left(\mathrm{CH}_{2}, \mathrm{C}-5\right), 25.3\left(\mathrm{CH}_{2}, \mathrm{C}-1\right.$ ' or $\mathrm{C}-$ $\left.2^{\prime}\right), 24.7\left(\mathrm{CH}_{2}, \mathrm{C}-1\right.$ ' or $\mathrm{C}-2$ ' $), 17.43\left(\mathrm{CH}_{3},{ }^{i} \mathrm{Pr}\right), 17.31\left(3 \times \mathrm{CH}_{3},{ }^{i} \mathrm{Pr}\right)$, $17.19\left(\mathrm{CH}_{3},{ }^{i} \mathrm{Pr}\right), 17.02\left(2 \times \mathrm{CH}_{3},{ }^{i} \mathrm{Pr}\right), 16.94\left(\mathrm{CH}_{3},{ }^{i} \mathrm{Pr}\right), 13.4(\mathrm{CH}$, $\left.{ }^{i} \mathrm{Pr}\right), 13.2\left(\mathrm{CH},{ }^{i} \mathrm{Pr}\right), 12.8\left(\mathrm{CH},{ }^{i} \mathrm{Pr}\right), 12.6 \mathrm{ppm}\left(\mathrm{CH},{ }^{i} \mathrm{Pr}\right) . \mathrm{IR}$ $\left(\mathrm{CHCl}_{3}\right): \nu=3546,2948,1791,1733,1467,1039 \mathrm{~cm}^{-1}$. MS (ESI) $m / z(\%)=602(100)[\mathrm{M}+\mathrm{Na}]^{+}$. HRMS (ESI) $m / z:[\mathrm{M}+\mathrm{Na}]^{+}$ calcd for $\mathrm{C}_{28} \mathrm{H}_{45} \mathrm{NNaO}_{8} \mathrm{Si}_{2}$ 602.2581; found 602.2585 .

General Procedure to Give Acetyl Derivatives 1, 3, 5, 7, and 9. The phthalimide $(1 \mathrm{mmol})$ was dissolved in dry pyridine $(3.83$ $\mathrm{mL})$, and acetyl anhydride $(1.1 \mathrm{~mL})$ and DMAP $(12.6 \mathrm{mg}, 0.1 \mathrm{mmol})$ were added at $0{ }^{\circ} \mathrm{C}$ under a $\mathrm{N}_{2}$ atmosphere. The mixture was stirred at room temperature for $1 \mathrm{~h}$. Then, the reaction was evaporated on the high vacuum rotovap, and the crude was quenched with $\mathrm{HCl} 10 \%$ and extracted with $\mathrm{CH}_{2} \mathrm{Cl}_{2}$. The organic extracts were washed with a saturated aqueous $\mathrm{NaHCO}_{3}$ solution, dried over $\mathrm{Na}_{2} \mathrm{SO}_{4}$, and concentrated under reduced pressure. Column chromatography of the residue (hexanes-EtOAc) gave the corresponding acetyl compound.

3-C-(2-O-Acetyl-3,4,6-tri-O-benzyl- $\alpha$-D-glucopyranosyl) 1-propoxyphthalimide (1). Following the general procedure starting from phthalimide $64(39.2 \mathrm{mg}, 0.06 \mathrm{mmol})$ and purification by column chromatography (hexanes-EtOAc, 85:15), product $1(30 \mathrm{mg}, 0.04$ mmol, $72 \%)$ was obtained as a crystalline solid: $\mathrm{mp} 99.7-100.5{ }^{\circ} \mathrm{C}$ $\left(n\right.$-hexane-EtOAc); $[\alpha]_{\mathrm{D}}=+46.8\left(c=0.31, \mathrm{CHCl}_{3}\right) .{ }^{1} \mathrm{H}$ NMR $(500$ $\mathrm{MHz}, \mathrm{CDCl}_{3}$, simulated ring coupling constants using DAISY) $\delta_{\mathrm{H}}$ 7.83-7.80 (m, 2H, Ar), 7.74-7.71 (m, 2H, Ar), 7.34-7.15 (m, $15 \mathrm{H}$, Ar), $5.08(\mathrm{dd}, J=9.0,5.5 \mathrm{~Hz}, 1 \mathrm{H}, 2-\mathrm{H}), 4.78(\mathrm{~d}, J=11.7 \mathrm{~Hz}, 1 \mathrm{H}$, OBn), 4.75 (d, $J=11.1 \mathrm{~Hz}, 1 \mathrm{H}, \mathrm{OBn}), 4.74(\mathrm{~d}, J=11.4 \mathrm{~Hz}, 1 \mathrm{H}$, OBn $), 4.60(\mathrm{~d}, J=12.3 \mathrm{~Hz}, 1 \mathrm{H}, \mathrm{OBn}), 4.50(\mathrm{~d}, J=11.1 \mathrm{~Hz}, 1 \mathrm{H}$, $\mathrm{OBn}), 4.48(\mathrm{~d}, J=12.0 \mathrm{~Hz}, 1 \mathrm{H}, \mathrm{OBn}), 4.24(\mathrm{dd}, J=6.0,6.0 \mathrm{~Hz}, 2 \mathrm{H}$, 3'- $\mathrm{H}_{2}$ ), 4.17 (ddd, $J=11.0,5.5,3.1 \mathrm{~Hz}, 1 \mathrm{H}, 1-\mathrm{H}$ ), 3.87 (dd, $J=9.0$,
$7.8 \mathrm{~Hz}, 1 \mathrm{H}, 3-\mathrm{H}), 3.73-3.65\left(\mathrm{~m}, 4 \mathrm{H}, 4-\mathrm{H}, 5-\mathrm{H}, 6-\mathrm{H}_{2}\right), 2.04(\mathrm{~s}, 3 \mathrm{H}$, OAc), $1.99-1.90\left(\mathrm{~m}, 2 \mathrm{H}, 1^{\prime}-\mathrm{H}_{\mathrm{b}}, 2^{\prime}-\mathrm{H}_{\mathrm{b}}\right), 1.84-1.73 \mathrm{ppm}\left(\mathrm{m}, 2 \mathrm{H}, 1^{\prime}-\right.$ $\left.\mathrm{H}_{\mathrm{a}}, 2^{\prime}-\mathrm{H}_{\mathrm{a}}\right) \cdot{ }^{13} \mathrm{C}\left\{{ }^{1} \mathrm{H}\right\}$ NMR $\left(100.6 \mathrm{MHz}, \mathrm{CDCl}_{3}\right) \delta_{\mathrm{C}} 170.1$ (C, OAc), $163.5(2 \times \mathrm{C}, \mathrm{CO}), 138.5$ (C, Ar), $138.1(2 \times \mathrm{C}, \mathrm{Ar}), 134.4(2 \times \mathrm{CH}$, $\mathrm{Ar}), 129.0(2 \times \mathrm{C}, \mathrm{Ar}), 128.4(2 \times \mathrm{CH}, \mathrm{Ar}), 128.34(2 \times \mathrm{CH}, \mathrm{Ar})$, $128.31(2 \times \mathrm{CH}, \mathrm{Ar}), 127.9(2 \times \mathrm{CH}, \mathrm{Ar}), 127.73(2 \times \mathrm{CH}, \mathrm{Ar})$, 127.69 (CH, Ar), $127.61(\mathrm{CH}, \mathrm{Ar}), 127.59(2 \times \mathrm{CH}, \mathrm{Ar}), 127.5(\mathrm{CH}$, $\mathrm{Ar}), 123.4(2 \times \mathrm{CH}, \mathrm{Ar}), 80.1(\mathrm{CH}, \mathrm{C}-3), 77.9\left(\mathrm{CH}_{2}, \mathrm{C}-3\right), 77.6$ (CH, C-4 or C-5), $74.8\left(\mathrm{CH}_{2}, \mathrm{OBn}\right), 74.6\left(\mathrm{CH}_{2}, \mathrm{OBn}\right), 73.5\left(\mathrm{CH}_{2}\right.$, $\mathrm{OBn}), 73.0(\mathrm{CH}, \mathrm{C}-2), 72.1(\mathrm{CH}, \mathrm{C}-1), 71.9(\mathrm{CH}, \mathrm{C}-4$ or $\mathrm{C}-5), 69.0$ $\left(\mathrm{CH}_{2}, \mathrm{C}-6\right), 24.3\left(\mathrm{CH}_{2}, \mathrm{C}-1^{\prime}\right.$ or C-2' $), 22.1\left(\mathrm{CH}_{2}, \mathrm{C}-1^{\prime}\right.$ or $\left.\mathrm{C}-2^{\prime}\right), 20.9$ ppm $\left(\mathrm{CH}_{3}, \mathrm{OAc}\right)$. IR $\left(\mathrm{CHCl}_{3}\right): \nu=3013,2870,1790,1734,1236$ $\mathrm{cm}^{-1}$. MS (ESI) $m / z(\%)=702(100)[\mathrm{M}+\mathrm{Na}]^{+}$. HRMS (ESI) $m / z$ : $[\mathrm{M}+\mathrm{Na}]^{+}$calcd for $\mathrm{C}_{40} \mathrm{H}_{41} \mathrm{NNaO}_{9}$ 702.2679; found 702.2680. Anal. calcd for $\mathrm{C}_{40} \mathrm{H}_{41} \mathrm{NO}_{9}$ : C, 70.68; H, 6.08; N, 2.06. Found C, 70.55; H, 6.07; $\mathrm{N}, 2.24$

3-C-(2-O-Acetyl-3,4,6-tri-O-benzyl- $\beta$-D-glucopyranosyl) 1-propoxyphthalimide (3). Following the general procedure starting from phthalimide $67(790 \mathrm{mg}, 1.26 \mathrm{mmol})$ and purification by column chromatography (hexanes-EtOAc, 7:3 to $1: 1)$, product $3(600 \mathrm{mg}$, $0.88 \mathrm{mmol}, 71 \%)$ was obtained as a colorless oil: $[\alpha]_{\mathrm{D}}=+17.7(c=$ $\left.0.62, \mathrm{CHCl}_{3}\right) .{ }^{1} \mathrm{H}$ NMR $\left(400 \mathrm{MHz}, \mathrm{CDCl}_{3}\right) \delta_{\mathrm{H}} 7.82-7.78(\mathrm{~m}, 2 \mathrm{H}$, Ar), 7.74-7.70 (m, 2H, Ar), 7.33-7.16 (m, 15H, Ar), 4.90 (dd, $J=$ 9.5, $9.5 \mathrm{~Hz}, 1 \mathrm{H}, 2-\mathrm{H}), 4.82(\mathrm{~d}, J=11.6 \mathrm{~Hz}, 1 \mathrm{H}, \mathrm{OBn}), 4.78(\mathrm{~d}, J=$ $10.8 \mathrm{~Hz}, 1 \mathrm{H}, \mathrm{OBn}), 4.67(\mathrm{~d}, J=11.4 \mathrm{~Hz}, 1 \mathrm{H}, \mathrm{OBn}), 4.60(\mathrm{~d}, J=12.3$ $\mathrm{Hz}, 1 \mathrm{H}, \mathrm{OBn}), 4.55(\mathrm{~d}, J=12.6 \mathrm{~Hz}, 1 \mathrm{H}, \mathrm{OBn}), 4.51(\mathrm{~d}, J=12.3 \mathrm{~Hz}$, $1 \mathrm{H}, \mathrm{OBn}), 4.25\left(\mathrm{~m}, 1 \mathrm{H}, 3^{\prime}-\mathrm{H}_{\mathrm{b}}\right), 4.17\left(\mathrm{~m}, 1 \mathrm{H}, 3^{\prime}-\mathrm{H}_{\mathrm{a}}\right), 3.74-3.64(\mathrm{~m}$, $4 \mathrm{H}, 3-\mathrm{H}, 4-\mathrm{H}, 6-\mathrm{H}_{2}$ ), 3.44 (ddd, $J=8.0,4.0,2.2 \mathrm{~Hz}, 1 \mathrm{H}, 5-\mathrm{H}$ ), 3.38 (ddd, $J=9.2,9.2,1.9 \mathrm{~Hz}, 1 \mathrm{H}, 1-\mathrm{H}), 2.01\left(\mathrm{~m}, 1 \mathrm{H}, 2^{\prime}-\mathrm{H}_{\mathrm{b}}\right), 1.99(\mathrm{~s}, 3 \mathrm{H}$, OAc), $1.89-1.81\left(\mathrm{~m}, 2 \mathrm{H}, 1^{\prime}-\mathrm{H}_{\mathrm{b}}, 2^{\prime}-\mathrm{H}_{\mathrm{a}}\right), 1.60 \mathrm{ppm}\left(\mathrm{m}, 1 \mathrm{H}, 1^{\prime}-\mathrm{H}_{\mathrm{a}}\right)$. ${ }^{13} \mathrm{C}\left\{{ }^{1} \mathrm{H}\right\}$ NMR (100.6 MHz, CDCl $\left.{ }_{3}\right) \delta_{\mathrm{C}} 170.0(\mathrm{C}, \mathrm{OAc}), 163.5(2 \times$ C, CO), 138.4 (C, Ar), 138.2 (C, Ar), 138.1 (C, Ar), 134.3 (2 × CH, $\mathrm{Ar}), 129.0(2 \times \mathrm{C}, \mathrm{Ar}), 128.4(2 \times \mathrm{CH}, \mathrm{Ar}), 128.33(2 \times \mathrm{CH}, \mathrm{Ar})$, $128.28(2 \times \mathrm{CH}, \mathrm{Ar}), 127.9(2 \times \mathrm{CH}, \mathrm{Ar}), 127.7(\mathrm{CH}, \mathrm{Ar}), 127.64(4$ $\times \mathrm{CH}, \mathrm{Ar}), 127.56(\mathrm{CH}, \mathrm{Ar}), 127.5(\mathrm{CH}, \mathrm{Ar}), 123.4(2 \times \mathrm{CH}, \mathrm{Ar})$, 84.7 (CH, C-2), 79.1 (CH, C-3), $78.4(\mathrm{CH}, \mathrm{C}-4), 78.1\left(\mathrm{CH}_{2}, \mathrm{C}-3\right.$ '), $77.4(\mathrm{CH}, \mathrm{C}-5), 75.1\left(\mathrm{CH}_{2}, \mathrm{OBn}\right), 74.9\left(\mathrm{CH}_{2}, \mathrm{OBn}\right), 73.8(\mathrm{CH}, \mathrm{C}-$ 1), $73.4\left(\mathrm{CH}_{2}, \mathrm{OBn}\right), 69.0\left(\mathrm{CH}_{2}, \mathrm{C}-6\right), 27.5\left(\mathrm{CH}_{2}, \mathrm{C}-1^{\prime}\right), 24.0\left(\mathrm{CH}_{2}\right.$, C-2'), $20.9 \mathrm{ppm}\left(\mathrm{CH}_{3}, \mathrm{OAc}\right)$. IR $\left(\mathrm{CHCl}_{3}\right): \nu=3032,2926,2863$, $1792,1737,1455,1371,1231,1103 \mathrm{~cm}^{-1}$. MS (ESI) $\mathrm{m} / z(\%)=702$ (100) $[\mathrm{M}+\mathrm{Na}]^{+}$. HRMS (ESI) $\mathrm{m} / z:[\mathrm{M}+\mathrm{Na}]^{+}$calcd for $\mathrm{C}_{40} \mathrm{H}_{41} \mathrm{NNaO}_{9}$ 702.2679; found 702.2689. Anal. calcd for $\mathrm{C}_{40} \mathrm{H}_{41} \mathrm{NO}_{9}$ : C, 70.68; H, 6.08; N, 2.06. Found: C, 70.69; H, 6.20; $\mathrm{N}, 2.33$.

3-C-(2-O-Acetyl-3,4,6-tri-O-benzyl- $\alpha$-D-mannopyranosyl) 1-propoxyphthalimide (5). Following the general procedure starting from phthalimide $70(99.2 \mathrm{mg}, 0.16 \mathrm{mmol})$ and purification by column chromatography (hexanes-EtOAc, 6:4), product $5(90.7 \mathrm{mg}, 0.13$ mmol, $86 \%)$ was obtained as a colorless oil: $[\alpha]_{\mathrm{D}}=+5.4(c=0.43$, $\left.\mathrm{CHCl}_{3}\right) .{ }^{1} \mathrm{H}$ NMR $\left(500 \mathrm{MHz}, \mathrm{CDCl}_{3}\right.$, simulated ring coupling constants using DAISY) $\delta_{\mathrm{H}} 7.83-7.81(\mathrm{~m}, 2 \mathrm{H}, \mathrm{Ar}), 7.74-7.72(\mathrm{~m}$, $2 \mathrm{H}, \mathrm{Ar}), 7.33-7.17(\mathrm{~m}, 15 \mathrm{H}, \mathrm{Ar}), 5.30(\mathrm{dd}, J=3.4,2.6 \mathrm{~Hz}, 1 \mathrm{H}, 2-\mathrm{H})$, $4.82(\mathrm{~d}, J=11.0 \mathrm{~Hz}, 1 \mathrm{H}, \mathrm{OBn}), 4.69(\mathrm{~d}, J=11.4 \mathrm{~Hz}, 1 \mathrm{H}, \mathrm{OBn}), 4.63$ $(\mathrm{d}, J=12.0 \mathrm{~Hz}, 1 \mathrm{H}, \mathrm{OBn}), 4.54(\mathrm{~d}, J=11.4 \mathrm{~Hz}, 1 \mathrm{H}, \mathrm{OBn}), 4.50(\mathrm{~d}, J$ $=12.3 \mathrm{~Hz}, 1 \mathrm{H}, \mathrm{OBn}), 4.49(\mathrm{~d}, J=11.1 \mathrm{~Hz}, 1 \mathrm{H}, \mathrm{OBn}), 4.27-4.19(\mathrm{~m}$, $2 \mathrm{H}, 3$ ' $-\mathrm{H}_{2}$ ), 4.03 (ddd, $\left.J=10.9,4.1,2.6 \mathrm{~Hz}, 1 \mathrm{H}, 1-\mathrm{H}\right), 3.93$ (dd, $J=$ $8.7,3.4 \mathrm{~Hz}, 1 \mathrm{H}, 3-\mathrm{H}), 3.85(\mathrm{dd}, J=8.7,8.4 \mathrm{~Hz}, 1 \mathrm{H}, 4-\mathrm{H}), 3.78-3.69$ (m, 3H, 5- H, 6- $\left.\mathrm{H}_{2}\right), 2.14$ (s, 3H, OAc), 2.01-1.91 (m, 2H, 1'- $\left.\mathrm{H}_{2}\right)$, 1.87-1.77 ppm (m, 2H, 2'- $\left.\mathrm{H}_{2}\right) .{ }^{13} \mathrm{C}\left\{{ }^{1} \mathrm{H}\right\}$ NMR $\left(100.6 \mathrm{MHz}, \mathrm{CDCl}_{3}\right)$ $\delta_{\mathrm{C}} 170.6(\mathrm{C}, \mathrm{OAc}), 163.6(2 \times \mathrm{C}, \mathrm{CO}), 138.4(2 \times \mathrm{C}, \mathrm{Ar}), 137.9(\mathrm{C}$, $\mathrm{Ar}), 134.4(2 \times \mathrm{CH}, \mathrm{Ar}), 129.0(2 \times \mathrm{C}, \mathrm{Ar}), 128.4(2 \times \mathrm{CH}, \mathrm{Ar})$, $128.31(2 \times \mathrm{CH}, \mathrm{Ar}), 128.29(2 \times \mathrm{CH}, \mathrm{Ar}), 128.1(2 \times \mathrm{CH}, \mathrm{Ar})$, $127.9(2 \times \mathrm{CH}, \mathrm{Ar}), 127.8(\mathrm{CH}, \mathrm{Ar}), 127.7(2 \times \mathrm{CH}, \mathrm{Ar}), 127.6$ $(\mathrm{CH}, \mathrm{Ar}), 127.5(\mathrm{CH}, \mathrm{Ar}), 123.5(2 \times \mathrm{CH}, \mathrm{Ar}), 77.9(\mathrm{CH}, \mathrm{C}-3), 77.8$ $\left(\mathrm{CH}_{2}, \mathrm{C}-3^{\prime}\right), 75.0(\mathrm{CH}, \mathrm{C}-4), 74.8(\mathrm{CH}, \mathrm{C}-5), 74.7\left(\mathrm{CH}_{2}, \mathrm{OBn}\right), 73.5$ $\left(\mathrm{CH}_{2}, \mathrm{OBn}\right), 72.8(\mathrm{CH}, \mathrm{C}-1), 72.0\left(\mathrm{CH}_{2}, \mathrm{OBn}\right), 70.7(\mathrm{CH}, \mathrm{C}-2)$, 69.4 $\left(\mathrm{CH}_{2}, \mathrm{C}-6\right), 25.0\left(\mathrm{CH}_{2}, \mathrm{C}-1\right.$ ' or C-2'), $24.8\left(\mathrm{CH}_{2}, \mathrm{C}-1\right.$ ' or C-2'), $21.2 \mathrm{ppm}\left(\mathrm{CH}_{3}, \mathrm{OAc}\right)$. IR $\left(\mathrm{CHCl}_{3}\right): \nu=3034,2929,1790,1734$, $1189 \mathrm{~cm}^{-1}$. MS (ESI) $\mathrm{m} / z(\%)=702(100)[\mathrm{M}+\mathrm{Na}]^{+}$. HRMS (ESI) $m / z:[\mathrm{M}+\mathrm{Na}]^{+}$calcd for $\mathrm{C}_{40} \mathrm{H}_{41} \mathrm{NNaO}_{9} 702.2679$; found 702.2687. 
Anal. calcd for $\mathrm{C}_{40} \mathrm{H}_{41} \mathrm{NO}_{9}$ : C, 70.68; H, 6.08; N, 2.06. Found: C, 70.74; H, 6.12; N, 2.04.

3-C-(2-O-Acetyl-3,4,6-tri-O-benzyl- $\beta$-D-mannopyranosyl) 1-propoxyphthalimide (7). Following the general procedure starting from phthalimide $73(77 \mathrm{mg}, 0.11 \mathrm{mmol})$ and purification by column chromatography (hexanes-EtOAc, 8:2), product $7(60 \mathrm{mg}, 0.09$ mmol, $80 \%)$ was obtained as a colorless oil: $[\alpha]_{\mathrm{D}}=-16.7(c=0.63$, $\left.\mathrm{CHCl}_{3}\right) .{ }^{1} \mathrm{H}$ NMR $\left(500 \mathrm{MHz}, \mathrm{CDCl}_{3}\right.$, simulated coupling constants using DAISY) $\delta_{\mathrm{H}} 7.82-7.79(\mathrm{~m}, 2 \mathrm{H}, \mathrm{Ar}), 7.74-7.72(\mathrm{~m}, 2 \mathrm{H}, \mathrm{Ar})$, 7.34-7.16 (m, 15H, Ar), $5.52(\mathrm{dd}, J=3.3,1.0 \mathrm{~Hz}, 1 \mathrm{H}, 2-\mathrm{H}), 4.86(\mathrm{~d}$, $J=10.8 \mathrm{~Hz}, 1 \mathrm{H}, \mathrm{OBn}), 4.77(\mathrm{~d}, J=11.1 \mathrm{~Hz}, 1 \mathrm{H}, \mathrm{OBn}), 4.64(\mathrm{~d}, J=$ $12.3 \mathrm{~Hz}, 1 \mathrm{H}, \mathrm{OBn}$ ), $4.52(\mathrm{~d}, J=11.7 \mathrm{~Hz}, 1 \mathrm{H}, \mathrm{OBn}), 4.50(\mathrm{~d}, J=10.4$ $\mathrm{Hz}, 1 \mathrm{H}, \mathrm{OBn}$ ), 4.50 (d, $J=10.4 \mathrm{~Hz}, 1 \mathrm{H}, \mathrm{OBn}), 4.25-4.18$ (m, 2H, 3'$\mathrm{H}_{2}$ ), 3.77 (dd, $\left.J=9.8,9.3 \mathrm{~Hz}, 1 \mathrm{H}, 4-\mathrm{H}\right), 3.74\left(\mathrm{~m}, 2 \mathrm{H}, 6-\mathrm{H}_{2}\right), 3.70$ (dd, $J=9.3,3.3 \mathrm{~Hz}, 1 \mathrm{H}, 3-\mathrm{H}), 3.63(\mathrm{ddd}, J=8.3,4.7,1.0 \mathrm{~Hz}, 1 \mathrm{H}, 1-$ H), 3.47 (ddd, $J=9.8,5.3,2.1 \mathrm{~Hz}, 1 \mathrm{H}, 5-\mathrm{H}), 2.19$ (s, 3H, OAc), 1.97 $\left(\mathrm{m}, 1 \mathrm{H}, 2^{\prime}-\mathrm{H}_{\mathrm{b}}\right), 1.90-1.83\left(\mathrm{~m}, 2 \mathrm{H}, \mathrm{l}^{\prime}-\mathrm{H}_{\mathrm{b}}, 2^{\prime}-\mathrm{H}_{\mathrm{a}}\right), 1.72 \mathrm{ppm}(\mathrm{m}, 1 \mathrm{H}$, $\left.1^{\prime}-\mathrm{H}_{\mathrm{a}}\right) .{ }^{13} \mathrm{C}\left\{{ }^{1} \mathrm{H}\right\}$ NMR (125.7 MHz, $\left.\mathrm{CDCl}_{3}\right) \delta_{\mathrm{C}} 170.8$ (C, OAc), 163.6 ( $2 \times \mathrm{C}, \mathrm{CO}), 138.4$ (C, Ar), 138.3 (C, Ar), 137.9 (C, Ar), $134.4(2 \times \mathrm{CH}, \mathrm{Ar}), 128.9(2 \times \mathrm{C}, \mathrm{Ar}), 128.34(2 \times \mathrm{CH}, \mathrm{Ar}), 128.26$ $(4 \times \mathrm{CH}, \mathrm{Ar}), 128.1(2 \times \mathrm{CH}, \mathrm{Ar}), 127.9(2 \times \mathrm{CH}, \mathrm{Ar}), 127.8(2 \times$ $\mathrm{CH}, \mathrm{Ar}), 127.7(\mathrm{CH}, \mathrm{Ar}), 127.6(\mathrm{CH}, \mathrm{Ar}), 127.5(\mathrm{CH}, \mathrm{Ar}), 123.4(2$ $\times \mathrm{CH}, \mathrm{Ar}), 81.9(\mathrm{CH}, \mathrm{C}-3), 79.4(\mathrm{CH}, \mathrm{C}-5), 78.0\left(\mathrm{CH}_{2}, \mathrm{C}-3{ }^{\prime}\right), 76.2$ $(\mathrm{CH}, \mathrm{C}-1), 75.1\left(\mathrm{CH}_{2}, \mathrm{OBn}\right), 74.6(\mathrm{CH}, \mathrm{C}-4), 73.4\left(\mathrm{CH}_{2}, \mathrm{OBn}\right)$, $71.5\left(\mathrm{CH}_{2}, \mathrm{OBn}\right), 69.4\left(\mathrm{CH}_{2}, \mathrm{C}-6\right), 69.2(\mathrm{CH}, \mathrm{C}-2), 27.2\left(\mathrm{CH}_{2}, \mathrm{C}-1^{\prime}\right.$ or C-2'), $24.4\left(\mathrm{CH}_{2}, \mathrm{C}-1^{\prime}\right.$ or $\left.\mathrm{C}-2^{\prime}\right), 21.0 \mathrm{ppm}\left(\mathrm{CH}_{3}, \mathrm{OAc}\right)$. IR $\left(\mathrm{CHCl}_{3}\right): \nu=3033,2951,2866,1792,1737,1237,1120 \mathrm{~cm}^{-1}$. MS (ESI) $\mathrm{m} / z(\%)=702(100)[\mathrm{M}+\mathrm{Na}]^{+}$. HRMS (ESI) $\mathrm{m} / z:[\mathrm{M}+$ $\mathrm{Na}]^{+}$calcd for $\mathrm{C}_{40} \mathrm{H}_{41} \mathrm{NNaO}_{9}$ 702.2679; found 702.2675. Anal. calcd for $\mathrm{C}_{40} \mathrm{H}_{41} \mathrm{NO}_{9}$ : C, 70.68; H, 6.08; N, 2.06. Found: C, 70.77; H, 6.05; $\mathrm{N}, 2.10$.

3-C-(2-O-Acetyl-3,4-di-O-benzyl- $\alpha$-L-fucopyranosyl) 1-propoxyphthalimide (9). Following the general procedure starting from phthalimide $77(247.4 \mathrm{mg}, 0.46 \mathrm{mmol})$ and purification by column chromatography (hexanes-EtOAc, 9:1 to7:3), product $9(153.6 \mathrm{mg}$, $0.27 \mathrm{mmol}, 58 \%)$ was obtained as a colorless oil: $[\alpha]_{\mathrm{D}}=-21.6(c=$ $\left.0.74, \mathrm{CHCl}_{3}\right) .{ }^{1} \mathrm{H}$ NMR $\left(500 \mathrm{MHz}, \mathrm{CDCl}_{3}\right.$, simulated coupling constants using DAISY) $\delta_{\mathrm{H}} 7.84-7.81(\mathrm{~m}, 2 \mathrm{H}, \mathrm{Ar}), 7.74-7.73(\mathrm{~m}$, $2 \mathrm{H}, \mathrm{Ar}), 7.39-7.25$ (m, 10H, Ar), $5.16(\mathrm{dd}, J=5.9,3.0 \mathrm{~Hz}, 1 \mathrm{H}, 2-\mathrm{H})$, $4.75(\mathrm{~d}, J=12.0 \mathrm{~Hz}, 1 \mathrm{H}, \mathrm{OBn}), 4.70(\mathrm{~d}, J=12.0 \mathrm{~Hz}, 1 \mathrm{H}, \mathrm{OBn}), 4.66$ $(\mathrm{d}, J=12.0 \mathrm{~Hz}, 1 \mathrm{H}, \mathrm{OBn}), 4.55(\mathrm{~d}, J=12.0 \mathrm{~Hz}, 1 \mathrm{H}, \mathrm{OBn}), 4.22(\mathrm{dd}$, $\left.J=5.0,5.0 \mathrm{~Hz}, 2 \mathrm{H}, 3{ }^{\prime}-\mathrm{H}_{2}\right), 4.15$ (ddd, $\left.J=9.4,4.1,3.0 \mathrm{~Hz}, 1 \mathrm{H}, 1-\mathrm{H}\right)$, 4.06 (dddd, $J=6.7,6.7,6.7,4.5 \mathrm{~Hz}, 1 \mathrm{H}, 5-\mathrm{H}), 3.82(\mathrm{dd}, J=5.9,3.2$ $\mathrm{Hz}, 1 \mathrm{H}, 3-\mathrm{H}), 3.74(\mathrm{dd}, J=4.5,3.2 \mathrm{~Hz}, 1 \mathrm{H}, 4-\mathrm{H}), 2.08(\mathrm{~s}, 3 \mathrm{H}, \mathrm{OAc})$, $1.91\left(\mathrm{~m}, 1 \mathrm{H}, 2^{\prime}-\mathrm{H}_{\mathrm{b}}\right), 1.81-1.74\left(\mathrm{~m}, 2 \mathrm{H}, 1^{\prime}-\mathrm{H}_{\mathrm{b}}, 2^{\prime}-\mathrm{H}_{\mathrm{a}}\right), 1.66(\mathrm{~m}, 1 \mathrm{H}$, $\left.1^{\prime}-\mathrm{H}_{\mathrm{a}}\right), 1.36 \mathrm{ppm}\left(\mathrm{d}, J=6.7 \mathrm{~Hz}, 3 \mathrm{H}, 6-\mathrm{H}_{3}\right) .{ }^{13} \mathrm{C}\left\{{ }^{1} \mathrm{H}\right\}$ NMR $(125.7$ $\left.\mathrm{MHz}, \mathrm{CDCl}_{3}\right) \delta_{\mathrm{C}} 170.3(\mathrm{C}, \mathrm{OAc}) .163 .6(2 \times \mathrm{C}, \mathrm{CO}), 138.4(\mathrm{C}, \mathrm{Ar})$, 138.3 (C, Ar), $134.4(2 \times \mathrm{CH}, \mathrm{Ar}), 128.9(2 \times \mathrm{C}, \mathrm{Ar}), 128.28(2 \times$ $\mathrm{CH}, \mathrm{Ar}), 128.26(2 \times \mathrm{CH}, \mathrm{Ar}), 127.7(2 \times \mathrm{CH}, \mathrm{Ar}), 127.6(\mathrm{CH}, \mathrm{Ar})$, $127.51(\mathrm{CH}, \mathrm{Ar}), 127.46(2 \times \mathrm{CH}, \mathrm{Ar}), 123.4(2 \times \mathrm{CH}, \mathrm{Ar}), 78.1$ $\left(\mathrm{CH}_{2}, \mathrm{C}-3^{\prime}\right), 75.6(\mathrm{CH}, \mathrm{C}-3), 74.6(\mathrm{CH}, \mathrm{C}-4), 72.9\left(\mathrm{CH}_{2}, \mathrm{OBn}\right), 72.2$ $\left(\mathrm{CH}_{2}, \mathrm{OBn}\right), 71.4(\mathrm{CH}, \mathrm{C}-2), 69.5(\mathrm{CH}, \mathrm{C}-5), 67.6(\mathrm{CH}, \mathrm{C}-1), 24.9$ $\left(\mathrm{CH}_{2}, \mathrm{C}-1^{\prime}\right), 24.7\left(\mathrm{CH}_{2}, \mathrm{C}-2^{\prime}\right), 21.0\left(\mathrm{CH}_{3}, \mathrm{OAc}\right), 14.6 \mathrm{ppm}\left(\mathrm{CH}_{3}, \mathrm{C}-\right.$ 6). IR $\left(\mathrm{CHCl}_{3}\right): \nu=3029,1791,1734,1214 \mathrm{~cm}^{-1}$. MS (ESI) $\mathrm{m} / z$ $(\%)=596(100)[\mathrm{M}+\mathrm{Na}]^{+}$. HRMS (ESI) $\mathrm{m} / z:[\mathrm{M}+\mathrm{Na}]^{+}$calcd for $\mathrm{C}_{33} \mathrm{H}_{35} \mathrm{NNaO}_{8}$ 596.2260; found 596.2264. Anal. calcd for $\mathrm{C}_{33} \mathrm{H}_{35} \mathrm{NO}_{8}$ : C, 69.10; H, 6.15; N, 2.44. Found: C, 69.25; H, 6.41; $\mathrm{N}, 2.58$.

General Procedure to Give Diphenoxyphosphoryl Derivatives $2,4,6,8,10$, and 15 . The phthalimide ( $1 \mathrm{mmol})$ was dissolved in dry $\mathrm{CH}_{2} \mathrm{Cl}_{2}(7.5 \mathrm{~mL})$ under a $\mathrm{N}_{2}$ atmosphere. $\mathrm{ClPO}(\mathrm{OPh})_{2}(1 \mathrm{~mL}, 4.7 \mathrm{mmol})$ and DMAP $(580 \mathrm{mg}, 4.75 \mathrm{mmol})$ were added at $0{ }^{\circ} \mathrm{C}$, and after $5 \mathrm{~min}$, the mixture was stirred at room temperature for $2 \mathrm{~h}$. The reaction was quenched with a saturated aqueous $\mathrm{NH}_{4} \mathrm{Cl}$ solution and extracted with $\mathrm{CH}_{2} \mathrm{Cl}_{2}$. The organic extracts were dried over $\mathrm{Na}_{2} \mathrm{SO}_{4}$ and concentrated under reduced pressure. Column chromatography of the residue (hexanes-EtOAc) gave the phosphatyl precursor.

3-C-(3,4,6-Tri-O-benzyl-2-O-diphenoxyphosphoryl- $\alpha$-Dglucopyranosyl)1-propoxyphthalimide (2). Following the general procedure starting from phthalimide $64(129.8 \mathrm{mg}, 0.20 \mathrm{mmol})$ and purification by column chromatography (hexanes-EtOAc, 75:25), product $2(132.6 \mathrm{mg}, 0.15 \mathrm{mmol}, 76 \%)$ was obtained as a colorless oil: $[\alpha]_{\mathrm{D}}=+40.5\left(c=0.44, \mathrm{CHCl}_{3}\right) .{ }^{1} \mathrm{H} \mathrm{NMR}\left(500 \mathrm{MHz}, \mathrm{CDCl}_{3}\right) \delta_{\mathrm{H}}$ 7.83-7.81 (m, 2H, Ar), 7.74-7.72 (m, 2H, Ar), 7.30-7.10 (m, $25 \mathrm{H}$, Ar), 4.83 (d, $J=11.0 \mathrm{~Hz}, 1 \mathrm{H}, \mathrm{OBn}), 4.80$ (ddd, $J=10.5,5.5 \mathrm{~Hz},{ }^{3} J_{\mathrm{PH}}$ $=8.6 \mathrm{~Hz}, 1 \mathrm{H}, 2-\mathrm{H}), 4.75(\mathrm{~d}, J=11.0 \mathrm{~Hz}, 1 \mathrm{H}, \mathrm{OBn}), 4.74(\mathrm{~d}, J=11.1$ $\mathrm{Hz}, 1 \mathrm{H}, \mathrm{OBn}), 4.60(\mathrm{~d}, J=12.0 \mathrm{~Hz}, 1 \mathrm{H}, \mathrm{OBn}), 4.46(\mathrm{~d}, J=11.0 \mathrm{~Hz}$, $1 \mathrm{H}, \mathrm{OBn}), 4.45$ (d, $J=12.0 \mathrm{~Hz}, 1 \mathrm{H}, \mathrm{OBn}), 4.20-4.15(\mathrm{~m}, 2 \mathrm{H}, 3$ '$\mathrm{H}_{2}$ ), 4.12 (ddd, $\left.J=9.4,7.2,5.5 \mathrm{~Hz}, 1 \mathrm{H}, 1-\mathrm{H}\right), 3.91$ (ddd, $J=10.5,5.7$ $\left.\mathrm{Hz},{ }^{4} J_{\mathrm{PH}}=2.9 \mathrm{~Hz}, 1 \mathrm{H}, 3-\mathrm{H}\right), 3.71-3.64\left(\mathrm{~m}, 4 \mathrm{H}, 4-\mathrm{H}, 5-\mathrm{H}, 6-\mathrm{H}_{2}\right)$, $1.97\left(\mathrm{~m}, 1 \mathrm{H}, 1^{\prime}-\mathrm{H}_{\mathrm{b}}\right.$ or $\left.2^{\prime}-\mathrm{H}_{\mathrm{b}}\right), 1.90\left(\mathrm{~m}, 1 \mathrm{H}, 1^{\prime}-\mathrm{H}_{\mathrm{b}}\right.$ or $\left.2^{\prime}-\mathrm{H}_{\mathrm{b}}\right), 1.72(\mathrm{~m}$, $1 \mathrm{H}, 1^{\prime}-\mathrm{H}_{\mathrm{a}}$ or $\left.2^{\prime}-\mathrm{H}_{\mathrm{a}}\right), 1.63 \mathrm{ppm}\left(\mathrm{m}, 1 \mathrm{H}, 1^{\prime}-\mathrm{H}_{\mathrm{a}}\right.$ or $\left.2^{\prime}-\mathrm{H}_{\mathrm{a}}\right) .{ }^{13} \mathrm{C}\left\{{ }^{1} \mathrm{H}\right\} \mathrm{NMR}$ $\left(125.7 \mathrm{MHz}, \mathrm{CDCl}_{3}\right) \delta_{\mathrm{C}} 163.5(2 \times \mathrm{C}, \mathrm{CO}) .150 .5\left(\mathrm{~d},{ }^{2} \mathrm{~J}_{\mathrm{PC}}=7.0 \mathrm{~Hz}\right.$, C, Ar), 150.4 (d, $\left.{ }^{2} J_{\mathrm{PC}}=6.4 \mathrm{~Hz}, \mathrm{C}, \mathrm{Ar}\right), 138.1$ (C, Ar), 138.0 (C, Ar), 137.9 (C, Ar), $134.4(2 \times \mathrm{CH}, \mathrm{Ar}), 129.8(2 \times \mathrm{CH}, \mathrm{Ar}), 129.7(2 \times$ $\mathrm{CH}, \mathrm{Ar}), 128.9(2 \times \mathrm{C}, \mathrm{Ar}), 128.3(4 \times \mathrm{CH}, \mathrm{Ar}), 128.2(2 \times \mathrm{CH}, \mathrm{Ar})$, $127.8(6 \times \mathrm{CH}, \mathrm{Ar}), 127.7(\mathrm{CH}, \mathrm{Ar}), 127.6(\mathrm{CH}, \mathrm{Ar}), 127.5(\mathrm{CH}$, $\mathrm{Ar}), 125.4(\mathrm{CH}, \mathrm{Ar}), 125.2(\mathrm{CH}, \mathrm{Ar}), 123.4(2 \times \mathrm{CH}, \mathrm{Ar}), 120.22$ (CH, Ar), 120.18 (CH, Ar), $119.99(\mathrm{CH}, \mathrm{Ar}), 119.95(\mathrm{CH}, \mathrm{Ar}), 80.5$ $\left(\mathrm{d},{ }^{3} J_{\mathrm{PC}}=6.3 \mathrm{~Hz}, \mathrm{CH}, \mathrm{C}-3\right), 78.4\left(\mathrm{~d},{ }^{2} J_{\mathrm{PC}}=7.4 \mathrm{~Hz}, \mathrm{CH}, \mathrm{C}-2\right), 77.9$ $\left(\mathrm{CH}_{2}, \mathrm{C}-3\right.$ '), $77.8(\mathrm{CH}, \mathrm{C}-1), 75.1\left(\mathrm{CH}_{2}, \mathrm{OBn}\right), 74.8\left(\mathrm{CH}_{2}, \mathrm{OBn}\right)$, $73.7(\mathrm{CH}, \mathrm{C}-4$ or $\mathrm{C}-5), 73.5\left(\mathrm{CH}_{2}, \mathrm{OBn}\right), 71.4(\mathrm{CH}, \mathrm{C}-4$ or $\mathrm{C}-5)$, $68.9\left(\mathrm{CH}_{2}, \mathrm{C}-6\right), 24.3\left(\mathrm{CH}_{2}, \mathrm{C}-1^{\prime}\right.$ or C-2'), $21.1 \mathrm{ppm}\left(\mathrm{CH}_{2}, \mathrm{C}-1^{\prime}\right.$ or C-2'). IR $\left(\mathrm{CHCl}_{3}\right): \nu=3021,2946,1790,1734,1213 \mathrm{~cm}^{-1}$. MS (ESI) $m / z(\%)=892(100)[\mathrm{M}+\mathrm{Na}]^{+}$. HRMS (ESI) $m / z:[\mathrm{M}+$ $\mathrm{Na}]^{+}$calcd for $\mathrm{C}_{50} \mathrm{H}_{48} \mathrm{NNaO}_{11} \mathrm{P}$ 892.2863; found 892.2856. Anal. calcd for $\mathrm{C}_{50} \mathrm{H}_{48} \mathrm{NO}_{11} \mathrm{P}$ : C, 69.04; $\mathrm{H}$; 5.56; N, 1.61. Found: C, 69.39; $\mathrm{H}, 5.74 ; \mathrm{N}, 1.71$.

3-C-(3,4,6-Tri-O-benzyl-2-O-diphenoxyphosphoryl- $\beta$-Dglucopyranosyl)1-propoxyphthalimide (4). Following the general procedure starting from phthalimide $67(327 \mathrm{mg}, 0.51 \mathrm{mmol})$ and purification by column chromatography (hexanes-EtOAc, 7:3), product $4(224 \mathrm{mg}, 0.26 \mathrm{mmol}, 51 \%)$ was obtained as a colorless oil: $[\alpha]_{\mathrm{D}}=+9.9\left(c=0.83, \mathrm{CHCl}_{3}\right) .{ }^{1} \mathrm{H} \operatorname{NMR}\left(500 \mathrm{MHz}, \mathrm{CDCl}_{3}\right.$, simulated coupling constants using DAISY) $\delta_{\mathrm{H}} 7.83-7.80(\mathrm{~m}, 2 \mathrm{H}$, $\mathrm{Ar}), 7.74-7.70(\mathrm{~m}, 2 \mathrm{H}, \mathrm{Ar}), 7.30-7.06(\mathrm{~m}, 25 \mathrm{H}, \mathrm{Ar}), 4.88(\mathrm{~d}, J=$ $10.8 \mathrm{~Hz}, 1 \mathrm{H}, \mathrm{OBn}), 4.83$ (d, $J=10.8 \mathrm{~Hz}, 1 \mathrm{H}, \mathrm{OBn}), 4.74$ (d, $J=11.1$ $\mathrm{Hz}, 1 \mathrm{H}, \mathrm{OBn}), 4.58(\mathrm{~d}, J=12.3 \mathrm{~Hz}, 1 \mathrm{H}, \mathrm{OBn}), 4.54(\mathrm{~d}, J=10.7 \mathrm{~Hz}$, $1 \mathrm{H}, \mathrm{OBn}), 4.49(\mathrm{~d}, J=12.3 \mathrm{~Hz}, 1 \mathrm{H}, \mathrm{OBn}), 4.43(\mathrm{ddd}, J=9.5,8.9 \mathrm{~Hz}$, $\left.{ }^{3} J_{\mathrm{PH}}=8.2 \mathrm{~Hz}, 1 \mathrm{H}, 2-\mathrm{H}\right), 4.13\left(\mathrm{ddd}, J=8.8,7.6,6.3 \mathrm{~Hz}, 1 \mathrm{H}, 3{ }^{\prime}-\mathrm{H}_{\mathrm{b}}\right)$, 4.04 (ddd, $\left.J=8.8,7.6,6.6 \mathrm{~Hz}, 1 \mathrm{H}, 3-\mathrm{H}_{\mathrm{a}}\right), 3.78(\mathrm{dd}, J=9.2,9.2 \mathrm{~Hz}$, $1 \mathrm{H}, 3-\mathrm{H}), 3.72-3.66\left(\mathrm{~m}, 2 \mathrm{H}, 6-\mathrm{H}_{2}\right), 3.70(\mathrm{dd}, J=9.5,9.3 \mathrm{~Hz}, 1 \mathrm{H}, 4-$ $\mathrm{H}$ ), 3.48 (dddd, $J=9.5,8.2,8.1,2.5 \mathrm{~Hz}, 1 \mathrm{H}, 1-\mathrm{H}), 3.45$ (ddd, $J=9.5$, 3.8, $1.8 \mathrm{~Hz}, 1 \mathrm{H}, 5-\mathrm{H}), 2.00\left(\mathrm{~m}, 1 \mathrm{H}, 2^{\prime}-\mathrm{H}_{\mathrm{b}}\right), 1.89\left(\mathrm{~m}, 1 \mathrm{H}, 2^{\prime}-\mathrm{H}_{\mathrm{a}}\right), 1.83$ $\left(\mathrm{m}, 1 \mathrm{H}, 11^{\prime}-\mathrm{H}_{\mathrm{b}}\right), 1.57 \mathrm{ppm}\left(\mathrm{m}, 1 \mathrm{H}, 11^{\prime}-\mathrm{H}_{\mathrm{a}}\right) .{ }^{13} \mathrm{C}\left\{{ }^{1} \mathrm{H}\right\} \operatorname{NMR}(100.6$ $\left.\mathrm{MHz}_{\mathrm{CDCl}}\right) \delta_{\mathrm{C}} 163.4(2 \times \mathrm{C}, \mathrm{CO}), 150.6\left(\mathrm{~d},{ }^{2} \mathrm{~J}_{\mathrm{PC}}=7.1 \mathrm{~Hz}, \mathrm{C}, \mathrm{Ar}\right)$, $150.5\left(\mathrm{~d},{ }^{2} J_{\mathrm{PC}}=7.1 \mathrm{~Hz}, \mathrm{C}, \mathrm{Ar}\right), 138.3(\mathrm{C}, \mathrm{Ar}), 138.1$ (C, Ar), 138.0 (C, Ar), $134.3(2 \times \mathrm{CH}, \mathrm{Ar}), 129.6(2 \times \mathrm{CH}, \mathrm{Ar}), 129.5(2 \times \mathrm{CH}$, Ar), $129.0(2 \times \mathrm{C}, \mathrm{Ar}), 128.28(2 \times \mathrm{CH}, \mathrm{Ar}), 128.26(2 \times \mathrm{CH}, \mathrm{Ar})$, $128.0(2 \times \mathrm{CH}, \mathrm{Ar}), 127.7(2 \times \mathrm{CH}, \mathrm{Ar}), 127.6(3 \times \mathrm{CH}, \mathrm{Ar}), 127.5$ $(3 \times \mathrm{CH}, \mathrm{Ar}), 127.2(\mathrm{CH}, \mathrm{Ar}), 125.3(\mathrm{CH}, \mathrm{Ar}), 125.0(\mathrm{CH}, \mathrm{Ar})$, $123.3(2 \times \mathrm{CH}, \mathrm{Ar}), 120.4(\mathrm{CH}, \mathrm{Ar}), 120.3(\mathrm{CH}, \mathrm{Ar}), 120.1(\mathrm{CH}$, $\mathrm{Ar}), 120.0(\mathrm{CH}, \mathrm{Ar}), 84.7\left(\mathrm{~d},{ }^{3} \mathrm{JC}_{\mathrm{PC}}=2.1 \mathrm{~Hz}, \mathrm{CH}, \mathrm{C}-3\right), 80.6\left(\mathrm{~d},{ }^{2} J_{\mathrm{PC}}=\right.$ $7.7 \mathrm{~Hz}, \mathrm{CH}, \mathrm{C}-2), 79.0$ (CH, C-4), $78.5(\mathrm{CH}, \mathrm{C}-5), 78.0\left(\mathrm{CH}_{2}, \mathrm{C}-3\right.$ '), $77.8\left(\mathrm{~d},{ }^{3} \mathrm{JPC}_{\mathrm{PC}}=4.9 \mathrm{~Hz}, \mathrm{CH}, \mathrm{C}-1\right), 75.0\left(\mathrm{CH}_{2}, \mathrm{OBn}\right), 74.8\left(\mathrm{CH}_{2}\right.$, $\mathrm{OBn}), 73.4\left(\mathrm{CH}_{2}, \mathrm{OBn}\right), 68.8\left(\mathrm{CH}_{2}, \mathrm{C}-6\right), 27.2\left(\mathrm{CH}_{2}, \mathrm{C}-1\right.$ ') $), 23.9$ ppm $\left(\mathrm{CH}_{2}, \mathrm{C}-2^{\prime}\right)$. IR $\left(\mathrm{CHCl}_{3}\right): \nu=3013,2870,1776,1736,1240$, $1090 \mathrm{~cm}^{-1}$. MS (ESI) $\mathrm{m} / z(\%)=892(100)[\mathrm{M}+\mathrm{Na}]^{+}$. HRMS (ESI) $m / z:[\mathrm{M}+\mathrm{Na}]^{+}$calcd for $\mathrm{C}_{50} \mathrm{H}_{48} \mathrm{NNaO}_{11} \mathrm{P}$ 892.2863; found 892.2879. Anal. calcd for $\mathrm{C}_{50} \mathrm{H}_{48} \mathrm{NO}_{11} \mathrm{P}: \mathrm{C}, 69.04 ; \mathrm{H}, 5.56 ; \mathrm{N}, 1.61$. Found: C, 69.15; H, 5.64; N, 1.99 .

3-C-(3,4,6-Tri-O-benzyl-2-O-diphenoxyphosphoryl- $\alpha-D-$ mannopyranosyl)1-propoxyphthalimide (6). Following the general procedure starting from phthalimide $70(109.2 \mathrm{mg}, 0.17 \mathrm{mmol})$ and purification by column chromatography (hexanes-EtOAc, 65:35), product $6(110.6 \mathrm{mg}, 0.13 \mathrm{mmol}, 74 \%)$ was obtained as a colorless oil: $[\alpha]_{\mathrm{D}}=-10.7\left(c=0.54, \mathrm{CHCl}_{3}\right) .{ }^{1} \mathrm{H} \mathrm{NMR}\left(500 \mathrm{MHz}, \mathrm{CDCl}_{3}\right) \delta_{\mathrm{H}}$ 7.80-7.79 (m, 2H, Ar), 7.74-7.70 (m, 2H, Ar), 7.34-7.06 (m, 25H, Ar), $4.96\left(\mathrm{ddd}, J=2.9,2.9 \mathrm{~Hz},{ }^{3} J_{\mathrm{PH}}=8.2 \mathrm{~Hz}, 1 \mathrm{H}, 2-\mathrm{H}\right), 4.81(\mathrm{~d}, J=$ 
$11.1 \mathrm{~Hz}, 1 \mathrm{H}, \mathrm{OBn}$ ), 4.67 (d, $J=11.0 \mathrm{~Hz}, 1 \mathrm{H}, \mathrm{OBn}), 4.57$ (d, $J=12.0$ $\mathrm{Hz}, 1 \mathrm{H}, \mathrm{OBn}), 4.53(\mathrm{~d}, J=11.4 \mathrm{~Hz}, 1 \mathrm{H}, \mathrm{OBn}), 4.49(\mathrm{~d}, J=12.3 \mathrm{~Hz}$, $1 \mathrm{H}, \mathrm{OBn}$ ), 4.40 (d, $J=11.0 \mathrm{~Hz}, 1 \mathrm{H}, \mathrm{OBn}), 4.21-4.16\left(\mathrm{~m}, 2 \mathrm{H}, 3^{\prime}-\right.$ $\mathrm{H}_{2}$ ), 4.07 (ddd, $\left.J=9.8,3.8,3.8 \mathrm{~Hz}, 1 \mathrm{H}, 1-\mathrm{H}\right), 3.92(\mathrm{~m}, 1 \mathrm{H}, 3-\mathrm{H})$, 3.74-3.66 (m, 4H, 4-H, 5-H, 6- $\left.\mathrm{H}_{2}\right), 1.94-1.85\left(\mathrm{~m}, 2 \mathrm{H}, 1^{\prime}-\mathrm{H}_{\mathrm{b}}, 2^{\prime}-\mathrm{H}_{\mathrm{b}}\right)$, 1.81-1.70 ppm (m, 2H, 1'- $\left.\mathrm{H}_{\mathrm{a}}, 2^{\prime}-\mathrm{H}_{\mathrm{a}}\right) \cdot{ }^{13} \mathrm{C}\left\{{ }^{1} \mathrm{H}\right\}$ NMR $(125.7 \mathrm{MHz}$, $\left.\mathrm{CDCl}_{3}\right) \delta_{\mathrm{C}} 163.5(2 \times \mathrm{C}, \mathrm{CO}), 150.7\left(\mathrm{~d},{ }^{2} J_{\mathrm{PC}}=7.4 \mathrm{~Hz}, \mathrm{C}, \mathrm{Ar}\right), 150.5$ $\left(\mathrm{d},{ }^{2} J_{\mathrm{PC}}=7.4 \mathrm{~Hz}, \mathrm{C}, \mathrm{Ar}\right), 138.3$ (C, Ar), 138.1 (C, Ar), 137.7 (C, Ar), 134.3 $(2 \times \mathrm{CH}, \mathrm{Ar}), 129.7(2 \times \mathrm{CH}, \mathrm{Ar}), 129.5(2 \times \mathrm{CH}, \mathrm{Ar}), 128.9$ $(2 \times \mathrm{C}, \mathrm{Ar}), 128.3(2 \times \mathrm{CH}, \mathrm{Ar}), 128.24(4 \times \mathrm{CH}, \mathrm{Ar}), 128.19(2 \times$ $\mathrm{CH}, \mathrm{Ar}), 127.9(2 \times \mathrm{CH}, \mathrm{Ar}), 127.64(2 \times \mathrm{CH}, \mathrm{Ar}), 127.59(2 \times \mathrm{CH}$, $\mathrm{Ar}), 127.4(\mathrm{CH}, \mathrm{Ar}), 125.2(\mathrm{CH}, \mathrm{Ar}), 125.0(\mathrm{CH}, \mathrm{Ar}), 123.4(2 \times$ $\mathrm{CH}, \mathrm{Ar}), 120.3(\mathrm{CH}, \mathrm{Ar}), 120.24(\mathrm{CH}, \mathrm{Ar}), 120.18(\mathrm{CH}, \mathrm{Ar}), 120.1$ $(\mathrm{CH}, \mathrm{Ar}), 77.8\left(\mathrm{~d},{ }^{3} \mathrm{~J}_{\mathrm{PC}}=3.2 \mathrm{~Hz}, \mathrm{CH}, \mathrm{C}-3\right), 77.7\left(\mathrm{CH}_{2}, \mathrm{C}-3\right), 77.5$ $\left(\mathrm{d},{ }^{2} J_{\mathrm{PC}}=6.4 \mathrm{~Hz}, \mathrm{CH}, \mathrm{C}-2\right), 74.9(\mathrm{br} \mathrm{s}, \mathrm{CH}, \mathrm{C}-1), 74.5\left(\mathrm{CH}_{2}, \mathrm{OBn}\right)$, $74.3\left(\mathrm{CH}, \mathrm{C}-4\right.$ or C-5), $73.3\left(\mathrm{CH}_{2}, \mathrm{OBn}\right), 73.0(\mathrm{CH}, \mathrm{C}-4$ or $\mathrm{C}-5)$, $72.1\left(\mathrm{CH}_{2}, \mathrm{OBn}\right), 69.1\left(\mathrm{CH}_{2}, \mathrm{C}-6\right), 24.8\left(\mathrm{CH}_{2}, \mathrm{C}-1\right.$ ' or C-2'), 24.6 ppm $\left(\mathrm{CH}_{2}, \mathrm{C}-1^{\prime}\right.$ or C-2'). IR $\left(\mathrm{CHCl}_{3}\right): \nu=2929,2858,1793,1737$, $1491,1191 \mathrm{~cm}^{-1}$. MS (ESI) $m / z(\%)=892(100)[\mathrm{M}+\mathrm{Na}]^{+}$. HRMS (ESI) $m / z:[\mathrm{M}+\mathrm{Na}]^{+}$calcd for $\mathrm{C}_{50} \mathrm{H}_{48} \mathrm{NNaO}_{11} \mathrm{P}$ 892.2863; found 892.2864. Anal. calcd for $\mathrm{C}_{50} \mathrm{H}_{48} \mathrm{NO}_{11} \mathrm{P}: \mathrm{C}, 69.04 ; \mathrm{H}, 5.56 ; \mathrm{N}, 1.61$. Found: C, 68.91; H, 5.95; N, 1.91 .

3-C-(3,4,6-Tri-O-benzyl-2-O-diphenoxyphosphoryl- $\beta$ - $D$ mannopyranosyl)1-propoxyphthalimide (8). Following the general procedure starting from phthalimide $73(220 \mathrm{mg}, 0.34 \mathrm{mmol})$ and purification by column chromatography (hexanes-EtOAc, $7: 3$ ), product $8(242.7 \mathrm{mg}, 0.28 \mathrm{mmol}, 81 \%)$ was obtained as a colorless oil: $[\alpha]_{\mathrm{D}}=-16.6\left(c=0.53, \mathrm{CHCl}_{3}\right) .{ }^{1} \mathrm{H} \mathrm{NMR}\left(500 \mathrm{MHz}, \mathrm{CDCl}_{3}\right) \delta_{\mathrm{H}}$ 7.83-7.80 (m, 2H, Ar), 7.76-7.72 (m, 2H, Ar), 7.39-7.12 (m, 25H, Ar), 5.09 (br dd, $\left.J=1.9 \mathrm{~Hz},{ }^{3} J_{\mathrm{PH}}=8.8 \mathrm{~Hz}, 1 \mathrm{H}, 2-\mathrm{H}\right), 4.94(\mathrm{~d}, J=11.4$ $\mathrm{Hz}, 1 \mathrm{H}, \mathrm{OBn}), 4.63(\mathrm{~d}, J=10.7 \mathrm{~Hz}, 1 \mathrm{H}, \mathrm{OBn}), 4.62(\mathrm{~d}, J=12.9 \mathrm{~Hz}$, $1 \mathrm{H}, \mathrm{OBn}), 4.52(\mathrm{~d}, J=11.7 \mathrm{~Hz}, 1 \mathrm{H}, \mathrm{OBn}), 4.52(\mathrm{~d}, J=11.7 \mathrm{~Hz}, 1 \mathrm{H}$, $\mathrm{OBn}), 4.31(\mathrm{~d}, J=10.8 \mathrm{~Hz}, 1 \mathrm{H}, \mathrm{OBn}), 4.08(\mathrm{dd}, J=6.4,6.4 \mathrm{~Hz}, 2 \mathrm{H}$, $\left.3^{\prime}-\mathrm{H}_{2}\right), 3.68\left(\mathrm{br} \mathrm{d}, J=3.2 \mathrm{~Hz}, 2 \mathrm{H}, 6-\mathrm{H}_{2}\right), 3.63(\mathrm{~m}, 2 \mathrm{H}, 3-\mathrm{H}, 4-\mathrm{H})$, $3.56(\mathrm{~m}, 1 \mathrm{H}, 1-\mathrm{H}), 3.42(\mathrm{~m}, 1 \mathrm{H}, 5-\mathrm{H}), 1.86\left(\mathrm{~m}, 1 \mathrm{H}, 2^{\prime}-\mathrm{H}_{\mathrm{b}}\right), 1.81-$ $1.73\left(\mathrm{~m}, 2 \mathrm{H}, \mathrm{l}^{\prime}-\mathrm{H}_{\mathrm{b}}, 2^{\prime}-\mathrm{H}_{\mathrm{a}}\right), 1.68 \mathrm{ppm}\left(\mathrm{m}, 1 \mathrm{H}, \mathrm{1}^{\prime}-\mathrm{H}_{\mathrm{a}}\right) \cdot{ }^{13} \mathrm{C}\left\{{ }^{1} \mathrm{H}\right\} \mathrm{NMR}$ $\left(100.6 \mathrm{MHz}, \mathrm{CDCl}_{3}\right) \delta_{\mathrm{C}} 163.5(2 \times \mathrm{C}, \mathrm{CO}), 150.9\left(\mathrm{~d},{ }^{2} J_{\mathrm{PC}}=7.8 \mathrm{~Hz}\right.$, C, Ar), 150.7 (d, $\left.{ }^{2} J_{\mathrm{PC}}=7.0 \mathrm{~Hz}, \mathrm{C}, \mathrm{Ar}\right), 138.5$ (C, Ar), 138.4 (C, Ar), 137.8 (C, Ar), $134.4(2 \times \mathrm{CH}, \mathrm{Ar}), 129.7(2 \times \mathrm{CH}, \mathrm{Ar}), 129.3(2 \times$ $\mathrm{CH}, \mathrm{Ar}), 129.0(2 \times \mathrm{C}, \mathrm{Ar}), 128.4(2 \times \mathrm{CH}, \mathrm{Ar}), 128.3(4 \times \mathrm{CH}, \mathrm{Ar})$, $128.2(2 \times \mathrm{CH}, \mathrm{Ar}), 127.9(2 \times \mathrm{CH}, \mathrm{Ar}), 127.7(2 \times \mathrm{CH}, \mathrm{Ar}), 127.6$ $(\mathrm{CH}, \mathrm{Ar}), 127.5(\mathrm{CH}, \mathrm{Ar}), 127.4(\mathrm{CH}, \mathrm{Ar}), 125.2(\mathrm{CH}, \mathrm{Ar}), 124.7$ $(\mathrm{CH}, \mathrm{Ar}), 123.4(2 \times \mathrm{CH}, \mathrm{Ar}), 120.4(\mathrm{CH}, \mathrm{Ar}), 120.3(\mathrm{CH}, \mathrm{Ar})$, $120.23(\mathrm{CH}, \mathrm{Ar}), 120.18(\mathrm{CH}, \mathrm{Ar}), 82.0(\mathrm{CH}, \mathrm{C}-3), 79.5(\mathrm{CH}, \mathrm{C}-5)$, $77.9\left(\mathrm{CH}_{2}, \mathrm{C}-3\right.$ ') $, 77.0(\mathrm{CH}, \mathrm{C}-2), 76.4\left(\mathrm{~d},{ }^{3} J_{\mathrm{PC}}=5.6 \mathrm{~Hz}, \mathrm{CH}, \mathrm{C}-1\right)$, $75.1\left(\mathrm{CH}_{2}, \mathrm{OBn}\right), 74.2(\mathrm{CH}, \mathrm{C}-4), 73.4\left(\mathrm{CH}_{2}, \mathrm{OBn}\right), 71.8\left(\mathrm{CH}_{2}\right.$, OBn $), 69.3\left(\mathrm{CH}_{2}, \mathrm{C}-6\right), 27.2\left(\mathrm{CH}_{2}, \mathrm{C}-1^{\prime}\right), 24.3 \mathrm{ppm}\left(\mathrm{CH}_{2}, \mathrm{C}-2\right.$ '). IR $\left(\mathrm{CHCl}_{3}\right): \nu=3013,2869,1789,1733,1193 \mathrm{~cm}^{-1}$. MS (ESI) $\mathrm{m} / z(\%)$ $=892(100)[\mathrm{M}+\mathrm{Na}]^{+}$. HRMS (ESI) $\mathrm{m} / z:[\mathrm{M}+\mathrm{Na}]^{+}$calcd for $\mathrm{C}_{50} \mathrm{H}_{48} \mathrm{NNaO}_{11} \mathrm{P}$ 892.2863; found 892.2861. Anal. calcd for $\mathrm{C}_{50} \mathrm{H}_{48} \mathrm{NO}_{11} \mathrm{P}: \mathrm{C}, 69.04 ; \mathrm{H}, 5.56 ; \mathrm{N}, 1.61$. Found: $\mathrm{C}, 68.94 ; \mathrm{H}$, $5.83 ; \mathrm{N}, 1.67$.

3-C-(3,4-Di-O-benzyl-2-O-diphenoxyphosphoryl- $\alpha$-Lfucopyranosyl)1-propoxyphthalimide (10). Following the general procedure starting from phthalimide $77(253 \mathrm{mg}, 0.48 \mathrm{mmol})$ and purification by column chromatography (hexanes-EtOAc, 7:3), product $10(205 \mathrm{mg}, 0.27 \mathrm{mmol}, 56 \%)$ was obtained as a colorless oil: $[\alpha]_{\mathrm{D}}=-19.6\left(c=0.43, \mathrm{CHCl}_{3}\right) .{ }^{1} \mathrm{H} \mathrm{NMR}\left(500 \mathrm{MHz}, \mathrm{CDCl}_{3}\right.$, simulated ring coupling constants using DAISY) $\delta_{\mathrm{H}} 7.83-7.80(\mathrm{~m}$, $2 \mathrm{H}, \mathrm{Ar}$ ), 7.75-7.73 (m, 2H, Ar), 7.37-7.13 (m, 20H, Ar), 4.91 (ddd, $\left.J=6.3,3.3 \mathrm{~Hz},{ }^{3} J_{\mathrm{PH}}=7.5 \mathrm{~Hz}, 1 \mathrm{H}, 2-\mathrm{H}\right), 4.69(\mathrm{~d}, J=12.0 \mathrm{~Hz}, 1 \mathrm{H}$, OBn), $4.66(\mathrm{~d}, J=12.0 \mathrm{~Hz}, 1 \mathrm{H}, \mathrm{OBn}), 4.57(\mathrm{~d}, J=11.7 \mathrm{~Hz}, 1 \mathrm{H}$, OBn), 4.42 (d, $J=11.7 \mathrm{~Hz}, 1 \mathrm{H}, \mathrm{OBn}), 4.17-4.09$ (m, 3H, 1-H, 3'$\mathrm{H}_{2}$ ), 4.01 (dddd, $\left.J=6.7,6.7,6.7,4.0 \mathrm{~Hz}, 1 \mathrm{H}, 5-\mathrm{H}\right), 3.95$ (dd, $J=6.3$, $3.1 \mathrm{~Hz}, 1 \mathrm{H}, 3-\mathrm{H}), 3.72(\mathrm{dd}, J=4.1,3.1 \mathrm{~Hz}, 1 \mathrm{H}, 4-\mathrm{H}), 1.90-1.76(\mathrm{~m}$, $2 \mathrm{H}, 1^{\prime}-\mathrm{H}_{2}$ or 2' $\left.-\mathrm{H}_{2}\right), 1.74-1.60\left(\mathrm{~m}, 2 \mathrm{H}, 1^{\prime}-\mathrm{H}_{2}\right.$ or 2' $\left.-\mathrm{H}_{2}\right), 1.31 \mathrm{ppm}(\mathrm{d}$, $\left.J=6.7 \mathrm{~Hz}, 3 \mathrm{H}, 6-\mathrm{H}_{3}\right) .{ }^{13} \mathrm{C}\left\{{ }^{1} \mathrm{H}\right\} \mathrm{NMR}\left(125.7 \mathrm{MHz}, \mathrm{CDCl}_{3}\right) \delta_{\mathrm{C}} 163.5$ $(2 \times \mathrm{C}, \mathrm{CO}), 150.52\left(\mathrm{~d},{ }^{2} J_{\mathrm{PC}}=7.4 \mathrm{~Hz}, \mathrm{C}, \mathrm{Ar}\right), 150.48\left(\mathrm{~d},{ }^{2} J_{\mathrm{PC}}=7.4\right.$ $\mathrm{Hz}, \mathrm{C}, \mathrm{Ar}), 138.3$ (C, Ar), 138.1 (C, Ar), 134.4 (2× CH, Ar), 129.7 $(4 \times \mathrm{CH}, \mathrm{Ar}), 128.9(2 \times \mathrm{C}, \mathrm{Ar}), 128.3(2 \times \mathrm{CH}, \mathrm{Ar}), 128.2(2 \times$
$\mathrm{CH}, \mathrm{Ar}), 127.6(4 \times \mathrm{CH}, \mathrm{Ar}), 127.5(2 \times \mathrm{CH}, \mathrm{Ar}), 125.4(\mathrm{CH}, \mathrm{Ar})$, $125.3(\mathrm{CH}, \mathrm{Ar}), 123.4(2 \times \mathrm{CH}, \mathrm{Ar}), 120.2(\mathrm{CH}, \mathrm{Ar}), 120.12(\mathrm{CH}$, $\mathrm{Ar}), 120.08$ ( $\mathrm{CH}, \mathrm{Ar}), 120.0(\mathrm{CH}, \mathrm{Ar}), 78.1\left(\mathrm{CH}_{2}, \mathrm{C}-3^{\prime}\right), 77.2(\mathrm{CH}$, $\mathrm{C}-2), 76.1(\mathrm{CH}, \mathrm{C}-3), 75.0(\mathrm{CH}, \mathrm{C}-4), 73.1\left(\mathrm{CH}_{2}, \mathrm{OBn}\right), 72.6\left(\mathrm{CH}_{2}\right.$, OBn), $69.2(\mathrm{CH}, \mathrm{C}-5), 68.9(\mathrm{CH}, \mathrm{C}-1), 24.7\left(\mathrm{CH}_{2}, \mathrm{C}-1^{\prime}\right), 24.1\left(\mathrm{CH}_{2}\right.$, C-2'), $14.8 \mathrm{ppm}\left(\mathrm{CH}_{3}, \mathrm{C}-6\right)$. IR $\left(\mathrm{CHCl}_{3}\right): \nu=3027,1791,1733$, $1214 \mathrm{~cm}^{-1}$. MS (ESI) $m / z(\%)=786(100)[\mathrm{M}+\mathrm{Na}]^{+}$. HRMS (ESI) $m / z:[\mathrm{M}+\mathrm{Na}]^{+}$calcd for $\mathrm{C}_{43} \mathrm{H}_{42} \mathrm{NNaO}_{10} \mathrm{P}$ 786.2444; found 786.2448. Anal. calcd for $\mathrm{C}_{43} \mathrm{H}_{42} \mathrm{NO}_{10} \mathrm{P}: \mathrm{C}, 67.62 ; \mathrm{H}, 5.54 ; \mathrm{N}, 1.83$. Found: C, 67.81; H, 5.88; N, 1.48 .

3-C-(2-O-Diphenoxyphosphoryl-3,5-di-O-1,1,3,3-tetraisopropyldisiloxanyl- $\alpha$-D-ribofuranosyl) 1-propoxyphthalimide (15). Following the general procedure starting from phthalimide 88 (351 $\mathrm{mg}, 0.61$ mmol) and purification by column chromatography (hexanesEtOAc, $8: 2$ to $7: 3$ ), product $15(443 \mathrm{mg}, 0.55 \mathrm{mmol}, 90 \%)$ was obtained as a colorless oil: $[\alpha]_{\mathrm{D}}=+15.8\left(c=0.83, \mathrm{CHCl}_{3}\right) .{ }^{1} \mathrm{H}$ NMR $\left(500 \mathrm{MHz}, \mathrm{CDCl}_{3}\right.$, simulated ring coupling constants using DAISY) $\delta_{\mathrm{H}} 7.83-7.73(\mathrm{~m}, 4 \mathrm{H}, \mathrm{Ar}), 7.32-7.12(\mathrm{~m}, 10 \mathrm{H}, \mathrm{Ar}), 5.15$ (ddd, $J=$ $\left.3.9,2.9 \mathrm{~Hz},{ }^{3} J_{\mathrm{PH}}=7.9 \mathrm{~Hz}, 1 \mathrm{H}, 2-\mathrm{H}\right), 4.47\left(\mathrm{ddd}, J=9.1,3.9 \mathrm{~Hz},{ }^{4} J_{\mathrm{PH}}=\right.$ $1.5 \mathrm{~Hz}, 1 \mathrm{H}, 3-\mathrm{H}), 4.19(\mathrm{~m}, 1 \mathrm{H}, 1-\mathrm{H}), 4.08(\mathrm{br} \mathrm{dd}, J=6.3,6.3 \mathrm{~Hz}, 2 \mathrm{H}$, 3' $\left.-\mathrm{H}_{2}\right), 3.99\left(\mathrm{dd}, J=12.8,3.1 \mathrm{~Hz}, 1 \mathrm{H}, 5-\mathrm{H}_{\mathrm{b}}\right), 3.94-3.90(\mathrm{~m}, 2 \mathrm{H}, 4-\mathrm{H}$, 5- $\left.\mathrm{H}_{\mathrm{a}}\right), 1.88\left(\mathrm{~m}, 1 \mathrm{H}, 2^{\prime}-\mathrm{H}_{\mathrm{b}}\right), 1.71\left(\mathrm{~m}, 1 \mathrm{H}, 2^{\prime}-\mathrm{H}_{\mathrm{a}}\right), 1.65-1.57(\mathrm{~m}, 2 \mathrm{H}$, $\left.1^{\prime}-\mathrm{H}_{2}\right), 1.10-0.97 \mathrm{ppm}\left(\mathrm{m}, 28 \mathrm{H},{ }^{i} \mathrm{Pr}\right) .{ }^{13} \mathrm{C}\left\{{ }^{1} \mathrm{H}\right\} \operatorname{NMR}(125.7 \mathrm{MHz}$, $\left.\mathrm{CDCl}_{3}\right) \delta_{\mathrm{C}} 163.6(2 \times \mathrm{C}, \mathrm{CO}), 150.9\left(\mathrm{~d},{ }^{2} J_{\mathrm{PC}}=7.4 \mathrm{~Hz}, \mathrm{C}, \mathrm{Ar}\right), 150.6$ $\left(\mathrm{d},{ }^{2} J_{\mathrm{PC}}=6.4 \mathrm{~Hz}, \mathrm{C}, \mathrm{Ar}\right), 134.4(2 \times \mathrm{CH}, \mathrm{Ar}), 129.7(2 \times \mathrm{CH}, \mathrm{Ar})$, $129.6(2 \times \mathrm{CH}, \mathrm{Ar}), 129.0(2 \times \mathrm{C}, \mathrm{Ar}), 125.2(\mathrm{CH}, \mathrm{Ar}), 125.0(\mathrm{CH}$, Ar), $123.4(2 \times \mathrm{CH}, \mathrm{Ar}), 120.23(\mathrm{CH}, \mathrm{Ar}), 120.18(\mathrm{CH}, \mathrm{Ar}), 120.05$ (CH, Ar), $120.00(\mathrm{CH}, \mathrm{Ar}), 81.5\left(\mathrm{~d},{ }^{2} J_{\mathrm{PC}}=6.4 \mathrm{~Hz}, \mathrm{CH}, \mathrm{C}-2\right), 79.6$ (CH, C-4), $78.9\left(\mathrm{~d},{ }^{3} J_{\mathrm{PC}}=5.3 \mathrm{~Hz}, \mathrm{CH}, \mathrm{C}-1\right), 77.9\left(\mathrm{CH}_{2}, \mathrm{C}-3\right.$ ) $), 71.7$ (CH, C-3), 61.0 ( $\left.\mathrm{CH}_{2}, \mathrm{C}-5\right), 26.1\left(\mathrm{CH}_{2}, \mathrm{C}-1{ }^{\prime}\right), 24.6\left(\mathrm{CH}_{2}, \mathrm{C}-2^{\prime}\right)$, $17.4\left(\mathrm{CH}_{3},{ }^{i} \mathrm{Pr}\right), 17.3\left(\mathrm{CH}_{3},{ }^{i} \mathrm{Pr}\right), 17.28\left(\mathrm{CH}_{3},{ }^{i} \mathrm{Pr}\right), 17.26\left(\mathrm{CH}_{3},{ }^{i} \mathrm{Pr}\right)$, $17.04\left(\mathrm{CH}_{3},{ }^{i} \mathrm{Pr}\right), 17.01\left(\mathrm{CH}_{3},{ }^{i} \mathrm{Pr}\right), 16.8\left(\mathrm{CH}_{3},{ }^{i} \mathrm{Pr}\right), 16.7\left(\mathrm{CH}_{3},{ }^{i} \mathrm{Pr}\right)$, $13.5\left(\mathrm{CH},{ }^{i} \mathrm{Pr}\right), 13.1\left(\mathrm{CH},{ }^{i} \mathrm{Pr}\right), 12.7\left(\mathrm{CH},{ }^{i} \mathrm{Pr}\right), 12.4 \mathrm{ppm}\left(\mathrm{CH},{ }^{i} \mathrm{Pr}\right)$. IR $\left(\mathrm{CHCl}_{3}\right): \nu=2948,1791,1733,1490,1212 \mathrm{~cm}^{-1}$. MS (ESI) $\mathrm{m} / z$ $(\%)=834(100)[\mathrm{M}+\mathrm{Na}]^{+}$. HRMS (ESI) $\mathrm{m} / z:[\mathrm{M}+\mathrm{Na}]^{+}$calcd for $\mathrm{C}_{40} \mathrm{H}_{54} \mathrm{NNaO}_{11} \mathrm{PSi}_{2}$ 834.2871; found 834.2874. Anal. calcd for $\mathrm{C}_{40} \mathrm{H}_{54} \mathrm{NO}_{11} \mathrm{PSi}_{2}$ : C, 59.17; H, 6.70; N, 1.72. Found: C, 59.00; H, 6.94; N, 1.67

3-C-(2-O-Diphenoxyphosphoryl- $\beta$-D-arabinopyranosyl) 1-propoxyphthalimide (12). Compound $11(227 \mathrm{mg}, 0.35 \mathrm{mmol})$ was dissolved in $\mathrm{CH}_{2} \mathrm{Cl}_{2}(1.6 \mathrm{~mL})$, and TFA $/ \mathrm{H}_{2} \mathrm{O}(230 \mu \mathrm{L}, 80 \%)$ was dropwise added at $0{ }^{\circ} \mathrm{C}$. After $1 \mathrm{~h}$, the mixture was neutralized with a saturated aqueous solution of $\mathrm{NaHCO}_{3}$ and extracted with $\mathrm{CH}_{2} \mathrm{Cl}_{2}$. The organic extracts were dried over $\mathrm{Na}_{2} \mathrm{SO}_{4}$ and concentrated under reduced pressure. Column chromatography of the residue (hexanesEtOAc, 3:7) gave $12(161.7 \mathrm{mg}, 0.28 \mathrm{mmol}, 81 \%)$ as a colorless oil: $[\alpha]_{\mathrm{D}}=+4.6\left(c=0.56, \mathrm{CHCl}_{3}\right) .{ }^{1} \mathrm{H}$ NMR $\left(500 \mathrm{MHz}, \mathrm{CDCl}_{3}\right) \delta_{\mathrm{H}}$ 7.83-7.80 (m, 2H, Ar), 7.75-7.72 (m, 2H, Ar), 7.35-7.17 (m, 10H, Ar), 4.60 (ddd, $J=4.1,1.3 \mathrm{~Hz},{ }^{3} J_{\mathrm{PH}}=8.2 \mathrm{~Hz}, 1 \mathrm{H}, 2-\mathrm{H}$ ), $4.16-4.05$ $\left(\mathrm{m}, 3 \mathrm{H}, 3-\mathrm{H}, 3-\mathrm{H}_{2}\right), 3.86-3.81(\mathrm{~m}, 2 \mathrm{H}, 1-\mathrm{H}, 4-\mathrm{H}), 3.74(\mathrm{dd}, J=$ $\left.11.1,5.1 \mathrm{~Hz}, 1 \mathrm{H}, 5-\mathrm{H}_{\mathrm{b}}\right), 3.48\left(\mathrm{dd}, J=10.7,10.7 \mathrm{~Hz}, 1 \mathrm{H}, 5-\mathrm{H}_{\mathrm{a}}\right), 2.75$ (br d, $J=8.2 \mathrm{~Hz}, 1 \mathrm{H}, \mathrm{OH}), 2.01($ br s, $1 \mathrm{H}, \mathrm{OH}), 1.86\left(\mathrm{~m}, 1 \mathrm{H}, 2^{\prime}-\mathrm{H}_{\mathrm{b}}\right.$ ), $1.75\left(\mathrm{~m}, 1 \mathrm{H}, 2^{\prime}-\mathrm{H}_{\mathrm{a}}\right), 1.68\left(\mathrm{~m}, 1 \mathrm{H}, \mathrm{l}^{\prime}-\mathrm{H}_{\mathrm{b}}\right), 1.58 \mathrm{ppm}\left(\mathrm{m}, 1 \mathrm{H}, 1^{\prime}-\mathrm{H}_{\mathrm{a}}\right)$. ${ }^{13} \mathrm{C}\left\{{ }^{1} \mathrm{H}\right\}$ NMR $\left(125.7 \mathrm{MHz}, \mathrm{CDCl}_{3}\right) \delta_{\mathrm{C}} 163.6(2 \times \mathrm{C}, \mathrm{CO}), 150.3(\mathrm{~d}$, $\left.{ }^{2} J_{\mathrm{PC}}=7.4 \mathrm{~Hz}, \mathrm{C}, \mathrm{Ar}\right), 150.2\left(\mathrm{~d},{ }^{2} J_{\mathrm{PC}}=8.4 \mathrm{~Hz}, \mathrm{C}, \mathrm{Ar}\right), 134.4(2 \times \mathrm{CH}$, Ar), $129.9(4 \times \mathrm{CH}, \mathrm{Ar}), 128.9(2 \times \mathrm{C}, \mathrm{Ar}), 125.7(\mathrm{CH}, \mathrm{Ar}), 125.6$ (CH, Ar), $123.5(2 \times \mathrm{CH}, \mathrm{Ar}), 120.09(\mathrm{CH}, \mathrm{Ar}), 120.06(\mathrm{CH}, \mathrm{Ar})$, $120.04(\mathrm{CH}, \mathrm{Ar}), 120.00(\mathrm{CH}, \mathrm{Ar}), 79.1\left(\mathrm{~d},{ }^{2} \mathrm{~J}_{\mathrm{PC}}=6.4 \mathrm{~Hz}, \mathrm{CH}, \mathrm{C}-2\right)$, $78.0\left(\mathrm{CH}_{2}, \mathrm{C}-3^{\prime}\right), 72.4\left(\mathrm{~d},{ }^{3} \mathrm{JPC}_{\mathrm{PC}}=6.4 \mathrm{~Hz}, \mathrm{CH}, \mathrm{C}-1\right), 68.0(\mathrm{CH}, \mathrm{C}-3)$, $65.9\left(\mathrm{CH}_{2}, \mathrm{C}-5\right), 64.2(\mathrm{CH}, \mathrm{C}-4), 26.0\left(\mathrm{CH}_{2}, \mathrm{C}-1{ }^{\prime}\right), 24.5 \mathrm{ppm}\left(\mathrm{CH}_{2}\right.$, C-2'). IR $\left(\mathrm{CHCl}_{3}\right): \nu=3688,3557,3393,3026,1790,1733,1211$ $\mathrm{cm}^{-1}$. MS (ESI) $m / z(\%)=592(100)[\mathrm{M}+\mathrm{Na}]^{+}$. HRMS (ESI) $m / z$ : $[\mathrm{M}+\mathrm{Na}]^{+}$calcd for $\mathrm{C}_{28} \mathrm{H}_{28} \mathrm{NNaO}_{10} \mathrm{P}$ 592.1349; found 592.1341. Anal. calcd for $\mathrm{C}_{28} \mathrm{H}_{28} \mathrm{NO}_{10} \mathrm{P}$ : C, 59.05; H, 4.96; N, 2.46. Found: C, 59.26; H, 4.99; N, 2.48.

General Procedure to Give Acetyl Derivatives 90 and 97. The corresponding alcohol $(1 \mathrm{mmol})$ was dissolved in dry pyridine $(3.7 \mathrm{~mL})$, and $\mathrm{Ac}_{2} \mathrm{O}(1.2 \mathrm{~mL})$ and DMAP $(13.7 \mathrm{mg}, 0.11 \mathrm{mmol})$ were added. The reaction was stirred at room temperature for $0.5 \mathrm{~h}$, and then it was evaporated in a high vacuum rotovap, quenched with an 
aqueous solution of $\mathrm{HCl} 10 \%$, and extracted with $\mathrm{CH}_{2} \mathrm{Cl}_{2}$. The combined organic extracts were dried over $\mathrm{Na}_{2} \mathrm{SO}_{4}$ and concentrated under reduced pressure. Column chromatography of the residue (hexanes-EtOAc) gave the acetyl derivative.

Methyl 4-O-Acetyl-6-O-tert-butyldiphenylsilyl-2,3-di-O-methyl$\alpha$-D-glucopyranoside (90). Following the general procedure starting from methyl 6-O-tert-butyldiphenylsilyl-2,3-di-O-methyl- $\alpha$-D-glucopyranoside $(89)^{64}(119.7 \mathrm{mg}, 0.27 \mathrm{mmol})$ and purification by column chromatography (hexanes-EtOAc, 7:3), product 90 (122.7 $\mathrm{mg}, 0.25 \mathrm{mmol}, 94 \%)$ was obtained as a colorless oil: $[\alpha]_{\mathrm{D}}=+65.3(c$ $\left.=0.80, \mathrm{CHCl}_{3}\right) .{ }^{1} \mathrm{H} \mathrm{NMR}\left(400 \mathrm{MHz}, \mathrm{CDCl}_{3}\right) \delta_{\mathrm{H}} 7.69-7.67 \mathrm{ppm}(\mathrm{m}$, $4 \mathrm{H}, \mathrm{Ar}), 7.45-7.36(\mathrm{~m}, 6 \mathrm{H}, \mathrm{Ar}), 4.87(\mathrm{~d}, J=3.5 \mathrm{~Hz}, 1 \mathrm{H}, 1-\mathrm{H}), 4.87$ (dd, $J=9.5,9.5 \mathrm{~Hz}, 1 \mathrm{H}, 4-\mathrm{H}$ ), 3.75 (ddd, $J=10.1,5.7,2.5 \mathrm{~Hz}, 1 \mathrm{H}, 5$ $\mathrm{H}), 3.71-3.62\left(\mathrm{~m}, 2 \mathrm{H}, 6-\mathrm{H}_{2}\right), 3.58(\mathrm{dd}, J=9.5,9.5 \mathrm{~Hz}, 1 \mathrm{H}, 3-\mathrm{H})$, $3.54(\mathrm{~s}, 3 \mathrm{H}, \mathrm{OMe}), 3.51$ (s, 3H, OMe), 3.47 (s, $3 \mathrm{H}, \mathrm{OMe}), 3.30$ (dd, $J=9.6,3.7 \mathrm{~Hz}, 1 \mathrm{H}, 2-\mathrm{H}), 1.95$ (s, 3H, OAc), $1.05 \mathrm{ppm}\left(\mathrm{s}, 9 \mathrm{H},{ }^{t} \mathrm{Bu}\right)$. ${ }^{13} \mathrm{C}\left\{{ }^{1} \mathrm{H}\right\}$ NMR $\left(100.6 \mathrm{MHz}, \mathrm{CDCl}_{3}\right) \delta_{\mathrm{C}} 169.5$ (C, OAc), $135.7(2 \times$ $\mathrm{CH}, \mathrm{Ar}), 135.6(2 \times \mathrm{CH}, \mathrm{Ar}), 133.4$ (C, Ar), 133.3 (C, Ar), 129.62 $(\mathrm{CH}, \mathrm{Ar}), 129.60(\mathrm{CH}, \mathrm{Ar}), 127.62(2 \times \mathrm{CH}, \mathrm{Ar}), 127.59(2 \times \mathrm{CH}$, $\mathrm{Ar}), 97.3(\mathrm{CH}, \mathrm{C}-1), 81.5(\mathrm{CH}, \mathrm{C}-2$ or C-5), $81.1(\mathrm{CH}, \mathrm{C}-2$ or $\mathrm{C}-5)$, $70.6\left(\mathrm{CH}, \mathrm{C}-3\right.$ or C-4), 70.5 (CH, C-3 or C-4), $63.2\left(\mathrm{CH}_{2}, \mathrm{C}-6\right), 60.6$ $\left(\mathrm{CH}_{3}, \mathrm{OMe}\right), 59.1\left(\mathrm{CH}_{3}, \mathrm{OMe}\right), 55.1\left(\mathrm{CH}_{3}, \mathrm{OMe}\right), 26.7\left(3 \times \mathrm{CH}_{3}\right.$, DPS), $20.8\left(\mathrm{CH}_{3}, \mathrm{OAc}\right), 19.2 \mathrm{ppm}(\mathrm{C}$, DPS $)$. IR $\left(\mathrm{CHCl}_{3}\right): \nu=2932$, $1748,1233,1046 \mathrm{~cm}^{-1}$. MS (ESI) $\mathrm{m} / z(\%)=525(100)[\mathrm{M}+\mathrm{Na}]^{+}$. HRMS (ESI) $m / z:[\mathrm{M}+\mathrm{Na}]^{+}$calcd for $\mathrm{C}_{27} \mathrm{H}_{38} \mathrm{NaO}_{7} \mathrm{Si} 525.2285$; found 525.2272. Anal. calcd for $\mathrm{C}_{27} \mathrm{H}_{38} \mathrm{O}_{7} \mathrm{Si}: \mathrm{C}, 64.51 ; \mathrm{H}, 7.62$. Found: C, 64.45; H, 7.85 .

Methyl 4-O-Acetyl-6-O-tert-butyldiphenylsilyl-2,3-di-O-methyl$\alpha$-D-galactopyranoside (97). Following the general procedure starting from methyl 6-O-tert-butyldiphenylsilyl-2,3-di-O-methyl- $\alpha$-Dgalactopyranoside $(96)^{64}(2.15 \mathrm{~g}, 4.80 \mathrm{mmol})$ and purification by column chromatography (hexanes-EtOAc, 7:3), product $97(2.19 \mathrm{~g}$, $4.36 \mathrm{mmol}, 91 \%)$ was obtained as a colorless oil: $[\alpha]_{\mathrm{D}}=+65.5(c=$ $\left.1.45, \mathrm{CHCl}_{3}\right) .{ }^{1} \mathrm{H}$ NMR $\left(400 \mathrm{MHz}, \mathrm{CDCl}_{3}\right) \delta_{\mathrm{H}} 7.69-7.64(\mathrm{~m}, 4 \mathrm{H}$, $\mathrm{Ar}), 7.45-7.36(\mathrm{~m}, 6 \mathrm{H}, \mathrm{Ar}), 5.58(\mathrm{dd}, J=3.2,1.0 \mathrm{~Hz}, 1 \mathrm{H}, 4-\mathrm{H}), 4.88$ (d, $J=3.6 \mathrm{~Hz}, 1 \mathrm{H}, 1-\mathrm{H}), 3.93$ (ddd, $J=6.9,6.9,0.9 \mathrm{~Hz}, 1 \mathrm{H}, 5-\mathrm{H})$, $3.69\left(\mathrm{dd}, J=10.2,6.3 \mathrm{~Hz}, 1 \mathrm{H}, 6-\mathrm{H}_{\mathrm{b}}\right), 3.64(\mathrm{dd}, J=10.4,7.0 \mathrm{~Hz}, 1 \mathrm{H}$, 6- $\left.\mathrm{H}_{\mathrm{a}}\right), 3.60(\mathrm{dd}, J=10.1,3.4 \mathrm{~Hz}, 1 \mathrm{H}, 3-\mathrm{H}), 3.51(\mathrm{~s}, 3 \mathrm{H}, \mathrm{OMe}), 3.50$ $(\mathrm{dd}, J=10.1,6.4 \mathrm{~Hz}, 1 \mathrm{H}, 2-\mathrm{H}), 3.43(\mathrm{~s}, 3 \mathrm{H}, \mathrm{OMe}), 3.40(\mathrm{~s}, 3 \mathrm{H}$, OMe), 2.03 (s, 3H, OAc), $1.06 \mathrm{ppm}\left(\mathrm{s}, 9 \mathrm{H},{ }^{t} \mathrm{Bu}\right) .{ }^{13} \mathrm{C}\left\{{ }^{1} \mathrm{H}\right\} \mathrm{NMR}$ $\left(100.6 \mathrm{MHz}, \mathrm{CDCl}_{3}\right) \delta_{\mathrm{C}} 169.9 \mathrm{ppm}(\mathrm{C}, \mathrm{OAc}), 135.5(4 \times \mathrm{CH}, \mathrm{Ar})$, 133.2 (C, Ar), 133.1 (C, Ar), 129.7 (CH, Ar), $129.6(\mathrm{CH}, \mathrm{Ar}), 127.7$ $(2 \times \mathrm{CH}, \mathrm{Ar}), 127.6(2 \times \mathrm{CH}, \mathrm{Ar}), 97.7(\mathrm{CH}, \mathrm{C}-1), 78.1(\mathrm{CH}, \mathrm{C}-2$ or $\mathrm{C}-5), 77.3(\mathrm{CH}, \mathrm{C}-2$ or C-5), $69.2(\mathrm{CH}, \mathrm{C}-3$ or C-4), $66.9(\mathrm{CH}, \mathrm{C}-3$ or C-4), $62.2\left(\mathrm{CH}_{2}, \mathrm{C}-6\right), 59.0\left(\mathrm{CH}_{3}, \mathrm{OMe}\right), 57.6\left(\mathrm{CH}_{3}, \mathrm{OMe}\right), 55.2$ $\left(\mathrm{CH}_{3}, \mathrm{OMe}\right), 26.7\left(3 \times \mathrm{CH}_{3}, \mathrm{DPS}\right), 20.6\left(\mathrm{CH}_{3}, \mathrm{OAc}\right), 19.0 \mathrm{ppm}(\mathrm{C}$, DPS). IR $\left(\mathrm{CHCl}_{3}\right): \nu=2934,1741,1239,1106 \mathrm{~cm}^{-1}$. MS (ESI) $\mathrm{m} / z$ $(\%)=525(100)[\mathrm{M}+\mathrm{Na}]^{+}$. HRMS (ESI) $\mathrm{m} / z:[\mathrm{M}+\mathrm{Na}]^{+}$calcd for $\mathrm{C}_{27} \mathrm{H}_{38} \mathrm{NaO}_{7} \mathrm{Si}$ 525.2285; found 525.2279. Anal. calcd for $\mathrm{C}_{27} \mathrm{H}_{38} \mathrm{O}_{7} \mathrm{Si}: \mathrm{C}, 64.51 ; \mathrm{H}, 7.62$. Found: C, 64.72; H, 7.50.

General Procedure to Give Allenyl Derivatives 91, 98, and 107. The precursor $(1 \mathrm{mmol})$ was dissolved in $\mathrm{CH}_{3} \mathrm{CN}(11 \mathrm{~mL})$ under a $\mathrm{N}_{2}$ atmosphere, and freshly prepared propargyl trimethylsilane $/ \mathrm{Et}_{2} \mathrm{O}^{65} 39 \% \mathrm{v} / \mathrm{v}(0.77 \mathrm{~mL}, 2 \mathrm{mmol})$ and TMSOTf $(0.4 \mathrm{~mL}, 2.27$ $\mathrm{mmol})$ were dropwise added. The reaction was sonicated in an ultrasonic bath for $1.5-3 \mathrm{~h}$, and then it was poured over a saturated aqueous solution of $\mathrm{NaHCO}_{3}$ and extracted with EtOAc. The organic phase was dried over $\mathrm{Na}_{2} \mathrm{SO}_{4}$, filtered, and evaporated. The crude was dissolved in DMF $(2 \mathrm{~mL})$, and DPSCl $(176 \mu \mathrm{L}, 38.68 \mathrm{mmol})$ and imidazole $(102 \mathrm{mg}, 22.42 \mathrm{mmol})$ were added. The reaction was stirred at room temperature for $2 \mathrm{~h}$, evaporated under reduced high pressure, and purified by column chromatography (hexanes-EtOAc) to give the expected allenyl derivative.

C-(4-O-Acetyl-6-O-tert-butyldiphenylsilyl-2,3-di-O-methyl- $\alpha$-Dglucopyranosyl)allene (91). Following the general procedure starting from $90(2.23 \mathrm{~g}, 4.55 \mathrm{mmol})$ and purification by column chromatography (hexanes-EtOAc, 9:1), product 91 (991.3 mg, $1.79 \mathrm{mmol}, 39 \%)$ was obtained as a colorless oil: $[\alpha]_{\mathrm{D}}=+91.4(c=$ $\left.0.80, \mathrm{CHCl}_{3}\right) .{ }^{1} \mathrm{H}$ NMR $\left(400 \mathrm{MHz}, \mathrm{CDCl}_{3}\right) \delta_{\mathrm{H}} 7.70-7.66(\mathrm{~m}, 4 \mathrm{H}$, Ar), 7.44-7.35 (m, 6H, Ar), 5.38 (ddd, $J=6.7,6.7,6.7 \mathrm{~Hz}, 1 \mathrm{H}, 1$ '- $-\mathrm{H}$ ), $4.93(\mathrm{dd}, J=9.2,9.2 \mathrm{~Hz}, 1 \mathrm{H}, 3-\mathrm{H}), 4.87$ (dd, $J=6.7,0.0 \mathrm{~Hz}, 1 \mathrm{H}, 3^{\prime}-$ $\left.\mathrm{H}_{\mathrm{b}}\right), 4.86\left(\mathrm{dd}, J=6.7,0.0 \mathrm{~Hz}, 1 \mathrm{H}, 3-\mathrm{H}_{\mathrm{a}}\right), 4.78(\mathrm{~m}, 1 \mathrm{H}, 1-\mathrm{H}), 3.85-$ $3.80\left(\mathrm{~m}, 2 \mathrm{H}, 4-\mathrm{H}, 6-\mathrm{H}_{\mathrm{b}}\right), 3.69-3.67\left(\mathrm{~m}, 2 \mathrm{H}, 5-\mathrm{H}, 6-\mathrm{H}_{\mathrm{a}}\right), 3.51(\mathrm{~s}, 3 \mathrm{H}$, OMe), 3.48 (s, 3H, OMe), $3.43(\mathrm{dd}, J=9.5,6.3 \mathrm{~Hz}, 1 \mathrm{H}, 2-\mathrm{H}), 1.96$ (s, 3H, OAc), $1.06 \mathrm{ppm}\left(\mathrm{s}, 9 \mathrm{H},{ }^{t} \mathrm{Bu}\right) .{ }^{13} \mathrm{C}\left\{{ }^{1} \mathrm{H}\right\} \mathrm{NMR}(100.6 \mathrm{MHz}$, $\left.\mathrm{CDCl}_{3}\right) \delta_{\mathrm{C}} 209.5(\mathrm{C}, \mathrm{C}-2$ '), 169.5 (C, OAc), 135.7 (4 × CH, Ar), 133.6 (C, Ar), 133.4 (C, Ar), 129.6 (CH, Ar), 129.5 (CH, Ar), 127.57 $(2 \times \mathrm{CH}, \mathrm{Ar}), 127.55(2 \times \mathrm{CH}, \mathrm{Ar}), 85.5\left(\mathrm{CH}, \mathrm{C}-1^{\prime}\right), 81.0(2 \times \mathrm{CH}$, C-2, C-5), $76.7\left(\mathrm{CH}_{2}, \mathrm{C}-3\right.$ '), $72.8(\mathrm{CH}, \mathrm{C}-4), 71.1(\mathrm{CH}, \mathrm{C}-1), 70.5$ (CH, C-3), $63.6\left(\mathrm{CH}_{2}, \mathrm{C}-6\right), 60.0\left(\mathrm{CH}_{3}, \mathrm{OMe}\right), 58.4\left(\mathrm{CH}_{3}, \mathrm{OMe}\right)$, $26.8\left(3 \times \mathrm{CH}_{3}, \mathrm{DPS}\right), 20.9\left(\mathrm{CH}_{3}, \mathrm{OAc}\right), 19.2 \mathrm{ppm}$ (C, DPS). IR $\left(\mathrm{CHCl}_{3}\right): \nu=3024,2934,1956,1736,1208 \mathrm{~cm}^{-1}$. MS (ESI) $\mathrm{m} / z(\%)$ $=533(100)[\mathrm{M}+\mathrm{Na}]^{+}$. HRMS (ESI) $m / z:[\mathrm{M}+\mathrm{Na}]^{+}$calcd for $\mathrm{C}_{29} \mathrm{H}_{38} \mathrm{NaO}_{6} \mathrm{Si}$ 533.2335; found 533.2333.

C-(4-O-Acetyl-6-O-tert-butyldiphenylsilyl-2,3-di-O-methyl- $\alpha-D-$ galactopyranosyl)allene (98). Following the general procedure starting from 97 (1.91 g, $3.80 \mathrm{mmol}$ ) and purification by column chromatography (hexanes-EtOAc, 8:2), product 98 (1.27 g, 2.49 mmol, $59 \%)$ was obtained as a yellow oil: $[\alpha]_{\mathrm{D}}=+101.0(c=0.81$, $\mathrm{CHCl}_{3}$ ). ${ }^{1} \mathrm{H}$ NMR $\left(400 \mathrm{MHz}, \mathrm{CDCl}_{3}\right) \delta_{\mathrm{H}} 7.68-7.63(\mathrm{~m}, 4 \mathrm{H}, \mathrm{Ar})$, 7.45-7.35 (m, 6H, Ar), $5.67(\mathrm{dd}, J=2.9,1.2 \mathrm{~Hz}, 1 \mathrm{H}, 4-\mathrm{H}), 5.33(\mathrm{~m}$, $\left.1 \mathrm{H}, 1^{\prime}-\mathrm{H}\right), 4.85-4.79\left(\mathrm{~m}, 3 \mathrm{H}, 1-\mathrm{H}, 3\right.$ ' $\left.-\mathrm{H}_{2}\right), 3.98$ (ddd, $J=7.6,5.7,1.5$ $\mathrm{Hz}, 1 \mathrm{H}, 5-\mathrm{H}), 3.67$ (dd, $\left.J=10.1,5.8 \mathrm{~Hz}, 1 \mathrm{H}, 6-\mathrm{H}_{\mathrm{b}}\right), 3.66(\mathrm{dd}, J=$ 10.5, 5.7 Hz, 1H, 2-H), 3.59 (dd, $\left.J=9.8,7.9 \mathrm{~Hz}, 1 \mathrm{H}, 6-\mathrm{H}_{\mathrm{a}}\right), 3.47$ (s, $3 \mathrm{H}, \mathrm{OMe}$ ), 3.46 (s, 3H, OMe), 3.40 (dd, $J=9.9,3.6 \mathrm{~Hz}, 1 \mathrm{H}, 3-\mathrm{H})$, 2.06 (s, 3H, OAc), $1.06 \mathrm{ppm}\left(\mathrm{s}, 9 \mathrm{H},{ }^{t} \mathrm{Bu}\right) .{ }^{13} \mathrm{C}\left\{{ }^{1} \mathrm{H}\right\}$ NMR $(100.6$ $\left.\mathrm{MHz}, \mathrm{CDCl}_{3}\right) \delta_{\mathrm{C}} 209.0$ (C, C-2'). 169.8 (C, OAc), $135.60(2 \times \mathrm{CH}$, $\mathrm{Ar}), 135.56(2 \times \mathrm{CH}, \mathrm{Ar}), 133.4(\mathrm{C}, \mathrm{Ar}), 133.2(\mathrm{C}, \mathrm{Ar}), 129.72(\mathrm{CH}$, Ar), $129.67(\mathrm{CH}, \mathrm{Ar}), 127.7(2 \times \mathrm{CH}, \mathrm{Ar}), 127.6(2 \times \mathrm{CH}, \mathrm{Ar}), 85.2$ (CH, C-1'), 78.8 (CH, C-5), 77.1 (CH, C-2), $76.9\left(\mathrm{CH}_{2}, \mathrm{C}-3\right.$ '), 71.5 (CH, C-4), $71.0(\mathrm{CH}, \mathrm{C}-1), 66.6(\mathrm{CH}, \mathrm{C}-3), 61.8\left(\mathrm{CH}_{2}, \mathrm{C}-6\right), 58.5$ $\left(\mathrm{CH}_{3}, \mathrm{OMe}\right), 57.7\left(\mathrm{CH}_{3}, \mathrm{OMe}\right), 26.7\left(3 \times \mathrm{CH}_{3}, \mathrm{DPS}\right), 20.7\left(\mathrm{CH}_{3}\right.$, OAc), $19.1 \mathrm{ppm}$ (C, DPS). IR $\left(\mathrm{CHCl}_{3}\right): \nu=3016,2934,1955,1741$, $1208 \mathrm{~cm}^{-1}$. MS (ESI) $m / z(\%)=533(100)[\mathrm{M}+\mathrm{Na}]^{+}$. HRMS (ESI) $m / z:[\mathrm{M}+\mathrm{Na}]^{+}$calcd for $\mathrm{C}_{29} \mathrm{H}_{38} \mathrm{NaO}_{6} \mathrm{Si}$ 533.2335; found 533.2328. Anal. calcd for $\mathrm{C}_{29} \mathrm{H}_{38} \mathrm{O}_{6} \mathrm{Si}$ : C, 68.20; H, 7.50. Found: C, 68.24; H, 7.70 .

C-(2,3,4-Tri-O-acetyl- $\alpha$-L-fucopyranosyl)allene (107). Following the general procedure starting from peracetyl L-fucose (106) (877 $\mathrm{mg}, \quad 2.64 \mathrm{mmol}$ ) and purification by column chromatography (hexanes-EtOAc, 8:2), product 107 (601.8 mg, $1.93 \mathrm{mmol}, 73 \%$ ) was obtained as a yellow oil: $[\alpha]_{\mathrm{D}}=-170.0\left(c=0.92, \mathrm{CHCl}_{3}\right) .{ }^{1} \mathrm{H}$ $\operatorname{NMR}\left(500 \mathrm{MHz}, \mathrm{C}_{6} \mathrm{D}_{6}\right) \delta_{\mathrm{H}} 5.68(\mathrm{dd}, J=11.0,6.0 \mathrm{~Hz}, 1 \mathrm{H}, 2-\mathrm{H}), 5.46$ (dd, $J=11.0,3.5 \mathrm{~Hz}, 1 \mathrm{H}, 3-\mathrm{H}), 5.37$ (dd, $J=3.5,1.3 \mathrm{~Hz}, 1 \mathrm{H}, 4-\mathrm{H})$, 5.17 (ddd, $J=6.9,6.9,6.9 \mathrm{~Hz}, 1 \mathrm{H}, 1^{\prime}-\mathrm{H}$ ), 5.04 (dddd, $J=6.1,6.1,3.2$, $3.2 \mathrm{~Hz}, 1 \mathrm{H}, 1-\mathrm{H}), 4.59$ (ddd, $\left.J=11.3,6.6,3.2 \mathrm{~Hz}, 1 \mathrm{H}, 3^{\prime}-\mathrm{H}_{\mathrm{b}}\right), 4.55$ (ddd, $J=11.7,6.9,3.5 \mathrm{~Hz}, 1 \mathrm{H}, 3^{\prime}-\mathrm{H}_{\mathrm{a}}$ ), 3.67 (dddd, $J=6.3,6.3,6.3$, $1.0 \mathrm{~Hz}, 1 \mathrm{H}, 5-\mathrm{H}), 1.75$ (s, 3H, OAc), 1.65 (s, 3H, OAc), 1.59 (s, 3H, OAc), $0.97 \mathrm{ppm}\left(\mathrm{d}, J=6.3 \mathrm{~Hz}, 3 \mathrm{H}, 6-\mathrm{H}_{3}\right) .{ }^{13} \mathrm{C}\left\{{ }^{1} \mathrm{H}\right\}$ NMR $(125.7$ $\left.\mathrm{MHz}, \mathrm{C}_{6} \mathrm{D}_{6}\right) \delta_{\mathrm{C}} 209.8\left(\mathrm{CH}, \mathrm{C}-2^{\prime}\right), 170.2(\mathrm{C}, \mathrm{OAc}), 169.8$ (C, OAc), 169.4 (C, OAc), 85.4 ( $\left.\mathrm{CH}, \mathrm{C}-1^{\prime}\right), 76.6\left(\mathrm{CH}_{2}, \mathrm{C}-3^{\prime}\right), 71.7(\mathrm{CH}, \mathrm{C}-1)$, $71.5(\mathrm{CH}, \mathrm{C}-4), 69.1(\mathrm{CH}, \mathrm{C}-3), 68.4(\mathrm{CH}, \mathrm{C}-2), 66.6(\mathrm{CH}, \mathrm{C}-5)$, $20.4\left(\mathrm{CH}_{3}, \mathrm{OAc}\right), 20.2\left(\mathrm{CH}_{3}, \mathrm{OAc}\right), 20.0\left(\mathrm{CH}_{3}, \mathrm{OAc}\right), 16.3 \mathrm{ppm}$ $\left(\mathrm{CH}_{3}, \mathrm{C}-6\right)$. IR $\left(\mathrm{CHCl}_{3}\right): \nu=2989,2944,1957,1750,1373,1230$, $1062 \mathrm{~cm}^{-1}$. MS (ESI) $\mathrm{m} / z(\%)=335(100)[\mathrm{M}+\mathrm{Na}]^{+}$. HRMS (ESI) $m / z:[\mathrm{M}+\mathrm{Na}]^{+}$calcd for $\mathrm{C}_{15} \mathrm{H}_{20} \mathrm{NaO}_{7}$ 335.1107; found 335.1101. Anal. calcd for $\mathrm{C}_{15} \mathrm{H}_{20} \mathrm{O}_{7}$ : C, 57.69; H, 6.45. Found: C, 58.07; H, 6.79 .

C-(2,3,4-Tri-O-acetyl- $\alpha$-L-rhamnopyranosyl)allene (101) and C(2,3,4-Tri-O-acetyl- $\beta$-L-rhamnopyranosyl)allene (102). $\mathrm{BF}_{3} \bullet \mathrm{OEt}_{2}$ $(6.40 \mathrm{~mL}, 51.86 \mathrm{mmol})$ and TMSOTf $(6.20 \mathrm{~mL}, 34.66 \mathrm{mmol})$ were dropwise added to a solution of peracetyl L-rhamnose (100) (6 $\mathrm{g}, 18.0 \mathrm{mmol})$ and fresly prepared propargyl trimethylsilane $(5.11 \mathrm{~mL}$, $34.28 \mathrm{mmol})$ in dry $\mathrm{CH}_{3} \mathrm{CN}(39 \mathrm{~mL})$ at $0{ }^{\circ} \mathrm{C}$, and the mixture was stirred at room temperature for $15 \mathrm{~h}$. Then, it was poured over $\mathrm{HCl}$ $10 \%$ and extracted with EtOAc. The organic extracts were washed with saturated aqueous solutions of $\mathrm{NaHCO}_{3}$ and $\mathrm{NaCl}$, dried over $\mathrm{Na}_{2} \mathrm{SO}_{4}$, and concentrated to dryness under reduced pressure. Column chromatography of the residue (hexanes-EtOAc, 95:5) gave 101 ( $3.80 \mathrm{~g}, 12.18 \mathrm{mmol}, 67 \%)$ and $102(1.40 \mathrm{~g}, 4.49 \mathrm{mmol}$, 
$25 \%)$ that could not be completely purified and was isolated as a mixture (1:1) with 101. Compound 101: colorless oil, $[\alpha]_{\mathrm{D}}=-16.7$ $\left(c=0.42, \mathrm{CHCl}_{3}\right) .{ }^{1} \mathrm{H}$ NMR $\left(500 \mathrm{MHz}, \mathrm{CDCl}_{3}\right) \delta_{\mathrm{H}} 5.40(\mathrm{dd}, J=3.3$, $2.0 \mathrm{~Hz}, 1 \mathrm{H}, 2-\mathrm{H}), 5.26(\mathrm{dd}, J=9.8,3.5 \mathrm{~Hz}, 1 \mathrm{H}, 3-\mathrm{H}), 5.21(\mathrm{ddd}, J=$ $\left.6.8,6.8,4.4 \mathrm{~Hz}, 1 \mathrm{H}, 1^{\prime}-\mathrm{H}\right), 5.07$ (dd, $\left.J=9.5,9.5 \mathrm{~Hz}, 1 \mathrm{H}, 4-\mathrm{H}\right), 5.05$ (ddd, $\left.J=12.3,6.9,4.1 \mathrm{~Hz}, 1 \mathrm{H}, 3^{\prime}-\mathrm{H}_{\mathrm{b}}\right), 4.99$ (ddd, $J=11.4,6.9,4.4$ $\mathrm{Hz}, 1 \mathrm{H}, 3^{\prime}-\mathrm{H}_{\mathrm{a}}$ ), 4.57 (dddd, $J=4.1,4.1,4.1,1.9 \mathrm{~Hz}, 1 \mathrm{H}, 1-\mathrm{H}$ ), 3.84 (dddd, $J=9.5,6.2,6.2,6.2 \mathrm{~Hz}, 1 \mathrm{H}, 5-\mathrm{H}), 2.14(\mathrm{~s}, 3 \mathrm{H}, \mathrm{OAc}), 2.04$ (s, $3 \mathrm{H}, \mathrm{OAc}), 1.97$ (s, 3H, OAc), $1.21 \mathrm{ppm}\left(\mathrm{d}, J=6.0 \mathrm{~Hz}, 3 \mathrm{H}, 6-\mathrm{H}_{3}\right)$. ${ }^{13} \mathrm{C}\left\{{ }^{1} \mathrm{H}\right\} \operatorname{NMR}\left(125.7 \mathrm{MHz}, \mathrm{CDCl}_{3}\right) \delta_{\mathrm{C}} 207.7\left(\mathrm{CH}, \mathrm{C}-2^{\prime}\right), 170.3(\mathrm{C}$, OAc), 170.0 (C, OAc), 169.9 (C, OAc), $87.8\left(\mathrm{CH}, \mathrm{C}-1^{\prime}\right), 78.7\left(\mathrm{CH}_{2}\right.$, C-3'), 73.3 (CH, C-1), $71.6(\mathrm{CH}, \mathrm{C}-4), 70.3(\mathrm{CH}, \mathrm{C}-2), 69.3(\mathrm{CH}$, C-3), 68.8 (CH, C-5), 21.0 ( $\left.\mathrm{CH}_{3}, \mathrm{OAc}\right), 20.8\left(\mathrm{CH}_{3}, \mathrm{OAc}\right), 20.6$ $\left(\mathrm{CH}_{3}, \mathrm{OAc}\right), 17.6 \mathrm{ppm}\left(\mathrm{CH}_{3}, \mathrm{C}-6\right)$. IR $\left(\mathrm{CHCl}_{3}\right): \nu=2981,2933$, $1955,1746,1369,1221 \mathrm{~cm}^{-1}$. MS (ESI) $\mathrm{m} / z(\%)=335(100)[\mathrm{M}+$ $\mathrm{Na}]^{+}$. HRMS (ESI) $m / z:[\mathrm{M}+\mathrm{Na}]^{+}$calcd for $\mathrm{C}_{15} \mathrm{H}_{20} \mathrm{NaO}_{7} 335.1107$; found 335.1107. Anal. calcd for $\mathrm{C}_{15} \mathrm{H}_{20} \mathrm{O}_{7}: \mathrm{C}, 57.69 ; \mathrm{H}, 6.45$. Found: C, 58.07; H, 6.79. Compound 102: ${ }^{1} \mathrm{H}$ NMR (500 MHz, $\mathrm{CDCl}_{3}$, signals taken from a spectrum of the mix of 101 and 102) $\delta_{\mathrm{H}} 5.39$ (dd, $J=2.9,1.3 \mathrm{~Hz}, 1 \mathrm{H}, 2-\mathrm{H}), 5.14$ (ddd, $J=6.6,6.6,6.6 \mathrm{~Hz}, 1 \mathrm{H}, 1^{\prime}$ $\mathrm{H}), 5.05(\mathrm{~m}, 1 \mathrm{H}, 3-\mathrm{H}), 4.98(\mathrm{dd}, J=6.7,4.4 \mathrm{~Hz}, 1 \mathrm{H}, 4-\mathrm{H}), 4.86$ (ddd, $\left.J=11.4,6.6,2.2 \mathrm{~Hz}, 1 \mathrm{H}, 3^{\prime}-\mathrm{H}_{\mathrm{b}}\right), 4.81(\mathrm{ddd}, J=11.7,6.9,2.2 \mathrm{~Hz}, 1 \mathrm{H}$, $\left.3^{\prime}-\mathrm{H}_{\mathrm{a}}\right), 4.19$ (dddd, $\left.J=6.9,2.2,2.2,1.3 \mathrm{~Hz}, 1 \mathrm{H}, 1-\mathrm{H}\right), 3.54$ (dddd, $J=$ 9.1, 6.3, 6.3, 6.3 Hz, 1H, 5-H), 2.15 (s, 3H, OAc), 2.04 (s, 3H, OAc), $1.96(\mathrm{~s}, 3 \mathrm{H}, \mathrm{OAc}), 1.25 \mathrm{ppm}\left(\mathrm{d}, J=6.3 \mathrm{~Hz}, 3 \mathrm{H}, 6-\mathrm{H}_{3}\right) .{ }^{13} \mathrm{C}\left\{{ }^{1} \mathrm{H}\right\}$ NMR (125.7 MHz, $\mathrm{CDCl}_{3}$, signals taken from a spectrum of the mix of 101 and 102) $\delta_{\mathrm{C}} 208.4$ ( $\left.\mathrm{CH}, \mathrm{C}-2^{\prime}\right), 170.4(\mathrm{C}, \mathrm{OAc}), 170.3(\mathrm{C}$, OAc), 169.9 (C, OAc), 87.3 (CH, C-1'), $77.4\left(\mathrm{CH}_{2}, \mathrm{C}-3^{\prime}\right), 75.1(\mathrm{CH}$, $\mathrm{C}-1), 74.6(\mathrm{CH}, \mathrm{C}-5), 72.3(\mathrm{CH}, \mathrm{C}-4), 70.6(\mathrm{CH}, \mathrm{C}-3), 70.4(\mathrm{CH}, \mathrm{C}-$ 2), $21.0\left(\mathrm{CH}_{3}, \mathrm{OAc}\right), 20.7\left(\mathrm{CH}_{3}, \mathrm{OAc}\right), 20.6\left(\mathrm{CH}_{3}, \mathrm{OAc}\right), 17.7 \mathrm{ppm}$ $\left(\mathrm{CH}_{3}, \mathrm{C}-6\right)$. IR $\left(\mathrm{CHCl}_{3}\right): \nu=2981,2937,1959,1750,1373,1225$, $1054 \mathrm{~cm}^{-1}$. MS (ESI) $\mathrm{m} / z(\%)=335(100)[\mathrm{M}+\mathrm{Na}]^{+}$. HRMS (ESI) $m / z:[\mathrm{M}+\mathrm{Na}]^{+}$calcd for $\mathrm{C}_{15} \mathrm{H}_{20} \mathrm{NaO}_{7} 335.1107$; found 335.1107 .

C-(4-O-Benzyl-2,3-O-isopropylidene- $\alpha$-L-rhamnopyranosyl)allene (103). To a solution of $101(1.89 \mathrm{~g}, 6.06 \mathrm{mmol})$ in dry $\mathrm{MeOH}$ $(45 \mathrm{~mL})$ was added $\mathrm{K}_{2} \mathrm{CO}_{3}(1.35 \mathrm{~g}, 9.78 \mathrm{mmol})$, and the mixture was stirred at room temperature for $3 \mathrm{~h}$. Then, it was filtered and neutralized with the Amberlyst $15 \mathrm{H}^{+}$ion exchange resin. It was filtered again under vacuum and evaporated. The resulting organic crude was dissolved in acetone $(60 \mathrm{~mL})$, and 2,2-dimethoxypropane $(1.9 \mathrm{~mL}, 15.15 \mathrm{mmol})$ and $p-\mathrm{TsOH} \cdot \mathrm{H}_{2} \mathrm{O}(692 \mathrm{mg}, 3.64 \mathrm{mmol})$ were subsequently added while stirring at room temperature for $3 \mathrm{~h}$. The acetone was evaporated, and the residue was poured over a saturated aqueous solution of $\mathrm{NaHCO}_{3}$ and extracted with $\mathrm{CH}_{2} \mathrm{Cl}_{2}$. The organic phase was dried over $\mathrm{Na}_{2} \mathrm{SO}_{4}$ and concentrated to dryness under reduced pressure. The organic crude was dissolved in dry DMF (73 mL) under a $\mathrm{N}_{2}$ atmosphere, and $\mathrm{NaH} 60 \%$ in mineral oil (364 $\mathrm{mg}, 9.09 \mathrm{mmol})$ was slowly added at $0{ }^{\circ} \mathrm{C}$. After $20 \mathrm{~min}, \mathrm{BnBr}(1.4$ $\mathrm{mL}, 12.12 \mathrm{mmol}$ ) was dropwise added and stirring was continued at 0 ${ }^{\circ} \mathrm{C}$ for $3 \mathrm{~h}$. Ice-water was used to destroy the excess of $\mathrm{NaH}$, and the mixture was evaporated in a high vacuum rotovap, poured over a saturated solution of $\mathrm{NH}_{4} \mathrm{Cl}$, and extracted with $\mathrm{CH}_{2} \mathrm{Cl}_{2}$. The combined extracts were dried over $\mathrm{Na}_{2} \mathrm{SO}_{4}$ and concentrated to dryness under reduced pressure. Column chromatography of the residue (hexanes-EtOAc, 95:5) gave $103(1.03 \mathrm{~g}, 3.27 \mathrm{mmol}, 54 \%)$ as a yellow oil: $[\alpha]_{\mathrm{D}}=+37.0\left(c=0.74, \mathrm{CHCl}_{3}\right) .{ }^{1} \mathrm{H} \mathrm{NMR}(500 \mathrm{MHz}$, $\left.\mathrm{CDCl}_{3}\right) \delta_{\mathrm{H}} 7.36-7.24(\mathrm{~m}, 5 \mathrm{H}, \mathrm{Ar}), 5.23(\mathrm{ddd}, J=7.0,7.0,5.1 \mathrm{~Hz}$, $\left.1 \mathrm{H}, 1^{\prime}-\mathrm{H}\right), 4.90-4.86\left(\mathrm{~m}, 3 \mathrm{H}, \mathrm{OBn}, 3^{\prime}-\mathrm{H}_{2}\right), 4.73(\mathrm{~m}, 1 \mathrm{H}, 1-\mathrm{H}), 4.62$ (d, $J=11.7 \mathrm{~Hz}, 1 \mathrm{H}, \mathrm{OBn}), 4.29$ (dd, $J=5.4,1.6 \mathrm{~Hz}, 1 \mathrm{H}, 2-\mathrm{H}), 4.19$ (dd, $J=7.0,5.6 \mathrm{~Hz}, 1 \mathrm{H}, 3-\mathrm{H}), 3.61$ (dddd, $J=9.5,6.0,6.0,6.0 \mathrm{~Hz}$, $1 \mathrm{H}, 5-\mathrm{H}), 3.26$ (dd, $J=9.8,7.3 \mathrm{~Hz}, 1 \mathrm{H}, 4-\mathrm{H}), 1.52$ (s, 3H, Me), 1.37 (s, 3H, Me), $1.26 \mathrm{ppm}\left(\mathrm{d}, J=6.3 \mathrm{~Hz}, 3 \mathrm{H}, 6-\mathrm{H}_{3}\right) .{ }^{13} \mathrm{C}\left\{{ }^{1} \mathrm{H}\right\} \mathrm{NMR}$ $\left(125.7 \mathrm{MHz}, \mathrm{CDCl}_{3}\right) \delta_{\mathrm{C}} 208.0\left(\mathrm{C}, \mathrm{C}-2^{\prime}\right), 138.2(\mathrm{C}, \mathrm{Ar}), 128.1(2 \times$ $\mathrm{CH}, \mathrm{Ar}), 127.9(2 \times \mathrm{CH}, \mathrm{Ar}), 127.5(\mathrm{CH}, \mathrm{Ar}), 108.6(\mathrm{C}$, isopropylidene), 89.5 ( $\mathrm{CH}, \mathrm{C}-1$ '), $81.5(\mathrm{CH}, \mathrm{C}-4), 78.0(\mathrm{CH}, \mathrm{C}-3)$, $77.4\left(\mathrm{CH}_{2}, \mathrm{C}-3\right.$ ') $75.5(\mathrm{CH}, \mathrm{C}-2), 72.9\left(\mathrm{CH}_{2}, \mathrm{OBn}\right), 70.7(\mathrm{CH}, \mathrm{C}-1)$, $67.0(\mathrm{CH}, \mathrm{C}-5), 28.0\left(\mathrm{CH}_{3}, \mathrm{Me}\right), 26.3\left(\mathrm{CH}_{3}, \mathrm{Me}\right), 18.0 \mathrm{ppm}\left(\mathrm{CH}_{3}\right.$, C-6). IR $\left(\mathrm{CHCl}_{3}\right): \nu=3016,2993,1955,1228,1074 \mathrm{~cm}^{-1}$. MS (ESI) $m / z(\%)=339(100)[\mathrm{M}+\mathrm{Na}]^{+}$. HRMS (ESI) $m / z:[\mathrm{M}+\mathrm{Na}]^{+}$ calcd for $\mathrm{C}_{19} \mathrm{H}_{24} \mathrm{NaO}_{4}$ 339.1572; found 339.1567. Anal. calcd for $\mathrm{C}_{19} \mathrm{H}_{24} \mathrm{O}_{4}$ : C, 72.13; H, 7.65. Found: C, 72.06; H, 7.74.

C-(2,3-O-[(2S,3S)-2,3-Dimethoxybutane-2,3-diyl]- $\alpha-L-$ fucopyranosyl)allene (108). To a solution of 107 (5.07 g, 16.25 $\mathrm{mmol})$ in dry $\mathrm{MeOH}(244 \mathrm{~mL})$ was added $\mathrm{K}_{2} \mathrm{CO}_{3}(3.6 \mathrm{~g}, 26 \mathrm{mmol})$, and the mixture was stirred at room temperature for $3 \mathrm{~h}$. Then, it was filtered and neutralized with the Amberlyst $15 \mathrm{H}^{+}$ion exchange resin. It was filtered again under vacuum and evaporated. The resulting organic crude was dissolved in dry $\mathrm{MeOH}(81.3 \mathrm{~mL})$, and 2,3butanedione $(2.85 \mathrm{~mL}, 32.5 \mathrm{mmol}),(\mathrm{MeO})_{3} \mathrm{CH}(7.1 \mathrm{~mL}, 65 \mathrm{mmol})$, and $\mathrm{BF}_{3} \bullet \mathrm{Et}_{2} \mathrm{O}(3.4 \mathrm{~mL}, 30.88 \mathrm{mmol})$ were added dropwise. The mixture was stirred at $60{ }^{\circ} \mathrm{C}$ for $4.5 \mathrm{~h}$. A few pipettes of $\mathrm{Et}_{3} \mathrm{~N}$ were added while stirring at room temperature for $15 \mathrm{~min}$. Then, it was evaporated to dryness. Column chromatography of the residue (hexanes-EtOAc, 7:3) gave $108(3.18 \mathrm{~g}, 10.6 \mathrm{mmol}, 65 \%)$ as a colorless oil: $[\alpha]_{\mathrm{D}}=-16.9\left(c=1.15, \mathrm{CHCl}_{3}\right) .{ }^{1} \mathrm{H} \mathrm{NMR}(500 \mathrm{MHz}$, $\left.\mathrm{CDCl}_{3}\right) \delta_{\mathrm{H}} 5.38$ (ddd, $\left.J=6.8,6.8,4.4 \mathrm{~Hz}, 1 \mathrm{H}, 1^{\prime}-\mathrm{H}\right), 4.85$ (dd, $J=$ $\left.6.9,4.1 \mathrm{~Hz}, 2 \mathrm{H}, 3{ }^{\prime}-\mathrm{H}_{2}\right), 4.61$ (dddd, $\left.J=6.0,4.1,4.1,4.1 \mathrm{~Hz}, 1 \mathrm{H}, 1-\mathrm{H}\right)$, 4.29 (dd, $J=10.6,6.0 \mathrm{~Hz}, 1 \mathrm{H}, 2-\mathrm{H}), 4.00$ (dddd, $J=6.3,6.3,6.3,0.0$ Hz, $1 \mathrm{H}, 5-\mathrm{H}), 3.85$ (dd, $J=10.6,3.1 \mathrm{~Hz}, 1 \mathrm{H}, 3-\mathrm{H}), 3.72$ (br s, $1 \mathrm{H}, 4-$ $\mathrm{H}), 3.24$ (s, 3H, OMe), 3.23 (s, 3H, OMe), 2.39 (br s, $1 \mathrm{H}, \mathrm{OH}), 1.31$ (s, $3 \mathrm{H}, \mathrm{Me}), 1.26\left(\mathrm{~d}, J=6.3 \mathrm{~Hz}, 3 \mathrm{H}, 6-\mathrm{H}_{3}\right), 1.25 \mathrm{ppm}(\mathrm{s}, 3 \mathrm{H}, \mathrm{Me})$. ${ }^{13} \mathrm{C}\left\{{ }^{1} \mathrm{H}\right\}$ NMR $\left(125.7 \mathrm{MHz}, \mathrm{CDCl}_{3}\right) \delta_{\mathrm{C}} 208.7\left(\mathrm{C}, \mathrm{C}-2^{\prime}\right), 100.1(\mathrm{C})$, 99.8 (C), 85.8 (CH, C-1'), $77.0\left(\mathrm{CH}_{2}, \mathrm{C}-3^{\prime}\right), 71.5(\mathrm{CH}, \mathrm{C}-1), 70.8$ $(\mathrm{CH}, \mathrm{C}-4), 68.4(\mathrm{CH}, \mathrm{C}-5), 67.9(\mathrm{CH}, \mathrm{C}-3), 64.0(\mathrm{CH}, \mathrm{C}-2), 47.9(2$ $\left.\times \mathrm{CH}_{3}, 2 \times \mathrm{OMe}\right), 17.6\left(2 \times \mathrm{CH}_{3}, \mathrm{Me}, \mathrm{C}-6\right), 16.5 \mathrm{ppm}\left(\mathrm{CH}_{3}, \mathrm{Me}\right)$. IR $\left(\mathrm{CHCl}_{3}\right): \nu=3672,3583,3010,2942,1957,1226 \mathrm{~cm}^{-1}$. MS (ESI) $m / z(\%)=323(100)[\mathrm{M}+\mathrm{Na}]^{+}$. HRMS (ESI) $m / z:[\mathrm{M}+\mathrm{Na}]^{+}$ calcd for $\mathrm{C}_{15} \mathrm{H}_{24} \mathrm{NaO}_{6} 323.1471$; found 323.1476. Anal. calcd for $\mathrm{C}_{15} \mathrm{H}_{24} \mathrm{O}_{6}$ : C, 59.98; H, 8.05. Found: C, 59.90; H, 8.35.

General Procedure to Give Diphenoxyphosphoryl Derivatives 92, 95, and 99. The corresponding alcohol $(1 \mathrm{mmol})$ in dry $\mathrm{CH}_{2} \mathrm{Cl}_{2}(58 \mathrm{~mL})$ was treated with DMAP $(562 \mathrm{mg}, 4.61 \mathrm{mmol})$ and $\mathrm{ClPO}(\mathrm{OPh})_{2}(0.95 \mathrm{~mL}, 4.61 \mathrm{mmol})$ at room temperature for $2 \mathrm{~h}$. The reaction was quenched with a saturated aqueous solution of $\mathrm{NH}_{4} \mathrm{Cl}$ and extracted with $\mathrm{CH}_{2} \mathrm{Cl}_{2}$. The organic phase was dried over $\mathrm{Na}_{2} \mathrm{SO}_{4}$, filtered, and evaporated. Column chromatography of the residue (hexanes-EtOAc) gave the diphenoxyphosphoryl derivatives.

C-(6-O-tert-Butyldiphenylsilyl-4-O-diphenoxyphosphoryl-2,3-diO-methyl- $\alpha$-D-glucopyranosyl)allene (92). To a solution of the allene 91 (97.4 mg, $0.19 \mathrm{mmol})$ in dry $\mathrm{MeOH}(0.95 \mathrm{~mL})$ was added $\mathrm{K}_{2} \mathrm{CO}_{3}(2.1 \mathrm{mg}, 0.015 \mathrm{mmol}$ ), and the mixture was stirred at room temperature overnight. The residue was evaporated to afford the intermediate alcohol that was submitted to the general procedure to give the diphenoxylphosphoryl derivative for $2.5 \mathrm{~h}$. Column chromatography (hexanes-EtOAc, 85:15) gave $92(96.5 \mathrm{mg}, 0.14$ mmol, $73 \%)$ as a colorless oil: $[\alpha]_{\mathrm{D}}=+45.6\left(c=0.45, \mathrm{CHCl}_{3}\right) .{ }^{1} \mathrm{H}$ $\operatorname{NMR}\left(500 \mathrm{MHz}, \mathrm{CDCl}_{3}\right) \delta_{\mathrm{H}} 7.68-7.07(\mathrm{~m}, 20 \mathrm{H}, \mathrm{Ar}), 5.36$ (ddd, $J=$ 8.3, 8.3, $\left.8.3 \mathrm{~Hz}, 1 \mathrm{H}, 1^{\prime}-\mathrm{H}\right), 4.84$ (dd, $\left.J=8.6,0.0 \mathrm{~Hz}, 1 \mathrm{H}, 3{ }^{\prime}-\mathrm{H}_{\mathrm{b}}\right), 4.83$ $\left(\mathrm{dd}, J=8.4,0.0 \mathrm{~Hz}, 1 \mathrm{H}, 3^{\prime}-\mathrm{H}_{\mathrm{a}}\right), 4.74(\mathrm{~m}, 1 \mathrm{H}, 1-\mathrm{H}), 4.62(\mathrm{ddd}, J=8.5$, $\left.8.5 \mathrm{~Hz},{ }^{3} \mathrm{~J}_{\mathrm{PH}}=8.5 \mathrm{~Hz}, 1 \mathrm{H}, 4-\mathrm{H}\right), 3.96-3.87\left(\mathrm{~m}, 2 \mathrm{H}, 5-\mathrm{H}, 6-\mathrm{H}_{\mathrm{b}}\right), 3.79$ $\left(\mathrm{dd}, J=14.9,6.7 \mathrm{~Hz}, 1 \mathrm{H}, 6-\mathrm{H}_{\mathrm{a}}\right), 3.57(\mathrm{dd}, J=10.9,8.3 \mathrm{~Hz}, 1 \mathrm{H}, 3-\mathrm{H})$, $3.46(\mathrm{~m}, 1 \mathrm{H}, 2-\mathrm{H}), 3.45(\mathrm{~s}, 6 \mathrm{H}, \mathrm{OMe}), 1.04 \mathrm{ppm}\left(\mathrm{s}, 9 \mathrm{H},{ }^{t} \mathrm{Bu}\right)$. ${ }^{13} \mathrm{C}\left\{{ }^{1} \mathrm{H}\right\}$ NMR $\left(100.6 \mathrm{MHz}, \mathrm{CDCl}_{3}\right) \delta_{\mathrm{C}} 209.6\left(\mathrm{C}, \mathrm{C}-2^{\prime}\right), 150.8(\mathrm{~d}$, $\left.{ }^{2} J_{\mathrm{PC}}=7.0 \mathrm{~Hz}, \mathrm{C}, \mathrm{Ar}\right), 150.6\left(\mathrm{~d},{ }^{2} J_{\mathrm{PC}}=7.8 \mathrm{~Hz}, \mathrm{C}, \mathrm{Ar}\right), 133.6(2 \times \mathrm{C}$, $\mathrm{Ar}), 119.9-135.7(20 \times \mathrm{CH}, \mathrm{Ar}), 85.2(\mathrm{CH}, \mathrm{C}-1$ '), $81.6(\mathrm{CH}, \mathrm{C}-2)$, 81.1 (CH, C-3), $76.7\left(\mathrm{CH}_{2}, \mathrm{C}-3\right)$ ), $76.6(\mathrm{CH}, \mathrm{C}-4), 72.8\left(\mathrm{~d},{ }^{3} J_{\mathrm{PC}}=7.0\right.$ $\mathrm{Hz}, \mathrm{CH}, \mathrm{C}-5), 70.9(\mathrm{CH}, \mathrm{C}-1), 63.0\left(\mathrm{CH}_{2}, \mathrm{C}-6\right), 60.2\left(\mathrm{CH}_{3}, \mathrm{OMe}\right)$, $58.2\left(\mathrm{CH}_{3}, \mathrm{OMe}\right), 26.7\left(3 \times \mathrm{CH}_{3}, \mathrm{DPS}\right), 19.3 \mathrm{ppm}$ (C, DPS). IR $\left(\mathrm{CHCl}_{3}\right): \nu=3015,2933,1955,1592,1490,1191 \mathrm{~cm}^{-1}$. MS (ESI) $m / z(\%)=723(100)[\mathrm{M}+\mathrm{Na}]^{+}$. HRMS $(\mathrm{ESI}) \mathrm{m} / z:[\mathrm{M}+\mathrm{Na}]^{+}$ calcd for $\mathrm{C}_{39} \mathrm{H}_{45} \mathrm{NaO}_{8} \mathrm{PSi}$ 723.2519; found 723.2510. Anal. calcd for $\mathrm{C}_{39} \mathrm{H}_{45} \mathrm{O}_{8}$ Psi: C, 66.84; H, 6.47. Found: C, 66.90; H, 6.42.

C-(4,6-Bis-O-diphenoxyphosphoryl-2,3-di-O-methyl- $\alpha-D-$ glucopyranosyl)methanol (95). Compound $48(620 \mathrm{mg}, 1.24 \mathrm{mmol})$ was dissolved in $\mathrm{CH}_{2} \mathrm{Cl}_{2}(6.2 \mathrm{~mL})$, and DHP $(282 \mu \mathrm{L}, 3.09 \mathrm{mmol})$ and $p-\mathrm{TsOH} \cdot \mathrm{H}_{2} \mathrm{O}(15 \mathrm{mg}, 0.08 \mathrm{mmol})$ were added while stirring at room temperature for $2 \mathrm{~h}$. The reaction was poured over a saturated aqueous solution of $\mathrm{NaHCO}_{3}$ and extracted with $\mathrm{CH}_{2} \mathrm{Cl}_{2}$. The organic extract was dried over $\mathrm{Na}_{2} \mathrm{SO}_{4}$, filtered, and evaporated. The 
crude in dry THF $(25 \mathrm{~mL})$ was treated with TBAF/THF $1 \mathrm{M}$ solution $(1.9 \mathrm{~mL}, 1.9 \mathrm{mmol})$ for $3 \mathrm{~h}$ at room temperature. Then, the mixture was evaporated to dryness and quickly chromatographed (hexanes-EtOAc 3:7) to obtain the corresponding alcohol $(315 \mathrm{mg}$, $0.91 \mathrm{mmol}, 73 \%)$ as an orange oil that was saponified with $\mathrm{K}_{2} \mathrm{CO}_{3}(25$ $\mathrm{mg}, 0.18 \mathrm{mmol})$ in $\mathrm{MeOH}(4.6 \mathrm{~mL})$ at room temperature for $4 \mathrm{~h}$, filtered over a pad of Celite, and concentrated. The resulting diol was treated with $\mathrm{ClPO}(\mathrm{OPh})_{2}(1.7 \mathrm{~mL}, 8.19 \mathrm{mmol})$ and dry pyridine $(9.1$ $\mathrm{mL}, 112.5 \mathrm{mmol})$ at room temperature overnight. The reaction was evaporated in a high vacuum rotovap, quenched with an aqueous solution of $\mathrm{HCl} 10 \%$, and extracted with $\mathrm{CH}_{2} \mathrm{Cl}_{2}$. The organic phase was washed with a saturated aqueous solution of $\mathrm{NaHCO}_{3}$, dried over $\mathrm{Na}_{2} \mathrm{SO}_{4}$, and evaporated. Finally, the THP protecting group was hydrolyzed by treatment with $p-\mathrm{TsOH} \cdot \mathrm{H}_{2} \mathrm{O}(17.3 \mathrm{mg}, 0.091 \mathrm{mmol})$ in $\mathrm{MeOH}(1.8 \mathrm{~mL})$ at room temperature for $2 \mathrm{~h}$. The mixture was poured over a saturated aqueous solution of $\mathrm{NaHCO}_{3}$ and extracted with $\mathrm{CH}_{2} \mathrm{Cl}_{2}$. The organic phase was dried over $\mathrm{Na}_{2} \mathrm{SO}_{4}$, filtered, and evaporated. Column chromatography of the residue (hexanesEtOAc, $4: 6)$ gave $95(315.3 \mathrm{mg}, 0.46 \mathrm{mmol}, 37 \%$ overall yield) as a colorless oil: $[\alpha]_{\mathrm{D}}=+20.6\left(c=1.25, \mathrm{CHCl}_{3}\right) .{ }^{1} \mathrm{H}$ NMR $(500 \mathrm{MHz}$, $\left.\mathrm{CDCl}_{3}\right) \delta_{\mathrm{H}} 7.34-7.11(\mathrm{~m}, 20 \mathrm{H}, \mathrm{Ar}), 4.33-4.20\left(\mathrm{~m}, 3 \mathrm{H}, 4-\mathrm{H}, 6-\mathrm{H}_{2}\right)$, 4.14-4.09 (m, 2H, 1-H, 5-H), $3.90\left(\mathrm{dd}, J=12.6,8.8 \mathrm{~Hz}, 1 \mathrm{H}, 1^{\prime}-\mathrm{H}_{\mathrm{b}}\right.$ ), $3.70\left(\mathrm{dd}, J=12.6,3.5 \mathrm{~Hz}, 1 \mathrm{H}, 1^{\prime}-\mathrm{H}_{\mathrm{a}}\right), 3.51(\mathrm{dd}, J=8.2,8.2 \mathrm{~Hz}, 1 \mathrm{H}, 3-$ $\mathrm{H}), 3.42(\mathrm{~s}, 6 \mathrm{H}, 2 \times \mathrm{OMe}), 3.40 \mathrm{ppm}(\mathrm{dd}, J=8.2,5.7 \mathrm{~Hz}, 1 \mathrm{H}, 2-\mathrm{H})$, $1 \mathrm{H}$ from $\mathrm{OH}$ is missing. ${ }^{13} \mathrm{C}\left\{{ }^{1} \mathrm{H}\right\}$ NMR $\left(125.7 \mathrm{MHz}, \mathrm{CDCl}_{3}\right) \delta_{\mathrm{C}}$ $150.7\left(\mathrm{~d},{ }^{2} J_{\mathrm{PC}}=7.4 \mathrm{~Hz}, \mathrm{C}, \mathrm{Ar}\right), 150.6\left(\mathrm{~d},{ }^{2} J_{\mathrm{PC}}=7.4 \mathrm{~Hz}, \mathrm{C}, \mathrm{Ar}\right), 150.5$ $\left(\mathrm{d},{ }^{2} J_{\mathrm{PC}}=7.4 \mathrm{~Hz}, \mathrm{C}, \mathrm{Ar}\right), 150.3\left(\mathrm{~d},{ }^{2} J_{\mathrm{PC}}=7.4 \mathrm{~Hz}, \mathrm{C}, \mathrm{Ar}\right), 120.0-129.8$ $(20 \times \mathrm{CH}, \mathrm{Ar}), 80.4(2 \times \mathrm{CH}, \mathrm{C}-2, \mathrm{C}-3), 75.9\left(\mathrm{~d},{ }^{2} J_{\mathrm{PC}}=6.4 \mathrm{~Hz}, \mathrm{CH}\right.$, C-4), $74.1(\mathrm{CH}, \mathrm{C}-1), 70.9(\mathrm{CH}, \mathrm{C}-5), 68.3\left(\mathrm{~d},{ }^{2} J_{\mathrm{PC}}=7.4 \mathrm{~Hz}, \mathrm{CH}_{2}\right.$, $\left.\mathrm{C}-6), 60.3\left(\mathrm{CH}_{3}, \mathrm{OMe}\right), 58.8\left(\mathrm{CH}_{3}, \mathrm{OMe}\right), 58.3 \mathrm{ppm}\left(\mathrm{CH}_{2}, \mathrm{C}^{\prime}\right)^{\prime}\right)$. IR $\left(\mathrm{CHCl}_{3}\right): \nu=3690,3620,3024,2401,1491,1226 \mathrm{~cm}^{-1}$. MS (ESI) $m / z(\%)=709(100)[\mathrm{M}+\mathrm{Na}]^{+}$. HRMS (ESI) $m / z:[\mathrm{M}+\mathrm{Na}]^{+}$ calcd for $\mathrm{C}_{33} \mathrm{H}_{36} \mathrm{NaO}_{12} \mathrm{P}_{2}$ 709.1580; found 709.1582. Anal. calcd for $\mathrm{C}_{33} \mathrm{H}_{36} \mathrm{O}_{12} \mathrm{P}_{2}$ : C, 57.73; H, 5.28. Found: C, 57.82; H, 5.62.

C-(6-O-tert-Butyldiphenylsilyl-4-O-diphenoxyphosphoryl-2,3-di$O$-methyl- $\alpha$-D-galactopyranosyl)allene (99). To a solution of the acetyl derivative $98(749.5 \mathrm{mg}, 1.47 \mathrm{mmol})$ in $\mathrm{MeOH}(7.3 \mathrm{~mL})$ was added $\mathrm{K}_{2} \mathrm{CO}_{3}(16.2 \mathrm{mg}, 0.12 \mathrm{mmol})$, and it was stirred at room temperature overnight. The mixture was filtered, evaporated, and submitted to the general procedure to give the diphenoxyphosphoryl derivative. Column chromatography (hexanes-EtOAc, 8:2) of the residue afforded $99(928.6 \mathrm{mg}, 1.32 \mathrm{mmol}, 90 \%)$ as a colorless oil: $[\alpha]_{\mathrm{D}}=+74.6\left(c=0.46, \mathrm{CHCl}_{3}\right) .{ }^{1} \mathrm{H} \mathrm{NMR}\left(400 \mathrm{MHz}, \mathrm{CDCl}_{3}\right) \delta_{\mathrm{H}}$ $7.08-7.65(\mathrm{~m}, 20 \mathrm{H}, \mathrm{Ar}), 5.28-5.24\left(\mathrm{~m}, 2 \mathrm{H}, 1-\mathrm{H}, 1^{\prime}-\mathrm{H}\right), 4.79-4.67$ $\left(\mathrm{m}, 3 \mathrm{H}, 4-\mathrm{H}, 3{ }^{\prime}-\mathrm{H}_{2}\right), 3.85(\mathrm{~m}, 1 \mathrm{H}, 5-\mathrm{H}), 3.79$ (dd, $J=10.1,7.4 \mathrm{~Hz}$, $\left.1 \mathrm{H}, 6-\mathrm{H}_{\mathrm{b}}\right), 3.69\left(\mathrm{dd}, J=10.1,6.1 \mathrm{~Hz}, 1 \mathrm{H}, 6-\mathrm{H}_{\mathrm{a}}\right), 3.61(\mathrm{dd}, J=9.6,5.6$ $\mathrm{Hz}, 1 \mathrm{H}, 3-\mathrm{H}), 3.41$ (s, 3H, OMe), 3.38 (s, 3H, OMe), 3.32 (dd, $J=$ 9.6, 1.3 Hz, 1H, 2-H), $1.03 \mathrm{ppm}\left(\mathrm{s}, 9 \mathrm{H},{ }^{t} \mathrm{Bu}\right) .{ }^{13} \mathrm{C}\left\{{ }^{1} \mathrm{H}\right\}$ NMR $(100.6$ $\left.\mathrm{MHz}, \mathrm{CDCl}_{3}\right) \delta_{\mathrm{C}} 208.8\left(\mathrm{C}, \mathrm{C}-2{ }^{\prime}\right), 150.8\left(\mathrm{~d},{ }^{2} J_{\mathrm{PC}}=7.8 \mathrm{~Hz}, \mathrm{C}, \mathrm{Ar}\right)$, $150.7\left(\mathrm{~d},{ }^{2} J_{\mathrm{PC}}=7.1 \mathrm{~Hz}, \mathrm{C}, \mathrm{Ar}\right), 135.61(2 \times \mathrm{CH}, \mathrm{Ar}), 135.56(2 \times$ $\mathrm{CH}, \mathrm{Ar}), 133.5$ (C, Ar), 133.3 (C, Ar), 129.8 (CH, Ar), $129.7(\mathrm{CH}$, $\mathrm{Ar}), 129.6(2 \times \mathrm{CH}, \mathrm{Ar}), 129.3(2 \times \mathrm{CH}, \mathrm{Ar}), 127.64(2 \times \mathrm{CH}, \mathrm{Ar})$, $127.59(2 \times \mathrm{CH}, \mathrm{Ar}), 125.0(\mathrm{CH}, \mathrm{Ar}), 124.9(\mathrm{CH}, \mathrm{Ar}), 120.52(\mathrm{CH}$, $\mathrm{Ar}), 120.45(\mathrm{CH}, \mathrm{Ar}), 120.12(\mathrm{CH}, \mathrm{Ar}), 120.07(\mathrm{CH}, \mathrm{Ar}), 85.3(\mathrm{CH}$, C-1'), 79.1 ( $\mathrm{CH}, \mathrm{C}-2), 76.7\left(\mathrm{CH}_{2}, \mathrm{C}-3^{\prime}\right), 76.7(\mathrm{CH}, \mathrm{C}-3), 74.5$ (d, $\left.{ }^{2} J_{\mathrm{PC}}=6.3 \mathrm{~Hz}, \mathrm{CH}, \mathrm{C}-4\right), 71.7\left(\mathrm{~d},{ }^{3} \mathrm{~J}_{\mathrm{PC}}=5.6 \mathrm{~Hz}, \mathrm{CH}, \mathrm{C}-5\right), 71.3(\mathrm{CH}$, $\mathrm{C}-1), 62.1\left(\mathrm{CH}_{2}, \mathrm{C}-6\right), 59.0\left(\mathrm{CH}_{3}, \mathrm{OMe}\right), 57.5\left(\mathrm{CH}_{3}, \mathrm{OMe}\right), 26.7$ (3 $\times \mathrm{CH}_{3}$, DPS $), 19.1 \mathrm{ppm}$ (C, DPS). IR $\left(\mathrm{CHCl}_{3}\right): \nu=3016,2933$, $1956,1592,1490,1112 \mathrm{~cm}^{-1}$. MS (ESI) $\mathrm{m} / z(\%)=723(100)[\mathrm{M}+$ $\mathrm{Na}]^{+}$. HRMS (ESI) $m / z:[\mathrm{M}+\mathrm{Na}]^{+}$calcd for $\mathrm{C}_{39} \mathrm{H}_{45} \mathrm{NaO}_{8} \mathrm{PSi}$ 723.2519; found 723.2513. Anal. calcd for $\mathrm{C}_{39} \mathrm{H}_{45} \mathrm{O}_{8} \mathrm{PSi}$ : C, 66.84; $\mathrm{H}$, 6.47. Found: C, 66.80; H, 6.48 .

C-(6-O-tert-Butyldiphenylsilyl-2,3-di-O-methyl-4-O-tosyl- $\alpha-D-$ glucopyranosyl)allene (93). To a solution of the allene $91(301 \mathrm{mg}$, $0.59 \mathrm{mmol})$ in dry $\mathrm{MeOH}(3 \mathrm{~mL}), \mathrm{K}_{2} \mathrm{CO}_{3}(6 \mathrm{mg}, 0.04 \mathrm{mmol})$ was added and the mixture was stirred at room temperature overnight. The residue was evaporated to afford the intermediate alcohol that was dissolved in dry pyridine $(6 \mathrm{~mL})$ and treated with $\mathrm{TsCl}(343 \mathrm{mg}$, $1.8 \mathrm{mmol}$ ) overnight. The reaction was evaporated at high vacuum rotovap, quenched with $\mathrm{HCl} 10 \%$, and extracted with $\mathrm{CH}_{2} \mathrm{Cl}_{2}$. The organic phase was washed with a saturated aqueous solution of $\mathrm{NaHCO}_{3}$, dried over $\mathrm{Na}_{2} \mathrm{SO}_{4}$, filtered, and evaporated. Column chromatography (hexanes-EtOAc, 9:1) gave 93 (165.1 mg, 0.27 mmol, $45 \%)$ as a colorless oil: $[\alpha]_{\mathrm{D}}=+69.6\left(c=0.46, \mathrm{CHCl}_{3}\right) .{ }^{1} \mathrm{H}$ $\operatorname{NMR}\left(500 \mathrm{MHz}, \mathrm{CDCl}_{3}\right) \delta_{\mathrm{H}} 7.78-7.18(\mathrm{~m}, 14 \mathrm{H}, \mathrm{Ar}), 5.31$ (ddd, $J=$ 6.7, 6.7, $\left.6.7 \mathrm{~Hz}, 1 \mathrm{H}, 1^{\prime}-\mathrm{H}\right), 4.82$ (dd, $\left.J=6.7,0.0 \mathrm{~Hz}, 1 \mathrm{H}, 3^{\prime}-\mathrm{H}_{\mathrm{b}}\right), 4.81$ (dd, $\left.J=6.9,0.0 \mathrm{~Hz}, 1 \mathrm{H}, 3-\mathrm{H}_{\mathrm{a}}\right), 4.75-4.70(\mathrm{~m}, 2 \mathrm{H}, 1-\mathrm{H}, 3-\mathrm{H}), 3.88$ $\left(\mathrm{dd}, J=11.4,2.2 \mathrm{~Hz}, 1 \mathrm{H}, 6-\mathrm{H}_{\mathrm{b}}\right), 3.82(\mathrm{~m}, 1 \mathrm{H}, 4-\mathrm{H}), 3.76(\mathrm{dd}, J=$ 11.0, $\left.4.7 \mathrm{~Hz}, 1 \mathrm{H}, 6-\mathrm{H}_{\mathrm{a}}\right), 3.46(\mathrm{~s}, 3 \mathrm{H}, \mathrm{OMe}), 3.42-3.40(\mathrm{~m}, 2 \mathrm{H}, 2-\mathrm{H}$, 5-H), 3.23 (s, 3H, OMe), 2.39 (s, 3H, OTs), $1.09 \mathrm{ppm}\left(\mathrm{s}, 9 \mathrm{H},{ }^{t} \mathrm{Bu}\right)$. ${ }^{13} \mathrm{C}\left\{{ }^{1} \mathrm{H}\right\}$ NMR $\left(125.7 \mathrm{MHz}, \mathrm{CDCl}_{3}\right) \delta_{\mathrm{C}} 209.5(\mathrm{C}, \mathrm{C}-2$ ) $), 144.2(\mathrm{C}$, Ar), 135.0 (C, Ar), 133.7 (C, Ar), 133.6 (C, Ar), 127.5-135.9 (14 × $\mathrm{CH}, \mathrm{Ar}), 85.3(\mathrm{CH}, \mathrm{C}-1$ '), $81.7(\mathrm{CH}, \mathrm{C}-2$ or C-5), $80.7(\mathrm{CH}, \mathrm{C}-2$ or C-5), $77.9(\mathrm{CH}, \mathrm{C}-1), 76.8\left(\mathrm{CH}_{2}, \mathrm{C}-3^{\prime}\right), 72.4(\mathrm{CH}, \mathrm{C}-4), 70.8(\mathrm{CH}$, $\mathrm{C}-3), 62.8\left(\mathrm{CH}_{2}, \mathrm{C}-6\right), 60.3\left(\mathrm{CH}_{3}, \mathrm{OMe}\right), 58.4\left(\mathrm{CH}_{3}, \mathrm{OMe}\right), 26.8(3$ $\times \mathrm{CH}_{3}$, DPS $), 21.6\left(\mathrm{CH}_{3}\right.$, OTs), $19.4 \mathrm{ppm}$ (C, DPS). IR $\left(\mathrm{CHCl}_{3}\right): \nu$ $=3015,2933,1955,1599,1373,1112 \mathrm{~cm}^{-1}$. MS (ESI) $\mathrm{m} / z(\%)=$ $645(100)[\mathrm{M}+\mathrm{Na}]^{+}$. HRMS (ESI) $m / z:[\mathrm{M}+\mathrm{Na}]^{+}$calcd for $\mathrm{C}_{34} \mathrm{H}_{42} \mathrm{NaO}_{7} \mathrm{SSi}$ 645.2318; found 645.2321. Anal. calcd for $\mathrm{C}_{34} \mathrm{H}_{42} \mathrm{O}_{7} \mathrm{SSi}$ : C, 65.56; H, 6.80. Found: C, 65.40; H, 7.05.

General Procedure for the Synthesis of the Hydroxymethyl Derivatives 48, 49, 51, 52, 58, 59, 94, 104, and 109. A solution of the allene $(1 \mathrm{mmol})$ in $\mathrm{CH}_{2} \mathrm{Cl}_{2}-\mathrm{MeOH}(30 \mathrm{~mL}, 4: 1)$ was cooled to $-78{ }^{\circ} \mathrm{C}$, and ozone was bubbled into the solution until it became blue. Then, nitrogen was introduced through the mixture to expel the excess of ozone, and it was heated to $0{ }^{\circ} \mathrm{C}$. Afterward, $\mathrm{NaBH}_{4}(75.3$ $\mathrm{mg}, 1.99 \mathrm{mmol}$ ) was added slowly and the solution was stirred for $2 \mathrm{~h}$ at room temperature. The reaction mixture was then poured into brine, extracted with $\mathrm{CH}_{2} \mathrm{Cl}_{2}$, dried over $\mathrm{Na}_{2} \mathrm{SO}_{4}$, and concentrated. Column chromatography (hexanes-EtOAc) of the residue afforded the title alcohol.

C-(4-O-Acetyl-6-O-tert-butyldiphenylsilyl-2,3-di-O-methyl- $\alpha$-Dglucopyranosyl)methanol (48). Following the general procedure starting from allene $91(524 \mathrm{mg}, 1.03 \mathrm{mmol})$ and purification by column chromatography (hexanes-EtOAc, 25:75), alcohol 48 (332.7 $\mathrm{mg}, 0.66 \mathrm{mmol}, 64 \%)$ was obtained as a colorless oil: $[\alpha]_{\mathrm{D}}=+19.0(c$ $\left.=0.39, \mathrm{CHCl}_{3}\right) .{ }^{1} \mathrm{H}$ NMR $\left(500 \mathrm{MHz} \mathrm{CDCl}_{3}, 2-\mathrm{H}\right.$ and $3-\mathrm{H}$ are strongly coupled; therefore, $4-\mathrm{H}$ and $1-\mathrm{H}$ show virtual coupling effects; the chemical shifts and coupling constants shown below were obtained using DAISY) $\delta_{\mathrm{H}} 7.70-7.65 \mathrm{ppm}(\mathrm{m}, 4 \mathrm{H}, \mathrm{Ar}), 7.45-7.36$ $(\mathrm{m}, 6 \mathrm{H}, \mathrm{Ar}), 4.89(\mathrm{dd}, J=8.0,5.8 \mathrm{~Hz}, 1 \mathrm{H}, 4-\mathrm{H}), 4.13$ (ddd, $J=8.6$, $4.8,4.6 \mathrm{~Hz}, 1 \mathrm{H}, 1-\mathrm{H}), 3.91\left(\mathrm{dd}, J=12.0,8.6 \mathrm{~Hz}, 1 \mathrm{H}, 1^{\prime}-\mathrm{H}_{\mathrm{b}}\right), 3.77(\mathrm{dd}$, $\left.J=12.0,4.6 \mathrm{~Hz}, 1 \mathrm{H}, 1^{\prime}-\mathrm{H}_{\mathrm{a}}\right), 3.76(\mathrm{ddd}, J=8.0,5.8,4.1 \mathrm{~Hz}, 1 \mathrm{H}, 5-\mathrm{H})$, $3.72\left(\mathrm{dd}, J=10.8,4.1 \mathrm{~Hz}, 1 \mathrm{H}, 6-\mathrm{H}_{\mathrm{b}}\right), 3.71(\mathrm{dd}, J=10.8,5.8 \mathrm{~Hz}, 1 \mathrm{H}$, 6- $\mathrm{H}_{\mathrm{a}}$ ), $3.480(\mathrm{~s}, 3 \mathrm{H}, \mathrm{OMe}), 3.478(\mathrm{~s}, 3 \mathrm{H}, \mathrm{OMe}), 3.467$ (dd, $J=7.8$, $7.1 \mathrm{~Hz}, 1 \mathrm{H}, 3-\mathrm{H}), 3.467(\mathrm{dd}, J=7.1,4.8 \mathrm{~Hz}, 1 \mathrm{H}, 2-\mathrm{H}), 1.98(\mathrm{~s}, 3 \mathrm{H}$, $\mathrm{OAc}), 1.06 \mathrm{ppm}\left(\mathrm{s}, 9 \mathrm{H},{ }^{t} \mathrm{Bu}\right), 1 \mathrm{H}$ from $\mathrm{OH}$ is missing. ${ }^{1} \mathrm{H}$ NMR $(500$ $\left.\mathrm{MHz}, \mathrm{C}_{6} \mathrm{D}_{6}\right) \delta_{\mathrm{H}} 7.85-7.82(\mathrm{~m}, 4 \mathrm{H}, \mathrm{Ar}), 7.26-7.21(\mathrm{~m}, 6 \mathrm{H}, \mathrm{Ar}), 5.21$ (dd, $J=8.5,8.5 \mathrm{~Hz}, 1 \mathrm{H}, 4-\mathrm{H}), 4.07$ (ddd, $J=5.0,5.0,8.3 \mathrm{~Hz}, 1 \mathrm{H}, 1-$ $\mathrm{H})$, 3.84-3.79 (m, 5H, 1'- $\left.\mathrm{H}_{2}, 5-\mathrm{H}, 6-\mathrm{H}_{2}\right), 3.44(\mathrm{dd}, J=8.4,8.4 \mathrm{~Hz}$, $1 \mathrm{H}, 3-\mathrm{H}), 3.31(\mathrm{~s}, 3 \mathrm{H}, \mathrm{OMe}), 3.26(\mathrm{dd}, J=8.5,5.9 \mathrm{~Hz}, 1 \mathrm{H}, 2-\mathrm{H})$, 2.99 (s, 3H, OMe), 1.63 (s, 3H, OAc), $1.23 \mathrm{ppm}\left(\mathrm{s}, 9 \mathrm{H},{ }^{t} \mathrm{Bu}\right)$. ${ }^{13} \mathrm{C}\left\{{ }^{1} \mathrm{H}\right\}$ NMR $\left(100.6 \mathrm{MHz}, \mathrm{CDCl}_{3}\right) \delta_{\mathrm{C}} 169.7$ (C, OAc), $135.7(2 \times$ $\mathrm{CH}, \mathrm{Ar}), 135.6$ ( $2 \times \mathrm{CH}, \mathrm{Ar}), 133.3$ (C, Ar), 133.2 (C, Ar), 129.72 (2 $\times \mathrm{CH}, \mathrm{Ar}), 129.70(2 \times \mathrm{CH}, \mathrm{Ar}), 127.69(2 \times \mathrm{CH}, \mathrm{Ar}), 127.67(2 \times$ $\mathrm{CH}, \mathrm{Ar}), 79.9(\mathrm{CH}, \mathrm{C}-2$ or C-5), $79.8(\mathrm{CH}, \mathrm{C}-2$ or C-5), $73.4(\mathrm{CH}$, $\mathrm{C}-4), 72.5$ (CH, C-1), $69.6(\mathrm{CH}, \mathrm{C}-3), 63.2\left(\mathrm{CH}_{2}, \mathrm{C}-6\right), 59.8\left(\mathrm{CH}_{3}\right.$, $\mathrm{OMe})$, $59.6\left(\mathrm{CH}_{2}, \mathrm{C}-1^{\prime}\right), 58.9\left(\mathrm{CH}_{3}, \mathrm{OMe}\right), 26.8\left(3 \times \mathrm{CH}_{3}, \mathrm{DPS}\right)$, $20.9\left(\mathrm{CH}_{3}, \mathrm{OAc}\right), 19.2 \mathrm{ppm}(\mathrm{C}, \mathrm{DPS})$. IR $\left(\mathrm{CHCl}_{3}\right): \nu=3680,3553$, $2934,1742,1217 \mathrm{~cm}^{-1}$. MS (ESI) $\mathrm{m} / z(\%)=525(100)[\mathrm{M}+\mathrm{Na}]^{+}$. HRMS (ESI) $m / z$ : $[\mathrm{M}+\mathrm{Na}]^{+}$calcd for $\mathrm{C}_{27} \mathrm{H}_{38} \mathrm{NaO}_{7} \mathrm{Si} 525.2285$; found 525.2286 .

C-(6-O-tert-Butyldiphenylsilyl-2,3-di-O-methyl-4-O-tosyl- $\alpha$-Dglucopyranosyl)methanol (49). Following the general procedure starting from allene $93(226.2 \mathrm{mg}, 0.36 \mathrm{mmol})$ and purification by column chromatography (hexanes-EtOAc, 6:4), alcohol 49 (147.9 $\mathrm{mg}, 0.24 \mathrm{mmol}, 67 \%)$ was obtained as a colorless oil: $[\alpha]_{\mathrm{D}}=+15.2(c$ $\left.=0.54, \mathrm{CHCl}_{3}\right) .{ }^{1} \mathrm{H}$ NMR $\left(500 \mathrm{MHz}, \mathrm{CDCl}_{3}\right.$, simulated coupling constants using DAISY) $\delta_{\mathrm{H}} 7.74-7.17(\mathrm{~m}, 14 \mathrm{H}, \mathrm{Ar}), 4.64(\mathrm{dd}, J=7.6$, $6.8 \mathrm{~Hz}, 1 \mathrm{H}, 4-\mathrm{H}$ ), 4.04 (ddd, $J=8.5,5.1,4.4 \mathrm{~Hz}, 1 \mathrm{H}, 1-\mathrm{H}$ ), 3.834 (dd, 
$\left.J=10.9,3.7 \mathrm{~Hz}, 1 \mathrm{H}, 6-\mathrm{H}_{\mathrm{b}}\right), 3.831\left(\mathrm{dd}, J=12.1,8.5 \mathrm{~Hz}, 1 \mathrm{H}, 1^{\prime}-\mathrm{H}_{\mathrm{b}}\right)$, 3.73 (ddd, $J=7.6,6.0,3.7 \mathrm{~Hz}, 1 \mathrm{H}, 5-\mathrm{H}), 3.69$ (dd, $J=12.1,4.4 \mathrm{~Hz}$, $\left.1 \mathrm{H}, 1^{\prime}-\mathrm{H}_{\mathrm{a}}\right), 3.66\left(\mathrm{dd}, J=10.9,6.0 \mathrm{~Hz}, 1 \mathrm{H}, 6-\mathrm{H}_{\mathrm{a}}\right), 3.51(\mathrm{dd}, J=7.2,6.8$ $\mathrm{Hz}, 1 \mathrm{H}, 3-\mathrm{H}), 3.47(\mathrm{~s}, 3 \mathrm{H}, \mathrm{OMe}), 3.43(\mathrm{dd}, J=7.2,5.1 \mathrm{~Hz}, 1 \mathrm{H}, 2-\mathrm{H})$, 3.29 (s, 3H, OMe), 2.36 (s, 3H, OTs), $1.05 \mathrm{ppm}\left(\mathrm{s}, 9 \mathrm{H},{ }^{t} \mathrm{Bu}\right), 1 \mathrm{H}$ from $\mathrm{OH}$ is missing. ${ }^{13} \mathrm{C}\left\{{ }^{1} \mathrm{H}\right\}$ NMR $\left(100.6 \mathrm{MHz}, \mathrm{CDCl}_{3}\right) \delta_{\mathrm{C}} 144.5$ (C, OTs), 134.3 (C, OTs), 133.3 (C, Ar), 133.2 (C, Ar), 127.6$135.6(14 \times \mathrm{CH}, \mathrm{Ar}), 79.8(\mathrm{CH}, \mathrm{C}-2), 79.1(\mathrm{CH}, \mathrm{C}-3), 76.3(\mathrm{CH}, \mathrm{C}-$ 4), $73.0(\mathrm{CH}, \mathrm{C}-5), 72.2(\mathrm{CH}, \mathrm{C}-1), 62.5\left(\mathrm{CH}_{2}, \mathrm{C}-6\right), 59.8\left(\mathrm{CH}_{3}\right.$, $\mathrm{OMe}), 59.7\left(\mathrm{CH}_{2}, \mathrm{C}-1^{\prime}\right), 58.7\left(\mathrm{CH}_{3}, \mathrm{OMe}\right), 26.7\left(3 \times \mathrm{CH}_{3}, \mathrm{DPS}\right)$, $21.6\left(\mathrm{CH}_{3}, \mathrm{OTs}\right), 19.2 \mathrm{ppm}(\mathrm{C}, \mathrm{DPS})$. IR $\left(\mathrm{CHCl}_{3}\right): \nu=3694,3574$, $3024,2934,1600,1104 \mathrm{~cm}^{-1}$. MS (ESI) $m / z(\%)=637(100)[\mathrm{M}+$ $\mathrm{Na}]^{+}$. HRMS (ESI) $m / z:[\mathrm{M}+\mathrm{Na}]^{+}$calcd for $\mathrm{C}_{32} \mathrm{H}_{42} \mathrm{NaO}_{8} \mathrm{SSi}$ 637.2267; found 637.2257. Anal. calcd for $\mathrm{C}_{32} \mathrm{H}_{42} \mathrm{O}_{8} \mathrm{SSi}$ : C, 62.51; $\mathrm{H}$, 6.89; S, 5.22. Found: C, 62.29; H, 7.09; S, 4.86 .

C-(4-O-Acetyl-6-O-tert-butyldiphenylsilyl-2,3-di-O-methyl- $\alpha-D-$ galactopyranosyl)methanol (51). Following the general procedure starting from allene $98(461.5 \mathrm{mg}, 0.90 \mathrm{mmol})$ and purification by column chromatography (hexanes-EtOAc, 25:75), alcohol 51 (316.3 $\mathrm{mg}, 0.63 \mathrm{mmol}, 70 \%)$ was obtained as a colorless oil: $[\alpha]_{\mathrm{D}}=+30.6(c$ $\left.=0.66, \mathrm{CHCl}_{3}\right) .{ }^{1} \mathrm{H}$ NMR $\left(500 \mathrm{MHz}, \mathrm{CDCl}_{3}\right.$, simulated ring coupling constants using DAISY) $\delta_{\mathrm{H}} 7.67-7.64(\mathrm{~m}, 4 \mathrm{H}, \mathrm{Ar}), 7.46-7.37(\mathrm{~m}$, $6 \mathrm{H}, \mathrm{Ar}$ ), $5.52(\mathrm{dd}, J=3.3,1.9 \mathrm{~Hz}, 1 \mathrm{H}, 4-\mathrm{H}), 4.20$ (ddd, $J=8.4,6.1$, $4.8 \mathrm{~Hz}, 1 \mathrm{H}, 1-\mathrm{H}), 3.83$ (ddd, $J=6.6,6.2,1.9 \mathrm{~Hz}, 1 \mathrm{H}, 5-\mathrm{H}), 3.82(\mathrm{dd}$, $\left.J=12.0,8.4 \mathrm{~Hz}, 1 \mathrm{H}, 1^{\prime}-\mathrm{H}_{\mathrm{b}}\right), 3.77\left(\mathrm{dd}, J=12.0,5.1 \mathrm{~Hz}, 1 \mathrm{H}, 1^{\prime}-\mathrm{H}_{\mathrm{a}}\right)$, $3.73\left(\mathrm{dd}, J=10.4,6.6 \mathrm{~Hz}, 1 \mathrm{H}, 6-\mathrm{H}_{\mathrm{b}}\right), 3.66(\mathrm{dd}, J=9.1,6.1 \mathrm{~Hz}, 1 \mathrm{H}, 2-$ $\mathrm{H}), 3.61\left(\mathrm{dd}, J=10.4,6.2 \mathrm{~Hz}, 1 \mathrm{H}, 6-\mathrm{H}_{\mathrm{a}}\right), 3.48(\mathrm{~s}, 3 \mathrm{H}, \mathrm{OMe}), 3.42$ (dd, $J=9.1,3.3 \mathrm{~Hz}, 1 \mathrm{H}, 3-\mathrm{H}), 3.41$ (s, 3H, OMe), 2.02 (s, 3H, OAc), $1.06 \mathrm{ppm}\left(\mathrm{s}, 9 \mathrm{H},{ }^{t} \mathrm{Bu}\right), 1 \mathrm{H}$ from $\mathrm{OH}$ is missing. ${ }^{13} \mathrm{C}\left\{{ }^{1} \mathrm{H}\right\}$ NMR $(100.6$ $\left.\mathrm{MHz}, \mathrm{CDCl}_{3}\right) \delta_{\mathrm{C}} 169.9(\mathrm{C}, \mathrm{OAc}), 135.6(4 \times \mathrm{CH}, \mathrm{Ar}), 133.2(\mathrm{C}, \mathrm{Ar})$, $133.0(\mathrm{C}, \mathrm{Ar}), 129.8(2 \times \mathrm{CH}, \mathrm{Ar}), 127.7(4 \times \mathrm{CH}, \mathrm{Ar}), 79.0(\mathrm{CH}, \mathrm{C}-$ 2 or C-5), $77.5(\mathrm{CH}, \mathrm{C}-2$ or C-5), $73.4(\mathrm{CH}, \mathrm{C}-4), 71.9(\mathrm{CH}, \mathrm{C}-1)$, $66.6(\mathrm{CH}, \mathrm{C}-3), 62.2\left(\mathrm{CH}_{2}, \mathrm{C}-6\right), 59.4\left(\mathrm{CH}_{3}, \mathrm{OMe}\right), 59.1\left(\mathrm{CH}_{2}, \mathrm{C}-\right.$ 1'), $57.7\left(\mathrm{CH}_{3}, \mathrm{OMe}\right), 26.8\left(3 \times \mathrm{CH}_{3}, \mathrm{DPS}\right), 20.7\left(\mathrm{CH}_{3}, \mathrm{OAc}\right), 19.1$ $\mathrm{ppm}(\mathrm{C}, \mathrm{DPS})$. IR $\left(\mathrm{CHCl}_{3}\right): \nu=3686,3620,3015,2975,1742,1229$ $\mathrm{cm}^{-1}$. MS (ESI) $\mathrm{m} / z(\%)=525(100)[\mathrm{M}+\mathrm{Na}]^{+}$. HRMS (ESI) $\mathrm{m} / z$ : $[\mathrm{M}+\mathrm{Na}]^{+}$calcd for $\mathrm{C}_{27} \mathrm{H}_{38} \mathrm{NaO}_{7} \mathrm{Si}$ 525.2285; found 525.2284. Anal. calcd for $\mathrm{C}_{27} \mathrm{H}_{38} \mathrm{O}_{7} \mathrm{Si}$ : C, 64.51; H, 7.62. Found: C, 64.20; H, 7.65.

C-(6-O-tert-Butyldiphenylsilyl-4-O-diphenoxyphosphoryl-2,3-di$O$-methyl- $\alpha$-D-galactopyranosyl)methanol (52). Following the general procedure starting from allene $99(896.2 \mathrm{~g}, 1.28 \mathrm{mmol})$ and purification by column chromatography (hexanes-EtOAc, 6:4), alcohol 52 (567.3 mg, $0.82 \mathrm{mmol}, 64 \%)$ was obtained as a colorless oil: $[\alpha]_{\mathrm{D}}=+25.0\left(c=0.32, \mathrm{CHCl}_{3}\right) .{ }^{1} \mathrm{H} \mathrm{NMR}\left(500 \mathrm{MHz}, \mathrm{CDCl}_{3}\right) \delta_{\mathrm{H}}$ $7.65-7.05$ (m, 20H, Ar), 5.06 (ddd, $J=2.6,1.6 \mathrm{~Hz},{ }^{3} J_{\mathrm{PH}}=8.5 \mathrm{~Hz}$, $1 \mathrm{H}, 4-\mathrm{H}), 4.09$ (ddd, $J=7.3,5.7,5.7 \mathrm{~Hz}, 1 \mathrm{H}, 1-\mathrm{H}), 3.80-3.68(\mathrm{~m}$, $\left.5 \mathrm{H}, 5-\mathrm{H}, 6-\mathrm{H}_{2}, 1^{\prime}-\mathrm{H}_{2}\right), 3.57$ (dd, $\left.J=8.8,5.6 \mathrm{~Hz}, 1 \mathrm{H}, 2-\mathrm{H}\right), 3.389(\mathrm{~s}$, $3 \mathrm{H}, \mathrm{OMe}$ ), 3.38 (dd, $J=8.8,2.6 \mathrm{~Hz}, 1 \mathrm{H}, 3-\mathrm{H}), 3.35$ (s, $3 \mathrm{H}, \mathrm{OMe})$, 1.93 (br s, $1 \mathrm{H}, \mathrm{OH}), 1.03 \mathrm{ppm}\left(\mathrm{s}, 9 \mathrm{H},{ }^{t} \mathrm{Bu}\right) .{ }^{13} \mathrm{C}\left\{{ }^{1} \mathrm{H}\right\}$ NMR $(125.7$ $\left.\mathrm{MHz}, \mathrm{CDCl}_{3}\right) \delta_{\mathrm{C}} 150.8\left(\mathrm{~d},{ }^{2} J_{\mathrm{PC}}=7.4 \mathrm{~Hz}, \mathrm{C}, \mathrm{Ar}\right), 150.5\left(\mathrm{~d},{ }^{2} J_{\mathrm{PC}}=7.4\right.$ $\mathrm{Hz}, \mathrm{C}, \mathrm{Ar}), 135.6(2 \times \mathrm{CH}, \mathrm{Ar}), 135.5(2 \times \mathrm{CH}, \mathrm{Ar}), 133.3(\mathrm{C}, \mathrm{Ar})$, 133.2 (C, Ar), $129.74(\mathrm{CH}, \mathrm{Ar}), 129.72(\mathrm{CH}, \mathrm{Ar}), 129.6(2 \times \mathrm{CH}$, $\mathrm{Ar}), 129.4(2 \times \mathrm{CH}, \mathrm{Ar}), 127.7(4 \times \mathrm{CH}, \mathrm{Ar}), 125.2(\mathrm{CH}, \mathrm{Ar}), 125.1$ $(\mathrm{CH}, \mathrm{Ar}), 120.33(\mathrm{CH}, \mathrm{Ar}), 120.28(\mathrm{CH}, \mathrm{Ar}), 120.00(\mathrm{CH}, \mathrm{Ar})$, $119.96(\mathrm{CH}, \mathrm{Ar}), 78.9$ (CH, C-3), $77.0(\mathrm{CH}, \mathrm{C}-2), 74.2\left(\mathrm{~d},{ }^{2} J_{\mathrm{PC}}=6.3\right.$ $\mathrm{Hz}, \mathrm{CH}, \mathrm{C}-4), 72.8\left(\mathrm{~d},{ }^{3} \mathrm{~J}_{\mathrm{PC}}=6.3 \mathrm{~Hz}, \mathrm{CH}, \mathrm{C}-5\right), 72.6(\mathrm{CH}, \mathrm{C}-1), 62.2$ $\left(\mathrm{CH}_{2}, \mathrm{C}-6\right), 59.58\left(\mathrm{CH}_{3}, \mathrm{OMe}\right), 59.2\left(\mathrm{CH}_{2}, \mathrm{C}-1\right), 57.7\left(\mathrm{CH}_{3}, \mathrm{OMe}\right)$, $26.7\left(3 \times \mathrm{CH}_{3}, \mathrm{DPS}\right), 19.0 \mathrm{ppm}(\mathrm{C}, \mathrm{DPS})$. IR $\left(\mathrm{CHCl}_{3}\right): \nu=3690$, $3546,3023,2934,1592,1206 \mathrm{~cm}^{-1}$. MS (ESI) $\mathrm{m} / z(\%)=715(100)$ $[\mathrm{M}+\mathrm{Na}]^{+}$. HRMS (ESI) $m / z:[\mathrm{M}+\mathrm{Na}]^{+}$calcd for $\mathrm{C}_{37} \mathrm{H}_{45} \mathrm{NaO}_{9} \mathrm{PSi}$ 715.2468; found 715.2475. Anal. calcd for $\mathrm{C}_{37} \mathrm{H}_{45} \mathrm{O}_{9} \mathrm{PSi}$ : C, 64.14; $\mathrm{H}$, 6.55. Found: C, 64.02; H, 6.67.

C-(4-O-Acetyl-2,3-di-O-methyl- $\alpha$-D-fucopyranosyl)methanol (58). Alcohol 109 (200 mg, $0.68 \mathrm{mmol})$ was dissolved in dry DMF $(2.7 \mathrm{~mL})$, and DPSCl $(238 \mu \mathrm{L}, 1.01 \mathrm{mmol})$ and imidazole $(138 \mathrm{mg}$, $2.03 \mathrm{mmol}$ ) were added at room temperature. After $3 \mathrm{~h}$, the reaction was evaporated in a high vacuum rotovap, quenched with $\mathrm{H}_{2} \mathrm{O}$, and extracted with $\mathrm{CH}_{2} \mathrm{Cl}_{2}$. The organic phase was dried over $\mathrm{Na}_{2} \mathrm{SO}_{4}$, filtered, and evaporated. To a solution of the resulting crude in dry EtOAc $(16 \mathrm{~mL})$ was added Pd/C 10\% (152 mg), and the mixture was submitted to $\mathrm{H}_{2}$ atmosphere overnight. The reaction was filtered over a pad of Celite and evaporated. The alcohol was protected by treatment with dry pyridine $(2.6 \mathrm{~mL}), \mathrm{Ac}_{2} \mathrm{O}(0.9 \mathrm{~mL})$, and DMAP $(8.3 \mathrm{mg}, 0.068 \mathrm{mmol})$ for $0.5 \mathrm{~h}$. The mixture was evaporated in a high vacuum rotovap, quenched with aqueous $\mathrm{HCl} 10 \%$, and extracted with $\mathrm{CH}_{2} \mathrm{Cl}_{2}$. The organic phase was washed with a saturated aqueous solution of $\mathrm{NaHCO}_{3}$, dried with $\mathrm{Na}_{2} \mathrm{SO}_{4}$, filtered, and concentrated to dryness. Finally, the silyl group was deprotected by treatment with a 1 $\mathrm{M}$ solution of TBAF/THF $(1.4 \mathrm{~mL}, 1.4 \mathrm{mmol})$ in dry THF (13.6 $\mathrm{mL}$ ) for $4 \mathrm{~h}$ at room temperature. The mixture was evaporated, and the residue was chromatographed in a silica gel column (hexanesEtOAc, $4: 6$ to $2: 8)$ to give $58(111 \mathrm{mg}, 0.45 \mathrm{mmol}, 66 \%)$ as a colorless oil: $[\alpha]_{\mathrm{D}}=-76.0\left(c=0.65, \mathrm{CHCl}_{3}\right) .{ }^{1} \mathrm{H}$ NMR $(500 \mathrm{MHz}$, $\mathrm{CDCl}_{3}$, simulated ring coupling constants using DAISY) $\delta_{\mathrm{H}} 5.33(\mathrm{dd}$, $J=3.5,2.0 \mathrm{~Hz}, 1 \mathrm{H}, 4-\mathrm{H}), 4.23$ (ddd, $J=8.0,5.9,4.7 \mathrm{~Hz}, 1 \mathrm{H}, 1-\mathrm{H})$, 3.96 (dddd, $J=6.5,6.5,6.5,2.0 \mathrm{~Hz}, 1 \mathrm{H}, 5-\mathrm{H}$ ), 3.89 (dd, $J=12.1,8.0$ $\left.\mathrm{Hz}, 1 \mathrm{H}, 1^{\prime}-\mathrm{H}_{\mathrm{b}}\right), 3.85$ (dd, $J=12.1,4.7 \mathrm{~Hz}, 1 \mathrm{H}, 1$ '- $\left.-\mathrm{H}_{\mathrm{a}}\right), 3.71(\mathrm{dd}, J=$ $8.2,5.9 \mathrm{~Hz}, 1 \mathrm{H}, 2-\mathrm{H}), 3.50(\mathrm{dd}, J=8.2,3.5 \mathrm{~Hz}, 1 \mathrm{H}, 3-\mathrm{H}), 3.50(\mathrm{~s}, 3 \mathrm{H}$, $\mathrm{OMe}$ ), 3.42 (s, 3H, OMe), 2.17 (s, 3H, OAc), 2.10 (br s, $1 \mathrm{H}, \mathrm{OH})$, $1.17 \mathrm{ppm}\left(\mathrm{d}, J=6.5 \mathrm{~Hz}, 3 \mathrm{H}, 6-\mathrm{H}_{3}\right) .{ }^{13} \mathrm{C}\left\{{ }^{1} \mathrm{H}\right\} \mathrm{NMR}(125.7 \mathrm{MHz}$, $\left.\mathrm{CDCl}_{3}\right) \delta_{\mathrm{C}} 170.6(\mathrm{C}, \mathrm{OAc}), 78.7(\mathrm{CH}, \mathrm{C}-3), 77.1(\mathrm{CH}, \mathrm{C}-2), 73.4$ (CH, C-1), 69.5 (CH, C-4), $67.0(\mathrm{CH}, \mathrm{C}-5), 59.5\left(\mathrm{CH}_{2}, \mathrm{C}-1^{\prime}\right), 59.3$ $\left(\mathrm{CH}_{3}, \mathrm{OMe}\right), 57.5\left(\mathrm{CH}_{3}, \mathrm{OMe}\right), 20.8\left(\mathrm{CH}_{3}, \mathrm{OAc}\right), 16.5 \mathrm{ppm}\left(\mathrm{CH}_{3}\right.$, C-6). IR $\left(\mathrm{CHCl}_{3}\right): \nu=3677,3502,3018,2939,1739,1239 \mathrm{~cm}^{-1}$. MS (ESI) $\mathrm{m} / z(\%)=271(100)[\mathrm{M}+\mathrm{Na}]^{+}$. HRMS (ESI) $\mathrm{m} / z:[\mathrm{M}+$ $\mathrm{Na}]^{+}$calcd for $\mathrm{C}_{11} \mathrm{H}_{20} \mathrm{NaO}_{6}$ 271.1158; found 271.1159.

C-(4-O-Diphenoxyphosphoryl-2,3-di-O-methyl- $\alpha-D-$ fucopyranosyl)methanol (59). Alcohol 109 (1.4 g, $4.73 \mathrm{mmol})$ was dissolved in dry DMF $(18.9 \mathrm{~mL})$, and imidazole $(960 \mathrm{mg}, 14.1 \mathrm{mmol})$ and DPSCl $(1.7 \mathrm{~mL}, 7.25 \mathrm{mmol})$ were added at room temperature. After $3 \mathrm{~h}$, the reaction was evaporated in a high vacuum rotovap, quenched with $\mathrm{H}_{2} \mathrm{O}$, and extracted with $\mathrm{CH}_{2} \mathrm{Cl}_{2}$. The organic phase was dried over $\mathrm{Na}_{2} \mathrm{SO}_{4}$, filtered, and evaporated. To a solution of the resulting crude in dry EtOAc $(111 \mathrm{~mL})$ was added Pd/C 10\% (1.06 $\mathrm{g}$ ), and the mixture was submitted to $\mathrm{H}_{2}$ atmosphere overnight. The reaction was filtered over a pad of Celite and evaporated. The alcohol was protected without purification by treatment with DMAP $(2.66 \mathrm{~g}$, $21.7 \mathrm{mmol})$ and $\mathrm{ClPO}(\mathrm{OPh})_{2}(2.82 \mathrm{~mL}, 21.7 \mathrm{mmol})$ in dry $\mathrm{CH}_{2} \mathrm{Cl}_{2}$ $(110 \mathrm{~mL})$ for $3.5 \mathrm{~h}$. The mixture was quenched with a saturated aqueous $\mathrm{NH}_{4} \mathrm{Cl}$ solution and extracted with $\mathrm{CH}_{2} \mathrm{Cl}_{2}$. The organic phase was dried with $\mathrm{Na}_{2} \mathrm{SO}_{4}$, filtered, and concentrated to dryness. Finally, the silyl group was deprotected by treatment with a $1 \mathrm{M}$ solution of TBAF/THF $(9.46 \mathrm{~mL}, 9.46 \mathrm{mmol})$ in dry THF $(95 \mathrm{~mL})$ for $3.5 \mathrm{~h}$ at room temperature. The mixture was evaporated, and the residue was chromatographed on a silica gel column (hexanesEtOAc, 1:1) to give $\mathbf{5 9}(1.24 \mathrm{~g}, 2.84 \mathrm{mmol}, 60 \%)$ as a colorless oil: $[\alpha]_{\mathrm{D}}=-41.8\left(c=0.49, \mathrm{CHCl}_{3}\right) .{ }^{1} \mathrm{H} \mathrm{NMR}\left(400 \mathrm{MHz}, \mathrm{CDCl}_{3}\right.$, simulated coupling constants using DAISY) $\delta_{\mathrm{H}} 7.37-7.17(\mathrm{~m}, 10 \mathrm{H}$, Ar), 4.92 (ddd, $\left.J=3.0,2.5 \mathrm{~Hz},{ }^{3} J_{\mathrm{PH}}=9.1 \mathrm{~Hz}, 1 \mathrm{H}, 4-\mathrm{H}\right), 4.14$ (ddd, $J$ $=7.5,5.7,5.2 \mathrm{~Hz}, 1 \mathrm{H}, 1-\mathrm{H}$ ), 3.96 (ddddd, $J=6.5,6.5,6.5,2.5 \mathrm{~Hz}$, $\left.{ }^{4} J_{\mathrm{PH}}=1.9 \mathrm{~Hz}, 1 \mathrm{H}, 5-\mathrm{H}\right), 3.87-3.77\left(\mathrm{~m}, 2 \mathrm{H}, 1^{\prime}-\mathrm{H}_{2}\right), 3.60$ (dd, $J=8.4$, $5.2 \mathrm{~Hz}, 1 \mathrm{H}, 2-\mathrm{H}$ ), 3.53 (ddd, $J=8.4,3.0, \mathrm{~Hz},{ }^{4} J_{\mathrm{PH}}=1.0 \mathrm{~Hz}, 1 \mathrm{H}, 3-\mathrm{H}$ ), $3.43(\mathrm{~s}, 3 \mathrm{H}, \mathrm{OMe}), 3.41(\mathrm{~s}, 3 \mathrm{H}, \mathrm{OMe}), 1.22 \mathrm{ppm}(\mathrm{d}, J=6.5 \mathrm{~Hz}, 3 \mathrm{H}$, 6- $\left.\mathrm{H}_{3}\right), 1 \mathrm{H}$ from $\mathrm{OH}$ is missing. ${ }^{13} \mathrm{C}\left\{{ }^{1} \mathrm{H}\right\}$ NMR $\left(125.7 \mathrm{MHz}, \mathrm{CDCl}_{3}\right)$ $\delta_{\mathrm{C}} 150.8\left(\mathrm{~d},{ }^{2} J_{\mathrm{PC}}=8.5 \mathrm{~Hz}, \mathrm{C}, \mathrm{Ar}\right), 150.6\left(\mathrm{~d},{ }^{2} J_{\mathrm{PC}}=7.4 \mathrm{~Hz}, \mathrm{C}, \mathrm{Ar}\right)$, $129.7(2 \times \mathrm{CH}, \mathrm{Ar}), 129.3(2 \times \mathrm{CH}, \mathrm{Ar}), 125.3(\mathrm{CH}, \mathrm{Ar}), 125.1$ $(\mathrm{CH}, \mathrm{Ar}), 120.32(\mathrm{CH}, \mathrm{Ar}), 120.28(\mathrm{CH}, \mathrm{Ar}), 120.11(\mathrm{CH}, \mathrm{Ar})$, $120.07(\mathrm{CH}, \mathrm{Ar}), 78.7(\mathrm{CH}, \mathrm{C}-3), 76.9(\mathrm{CH}, \mathrm{C}-2), 76.8\left(\mathrm{~d},{ }^{3} \mathrm{~J}_{\mathrm{PC}}=6.3\right.$ $\mathrm{Hz}, \mathrm{CH}, \mathrm{C}-4), 72.3(\mathrm{CH}, \mathrm{C}-1), 67.9\left(\mathrm{~d},{ }^{3} J_{\mathrm{PC}}=5.3 \mathrm{~Hz}, \mathrm{CH}, \mathrm{C}-5\right), 60.1$ $\left(\mathrm{CH}_{2}, \mathrm{C}-1^{\prime}\right), 59.5\left(\mathrm{CH}_{3}, \mathrm{OMe}\right), 57.8\left(\mathrm{CH}_{3}, \mathrm{OMe}\right), 15.9 \mathrm{ppm}\left(\mathrm{CH}_{3}\right.$, C-6). IR $\left(\mathrm{CHCl}_{3}\right): \nu=3694,3018,2938,1490,1218 \mathrm{~cm}^{-1}$. MS (ESI) $m / z(\%)=461(100)[\mathrm{M}+\mathrm{Na}]^{+}$. HRMS (ESI) $m / z:[\mathrm{M}+\mathrm{Na}]^{+}$ calcd for $\mathrm{C}_{21} \mathrm{H}_{27} \mathrm{NaO}_{8} \mathrm{P}$ 461.1341; found 461.1342. Anal. calcd for $\mathrm{C}_{21} \mathrm{H}_{27} \mathrm{O}_{8} \mathrm{P}: \mathrm{C}$, 57.53; H, 6.21. Found: C, 57.62; H, 6.49.

C-(6-O-tert-Butyldiphenylsilyl-4-O-diphenoxyphosphoryl-2,3-diO-methyl- $\alpha$-D-glucopyranosyl)methanol (94). Following the general procedure starting from allene $92(1.08 \mathrm{~g}, 1.54 \mathrm{mmol})$ and purification by column chromatography (hexanes-EtOAc, 6:4), alcohol $94(556.2 \mathrm{mg}, 0.80 \mathrm{mmol}, 52 \%)$ was obtained as a colorless oil: $[\alpha]_{\mathrm{D}}=+30.3\left(c=0.33, \mathrm{CHCl}_{3}\right) .{ }^{1} \mathrm{H} \operatorname{NMR}\left(500 \mathrm{MHz}, \mathrm{CDCl}_{3}\right.$, 
simulated coupling constants using DAISY) $\delta_{\mathrm{H}} 7.65-7.07(\mathrm{~m}, 20 \mathrm{H}$, Ar), 4.56 (ddd, $\left.J=8.5,7.6 \mathrm{~Hz},{ }^{3} J_{\mathrm{PH}}=9.4 \mathrm{~Hz}, 1 \mathrm{H}, 4-\mathrm{H}\right), 4.13$ (ddd, $J$ $=8.8,5.7,4.6 \mathrm{~Hz}, 1 \mathrm{H}, 1-\mathrm{H}), 3.91\left(\mathrm{dd}, J=11.0,32.8 \mathrm{~Hz}, 1 \mathrm{H}, 6-\mathrm{H}_{\mathrm{b}}\right)$, $3.90\left(\mathrm{dd}, J=12.1,8.8 \mathrm{~Hz}, 1 \mathrm{H}, 1^{\prime}-\mathrm{H}_{\mathrm{b}}\right), 3.83(\mathrm{ddd}, J=8.5,5.9,2.8 \mathrm{~Hz}$, $1 \mathrm{H}, 5-\mathrm{H}), 3.76\left(\mathrm{dd}, J=11.0,5.9 \mathrm{~Hz}, 1 \mathrm{H}, 6-\mathrm{H}_{\mathrm{a}}\right), 3.75(\mathrm{dd}, J=12.1,4.6$ $\mathrm{Hz}, 1 \mathrm{H}, 1$ ' $-\mathrm{H}_{\mathrm{a}}$ ), 3.57 (dd, $\left.J=7.9,7.6 \mathrm{~Hz}, 1 \mathrm{H}, 3-\mathrm{H}\right), 3.50$ (dd, $J=7.9$, $5.7 \mathrm{~Hz}, 1 \mathrm{H}, 2-\mathrm{H}), 3.45$ (s, 3H, OMe), 3.42 (s, 3H, OMe), $1.03 \mathrm{ppm}$ $\left(\mathrm{s}, 9 \mathrm{H},{ }^{t} \mathrm{Bu}\right), 1 \mathrm{H}$ from $\mathrm{OH}$ is missing. ${ }^{13} \mathrm{C}\left\{{ }^{\mathrm{l}} \mathrm{H}\right\}$ NMR $(100.6 \mathrm{MHz}$, $\left.\mathrm{CDCl}_{3}\right) \delta_{\mathrm{C}} 150.7\left(\mathrm{~d},{ }^{2} J_{\mathrm{PC}}=7.0 \mathrm{~Hz}, \mathrm{C}, \mathrm{Ar}\right), 150.5\left(\mathrm{~d},{ }^{2} J_{\mathrm{PC}}=7.1 \mathrm{~Hz}, \mathrm{C}\right.$, $\mathrm{Ar}), 133.3(2 \times \mathrm{C}, \mathrm{Ar}), 120.0-135.7(20 \times \mathrm{CH}, \mathrm{Ar}), 80.6(\mathrm{CH}, \mathrm{C}-2$ or C-3), $80.4\left(\mathrm{CH}, \mathrm{C}-2\right.$ or C-3), $75.7\left(\mathrm{~d},{ }^{2} \mathrm{~J}_{\mathrm{PC}}=6.3 \mathrm{~Hz}, \mathrm{CH}, \mathrm{C}-4\right)$, $73.3\left(\mathrm{~d},{ }^{3} \mathrm{~J}_{\mathrm{PC}}=6.3 \mathrm{~Hz}, \mathrm{CH}, \mathrm{C}-5\right), 72.6(\mathrm{CH}, \mathrm{C}-1), 62.9\left(\mathrm{CH}_{2}, \mathrm{C}-6\right)$, $59.9\left(\mathrm{CH}_{3}, \mathrm{OMe}\right), 59.3\left(\mathrm{CH}_{2}, \mathrm{C}-1^{\prime}\right), 58.8\left(\mathrm{CH}_{3}, \mathrm{OMe}\right), 26.8(3 \times$ $\mathrm{CH}_{3}$, DPS), $19.2 \mathrm{ppm}$ (C, DPS). IR $\left(\mathrm{CHCl}_{3}\right): \nu=3676,3532,3016$, 2934, 1591, $1490 \mathrm{~cm}^{-1}$. MS (ESI) $\mathrm{m} / z(\%)=715(100)[\mathrm{M}+\mathrm{Na}]^{+}$. HRMS (ESI) $m / z:[\mathrm{M}+\mathrm{Na}]^{+}$calcd for $\mathrm{C}_{37} \mathrm{H}_{45} \mathrm{NaO}_{9} \mathrm{PSi}$ 715.2468; found 715.2469. Anal. calcd for $\mathrm{C}_{37} \mathrm{H}_{45} \mathrm{O}_{9} \mathrm{PSi}$ : C, 64.14; $\mathrm{H}, 6.55$. Found: C, 64.06; H, 6.36 .

C-(4-O-Benzyl-2,3-di-O-methyl- $\alpha$-L-rhamnopyranosyl)methanol (104). Allene 103 (154 mg, $0.49 \mathrm{mmol}$ ) in TFA $/ \mathrm{H}_{2} \mathrm{O}$ (4.5 mL, 4:6) was stirred at room temperature for $2 \mathrm{~h}$. The solution was evaporated in a high vacuum rotovap, poured over a saturated aqueous solution of $\mathrm{NaHCO}_{3}$, extracted with $\mathrm{CH}_{2} \mathrm{Cl}_{2}$, dried over $\mathrm{Na}_{2} \mathrm{SO}_{4}$, and concentrated. The crude was dissolved in dry DMF $(5.9 \mathrm{~mL})$ under a $\mathrm{N}_{2}$ atmosphere, and $\mathrm{NaH} 60 \%$ in mineral oil $(58.8 \mathrm{mg}, 1.47 \mathrm{mmol})$ was slowly added at $0{ }^{\circ} \mathrm{C}$. After $20 \mathrm{~min}, \mathrm{MeI}(122 \mu \mathrm{L}, 1.96 \mathrm{mmol})$ was dropwise added and stirring was continued at $0{ }^{\circ} \mathrm{C}$ for $1 \mathrm{~h}$. Icewater was used to destroy the excess of $\mathrm{NaH}$, and the mixture was evaporated in a high vacuum rotovap, poured over a saturated solution of $\mathrm{NH}_{4} \mathrm{Cl}$, and extracted with $\mathrm{CH}_{2} \mathrm{Cl}_{2}$. The combined organic extracts were dried over $\mathrm{Na}_{2} \mathrm{SO}_{4}$ and concentrated to dryness under reduced pressure. The organic residue was then submitted to the general procedure for the synthesis of hydroxymethyl derivatives. Column chromatography of the residue (hexanes-EtOAc, 4:6) gave $104(65.4 \mathrm{mg}, 0.22 \mathrm{mmol}, 45 \%$, three steps $)$ as a colorless oil: $[\alpha]_{\mathrm{D}}=$ $-22.4\left(c=0.293, \mathrm{CHCl}_{3}\right) .{ }^{1} \mathrm{H}$ NMR $\left(500 \mathrm{MHz}, \mathrm{CDCl}_{3}\right) \delta_{\mathrm{H}} 7.37-$ $7.29(\mathrm{~m}, 5 \mathrm{H}, \mathrm{Ar}), 4.70(\mathrm{~d}, J=11.7 \mathrm{~Hz}, 1 \mathrm{H}, \mathrm{OBn}), 4.65(\mathrm{~d}, J=11.7$ $\mathrm{Hz}, 1 \mathrm{H}, \mathrm{OBn}$ ), 3.99 (m, 1H, 1-H), 3.89 (dddd, $J=6.6,6.6,6.6,4.7$ $\mathrm{Hz}, 1 \mathrm{H}, 5-\mathrm{H}), 3.80\left(\mathrm{dd}, J=11.7,7.0 \mathrm{~Hz}, 1 \mathrm{H}, \mathrm{l}^{\prime}-\mathrm{H}_{\mathrm{b}}\right), 3.70(\mathrm{dd}, J=$ $\left.11.4,4.5 \mathrm{~Hz}, 1 \mathrm{H}, 1^{\prime}-\mathrm{H}_{\mathrm{a}}\right), 3.60-3.56(\mathrm{~m}, 2 \mathrm{H}, 2-\mathrm{H}, 3-\mathrm{H}), 3.52(\mathrm{dd}, J=$ 5.4, $4.7 \mathrm{~Hz}, 1 \mathrm{H}, 4-\mathrm{H}), 3.45$ (s, 3H, OMe), 3.44 (s, 3H, OMe), 2.07 (br s, $1 \mathrm{H}, \mathrm{OH}), 1.34 \mathrm{ppm}\left(\mathrm{d}, J=7.0 \mathrm{~Hz}, 3 \mathrm{H}, 6-\mathrm{H}_{3}\right) .{ }^{13} \mathrm{C}\left\{{ }^{1} \mathrm{H}\right\} \mathrm{NMR}$ $\left(125.7 \mathrm{MHz}, \mathrm{CDCl}_{3}\right) \delta_{\mathrm{C}} 138.2(\mathrm{C}, \mathrm{Ar}), 128.4(2 \times \mathrm{CH}, \mathrm{Ar}), 127.8(3$ $\times \mathrm{CH}, \mathrm{Ar}), 78.4(\mathrm{CH}, \mathrm{C}-2$ or C-3), $78.0(\mathrm{CH}, \mathrm{C}-4), 75.7(\mathrm{CH}, \mathrm{C}-2$ or $\mathrm{C}-3), 73.4\left(\mathrm{CH}_{2}, \mathrm{OBn}\right), 70.7(\mathrm{CH}, \mathrm{C}-1), 70.6(\mathrm{CH}, \mathrm{C}-5), 61.7\left(\mathrm{CH}_{2}\right.$, C-1'), $58.0\left(\mathrm{CH}_{3}, \mathrm{OMe}\right), 57.4\left(\mathrm{CH}_{3}, \mathrm{OMe}\right), 17.2 \mathrm{ppm}\left(\mathrm{CH}_{3}, \mathrm{C}-6\right)$. IR $\left(\mathrm{CHCl}_{3}\right): \nu=3587,3478,2587,2478,2015,2935,1455,1088$ $\mathrm{cm}^{-1}$. MS (ESI) $m / z(\%)=319(100)[\mathrm{M}+\mathrm{Na}]^{+}$. HRMS (ESI) $\mathrm{m} / z$ : $[\mathrm{M}+\mathrm{Na}]^{+}$calcd for $\mathrm{C}_{16} \mathrm{H}_{24} \mathrm{NaO}_{5} 319.1521$; found 319.1525. Anal. calcd for $\mathrm{C}_{16} \mathrm{H}_{24} \mathrm{O}_{5}$ : C, 64.84; H, 8.16. Found: C, 64.61; H, 8.37.

$C$-(4-O-Benzyl-2,3-di-O-methyl- $\alpha$-D-fucopyranosyl)methanol (109). Alcohol 108 (251.4 mg, $0.84 \mathrm{mmol})$ was dissolved in dry DMF $\left(10 \mathrm{~mL}\right.$ ) under a $\mathrm{N}_{2}$ atmosphere, and $\mathrm{NaH} 60 \%$ in mineral oil (50.4 $\mathrm{mg}, 1.26 \mathrm{mmol})$ was slowly added at $0{ }^{\circ} \mathrm{C}$. After $20 \mathrm{~min}, \mathrm{BnBr}(200$ $\mu \mathrm{L}, 1.68 \mathrm{mmol}$ ) was dropwise added and stirring was continued at 0 ${ }^{\circ} \mathrm{C}$ for $2 \mathrm{~h}$. Ice-water was used to destroy the $\mathrm{NaH}$ in excess, and the mixture was evaporated in a high vacuum rotovap, poured over a saturated solution of $\mathrm{NH}_{4} \mathrm{Cl}$, and extracted with $\mathrm{CH}_{2} \mathrm{Cl}_{2}$. The combined extracts were dried over $\mathrm{Na}_{2} \mathrm{SO}_{4}$ and concentrated to dryness under reduced pressure. The crude was dissolved in TFA/ $\mathrm{H}_{2} \mathrm{O}(7.7 \mathrm{~mL}, 4: 6)$ and stirred at $40{ }^{\circ} \mathrm{C}$ overnight. The solution was evaporated in a high vacuum rotovap, quenched with a saturated aqueous solution of $\mathrm{NaHCO}_{3}$, and extracted with $\mathrm{CH}_{2} \mathrm{Cl}_{2}$. The organic residue was submitted to methyl protection by treatment with $\mathrm{NaH} 60 \%(100.8 \mathrm{mg}, 2.52 \mathrm{mmol})$ and $\mathrm{MeI}(209 \mu \mathrm{L}, 3.36 \mathrm{mmol})$ in DMF $(10 \mathrm{~mL})$ for $2 \mathrm{~h}$ from $0{ }^{\circ} \mathrm{C}$ until room temperature. Ice-water was used to destroy the excess of $\mathrm{NaH}$, and the mixture was evaporated in a high vacuum rotovap, poured over a saturated solution of $\mathrm{NH}_{4} \mathrm{Cl}$, and extracted with $\mathrm{CH}_{2} \mathrm{Cl}_{2}$. The organic phase was dried over $\mathrm{Na}_{2} \mathrm{SO}_{4}$ and concentrated to dryness under reduced pressure. Finally, the crude was submitted to the general procedure to give hydroxymethyl derivatives. Column chromatography of the residue (hexanes-EtOAc, 4:6) gave $109(106.6 \mathrm{mg}, 0.36 \mathrm{mmol}, 43 \%)$ as a colorless oil: $[\alpha]_{\mathrm{D}}=-30.2\left(c=1.35, \mathrm{CHCl}_{3}\right) .{ }^{1} \mathrm{H}$ NMR $(400$ $\mathrm{MHz}, \mathrm{CDCl}_{3}$, simulated ring coupling constants using DAISY) $\delta_{\mathrm{H}}$ 7.39-7.27 (m, 5H, Ar), 4.77 (d, $J=11.8 \mathrm{~Hz}, 1 \mathrm{H}, \mathrm{OBn}), 4.63$ (d, $J=$ $11.8 \mathrm{~Hz}, 1 \mathrm{H}, \mathrm{OBn}$ ), 4.13 (ddd, $J=7.9,4.7,4.2 \mathrm{~Hz}, 1 \mathrm{H}, 1-\mathrm{H}$ ), 3.97 (dddd, $J=6.7,6.7,6.7,3.6 \mathrm{~Hz}, 1 \mathrm{H}, 5-\mathrm{H}), 3.83(\mathrm{dd}, J=11.6,7.9 \mathrm{~Hz}$, $\left.1 \mathrm{H}, 1^{\prime}-\mathrm{H}_{\mathrm{b}}\right), 3.76(\mathrm{dd}, J=3.6,2.9 \mathrm{~Hz}, 1 \mathrm{H}, 4-\mathrm{H}), 3.74(\mathrm{dd}, J=11.6,4.7$ $\left.\mathrm{Hz}, 1 \mathrm{H}, 1^{\prime}-\mathrm{Ha}\right), 3.69(\mathrm{dd}, J=7.0,4.2 \mathrm{~Hz}, 1 \mathrm{H}, 2-\mathrm{H}), 3.53(\mathrm{dd}, J=7.0$, $2.9 \mathrm{~Hz}, 1 \mathrm{H}, 3-\mathrm{H}), 3.50$ (s, 3H, OMe), 3.45 (s, 3H, OMe), 2.11 (br s, $1 \mathrm{H}, \mathrm{OH}), 1.28 \mathrm{ppm}\left(\mathrm{d}, J=6.7 \mathrm{~Hz}, 3 \mathrm{H}, 6-\mathrm{H}_{3}\right) .{ }^{13} \mathrm{C}\left\{{ }^{1} \mathrm{H}\right\}$ NMR $(100.6$ $\left.\mathrm{MHz}, \mathrm{CDCl}_{3}\right) \delta_{\mathrm{C}} 138.4(\mathrm{C}, \mathrm{Ar}), 128.3(2 \times \mathrm{CH}, \mathrm{Ar}), 128.0(2 \times \mathrm{CH}$, Ar), $127.7(\mathrm{CH}, \mathrm{Ar}), 79.4(\mathrm{CH}, \mathrm{C}-3), 78.1(\mathrm{CH}, \mathrm{C}-2), 75.0(\mathrm{CH}, \mathrm{C}-$ 4), $73.4\left(\mathrm{CH}_{2}, \mathrm{OBn}\right), 70.7(\mathrm{CH}, \mathrm{C}-1), 69.4(\mathrm{CH}, \mathrm{C}-5), 60.8\left(\mathrm{CH}_{2}, \mathrm{C}-\right.$ 1'), $58.9\left(\mathrm{CH}_{3}, \mathrm{OMe}\right), 58.6\left(\mathrm{CH}_{3}, \mathrm{OMe}\right), 15.5 \mathrm{ppm}\left(\mathrm{CH}_{3}, \mathrm{C}-6\right)$. IR $\left(\mathrm{CHCl}_{3}\right): \nu=3676,3588,3012,2937,1101 \mathrm{~cm}^{-1}$. MS (ESI) $\mathrm{m} / z(\%)$ $=319(100)[\mathrm{M}+\mathrm{Na}]^{+}$. HRMS (ESI) $m / z:[\mathrm{M}+\mathrm{Na}]^{+}$calcd for $\mathrm{C}_{16} \mathrm{H}_{24} \mathrm{NaO}_{5} 319.1521$; found 319.1519. Anal. calcd for $\mathrm{C}_{16} \mathrm{H}_{24} \mathrm{O}_{5}$ : C, 64.84; H, 8.16; found: C, 64.89; H, 8.20.

General Procedure for the Synthesis of Phthalimide Derivatives 16, 17, 18, 19, 20, 21, 22, 23, 24, and 105. DEAD $(394 \mu \mathrm{L}, 2.50 \mathrm{mmol})$ was added dropwise to a stirred solution of the alcohol ( $1 \mathrm{mmol}), N$-hydroxyphthalimide $(408 \mathrm{mg}, 2.5 \mathrm{mmol})$, and $\mathrm{PPh}_{3}(656 \mathrm{mg}, 2.5 \mathrm{mmol})$ in dry THF $(10 \mathrm{~mL})$, and the resulting solution was stirred at $0{ }^{\circ} \mathrm{C}$ for $0.5-2.5 \mathrm{~h}$. Then, the solvent was removed and the crude was quenched with water and extracted with $\mathrm{CHCl}_{3}$. The combined organic extracts were dried over $\mathrm{Na}_{2} \mathrm{SO}_{4}$ and concentrated under reduced pressure. Column chromatography of the residue (hexanes-EtOAc) gave the corresponding phthalimides.

C-(4-O-Acetyl-6-O-tert-Butyldiphenylsilyl-2,3-di-O-methyl- $\alpha$-Dglucopyranosyl)N-methoxyphthalimide (16). Following the general procedure starting from alcohol $48(332 \mathrm{mg}, 0.66 \mathrm{mmol})$ and purification by column chromatography (hexanes-EtOAc, 8:2), product $16(281 \mathrm{mg}, 0.43 \mathrm{mmol}, 66 \%)$ was obtained as an amorphous solid: $[\alpha]_{\mathrm{D}}=+10.4\left(c=0.53, \mathrm{CHCl}_{3}\right) .{ }^{1} \mathrm{H}$ NMR $(500$ $\mathrm{MHz}, \mathrm{CDCl}_{3}$, simulated coupling constants using DAISY) $\delta_{\mathrm{H}} 7.74-$ $7.62(\mathrm{~m}, 8 \mathrm{H}, \mathrm{Ar}), 7.43-7.34(\mathrm{~m}, 6 \mathrm{H}, \mathrm{Ar}), 5.17(\mathrm{dd}, J=6.8,6.5 \mathrm{~Hz}$, $1 \mathrm{H}, 4-\mathrm{H}), 4.58\left(\mathrm{dd}, J=10.8,7.5 \mathrm{~Hz}, 1 \mathrm{H}, 1^{\prime}-\mathrm{H}_{\mathrm{b}}\right), 4.48$ (ddd, $J=7.5$, 4.6, $3.9 \mathrm{~Hz}, 1 \mathrm{H}, 1-\mathrm{H}$ ), 4.37 (dd, $J=10.8,3.9 \mathrm{~Hz}, 1 \mathrm{H}, 1^{\prime}-\mathrm{H}_{\mathrm{a}}$ ), 3.88 (ddd, $J=6.8,5.0,4.6 \mathrm{~Hz}, 1 \mathrm{H}, 5-\mathrm{H}), 3.77(\mathrm{dd}, J=11.0,4.6 \mathrm{~Hz}, 1 \mathrm{H}, 6-$ $\left.\mathrm{H}_{\mathrm{b}}\right), 3.71\left(\mathrm{dd}, J=11.0,5.0 \mathrm{~Hz}, 1 \mathrm{H}, 6-\mathrm{H}_{\mathrm{a}}\right), 3.52(\mathrm{dd}, J=7.1,4.6 \mathrm{~Hz}$, $1 \mathrm{H}, 2-\mathrm{H}), 3.51(\mathrm{~s}, 3 \mathrm{H}, \mathrm{OMe}), 3.49(\mathrm{~s}, 3 \mathrm{H}, \mathrm{OMe}), 3.46(\mathrm{dd}, J=7.1$, $6.5 \mathrm{~Hz}, 1 \mathrm{H}, 3-\mathrm{H}), 2.03(\mathrm{~s}, 3 \mathrm{H}, \mathrm{OAc}), 1.03 \mathrm{ppm}\left(\mathrm{s}, 9 \mathrm{H},{ }^{t} \mathrm{Bu}\right) .{ }^{13} \mathrm{C}\left\{{ }^{1} \mathrm{H}\right\}$ NMR $\left(125.7 \mathrm{MHz}, \mathrm{CDCl}_{3}\right) \delta_{\mathrm{C}} 169.7$ (C, OAc), $163.1(2 \times \mathrm{C}, \mathrm{CO})$, $135.64(2 \times \mathrm{CH}, \mathrm{Ar}), 135.60(2 \times \mathrm{CH}, \mathrm{Ar}), 134.3(2 \times \mathrm{CH}, \mathrm{Ar})$, 133.4 (C, Ar), 133.3 (C, Ar), 129.5 (CH, Ar), 129.4 (CH, Ar), 128.8 $(2 \times \mathrm{C}, \mathrm{Ar}), 127.5(4 \times \mathrm{CH}, \mathrm{Ar}), 123.4(2 \times \mathrm{CH}, \mathrm{Ar}), 78.3(\mathrm{CH}, \mathrm{C}-2$ or C-3), $78.0\left(\mathrm{CH}, \mathrm{C}-2\right.$ or C-3), $74.5\left(\mathrm{CH}_{2}, \mathrm{C}-1^{\prime}\right), 73.6(\mathrm{CH}, \mathrm{C}-5)$, $70.3(\mathrm{CH}, \mathrm{C}-1), 68.2(\mathrm{CH}, \mathrm{C}-4), 62.3\left(\mathrm{CH}_{2}, \mathrm{C}-6\right), 59.3\left(\mathrm{CH}_{3}, \mathrm{OMe}\right)$, $58.7\left(\mathrm{CH}_{3}, \mathrm{OMe}\right), 26.7\left(3 \times \mathrm{CH}_{3}, \mathrm{DPS}\right), 21.0\left(\mathrm{CH}_{3}, \mathrm{OAc}\right), 19.1 \mathrm{ppm}$ (C, DPS). IR $\left(\mathrm{CHCl}_{3}\right): \nu=2934,1792,1735,1236 \mathrm{~cm}^{-1}$. MS (ESI) $m / z(\%)=670(100)[\mathrm{M}+\mathrm{Na}]^{+}$. HRMS (ESI) $m / z:[\mathrm{M}+\mathrm{Na}]^{+}$ calcd for $\mathrm{C}_{35} \mathrm{H}_{41} \mathrm{NNaO}_{9} \mathrm{Si}$ 670.2448; found 670.2441. Anal. calcd for $\mathrm{C}_{35} \mathrm{H}_{41} \mathrm{NO}_{9} \mathrm{Si}$ : C, 64.89; H, 6.38; N, 2.16. Found: C, 65.05; H, 6.40; $\mathrm{N}, 2.18$.

C-(6-O-tert-Butyldiphenylsilyl-4-O-diphenoxyphosphoryl-2,3-di$O$-methyl- $\alpha$-D-glucopyranosyl) $N$-methoxyphthalimide (17). Following the general procedure starting from alcohol $94(507.2 \mathrm{mg}, 0.73$ $\mathrm{mmol}$ ) and purification by column chromatography (hexanesEtOAc, 75:25), product $17(331.3 \mathrm{mg}, 0.40 \mathrm{mmol}, 54 \%)$ was obtained as a an amorphous solid: $[\alpha]_{\mathrm{D}}=+14.9\left(c=0.47, \mathrm{CHCl}_{3}\right)$. ${ }^{1} \mathrm{H}$ NMR $\left(500 \mathrm{MHz}, \mathrm{CDCl}_{3}\right.$, simulated coupling constants using DAISY) $\delta_{\mathrm{H}} 7.73-7.11(\mathrm{~m}, 24 \mathrm{H}, \mathrm{Ar}), 4.87\left(\mathrm{ddd}, J=7.0,7.0 \mathrm{~Hz},{ }^{3} J_{\mathrm{PH}}=\right.$ $9.4 \mathrm{~Hz}, 1 \mathrm{H}, 4-\mathrm{H}), 4.58$ (dd, $J=10.9,7.6 \mathrm{~Hz}, 1 \mathrm{H}, 1^{\prime}-\mathrm{H}_{\mathrm{b}}$ ), 4.48 (ddd, $J$ $=7.6,5.3,3.6 \mathrm{~Hz}, 1 \mathrm{H}, 1-\mathrm{H}), 4.36\left(\mathrm{dd}, J=10.9,3.6 \mathrm{~Hz}, 1 \mathrm{H}, 1\right.$ '- $\left.-\mathrm{H}_{\mathrm{a}}\right)$, 3.95 (ddd, $J=7.0,4.0,4.0 \mathrm{~Hz}, 1 \mathrm{H}, 5-\mathrm{H}), 3.77(\mathrm{dd}, J=4.0,4.0 \mathrm{~Hz}$, $\left.2 \mathrm{H}, 6-\mathrm{H}_{2}\right), 3.60(\mathrm{dd}, J=7.4,7.0 \mathrm{~Hz}, 1 \mathrm{H}, 3-\mathrm{H}), 3.51(\mathrm{dd}, J=7.4,5.3$ $\mathrm{Hz}, 1 \mathrm{H}, 2-\mathrm{H}), 3.48(\mathrm{~s}, 3 \mathrm{H}, \mathrm{OMe}), 3.44(\mathrm{~s}, 3 \mathrm{H}, \mathrm{OMe}), 1.00 \mathrm{ppm}(\mathrm{s}$, $\left.9 \mathrm{H},{ }^{t} \mathrm{Bu}\right) \cdot{ }^{13} \mathrm{C}\left\{{ }^{1} \mathrm{H}\right\}$ NMR $\left(100.6 \mathrm{MHz}, \mathrm{CDCl}_{3}\right) \delta_{\mathrm{C}} 163.2(2 \times \mathrm{C}$, 
CO), 150.7 (d, $\left.{ }^{2} J_{\mathrm{PC}}=7.0 \mathrm{~Hz}, \mathrm{C}, \mathrm{Ar}\right), 150.6\left(\mathrm{~d},{ }^{2} J_{\mathrm{PC}}=7.8 \mathrm{~Hz}, \mathrm{C}, \mathrm{Ar}\right)$, 133.5 (C, Ar), 133.4 (C, Ar), 128.8 ( $2 \times$ C, Ar), 119.9-135.7 (24 $\times$ $\mathrm{CH}, \mathrm{Ar}), 79.3(\mathrm{CH}, \mathrm{C}-3), 78.8(\mathrm{CH}, \mathrm{C}-2), 74.5\left(\mathrm{~d},{ }^{2} \mathrm{~J}_{\mathrm{PC}}=7.0 \mathrm{~Hz}\right.$, $\mathrm{CH}, \mathrm{C}-4), 74.0\left(\mathrm{CH}_{2}, \mathrm{C}-1\right.$ ') $73.7\left(\mathrm{~d},{ }^{3} \mathrm{~J}_{\mathrm{PC}}=7.1 \mathrm{~Hz}, \mathrm{CH}, \mathrm{C}-5\right), 70.6$ (CH, C-1), 62.1 $\left(\mathrm{CH}_{2}, \mathrm{C}-6\right), 59.6\left(\mathrm{CH}_{3}, \mathrm{OMe}\right), 58.7\left(\mathrm{CH}_{3}, \mathrm{OMe}\right)$, $26.7\left(3 \times \mathrm{CH}_{3}\right.$, DPS $), 19.2 \mathrm{ppm}$ (C, DPS). IR $\left(\mathrm{CHCl}_{3}\right): \nu=2934$, $1792,1735,1490,1190 \mathrm{~cm}^{-1}$. MS (ESI) $\mathrm{m} / z(\%)=860(100)[\mathrm{M}+$ $\mathrm{Na}]^{+}$. HRMS (ESI) $m / z$ : $[\mathrm{M}+\mathrm{Na}]^{+}$calcd for $\mathrm{C}_{45} \mathrm{H}_{48} \mathrm{NNaO}_{11} \mathrm{PSi}$ 860.2632; found 860.2628. Anal. calcd for $\mathrm{C}_{45} \mathrm{H}_{48} \mathrm{NO}_{11} \mathrm{PSi}$ : C, 64.50; $\mathrm{H}, 5.77$; N, 1.67. Found: C, 64.42; H, 5.61; N, 1.82 .

C-(6-O-tert-Butyldiphenylsilyl-2,3-di-O-methyl-4-O-tosyl- $\alpha$-Dglucopyranosyl)N-methoxyphthalimide (18). Following the general procedure starting from alcohol $49(124.5 \mathrm{mg}, 0.20 \mathrm{mmol})$ and purification by column chromatography (hexanes-EtOAc, 75:25), phthalimide $18(121.5 \mathrm{mg}, 0.16 \mathrm{mmol}, 80 \%)$ was obtained as a colorless oil: $[\alpha]_{\mathrm{D}}=+5.8\left(c=0.36, \mathrm{CHCl}_{3}\right) .{ }^{1} \mathrm{H}$ NMR $(500 \mathrm{MHz}$, $\mathrm{CDCl}_{3}$, simulated ring coupling constants using DAISY) $\delta_{\mathrm{H}} 7.78-$ $7.13(\mathrm{~m}, 18 \mathrm{H}, \mathrm{Ar}), 4.96(\mathrm{dd}, J=6.2,5.8 \mathrm{~Hz}, 1 \mathrm{H}, 4-\mathrm{H}), 4.50(\mathrm{dd}, J=$ $11.0,7.4 \mathrm{~Hz}, 1 \mathrm{H}, 1$ ' $-\mathrm{H}_{\mathrm{b}}$ ), 4.39 (ddd, $\left.J=7.4,4.3,3.8 \mathrm{~Hz}, 1 \mathrm{H}, 1-\mathrm{H}\right)$, $4.32\left(\mathrm{dd}, J=11.0,3.8 \mathrm{~Hz}, 1 \mathrm{H}, 1^{\prime}-\mathrm{H}_{\mathrm{a}}\right), 3.86-3.80\left(\mathrm{~m}, 2 \mathrm{H}, 5-\mathrm{H}, 6-\mathrm{H}_{\mathrm{b}}\right)$, $3.71\left(\mathrm{~m}, 1 \mathrm{H}, 6-\mathrm{H}_{\mathrm{a}}\right), 3.60(\mathrm{dd}, J=6.4,5.8 \mathrm{~Hz}, 1 \mathrm{H}, 3-\mathrm{H}), 3.51(\mathrm{~s}, 3 \mathrm{H}$, OMe), 3.48 (dd, $J=6.4,4.3 \mathrm{~Hz}, 1 \mathrm{H}, 2-\mathrm{H}), 3.36(\mathrm{~s}, 3 \mathrm{H}, \mathrm{OMe}), 2.35$ (s, 3H, OTs), $1.05 \mathrm{ppm}\left(\mathrm{s}, 9 \mathrm{H},{ }^{t} \mathrm{Bu}\right) .{ }^{13} \mathrm{C}\left\{{ }^{1} \mathrm{H}\right\}$ NMR $(100.6 \mathrm{MHz}$, $\left.\mathrm{CDCl}_{3}\right) \delta_{\mathrm{C}} 163.1(2 \times \mathrm{C}, \mathrm{CO}), 144.3$ (C, OTs), 134.3 (C, OTs), 133.5 (C, Ar), 133.2 (C, Ar), 128.7 ( $2 \times \mathrm{C}, \mathrm{Ar}), 123.4-135.6(18 \times$ $\mathrm{CH}, \mathrm{Ar}), 77.8$ (CH, C-2), 77.6 ( $\mathrm{CH}, \mathrm{C}-3), 74.8\left(\mathrm{CH}_{2}, \mathrm{C}-1^{\prime}\right), 74.7$ (CH, C-4), $73.5(\mathrm{CH}, \mathrm{C}-5), 69.9(\mathrm{CH}, \mathrm{C}-1), 61.7\left(\mathrm{CH}_{2}, \mathrm{C}-6\right), 59.3$ $\left(\mathrm{CH}_{3}, \mathrm{OMe}\right), 58.5\left(\mathrm{CH}_{3}, \mathrm{OMe}\right), 26.7\left(3 \times \mathrm{CH}_{3}, \mathrm{DPS}\right), 21.5\left(\mathrm{CH}_{3}\right.$, OTs), $19.2 \mathrm{ppm}$ (C, DPS). IR $\left(\mathrm{CHCl}_{3}\right): \nu=3024,2934,1792,1735$, $1215,1106 \mathrm{~cm}^{-1}$. MS (ESI) $\mathrm{m} / z(\%)=782(100)[\mathrm{M}+\mathrm{Na}]^{+}$. HRMS (ESI) $m / z:[\mathrm{M}+\mathrm{Na}]^{+}$calcd for $\mathrm{C}_{40} \mathrm{H}_{45} \mathrm{NNaO}_{10} \mathrm{SSi} 782.2431$; found 782.2434. Anal. calcd for $\mathrm{C}_{40} \mathrm{H}_{45} \mathrm{NO}_{10} \mathrm{SSi}$ : C, 63.22; $\mathrm{H}, 5.97 ; \mathrm{N}, 1.84$; S, 4.22. Found: C, 63.35; H, 6.17; N, 1.75; S, 4.43.

C-(4,6-Bis-O-diphenoxyphosphoryl-2,3-di-O-methyl- $\alpha$-D-glucopyranosyl) N-methoxyphthalimide (19). Following the general procedure starting from alcohol $95(315 \mathrm{mg}, 0.46 \mathrm{mmol})$ and purification by column chromatography (hexanes-EtOAc, 1:1), product $19(235.8 \mathrm{mg}, 0.28 \mathrm{mmol}, 62 \%)$ was obtained as a colorless oil: $[\alpha]_{\mathrm{D}}=+13.5\left(c=1.90, \mathrm{CHCl}_{3}\right) .{ }^{1} \mathrm{H} \mathrm{NMR}\left(500 \mathrm{MHz}, \mathrm{CDCl}_{3}\right) \delta_{\mathrm{H}}$ 7.83-7.81 (m, 2H, Ar), 7.74-7.73 (m, 2H, Ar), 7.33-7.11 (m, 20H, Ar), 4.53 (ddd, $\left.J=7.9,7.9 \mathrm{~Hz},{ }^{3} J_{\mathrm{PH}}=7.9 \mathrm{~Hz}, 1 \mathrm{H}, 4-\mathrm{H}\right), 4.49$ (dd, $J=$ $\left.10.1,6.6 \mathrm{~Hz}, 1 \mathrm{H}, 1^{\prime}-\mathrm{H}_{\mathrm{b}}\right), 4.42-4.33\left(\mathrm{~m}, 3 \mathrm{H}, 1-\mathrm{H}, 6-\mathrm{H}_{\mathrm{b}}, 1^{\prime}-\mathrm{H}_{\mathrm{a}}\right), 4.26$ (ddd, $\left.J=12.3,4.7 \mathrm{~Hz},{ }^{3} J_{\mathrm{PH}}=7.9 \mathrm{~Hz}, 1 \mathrm{H}, 6-\mathrm{H}_{\mathrm{a}}\right), 4.14(\mathrm{~m}, 1 \mathrm{H}, 5-\mathrm{H})$, 3.58 (dd, $J=7.3,7.3 \mathrm{~Hz}, 1 \mathrm{H}, 3-\mathrm{H}), 3.44$ (s, 3H, OMe), $3.43(\mathrm{~s}, 3 \mathrm{H}$, OMe), $3.41 \mathrm{ppm}(\mathrm{dd}, J=7.6,4.7 \mathrm{~Hz}, 1 \mathrm{H}, 2-\mathrm{H}) .{ }^{13} \mathrm{C}\left\{{ }^{1} \mathrm{H}\right\}$ NMR $\left(125.7 \mathrm{MHz}, \mathrm{CDCl}_{3}\right) \delta_{\mathrm{C}} 163.3(2 \times \mathrm{C}, \mathrm{CO}), 150.6\left(\mathrm{~d},{ }^{2} J_{\mathrm{PC}}=6.3 \mathrm{~Hz}\right.$, $2 \times \mathrm{C}, \mathrm{Ar}), 150.5\left(\mathrm{~d},{ }^{2} J_{\mathrm{PC}}=7.4 \mathrm{~Hz}, \mathrm{C}, \mathrm{Ar}\right), 150.4\left(\mathrm{~d},{ }^{2} J_{\mathrm{PC}}=8.5 \mathrm{~Hz}, \mathrm{C}\right.$, $\mathrm{Ar}), 128.8(2 \times \mathrm{C}, \mathrm{Ar}), 120.0-134.5(24 \times \mathrm{CH}, \mathrm{Ar}), 79.3(\mathrm{CH}, \mathrm{C}-3)$, $78.4(\mathrm{CH}, \mathrm{C}-2), 74.4\left(\mathrm{~d},{ }^{2} \mathrm{~J}_{\mathrm{PC}}=6.4 \mathrm{~Hz}, \mathrm{CH}, \mathrm{C}-4\right), 74.1\left(\mathrm{CH}_{2}, \mathrm{C}-1^{\prime}\right)$, $72.8\left(\mathrm{dd},{ }^{3} J_{\mathrm{PC}}=6.1,6.1 \mathrm{~Hz}, \mathrm{CH}, \mathrm{C}-5\right), 70.4(\mathrm{CH}, \mathrm{C}-1), 66.5\left(\mathrm{~d},{ }^{2}\right)_{\mathrm{PC}}$ $\left.=6.3 \mathrm{~Hz}, \mathrm{CH}_{2}, \mathrm{C}-6\right), 59.8\left(\mathrm{CH}_{3}, \mathrm{OMe}\right), 58.8 \mathrm{ppm}\left(\mathrm{CH}_{3}, \mathrm{OMe}\right)$. IR $\left(\mathrm{CHCl}_{3}\right): \nu=3018,2937,1736,1214 \mathrm{~cm}^{-1}$. MS (ESI) $\mathrm{m} / z(\%)=$ $854(100)[\mathrm{M}+\mathrm{Na}]^{+}$. HRMS (ESI) $\mathrm{m} / z:[\mathrm{M}+\mathrm{Na}]^{+}$calcd for $\mathrm{C}_{41} \mathrm{H}_{39} \mathrm{NNaO}_{14} \mathrm{P}_{2}$ : 854.1744; found 854.1743. Anal. calcd for $\mathrm{C}_{41} \mathrm{H}_{39} \mathrm{NO}_{14} \mathrm{P}_{2}$ : C, 59.21; H, 4.73; N, 1.68. Found: C, 58.99; H, 4.89; N, 2.08 .

C-(4-O-Acetyl-6-O-tert-butyldiphenylsilyl-2,3-di-O-methyl- $\alpha$-Dgalactopyranosyl) N-methoxyphthalimide (20). Following the general procedure starting from alcohol $51(285.9 \mathrm{mg}, 0.57 \mathrm{mmol})$ and purification by column chromatography (hexanes-EtOAc, 75:25), product $20(318.2 \mathrm{mg}, 0.49 \mathrm{mmol}, 86 \%)$ was obtained as a crystalline solid: $\mathrm{mp} 48.9-50.0{ }^{\circ} \mathrm{C}(n$-hexane-EtOAc $) ;[\alpha]_{\mathrm{D}}=+6.7(c=0.43$, $\left.\mathrm{CHCl}_{3}\right)$. ${ }^{1} \mathrm{H}$ NMR $\left(500 \mathrm{MHz}, \mathrm{CDCl}_{3}\right.$, simulated ring coupling constants using DAISY) $\delta_{\mathrm{H}} 7.74-7.61(\mathrm{~m}, 8 \mathrm{H}, \mathrm{Ar}), 7.43-7.35(\mathrm{~m}$, $6 \mathrm{H}, \mathrm{Ar}), 5.68(\mathrm{dd}, J=3.2,2.1 \mathrm{~Hz}, 1 \mathrm{H}, 4-\mathrm{H}), 4.62-4.58(\mathrm{~m}, 2 \mathrm{H}, 1-\mathrm{H}$, $\left.1^{\prime}-\mathrm{H}_{\mathrm{b}}\right), 4.38\left(\mathrm{~m}, 1 \mathrm{H}, 1^{\prime}-\mathrm{H}_{\mathrm{a}}\right), 4.09$ (ddd, $\left.J=8.1,5.7,2.1 \mathrm{~Hz}, 1 \mathrm{H}, 5-\mathrm{H}\right)$, $3.69(\mathrm{dd}, J=9.1,5.4 \mathrm{~Hz}, 1 \mathrm{H}, 2-\mathrm{H}), 3.64(\mathrm{dd}, J=10.0,5.7 \mathrm{~Hz}, 1 \mathrm{H}, 6-$ $\mathrm{H}_{\mathrm{b}}$ ), $3.54\left(\mathrm{dd}, J=10.0,8.1 \mathrm{~Hz}, 1 \mathrm{H}, 6-\mathrm{H}_{\mathrm{a}}\right), 3.50(\mathrm{~s}, 3 \mathrm{H}, \mathrm{OMe}), 3.46$ (dd, $J=9.1,3.2 \mathrm{~Hz}, 1 \mathrm{H}, 3-\mathrm{H}), 3.44(\mathrm{~s}, 3 \mathrm{H}, \mathrm{OMe}), 2.02$ (s, 3H, OAc), $1.03 \mathrm{ppm}\left(\mathrm{s}, 9 \mathrm{H},{ }^{t} \mathrm{Bu}\right) .{ }^{13} \mathrm{C}\left\{{ }^{1} \mathrm{H}\right\} \mathrm{NMR}\left(125.7 \mathrm{MHz}, \mathrm{CDCl}_{3}\right) \delta_{\mathrm{C}} 169.7$
(C, OAc), $163.2(2 \times \mathrm{C}, \mathrm{CO}), 135.60(2 \times \mathrm{CH}, \mathrm{Ar}), 135.56(2 \times$ $\mathrm{CH}, \mathrm{Ar}), 134.4(2 \times \mathrm{CH}, \mathrm{Ar}), 133.3$ (C, Ar), 133.1 (C, Ar), 129.7 $(\mathrm{CH}, \mathrm{Ar}), 129.6(\mathrm{CH}, \mathrm{Ar}), 128.8(2 \times \mathrm{C}, \mathrm{Ar}), 127.67(2 \times \mathrm{CH}, \mathrm{Ar})$, $127.66(2 \times \mathrm{CH}, \mathrm{Ar}), 123.4(2 \times \mathrm{CH}, \mathrm{Ar}), 78.9(\mathrm{CH}, \mathrm{C}-3), 76.3$ $(\mathrm{CH}, \mathrm{C}-2), 72.8\left(\mathrm{CH}_{2}, \mathrm{C}-1\right.$ '), $72.2(\mathrm{CH}, \mathrm{C}-1), 71.5(\mathrm{CH}, \mathrm{C}-5), 66.1$ $(\mathrm{CH}, \mathrm{C}-4), 61.1\left(\mathrm{CH}_{2}, \mathrm{C}-6\right), 59.3\left(\mathrm{CH}_{3}, \mathrm{OMe}\right), 57.7\left(\mathrm{CH}_{3}, \mathrm{OMe}\right)$, $26.7\left(3 \times \mathrm{CH}_{3}, \mathrm{DPS}\right), 20.8\left(\mathrm{CH}_{3}, \mathrm{OAc}\right), 19.0 \mathrm{ppm}$ (C, DPS). IR $\left(\mathrm{CHCl}_{3}\right): \nu=2933,1792,1734,1226 \mathrm{~cm}^{-1}$. MS (ESI) $\mathrm{m} / z(\%)=$ $670(100)[\mathrm{M}+\mathrm{Na}]^{+}$. HRMS (ESI) $m / z:[\mathrm{M}+\mathrm{Na}]^{+}$calcd for $\mathrm{C}_{35} \mathrm{H}_{41} \mathrm{NNaO}_{9} \mathrm{Si}$ : 670.2448; found 670.2443. Anal. calcd for $\mathrm{C}_{35} \mathrm{H}_{41} \mathrm{NO}_{9} \mathrm{Si}$ : C, 64.89; H, 6.38; N, 2.16. Found: C, 64.67; H, $6.41 ; \mathrm{N}, 2.23$

C-(6-O-tert-Butyldiphenylsilyl-4-O-diphenoxyphosphoryl-2,3-di0 -methyl- $\alpha$-D-galactopyranosyl) $N$-methoxyphthalimide (21). Following the general procedure starting from alcohol $\mathbf{5 2}(510 \mathrm{mg}, 0.74$ $\mathrm{mmol}$ ) (327 $\mathrm{mg}, 0.51 \mathrm{mmol}$ ) and purification by column chromatography (hexanes-EtOAc, 8:2), product 21 (574 mg, 0.68 mmol, $93 \%)$ was obtained as a colorless oil: $[\alpha]_{\mathrm{D}}=-9.1(c=0.67$, $\left.\mathrm{CHCl}_{3}\right) \cdot{ }^{1} \mathrm{H}$ NMR $\left(500 \mathrm{MHz}, \mathrm{CDCl}_{3}\right.$, simulated ring coupling constants using DAISY) $\delta_{\mathrm{H}} 7.74-7.11(\mathrm{~m}, 24 \mathrm{H}, \mathrm{Ar}), 5.24$ (ddd, $J=$ $\left.2.9,2.7 \mathrm{~Hz},{ }^{3} J_{\mathrm{PH}}=8.7 \mathrm{~Hz}, 1 \mathrm{H}, 4-\mathrm{H}\right), 4.47-4.41\left(\mathrm{~m}, 2 \mathrm{H}, 1-\mathrm{H}, 1^{\prime}-\mathrm{H}_{\mathrm{b}}\right)$, $4.33\left(\mathrm{~m}, 1 \mathrm{H}, 1^{\prime}-\mathrm{H}_{\mathrm{a}}\right), 4.03$ (dddd, $J=6.7,5.8,2.7 \mathrm{~Hz},{ }^{4} J_{\mathrm{PH}}=2.3 \mathrm{~Hz}$, $1 \mathrm{H}, 5-\mathrm{H}), 3.77\left(\mathrm{dd}, J=10.6,5.8 \mathrm{~Hz}, 1 \mathrm{H}, 6-\mathrm{H}_{\mathrm{b}}\right), 3.74(\mathrm{dd}, J=10.6,6.7$ $\left.\mathrm{Hz}, 1 \mathrm{H}, 6-\mathrm{H}_{\mathrm{a}}\right), 3.66(\mathrm{dd}, J=8.2,5.6 \mathrm{~Hz}, 1 \mathrm{H}, 2-\mathrm{H}), 3.42(\mathrm{~s}, 3 \mathrm{H}$, $\mathrm{OMe}$ ), 3.39 (dd, $J=8.2,2.9 \mathrm{~Hz}, 1 \mathrm{H}, 3-\mathrm{H}), 3.35(\mathrm{~s}, 3 \mathrm{H}, \mathrm{OMe}), 1.00$ ppm $\left(\mathrm{s}, 9 \mathrm{H},{ }^{t} \mathrm{Bu}\right) .{ }^{13} \mathrm{C}\left\{{ }^{1} \mathrm{H}\right\} \mathrm{NMR}\left(100.6 \mathrm{MHz}, \mathrm{CDCl}_{3}\right) \delta_{\mathrm{C}} 163.2(2 \times$ C, CO), $150.7\left(\mathrm{~d},{ }^{2} J_{\mathrm{PC}}=7.4 \mathrm{~Hz}, \mathrm{C}, \mathrm{Ar}\right), 150.6\left(\mathrm{~d},{ }^{2} J_{\mathrm{PC}}=6.4 \mathrm{~Hz}, \mathrm{C}\right.$, $\mathrm{Ar}), 135.58(2 \times \mathrm{CH}, \mathrm{Ar}), 135.55(2 \times \mathrm{CH}, \mathrm{Ar}), 134.4(2 \times \mathrm{CH}, \mathrm{Ar})$, 133.5 (C, Ar), 133.3 (C, Ar), $129.7(2 \times \mathrm{CH}, \mathrm{Ar}), 129.6(\mathrm{CH}, \mathrm{Ar})$, $129.5(\mathrm{CH}, \mathrm{Ar}), 129.4(2 \times \mathrm{CH}, \mathrm{Ar}), 128.8(2 \times \mathrm{C}, \mathrm{Ar}), 127.7(2 \times$ $\mathrm{CH}, \mathrm{Ar}), 127.6(2 \times \mathrm{CH}, \mathrm{Ar}), 125.1(\mathrm{CH}, \mathrm{Ar}), 125.0(\mathrm{CH}, \mathrm{Ar}), 123.4$ $(2 \times \mathrm{CH}, \mathrm{Ar}), 120.4(\mathrm{CH}, \mathrm{Ar}), 120.3(\mathrm{CH}, \mathrm{Ar}), 120.1(\mathrm{CH}, \mathrm{Ar})$, $120.0(\mathrm{CH}, \mathrm{Ar}), 78.3(\mathrm{CH}, \mathrm{C}-3), 75.7(\mathrm{CH}, \mathrm{C}-2), 73.8\left(\mathrm{~d},{ }^{2} J_{\mathrm{PC}}=6.3\right.$ $\mathrm{Hz}, \mathrm{CH}, \mathrm{C}-4), 73.5\left(\mathrm{CH}_{2}, \mathrm{C}-1\right.$ '), 73.1 (CH, C-1), 70.6 (br s, CH, C5), $60.9\left(\mathrm{CH}_{2}, \mathrm{C}-6\right), 59.5\left(\mathrm{CH}_{3}, \mathrm{OMe}\right), 57.8\left(\mathrm{CH}_{3}, \mathrm{OMe}\right), 26.7(3 \times$ $\mathrm{CH}_{3}$, DPS $), 19.1 \mathrm{ppm}(\mathrm{C}, \mathrm{DPS})$. IR $\left(\mathrm{CHCl}_{3}\right): \nu=3015,2933,1792$, $1730,1490,1190 \mathrm{~cm}^{-1}$. MS (ESI) $\mathrm{m} / z(\%)=860(100)[\mathrm{M}+\mathrm{Na}]^{+}$. HRMS (ESI) $m / z:[\mathrm{M}+\mathrm{Na}]^{+}$calcd for $\mathrm{C}_{45} \mathrm{H}_{48} \mathrm{NNaO}_{11} \mathrm{PSi}$ : 860.2632; found 860.2648. Anal. calcd for $\mathrm{C}_{45} \mathrm{H}_{48} \mathrm{NO}_{11} \mathrm{PSi}$ : C, 64.50; H, 5.77; N, 1.67. Found: C, 64.82; H, 6.01; N, 1.92 .

C-(4-O-Diphenoxyphosphoryl-2,3-di-O-methyl- $\alpha$-L-rhamnopyranosyl) N-methoxyphthalimide (22). Alcohol 105 (231.7 mg, 0.66 $\mathrm{mmol})$ in dry $\mathrm{CH}_{2} \mathrm{Cl}_{2}(38 \mathrm{~mL})$ was treated with DMAP $(371 \mathrm{mg}$, $3.04 \mathrm{mmol})$ and $\mathrm{ClPO}(\mathrm{OPh})_{2}(0.63 \mathrm{~mL}, 3.04 \mathrm{mmol})$ for $2 \mathrm{~h}$. The reaction was quenched with a saturated aqueous solution of $\mathrm{NH}_{4} \mathrm{Cl}$ and extracted with $\mathrm{CH}_{2} \mathrm{Cl}_{2}$. The organic phase was dried over $\mathrm{Na}_{2} \mathrm{SO}_{4}$, filtered, and evaporated. Column chromatography of the residue (hexanes-EtOAc, 6:4) gave the compound 22 (333 mg, 0.55 mmol, $84 \%)$ as an amorphous solid: $[\alpha]_{\mathrm{D}}=-0.1\left(c=0.41, \mathrm{CHCl}_{3}\right)$. ${ }^{1} \mathrm{H}$ NMR $\left(500 \mathrm{MHz}, \mathrm{CDCl}_{3}\right.$, simulated ring coupling constants using DAISY) $\delta_{\mathrm{H}} 7.84-7.82(\mathrm{~m}, 2 \mathrm{H}, \mathrm{Ar}), 7.75-7.73(\mathrm{~m}, 2 \mathrm{H}, \mathrm{Ar}), 7.37-$ $7.17(\mathrm{~m}, 10 \mathrm{H}, \mathrm{Ar}), 4.63$ (ddd, $J=5.2,3.2 \mathrm{~Hz},{ }^{3} J_{\mathrm{PH}}=9.2 \mathrm{~Hz}, 1 \mathrm{H}, 4-$ $\mathrm{H}), 4.46\left(\mathrm{dd}, J=10.9,3.2 \mathrm{~Hz}, 1 \mathrm{H}, 1^{\prime}-\mathrm{H}_{\mathrm{b}}\right), 4.38(\mathrm{dd}, J=10.9,6.2 \mathrm{~Hz}$, $1 \mathrm{H}, 1$ '- $\mathrm{H}_{\mathrm{a}}$ ), 4.23 (ddd, $\left.J=7.6,6.2,3.2 \mathrm{~Hz}, 1 \mathrm{H}, 1-\mathrm{H}\right), 4.01$ (dddd, $J=$ 6.9, 6.9, 6.9, $3.7 \mathrm{~Hz}, 1 \mathrm{H}, 5-\mathrm{H}), 3.71(\mathrm{dd}, J=5.2,3.2 \mathrm{~Hz}, 1 \mathrm{H}, 3-\mathrm{H})$, $3.61(\mathrm{dd}, J=7.6,3.2 \mathrm{~Hz}, 1 \mathrm{H}, 2-\mathrm{H}), 3.42(\mathrm{~s}, 3 \mathrm{H}, \mathrm{OMe}), 3.35(\mathrm{~s}, 3 \mathrm{H}$, $\mathrm{OMe}), 1.34 \mathrm{ppm}\left(\mathrm{d}, J=6.9 \mathrm{~Hz}, 3 \mathrm{H}, 6-\mathrm{H}_{3}\right) .{ }^{13} \mathrm{C}\left\{{ }^{1} \mathrm{H}\right\}$ NMR $(125.7$ $\left.\mathrm{MHz}, \mathrm{CDCl}_{3}\right) \delta_{\mathrm{C}} 163.2(2 \times \mathrm{C}, \mathrm{CO}), 150.5\left(\mathrm{~d},{ }^{2} J_{\mathrm{PC}}=7.4 \mathrm{~Hz}, \mathrm{C}, \mathrm{Ar}\right)$, $150.3\left(\mathrm{~d},{ }^{2} J_{\mathrm{PC}}=7.4 \mathrm{~Hz}, \mathrm{C}, \mathrm{Ar}\right), 134.4(2 \times \mathrm{CH}, \mathrm{Ar}), 129.8(2 \times \mathrm{CH}$, $\mathrm{Ar}), 129.7(2 \times \mathrm{CH}, \mathrm{Ar}), 128.9(2 \times \mathrm{C}, \mathrm{Ar}), 125.42(\mathrm{CH}, \mathrm{Ar}), 125.37$ $(\mathrm{CH}, \mathrm{Ar}), 123.4(2 \times \mathrm{CH}, \mathrm{Ar}), 120.2(\mathrm{CH}, \mathrm{Ar}), 120.12(\mathrm{CH}, \mathrm{Ar})$, $120.11(\mathrm{CH}, \mathrm{Ar}), 120.07(\mathrm{CH}, \mathrm{Ar}), 77.4\left(\mathrm{~d},{ }^{2} \mathrm{~J}_{\mathrm{PC}}=6.4 \mathrm{~Hz}, \mathrm{CH}, \mathrm{C}-4\right)$, $77.2\left(\mathrm{CH}_{2}, \mathrm{C}-1^{\prime}\right), 76.2\left(\mathrm{~d},{ }^{3} J_{\mathrm{PC}}=3.2 \mathrm{~Hz}, \mathrm{CH}, \mathrm{C}-3\right), 73.9(\mathrm{CH}, \mathrm{C}-2)$, $71.6\left(\mathrm{~d},{ }^{3} \mathrm{~J}_{\mathrm{PC}}=5.3 \mathrm{~Hz}, \mathrm{CH}, \mathrm{C}-5\right), 68.2(\mathrm{CH}, \mathrm{C}-1), 58.3\left(\mathrm{CH}_{3}, \mathrm{OMe}\right)$, $57.2\left(\mathrm{CH}_{3}, \mathrm{OMe}\right), 16.2 \mathrm{ppm}\left(\mathrm{CH}_{3}, \mathrm{C}-6\right)$. IR $\left(\mathrm{CHCl}_{3}\right): \nu=3015$, $2939,1792,1736,1490,1209 \mathrm{~cm}^{-1}$. MS (ESI) $\mathrm{m} / z(\%)=606(100)$ $[\mathrm{M}+\mathrm{Na}]^{+}$. HRMS (ESI) $m / z:[\mathrm{M}+\mathrm{Na}]^{+}$calcd for $\mathrm{C}_{29} \mathrm{H}_{30} \mathrm{NNaO}_{10} \mathrm{P}$ : 606.1505; found 606.1516. Anal. calcd for $\mathrm{C}_{29} \mathrm{H}_{30} \mathrm{NO}_{10} \mathrm{P}: \mathrm{C}, 59.69$; H, 5.18; N, 2.40. Found: C, 59.37; H, 5.40; N, 2.68 . 
C-(4-O-Acetyl-2,3-di-O-methyl- $\alpha$-L-fucopyranosyl)N-methoxyphthalimide (23). Following the general procedure starting from 58 (65.6 $\mathrm{mg}, 0.26 \mathrm{mmol}$ ) and purification by column chromatography (hexanes- $\left.\mathrm{Et}_{2} \mathrm{O}, 4: 6\right)$, product $23(52.4 \mathrm{mg}, 0.13 \mathrm{mmol}, 51 \%)$ was obtained as a white crystalline solid: $\mathrm{mp} 37.8-38.5^{\circ} \mathrm{C}$ ( $n$-hexaneEtOAc). $[\alpha]_{\mathrm{D}}=-42.5\left(c=0.72, \mathrm{CHCl}_{3}\right) .{ }^{1} \mathrm{H}$ NMR $(500 \mathrm{MHz}$, $\mathrm{CDCl}_{3}$, simulated ring coupling constants using DAISY) $\delta_{\mathrm{H}} 7.86-$ $7.84(\mathrm{~m}, 2 \mathrm{H}, \mathrm{Ar}), 7.78-7.76(\mathrm{~m}, 2 \mathrm{H}, \mathrm{Ar}), 5.31(\mathrm{dd}, J=3.4,2.0 \mathrm{~Hz}$, $1 \mathrm{H}, 4-\mathrm{H}), 4.67$ (ddd, $J=9.0,5.9,1.9 \mathrm{~Hz}, 1 \mathrm{H}, 1-\mathrm{H}), 4.63$ (dd, $J=10.7$, $\left.9.0 \mathrm{~Hz}, 1 \mathrm{H}, \mathrm{l}^{\prime}-\mathrm{H}_{\mathrm{b}}\right), 4.39$ (dd, $J=10.7,1.9 \mathrm{~Hz}, 1 \mathrm{H}, 1^{\prime}-\mathrm{H}_{\mathrm{a}}$ ), 4.09 (dddd, $J=6.4,6.4,6.4,2.0 \mathrm{~Hz}, 1 \mathrm{H}, 5-\mathrm{H}), 3.71(\mathrm{dd}, J=9.3,5.9 \mathrm{~Hz}, 1 \mathrm{H}, 2-\mathrm{H})$, 3.51 (s, 3H, OMe), 3.40 (s, 3H, OMe), $3.36(\mathrm{dd}, J=9.3,3.4 \mathrm{~Hz}, 1 \mathrm{H}$, 3-H), $2.16(\mathrm{~s}, 3 \mathrm{H}, \mathrm{OAc}), 1.11 \mathrm{ppm}\left(\mathrm{d}, J=6.4 \mathrm{~Hz}, 3 \mathrm{H}, 6-\mathrm{H}_{3}\right)$. ${ }^{13} \mathrm{C}\left\{{ }^{1} \mathrm{H}\right\}$ NMR $\left(125.7 \mathrm{MHz}, \mathrm{CDCl}_{3}\right) \delta_{\mathrm{C}} 170.6$ (C, OAc), $163.3(2 \times$ $\mathrm{C}, \mathrm{CO}), 134.5(2 \times \mathrm{CH}, \mathrm{Ar}), 128.8(2 \times \mathrm{C}, \mathrm{Ar}), 123.5(2 \times \mathrm{CH}, \mathrm{Ar})$, $78.6(\mathrm{CH}, \mathrm{C}-3), 76.1(\mathrm{CH}, \mathrm{C}-2), 72.8\left(\mathrm{CH}_{2}, \mathrm{C}-1^{\prime}\right), 71.8(\mathrm{CH}, \mathrm{C}-1)$, $69.4(\mathrm{CH}, \mathrm{C}-4), 67.0(\mathrm{CH}, \mathrm{C}-5), 59.2\left(\mathrm{CH}_{3}, \mathrm{OMe}\right), 57.6\left(\mathrm{CH}_{3}\right.$, $\mathrm{OMe}), 20.8\left(\mathrm{CH}_{3}, \mathrm{OAc}\right), 16.1 \mathrm{ppm}\left(\mathrm{CH}_{3}, \mathrm{C}-6\right)$. IR $\left(\mathrm{CHCl}_{3}\right): \nu=$ $3018,2936,1791,1744,1239 \mathrm{~cm}^{-1}$. MS (ESI) $\mathrm{m} / z(\%)=416(100)$ $[\mathrm{M}+\mathrm{Na}]^{+}$. HRMS (ESI) $m / z:[\mathrm{M}+\mathrm{Na}]^{+}$calcd for $\mathrm{C}_{19} \mathrm{H}_{23} \mathrm{NNaO}_{8}$ : 416.1321; found 416.1328. Anal. calcd for $\mathrm{C}_{19} \mathrm{H}_{23} \mathrm{NO}_{8}$ : C, 58.01; $\mathrm{H}$, 5.89; N, 3.56. Found: C, 58.33; H, 5.95; N, 3.83.

C-(4-O-Diphenoxyphosphoryl-2,3-di-O-methyl- $\alpha$-L-fucopyranosyl) N-methoxyphthalimide (24). Following the general procedure starting from $59(232 \mathrm{mg}, 0.53 \mathrm{mmol})$ and purification by column chromatography (hexanes-EtOAc, 6:4), product $24(52.4 \mathrm{mg}, 0.13$ $\mathrm{mmol}, 51 \%)$ was obtained as a colorless oil: $[\alpha]_{\mathrm{D}}=-19.0(c=0.57$, $\left.\mathrm{CHCl}_{3}\right)$. ${ }^{1} \mathrm{H}$ NMR $\left(500 \mathrm{MHz}, \mathrm{CDCl}_{3}\right.$, simulated ring coupling constants using DAISY) $\delta_{\mathrm{H}} 7.84-7.81(\mathrm{~m}, 2 \mathrm{H}, \mathrm{Ar}), 7.76-7.74(\mathrm{~m}$, $2 \mathrm{H}, \mathrm{Ar}), 7.34-7.16(\mathrm{~m}, 10 \mathrm{H}, \mathrm{Ar}), 4.91$ (ddd, $J=2.5,3.3 \mathrm{~Hz},{ }^{3} \mathrm{JH}_{\mathrm{PH}}=$ $9.0 \mathrm{~Hz}, 1 \mathrm{H}, 4-\mathrm{H}), 4.55-4.50\left(\mathrm{~m}, 2 \mathrm{H}, 1-\mathrm{H}, 1^{\prime}-\mathrm{H}_{\mathrm{b}}\right), 4.35\left(\mathrm{~m}, 1 \mathrm{H}, \mathrm{l}^{\prime}-\right.$ $\mathrm{H}_{\mathrm{a}}$ ), 4.08 (ddddd, $J=6.5,6.5,6.5,2.5 \mathrm{~Hz},{ }^{4} J_{\mathrm{PH}}=2.0 \mathrm{~Hz}, 1 \mathrm{H}, 5-\mathrm{H}$ ), $3.61(\mathrm{dd}, J=7.9,5.0 \mathrm{~Hz}, 1 \mathrm{H}, 2-\mathrm{H}), 3.43(\mathrm{dd}, J=7.9,3.3 \mathrm{~Hz}, 1 \mathrm{H}, 3-$ $\mathrm{H}), 3.42$ (s, $3 \mathrm{H}, \mathrm{OMe}), 3.39$ (s, $3 \mathrm{H}, \mathrm{OMe}), 1.19 \mathrm{ppm}(\mathrm{d}, J=6.5 \mathrm{~Hz}$, $\left.3 \mathrm{H}, 6-\mathrm{H}_{3}\right) \cdot{ }^{13} \mathrm{C}\left\{{ }^{1} \mathrm{H}\right\}$ NMR $\left(125.7 \mathrm{MHz}, \mathrm{CDCl}_{3}\right) \delta_{\mathrm{C}} 163.3(2 \times \mathrm{C}$, $\mathrm{CO}), 150.7\left(\mathrm{~d},{ }^{2} J_{\mathrm{PC}}=7.4 \mathrm{~Hz}, \mathrm{C}, \mathrm{Ar}\right), 150.5\left(\mathrm{~d},{ }^{2} \mathrm{~J}_{\mathrm{PC}}=6.4 \mathrm{~Hz}, \mathrm{C}, \mathrm{Ar}\right)$, 134.5 ( $2 \times \mathrm{CH}, \mathrm{Ar}), 129.7(2 \times \mathrm{CH}, \mathrm{Ar}), 129.5(2 \times \mathrm{CH}, \mathrm{Ar}), 128.8$ $(2 \times \mathrm{C}, \mathrm{Ar}), 125.2(\mathrm{CH}, \mathrm{Ar}), 125.1(\mathrm{CH}, \mathrm{Ar}), 123.5(2 \times \mathrm{CH}, \mathrm{Ar})$, $120.3(\mathrm{CH}, \mathrm{Ar}), 120.2(\mathrm{CH}, \mathrm{Ar}), 120.1(\mathrm{CH}, \mathrm{Ar}), 120.0(\mathrm{CH}, \mathrm{Ar})$, $78.4(\mathrm{CH}, \mathrm{C}-3), 76.5\left(\mathrm{~d},{ }^{2} J_{\mathrm{PC}}=6.4 \mathrm{~Hz}, \mathrm{CH}, \mathrm{C}-4\right), 75.9(\mathrm{CH}, \mathrm{C}-2)$, $73.8\left(\mathrm{CH}_{2}, \mathrm{C}-1^{\prime}\right), 70.3(\mathrm{CH}, \mathrm{C}-1), 68.1\left(\mathrm{~d},{ }^{3} \mathrm{~J}_{\mathrm{PC}}=6.3 \mathrm{~Hz}, \mathrm{CH}, \mathrm{C}-5\right)$, $59.4\left(\mathrm{CH}_{3}, \mathrm{OMe}\right), 57.9\left(\mathrm{CH}_{3}, \mathrm{OMe}\right), 15.4 \mathrm{ppm}\left(\mathrm{CH}_{3}, \mathrm{C}-6\right)$. IR $\left(\mathrm{CHCl}_{3}\right): \nu=3026,2938,1792,1734,1210 \mathrm{~cm}^{-1}$. MS (ESI) $\mathrm{m} / z(\%)$ $=606(100)[\mathrm{M}+\mathrm{Na}]^{+}$. HRMS $(\mathrm{ESI}) \mathrm{m} / z:[\mathrm{M}+\mathrm{Na}]^{+}$calcd for $\mathrm{C}_{29} \mathrm{H}_{30} \mathrm{NNaO}_{10} \mathrm{P}: 606.1505$; found 606.1500. Anal. calcd for $\mathrm{C}_{29} \mathrm{H}_{30} \mathrm{NO}_{10} \mathrm{P}: \mathrm{C}, 59.69 ; \mathrm{H}, 5.18 ; \mathrm{N}, 2.40$. Found: C, 59.61; H, $5.23 ; \mathrm{N}, 2.70$.

C-(2,3-Di-O-methyl- $\alpha$-L-rhamnopyranosyl) N-methoxyphthalimide (105). To a solution of $104(570 \mathrm{mg}, 1.92 \mathrm{mmol})$ in dry EtOAc (45 mL) was added $\mathrm{Pd} / \mathrm{C} 10 \%(430 \mathrm{mg}$ ), and the mixture was submitted to a $\mathrm{H}_{2}$ atmosphere overnight. The reaction was filtered over a pad of Celite and evaporated. The residue was then submitted to the general procedure to give phthalimide $105(383 \mathrm{mg}, 1.09$ mmol, 57\%) as a colorless oil: $[\alpha]_{\mathrm{D}}=-22.6\left(c=1.34, \mathrm{CHCl}_{3}\right) .{ }^{1} \mathrm{H}$ NMR $\left(500 \mathrm{MHz}, \mathrm{CDCl}_{3}\right) \delta_{\mathrm{H}} 7.84-7.82(\mathrm{~m}, 2 \mathrm{H}, \mathrm{Ar}), 7.76-7.74(\mathrm{~m}$, $2 \mathrm{H}, \mathrm{Ar}), 4.53$ (dd, $\left.J=11.0,4.9 \mathrm{~Hz}, 1 \mathrm{H}, 1^{\prime}-\mathrm{H}_{\mathrm{b}}\right), 4.32(\mathrm{dd}, J=11.0,4.7$ $\left.\mathrm{Hz}, 1 \mathrm{H}, 1^{\prime}-\mathrm{H}_{\mathrm{a}}\right), 4.24$ (ddd, $\left.J=4.7,4.7,4.7 \mathrm{~Hz}, 1 \mathrm{H}, 1-\mathrm{H}\right), 4.07$ (dd, $J=$ 5.1, $3.3 \mathrm{~Hz}, 1 \mathrm{H}, 2-\mathrm{H}), 3.70-3.67(\mathrm{~m}, 2 \mathrm{H}, 4-\mathrm{H}, 5-\mathrm{H}), 3.60(\mathrm{dd}, J=$ 7.2, $3.3 \mathrm{~Hz}, 1 \mathrm{H}, 3-\mathrm{H}), 3.52$ (s, $3 \mathrm{H}, \mathrm{OMe}$ ), 3.50 (s, $3 \mathrm{H}, \mathrm{OMe}), 1.28$ ppm $\left(\mathrm{d}, J=6.3 \mathrm{~Hz}, 3 \mathrm{H}, 6-\mathrm{H}_{3}\right), 1 \mathrm{H}$ from $\mathrm{OH}$ is missing. ${ }^{13} \mathrm{C}\left\{{ }^{1} \mathrm{H}\right\}$ NMR $\left(125.7 \mathrm{MHz}, \mathrm{CDCl}_{3}\right) \delta_{\mathrm{C}} 163.4(2 \times \mathrm{C}, \mathrm{CO}), 134.6(2 \times \mathrm{CH}$, $\mathrm{Ar}), 128.7(2 \times \mathrm{C}, \mathrm{Ar}), 123.6(2 \times \mathrm{CH}, \mathrm{Ar}), 79.5(\mathrm{CH}, \mathrm{C}-3), 76.4$ $\left(\mathrm{CH}_{2}, \mathrm{C}-1^{\prime}\right), 73.6(\mathrm{CH}, \mathrm{C}-2), 72.8(\mathrm{CH}, \mathrm{C}-4$ or C-5), $70.5(\mathrm{CH}, \mathrm{C}-4$ or C-5), $70.3(\mathrm{CH}, \mathrm{C}-1), 57.7\left(\mathrm{CH}_{3}, \mathrm{OMe}\right), 57.5\left(\mathrm{CH}_{3}, \mathrm{OMe}\right), 16.9$ ppm $\left(\mathrm{CH}_{3}, \mathrm{C}-6\right)$. IR $\left(\mathrm{CHCl}_{3}\right): \nu=3674,3501,3022,2937,1792$, $1735,1212 \mathrm{~cm}^{-1}$. MS (ESI) $m / z(\%)=374(100)[\mathrm{M}+\mathrm{Na}]^{+}$. HRMS (ESI) $m / z:[\mathrm{M}+\mathrm{Na}]^{+}$calcd for $\mathrm{C}_{17} \mathrm{H}_{21} \mathrm{NNaO}_{7}$ : 374.1216; found 374.1213. Anal. calcd for $\mathrm{C}_{17} \mathrm{H}_{21} \mathrm{NO}_{7}$ : C, 58.11; H, 6.02; N, 3.99. Found: C, 58.22; H, 6.18; N, 4.11.
Methyl 4-O-Benzyl-6-O-tert-butyldiphenylsilyl-2,3-di-O-methyl$\alpha-D-\left[4-\mathrm{O}-\mathrm{PhCH}-{ }^{2} \mathrm{H}\right]$ galactopyranoside $\left(\left[\mathrm{PhCH}-{ }^{2} \mathrm{H}\right] 97\right)$. A mixture of $96(47.4 \mathrm{mg}, 0.10 \mathrm{mmol})$ and benzyl $\alpha$ - $\left[{ }^{2} \mathrm{H}\right]$-4-methylbenzenesulfonate $^{52}\left(31.6 \mathrm{mg}, 0.12 \mathrm{mmol},{ }^{2} \mathrm{H} /{ }^{1} \mathrm{H}\right.$ 6.9:1) in DMF $(0.2 \mathrm{~mL})$ and $\mathrm{CH}_{2} \mathrm{Cl}_{2}(0.2 \mathrm{~mL})$ was cooled to $0{ }^{\circ} \mathrm{C}$. Then, sodium hydride $(60 \%$ dispersion in mineral oil, $8 \mathrm{mg}, 0.20 \mathrm{mmol}$ ) was added to the mixture, and the reaction was gradually warmed up to room temperature. After stirring for $1 \mathrm{~h}$, the Dowex 50WX4-200 was added to quench the reaction. The mixture was filtered through a pad of Celite, and the filtrate was evaporated under reduced pressure. The crude residue was purified by column chromatography (hexanes-EtOAc, 8:2 to 7:3) to obtain the product $\left[\mathrm{PhCH}^{2} \mathrm{H}\right] 97\left(28.6 \mathrm{mg}, 0.05 \mathrm{mmol}, 50 \%,{ }^{2} \mathrm{H} /{ }^{1} \mathrm{H}\right.$ 7:1) as a colorless oil: ${ }^{1} \mathrm{H}$ NMR $\left(500 \mathrm{MHz}, \mathrm{CDCl}_{3}\right) \delta_{\mathrm{H}} 7.67-7.60$ (m, 4H, Ar), 7.46-7.32 (m, 6H, Ar), 7.31-7.21 (m, 5H, Ar), 4.91 (d, $\left.J=11.4 \mathrm{~Hz}, 0.1 \mathrm{H}, \mathrm{O}-\mathrm{CH}_{2}-\mathrm{Ph}\right), 4.900($ br s, $0.4 \mathrm{H}, \mathrm{O}-\mathrm{CHD}-\mathrm{Ph}), 4.84$ $(\mathrm{d}, J=3.6 \mathrm{~Hz}, 1 \mathrm{H}, 1-\mathrm{H}), 4.60\left(\mathrm{~d}, J=11.5 \mathrm{~Hz}, 0.1 \mathrm{H}, \mathrm{O}-\mathrm{CH}_{2}-\mathrm{Ph}\right)$, 4.589 (br s, 0.4H, O-CHD-Ph), 4.01 (br s, $1 \mathrm{H}, 4-\mathrm{H}), 3.79-3.69(\mathrm{~m}$, $\left.4 \mathrm{H}, 6-\mathrm{H}_{2}, 2-\mathrm{H}, 5-\mathrm{H}\right), 3.56(\mathrm{dd}, J=10.1,2.8 \mathrm{~Hz}, 1 \mathrm{H}, 3-\mathrm{H}), 3.51(\mathrm{~s}$, $3 \mathrm{H}, 3-\mathrm{OMe}$ ), 3.51 (s, 3H, 2-OMe), 3.32 (s, 3H, 1-OMe), $1.06 \mathrm{ppm}$ (s, 9H). ${ }^{13} \mathrm{C}\left\{{ }^{1} \mathrm{H}\right\}$ NMR (125.7 MHz, $\left.\mathrm{CDCl}_{3}\right) \delta_{\mathrm{C}} 138.6$ (C, Ar), $135.56(2 \times \mathrm{CH}, \mathrm{Ar}), 135.53(2 \times \mathrm{CH}, \mathrm{Ar}), 133.4(2 \times \mathrm{C}, \mathrm{Ar})$, 129.74 (CH, Ar), 129.71 (CH, Ar), $128.2(2 \times \mathrm{CH}, \mathrm{Ar}), 128.1(2 \times$ $\mathrm{CH}, \mathrm{Ar}), 127.71(2 \times \mathrm{CH}, \mathrm{Ar}), 127.70(2 \times \mathrm{CH}, \mathrm{Ar}), 127.5(\mathrm{CH}$, $\mathrm{Ar}), 97.7(\mathrm{CH}, \mathrm{C}-1), 80.8(\mathrm{CH}, \mathrm{C}-3), 78.1(\mathrm{CH}, \mathrm{C}-2), 74.73$ $\left(0.12 \mathrm{CH}_{2}, \mathrm{O}-\mathrm{CH}_{2}-\mathrm{Ph}\right), 74.35\left(0.88 \mathrm{CH}, \mathrm{t}, J_{\mathrm{CD}}=22.1 \mathrm{~Hz}, \mathrm{O}-\mathrm{CHD}-\right.$ $\mathrm{Ph}), 73.64(0.12 \mathrm{CH}, \mathrm{C}-4), 73.59(0.44 \mathrm{CH}, \mathrm{C}-4), 73.56(0.44 \mathrm{CH}, \mathrm{C}-$ 4), $70.7(\mathrm{CH}, \mathrm{C}-5), 62.8\left(\mathrm{CH}_{2}, \mathrm{C}-6\right), 58.8\left(\mathrm{CH}_{3}, \mathrm{OMe}\right), 58.3\left(\mathrm{CH}_{3}\right.$, $\mathrm{OMe}), 55.0\left(\mathrm{CH}_{3}, \mathrm{OMe}\right), 26.9\left(3 \times \mathrm{CH}_{3}, \mathrm{DPS}\right), 19.2 \mathrm{ppm}(\mathrm{C}, \mathrm{DPS})$. ${ }^{1} \mathrm{H}$ NMR $\left(500 \mathrm{MHz}, \mathrm{C}_{6} \mathrm{D}_{6}\right) \delta_{\mathrm{H}} 7.84-7.79(\mathrm{~m}, 2 \mathrm{H}, \mathrm{Ar}), 7.79-7.74$ (m, 2H, Ar), 7.35 (m, 2H, Ar), 7.23-7.07 (m, 9H, Ar), 5.07 (d, J = $11.4 \mathrm{~Hz}, 0.1 \mathrm{H}, \mathrm{O}-\mathrm{CH}_{2}-\mathrm{Ph}$ ), 5.05 (br s, $\left.0.4 \mathrm{H}, \mathrm{O}-\mathrm{CHD}-\mathrm{Ph}\right), 4.82$ (d, $J$ $=3.6 \mathrm{~Hz}, 1 \mathrm{H}, 1-\mathrm{H}), 4.61\left(\mathrm{~d}, J=11.4 \mathrm{~Hz}, 0.1 \mathrm{H}, \mathrm{O}-\mathrm{CH}_{2}-\mathrm{Ph}\right), 4.58(\mathrm{br}$ s, $0.4 \mathrm{H}, \mathrm{O}-\mathrm{CHD}-\mathrm{Ph}), 4.105(\mathrm{dd}, J=10.0,6.6 \mathrm{~Hz}, 0.5 \mathrm{H}, 6-\mathrm{H}), 4.107$ (dd, $J=10.1,6.5 \mathrm{~Hz}, 0.5 \mathrm{H}, 6-\mathrm{H}), 4.06$ (dd, $J=10.1,3.7 \mathrm{~Hz}, 0.5 \mathrm{H}, 6-$ $\mathrm{H}), 4.05$ (dd, $J=10.2,3.8 \mathrm{~Hz}, 0.5 \mathrm{H}, 6-\mathrm{H}), 3.97-3.90(\mathrm{~m}, 3 \mathrm{H}, 5-\mathrm{H}, 4-$ $\mathrm{H}, 2-\mathrm{H}), 3.73(\mathrm{dd}, J=10.0,2.9 \mathrm{~Hz}, 1 \mathrm{H}, 3-\mathrm{H}), 3.35(\mathrm{~s}, 3 \mathrm{H}, 3-\mathrm{OMe})$, 3.232 (s, 1.5H, 2-OMe), 3.230 (s, 1.5H, 2-OMe), 3.21 (s, $3 \mathrm{H}, 1-$ OMe), $1.19 \mathrm{ppm}(\mathrm{s}, 9 \mathrm{H}) .{ }^{13} \mathrm{C}\left\{{ }^{1} \mathrm{H}\right\}$ NMR $\left(125.7 \mathrm{MHz}, \mathrm{C}_{6} \mathrm{D}_{6}\right) \delta_{\mathrm{C}}$ 139.98 (C, Ar), 136.41 ( $2 \times \mathrm{CH}, \mathrm{Ar}), 136.31(2 \times \mathrm{CH}, \mathrm{Ar}), 134.33$ (C, Ar), 134.16 (C, Ar), $130.42(\mathrm{CH}, \mathrm{Ar}), 130.40(\mathrm{CH}, \mathrm{Ar}), 98.93$ $(\mathrm{CH}, \mathrm{C}-1), 81.71(\mathrm{CH}, \mathrm{C}-3), 79.40(\mathrm{CH}, \mathrm{C}-2), 75.55(0.12 \mathrm{CH}, \mathrm{C}-4)$, $75.51(0.44 \mathrm{CH}, \mathrm{C}-4), 75.48(0.44 \mathrm{CH}, \mathrm{C}-4), 75.45\left(0.12 \mathrm{CH}_{2}, \mathrm{O}_{-} \mathrm{CH}_{2-}\right.$ $\mathrm{Ph}), 75.08\left(0.44 \mathrm{CH}, \mathrm{t}, J_{\mathrm{CD}}=22.1 \mathrm{~Hz}, \mathrm{O}-\mathrm{CHD}-\mathrm{Ph}\right), 75.05(0.44 \mathrm{CH}, \mathrm{t}$, $\left.J_{\mathrm{CD}}=21.1 \mathrm{~Hz}, \mathrm{O}-\mathrm{CHD}-\mathrm{Ph}\right), 71.89(\mathrm{CH}, \mathrm{C}-5), 64.14\left(\mathrm{CH}_{2}, \mathrm{C}-6\right)$, $59.00\left(\mathrm{CH}_{3}, \mathrm{OMe}\right), 58.59\left(\mathrm{CH}_{3}, \mathrm{OMe}\right), 55.26\left(\mathrm{CH}_{3}, \mathrm{OMe}\right), 27.46(3$ $\left.\times \mathrm{CH}_{3}, \mathrm{DPS}\right), 19.81 \mathrm{ppm}$ (C, DPS), some aromatic carbons were not observed. IR $\left(\mathrm{CHCl}_{3}\right): \nu=3020,2932,1471,1428,1220,1103 \mathrm{~cm}^{-1}$. MS (ESI) $m / z(\%)=574(100)[\mathrm{M}+\mathrm{Na}]^{+}, 573(13)[\mathrm{M}+\mathrm{Na}]^{+}$. HRMS (ESI) $m / z$ : $[\mathrm{M}+\mathrm{Na}]^{+}$calcd for $\mathrm{C}_{32} \mathrm{H}_{41}{ }^{2} \mathrm{HNaO}_{6} \mathrm{Si}$ 574.2711; found 574.2716; $[\mathrm{M}+\mathrm{Na}]^{+}$calcd for $\mathrm{C}_{32} \mathrm{H}_{42} \mathrm{NaO}_{6} \mathrm{Si}$ 573.2648; found 573.2653 .

\section{ASSOCIATED CONTENT}

\section{Supporting Information}

The Supporting Information is available free of charge at https://pubs.acs.org/doi/10.1021/acs.joc.1c01376.

Tables of calculated ring coupling constants $\left({ }^{3} \mathrm{~J}_{\mathrm{H}, \mathrm{H}}\right)$ of starting phthalimides and final bicyclic ketals; calculated long-range ${ }^{4} J_{\mathrm{w}}$ coupling constants; selected signals of ${ }^{1} \mathrm{H}$ and ${ }^{13} \mathrm{C}\{1 \mathrm{H}\}$ NMR spectra of labeled $\left[\mathrm{PhCH}^{2} \mathrm{H}\right] 31$ and $\left[\mathrm{PhCH}^{-2} \mathrm{H}\right] 97$; reactivity differences between LGs in the 1,5-hydrogen atom transfer/Surzur-Tanner rearrangement sequence; and copies of the ${ }^{1} \mathrm{H}$ and ${ }^{13} \mathrm{C}\{1 \mathrm{H}\}$ NMR spectra of all new compounds (PDF) 


\section{AUTHOR INFORMATION}

\section{Corresponding Authors}

Inés Pérez-Martín - Síntesis de Productos Naturales, Instituto de Productos Naturales y Agrobiología del CSIC, 38206 La Laguna, Tenerife, Spain; (1) orcid.org/0000-0001-77791830; Email: ines@ipna.csic.es

Ernesto Suárez - Síntesis de Productos Naturales, Instituto de Productos Naturales y Agrobiología del CSIC, 38206 La Laguna, Tenerife, Spain; Email: ersualo@gmail.com

\section{Authors}

Elisa I. León - Sintesis de Productos Naturales, Instituto de Productos Naturales y Agrobiología del CSIC, $38206 \mathrm{La}$ Laguna, Tenerife, Spain

Ángeles Martín - Síntesis de Productos Naturales, Instituto de Productos Naturales y Agrobiología del CSIC, $38206 \mathrm{La}$ Laguna, Tenerife, Spain; • orcid.org/0000-0001-55244828

Adrián S. Montes - Sintesis de Productos Naturales, Instituto de Productos Naturales y Agrobiología del CSIC, 38206 La Laguna, Tenerife, Spain; Doctoral and Postgraduate School, Universidad de La Laguna, 38200 La Laguna, Tenerife, Spain

María del Sol Rodríguez - Síntesis de Productos Naturales, Instituto de Productos Naturales y Agrobiología del CSIC, 38206 La Laguna, Tenerife, Spain; 이이.org/0000-00026491-2784

Complete contact information is available at:

https://pubs.acs.org/10.1021/acs.joc.1c01376

\section{Notes}

The authors declare no competing financial interest.

\section{ACKNOWLEDGMENTS}

The authors gratefully acknowledge the financial support from the Investigation Programs of the Ministerio de Economia y Competitividad (CTQ2010-18244), Fundación CajaCanarias (2015-BIO08), and the Gobierno de Canarias (ProID2017010017). A.S.M. is grateful to the CSIC JAE Predoc Program.

\section{REFERENCES}

(1) (a) Gillard, R. M.; Brimble, M. A. Benzannulated Spiroketal Natural Products: Isolation, Biological Activity, Biosynthesis, and Total Synthesis. Org. Biomol. Chem. 2019, 17, 8272-8307. (b) Zhang, F.-M.; Zhang, S.-Y.; Tu, Y.-Q. Recent Progress in the Isolation, Bioactivity, Biosynthesis, and Total Synthesis of Natural Spiroketals. Nat. Prod. Rep. 2018, 35, 75-104. (c) Quach, R.; Furkert, D. P.; Brimble, M. A. Gold Catalysis: Synthesis of Spiro, Bridged, and Fused Ketal Natural Products. Org. Biomol. Chem. 2017, 15, 3098-3104. (d) Brimble, M. A.; Stubbing, L. A. Synthesis of 5,6- and 6,6Spirocyclic Compounds. In Synthesis of saturated oxygenated heterocycles I; Springer: Berlin, Heidelberg, 2014, 35, 189-267, DOI: 10.1007/978-3-642-41473-2_5. (e) Palmes, J.; Aponick, A. Strategies for Spiroketal Synthesis Based on Transition-Metal Catalysis. Synthesis 2012, 44, 3699-3721. (f) Aho, J. E.; Pihko, P. M.; Rissa, T. K. Nonanomeric Spiroketals in Natural Products: Structures, Sources, and Synthetic Strategies. Chem. Rev. 2005, 105, 4406-4440. (g) Perron, F.; Albizati, K. F. Chemistry of Spiroketals. Chem. Rev. 1989, 89, 1617-1661.

(2) (a) Zhang, W.; Tong, R. Synthetic Approaches To Construct the 6,8-DOBCO Framework in Natural Products. J. Org. Chem. 2016, 81, 2203-2212. (b) Milroy, L.-G.; Zinzalla, G.; Prencipe, G.; Michel, P.; Ley, S. V.; Gunaratnam, M.; Beltran, M.; Neidle, S. Chemical
Variation of Natural-Product-Like Scaffolds: Design, Synthesis, and Biological Activity of Fused Bicyclic Acetal Derivatives. Angew. Chem., Int. Ed. 2007, 46, 2493-2496. (c) Jun, J.-G. Introduction of Bicyclic Ketal Chemistry: Synthesis and Transformation Reaction of 6,8Dioxabicyclo[3.2.1] Octane Skeletal System. Synlett 2003, 17591777. (d) Mundy, B. P.; Lipkowitz, K. B.; Dirks, G. W. Chemistry of the 6,8-Dioxabicyclo[3.2.1] Octane Series. Sources, Synthesis, Structures and Reactions. Heterocycles 1977, 51-76. For recent articles, see (e) Zeng, R.; Li, J. L.; Zhang, X.; Liu, Y. Q.; Jia, Z. Q.; Leng, H. J.; Huang, Q. W.; Liu, Y.; Li, Q. Z. Diastereoselective Construction of 6,8-Dioxabicyclo[3.2.1] Octane Frameworks from Vinylethylene Carbonates via Palladium-Organo Relay Catalysis. ACS Catal. 2019, 9, 8256-8262. (f) Hohol, R. E.; Arcure, H.; Witczak, Z. J.; Bielski, R.; Kirschbaum, K.; Andreana, P.; Mencer, D. E. One-Pot Synthesis of Carbohydrate Exo-Cyclic Enones and Hemiketals with 6,8-Dioxabicyclo-[3.2.1] Octane Moieties. Serendipitous Formation of a Spironolactone When 2-Pyridinecarboxaldehyde Is Used as the Reactant. Part II. Tetrahedron 2018, 74, 7303-7309. (g) Schmidt, E. Y.; Tatarinova, I. V.; Semenova, N. V.; Protsuk, N. I.; Ushakov, I. A.; Trofimov, B. A. Exploring Acetylene Chemistry: A Transition Metal-Free Route to Dienyl 6,8-Dioxabicyclo[3.2.1] Octanes from Ketones and Acetylenes. J. Org. Chem. 2018, 83, 10272-10280. (h) Schmidt, E. Y.; Bidusenko, I. A.; Ushakov, I. A.; Trofimov, B. A. Unfolding the Frontalin Chemistry: A Facile Selective Hydrogenation of 7-Methylidene-6,8Dioxabicyclo[3.2.1] Octanes, 2:2 Ensembles of Ketones and Acetylene. Mendeleev Commun. 2018, 28, 513-514. (i) Schmidt, E. Y.; Bidusenko, I. A.; Ushakov, I. A.; Protsuk, N. I.; Trofimov, B. A. An Easy Access to Sulfur Derivatives of 6,8-Dioxabicyclo[3.2.1] Octanes, Naturally Abundant Scaffolds. Synthesis 2018, 50, 2624-2630.

(3) (a) Mori, K. Protective Group-Free Syntheses of ( \pm )-Frontalin, $( \pm)$-endo-Brevicomin, ( \pm )-exo-Brevicomin, and ( \pm )-3,4-Dehydroexo-Brevicomin: Racemic Pheromones with a 6,8Dioxabicyclo[3.2.1] Octane Ring. Biosci., Biotechnol., Biochem. 2011, 75, 976-981. (b) Booth, Y. K.; Kitching, W.; De Voss, J. J. Biosynthesis of Insect Spiroacetals. Nat. Prod. Rep. 2009, 26, 490525. (c) Francke, W.; Kitching, W. Spiroacetals in Insects. Curr. Org. Chem. 2001, 5, 233-251. (d) Mori, K. The Synthesis of Insect Pheromones. In The Total Synthesis of Natural Products; 1981; pp. 1183, DOI: $10.1002 / 9780470129678 . c h 1$.

(4) For a study of the abundance of bicyclic acetals in natural products, see Lenci, E.; Menchi, G.; Saldívar-Gonzalez, F. I.; MedinaFranco, J. L.; Trabocchi, A. Bicyclic Acetals: Biological Relevance, Scaffold Analysis, and Applications in Diversity-Oriented Synthesis. Org. Biomol. Chem. 2019, 17, 1037-1052.

(5) For recent reviews, see (a) Iglesias-Arteaga, M. A.; Morzycki, J. W. Cephalostatins and Ritterazines. In The Alkaloids Chemistry and Biology; 2013; pp. 153-279, DOI: 10.1016/B978-0-12-4077744.00002-9. (b) Lee, S.; LaCour, T. G.; Fuchs, P. L. Chemistry of Trisdecacyclic Pyrazine Antineoplastics: The Cephalostatins and Ritterazines. Chem. Rev. 2009, 109, 2275-2314. (c) SimmonsBoyce, J. L.; Tinto, W. F. Steroidal Saponins and Sapogenins from the Agavaceae Family. Nat. Prod. Commun. 2007, 2, 99-114.

(6) Liu, H.; Lin, S.; Jacobsen, K. M.; Poulsen, T. B. Chemical Syntheses and Chemical Biology of Carboxyl Polyether Ionophores: Recent Highlights. Angew. Chem., Int. Ed. 2019, 58, 13630-13642.

(7) Carroll, A. R.; Copp, B. R.; Davis, R. A.; Keyzers, R. A.; Prinsep, M. R. Marine Natural Products. Nat. Prod. Rep. 2020, 37, 175-223. See also previous reviews of this series.

(8) (a) Beaumont, S.; Ilardi, E. A.; Tappin, N. D. C.; Zakarian, A. Marine Toxins with Spiroimine Rings: Total Synthesis of Pinnatoxin A. Eur. J. Org. Chem. 2010, 2010, 5743-5765. (b) Matsuura, F.; Peters, R.; Anada, M.; Harried, S. S.; Hao, J.; Kishi, Y. Unified Total Synthesis of Pteriatoxins and Their Diastereomers. J. Am. Chem. Soc. 2006, 128, 7463-7465.

(9) (a) Ren, J.; Wang, J.; Tong, R. Asymmetric Total Synthesis of (+)-Attenol B. Org. Lett. 2015, 17, 744-747. (b) Ren, J.; Tong, R. Asymmetric Total Synthesis of (+)-Didemniserinolipid B via Achmatowicz Rearrangement/Bicycloketalization. J. Org. Chem. 2014, 79, 6987-6995. (c) Schmidt, E. Y.; Trofimov, B. A.; Zorina, 
N. V.; Mikhaleva, A. I.; Ushakov, I. A.; Skital'tseva, E. V.; Kazheva, O. N.; Alexandrov, G. G.; Dyachenko, O. A. Synthesis of Functionalized 3,4-Dihydropyrans via Rearrangement of the Products of a One-Pot Diastereoselective Assembly of Ketones and Acetylene. Eur. J. Org. Chem. 2010, 2010, 6727-6730. (d) Jong-Gab Jun, J.-G.; Shin, D. G.; Shin, H. S.; Kim, S. H. Transformation of Bicyclic Ketal Compound to 1,2-Cyclopentanediol via 1,5-Diketone. Bull. Korean Chem. Soc. 1992, 13, 176-179.

(10) Somsák, L., Ed. Carbohydrate-Spiro-Heterocycles; Springer International Publishing, 2019.

(11) (a) Chen, Y.; Wang, X.; Wang, J.; Yang, Y. Synthesis of DManno-Heptulose via a Cascade Aldol/Hemiketalization Reaction. Beilstein J. Org. Chem. 2017, 13, 795-799. (b) Subrizi, F.; CárdenasFernández, M.; Lye, G. J.; Ward, J. M.; Dalby, P. A.; Sheppard, T. D.; Hailes, H. C. Transketolase Catalysed Upgrading of L-Arabinose: The One-Step Stereoselective Synthesis of L-Gluco-Heptulose. Green Chem. 2016, 18, 3158-3165. (c) Jacobsen, A.; Thiem, J. Contemporary Syntheses of 2-Ketoheptoses and Derivatives. Curr. Org. Chem. 2014, 18, 2833-2841. (d) Matsuda, S.; Yamanoi, T.; Watanabe, M. Syntheses of a Partially Benzylated Derivative of the Anhydro-D-Altro-Heptulose Found in Coriaria Japonica A and of Its Analogs. Tetrahedron 2008, 64, 8082-8088.

(12) (a) Martín, A.; Salazar, J. A.; Suárez, E. Synthesis of Chiral Spiroacetals from Carbohydrates. J. Org. Chem. 1996, 61, 3999-4006. (b) Dorta, R. L.; Martín, A.; Salazar, J. A.; Suárez, E.; Prangé, T. Syntheses of Chiral Dispiroacetals from Carbohydrates. J. Org. Chem. 1998, 63, 2251-2261.

(13) Francisco, C. G.; Herrera, A. J.; Suárez, E. Intramolecular Hydrogen Abstraction Reaction Promoted by Alkoxy Radicals in Carbohydrates. Synthesis of Chiral 2,7-Dioxabicyclo[2.2.1]Heptane and 6,8-Dioxabicyclo[3.2.1] Octane Ring Systems. J. Org. Chem. 2002, 67, 7439-7445.

(14) For a recent review on HAT reactions, see Nechab, M.; Mondal, S.; Bertrand, M. P. 1,n-Hydrogen-Atom Transfer (HAT) Reactions in Which $\mathrm{n} \neq 5$ : An Updated Inventory. Chem. - Eur. J. 2014, 20, 16034-16059. and references cited therein.

(15) For the interest of $C$-ketosides as versatile chiral synthons, see (a) Pérez-Martín, I.; Suárez, E. Radicals and Carbohydrates. In Encyclopedia of Radicals in Chemistry, Biology and Materials; John Wiley \& Sons, Ltd: Chichester, UK, 2012; pp. 1131-1174, DOI: $10.1002 / 9781119953678 . r a d 031$. For selected examples, see (b) Pal, A. P. J.; Mallick, A.; Reddy, Y. S.; Vankar, Y. D. Molecular Iodine-Promoted $\mathrm{N}$ - and C-Glycosylation of 1-C-Alkyl (or Phenyl)Glycopyranoses. Tetrahedron Lett. 2010, 51, 6334-6337. (c) Malapelle, A.; Coslovi, A.; Doisneau, G.; Beau, J. M. An Expeditious Synthesis of $N$-Acetylneuraminic Acid $\alpha$-C-Glycosyl Derivatives ( $~ \alpha-$ C-Glycosides)" from the Anomeric Acetates. Eur. J. Org. Chem. 2007, 2007, 3145-3157. (d) Majumder, U.; Cox, J. M.; Johnson, H. W.; Rainier, J. D. Total Synthesis of Gambierol: The Generation of the $\mathrm{A}-\mathrm{C}$ and $\mathrm{F}-\mathrm{H}$ Subunits by Using a $\mathrm{C}$-Glycoside Centered Strategy. Chem. - Eur. J. 2006, 12, 1736-1746. (e) Roberts, S. W.; Rainier, J. D. Substitution and Remote Protecting Group Influence on the Oxidation/Addition of $\alpha$-Substituted 1,2-Anhydroglycosides: A Novel Entry into C-Ketosides. Org. Lett. 2005, 7, 1141-1144.

(16) León, E. I.; Martín, Á.; Pérez-Martín, I.; Quintanal, L. M.; Suárez, E. C-C Bond Formation by Sequential Intramolecular Hydrogen Atom Transfer/Intermolecular Radical Allylation Reaction in Carbohydrate Systems. Eur. J. Org. Chem. 2012, 2012, 3818-3829. (17) (a) Takahashi, H.; Shida, T.; Hitomi, Y.; Iwai, Y.; Miyama, N.; Nishiyama, K.; Sawada, D.; Ikegami, S. Divergent Synthesis of LSugars and L-Iminosugars from D-Sugars. Chem. - Eur. J. 2006, 12, 5868-5877. (b) Hollingsworth, R.; Song, X. A Facile and General Synthesis of Rare L-Sugar Lactones. Synlett 2007, 2007, 1247-1250.

(c) Ermolenko, L.; Sasaki, N. A. Diastereoselective Synthesis of All Eight L-Hexoses from L-Ascorbic Acid. J. Org. Chem. 2006, 71, 693703.

(18) Wang, Z.; Shao, H.; Lacroix, E.; Wu, S.-H.; Jennings, H. J.; Zou, W. Epimerization of 2'-Carbonylalkyl-C-Glycosides via Enolation, $\beta$ -
Elimination and Intramolecular Cycloaddition. J. Org. Chem. 2003, 68, 8097-8105. And references cited therein.

(19) (a) Surzur, J. M.; Teissier, P. Addition Radicalaire de Esters Sur Les Alcools Ethyleniques. C. R. Acad. Sci. Fr. Ser. C 1967, 264, 19811984. (b) Surzur, J. M.; Teissier, P. Radical Addition to Unsaturated Alcohols. III. Addition on Ethylene Acetate: 1,2-Radical Migration of Acetoxy Group. Bull. Soc. Chim. Fr. 1970, 3060-3070.

(20) Tanner, D. D.; Law, F. C. P. Free-Radical Acetoxy Group Migration. J. Am. Chem. Soc. 1969, 91, 7535-7537.

(21) (a) Beckwith, A. L. J.; Duggan, P. J. The Mechanism of the $\beta$ (Acyloxy)alkyl Radical Rearrangement: Substituent and Solvent Effects. J. Am. Chem. Soc. 1996, 118, 12838-12839. (b) Zipse, H. $[1,2]$-Acyloxy Shifts in Radicals. A Computational Investigation of Substituent and Solvent Effects. J. Am. Chem. Soc. 1997, 119, 10871093. (c) Zipse, H.; Bootz, M. 1,2-Migration in $\beta$-(Acyloxy)ethyl Radicals Revisited-Concerted or Stepwise? J. Chem. Soc., Perkin Trans. 2 2001, 2, 1566-1572. For reviews, see (d) Beckwith, A. L. J.; Crich, D.; Duggan, P. J.; Yao, Q. Chemistry of $\beta$-(Acyloxy)Alkyl and $\beta$-(Phosphatoxy)Alkyl Radicals and Related Species: Radical and Radical Ionic Migrations and Fragmentations of Carbon-Oxygen Bonds. Chem. Rev. 1997, 97, 3273-3312. (e) Crich, D. Radical Rearrangements of Esters. In Radicals in Organic Synthesis; Renaud, P.; Sibi, M. P. Eds.; Wiley-VCH Verlag GmbH: Weinheim, Germany, 2001; pp. 188-206, DOI: 10.1002/9783527618293.ch35. (f) Sasaki, K.; Cumpstey, I.; Crich, D. Radical Rearrangements: EsterSubstituted Radicals and Hydrogen Atom Migrations. In Encyclopedia of Radicals in Chemistry, Biology and Materials; Chatgilialoglu, C., Studer, A. Eds.; John Wiley \& Sons, Ltd: Chichester, UK, 2012; pp. 125-146, DOI: $10.1002 / 9781119953678 . r a d 036$.

(22) (a) Crich, D.; Yao, Q. The $\beta$-(Phosphonooxy)Alkyl Radical Rearrangement. J. Am. Chem. Soc. 1993, 115, 1165-1166. (b) Koch, A.; Lamberth, C.; Wetterich, F.; Giese, B. Radical Rearrangement of 2-O-(Diphenylphosphoryl)Glycosyl Bromides. A New Synthesis for 2-Deoxy Disaccharides and 2-Deoxy Ribonucleosides. J. Org. Chem. 1993, 58, 1083-1089.

(23) Lacôte, E.; Renaud, P. Rate Enhancement of the Radical 1,2Acyloxy Shift (Surzur-Tanner Rearrangement) by Complexation with Lewis Acids. Angew. Chem., Int. Ed. 1998, 37, 2259-2262.

(24) (a) Giese, B.; Gröninger, K. S. 1,3,4,6-Tetra-O-Acetyl-2-Deoxy$\alpha$-D-Glucopyranose. Org. Synth. 1990, 69, 66-69. (b) Giese, B.; Gilges, S.; Groninger, K. S.; Lamberth, C.; Witzel, T. Synthesis of 2Deoxy Sugars. Liebigs Ann. der Chem. 1988, 615-617.

(25) Gimisis, T.; Ialongo, G.; Chatgilialoglu, C. Generation of C-1' Radicals through a $\beta$-(Acyloxy)Alkyl Rearrangement in Modified Purine and Pyrimidine Nucleosides. Tetrahedron 1998, 54, 573-592.

(26) (a) Paul, R.; Greenberg, M. M. Mechanistic Studies on RNA Strand Scission from a C2'-Radical. J. Org. Chem. 2016, 81, 91999205. (b) Taverna Porro, M. L.; Greenberg, M. M. DNA Double Strand Cleavage via Interstrand Hydrogen Atom Abstraction. J. Am. Chem. Soc. 2013, 135, 16368-16371. (c) Resendiz, M. J. E.; Pottiboyina, V.; Sevilla, M. D.; Greenberg, M. M. Direct Strand Scission in Double Stranded RNA via a C5-Pyrimidine Radical. J. Am. Chem. Soc. 2012, 134, 3917-3924. (d) Kerwin, S. M. DNA Damage Due to Diradical-Generating Cyclizations. In Radical and Radical Ion Reactivity in Nucleic Acid Chemistry; Greenberg, M. M., Ed.; John Wiley \& Sons, 2009; pp. 389-443. (e) Giese, B. Long-Distance Charge Transport in DNA: The Hopping Mechanism. Acc. Chem. Res. 2000, 33, 631-636. (f) Pogozelski, W. K.; Tullius, T. D. Oxidative Strand Scission of Nucleic Acids: Routes Initiated by Hydrogen Abstraction from the Sugar Moiety. Chem. Rev. 1998, 98, 1089-1108. and references cited therein.

(27) (a) For a recent review, see Schauer, R.; Kamerling, J. P. Exploration of the Sialic Acid World. Adv. Carbohydr. Chem. Biochem. 2018, 75, 1-213. For chemical synthesis of Kdo and its derivatives, see (b) Feng, Y.; Dong, J.; Xu, F.; Liu, A.; Wang, L.; Zhang, Q.; Chai, Y. Efficient Large Scale Syntheses of 3-Deoxy-D-Manno-2-Octulosonic Acid (Kdo) and Its Derivatives. Org. Lett. 2015, 17, 2388-2391. and references cited therein. For chemical synthesis of sialic acids, see (c) Ishizawa, K.; Majima, S.; Wei, X. F.; Mitsunuma, H.; Shimizu, Y.; 
Kanai, M. Copper(I)-Catalyzed Stereodivergent Propargylation of NAcetyl Mannosamine for Protecting Group Minimal Synthesis of C3Substituted Sialic Acids. J. Org. Chem. 2019, 84, 10615-10628. and references cited therein.

(28) (a) Sun, Z.; Winschel, G. A.; Borovika, A.; Nagorny, P. Chiral Phosphoric Acid-Catalyzed Enantioselective and Diastereoselective Spiroketalizations. J. Am. Chem. Soc. 2012, 134, 8074-8077. (b) Audrain, H.; Thorhauge, J.; Hazell, R. G.; Jørgensen, K. A. A Novel Catalytic and Highly Enantioselective Approach for the Synthesis of Optically Active Carbohydrate Derivatives. J. Org. Chem. 2000, 65, 4487-4497. (c) Izquierdo Cubero, I.; Plaza López-Espinosa, M. T.; Richardson, A. C.; Aamlid, K. H. Enantiospecific Synthesis of (R)-1,6-Dioxaspiro[4.5]Decane from a Derivative of D-Fructose. Carbohydr. Res. 1993, 242, 281-286.

(29) (a) Elshahawi, S. I.; Shaaban, K. A.; Kharel, M. K.; Thorson, J. S. A Comprehensive Review of Glycosylated Bacterial Natural Products. Chem. Soc. Rev. 2015, 44, 7591-7697. (b) Kirschning, A.; Jesberger, M.; Schöning, K. U. Concepts for the Total Synthesis of Deoxy Sugars. Synthesis 2001, 2001, 507-540. (c) Hanessian, S. Deoxy Sugars. Adv. Carbohydr. Chem. 1967, 21, 143-207.

(30) For early reports on the use of $n$ - $\mathrm{Bu}_{3} \mathrm{SnH} / \mathrm{AIBN}$ for the generation of alkoxyl radicals from $N$-alkoxyphthalimides, see: (a) Kim, S.; Lee, T. A.; Song, Y. Facile Generation of Alkoxy Radicals from N-Alkoxyphthalimides. Synlett 1998, 1998, 471-472. (b) Okada, K.; Okamoto, K.; Oda, M. A New and Practical Method of Decarboxylation: Photosensitized Decarboxylation of N-Acyloxyphthalimides via Electron-Transfer Mechanism. J. Am. Chem. Soc. 1988, 110, 8736-8738. (c) Martín, A.; Rodríguez, M. S.; Suárez, E. Synthesis of Alditols by Reductive Radical Fragmentation of NPhthalimido Glycosides. Preparation of Chiral Synthetic Intermediates. Tetrahedron Lett. 1999, 40, 7525-7528.

(31) (a) Jia, K.; Chen, Y. Visible-Light-Induced Alkoxyl Radical Generation for Inert Chemical Bond Cleavage/Functionalization. Chem. Commun. 2018, 54, 6105-6112. (b) Hu, X.-Q.; Chen, J.-R.; Xiao, W.-J. Controllable Remote $\mathrm{C}-\mathrm{H}$ Bond Functionalization by Visible-Light Photocatalysis. Angew. Chem., Int. Ed. 2017, 56, 19601962. (c) Studer, A.; Curran, D. P. Catalysis of Radical Reactions: A Radical Chemistry Perspective. Angew. Chem., Int. Ed. 2016, 55, 58102. (d) Huang, W.; Cheng, X. Hantzsch Esters as Multifunctional Reagents in Visible-Light Photoredox Catalysis. Synlett 2017, 28, 148-158. (e) Shaw, M. H.; Twilton, J.; MacMillan, D. W. C. Photoredox Catalysis in Organic Chemistry. J. Org. Chem. 2016, 81, 6898-6926. (f) Prier, C. K.; Rankic, D. A.; MacMillan, D. W. C. Visible Light Photoredox Catalysis with Transition Metal Complexes: Applications in Organic Synthesis. Chem. Rev. 2013, 113, 5322-5363. (32) (a) Dorigo, A. E.; McCarrick, M. A.; Loncharich, R. J.; Houk, K. N. Transition Structures for Hydrogen Atom Transfers to Oxygen. Comparisons of Intermolecular and Intramolecular Processes, and Open- and Closed-Shell Systems. J. Am. Chem. Soc. 1990, 112, 75087514. (b) Dorigo, A. E.; Houk, K. N. The Relationship between Proximity and Reactivity. An Ab Initio Study of the Flexibility of the $\mathrm{OH}^{\bullet}+\mathrm{CH}_{4}$ Hydrogen Abstraction Transition State and a Force-Field Model for the Transition States of Intramolecular Hydrogen Abstractions. J. Org. Chem. 1988, 53, 1650-1664. (c) Dorigo, A. E.; Houk, K. N. Transition Structures for Intramolecular HydrogenAtom Transfers: The Energetic Advantage of Seven-Membered over Six-Membered Transition Structures. J. Am. Chem. Soc. 1987, 109, 2195-2197.

(33) DAISY program simulator as implemented in TOPSPIN, version 4.0.6, for Bruker.

(34) Coupling constants were calculated from a generalization of the Karplus equation established by Haasnoot as implemented in Maestro version 9.0, Schrödinger, LLC, New York, NY, 2009. Haasnoot, C. A. G.; de Leeuw, F. A. A. M.; Altona, C. The Relationship between Proton-Proton NMR Coupling Constants and Substituent Electronegativities-I: An Empirical Generalization of the Karplus Equation. Tetrahedron 1980, 36, 2783-2792.

(35) Crich, D.; Huang, X.; Newcomb, M. Inter- and Intramolecular Pathways for the Formation of Tetrahydrofurans from $\beta$ -
(Phosphatoxy)Alkyl Radicals. Evidence for a Dissociative Mechanism. J. Org. Chem. 2000, 65, 523-529.

(36) Cortezano-Arellano, O.; Quintero, L.; Sartillo-Piscil, F. Total Synthesis of Cephalosporolide E via a Tandem Radical/Polar Crossover Reaction. The Use of the Radical Cations under Nonoxidative Conditions in Total Synthesis. J. Org. Chem. 2015, $80,2601-2608$.

(37) Martín, A.; Pérez-Martín, I.; Quintanal, L. M.; Suárez, E. Intramolecular 1,8-Hydrogen Atom Transfer. Stereoselectivity of the Hexopyranos-5'-yl Radical Reactions in Hexp-(1 $\rightarrow 4)$-Hexp Disaccharide Systems. J. Org. Chem 2008, 73, 7710-7720.

(38) Crich, D.; Suk, D.-H. The $\beta$-(Acyloxy)alkyl Radical Rearrangement Revisited. Can. J. Chem. 2004, 82, 75-79.

(39) For previous studies on migratory differences between axial and equatorial diphenoxyphosphatoxy groups in isolated S-T rearrangement, see (a) Koch, A.; Giese, B. Radical Rearrangements of 2-O(Diphenoxyphosphoryl)Glycosyl Bromides. Helv. Chim. Acta 1993 , 76, 1687-1701. (b) Crich, D.; Suk, D.-H.; Sun, S. Highly Diastereoselective Radical Cyclization of a Glucose-Derived Enol Ether Radical Cation/Phosphate Anion Pair. Tetrahedron: Asymmetry 2003, 14, 2861-2864.

(40) For comparison between the displacement of acetoxy and diphenoxyphosphatoxy groups in the S-T rearrangement, see Ref $21 \mathrm{~d}$.

(41) (a) Chatgilialoglu, C.; Lalevée, J. Recent Applications of the (TMS) ${ }_{3} \mathrm{SiH}$ Radical-Based Reagent. Molecules 2012, 17, 527-555. (b) Chatgilialoglu, C.; Ferreri, C.; Landais, Y.; Timokhin, V. I. Thirty Years of (TMS) ${ }_{3} \mathrm{SiH}$ : A Milestone in Radical-Based Synthetic Chemistry. Chem. Rev. 2018, 118, 6516-6572. (c) Ballestri, M.; Chatgilialoglu, C.; Clark, K. B.; Griller, D.; Giese, B.; Kopping, B. Tris(Trimethylsilyl)Silane as a Radical-Based Reducing Agent in Synthesis. J. Org. Chem. 1991, 56, 678-683.

(42) (a) Deslongchamps, P.; Rowan, D. D.; Pothier, N.; Sauvé, G.; Saunders, J. K. 1,7-Dioxaspiro[5.5]Undecanes. An Excellent System for the Study of Stereoelectronic Effects (Anomeric and ExoAnomeric Effects) in Acetals. Can. J. Chem. 1981, 59, 1105-1121. (b) Deslongchamps, P. Stereoelectronic Effects in Organic Chemistry; Pergamon Press: New York, 1983.

(43) (a) Beckwith, A. L. J.; Easton, C. J. Stereoelectronic Effects in Hydrogen Atom Abstraction from Substituted 1,3-Dioxanes. J. Am. Chem. Soc. 1981, 103, 615-619. (b) Hayday, K.; McKelvey, R. D. An Anomeric Effect in Photochemical Hydrogen Abstraction Reactions of Tetrahydropyranyl Ethers. J. Org. Chem. 1976, 41, 2222-2223.

(44) Chatgilialoglu, C.; Studer, A. Encyclopedia of Radicals in Chemistry, Biology and Materials; John Wiley \& Sons, Ltd: Chichester, UK, 2012; pp. 655-692, DOI: 10.1002/9781119953678.rad019.

(45) Although, examples of pure radical $\beta$-(ester)alkyl fragmentation with concomitant decarboxylation are scarce, we have used it as a simple mechanism to explain the partial loss of deuterium found in some of the 1,5-HAT-Surzur-Tanner rearrangement products. Alternative mechanisms like adventitious acid-catalyzed opening and recombination of the spiroketal ring or the use of $n-\mathrm{Bu}_{3} \mathrm{SnH}(\mathrm{D})$ as a potential external nucleophile for the trapping of the alkene radicalcation intermediate can also be considered. For references of pure radical $\beta$-(ester)alkyl fragmentation, see: (a) Motherwell, W. B.; Imboden, C. Decarboxylation via O-Acyl Thiohydroxamates. In Radicals in Organic Synthesis; Renaud, P.; Sibi, M. P., Eds.; WileyVCH Verlag GmbH: Weinheim, Germany, 2001; pp. 109-134, DOI: 10.1002/9783527618293.ch7. (b) Barton, D. H. R.; Dowlatshahi, H. A.; Motherwell, W. B.; Villemin, D. A New Radical Decarboxylation Reaction for the Conversion of Carboxylic-Acids into Hydrocarbons. J. Chem. Soc., Chem. Commun. 1980, 732-733. (c) Crich, D.; Mo, X.-S. Free-Radical Chemistry of Lactones: Fragmentation of $\beta$-Lactones. The Beneficial Effect of Catalytic Benzeneselenol on Chain Propagation. J. Org. Chem. 1997, 62, 86248625. (d) Crich, D.; Mo, X.-S. Free Radical Chemistry of $\beta$-Lactones. Arrhenius Parameters for the Decarboxylative Cleavage and Ring Expansion of 2-Oxetanon-4-ylcarbinyl Radicals. Facilitation of Chain Propagation by Catalytic Benzeneselenol. J. Am. Chem. Soc. 1998, 120, 8298-8304. See also Ref 21e. 
(46) Bruyère, I.; Tóth, Z.; Benyahia, H.; Xue, J. L.; Praly, J.-P. $\mathrm{NaBH}_{3} \mathrm{CN}$ and other Systems as Substitutes of Tin and Silicon Hydrides in the Light or Heat-initiated Reduction of Halosugars: a Tunable Access to either 2-Deoxy Sugars or 1,5-Anhydro-itols. Tetrahedron 2013, 69, 9656-9662.

(47) For a pioneer report, see: Zhang, J.; Li, Y.; Zhang, F.; Hu, C.; Chen, Y. Generation of Alkoxyl Radicals by Photoredox Catalysis Enables Selective C(Sp 3)-H Functionalization under Mild Reaction Conditions. Angew. Chem., Int. Ed. 2016, 55, 1872-1875.

(48) Abraham, R. J.; Gottschalck, H.; Paulsen, H.; Thomas, W. A. The Proton Magnetic Resonance Spectra and Conformations of Cyclic Compounds. Part II. The p.m.r. Spectra of the Conduritols. J. Chem. Soc. 1965, 6268-6277.

(49) For the synthesis of an analogous trans-fused bis(pyran) compound from a sugar, see: Oguri, H. Designed Hapten Aimed at Anti-Ciguatoxin Monoclonal Antibody: Synthesis, Immunization and Discrimination of the C2 Configuration. Synthesis 1999, 1999, 14311436.

(50) (a) Guyenne, S.; León, E. I.; Martín, A.; Pérez-Martín, I.; Suárez, E. Intramolecular 1,8-Hydrogen Atom Transfer Reactions in Disaccharide Systems Containing Furanose Units. J. Org. Chem. 2012, 77, 7371-7391. (b) León, E. I.; Martín, A.; Peréz-Martín, I.; Quintanal, L. M.; Suárez, E. Hydrogen Atom Transfer Experiments Provide Chemical Evidence for the Conformational Differences between C- and O-Disaccharides. Eur. J. Org. Chem. 2010, 2010, 5248-5262. (c) Boto, A.; Hernández, D.; Hernández, R.; Suárez, E. $\beta$-Fragmentation of Primary Alkoxyl Radicals versus Hydrogen Abstraction: Synthesis of Polyols and $\alpha, \omega$-Differently Substituted Cyclic Ethers from Carbohydrates. J. Org. Chem. 2003, 68, 53105319.

(51) (a) Lee, I.-C.; Zulueta, M. M. L.; Shie, C.-R.; Arco, S. D.; Hung, S.-C. Deuterium-Isotope Study on the Reductive Ring Opening of Benzylidene Acetals. Org. Biomol. Chem. 2011, 9, 7655-7658. (b) Johnsson, R.; Ohlin, M.; Ellervik, U. Reductive Openings of Benzylidene Acetals Revisited: A Mechanistic Scheme for Regio- and Stereoselectivity. J. Org. Chem. 2010, 75, 8003-8011. (c) Shie, C.-R.; Tzeng, Z.-H.; Kulkarni, S. S.; Uang, B.-J.; Hsu, C.-Y.; Hung, S.-C. $\mathrm{Cu}(\mathrm{OTf})_{2}$ as an Efficient and Dual-Purpose Catalyst in the Regioselective Reductive Ring Opening of Benzylidene Acetals. Angew. Chem., Int. Ed. 2005, 44, 1665-1668.

(52) Barnett, D. W.; Panigot, M. J.; Curley, R. W. Stereoselective Route to $15 \mathrm{~N}$-Labeled- $\beta$-Deuterated Amino Acids: Synthesis of $(2 S, 3 R)-\left[3-{ }^{2} \mathrm{H}, 15 \mathrm{~N}\right]-$ Phenylalanine. Tetrahedron: Asymmetry 2002, $13,1893-1900$.

(53) For references on the $\beta$-fragmentation of primary alkoxyl radicals in carbohydrates, see (a) Sánchez-Eleuterio, A.; Quintero, L.; Sartillo-Piscil, F. High 1,3-trans Stereoselectivity in Nucleophilic Substitution at the Anomeric Position and $\beta$-Fragmentation of the Primary Alkoxyl Radical in 3-Amino-3-deoxy-ribofuranose Derivatives: Application to the Synthesis of 2-epi-(-)-Jaspine B. J. Org. Chem. 2011, 76, 5466-5471. (b) Hernández-García, L.; Quintero, L.; Sánchez, M.; Sartillo-Piscil, F. Beneficial Effect of Internal Hydrogen Bonding Interactions on the $\beta$-Fragmentation of Primary Alkoxyl Radicals. Two-Step Conversion of D-Xylo- and D-Ribofuranoses into L-Threose and D-Erythrose, Respectively. J. Org. Chem. 2007, 72, 8196-8201.

(54) Cipolla, L.; Lay, L.; Nicotra, F. New and Easy Access to CGlycosides of Glucosamine and Mannosamine. J. Org. Chem. 1997, 62, 6678-6681.

(55) (a) Mitsunobu, O. The Use of Diethyl Azodicarboxylate and Triphenylphosphine in Synthesis and Transformation of Natural Products. Synthesis 1981, 1981, 1-28. (b) Grochowski, E.; Jurczak, J. A New Synthesis of O-Alkylhydroxylamines. Synthesis 1976, 1976, 682-684.

(56) (a) Bennek, J. A.; Gray, G. R. An Efficient Synthesis of Anhydroalditols and Allylic-Glycosides. J. Org. Chem. 1987, 52, 892897. (b) Kozikowski, A. P.; Sorgi, K. L.; Wang, B. C.; Xu, Z. An Improved Method for the Synthesis of Anomerically Allylated C-
Glycopyranosides and C-Glycofuranosides. Tetrahedron Lett. 1983, 24, 1563-1566.

(57) Li, X.; Li, J.; Mootoo, D. R. Synthesis of the ABCD Trioxadispiroketal Subunit of Azaspiracid-1: An IodoetherificationDehydroiodination Strategy for Complex Spiroketals. Org. Lett. 2007, 9, 4303-4306.

(58) (a) Jarikote, D. V.; O’Reilly, C.; Murphy, P. V. UltrasoundAssisted Synthesis of C-Glycosides. Tetrahedron Lett. 2010, 51, 67766778. (b) Zhu, Y.-H.; Vogel, P. Synthesis of a C-Disaccharide Analog of the Thomsen-Friedenreich (T) Epitope. Synlett 2001, 2001, 7981. (c) Schäfer, A.; Thiem, J. Synthesis of Novel Donor Mimetics of UDP-Gal, UDP-GlcNAc, and UDP-GalNAc as Potential Transferase Inhibitors. J. Org. Chem. 2000, 65, 24-29.

(59) (a) Grice, P.; Ley, S. V.; Pietruszka, J.; Priepke, H. W. M.; Warriner, S. L. Preparation, Structure, Derivatisation and NMR Data of Cyclohexane-1,2-Diacetal Protected Carbohydrates. J. Chem. Soc., Perkin Trans. 1 1997, 351-364. (b) Hense, A.; Ley, S. V.; Osborn, H. M.; Owen, D. R.; Poisson, J. F.; Warriner, S. L.; Wesson, K. E. Direct Preparation of Diacetals from 1,2-Diketones and Their Use as 1,2Diol Protecting Groups. J. Chem. Soc. Perkin Trans. 1 1997, 1, 20232032. (c) Ley, S. V.; Boons, G.-J.; Leslie, R.; Woods, M.; Hollinshead, D. M. Dispiroketals in Synthesis (Part 3): 1 Selective Protection of Diequatorial Vicinal Diols in Carbohydrates. Synthesis 1993, 1993, 689-692.

(60) (a) Pirrung, M. C. The Synthetic Organic Chemist's Companion; John Wiley \& Sons, Inc.: Hoboken, NJ, USA, 2007, pp. 171, DOI: 10.1002/9780470141045. (b) Long, D. D.; Smith, M. D.; Martín, A.; Wheatley, J. R.; Watkin, D. G.; Müller, M.; Fleet, G. W. J. Complex Tetrahydrofurans from Carbohydrate Lactones: THF Amino Acids as Building Blocks for Unnatural Biopolymers. J. Chem. Soc., Perkin Trans. 1 2002, 1982-1998.

(61) La Ferla, B.; Russo, L.; Airoldi, C.; Nicotra, F. Solid-Phase Supported Mimic of GDP-L-Galactose. Tetrahedron: Asymmetry 2009, $20,744-745$.

(62) Allavudeen, S. S.; Kuberan, B.; Loganathan, D. A Method for Obtaining Equilibrium Tautomeric Mixtures of Reducing Sugars via Glycosylamines Using Nonaqueous Media. Carbohydr. Res. 2002, 337, 965-968.

(63) Beignet, J.; Tiernan, J.; Woo, C. H.; Kariuki, B. M.; Cox, L. R. Stereoselective Synthesis of Allyl-C-Mannosyl Compounds: Use of a Temporary Silicon Connection in Intramolecular Allylation Strategies with Allylsilanes. J. Org. Chem. 2004, 69, 6341-6356.

(64) Martín, A.; Pérez-Martín, I.; Quintanal, L. M.; Suárez, E. Intramolecular 1,8- versus 1,6-Hydrogen Atom Transfer between Pyranose Units in a $(1 \rightarrow 4)$-Disaccharide Model Promoted by Alkoxyl Radicals. Conformational and Stereochemical Requirements. Org. Lett. 2007, 9, 1785-1788.

(65) (a) Kobertz, W. R.; Bertozzi, C. R.; Bednarski, M. D. An Efficient Method for the Synthesis of $\alpha$ - and $\beta$-C-Glycosyl Aldehydes. Tetrahedron Lett. 1992, 33, 737-740. (b) Brueckner, C.; Holzinger, H.; Reissig, H. U. Diastereoselective Syntheses of Highly Substituted Methyl Tetrahydrofuran-3-Carboxylates by Reaction of $\gamma$-Lactols with Silylated Nucleophiles. J. Org. Chem. 1988, 53, 2450-2456. (c) Babirad, S. A.; Wang, Y.; Kishi, Y. Synthesis of C-Disaccharides. J. Org. Chem. 1987, 52, 1370-1372. (d) Pornet, J.; Miginiac, L.; Jaworski, K.; Randrianoelina, B. Regiospecific Allenylation of Acetals with Propargyltrimethylsilanes Catalyzed by a Lewis Acid: Synthesis of $\alpha$-Allenyl Ethers and 3-Silylated 3,4-Dihydrofurans. Organometallics 1985, 4, 333-338. 